\title{
Southern Sea Otter (Enhydra lutris nereis) Population Biology at Big Sur and Monterey, California-Investigating the Consequences of Resource Abundance and Anthropogenic Stressors for Sea Otter Recovery
}

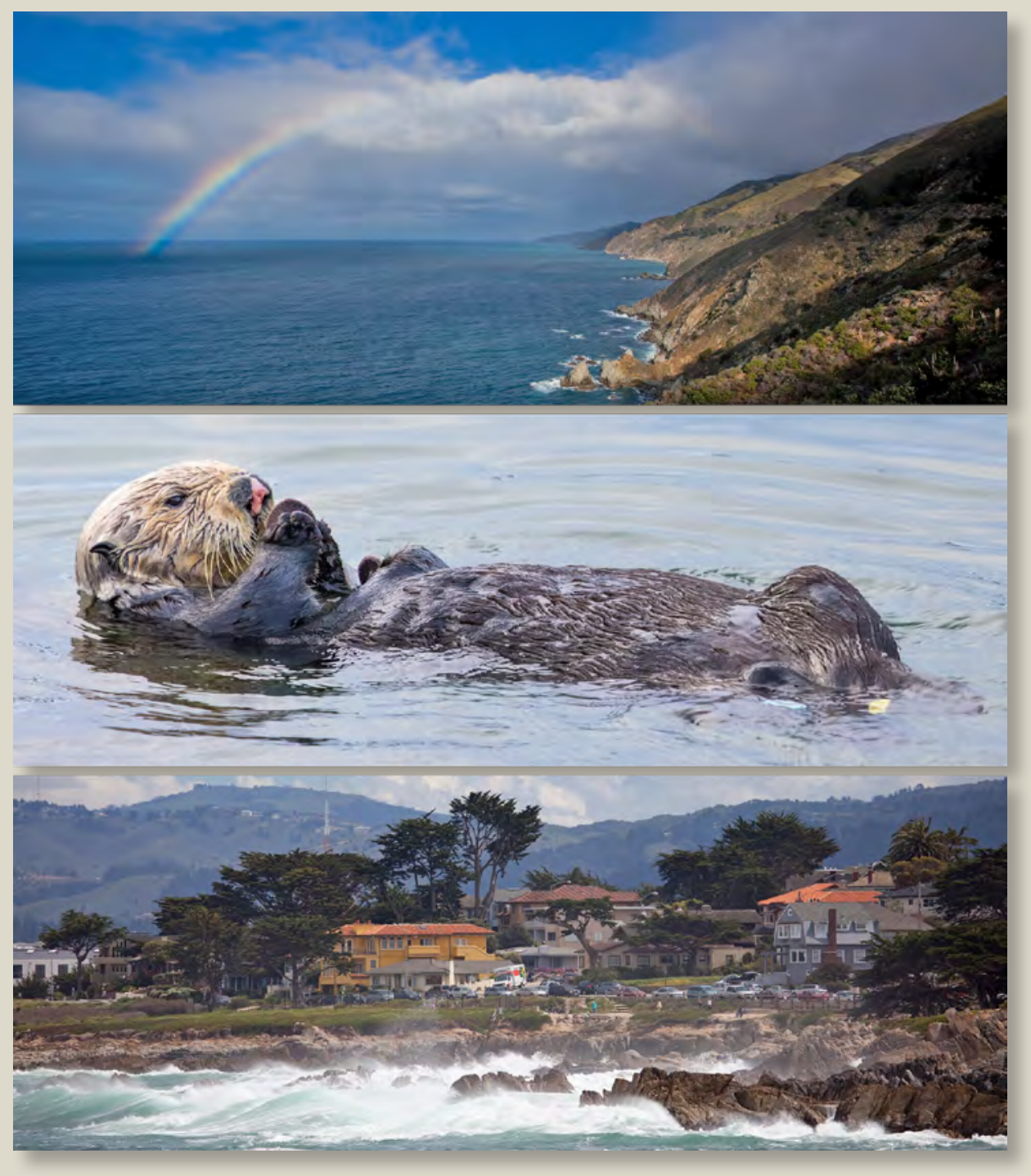

Open-File Report 2019-1022 
Cover: Photographs showing rugged and sparsely populated Big Sur Coast (top), tagged sea otter feeding on sand dollars in Monterey (middle), and the Monterey Peninsula with a high degree of coastal development and use (bottom).

Photographs by Joseph Tomoleoni, U.S. Geological Survey, March 8, 2010 (top), February 28, 2018 (middle), and March 31, 2010 (bottom). 


\section{Southern Sea Otter (Enhydra lutris nereis) Population Biology at Big Sur and Monterey, California-Investigating the Consequences of Resource Abundance and Anthropogenic Stressors for Sea Otter Recovery}

By M. Tim Tinker, Joseph A. Tomoleoni, Benjamin P. Weitzman, Michelle Staedler, Dave Jessup, Michael J. Murray, Melissa Miller, Tristan Burgess, Lizabeth Bowen, A. Keith Miles, Nicole Thometz, Lily Tarjan, Emily Golson, Francesca Batac, Erin Dodd, Eva Berberich, Jessica Kunz, Gena Bentall, Jessica Fujii, Teri Nicholson, Seth Newsome, Ann Melli, Nicole LaRoche, Holly MacCormick, Andy Johnson, Laird Henkel, Chris Kreuder-Johnson, and Pat Conrad

Open-File Report 2019-1022

U.S. Department of the Interior U.S. Geological Survey 


\title{
U.S. Department of the Interior DAVID BERNHARDT, Acting Secretary
}

\author{
U.S. Geological Survey \\ James F. Reilly II, Director
}

U.S. Geological Survey, Reston, Virginia: 2019

For more information on the USGS—-the Federal source for science about the Earth, its natural and living resources, natural hazards, and the environment-visit https://www.usgs.gov/ or call 1-888-ASK-USGS.

For an overview of USGS information products, including maps, imagery, and publications, visit https:/store.usgs.gov.

Any use of trade, firm, or product names is for descriptive purposes only and does not imply endorsement by the U.S. Government.

Although this information product, for the most part, is in the public domain, it also may contain copyrighted materials as noted in the text. Permission to reproduce copyrighted items must be secured from the copyright owner.

Suggested citation:

Tinker, M.T., Tomoleoni, J.A., Weitzman, B.P., Staedler, M., Jessup, D., Murray, M.J., Miller, M., Burgess, T., Bowen, L., Miles, A.K., Thometz, N., Tarjan, L., Golson, E., Batac, F., Dodd, E., Berberich, E., Kunz, J., Bentall, G., Fujii, J., Nicholson, T., Newsome, S., Melli, A., LaRoche, N., MacCormick, H., Johnson, A., Henkel, L., Kreuder-Johnson, C., and Conrad, P., 2019, Southern sea otter (Enhydra lutris nereis) population biology at Big Sur and Monterey, California-Investigating the consequences of resource abundance and anthropogenic stressors for sea otter recovery: U.S. Geological Survey Open-File Report 2019-1022, 225 p., https://doi.org/10.3133/ofr20191022.

ISSN 2331-1258 (online) 


\section{Contents}

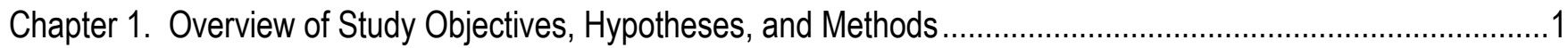

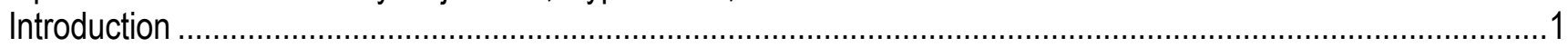

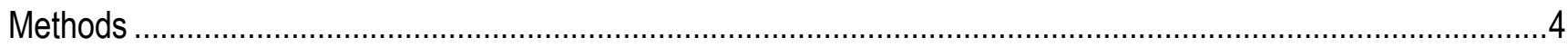

Live Sea Otter Captures ...........................................................................................................

Processing, Sampling, Health Assessments, and Tagging …………………………….....................

Live Sea Otter Surveillance ...............................................................................................................

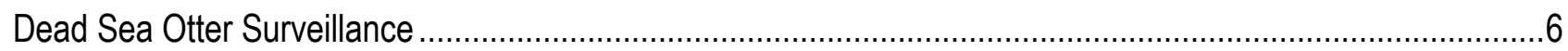

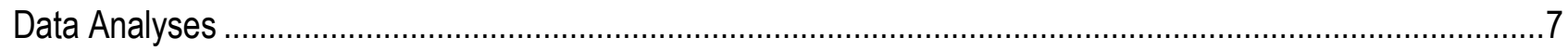

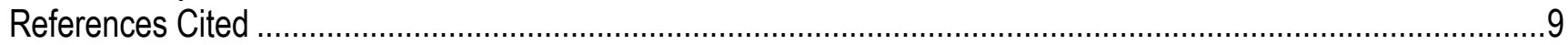

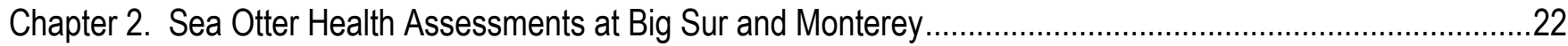

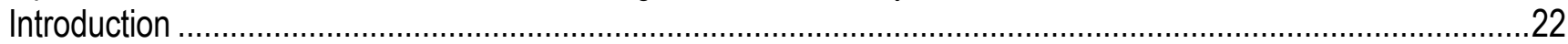

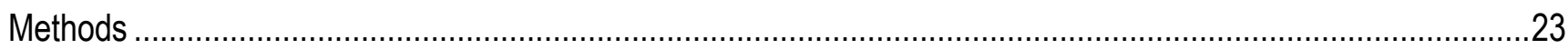

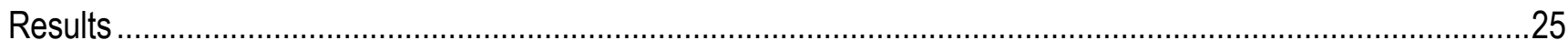

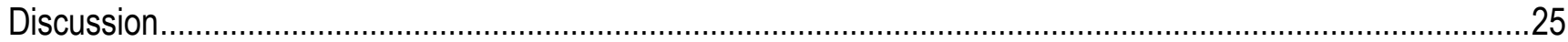

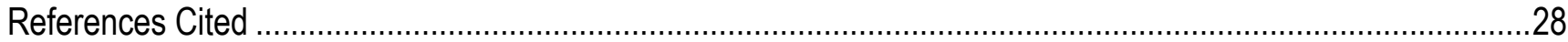

Chapter 3. Gene Transcription-Immune and Detoxification Function in California Sea Otters .............................47

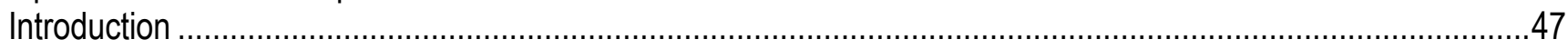

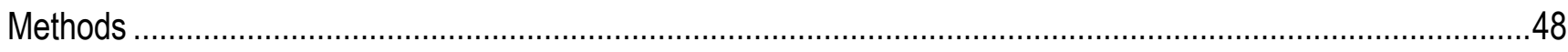

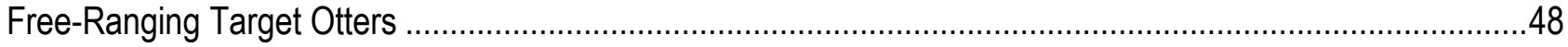

Captive Reference Otters .............................................................................................................

Blood Collection and Ribonucleic Acid Extraction .................................................................................48

Complementary Deoxyribonucleic Acid Creation......................................................................................49

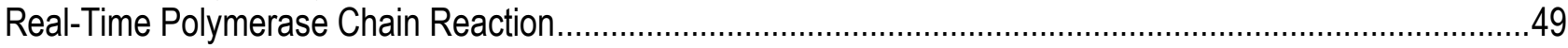

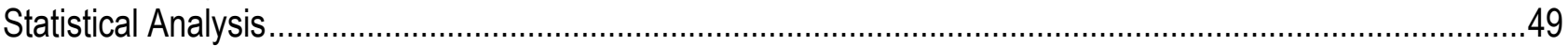

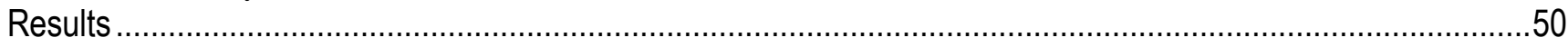

Transcription Pattern Analysis ...................................................................................................5

Within Site-Sex by Year Transcription Differences..............................................................................

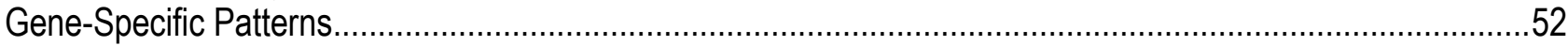

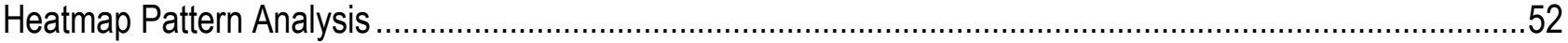

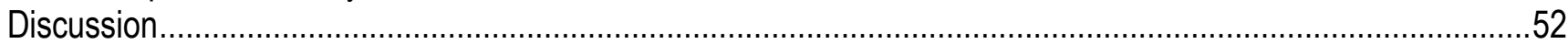

Transcription Pattern Analysis ....................................................................................................52

Within-Site Year by Sex Transcription Differences ................................................................................53

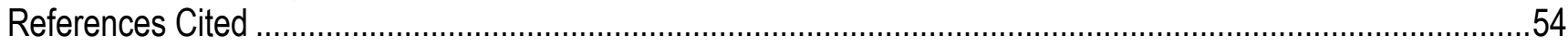

Chapter 4. Movement Behavior and Home Range Use of Sea Otters at Big Sur and Monterey ............................63

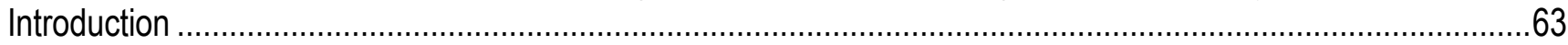

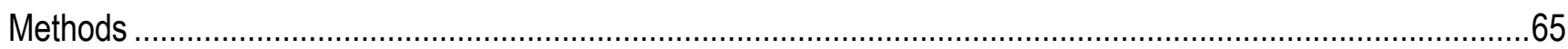

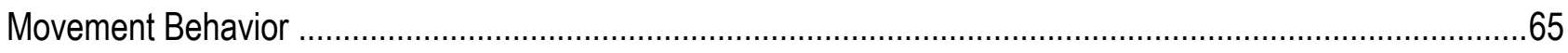

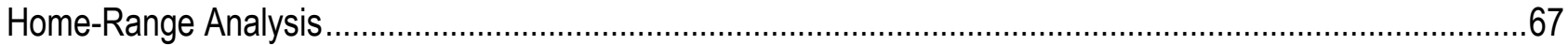

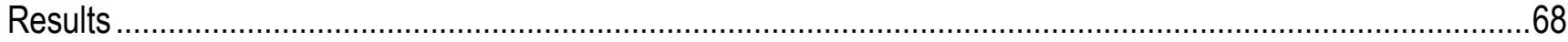

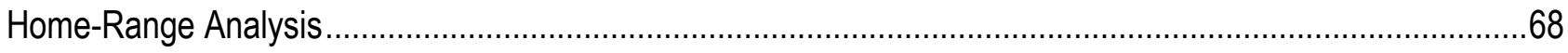

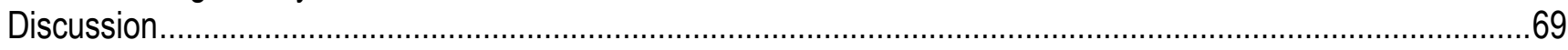

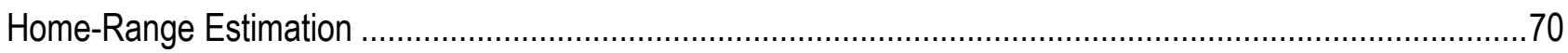

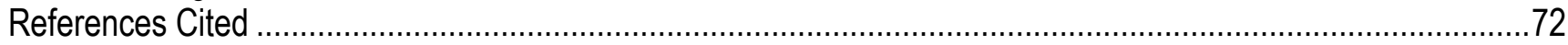

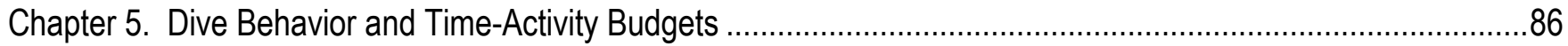

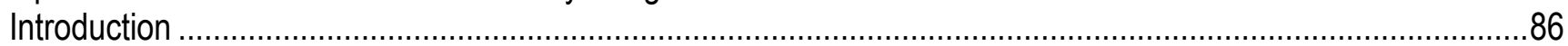




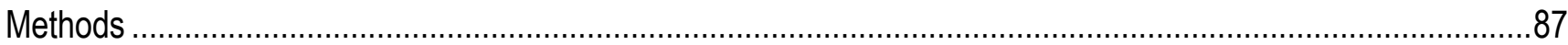

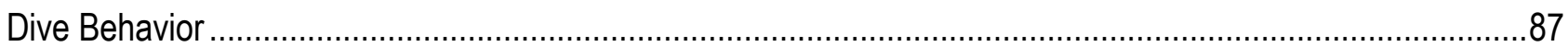

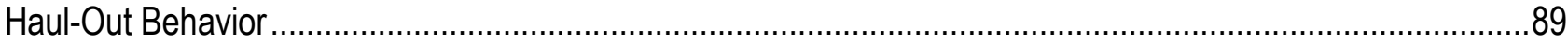

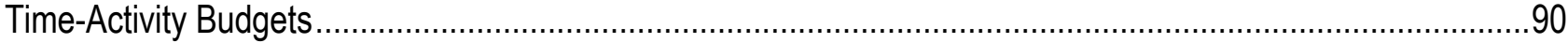

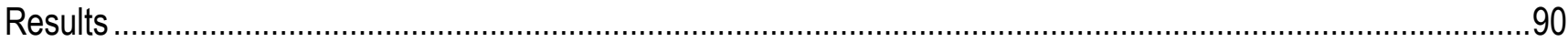

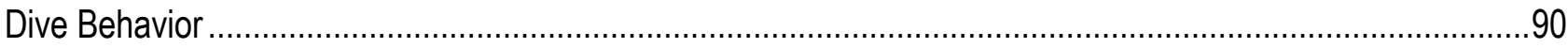

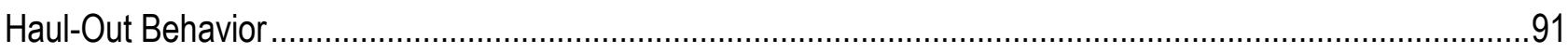

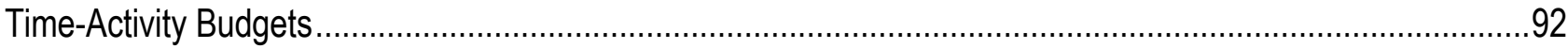

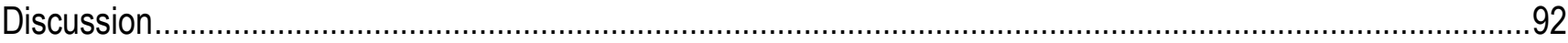

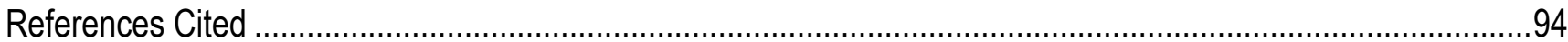

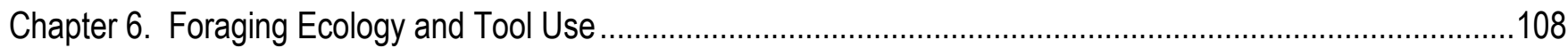

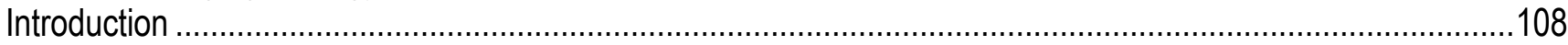

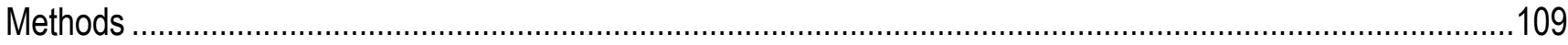

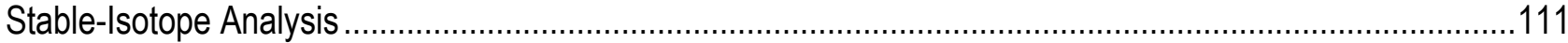

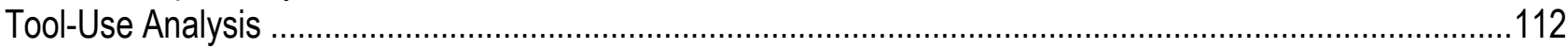

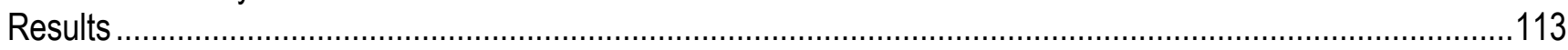

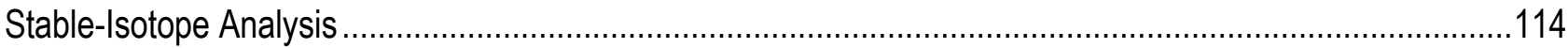

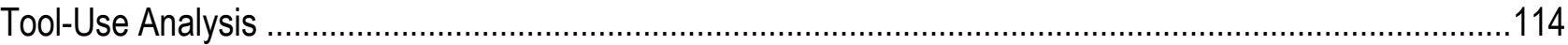

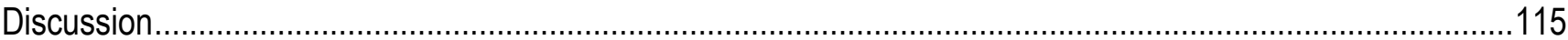

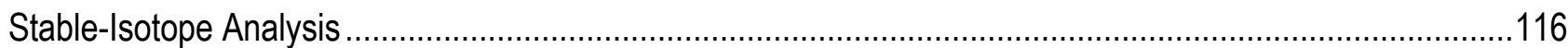

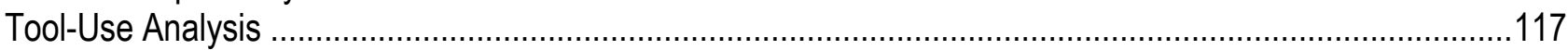

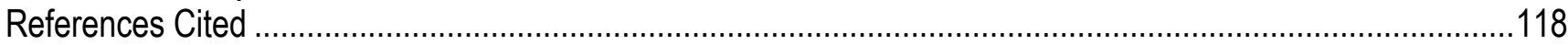

Chapter 7. Variation in Body Condition in California Sea Otters ...............................................................138

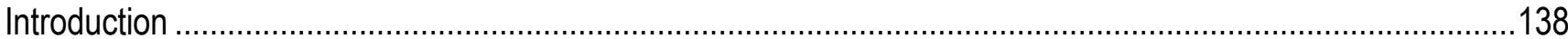

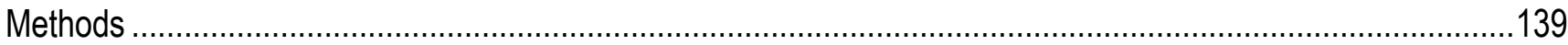

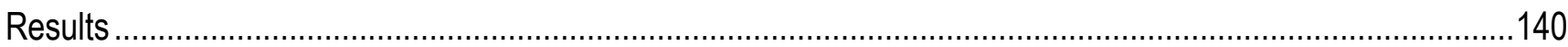

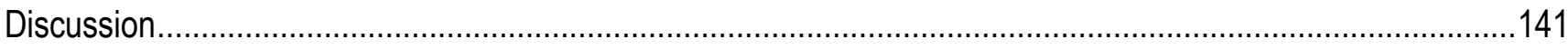

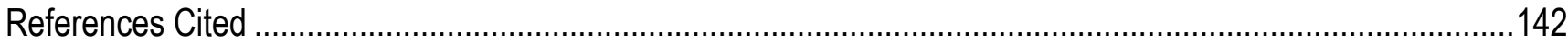

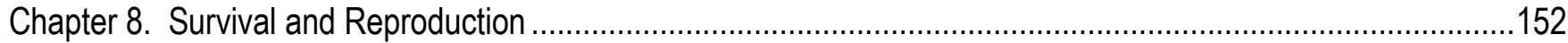

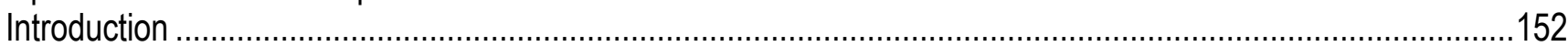

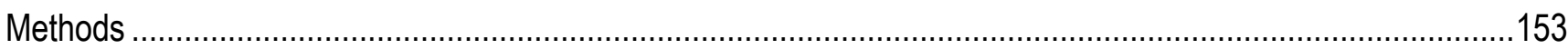

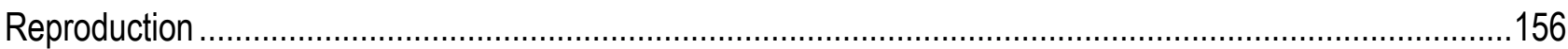

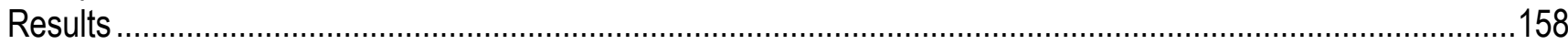

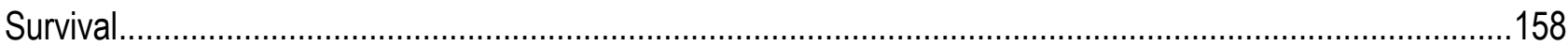

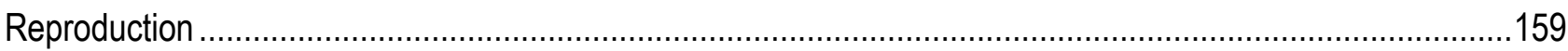

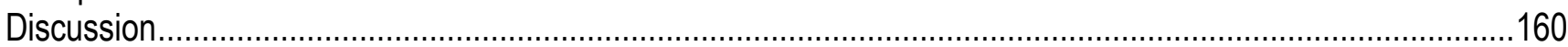

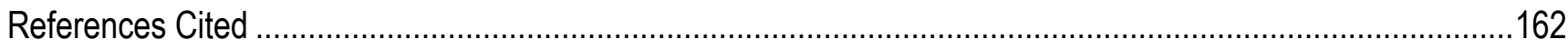

Chapter 9. Epidemiological Analysis of Protozoal Infections in Sea Otters at Big Sur and Monterey ...................179

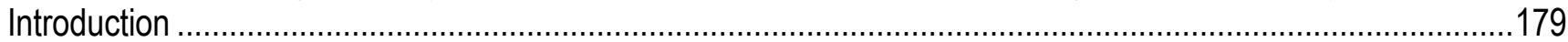

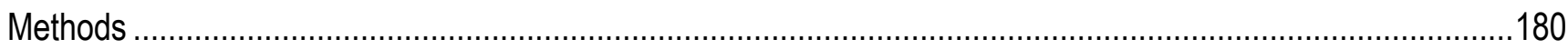

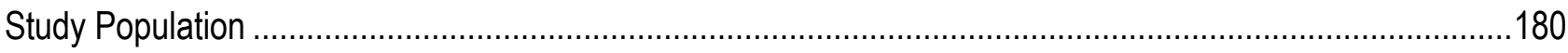

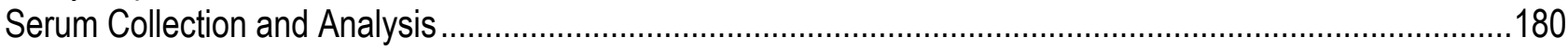

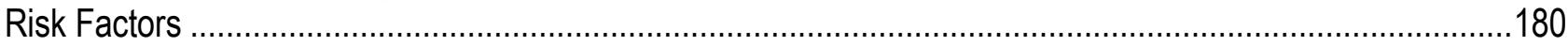

Univariate Analysis .............................................................................................................. 180

Logistic Regression Analysis .....................................................................................................181

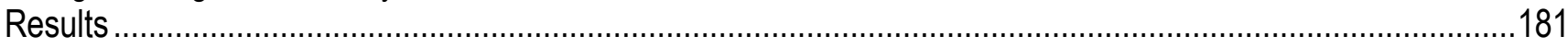

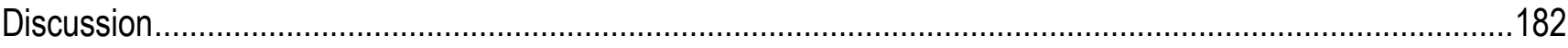


References Cited

Chapter 10. Preliminary Findings from Necropsy of Tagged Sea Otters from the Monterey-Big Sur Study ..........188

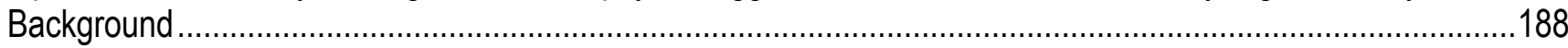

Summary of Lesions, Test Results, and Diagnoses ..............................................................................189

Congenital Malformations and Conditions with Undetermined Cause.............................................................189

Antemortem and Postmortem Bacterial Culture ……………………...................................................189

Antemortem and Postmortem Tests for Biotoxins ………………………………………………....190

Significant Findings from Gross Necropsy, Histopathology, and Diagnostic Testing ....................................191

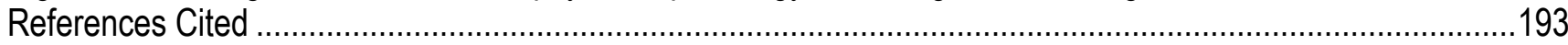

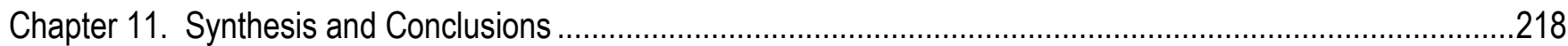

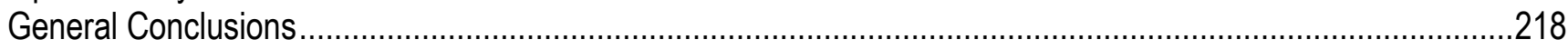

Density-Dependent Population Regulation ....................................................................................220

Variation in Environmental Stressors............................................................................................221

Synergistic Interactions between Resource Limitation and Environmental Stressors...................................221

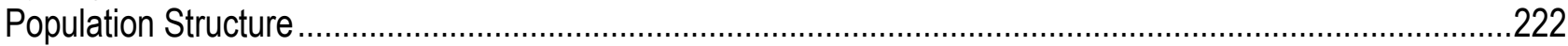

Resource Management Considerations ............................................................................................22

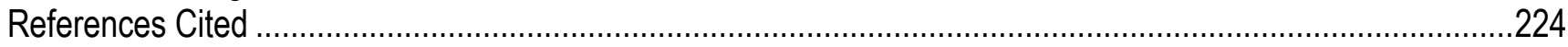

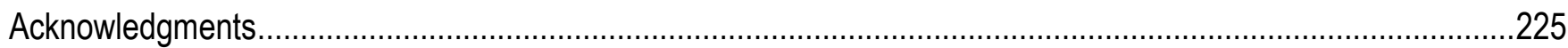

\section{Figures}

Figure 1. Schematic of the central California coast showing the current (2018) distribution of the southern sea otter and spatial variation in relative population density .................................................................16

Figure 2. Photographs showing segments of the coastline within each of the two study sites in central

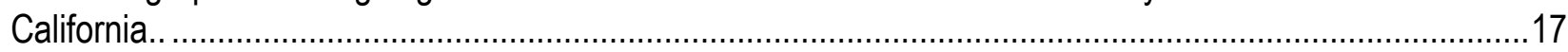

Figure 3. Photographs showing the scuba-based methods used to capture sea otters ......................................18

Figure 4. Photographs showing the health assessments and biosampling of anesthetized sea otters ..................19

Figure 5. Photographs showing the surgical procedure used to implant telemetry tracking devices in study animals

Figure 6. Photographs showing field monitoring of tagged study animals....................................................21

Figure 7. Graph showing multivariate, multi-dimensional scaling (MDS) analysis of gene transcription profiles of sea otters captured at four areas of the central California coast, 2008-13, in comparison to captive healthy sea otter

Figure 8. Gene expression profile showing transcription matrix of 13 target genes in clinically normal reference captive sea otters (CAP), and sea otters captured in Monterey Bay (MB), Big Sur (BS), San Luis Obispo (SLO), and Santa Barbara (SB), central California.

Figure 9. Graphs of (A) Weibull probability density functions used to model sea otter dispersal distance probabilities showing the effect of variation in the shape parameter $(b)$ on the degree of skew or leptokurtosis of the distribution, and (B) same as (A) but plotted on log-transformed y-axis to show how low values of $b$ are associated with higher probabilities of very short or very long dispersal distances

Figure 10. Schematics showing home-range estimate (red shaded areas on Monterey Bay) for sea otter 1317 (A) using kernel density estimation (smoothing parameter $\mathrm{h}=475 \mathrm{~m}, 90$-percent kernel) in geographic coordinate space (Monterey Peninsula, California), and (B) using local convex hull

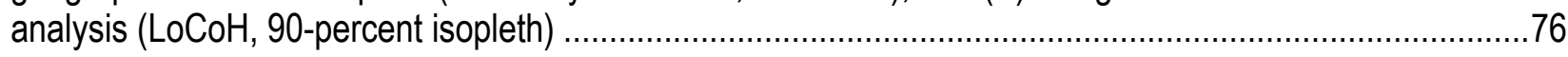

Figure 11. Geographic information system images showing sea otter resights and the results of the "permissible home range estimation" for two sea otters at the Monterey study site, central California 
Figure 12. Frequency histogram showing log-transformed values of "home-range coastal extent" (the number of kilometers of coastline contained within home-range polygons) for male otters monitored in this study ...78

Figure 13. Frequency histograms showing annual net linear displacement values, and associated Weibull probability density functions (fit using maximum likelihood estimates), for sea otters in this study, at Monterey and Big Sur, central California.

Figure 14. Boxplots showing maximum likelihood estimates for the two parameters of the Weibull probability functions fit to sea otter net linear displacement data (see fig. 13).

Figure 15. Graph showing results of a variance components analysis of sea otters showing the proportion of variation in annual net linear displacement values explained by four effects-between-site differences, age/sex class differences, among-individual differences, and within-individual variation.

Figure 16. Graphs showing difference between observed net linear displacement (NLDo) and expected net linear displacement $(\mathrm{NLD}$, as calculated from correlated random walk models using methods described in the body text) for male and female sea otters plotted as a function of time intervals over which NLD was estimated and measured, ranging from 4 weeks to 1 year

Figure 17. Graphs showing (A) proportion of sea otters with different numbers of centers of use, compared between study sites and sexes (MON, Monterey Peninsula; BSR, Big Sur coast), and (B) comparison of number of centers of use between females, M1 males, and M2 males, central California

Figure 18. Boxplots comparing sea otter females, M1 males (residents), and M2 males (transients) in terms of four home-range statistics-(A) home-range area, (B) distribution between centers of use, (C) coastline extent, and (D) range span, in central California. In all cases, distributions indicate logtransformed values

Figure 19. Graph showing coastline extent (in kilometers) plotted as a function of home-range area (in square kilometers) for otters in Monterey Bay and Big Sur, central California

Figure 20. Time-depth recorder trace showing dive behavior over a 24-hour period for a typical sea otter ..........102

Figure 21. Graph showing mean and maximum dive depths for southern sea otters in six classesNP, female with no pup; VP, female with very small pup; SP, female with small pup; LP. female with large pup; MA, male; SA, subadult

Figure 22. Graphs showing mean and maximum dive durations for southern sea otters in six classes$\mathrm{NP}$, female with no pup; VP, female with very small pup; SP, female with small pup; LP. female with large pup; MA, male; SA, subadult.

Figure 23. Box plots showing distributions of four southern sea otter dive attributes that varied as a function of individual diet specialization (diet groups 1-5) - (A) mean dive depth, (B) mean dive duration, (C) mean dive bottom time, and (D) mean ascent rate

Figure 24. Graphic representations of variation in the percentage of time female southern sea otters spent hauled out from Monterey Peninsula study site, central California

Figure 25. Graphs showing time-activity budgets, as estimated from time-depth recorder data, for female southern sea otters, central California

Figure 26. Histogram showing contribution of 20 prey types to the diets of sea otters at Big Sur coast and Monterey Peninsula study sites, central California

Figure 27. Graphs showing comparisons of the estimated rate of energy gain of sea otters while feeding (in kilocalories per minute [kcal/min]), corresponding to results of two-way analysis of variance with interaction effect (see body text for details).

Figure 28. Graph showing comparison of the mean rate of energy gain for sea otters in 16 sea otter populations from across their range in the North Pacific, based on field studies conducted using standardized methodologies

Figure 29. Graphical representations of results of a hierarchical cluster analysis of sea otter diet composition ...130 
Figure 30. Histogram showing contribution of 11 key prey types to the diets of sea otters belonging to each of 5 diet-type groups (as identified by cluster analysis) at Big Sur coast and Monterey Peninsula study sites, central California

Figure 31. Biplots showing comparison of $\delta^{13} \mathrm{C}$ to $\delta^{15 \mathrm{~N}}$ for California sea otter populations and their potential prey......

Figure 32. Graphs showing comparison of isotopic niche variation statistics at Big Sur coast (BSR) and Monterey Peninsula (MON) study sites, central California

Figure 33. Density plots showing population-level dietary niche specialization $(\varepsilon l)$ compared to individualpopulation dietary similarity $\left(S_{I}\right)$, based on stable isotope analysis, for sea otters at Monterey Peninsula (MON; top plot) and Big Sur coast (BSR; bottom plot) study sites, central California.

Figure 34. Graphs showing comparison of tool use frequency variation among individuals from Big Sur coast (top graph) and Monterey Peninsula (bottom graph) study sites, central California

Figure 35. Histograms showing individual tool use frequency by diet specialization group (see body text, table 19, and fig. 30). Proportion of the population (y-axis) refers to the percentage of the overall population (including non-tool users) at both study sites.

Figure 36. Graph showing average frequency of tool use on different types of prey by sea otters belonging to four different diet specialization groups-checkered, group 3 (mussels/urchins); solid gray, group 5 (clams/crabs/worms); lined, group 1 (Cancer crabs/urchins/miscellaneous); dotted, group 4 (snail specialists).

Figure 37. Graphs showing linear regression (A) and residuals from the linear regression (B) of sea otter body length minus tail length compared to body length including tail, for sea otters at three different California study sites

Figure 38. Scatterplots showing body length (in centimeters [cm]) compared to estimated age for sea otters at six different study sites in California

Figure 39. Scatterplots showing body mass (in kilograms $[\mathrm{kg}]$ ) compared to estimated age for sea otters at six different study sites in California

Figure 40. Boxplots showing residuals from length compared to age growth curves (see figure 38) plotted for sea otters at six different study sites in California

Figure 41. Boxplots showing residuals from mass compared to age growth curves (see figure 39) plotted for sea otters at six different study sites in California. Values represent an index of overall body condition (BCl).

Figure 42. Boxplots showing residuals from a regression of $\mathrm{BCl}$ compared to $\mathrm{SSI}$ values (that is, deviations from the expected mass-at-age compared to Length-at-age relationship) for sea otters at six different study sites in California

Figure 43. Regression plot showing average logit-transformed body condition index ( $\left(\mathrm{Cll}^{\prime}\right)$ compared to average estimated rate of energy gain (in kilocalories per minute [kcal/min]) for male and female sea otters at five sites in California

Figure 44. Graph showing average age-specific annual survival rates for female (top graph) and male (bottom graph) sea otters across all study sites, with mean estimated values indicated by the solid line and 95 -percent credible intervals $\left(\mathrm{Cl}_{95}\right)$ indicated by the shaded areas

Figure 45. Graphs showing Bayesian posterior distributions for model estimates of $\beta_{1}$, the effect of sea otter body condition (top graph), and $\beta_{2}$, the effect of reproductive status (bottom graph)

Figure 46. Graphs showing relationship between southern sea otter body condition (BCl', relative mass at age) and survival rates for females (top graph) and males (bottom graph), as estimated from data collected at six study sites throughout California.

Figure 47. Graph showing Bayesian posterior distributions for model estimates of random differences in sea otter survival at six study sites throughout California 
Figure 48. Graphs showing Bayesian posterior distributions for model estimates of adult (5-year old) female sea otter survival at four study sites in California (top graph), and posterior distribution for estimated difference in survival rates between adult females at Big Sur and Monterey (bottom graph; a negative value indicates lower survival at Big Sur relative to Monterey)

Figure 49. Graphs showing Bayesian posterior distributions for model estimates of adult (5-year old) male sea otter survival at four study sites in California (top graph), and posterior distribution for estimated difference in survival rates between adult males at Big Sur and Monterey (bottom graph; a negative value indicates lower survival at Big Sur relative to Monterey)

Figure 50. Graph showing log hazard rates for sea otter pups as a function of pup age (measured in weeks since birth). Lower log hazard rates indicate higher survival probability.....

Figure 51. Graph showing log hazard ratio for sea otter pups as a function of age of mother age, with a value of 0 indicating a hazard ratio of 1 relative to a 3-year-old female (baseline value).

Figure 52. Graph showing Bayesian posterior distribution for model estimates of $\beta_{3}$, the effect of the body condition of the sea otter mother on pup survival.

Figure 53. Graphs showing weaning success rates (sea otter pup survival) plotted as a function of age of mother (top graph) and body condition of mothers (body condition index, relative mass at age).....

Figure 54. Graphs showing Bayesian posterior distributions for model estimates of weaning success for an adult (5-year old) female sea otter at the Big Sur and Monterey study sites (top graph), and posterior distribution for estimated difference in weaning success rates between Big Sur and Monterey study sites (bottom graph; a negative value indicates lower pup survival at Big Sur relative to Monterey).

Figure 55. Photograph showing normal sea otter ovary (left), and missing ovary, (right). .206

Figure 56. Photograph showing intestinal content of sea otter providing insight on this animal's "last meal"--fragments of sea urchin exoskeleton and spines

Figure 57. Photograph showing Tegula operculum from a sea otter that had been eating snails 207

Figure 58. Photograph showing intestine of a sea otter with a colon impaction .................................................207

Figure 59. Photograph showing sea otter with a mild nose wound …............................................................208

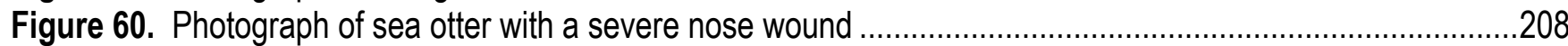

Figure 61. Photograph showing sea otter bacterial infections after mating-related trauma, which otters sometimes develop 209

Figure 62. Photograph showing old sea otter with severely worn and missing teeth 209

Figure 63. Photographs showing sea otter with a chest full of pressurized air or gas (pneumothorax), characterized by an abnormal, "barrel-shaped" chest (top photograph), and a diaphragm that is pushed back toward the abdomen (bottom photograph)

Figure 64. Photographs of same sea otter as in figure 63 , showing a severe bacterial infection of the chest cavity (top photograph) and a knee joint (bottom photograph).

Figure 65. Photographs showing normal sea otter heart (left photograph), compared with the heart of an otter with cardiomyopathy (right photograph, upper right center)

Figure 66. Photograph showing severely emaciated sea otter, typical of adult females dying with end-lactation syndrome

Figure 67. Photographs showing sea otter with a deep laceration and femur fracture due to boat propeller strike, as indicated by radiograph (top photograph) and gross photo (bottom photograph).

Figure 68. Photographs showing lesions suggestive of severe blood loss in sea otter due to trauma or other causes-Pale gums (top right photograph), small heart with collapsed atria and ventricles (top right photograph) and small, pale liver (bottom photograph)

Figure 69. Photographs showing normal lung tissue (top photograph), compared with an otter with severe pulmonary emphysema (bottom photograph). 
Figure 70. Photographs showing pulmonary edema, characterized by expansion of the spaces between pulmonary lobules by clear or blue-tinged fluid (top photograph), and build-up of pink or white froth in the trachea and bronchi (bottom photograph)

\section{Tables}

Table 1. Summary information for sea otters captured, sampled, tagged, and monitored as part of this study at the Big Sur and Monterey study sites, central California..

Table 2. Biological samples collected from sea otters captured at the Big Sur and Monterey study sites, central California

Table 3. Scoring matrix for sea otter subjective health assessment.

Table 4. Health assessment scores for 144 sea otters screened with respect to 10 parameters and captured at Monterey and Big Sur study sites, central California.

Table 5. Clinical pathology results for 143 sea otters captured at Monterey and Big Sur study sites, central California

Table 6. Documented function of 13 genes identified in sea otters

Table 7. Geometric mean, cycle threshold $\left(\mathrm{C}_{\mathrm{T}}\right)$ of 13 genes identified in free-ranging sea otters sampled in Monterey, Big Sur, San Luis Obispo, and Santa Barbara, and in clinically normal captive reference animals sampled, 2008-13

Table 8. Results of an analysis of annual net linear displacement for sea otters, grouped by sex and age-class, at Big Sur and Monterey, central California.

Table 9. Home-range characteristics across sexes and Big Sur coast and Monterey Peninsula study sites, central California

Table 10. Summary information on time-depth recorder deployments for study animals in the current study.........97

Table 11. Dive attributes of southern sea otters from Big Sur coast and Monterey Peninsula study sites, central California

Table 12. Dive attributes of southern sea otters in six classes, with data pooled from individuals for analysis from Big Sur coast and Monterey Peninsula study sites, central California

Table 13. Dive attributes of southern sea otters in six classes determined for Big Sur coast and Monterey Peninsula study sites, central California

Table 14. Comparison of mean percentages of time spent in three activity states (foraging, active-other, and inactive) for males, females, and sub-adult females at Big Sur coast and Monterey Peninsula study sites, central California.

Table 15. Summary of mean percentages of time spent in three activity states (foraging, active-other, and inactive) for female sea otters, grouped by Big Sur coast and Monterey Peninsula study sites, age class, and reproductive state, in central California

Table 16. Percentage of time and standard deviation for foraging, active-other, and inactive (resting) behaviors for all female sea otters in the study, grouped by reproductive status

Table 17. Stable Isotope characterization of sea otter prey types used in the analysis of isotopic niche space

Table 18. List of more than 75 prey species (or higher taxa) consumed by sea otters over the course of the study

Table 19. Summary of the five sea otter diet groups identified using hierarchical cluster analysis

Table 20. Results of discriminant analysis of individual sea otter diets used to evaluate the efficacy of diet-type groupings identified using hierarchical cluster analysis

Table 21. Generalized linear mixed effects models (GLMM) showing the relation between the probabilities of occurrence of tool use and various factors, including site (population ), prey type, sex, age, habitat, and diet specialization 
Table 22. Wald Test results for best-supported generalized linear mixed effects model explaining tool use probability as a function of diet specialization of the sea otter and prey type on a given dive.....

Table 23. Analysis of variance results for among-site contrasts of sea otter structural size index at six California study sites

Table 24. Analysis of variance results for among-site contrasts of sea otter body condition index at six California study sites

Table 25. Analysis of variance results for among-site contrasts of sea otter dynamic condition index, at six California study sites

Table 26. Parameter estimates for $\log$ (hazard) function from Bayesian survival analysis

Table 27. Adult (5-year-old) annual survival rate estimates for females (top) and males (bottom) at 6 study sites

Table 28. Mean annual age-specific sea otter survival rate estimates for females (top part of table) and males (bottom part of table) at six California study sites

Table 29. Weaning success rates for female sea otters at Big Sur and Monterey study sites

Table 30. Univariate analysis of putative categorical risk factors for Toxoplasma gondii antibody status in 159 sea otters sampled 2007-10 at Monterey and Big Sur, central California .

Table 31. Associations of continuous variables with Toxoplasma gondii positive antibody status in 159 sea otters sampled 2007-10 at Monterey and Big Sur, central California

Table 32. Ordinary logistic regression models predicting Toxoplasma gondii positive antibody status on the basis of body length, site, sex, diet type, and percentage of snails in the diet by biomass.

Table 33. Multivariable logistic regression model of risk factors for Toxoplasma gondii serum positive antibody status

Table 34. Stranding location, stranding date, and overall demographical information for tagged, necropsied sea otters from Monterey Peninsula and Big Sur coast study sites, central California.

Table 35. Aerobic and anaerobic bacterial culture findings for tagged, necropsied sea otters from Monterey Peninsula and Big Sur coast study sites, central California.

Table 36. Overview of implant history, and primary and contributing causes of death for tagged, necropsied sea otters from Monterey Peninsula and Big Sur coast study sites, central California

Table 37. Findings from antemortem and postmortem tests for domoic acid and cyanotoxins for tagged, necropsied sea otters from Monterey Peninsula and Big Sur coast study sites, central California.

Table 38. Findings from gross necropsy, histopathology, and diagnostic testing (including subjective weighting of relative lesion severity) for tagged, necropsied sea otters from Monterey Peninsula and Big Sur coast study sites, central California 


\section{Conversion Factors}

U.S. customary units to International System of Units

\begin{tabular}{lcl}
\hline \multicolumn{1}{c}{ Multiply } & By & \multicolumn{1}{c}{ To obtain } \\
\hline & Length & \\
\hline inch (in.) & 2.54 & centimeter $(\mathrm{cm})$ \\
inch (in.) & 25.4 & millimeter $(\mathrm{mm})$ \\
\hline & Flow rate & \\
\hline cubic foot per second $\left(\mathrm{ft}^{3} / \mathrm{s}\right)$ & 0.02832 & cubic meter per second $\left(\mathrm{m}^{3} / \mathrm{s}\right)$ \\
\hline
\end{tabular}

International System of Units to U.S. customary units

\begin{tabular}{|c|c|c|}
\hline Multiply & By & To obtain \\
\hline \multicolumn{3}{|c|}{ Length } \\
\hline millimeter $(\mathrm{mm})$ & 0.03937 & inch (in.) \\
\hline centimeter $(\mathrm{cm})$ & 0.3937 & inch (in.) \\
\hline meter $(\mathrm{m})$ & 3.281 & foot $(\mathrm{ft})$ \\
\hline kilometer $(\mathrm{km})$ & 0.6214 & mile (mi) \\
\hline \multicolumn{3}{|c|}{ Area } \\
\hline square kilometer $\left(\mathrm{km}^{2}\right)$ & 247.1 & acre \\
\hline square kilometer $\left(\mathrm{km}^{2}\right)$ & 0.3861 & square mile $\left(\mathrm{mi}^{2}\right)$ \\
\hline square hectometer $\left(\mathrm{hm}^{2}\right)$ & 0.003861 & section ( 640 acres or 1 square mile) \\
\hline hectare (ha) & 0.003861 & square mile $\left(\mathrm{mi}^{2}\right)$ \\
\hline \multicolumn{3}{|c|}{ Volume } \\
\hline microliter $(\mu \mathrm{L})$ & 0.000033814 & ounce, fluid (fl. oz) \\
\hline milliliter (mL) & 0.003814 & ounce, fluid (fl. oz) \\
\hline \multicolumn{3}{|c|}{ Flow rate } \\
\hline cubic foot per second $\left(\mathrm{ft}^{3} / \mathrm{s}\right)$ & 0.02832 & cubic meter per second $\left(\mathrm{m}^{3} / \mathrm{s}\right)$ \\
\hline meter per second $(\mathrm{m} / \mathrm{s})$ & 3.281 & foot per second (ft/s) \\
\hline \multicolumn{3}{|c|}{ Mass } \\
\hline microgram $(\mu \mathrm{g})$ & 0.0000003527 & ounce, avoirdupois (oz) \\
\hline milligram (mg)) & 0.00003527 & ounce, avoirdupois (oz) \\
\hline $\operatorname{gram}(\mathrm{g})$ & 0.03527 & ounce, avoirdupois (oz) \\
\hline kilogram $(\mathrm{kg})$ & 2.205 & pound avoirdupois (lb) \\
\hline
\end{tabular}

Temperature in degrees Celsius $\left({ }^{\circ} \mathrm{C}\right)$ may be converted to degrees Fahrenheit $\left({ }^{\circ} \mathrm{F}\right)$ as follows:

$$
{ }^{\circ} \mathrm{F}=\left(1.8 \times{ }^{\circ} \mathrm{C}\right)+32 \text {. }
$$

\section{Supplemental Information}

Note to USGS users: Use of hectare (ha) as an alternative name for square hectometer ( $\left.\mathrm{hm}^{2}\right)$ is restricted to the measurement of small land or water areas. 


\section{Abbreviations}

AIC Akaike information criterion

AICc Akaike information criterion, corrected

AN anatoxin-a

ANOVA analysis of variance

ATOS "As The Otter Swims"

$\mathrm{BCl} \quad$ body condition index

BCl' rescaled body condition index

BT mean bottom time

BT/DT mean ratio of bottom time to dive time

CAM Cambria/San Simeon

CAR conditional auto-regressive

CDFW California Department of Fish and Wildlife

$\mathrm{Cl}$

COU

CRW

CT

$\mathrm{DA}$

$\mathrm{DCl}$

DNA

DIC

DNase

dNTP

DT

ELISA

ELS

GIS

GLM

GLMM

GPS

$\mathrm{HAB}$

$\mathrm{Hgb}$

KDE

LC/MS

$\mathrm{LOCOH}$

LP

MA

MB1

$M C$

MCMC

MDL

MDS

MON

mRNA

confidence interval

center of use

correlated random walk

cycle threshold

discriminant analysis; domoic acid (in chapter 10 only)

dynamic condition index

deoxyribonucleic acid

deviance information criterion

deoxyribonuclease

deoxyribonucleotide triphosphate

mean dive duration

Enzyme-Linked Immunosorbent Assay

end-lactation syndrome

geographic information system

general linear model

generalized linear mixed effects model

Global Positioning System

harmful algal bloom

hemoglobin

kernel density estimation

liquid chromatography/mass spectrometry

local convex hull analysis

female with large pup (greater than 10 weeks old)

males

Monterey Bay Area

microcystin

Markov Chain Monte Carlo

minimum detection limit

multi-dimensional scaling

Monterey Peninsula

MWVCRC Marine Wildlife Veterinary Care and Research Center

NLD

$\mathrm{NLD} \mathrm{D}_{\mathrm{C}}$

NLDo

NO

net linear displacement

expected net linear displacement

observed net linear displacement

nodularin

NP female with no pup

$\mathrm{OA} \quad$ okadaic acid

PCR polymerase chain reaction

PCV packed cell volume

PDI mean post-dive interval

PHRE permissible home-range estimation

PS index of proportional similarity 


$\begin{array}{ll}\text { PTC } & \text { Point Conception } \\ \text { PVA } & \text { Population Viability Analyses } \\ \text { RMSSTD } & \text { root mean square standard deviation } \\ \text { R2 } & \text { coefficient of determination } \\ \text { RNA } & \text { ribonucleic acid } \\ \text { RNases } & \text { ribonucleases } \\ \text { SA } & \text { sub-adult females } \\ \text { SE } & \text { standard error } \\ \text { SNI } & \text { San Nicholas Island } \\ \text { SP } & \text { female with small pup (greater than } 3 \text { and less than or equal to 10 weeks old) } \\ \text { SSI } & \text { structural size index } \\ \text { STX } & \text { saxitoxin } \\ \text { TDFs } & \text { trophic discrimination factors } \\ \text { TDR } & \text { time-depth recorder } \\ \text { TNW } & \text { total niche width } \\ \text { USGS } & \text { U.S. Geological Survey } \\ \text { Variance PDI } & \text { variance in post-dive interval } \\ \text { VHF } & \text { very high frequency } \\ \text { VP } & \text { female with very small pup (less than or equal to } 3 \text { weeks old) } \\ \text { WBC } & \text { total white blood cell count } \\ \text { WIC } & \text { "within-individual component" of variation in dietary niche }\end{array}$


This page intentionally left blank. 


\title{
Chapter 1. Overview of Study Objectives, Hypotheses, and Methods
}

\author{
M. Tim Tinker ${ }^{1,2}$, Joseph A. Tomoleoni ${ }^{1}$, Dave Jessup ${ }^{3}$, and Andy Johnson ${ }^{4}$
}

\section{Introduction}

Southern sea otters (Enhydra lutris nereis) in California forage as an apex marine predator in nearshore habitat that occurs along a densely human-populated coastline. Sea otters are unusual among marine mammals in that they have an extremely high surface area-to-volume ratio, live outside of their thermal neutral zone, lack an insulating blubber layer used by most marine mammals, and, consequently, have one of the highest mass-specific metabolic demands of all marine mammals (Morrison and others, 1974; Costa and Kooyman 1982; Yeates and others, 2007). This trait, in conjunction with their distribution and the preponderance of filter-feeding benthic invertebrates in their diet, makes southern sea otters especially susceptible to naturally occurring and human-induced stressors in their environment, and as such, they are effective sentinels of the health of the California coastal ocean (Jessup and others, 2007). Their utility as a sentinel (or indicator) of coastal ecosystem health is further increased by their nearshore distribution, their extraordinary appeal to the general public (a fact that generates community support for monitoring efforts), and their tractability for observational study. In effect, sea otters can "tell us" how they encounter environmental stressors or acquire particular disease pathogens through detailed measurements of where they live and what they eat (Johnson and others, 2009). Sea otters also play a vital ecological role in coastal ecosystems as a keystone predator, and their relative abundance can have profound effects on the productivity and biodiversity of kelp forest communities (Kenyon, 1969; Estes and others, 1978, 2004). Southern sea otters are the only subspecies of sea otter found in California, and thus, any reference to "sea otters" in this report refers to the southern sea otter, unless otherwise stated.

\footnotetext{
${ }^{1}$ U.S. Geological Survey.

${ }^{2}$ University of California, Santa Cruz.

${ }^{3}$ California Department of Fish and Wildlife.

${ }^{4}$ Monterey Bay Aquarium.
} 
Research over the past 15 years has provided much information on a wide range of specific causes of mortality in southern sea otters, including starvation, predation, intra-specific aggression, domoic acid intoxication, toxic-exposure, and a variety of infectious diseases (Kreuder and others, 2003); however, important questions remain about the ultimate factors influencing these patterns, and about the nature of interactions among these causal factors. Studies of the diet, time-activity budgets, movements and behavioral patterns, demography (survival and reproduction), health metrics, and causes of death of tagged, free-ranging otters provide the core components of a research program aimed at clarifying the underlying drivers of population change. Comparisons of these metrics across space and time, and among populations that differ with respect to one or more factors of interest (for example low compared to high density, or low compared to high exposure to pollutants), allow for a "quasiexperimental" approach to testing hypotheses about the relative impacts of various factors on population recovery. The results of one such study, comparing sites with varying sea otter densities and per-capita prey abundance, highlighted the importance of prey resource availability in affecting sea otter behavior and health, and pointed towards density-dependent resource limitation as a key driver of population change in certain areas (Tinker and others, 2008, 2012). At the same time, comparative studies of sea otter exposure to land-derived pathogens showed that there was considerable spatial variation in rates of infection, and that these patterns potentially were related to point sources of pathogen pollution (Miller and others, 2002, 2007; Johnson and others, 2009; Miller, Byrne, and others, 2010). More recently, it was found that the biotoxins produced by harmful algal blooms (HABs) can have significant acute and chronic effects on sea otter health (Kreuder and others, 2005; Miller, Kudela, and others, 2010), and there is a possibility that land-based nutrient enrichment is contributing to increased frequency and severity of these HAB events.

Given these findings, the identification of the relative importance of (in terms of driving population change) and relations among food resource abundance, environmental conditions, and human-contributed increases in pathogens and pollutants (including nutrient-driven HAB events) is critical to reach a complete understanding of underlying drivers of population change. For example, although previous research suggests that food resource limitation and terrestrial input of pathogen pollution are contributing to elevated mortality, our ability to inform potential management actions has been hampered by uncertainty about the relative importance of these factors, their interaction, and the specific pathways of exposure to disease-causing pathogens.

The current study was designed to investigate causal links between the sluggish population performance of sea otters in central California and factors that could be driving variation in survival and reproduction, including food resource limitation and various types of terrestrial-based pollution (including pathogens). Our overall goal was to identify and inform the prioritization of conservation actions that can positively affect recovery of the southern sea otter and improve ecosystem health. To achieve this goal, we identified five specific objectives:

1. Describe the health status and basic ecology of sea otters at two study sites in central California, (a) an area of relatively dense human population that is heavily impacted by anthropogenic influences and urban and agricultural effluents ("high-impact" site), and (b) a more pristine area with minimal human impact ("low-impact" site).

2. Document patterns of mortality in the sea otter population, including spatial and temporal trends in the causes of death. Compare these patterns between high- and low-impact study sites.

3. Describe relations between specific health threats and putative risk factors (such as habitat use, movement behavior, diet, foraging success, body condition, and reproductive status), 
and contrast these between high- and low-impact sites. Conduct epidemiological analyses to pinpoint key risk factors for disease exposure.

4. Combine data from this study with similar data generated from previous studies, and conduct a comprehensive hazards analysis to identify the ultimate factors that are most important in affecting sea otter survival and thus limiting population growth.

5. Advise and assist with the science needed by the relevant management agencies to implement appropriate conservation, regulatory, and policy development actions.

We hypothesized that:

1. Sea otters living in areas adjacent to human population centers and areas heavily impacted by runoff or sewage are more likely to be exposed to pathogens and toxins (many of which also are relevant for public health) than those in more pristine areas.

2. Patterns of survival and causes of death will differ between heavily impacted and pristine environments, indicating differences in pathogen and pollutant exposure.

3. Environmental risk factors (proximity to point-source pollution, watershed inputs, oceanographic conditions, etc.) will vary between sites, corresponding to the differing land-use patterns.

4. Sea otters from high-density populations (and [or] areas that have been occupied longer) will have lower rates of foraging success due to prey resource depletion, and these patterns will be indicated by (a) greater percentage of time spent feeding, (b) more pronounced individual diet specialization, (c) poorer body condition, and (d) lower survival rates of adults and pups, compared to sea otters from low-density populations (and [or] areas where populations have been recently established).

Hypotheses 1-3 are premised on the notion that the high levels of infectious disease and contaminants found in some sea otters ultimately are related to elevated land-to-sea transport of the causal agents, which is associated with urban and agricultural activities and landscape alteration. Hypothesis 4 is premised upon the notion that density-dependent resource depletion is a primary factor limiting population growth in central California. None of these hypotheses are mutually exclusive, and our goal is to determine the relative degree to which each is supported or refuted by empirical data.

To test these hypotheses, we examined the health, survival and basic ecology of sea otters at two locations along the central California coast (fig. 1). The south end of Monterey Bay and Monterey Peninsula (MON) was selected as a site of high human impact, and the central segment of the Big Sur coast (BSR) was selected as a site of low human impact (fig. 2). Within the current range of the southern sea otter, these sites represent the extremes of the spectrum of anthropogenic impact (for example, urban, industrial and agricultural activities compared to relatively pristine). Another advantage was that these sites contain generally similar subtidal benthic habitat and both have supported highdensity sea otter populations for many years, thereby limiting the influence of confounding effects. Additionally, field studies of sea otters have been conducted in the past at both sites, providing us with historical datasets for temporal contrasts. We recognized that additional replicates of high and low human impact treatments would be desirable, but logistical constraints prevented such replication. Instead, we augmented data from the current comparative study with data from previously completed field studies (for example, Tinker and others, 2006, 2008), which spanned a range of human impacts and sea otter population densities, to account for the pseudo-replicated nature of the Big Sur-Monterey comparison. The latter study began in November 2008 and continued until February 2012. During the course of the study, a total of 98 sea otters were captured, radio-tagged, and monitored closely in the 
field for as many as 4 years, and another 37 were captured and sampled but not radio-tagged. From these study animals, we collected demographic, behavioral, dietary, and life-history data using telemetric methods; measured individual exposure to pathogens and other stressors; and performed necropsies and cause-of-death analyses for all study animals that died during the study period. These data, together with similar data from previous research projects, were subjected to extensive analyses designed to test our four hypotheses and to identify the ultimate factors most important in limiting population growth of southern sea otters in central California.

\section{Methods}

\section{Live Sea Otter Captures}

Study animals were captured using standardized sea otter capture techniques (Ames and others, 1983). Briefly, shore spotters with spotting scopes relayed information about potential target animals to dive crews operating out of small 17-20-ft skiffs. The otters must be resting (preferably sleeping) for this method to be successful. Divers worked in pairs, using closed-circuit oxygen rebreathers and electric propulsion vehicles with traps attached to maneuver the traps underneath the floating sea otters (fig. 3) and engulf them within the trap's net bag. The traps can hold one or two sea otters. The divers kept the animal and trap on the surface until the skiff arrived and the otter or otters could be transferred to a sliding-lid capture box. Once the otter or otters were secure within the capture box, the dive crew quickly transported them to veterinary facilities for anesthetic immobilization, health assessments, biosampling, surgical implantation with very high frequency (VHF) radio transmitters and time-depth recorders (TDRs), and tagging. The otters subsequently were transported back to the initial capture site and released (Ames and others, 1983; Williams and Siniff, 1983; Monson and others, 2001). The entire procedure, from capture to release, generally took from 2 to 3 hours.

At the Big Sur study site, four separate capture events occurred. The initial capture of 40 sea otters took place from 5 to 11 November 2008. A second capture event was conducted from 2 to 5 November 2009, which added 18 new sea otters to the study. From 22 to 27 September 2010, a third Big Sur capture event took place in which 11 new sea otters were captured and 5 previously captured sea otters were recaptured and resampled (TDRs were removed at this time). A fourth and final Big Sur capture event took place from 8 to 10 November 2011, with two animals recaptured and resampled (TDRs removed), and three new otters captured and flippers tagged but not implanted with instruments. A total of 72 individual sea otters were captured in Big Sur. Of those 72 otters, 45 were implanted with VHF radio transmitters and TDRs. The remaining 27 otters were determined not to be good candidates for surgery for various reasons (palpably pregnant females, young pups, or poor health); however, although not tagged, these otters received a full external "work up" (see section, "Processing, Sampling, Health Assessments and Tagging") and were included in all health analyses.

At the Monterey study site, a total of 63 sea otters were captured during multiple 1- to 2-day capture events occurring opportunistically between 2007 and 2011. Of that total, 53 individuals received VHF radios and TDRs. The remaining 10 otters were determined not to be good candidates for surgery for a various reasons (palpably pregnant females, young pups, or poor health). Summary information for all 135 animals captured for this study is provided in table 1.

\section{Processing, Sampling, Health Assessments, and Tagging}

Upon arrival at the veterinary station (the Animal Health Laboratory at the Monterey Bay Aquarium, an onshore mobile veterinary facility, or an onboard ship veterinary laboratory), sea otters were initially allowed to rest and cool within the capture box that was floated adjacent to a tender 
vessel. Following a 10-20 minute soak time, the animals were moved to the veterinary laboratory for sedation, physical examination, collection of morphometric data, and acquisition of a set of biological samples (fig.4A). The otters were chemically immobilized using standard doses of fentanyl citrate $(0.22-0.33 \mathrm{mg} / \mathrm{kg})$ and midazolam hydrochloride $(0.07-0.11 \mathrm{mg} / \mathrm{kg})$ administered intramuscularly (Monson and others, 2001). Once adequately sedated, typically after 6-10 minutes, the sea otter was removed from the capture box and processed. During the anesthetic period, vital signs (heart rate, respiratory rate, end-tidal carbon dioxide ( $\mathrm{CO} 2)$, blood pressure, blood oxygen saturation, and rectal body temperature) were monitored at 5-minute intervals. Anomalies were corrected as indicated (for example, otters were gently cooled or warmed, based on their temperature). Following completion of the examinations, measurements, sample collections, and tagging procedures, the effects of the opiate fentanyl were reversed with an intramuscular dose of naltrexone hydrochloride $(1.1-1.65 \mathrm{mg} / \mathrm{kg})$.

For all captured study animals, standardized health parameters were recorded, including weight, length, girth, fur condition and degree of grizzle, tooth-wear (and tooth-based age estimates; fig. 4B), and general body condition (see chapter 2). Biological samples were collected from each animal, as summarized in table 2, for analyses (including blood samples for clinical health assessments [chapter 2] and gene expression analyses [chapter 3], and vibrissae for stable isotope analyses [chapter 6]). The animals were then prepared for surgical implantation of VHF radio transmitters $(80 \times 22 \times 50 \mathrm{~mm}$, about $160 \mathrm{~g}$, Advanced Telemetry Systems, Isanti, Minnesota) and time depth recorders (TDRs, $67 \times 17$ $\times 17 \mathrm{~mm}$, about $27 \mathrm{~g}$, Wildlife Computers, Redmond, Washington) using standardized surgical techniques (fig. 5; Williams and Siniff, 1983). The VHF transmitter is used for telemetric monitoring and relocation of the otter post-release, and emits a radio signal at 1-second intervals on a unique frequency band that can be detected from as far as $10 \mathrm{~km}$ away. The TDR is a bio-logging instrument that records depth (using a scaled conversion of pressure transducer readings) and internal body temperature at 2-second intervals for a 2- to 3-year period, and allows for detailed analysis of sea otter dive behavior and time-activity budgets (see chapter 4). Each otter also was tagged with unique color/number coded polyethylene "Temple Tags "TM" (livestock ear tags, Temple, Texas) on their hind flippers (two tags per otter), to allow for visual identification by field observers, and received a coded, passive transponder chip, implanted subcutaneously in the inner thigh, for later identification should the temple tags be lost.

During recapture events of previously tagged study animals, the otters once again were anesthetized and a second surgical procedure was performed to retrieve the archival TDR for data collection. At that time, health parameters were reassessed, tissue samples were taken, and any missing flipper tags were replaced before release.

\section{Live Sea Otter Surveillance}

Tracking and observation of the study animals occurred daily for the duration of the study, which began with the first day of captures in Big Sur on November 1, 2008, and continued without interruption until February 1, 2012. Field personnel conducted shore-based surveys of both study sites, locating study animals using standard telemetric protocols (triangulation of radio signal and visual identification; fig. 6A) identical to the methods used in previous sea otter tracking studies (Siniff and Ralls, 1991; Tinker and others, 2006). Initial locations were determined by driving and (or) hiking throughout the study area while using a radio receiver and antenna to scan for telemetry signals. When a signal was obtained, trackers used a combination of binoculars and high-powered $(80 \times)$ spotting scopes (Questar Inc., New Hope, Pennsylvania) to positively identify individuals by their unique flipper tag color combination (fig. 6B). Once individual animals were located, observers recorded various biogeographic, behavioral, and environmental data, including Global Positioning System (GPS) position, survival, reproductive status, and instantaneous behavior. Aerial flights were conducted periodically to 
locate missing study animals. A total of 38,941 resights were recorded for tagged otters as part of this study (table 1).

To monitor time-activity budgets, detailed focal-animal observations (hereinafter "activity budgets") on behavior, diet, distance-to-shore, and fine-scale movements (habitat use) were collected for each study animal on a rotating schedule. These activity budgets were conducted during intense 6hour sessions, but spanned the entire 24-hour period of 1 day, with an emphasis on daylight hours when direct observational data could be collected.

Observational foraging data were collected from radio-tagged sea otters (fig. 6C) following wellestablished protocols (Ralls and others, 1995; Watt and others, 2000; Estes and others, 2003; Tinker and others, 2008). Field observations were collected 7 days per week throughout the study period, with teams of 1-2 observers making systematic searches of the study areas and sequentially targeting specific animals for foraging observations. Study animals were initially located by radio signal using standard telemetric techniques, and then visually monitored from shore using $50-80 \times$ spotting scopes. Foraging bouts (defined as contiguous sequences of feeding dives made by the focal otter) typically lasted 1-4 hours, and data were recorded throughout the entire bout or for as many dives as possible. The information recorded during these bouts included the following:

- Date and time,

- Precise location of each dive (determined by visual triangulation using GPS, compass, and laser range-finder),

- Duration of the subsurface dive interval ("DT") and the post-dive surface interval for each feeding dive (in seconds),

- Outcome of each dive (that is, whether or not prey was captured),

- Species of prey captured,

- Number and size of prey items,

- Per-item handling time (number of seconds required to handle and consume each item),

- Whether or not tools were used to handle the prey, and

- Ambient conditions (including sea-state, wind, etc.).

Prey size was recorded as the estimated diameter of the shell or maximum body dimension (excluding appendages), categorized in 5-cm size-classes. For observations where prey could not be reliably identified to species, the items in question were assigned to the lowest possible taxonomic unit. Any items that could not be reliably categorized to any taxonomic level were listed as "unidentified prey" (although size class and number of items were recorded for such items). Additional information recorded by observers included numbers of prey items that were stolen by or from the focal animal and, in the case of females with dependent pups, the number of items that were shared with the pups. All data were entered in GPS-enabled hand-held computers in the field and later transferred to a central relational database.

\section{Dead Sea Otter Surveillance}

Field personnel made every effort possible to locate and collect any animals that died during the course of the study. A number of animals disappeared from the focal study areas, and in these cases aerial telemetry was used in an attempt to locate them. If a carcass was located by shore-based trackers or by plane, researchers were dispatched by foot or boat to retrieve the carcass, which was placed on ice and transported immediately to the California Department of Fish and Wildlife Marine Wildlife Veterinary Care and Research Center (CDFW-MWVCRC) for necropsy. Every sea otter carcass retrieved was subject to detailed necropsies by a veterinary pathologist, following established protocols 
(see chapter 10). In addition to determining the primary and contributing causes of death, the pathologist supervised the collection of tissue samples for various otter and ecosystem health studies.

\section{Data Analyses}

After completion of field and laboratory-based activities, data were compiled in a relational Microsoft Access ${ }^{\circledR}$ database (the "Wild Sea Otter Database", or WSOD) and prepared for statistical analyses and syntheses. The following suites of analyses were conducted and are summarized in subsequent chapters of this report:

- Health Assessments (chapter 2). Objective and subjective data from the physical examinations and blood diagnostic analyses were summarized for all study animals and compared among study sites, to determine whether there were substantial differences in gross health or clinical pathology.

- Gene Transcription Analysis (chapter 3). Data on genetic biomarkers that measure expression of genes associated with specific physiological responses were summarized for all study animals and compared among study sites. This analysis was used to assess whether there was spatial (across sites) or temporal (across years) variation in response of study animals to environmental stressors.

- Movements and Home Range (chapter 4). Data on weekly movements, annual dispersal distance, and annual home range use were evaluated and compared among study sites to measure the degree of population spatial structure and to determine habitat use patterns, and whether these movement patterns varied as a function of physical differences between sites.

- Dive Behavior and Time-Activity Budgets (chapter 5). Archival TDR data were analyzed to compare dive depth, duration, and other characteristics of foraging bouts among animals from different age/sex classes and study sites. Time-activity budgets were estimated from each TDR record and compared to determine whether there were site differences or effects of reproductive status in terms of percentage of time spent feeding (foraging effort).

- Foraging Ecology (chapter 6). A comprehensive analysis of diet, feeding behavior, and foraging success (rate of energy gain) was conducted using both observational data and analysis of stable isotope ratios from collected vibrissae. Comparisons were made among study sites and with other populations around the North Pacific to infer the degree of prey resource abundance and how it varied among sites.

- Body Condition (chapter 7), Data on age-specific body length and body mass were analyzed using growth functions, and residuals from these functions were used to compute indices of relative body condition for each study site (BSR, MON, plus data from four past studies). Differences among sites were analyzed with respect to variation in foraging success.

- Survival and Reproduction (chapter 8). Bayesian non-parametric proportional hazards models were fit to data on adult and pup survival at all study sites (BSR, MON, plus data from 4 past studies) to estimate instantaneous hazard rates (and thereby annual survival rates) and to identify factors related to variation in these rates, such as sex, age, site effects, reproductive status, and body condition.

- Epidemiology (chapter 9). Serological analysis was used to determine whether study animals were infected with the protozoan parasite Toxoplasma gondii. Infection rates were then analyzed using logistic regression models to determine significant risk factors for disease exposure. Potential risk factors evaluated included sex, age, site effects, diet composition, and movement behavior. 
- Causes of Mortality (chapter 10). Data from necropsies of all tagged animals that died during the study (and whose carcasses were recovered) were analyzed to compare broad disease and mortality patterns among sea otters that were tagged in the Monterey and Big Sur coastal regions.

- Syntheses and Conclusions (chapter 11). The key results from the proceeding chapters are combined and integrated to evaluate the overall degree of support (or lack thereof) for the four main hypotheses. We discuss the implications of these results in understanding factors that are most important to sea otter health, reproductive output, and survival, and, thus, limiting population growth in central California. We then discuss implications for future management and conservation strategies. 


\section{References Cited}

Ames, J.A., Hardy, R.A., and Wendell. F.E., 1983, Tagging materials and methods for sea otters, Enhydra lutris: California Fish and Game, v. 69, p. 243-252.

Costa, D.P., and Kooyman, G.L., 1982. Oxygen consumption, thermoregulation, and the effect of fur oiling and washing on the sea otter, Enhydra lutris: Canadian Journal of Zoology, v. 60, p. 27612767.

Estes, J.A., Danner, E.M., Doak, D.F., Konar, B., Springer, A.M., Steinberg, P.D., Tinker, M.T., and Williams, T.M., 2004, Complex trophic interactions in kelp forest ecosystems: Bulletin of Marine Science, v. 74, p. 621-638.

Estes, J.A., Riedman, M.L., Staedler, M.M., Tinker, M.T., and Lyon, B.E., 2003, Individual variation in prey selection by sea otters-Patterns, causes and implications: Journal of Animal Ecology, v. 72, p. 144-155.

Estes, J.A., Smith, N.S., and Palmisano, J.F., 1978, Sea otter predation and community organization in the western Aleutian Islands, Alaska: Ecology, v. 59, p. 822-833.

Jessup, D.A., Miller, M.A., Kreuder-Johnson, C., Conrad, P.A., Tinker, M.T., Estes, J., and Mazet, J.A.K., 2007, Sea otters in a dirty ocean: JAVMA-Journal of the American Veterinary Medical Association, v. 231, p. 1648-1652.

Johnson, C.K., Tinker, M.T., Estes, J.A., Conrad, P.A., Staedler, M., Miller, M.A., Jessup D.A., and Mazet, 2009, Prey choice and habitat use drive sea otter pathogen exposure in a resource-limited coastal system: Proceedings of the National Academy of Sciences of the United States of America, v. 106, p. 2242-2247.

Kenyon, K.W., 1969, The sea otter in the eastern Pacific Ocean: North American Fauna, v. 68, p. 1352.

Kreuder, C., Miller, M.A., Jessup, D.A., Lowenstine, L.J., Harris, M.D., Ames, J.A., Carpenter, T.E., Conrad, P.A., and Mazet, J.A.K., 2003, Patterns of mortality in southern sea otters (Enhydra lutris nereis) from 1998-2001: Journal of Wildlife Diseases, v. 39, p. 495-509.

Kreuder, C., Miller, M.A., Lowenstine, L.J., Conrad, P.A., Carpenter, T.E., Jessup, D.A., and Mazet, J.A.K., 2005, Evaluation of cardiac lesions and risk factors associated with myocarditis and dilated cardiomyopathy in southern sea otters (Enhydra lutris nereis): American Journal of Veterinary Research, v. 66, p. 289-299.

Miller, M.A., Byrne, B.A., Jang, S.S., Dodd, E.M., Dorfmeier, E., Harris, M.D., Ames, J., Paradies, D., Worcester, K., Jessup, D.A., and Miller, W.A., 2010, Enteric bacterial pathogen detection in southern sea otters (Enhydra lutris nereis) is associated with coastal urbanization and freshwater runoff: Veterinary Research, v. 41, no. 1.

Miller, M.A., Gardner, I.A., Kreuder, C., Paradies, D.M., Worcester, K.R., Jessup, D.A., Dodd, E., Harris, M.D., Ames, J.A., Packham, A.E., and Conrad, P.A., 2002, Coastal freshwater runoff is a risk factor for Toxoplasma gondii infection of southern sea otters (Enhydra lutris nereis): International Journal for Parasitology, v. 32, p. 997-1006.

Miller, M.A., Grigg, M.E., Miller, W.A., Dabritz, H.A., James, E.R., Melli, A.C., Packham, A.E., Jessup, D., and Conrad, P.A., 2007, Toxoplasma gondii and Sarcocystis neurona infections of Pacific coastal sea otters in California, USA - Evidence for land-sea transfer of biological pathogens: Journal of Eukaryotic Microbiology, v. 54, p. 48S-49S.

Miller, M.A., Kudela, R.M., Mekebri, A., Crane, D., Oates, S.C., Tinker, M.T., Staedler, M., Miller, W.A., Toy-Choutka, S., Dominik, C., Hardin, D., Langlois, G., Murray, M., Ward, K., and Jessup, D.A., 2010, Evidence for a novel marine harmful algal bloom-Cyanotoxin (microcystin) transfer from land to sea otters: PLoS One, v. 5, no. 9, p. e12576. 
Monson, D.H., McCormick, C., and Ballachey, B.E., 2001, Chemical anesthesia of northern sea otters (Enhydra lutris) — Results of past field studies: Journal of Zoo and Wildlife Medicine, v. 32, p. 181189.

Morrison, P., Rosenmann, M., and Estes, J.A., 1974, Metabolism and thermoregulation in the sea otter: Physiological and Biochemical Zoology, v. 47, no. 4, p. 218-229.

Ralls, K., Hatfield, B.B., and Siniff, D.B., 1995, Foraging patterns of California sea otters as indicated by telemetry: Canadian Journal of Zoology, v. 73, p. 523-531.

Siniff, D.B., and Ralls, K., 1991, Reproduction, survival and tag loss in California sea otters: Marine Mammal Science, v. 7, p. 211-229.

Tinker, M.T., Bentall, G., and Estes, J.A., 2008, Food limitation leads to behavioral diversification and dietary specialization in sea otters: Proceedings of the National Academy of Sciences of the United States of America, v. 105, p. 560-565.

Tinker, M.T., Doak, D.F., Estes, J.A., Hatfield, B.B., Staedler, M.M., and Bodkin, J.L., 2006, Incorporating diverse data and realistic complexity into demographic estimation procedures for sea otters: Ecological Applications, v. 16, p. 2293-2312.

Tinker, M.T., Guimarães, P.R., Novak, M., Marquitti, F.M.D., Bodkin, J.L., Staedler, M., Bentall, G., and Estes, J.A., 2012, Structure and mechanism of diet specialisation-Testing models of individual variation in resource use with sea otters: Ecology Letters, v. 15, p. 475-483.

Watt, J., Siniff, D.B., and Estes, J.A., 2000, Inter-decadal patterns of population and dietary change in sea otters at Amchitka Island, Alaska: Oecologia (Berlin), v. 124, p. 289-298.

Williams, T., and Siniff, D.B., 1983, Surgical implantation of radio telemetry devices in the sea otter: Journal of the American Veterinary Medical Association, v. 11, p. 1290-1291.

Yeates, L.C., Williams, T.M., and Fink T.L., 2007, Diving and foraging energetics of the smallest marine mammal, the sea otter (Enhydra lutris): Journal of Experimental Biology, v. 210, p. 19601970. 
Table 1. Summary information for sea otters captured, sampled, tagged, and monitored as part of this study at the Big Sur and Monterey study sites, central California.

[Fields shown for each animal (each row in table) are the study site at which the otter was captured (BSR, Big Sur coast; MON, Monterey Peninsula), whether or not a very high frequency (VHF) transmitter was implanted, the sex (M, male; F, female) and identification (BRD) number, number of times the individual was captured and sampled, the date last captured, the capture site latitude and longitude, age class (p, pup; j, juvenile; s, sub-adult; a, adult; or o, old adult) and age estimate at time of initial capture (in years), and number of resights collected. For all rows, na = not applicable or data not collected]

\begin{tabular}{|c|c|c|c|c|c|c|c|c|c|c|}
\hline $\begin{array}{l}\text { Study } \\
\text { site }\end{array}$ & $\begin{array}{l}\text { VHF trans- } \\
\text { mitter? }\end{array}$ & Sex & $\begin{array}{c}\text { BRD } \\
\text { number }\end{array}$ & $\begin{array}{c}\text { Number of } \\
\text { samples }\end{array}$ & $\begin{array}{l}\text { Last } \\
\text { capture }\end{array}$ & $\begin{array}{l}\text { Capture } \\
\text { latitude }\end{array}$ & $\begin{array}{l}\text { Capture } \\
\text { longitude }\end{array}$ & $\begin{array}{l}\text { Age } \\
\text { class }\end{array}$ & $\begin{array}{c}\text { Age } \\
\text { estimate }\end{array}$ & $\begin{array}{c}\text { Number } \\
\text { of } \\
\text { resights }\end{array}$ \\
\hline$\overline{\mathrm{BSR}}$ & Yes & $\mathrm{F}$ & $1067-08$ & 2 & 23-Sep-10 & N $35^{\circ} 57.129$ & $\mathrm{~W} 121^{\circ} 29.767$ & $\mathrm{a}$ & 10 & 639 \\
\hline BSR & Yes & $\mathrm{F}$ & $1068-08$ & 1 & 05-Nov-08 & N $35^{\circ} 54.601$ & $\mathrm{~W} 121^{\circ} 28.354$ & $\mathrm{a}$ & 5 & 2 \\
\hline BSR & Yes & $\mathrm{F}$ & 1069-08 & 1 & 05-Nov-08 & N $35^{\circ} 54.601$ & $\mathrm{~W} 121^{\circ} 25.654$ & $\mathrm{a}$ & 8 & 163 \\
\hline BSR & Yes & $\mathrm{F}$ & $1070-08$ & 1 & 05-Nov-08 & $\mathrm{N} 36^{\circ} 00.660$ & $\mathrm{~W} 121^{\circ} 33.600$ & $\mathrm{a}$ & na & 72 \\
\hline BSR & Yes & $\mathrm{F}$ & $1073-08$ & 1 & 06-Nov-08 & $\mathrm{N} 36^{\circ} 02.463$ & W $121^{\circ} 35.206$ & $\mathrm{a}$ & 4 & 450 \\
\hline BSR & Yes & $\mathrm{F}$ & 1074-08 & 2 & 24-Sep-10 & N $36^{\circ} 07.829$ & W $121^{\circ} 39.366$ & $\mathrm{a}$ & 10 & 512 \\
\hline BSR & Yes & $\mathrm{F}$ & $1075-08$ & 1 & 06-Nov-08 & $\mathrm{N} 36^{\circ} 02.057$ & $\mathrm{~W} 121^{\circ} 35.015$ & $\mathrm{~s}$ & 3 & 608 \\
\hline BSR & Yes & $\mathrm{F}$ & $1079-08$ & 1 & 07-Nov-08 & $\mathrm{N} 36^{\circ} 02.489$ & W $121^{\circ} 35.191$ & $\mathrm{~s}$ & 1.5 & 638 \\
\hline BSR & Yes & $\mathrm{F}$ & $1081-08$ & 1 & 07-Nov-08 & N $36^{\circ} 05.628$ & W $121^{\circ} 37.424$ & $\mathrm{a}$ & 10 & 196 \\
\hline BSR & Yes & $\mathrm{F}$ & 1083-08 & 1 & 07-Nov-08 & N $36^{\circ} 09.218$ & W $121^{\circ} 09.218$ & $\mathrm{a}$ & 8 & 113 \\
\hline BSR & Yes & $\mathrm{F}$ & $1084-08$ & 1 & 07-Nov-08 & N $36^{\circ} 04.978$ & W $121^{\circ} 37.094$ & $\mathrm{a}$ & 6 & 152 \\
\hline BSR & Yes & $\mathrm{F}$ & $1086-08$ & 1 & 07-Nov-08 & N $36^{\circ} 06.091$ & W $121^{\circ} 37.685$ & $\mathrm{~s}$ & 2 & 609 \\
\hline BSR & Yes & $\mathrm{F}$ & $1088-08$ & 1 & $10-$ Nov-08 & N $36^{\circ} 07.435$ & W $121^{\circ} 38.688$ & $\mathrm{a}$ & 4 & 622 \\
\hline BSR & Yes & $\mathrm{F}$ & 1089-08 & 1 & 06-Nov-08 & $\mathrm{N} 36^{\circ} 02.057$ & W $121^{\circ} 35.015$ & $\mathrm{a}$ & 6 & 6 \\
\hline BSR & Yes & $\mathrm{F}$ & 1090-08 & 1 & 03-Nov-08 & N $36^{\circ} 56.605$ & W $121^{\circ} 29.074$ & o & 12 & 56 \\
\hline BSR & Yes & $\mathrm{F}$ & 1097-08 & 2 & 24-Sep-10 & N $36^{\circ} 11.291$ & W $121^{\circ} 42.715$ & $\mathrm{a}$ & 6 & 482 \\
\hline BSR & Yes & $\mathrm{F}$ & 1098-08 & 1 & 11-Nov-08 & N $36^{\circ} 11.718$ & $\mathrm{~W} 121^{\circ} 43.431$ & $\mathrm{~s}$ & 2 & 279 \\
\hline BSR & Yes & $\mathrm{F}$ & $1103-08$ & 2 & 09-Nov-11 & N $36^{\circ} 02.744$ & W $121^{\circ} 35.313$ & $\mathrm{a}$ & 10 & 215 \\
\hline BSR & Yes & $\mathrm{F}$ & $1105-08$ & 1 & 11-Nov-08 & $\mathrm{N} 36^{\circ} 10.029$ & $\mathrm{~W} 121^{\circ} 41.213$ & $\mathrm{a}$ & 4 & 2 \\
\hline BSR & Yes & $\mathrm{F}$ & 1106-08 & 1 & 11-Nov-08 & N $36^{\circ} 10.041$ & W $121^{\circ} 41.234$ & $\mathrm{a}$ & 9 & 47 \\
\hline BSR & Yes & $\mathrm{F}$ & 1135-09 & 2 & 09-Nov-11 & N $36^{\circ} 04.416$ & $\mathrm{~W} 121^{\circ} 36.752$ & $\mathrm{a}$ & 5 & 1 \\
\hline BSR & Yes & $\mathrm{F}$ & 1136-09 & 1 & 02-Nov-09 & $\mathrm{N} 36^{\circ} 2.170$ & W $121^{\circ} 35.025$ & $\mathrm{a}$ & 4 & 459 \\
\hline BSR & Yes & $\mathrm{F}$ & 1139-09 & 1 & 03-Nov-09 & na & na & na & 4 & 27 \\
\hline BSR & Yes & $\mathrm{F}$ & 1141-09 & 2 & 25-Sep-10 & N $36^{\circ} 06.629$ & W $121^{\circ} 37.982$ & $\mathrm{a}$ & 6 & 17 \\
\hline BSR & Yes & $\mathrm{F}$ & 1142-09 & 2 & 24-Sep-10 & $\mathrm{N} 36^{\circ} 09.221$ & $\mathrm{~W} 121^{\circ} 40.154$ & $\mathrm{a}$ & 4.5 & 323 \\
\hline BSR & Yes & $\mathrm{F}$ & 1146-09 & 1 & 04-Nov-09 & $\mathrm{N} 36^{\circ} 04.503$ & W $121^{\circ} 36.801$ & $\mathrm{j}$ & 1 & 17 \\
\hline BSR & Yes & $\mathrm{F}$ & $1148-09$ & 1 & 05-Nov-09 & $\mathrm{N} 36^{\circ} 05.625$ & $\mathrm{~W} 121^{\circ} 37.558$ & $\mathrm{a}$ & 5 & 76 \\
\hline BSR & Yes & $\mathrm{F}$ & $1159-10$ & 1 & 22-Sep-10 & N $36^{\circ} 00.365$ & $\mathrm{~W} 121^{\circ} 31.043$ & $\mathrm{a}$ & 5 & 250 \\
\hline BSR & Yes & $\mathrm{F}$ & $1161-10$ & 1 & $22-\mathrm{Sep}-10$ & $\mathrm{~N} 36^{\circ} 02.443$ & $\mathrm{~W} 121^{\circ} 35.182$ & $\mathrm{a}$ & 4.5 & 295 \\
\hline BSR & Yes & $\mathrm{F}$ & $1162-10$ & 1 & 23-Sep-10 & N $36^{\circ} 05.007$ & W $121^{\circ} 35.773$ & $\mathrm{a}$ & 3.5 & 254 \\
\hline BSR & Yes & $\mathrm{F}$ & $1166-10$ & 1 & 25-Sep-10 & N $36^{\circ} 06.768$ & W $121^{\circ} 38.293$ & $\mathrm{a}$ & 4 & 294 \\
\hline BSR & Yes & $\mathrm{F}$ & $1167-10$ & 1 & $25-$ Sep-10 & N $36^{\circ} 06.754$ & W $121^{\circ} 37.994$ & $\mathrm{~s}$ & 3 & 317 \\
\hline BSR & Yes & $\mathrm{F}$ & $1168-10$ & 1 & 27-Sep-10 & N $36^{\circ} 58.569$ & $\mathrm{~W} 121^{\circ} 29.502$ & a & 6 & 246 \\
\hline BSR & Yes & $\mathrm{F}$ & $1169-10$ & 1 & 27-Sep-10 & N $36^{\circ} 54.887$ & $\mathrm{~W} 121^{\circ} 28.597$ & $\mathrm{~s}$ & 3 & 257 \\
\hline BSR & Yes & M & $1066-08$ & 1 & 05-Nov-08 & $\mathrm{N} 35^{\circ} 57.129$ & $\mathrm{~W} 121^{\circ} 29.767$ & $\mathrm{a}$ & 10 & 31 \\
\hline
\end{tabular}




\begin{tabular}{|c|c|c|c|c|c|c|c|c|c|c|}
\hline $\begin{array}{l}\text { Study } \\
\text { site }\end{array}$ & $\begin{array}{l}\text { VHF trans- } \\
\text { mitter? }\end{array}$ & Sex & $\begin{array}{l}\text { BRD } \\
\text { number }\end{array}$ & $\begin{array}{l}\text { Number of } \\
\text { samples }\end{array}$ & $\begin{array}{l}\text { Last } \\
\text { capture }\end{array}$ & $\begin{array}{l}\text { Capture } \\
\text { latitude }\end{array}$ & $\begin{array}{l}\text { Capture } \\
\text { longitude }\end{array}$ & $\begin{array}{l}\text { Age } \\
\text { class }\end{array}$ & $\begin{array}{c}\text { Age } \\
\text { estimate }\end{array}$ & $\begin{array}{c}\text { Number } \\
\text { of } \\
\text { resights }\end{array}$ \\
\hline BSR & Yes & $\mathrm{M}$ & 1091-08 & 1 & 03-Nov-08 & N $36^{\circ} 04.595$ & W $121^{\circ} 36.662$ & $\mathrm{a}$ & 9 & 7 \\
\hline BSR & Yes & M & 1093-08 & 1 & 06-Nov-08 & N $36^{\circ} 02.463$ & W $121^{\circ} 35.206$ & $\mathrm{a}$ & 8 & 135 \\
\hline BSR & Yes & M & 1094-08 & 1 & 03-Nov-09 & $\mathrm{N} 36^{\circ} 05.628$ & W $121^{\circ} 37.424$ & a & 8 & 325 \\
\hline BSR & Yes & M & 1095-08 & 1 & 03-Nov-08 & N $36^{\circ} 00.660$ & W $121^{\circ} 33.600$ & $\mathrm{a}$ & 6 & 256 \\
\hline BSR & Yes & M & 1096-08 & 1 & 03-Nov-08 & N $36^{\circ} 01.490$ & W $121^{\circ} 34.693$ & $\mathrm{a}$ & 9 & 584 \\
\hline BSR & Yes & M & 1099-08 & 1 & 03-Nov-08 & N $36^{\circ} 06.091$ & W $121^{\circ} 37.685$ & $\mathrm{a}$ & 6 & 547 \\
\hline BSR & Yes & M & 1137-09 & 1 & 03-Nov-09 & $\mathrm{N} 36^{\circ} 1.754$ & W $121^{\circ} 34.834$ & $\mathrm{a}$ & 8 & 367 \\
\hline BSR & Yes & M & 1143-09 & 1 & 04-Nov-09 & N $36^{\circ} 06.764$ & W $121^{\circ} 38.086$ & a & 7 & 475 \\
\hline BSR & Yes & M & 1147-09 & 1 & 04-Nov-09 & $\mathrm{N} 36^{\circ} 04.503$ & W $121^{\circ} 36.807$ & $\mathrm{~s}$ & 2 & 410 \\
\hline BSR & Yes & M & $1165-10$ & 1 & 25 -Sep-10 & N $36^{\circ} 06.750$ & W $121^{\circ} 37.996$ & $\mathrm{a}$ & 7 & 72 \\
\hline BSR & No & $\mathrm{F}$ & 1071-08 & 1 & 05-Nov-08 & N $36^{\circ} 59.378$ & $\mathrm{~W} 121^{\circ} 30.035$ & $\mathrm{a}$ & 4 & 8 \\
\hline BSR & No & $\mathrm{F}$ & $1072-08$ & 1 & 05-Nov-08 & N $35^{\circ} 59.378$ & $\mathrm{~W} 121^{\circ} 30.035$ & a & 6 & 79 \\
\hline BSR & No & $\mathrm{F}$ & $1076-08$ & 1 & 06-Nov-08 & N $36^{\circ} 07.881$ & W $121^{\circ} 39.104$ & $\mathrm{a}$ & 9 & 8 \\
\hline BSR & No & $\mathrm{F}$ & $1077-08$ & 1 & 07-Nov-08 & N $36^{\circ} 07.881$ & W $121^{\circ} 39.115$ & a & 4 & 1 \\
\hline BSR & No & $\mathrm{F}$ & 1078-08 & 1 & 06-Nov-08 & N $36^{\circ} 07.881$ & W $121^{\circ} 39.115$ & o & 11 & 13 \\
\hline BSR & No & $\mathrm{F}$ & $1080-08$ & 1 & 07-Nov-08 & N $36^{\circ} 02.489$ & W $121^{\circ} 35.191$ & $\mathrm{a}$ & 5 & 17 \\
\hline BSR & No & $\mathrm{F}$ & $1082-08$ & 1 & 03-Nov-08 & N $36^{\circ} 09.210$ & W $121^{\circ} 40.262$ & $\mathrm{a}$ & 6 & 9 \\
\hline BSR & No & $\mathrm{F}$ & $1085-08$ & 1 & 08-Nov-08 & N $36^{\circ} 08.711$ & W $121^{\circ} 39.158$ & $\mathrm{a}$ & 6 & 116 \\
\hline BSR & No & $\mathrm{F}$ & $1087-08$ & 1 & 08-Nov-08 & na & na & na & na & 624 \\
\hline BSR & No & $\mathrm{F}$ & $1092-08$ & 1 & $11-$ Nov-08 & N $36^{\circ} 04.275$ & W $121^{\circ} 36.723$ & $\mathrm{a}$ & 8 & 2 \\
\hline BSR & No & $\mathrm{F}$ & 1104-08 & 1 & 11-Nov-08 & $\mathrm{N} 36^{\circ} 06.026$ & W $121^{\circ} 37.609$ & $\mathrm{a}$ & 6 & 108 \\
\hline BSR & No & $\mathrm{F}$ & $1129-08$ & 1 & 11-Nov-08 & N $36^{\circ} 10.029$ & $\mathrm{~W} 121^{\circ} 41.213$ & $\mathrm{p}$ & 0.4 & 1 \\
\hline BSR & No & $\mathrm{F}$ & 1138-09 & 1 & 03-Nov-09 & na & na & $\mathrm{a}$ & 4 & 75 \\
\hline BSR & No & $\mathrm{F}$ & $1140-09$ & 1 & 03-Nov-09 & N $36^{\circ} 06.629$ & W $121^{\circ} 37.982$ & a & 9 & 367 \\
\hline BSR & No & $\mathrm{F}$ & 1144-09 & 1 & 04-Nov-09 & $\mathrm{N} 36^{\circ} 1.456$ & W $121^{\circ} 34.786$ & a & 4 & 46 \\
\hline BSR & No & $\mathrm{F}$ & 1145-09 & 1 & 04-Nov-09 & $\mathrm{N} 36^{\circ} 02.428$ & W $121^{\circ} 57.976$ & $\mathrm{a}$ & 7 & 3 \\
\hline BSR & No & $\mathrm{F}$ & 1149-09 & 1 & 05-Nov-09 & N $36^{\circ} 05.625$ & $\mathrm{~W} 121^{\circ} 37.558$ & $\mathrm{p}$ & na & 520 \\
\hline BSR & No & $\mathrm{F}$ & 1150-09 & 1 & 05-Nov-09 & N $36^{\circ} 06.613$ & W $121^{\circ} 37.980$ & $\mathrm{a}$ & 5 & 37 \\
\hline BSR & No & $\mathrm{F}$ & 1151-09 & 1 & 05-Nov-09 & N $36^{\circ} 06.613$ & W $121^{\circ} 37.981$ & a & 9 & 86 \\
\hline BSR & No & $\mathrm{F}$ & $1160-10$ & 1 & 22-Sep-10 & N $35^{\circ} 59.494$ & $\mathrm{~W} 121^{\circ} 30.022$ & a & 8 & 22 \\
\hline BSR & No & $\mathrm{F}$ & $1163-10$ & 1 & 24-Sep-10 & N $36^{\circ} 08.130$ & W $121^{\circ} 39.294$ & $\mathrm{p}$ & 0.38 & 3 \\
\hline BSR & No & $\mathrm{F}$ & $1164-10$ & 1 & 24-Sep-10 & N $36^{\circ} 09.221$ & W $121^{\circ} 40.175$ & $\mathrm{a}$ & 8 & 112 \\
\hline BSR & No & $\mathrm{F}$ & $1177-11$ & 1 & 08-Nov-11 & N $36^{\circ} 06.153$ & W $121^{\circ} 37.543$ & o & 12 & 7 \\
\hline BSR & No & $\mathrm{F}$ & $1178-11$ & 1 & 08-Nov-11 & N $36^{\circ} 06.153$ & W $121^{\circ} 37.543$ & a & 7 & 13 \\
\hline BSR & No & $\mathrm{F}$ & $1179-11$ & 1 & 10-Nov-11 & N $36^{\circ} 06.75$ & $\mathrm{~W} 121^{\circ} 38.029$ & $\mathrm{a}$ & 4 & 19 \\
\hline BSR & No & M & $1100-08$ & 1 & $11-$ Nov-08 & na & na & na & na & 4 \\
\hline BSR & No & M & 1131-08 & 1 & 11-Nov-08 & N $36^{\circ} 11.273$ & W $121^{\circ} 42.680$ & $\mathrm{j}$ & 0.5 & 1 \\
\hline MON & Yes & $\mathrm{F}$ & $1000-05$ & 5 & 11-Jan-12 & N $36^{\circ} 37.885$ & W $121^{\circ} 55.191$ & o & 11 & 877 \\
\hline MON & Yes & $\mathrm{F}$ & 1011-06 & 2 & 28-Jul-10 & N $36^{\circ} 34.704$ & W $121^{\circ} 58.641$ & $\mathrm{a}$ & 9.5 & 248 \\
\hline MON & Yes & $\mathrm{F}$ & 1032-07 & 5 & 15-Dec-10 & N $36^{\circ} 37.172$ & W $121^{\circ} 53.903$ & $\mathrm{a}$ & 9 & 594 \\
\hline MON & Yes & $\mathrm{F}$ & 1033-07 & 2 & 15-Jul-08 & N $36^{\circ} 37.088$ & W $121^{\circ} 53.779$ & $\mathrm{a}$ & 6 & 220 \\
\hline MON & Yes & $\mathrm{F}$ & 1034-07 & 1 & 24-Sep-07 & $\mathrm{N} 36^{\circ} 54.787$ & W $121^{\circ} 93.930$ & $\mathrm{~s}$ & 3 & 684 \\
\hline MON & Yes & F & 1037-07 & 3 & $27-$ Oct- 10 & N $36^{\circ} 37.767$ & W $121^{\circ} 55.081$ & $\mathrm{a}$ & 9 & 296 \\
\hline
\end{tabular}




\begin{tabular}{|c|c|c|c|c|c|c|c|c|c|c|}
\hline $\begin{array}{l}\text { Study } \\
\text { site }\end{array}$ & $\begin{array}{l}\text { VHF trans- } \\
\text { mitter? }\end{array}$ & Sex & $\begin{array}{c}\text { BRD } \\
\text { number }\end{array}$ & $\begin{array}{c}\text { Number of } \\
\text { samples }\end{array}$ & $\begin{array}{c}\text { Last } \\
\text { capture }\end{array}$ & $\begin{array}{l}\text { Capture } \\
\text { latitude }\end{array}$ & $\begin{array}{l}\text { Capture } \\
\text { longitude }\end{array}$ & $\begin{array}{l}\text { Age } \\
\text { class }\end{array}$ & $\begin{array}{c}\text { Age } \\
\text { estimate }\end{array}$ & $\begin{array}{c}\text { Number } \\
\text { of } \\
\text { resights }\end{array}$ \\
\hline MON & Yes & $\mathrm{F}$ & $1038-07$ & 3 & 08-Sep-11 & N $36^{\circ} 34.704$ & W $121^{\circ} 58.641$ & $\mathrm{a}$ & 6 & 412 \\
\hline MON & Yes & $\mathrm{F}$ & $1042-07$ & 2 & 29-Jul-10 & N $36^{\circ} 34.704$ & W $121^{\circ} 58.641$ & $\mathrm{a}$ & 7 & 128 \\
\hline MON & Yes & $\mathrm{F}$ & 1043-07 & 1 & 26-Sep-07 & $\mathrm{N} 36^{\circ} 34.072$ & $\mathrm{~W} 121^{\circ} 57.087$ & a & 9.5 & 88 \\
\hline MON & Yes & $\mathrm{F}$ & 1044-07 & 1 & 26-Sep-07 & $\mathrm{N} 36^{\circ} 34.072$ & W $121^{\circ} 57.087$ & a & 7 & 169 \\
\hline MON & Yes & $\mathrm{F}$ & $1045-07$ & 2 & 10 -Sep-08 & $\mathrm{N} 36^{\circ} 34.517$ & W $121^{\circ} 58.502$ & o & 11 & 206 \\
\hline MON & Yes & $\mathrm{F}$ & $1046-07$ & 2 & 10-Jun-09 & N $36^{\circ} 34.549$ & W $121^{\circ} 58.494$ & o & 11 & 210 \\
\hline MON & Yes & $\mathrm{F}$ & 1049-07 & 1 & 27-Sep-07 & N $36^{\circ} 36.635$ & W $121^{\circ} 57.964$ & $\mathrm{a}$ & 5.5 & 568 \\
\hline MON & Yes & $\mathrm{F}$ & $1050-07$ & 1 & 27-Sep-07 & $\mathrm{N} 36^{\circ} 34.556$ & W $121^{\circ} 58.429$ & $\mathrm{a}$ & 5.5 & 158 \\
\hline MON & Yes & $\mathrm{F}$ & $1051-07$ & 1 & 27-Sep-07 & N $36^{\circ} 34.556$ & W $121^{\circ} 58.429$ & $\mathrm{a}$ & 4 & 279 \\
\hline MON & Yes & $\mathrm{F}$ & $1052-07$ & 1 & 27-Sep-07 & $\mathrm{N} 36^{\circ} 34.556$ & W $121^{\circ} 58.429$ & $\mathrm{a}$ & 3.5 & 508 \\
\hline MON & Yes & $\mathrm{F}$ & $1053-07$ & 4 & $13-F e b-12$ & $\mathrm{~N} 36^{\circ} 36.400$ & W $121^{\circ} 53.489$ & $\mathrm{a}$ & 7.5 & 177 \\
\hline MON & Yes & $\mathrm{F}$ & $1054-07$ & 2 & 04-Sep-08 & N $36^{\circ} 36.773$ & $\mathrm{~W} 121^{\circ} 53.785$ & $\mathrm{a}$ & 5 & 722 \\
\hline MON & Yes & $\mathrm{F}$ & $1056-08$ & 4 & 19-Apr-12 & $\mathrm{N} 36^{\circ} 36.754$ & W $121^{\circ} 53.716$ & $\mathrm{a}$ & 5 & 327 \\
\hline MON & Yes & $\mathrm{F}$ & 1057-08 & 3 & 02-Sep-11 & N $36^{\circ} 37.339$ & W $121^{\circ} 54.118$ & a & 3.11 & 34 \\
\hline MON & Yes & $\mathrm{F}$ & 1064-08 & 2 & 24-Aug-09 & N $36^{\circ} 38.527$ & W $121^{\circ} 56.194$ & $\mathrm{~s}$ & 1.11 & 205 \\
\hline MON & Yes & $\mathrm{F}$ & 1109-09 & 3 & 03-Feb-11 & N $36^{\circ} 37.450$ & W $121^{\circ} 55.260$ & a & 10 & 628 \\
\hline MON & Yes & $\mathrm{F}$ & 1110-09 & 2 & 27-Oct-10 & N $36^{\circ} 36.742$ & W $121^{\circ} 53.714$ & $\mathrm{a}$ & 8 & 682 \\
\hline MON & Yes & $\mathrm{F}$ & 1113-09 & 2 & 12-Jan-12 & $\mathrm{N} 36^{\circ} 34.704$ & W $121^{\circ} 58.627$ & a & 9 & 470 \\
\hline MON & Yes & $\mathrm{F}$ & 1115-09 & 2 & 01-Sep-11 & N363 37.842 & $\mathrm{~W}-121^{\circ} 55.114$ & a & 9 & 629 \\
\hline MON & Yes & $\mathrm{F}$ & 1117-09 & 2 & $28-O c t-10$ & $\mathrm{~N} 36^{\circ} 37.860$ & $\mathrm{~W} 121^{\circ} 54.931$ & $\mathrm{a}$ & 4 & 499 \\
\hline MON & Yes & $\mathrm{F}$ & 1119-09 & 1 & 09-Jun-09 & N $36^{\circ} 35.558$ & W $121^{\circ} 57.973$ & $\mathrm{~s}$ & 2 & 434 \\
\hline MON & Yes & $\mathrm{F}$ & $1122-09$ & 3 & 01-Sep-11 & N $36^{\circ} 38.086$ & W $121^{\circ} 55.260$ & a & 8 & 583 \\
\hline MON & Yes & $\mathrm{F}$ & 1123-09 & 2 & 02-Sep-11 & $\mathrm{N} 36^{\circ} 38.118$ & W $121^{\circ} 55.322$ & a & 6 & 576 \\
\hline MON & Yes & $\mathrm{F}$ & 1124-09 & 1 & 16-Jun-09 & N $36^{\circ} 36.634$ & W $121^{\circ} 57.243$ & $\mathrm{~s}$ & 3 & 563 \\
\hline MON & Yes & $\mathrm{F}$ & 1125-09 & 2 & 11-Nov-12 & N $36^{\circ} 36.202$ & W $121^{\circ} 52.883$ & $\mathrm{a}$ & 8.5 & 531 \\
\hline MON & Yes & $\mathrm{F}$ & 1126-09 & 3 & 29-Jul-10 & $\mathrm{N} 36^{\circ} 38.021$ & W $121^{\circ} 54.651$ & a & 10 & 510 \\
\hline MON & Yes & $\mathrm{F}$ & 1127-09 & 3 & 07-Jun-10 & N $36^{\circ} 37.059$ & W $121^{\circ} 53.873$ & a & 7 & 525 \\
\hline MON & Yes & $\mathrm{F}$ & 1128-09 & 2 & 28-Jul-10 & N $36^{\circ} 34.704$ & W $121^{\circ} 58.641$ & $\mathrm{a}$ & 6.5 & 492 \\
\hline MON & Yes & $\mathrm{F}$ & 1134-09 & 3 & 03-Feb-11 & N $36^{\circ} 37.400$ & W $121^{\circ} 53.500$ & $\mathrm{a}$ & 5.5 & 603 \\
\hline MON & Yes & $\mathrm{F}$ & $1153-10$ & 2 & 19-Apr-12 & $\mathrm{N} 36^{\circ} 36.738$ & W $121^{\circ} 53.727$ & $\mathrm{~s}$ & 5 & 345 \\
\hline MON & Yes & $\mathrm{F}$ & $1155-10$ & 1 & $01-F e b-10$ & $\mathrm{~N} 36^{\circ} 34.656$ & W $121^{\circ} 58.719$ & $\mathrm{a}$ & 6 & 62 \\
\hline MON & Yes & $\mathrm{F}$ & $1157-10$ & 2 & 02-Sep-11 & N $36^{\circ} 38.064$ & W $121^{\circ} 55.265$ & $\mathrm{a}$ & 8 & 310 \\
\hline MON & Yes & $\mathrm{F}$ & $1158-10$ & 2 & 12-Jan-12 & N $36^{\circ} 38.399$ & W $121^{\circ} 56.065$ & a & 10 & 297 \\
\hline MON & Yes & $\mathrm{F}$ & $946-03$ & 9 & $11-F e b-11$ & N $36^{\circ} 36.981$ & W $121^{\circ} 53.786$ & $\mathrm{a}$ & 10 & 1,557 \\
\hline MON & Yes & M & $1024-06$ & 2 & 09-Jun-09 & $\mathrm{N} 36^{\circ} 33.873$ & W $121^{\circ} 56.571$ & $\mathrm{a}$ & 11 & 375 \\
\hline MON & Yes & M & $1030-06$ & 3 & 16-Dec-10 & N $36^{\circ} 37.967$ & W $121^{\circ} 55.187$ & a & 10 & 1,008 \\
\hline MON & Yes & M & $1065-08$ & 2 & 22-Apr-09 & $\mathrm{N} 36^{\circ} 62.180$ & W $121^{\circ} 90.255$ & a & 7 & 4 \\
\hline MON & Yes & M & 1108-09 & 2 & 09-Jun-10 & N $36^{\circ} 37.870$ & W $121^{\circ} 55.203$ & $\mathrm{a}$ & 10.5 & 275 \\
\hline MON & Yes & M & 1114-09 & 4 & 02-Sep-11 & N $36^{\circ} 37.086$ & W $121^{\circ} 54.054$ & a & 9 & 738 \\
\hline MON & Yes & M & 1116-09 & 1 & 28-May-09 & N $36^{\circ} 38.141$ & W $121^{\circ} 56.697$ & $\mathrm{a}$ & 4 & 33 \\
\hline MON & Yes & M & 1118-09 & 1 & 28-May-09 & N $36^{\circ} 34.675$ & W $121^{\circ} 58.626$ & $\mathrm{a}$ & 5 & 35 \\
\hline MON & Yes & M & $1156-10$ & 2 & $11-F e b-11$ & $\mathrm{~N} 36^{\circ} 36.753$ & W $121^{\circ} 53.715$ & $\mathrm{a}$ & 7.5 & 526 \\
\hline MON & Yes & M & $1170-10$ & 1 & 16-Dec-10 & N $36^{\circ} 37.357$ & W $121^{\circ} 54.063$ & $\mathrm{a}$ & 4.5 & 241 \\
\hline
\end{tabular}




\begin{tabular}{|c|c|c|c|c|c|c|c|c|c|c|}
\hline $\begin{array}{l}\text { Study } \\
\text { site }\end{array}$ & $\begin{array}{l}\text { VHF trans- } \\
\text { mitter? }\end{array}$ & Sex & $\begin{array}{l}\text { BRD } \\
\text { number }\end{array}$ & $\begin{array}{l}\text { Number of } \\
\text { samples }\end{array}$ & $\begin{array}{l}\text { Last } \\
\text { capture }\end{array}$ & $\begin{array}{l}\text { Capture } \\
\text { latitude }\end{array}$ & $\begin{array}{l}\text { Capture } \\
\text { longitude }\end{array}$ & $\begin{array}{l}\text { Age } \\
\text { class }\end{array}$ & $\begin{array}{c}\text { Age } \\
\text { estimate }\end{array}$ & $\begin{array}{l}\text { Number } \\
\text { of } \\
\text { resights }\end{array}$ \\
\hline MON & Yes & M & $1172-11$ & 1 & 03-Feb-11 & N $36^{\circ} 38.058$ & W $121^{\circ} 55.146$ & $\mathrm{a}$ & 7 & 27 \\
\hline MON & Yes & M & $945-03$ & 4 & 10-Jun-09 & $\mathrm{N} 36^{\circ} 38.282$ & W $121^{\circ} 56.160$ & $\mathrm{a}$ & 8 & 440 \\
\hline MON & Yes & M & $985-04$ & 5 & 01-Sep-11 & $\mathrm{N} 36^{\circ} 37.539$ & W $121^{\circ} 54.755$ & $\mathrm{a}$ & 11 & 547 \\
\hline MON & Yes & M & $998-05$ & 2 & 26-Feb-08 & N $36^{\circ} 37.526$ & W $121^{\circ} 54.689$ & $\mathrm{a}$ & 8 & 1,225 \\
\hline MON & No & $\mathrm{F}$ & $1028-06$ & 3 & 14-Jul-08 & N $36^{\circ} 34.605$ & W $121^{\circ} 58.745$ & $\mathrm{~s}$ & 4.5 & 750 \\
\hline MON & No & $\mathrm{F}$ & 1111-09 & 2 & 08-Sep-11 & N $36^{\circ} 36.754$ & W $121^{\circ} 53.710$ & $\mathrm{a}$ & 8 & 186 \\
\hline MON & No & $\mathrm{F}$ & 1112-09 & 1 & 24-Apr-09 & N $36^{\circ} .576$ & W $121^{\circ} .979$ & $\mathrm{a}$ & na & 17 \\
\hline MON & No & F & 1120-09 & 1 & 09-Jun-09 & N $36^{\circ} 34.556$ & W $121^{\circ} 58.497$ & $\mathrm{a}$ & 8 & 72 \\
\hline MON & No & $\mathrm{F}$ & $1130-08$ & 1 & $11-$ Nov-08 & na & na & $\mathrm{a}$ & na & 1 \\
\hline MON & No & $\mathrm{F}$ & 1132-09 & 1 & 17-Jun-09 & N $36^{\circ} 34.704$ & W $121^{\circ} 58.641$ & $\mathrm{a}$ & 6 & 47 \\
\hline MON & No & $\mathrm{F}$ & 1133-09 & 1 & 08-Aug-09 & N $36^{\circ} 37.163$ & W $121^{\circ} 53.982$ & $\mathrm{a}$ & 8 & 66 \\
\hline MON & No & $\mathrm{F}$ & $1154-10$ & 1 & 01-Feb-10 & N $36^{\circ} 34.656$ & W $121^{\circ} 58.719$ & a & 5 & 4 \\
\hline MON & No & F & $941-03$ & 2 & 22-Apr-09 & na & na & $\mathrm{j}$ & 7 & 610 \\
\hline MON & No & M & 1121-09 & 1 & 10-Jun-09 & $\mathrm{N} 36^{\circ} 34.549$ & W $121^{\circ} 58.494$ & $\mathrm{a}$ & 5 & 102 \\
\hline
\end{tabular}


Table 2. Biological samples collected from sea otters captured at the Big Sur and Monterey study sites, central California.

\begin{tabular}{|c|l|}
\hline Sample type & \multicolumn{1}{c|}{ Use } \\
\hline Blood & $\begin{array}{l}\text { Hematology, clinical chemistry, infectious disease monitoring, gene } \\
\text { transcription biomarkers, contaminants }\end{array}$ \\
\hline $\begin{array}{c}\text { External swabs (integument, oral cavity, } \\
\text { rectum, genital orifice) }\end{array}$ & $\begin{array}{l}\text { Infectious disease (bacterial, fungal, parasitic, viral), contaminants, } \\
\text { genetics }\end{array}$ \\
\hline Saliva & Hormonal assays \\
\hline Feces & $\begin{array}{c}\text { Diet assessment, infectious disease (bacterial, fungal, parasitic, viral), } \\
\text { contaminants, biotoxin, hormonal assay }\end{array}$ \\
\hline Adipose tissue & Fatty acids, contaminants \\
\hline Liver biopsies & $\begin{array}{l}\text { Histopathology, toxicology, contaminants, genetics, chemical analysis } \\
\text { (that is, Vitamin A analysis) }\end{array}$ \\
\hline Skin plugs & Genetics \\
\hline Vibrissae & Stable isotope \\
\hline Tooth & Cementum aging \\
\hline Fur & Hormonal assays, toxins, contaminants \\
\hline
\end{tabular}




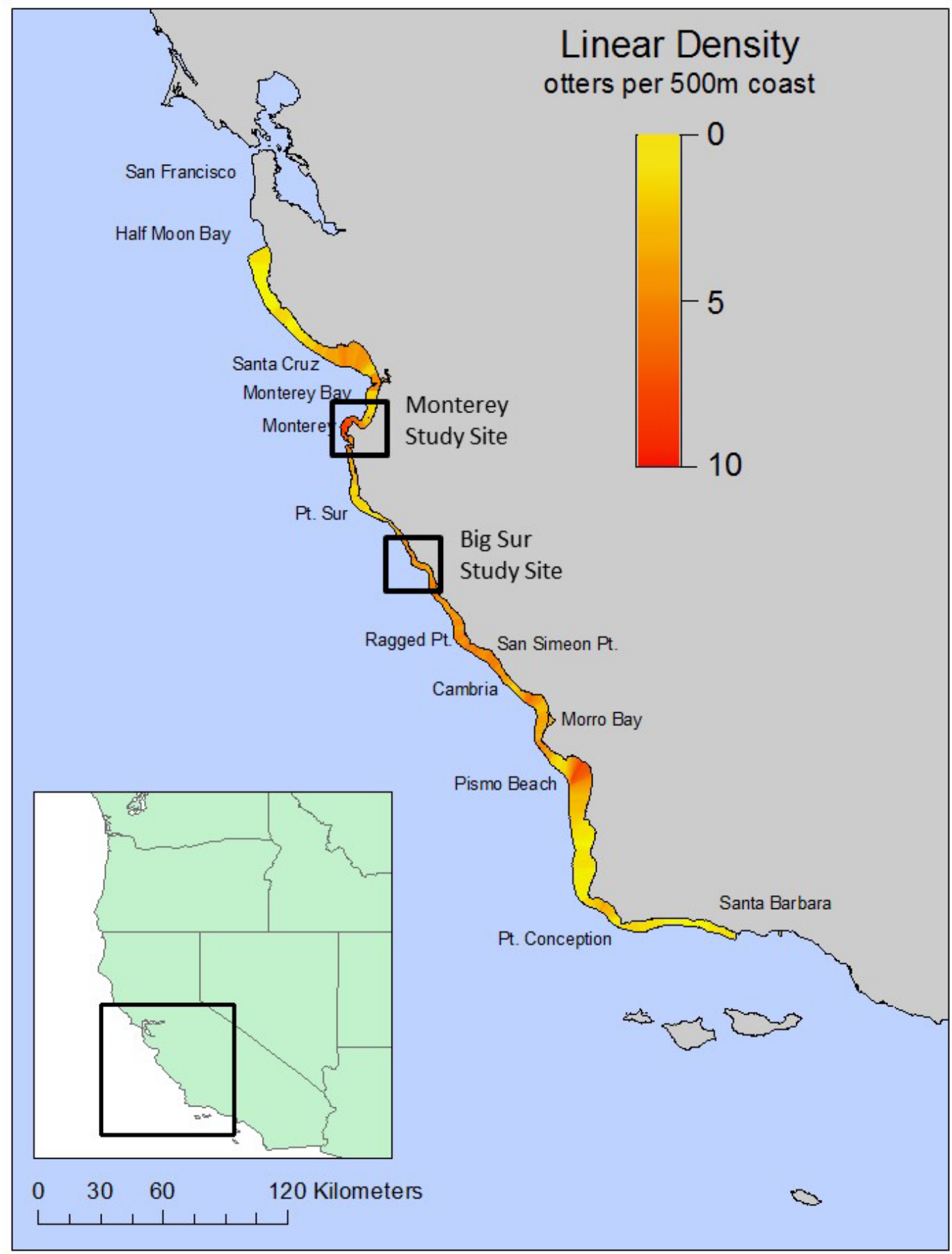

Figure 1. Schematic of the central California coast showing the current (2018) distribution of the southern sea otter and spatial variation in relative population density. Habitat currently used by sea otters is shown as a colored band along the coast, with color coding corresponding to the number of sea otters per 500 meters $(\mathrm{m})$ of coast. Locations of the two study sites for the current project are indicated with black squares. 

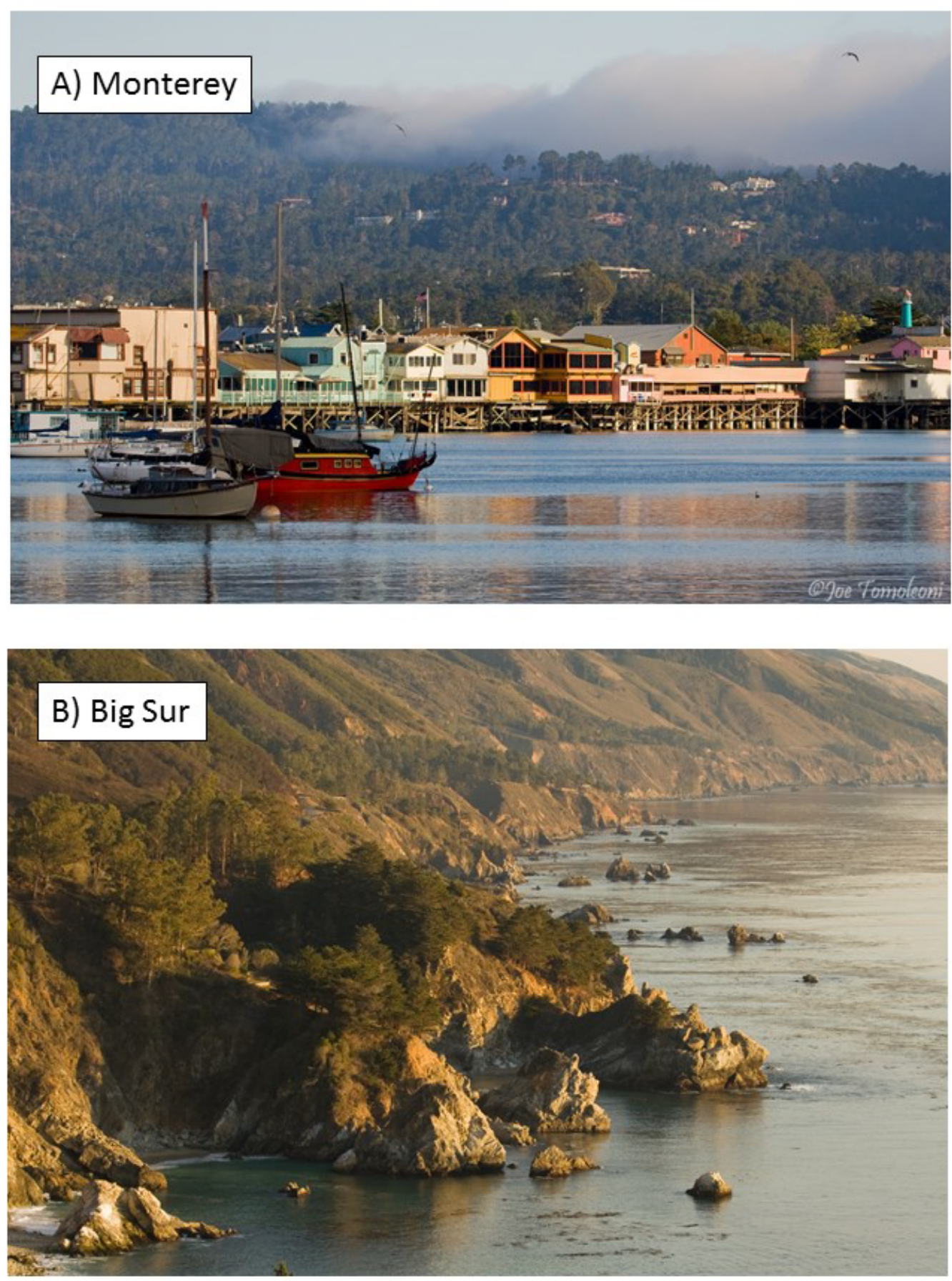

Figure 2. Photographs showing segments of the coastline within each of the two study sites in central California. A, Monterey study site along Cannery Row in Monterey, showing the high potential for human impacts on the nearshore marine ecosystem in this area. B, The Big Sur study site near McWay Falls in the center of the Big Sur study site, showing the low potential for human impacts on the nearshore marine ecosystem in this area. Photographs by Joseph Tomoleoni, U.S. Geological Survey, 2009. 

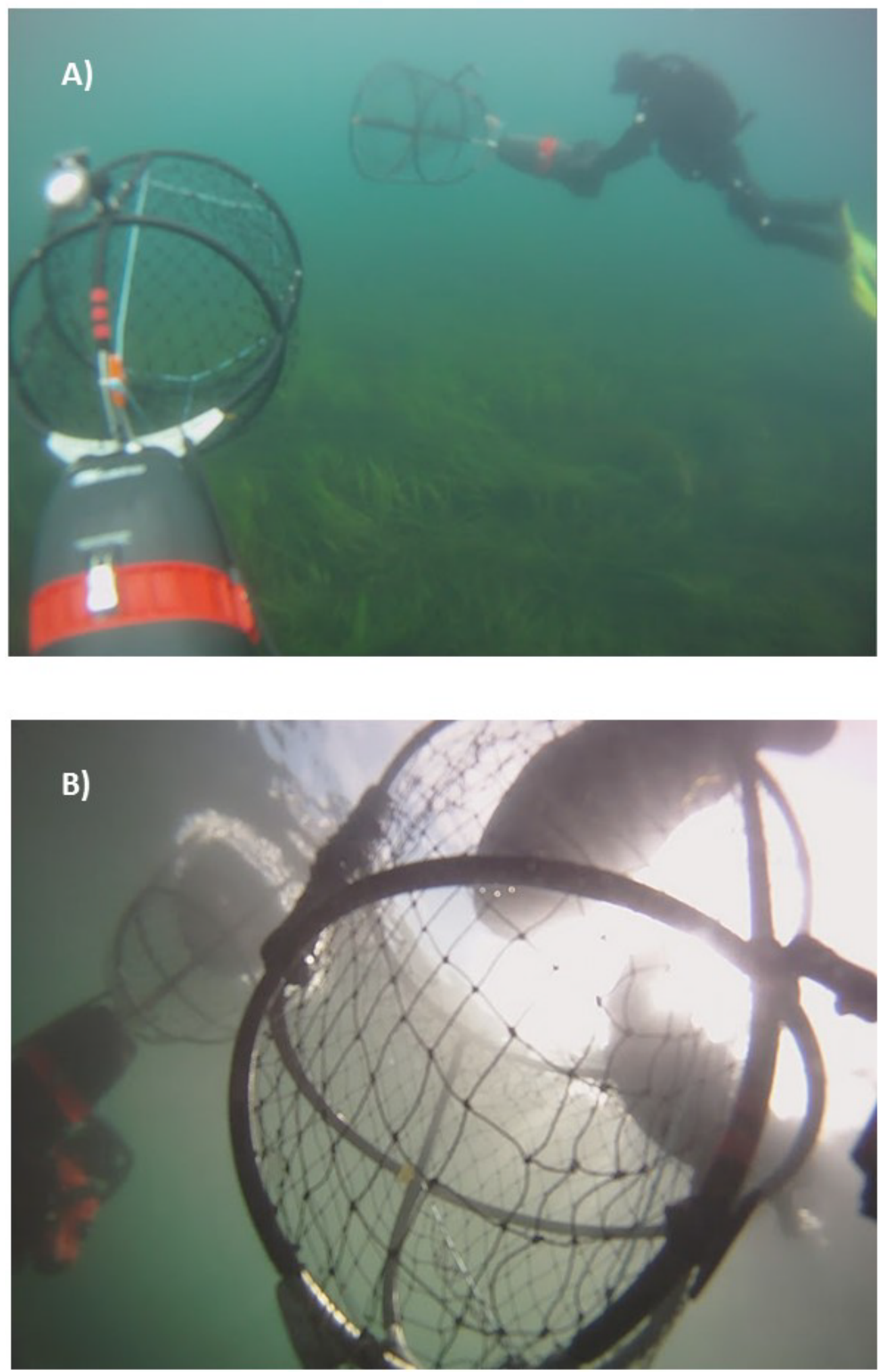

Figure 3. Photographs showing the scuba-based methods used to capture sea otters. A, Pair of divers travelling underwater with the Wilson traps propelled ahead of them using electric propulsion scooters. B, Resting sea otters being captured within the Wilson traps. Photographs by George Esslinger, U.S. Geological Survey, 2009. 

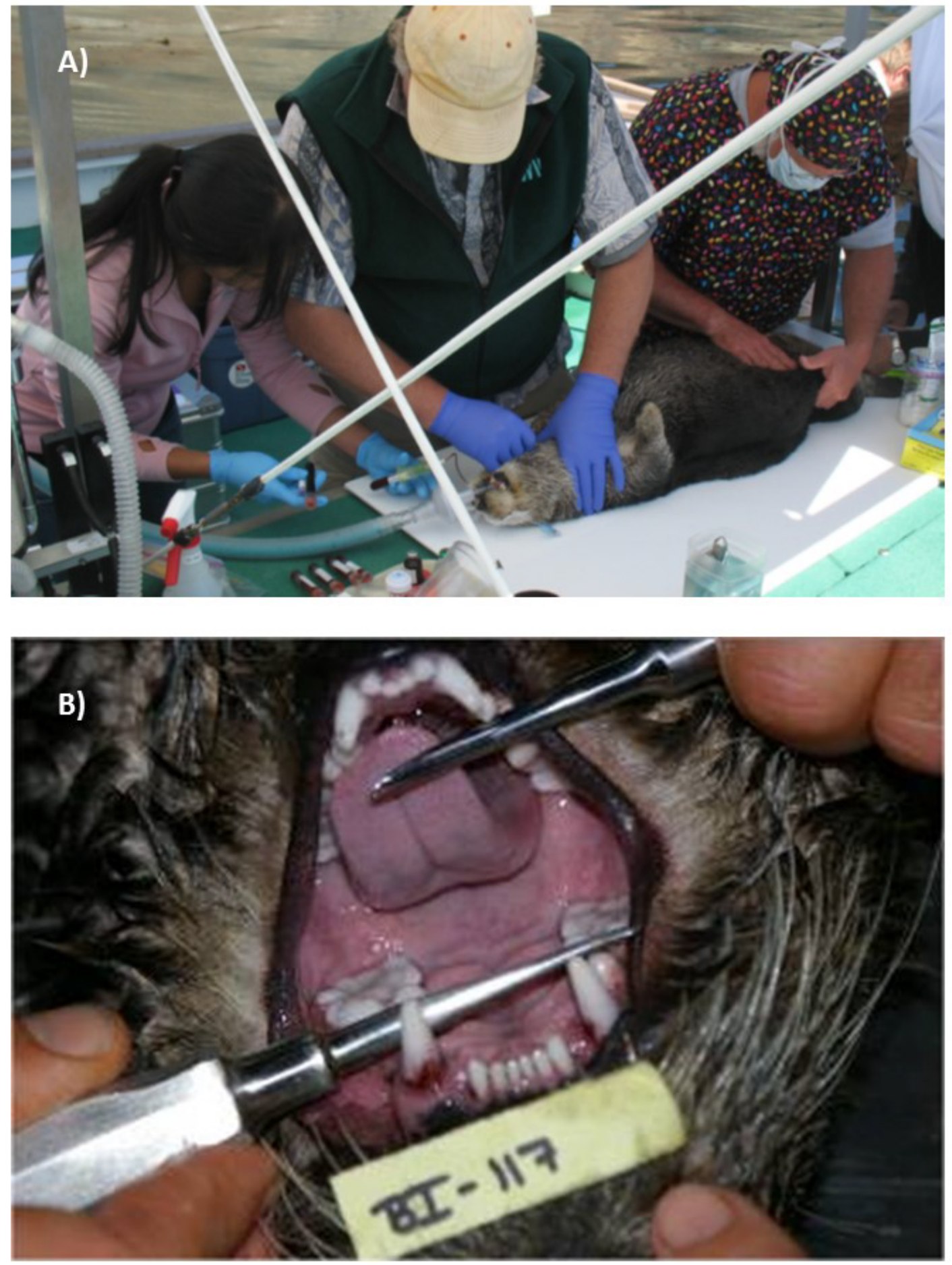

Figure 4. Photographs showing the health assessments and biosampling of anesthetized sea otters. A, Veterinarians conduct physical exams and collect blood samples. B, Veterinarian examines the dental and oral health of a sea otter, recording tooth wear, breakage, and the presence of oral lesions. Photograph A by Anne Tewksbury, Monterey Bay Aquarium, 2008. Photograph B by Dan Monson, U.S. Geological Survvey, 2006. 

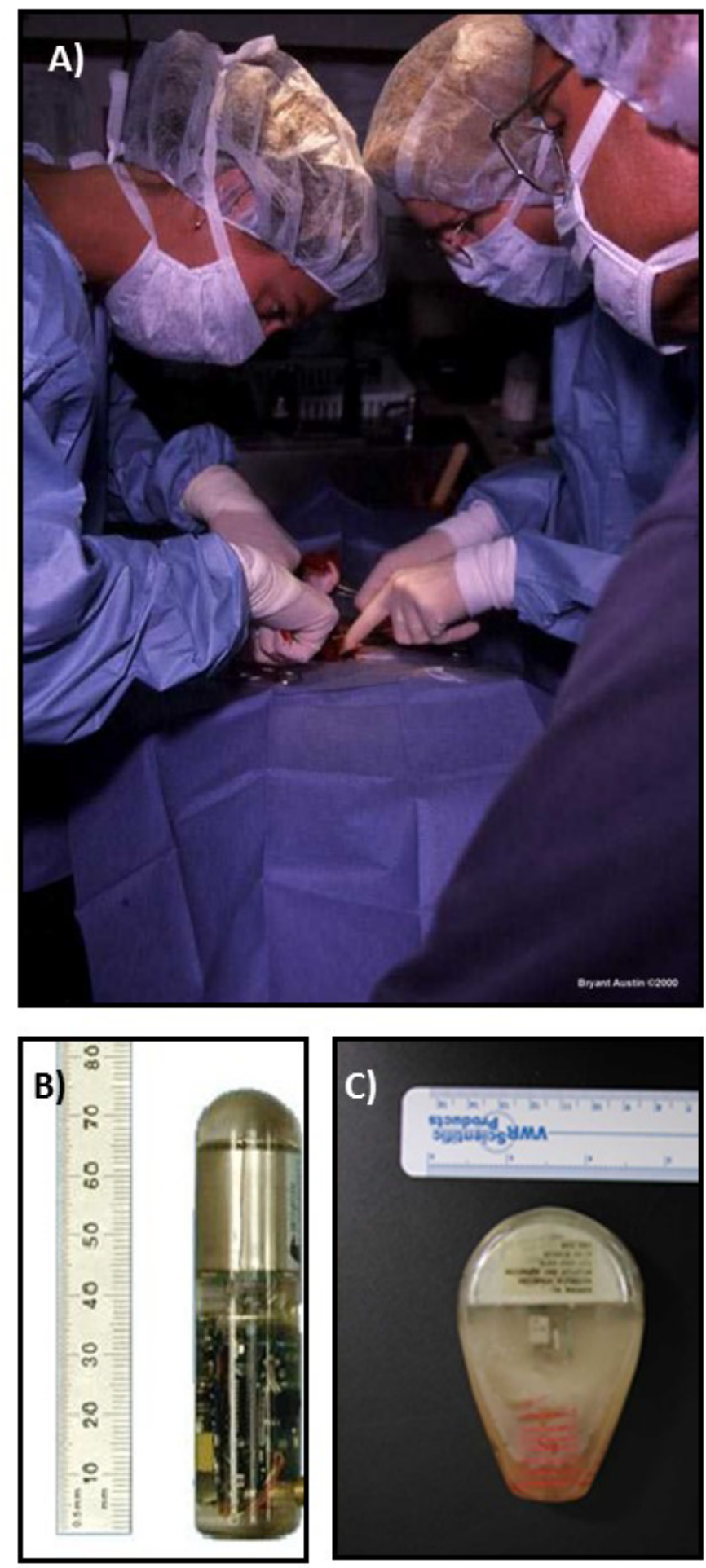

Figure 5. Photographs showing the surgical procedure used to implant telemetry tracking devices in study animals. A, Veterinary team performs abdominal surgery on an anaesthetized sea otter. B, Time-depth recorder (TDR) used to record diving behavior and internal temperature. C, Very high frequency (VHF) transmitter used to locate study animals in the wild using standard wildlife telemetry techniques. Photograph A by Bryant Austin, Studio Cosmos, 2000. Photographs B and C by Michelle Staedler, Monterey Bay Aquarium, 2005. 

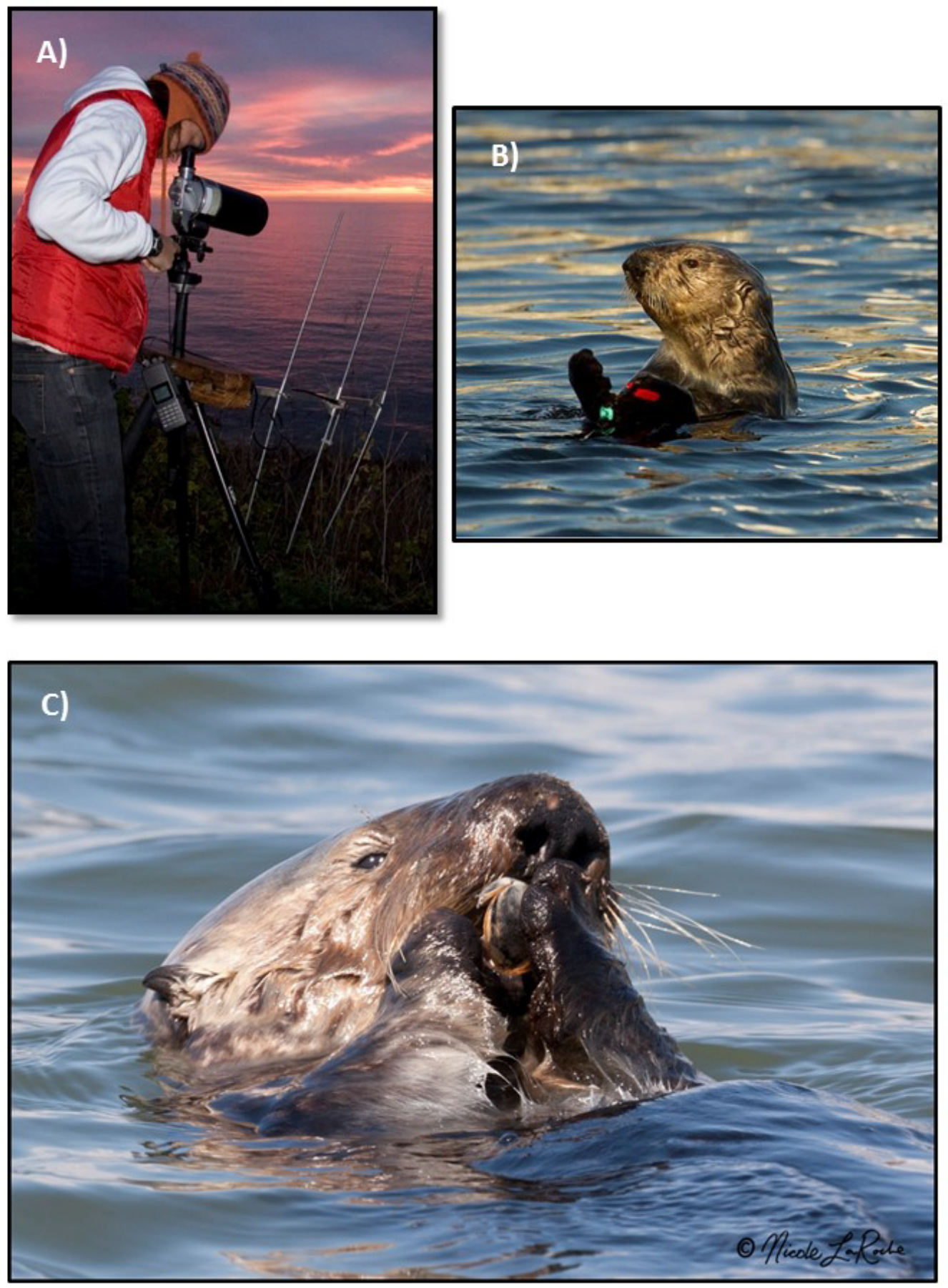

Figure 6. Photographs showing field monitoring of tagged study animals. A, Field observer uses a radio receiver and antenna to localize source of the very high frequency (VHF) signal from a study animal, and a 50-80x telescope to visually monitor the study animal. B, Study animal about to dive shows its uniquely colored flipper tags, used for visual identification. C, View of a study animal through a Questar ${ }^{\mathrm{TM}}$ spotting scope, showing the potential for a trained observer to visually identify prey and record feeding behavior (in this case a clam of size " $1 \mathrm{c}$ " (3.5-5 centimeters) is being consumed). Photographs by Nicole LaRoche, University of California, Santa Cruz, 2010 (A), 2013 (B and C). 


\title{
Chapter 2. Sea Otter Health Assessments at Big Sur and Monterey
}

\author{
Michael J. Murray ${ }^{1}$ (DVM) and M. Tim Tinker ${ }^{2,3}$
}

\section{Introduction}

This following question is one of the most readily apparent questions to ask when comparing populations of sea otters in "pristine" habitat with those from an area with significant anthropogenic impact: "Are the animals from the pristine habitat healthier?" We took advantage of the opportunity to conduct thorough examinations of sea otters from both geographic locations to answer this question. The determination of health status was made by integrating findings identified in the complete physical examination of sedated sea otters, with the results of complete blood count and serum chemistry panel analyses of blood samples submitted to a veterinary reference laboratory (IDEXX Laboratories, Westbrook, ME).

The nature of this study mandated making multiple changes in the clinical paradigm from the one traditionally practiced in domestic animal medicine. Much of veterinarian training and experience is found in the health management of individual patients. Assessments are made of numerous aspects of holistic picture, including clinical history, physical findings, and a myriad of laboratory and other ancillary tests of various body organs and systems, as well as specific infectious and non-infectious diseases. These assessments are then accumulated and considered together, a health assessment is made, appropriate therapeutic measures are recommended, and a prognosis is given. When dealing with populations of free-ranging wildlife, the "patient" becomes the population, and the various "organs and body systems" are individual animals within the population. Health of this "patient" is not simply a statistical analysis of the incidence of health and (or) disease within the population, but must also take into consideration many external factors, such as reproductive rates, resource availability, habitat use, and the effects of anthropogenic influences.

There are substantial shortcomings in this approach of applying individual animal data to a population-level health assessment. Similarly structured epidemiological studies in human medicine are bolstered by exponentially larger sample sizes numbering in the thousands instead of the approximately 150 individual otters in this study. Obviously, a larger " $n$ " results in a more robust statistical interpretation of the data. Another deficiency common to nearly all wildlife health studies is the reliance on single samples, thereby losing the perspective that one gains by evaluating trends in observed and measured data over a longer time frame. Despite these limitations, a good-faith effort has been made to interpret data as-is, without extrapolation towards potential future health status.

Nearly all spectra of infectious and non-infectious diseases have been recognized in either freeranging or captive sea otters. The use of serologic testing to look for historical exposure or infection, or antigen capture methods to evaluate the presence of pathogens or parts thereof in samples, were considered, but a decision was made to not include such tests in the general health assessment (however, refer to chapter 9 for a targeted epidemiological analysis of Toxoplasma gondii infections based on serology). This decision indicated uncertainty about which pathogens should be evaluated in a comprehensive health screening, which tests are validated in sea otters, what constitutes a "positive" test, and whether a positive test was consistent with disease. On a more practical level, funding limitations prohibited broad-based serological screening for pathogen exposure. Instead, reliance was

\footnotetext{
${ }^{1}$ Monterey Bay Aquarium.

${ }^{2}$ U.S. Geological Survey.

${ }^{3}$ University of California, Santa Cruz.
} 
placed on non-specific molecular genetic tests (gene expression analyses; see chapter 3) to provide insight into the role of infectious disease in the health of sampled sea otters. A similar evaluation and decision was reached with respect to testing for the myriad of potential contaminants in sea otters.

Limitations notwithstanding, complete physical examinations from "nose to toes" were performed on all sea otters using traditional clinical methods. Complete blood counts and serum chemistry panels submitted to a veterinary reference laboratory were evaluated using published reference ranges, bearing in mind the limitations associated with the interpretation of each parameter. Data from each sea otter were interpreted independently and health scores were assigned. The database of health scores was used in the assessment of population health between Monterey and Big Sur populations.

\section{Methods}

Once captured using standard techniques, sea otters were transported to the appropriate veterinary laboratory, the Animal Health Laboratory at the Monterey Bay Aquarium, an onshore mobile veterinary facility, or an onboard ship veterinary laboratory. Prior to further handling, otters were allowed to rest within the capture box that was floated adjacent to a tender vessel. This step served two major purposes. First, the movement of water in and out of the box through the capture box ventilation holes facilitated thermoregulation, as otters held out of water during transit from capture site to laboratory site often developed some degree of hyperthermia. Second, this step allowed the animal to float inside the box (a darkened, quiet environment), generally grooming and resting. This opportunity to "calm down" seems to reduce the effects of the catecholamine response from the initial capture. These endogenous hormones, epinephrine and norepinephrine, may have untoward effects on cardiac function during anesthesia and are known to artifactually alter clinical pathology data.

Following this 10- to 20-minute soak time, animals were moved to the veterinary laboratory for sedation, physical examination, collection of morphometric data, and acquisition of a set of biological specimens (see chapter 1). The physical examination was conducted in a standardized fashion following traditional clinical methods starting at the nose and ending at the tip of the tail. Particular attention was paid to the oral cavity examination; a complete dental inventory and evaluation were performed and results were recorded on dentition-specific data sheets. Other physical anomalies were recorded on standard data sheets by the attending clinician.

Blood samples were collected from the left or right external jugular vein into BD Vacutainer tubes using 19- or-21 gauge butterfly winged blood collection sets. Red/gray-topped serum separator tubes were used for serum chemistry panels and lavender-topped EDTA tubes were used for the complete blood counts. Blood smears were made directly from needles or syringes without anticoagulant effects. Serum was separated from the cellular components of the blood within 30 minutes of collection using a Clay Adams ${ }^{\mathrm{TM}}$ TRIAC $^{\circledR}$ centrifuge or its equivalent at a speed of 3,500 revolutions per minute (1,500 relative centrifugal force) for 5 minutes. After the serum was decanted from the tube, an aliquot of $1.0 \mathrm{~mL}$ was refrigerated or frozen until time of transport to the reference laboratory. The blood collected in the lavender-topped tube for the complete blood count (CBC) was inverted multiple times to assure adequate mixing of the anticoagulant and then refrigerated until shipment. Blood smears were air dried, placed in slide shipping containers, and accompanied the serum and whole blood to the laboratory.

$\mathrm{CBC}$ and serum chemistry evaluations were done by Idexx Laboratories, a nationwide veterinary reference laboratory used by many companion animal veterinary practices. Samples were taken by Idexx courier from a drop box to the reference laboratory for testing. A Sysmex ${ }^{\circledR}$ XT-2000i Automated Hematology Analyzer was used to perform the automated CBC. Serum chemistry parameters were analyzed using an Olympus Model AU 5400 Chemistry Immuno Analyzer. 
Evaluation of individual sea otter health was made by retrospectively reviewing objective and subjective data from the following aspects of the examinations:

- Physical exam,

- Dental exam (incisors, canines, premolar/molars),

- Packed cell volume (PCV),

- Hemoglobin (Hgb),

- Total white blood cell count (WBC),

- Differential white cell count,

- Serum chemistry panel, and

- "Subjective weight assessment".

The "subjective weight assessment" index was scored based on deviations from "typical" weight-at-age (see chapter 7 for detailed analytical methods), with a score of 1 indicating an animal at or greater than average weight, and a score of 5 indicating an animal far less than average weight (table 3 ). These specific parameters were selected because they indicate a paradigm for a typical health assessment of apparently healthy companion animals used in traditional clinical veterinary medicine, and this approach is consistent with the protocol used in the USGS Pacific Nearshore Project, to investigate biotic response to environmental variation in nearshore habitats of the northeast Pacific Ocean. The scoring matrix (table 4) is a subjective, qualitative ranking of status when compared to "normal" with higher scores indicating increased deviation. A higher score is a "less normal" appearance.

Interpretation of clinical pathology data was done by integrating the clinical interpretation of the data with comparisons to published reference ranges as well as the subjective, holistic evaluation of the entire suite of test results derived from the panels. The second evaluation, using the complete clinical pathology datasets, was a comparison between the two populations (Big Sur and Monterey), not between either or both of these populations and the reference ranges. To use the reference ranges in the second evaluation is not only outside of the scope of this project, but is subject to valid criticism, as the published reference ranges often involve different subspecies of sea otter, varying sample handling methods, and different analysis equipment, and all of these are potential sources for variability that were not addressed in this study.

Our overall goal was to answer two simple questions - (1) are there differences in sea otter health between study sites; and (2) if differences exist, which health parameters are most important in distinguishing between sites? The multivariate nature of the datasets precluded a univariate statistical approach to analyses; instead, we used multivariate discriminant analysis (DA) to compare Big Sur and Monterey study animals with respect to health parameters. We conducted DA on the matrixes of subjective health scores and clinical pathology results; for each dataset, the groups compared were sex/study site combinations (Big Sur females, Big Sur males, Monterey females, Monterey males). We used Wilks's lambda $\left(\lambda_{U}\right)$ to test for differences among groups with respect to the discriminant functions computed from the underlying variables, and also assessed the percentage of individuals that could be correctly assigned to study sites based on the discriminant classification functions (we present the raw classification and jackknife resampled classification matrixes). We evaluated the "F-to-remove" statistic $\left(\mathrm{F}_{\mathrm{TR}}\right)$ to determine which variables were most important for discriminating between groups. $\mathrm{F}_{\mathrm{TR}}$ is a partial multivariate $\mathrm{F}$ statistic that tests the significance of the decrease in discrimination power should that variable be removed from the model. We report on between-group differences for those variables with $\mathrm{F}_{\mathrm{TR}}$ values greater than $(>) 3$. 


\section{Results}

The health assessment dataset (table 4) consisted of a total of 144 sea otters, 75 from Monterey and 69 from Big Sur. At Monterey, the sample consisted of 17 males and 58 females, and at Big Sur, the sample consisted of 11 males and 58 females. Taken together, health profiles were significantly different between sexes and sites (Wilks's lambda $\lambda_{U}=0.642$, associated F statistic with 24/376 degrees of freedom $\mathrm{F}_{24,376}=2.66, \mathrm{P}=0.0001$ ), although a discriminant function was only able to classify otters correctly by sex and study site 49 percent of the time (Jackknife classification accuracy $=45$-percent). The variables most important in distinguishing between groups were subjective weight assessment ("Fto-remove" statistic FTR $=4.98)$, physical exam $\left(\mathrm{F}_{\mathrm{TR}}=9.27\right)$ and the blood differential counts $\left(\mathrm{F}_{\mathrm{TR}}=\right.$ 3.79). In terms of subjective weight assessments, Big Sur females and males had higher scores and thus were in poorer condition than their Monterey counterparts $\left(\mathrm{F}_{\mathrm{TR}}=1.67\right.$ and 2.18 for females and males at Big Sur, compared to 1.55 and 1.35 for females and males at Monterey), although the difference was greater for males (see chapter 7 for more detailed analysis of differences in weight and body condition). In terms of overall physical exam, Big Sur females and males, and Monterey females were all essentially equal $\left(\mathrm{F}_{\mathrm{TR}}=1.48,1.54,1.55\right.$ respectively), but Monterey males were in poorer condition $\left(F_{T R}=2.59\right)$. In terms of blood differential counts, Big Sur females showed more abnormalities than Monterey females $\left(F_{T R}=1.50\right.$ compared to 1.22), however Big Sur males showed fewer abnormalities than Monterey males $\left(\mathrm{F}_{\mathrm{TR}}=1.27\right.$ compared to 1.47$)$.

The complete clinical pathology panel dataset (table 5) represents 143 total samples composed of 119 individuals, 97 of which were sampled once, 20 of which were sampled twice, and 2 of which were sampled three times. The difference in sample size between the health assessment data and data for clinical pathology is attributable to two factors - (1) unacceptable logistical delay in getting samples delivered from collection site to laboratory (artifactually spurious results) or, (2) submission to a reference laboratory other than IDEXX (inconsistent methodology). Considered altogether, there were significant differences in clinical pathology statistics between sexes and sites $\left(\lambda_{U}=0.093, F_{24,376}=3.31\right.$, $\mathrm{P}<0.0001$ ), and a discriminant function was able to classify otters correctly by sex and study site 90percent of the time (Jackknife classification accuracy $=63$-percent). The variables most important in distinguishing between groups were serum creatinine $\left(\mathrm{F}_{\mathrm{TR}}=5.61\right)$, serum cholesterol $\left(\mathrm{F}_{\mathrm{TR}}=5.15\right)$, blood glucose $\left(\mathrm{F}_{\mathrm{TR}}=3.64\right)$, percent basophil $\left(\mathrm{F}_{\mathrm{TR}}=3.45\right)$ and absolute basophil counts $\left(\mathrm{F}_{\mathrm{TR}}=3.01\right)$. Serum creatinine was higher in Big Sur females and males compared to Monterey females and males $\left(\mathrm{F}_{\mathrm{TR}}=0.39\right.$ and 0.56 compared to 0.38 and 0.44 , respectively $)$, as were serum cholesterol $\left(\mathrm{F}_{\mathrm{TR}}=198.0\right.$ and 157.8 compared to 156.8 and 132.5 , respectively) and basophil metrics ( $F_{T R}=0.07$ and 0.31 compared to 0.03 and 0.17 for percent basophil, respectively, and $F_{T R}=3.96$ and 17.46 compared to 2.50 and 15.05 for basophil counts, respectively), whereas blood glucose in females was higher at Big Sur than at Monterey $\left(\mathrm{F}_{\mathrm{TR}}=137.75\right.$ compared to 117.54), but in males was lower at Big Sur $\left(\mathrm{F}_{\mathrm{TR}}=\right.$ 124.67 compared to 122.89$)$.

\section{Discussion}

We did not see substantial variation in the health assessment scores of sea otters at the two study sites, and what differences we did see were insufficiently large to reliably distinguish between sites. At Big Sur, otters scored slightly worse on assessments of physical weight (females and males), physical exams (males only), and blood differential counts (females only), although males at Monterey scored slightly worse than males at Big Sur on the latter metric. At both sites, individuals were in relatively poor body condition (we use the term "poor condition" in this case to refer to evidence of nutritional stress - that is, relatively low weight-at-age) as compared to sea otters from other populations in California and in Alaska, British Columbia, and Washington (see chapter 7). Most anomalies identified 
on physical examination were relatively minor in nature and not unexpected in populations that are at or near food-dictated carrying capacity. Male sea otters tended to have various peripheral limb injuries of varying degrees of seriousness likely associated with intra-specific trauma. Bite wounds, local and (or) regional cellulitis and associated lymph node reaction, and occasional septic arthritis or osteomyelitis predominated.

Female sea otters had similar peripheral wounds, but they did not seem as common. There were, however, a substantial number of injuries to the nose and muzzle of female otters. The injuries varied in nature from relatively minor and healing wounds to severe degloving injuries with loss of part or the entire nasal pad, maceration of tissue, and severe regional infection and inflammation. In some cases, the swelling associated with these wounds negatively impacted the ability of the otter to breathe nasally, forcing the animal to open-mouthed breathing. A number of females also showed systemic effects of long-term negative calorie balance associated with pup rearing. These females were substantially underweight, but also had physical and clinico-pathological changes associated with inanition, such as anemia, azotemia, and hypoproteinemia/hypoalbuminemia.

Dental disease seemed to be common, and the incidence was similar in both populations. The evidence seemed to suggest that the severity of the anomalies present in the oral cavity increased with the age of the animal; however, such a conclusion is significantly biased, as subjective age determination is determined in part by dental wear patterns. There also may be a relation between dental disease and diet, as it is not possible to differentiate wear patterns, particularly in the pre-molars and molars, between the normal physiologic wear of tooth surfaces as they occlude (attrition) and the abnormal wear of tooth surfaces from contact with external objects (abrasion). The nature of this relation, if it exists, remains to be described. Regardless, there is an apparent decline in the health and overall condition of the sea otter once dental health deteriorates to a certain point. Available data cannot describe the nature of this effect; however, the potential increased incidence of apical tooth abscessation, regional osteomyelitis, bacteremia and secondary endocarditis, and simply oral cavity pain are potential factors.

There were statistically significant differences in 5 of the 42 clinical pathology parameters either measured or calculated by the reference laboratory: creatinine, cholesterol, glucose, basophil percent, and basophil absolute count. Absolute counts are calculated by multiplying the total WBC by the percentage of a particular cell type; it is the absolute count that is of clinical significance even though the differential percentage is reported. Interestingly, although there are statistically significant differences between populations for these parameters, none of the values reported - despite some being greater than or less than published reference ranges - are considered clinically significant. This tends to imply that an explanation besides "disease" warrants consideration.

In this study, we determined that serum creatinine levels were significantly higher in Big Sur sea otters, both male and female, than in Monterey sea otters. In clinical medicine, serum creatinine is considered to be an indicator test for renal disease, specifically glomerular filtration rate. In dogs and cats, however, it seems to be a relatively insensitive measure of kidney disease, as values tend not to elevate until about 75 percent of renal function is compromised.

There are aspects of creatinine metabolism that may be influenced by sea otter diets and (or) general condition. Creatinine is a breakdown product of creatine phosphate found in muscle tissue. As a result, diets high in muscle tend to result in higher circulating creatinine. Because the compound tends to be produced endogenously at a relatively constant rate in muscle, animals with greater muscle mass tend to have higher levels. Conversely, decreased values are often seen in animals with muscle disease or systemic wasting. In this study, only one of the 41 sub-reference range results occurred in a male; the remainder were (not surprisingly) found in females. 
Serum cholesterol values also were higher in Big Sur males and females than in Monterey otters. This parameter is rarely used clinically in domestic carnivores, despite its importance in health screening in primates. Dogs and cats typically are resistant to atherosclerosis because of the absence of an important transfer protein. As a result, this parameter typically is not considered clinically significant. Its presence on standardized serum chemistry profiles likely is an indication of the manufacturer design emphasis on the human patient. There may be some degree of physiologic variation in serum cholesterol values based on the diet, body condition, athleticism (exercise), and reproductive status of the animal; one or more of these factors may explain the difference between Big Sur and Monterey, but we are unable to determine which factor or factors based on this study.

Blood glucose values were statistically higher in Big Sur females and Monterey males than in their respective counterparts. These differences, although statistically significant, did not appear to have any health-related relevance, as all values were within reference ranges. This parameter may be somewhat labile in free-ranging sea otters. One might anticipate some degree of postprandial elevation in blood glucose; however, the degree to which this occurs is tied closely to the amount of carbohydrate in the meal. In this study, a postprandial effect may be significant, as many of the animals are captured after feeding bouts. Another cause for physiologic elevations in blood glucose is the transient hyperglycemia associated with catecholamine release, the "fight or flight response." Differences in behavior between individuals, transport distance, and holding time may all influence the degree to which captured, free-ranging sea otters manifest this adaptive phenomenon.

Basophil counts, both differential percentage and absolute, also were higher in Big Sur sea otters than in Monterey otters. As described for the other three statistically significant parameters, most basophil counts (only one Monterey male was outside the reference range) were within the reference range. Basophils are the most uncommon cell type found in peripheral blood. They develop and mature under the influence of interleukin-3 (IL-3). The function of the basophil is not well understood, but it does appear to have a role in immediate and delayed hypersensitivity, as well as increasing in the face of parasitism. In the case of sea otters, there may be a relation between abundance of this cell and nasal mite (Halarachne sp.) infestation.

Evaluation of blood-based clinical pathology data collected one time from free-ranging sea otters is inherently problematic. In the case of the sea otter, published reference ranges are based on samples collected at least a decade ago, analyzed using different methodology, and from a different subspecies, the northern sea otter (Enhydra lutris kenyoni). Although the significance of this difference is difficult to quantify, its potential must be recognized. More importantly, however, the most effective use of clinicopathologic data involves the evaluation of parameters over time in an effort to detect and interpret trends in the patient. Obviously, this is not possible, or is at least highly impractical, for free-ranging wildlife. As a result, some of the information collected may represent clinically significant pathology in the earliest stages of disease, before the disease can be identified. This shortcoming is unavoidable in wildlife medicine.

In summary, there was little evidence to support a biologically meaningful difference in health assessments between sites nor evidence of any major disease process impeding health of individuals from either population. We recognize that this conclusion may to some degree indicate the lack of sensitivity associated with health evaluation based on physical examination and single, routine $\mathrm{CBC} /$ serum chemistry panels. We also recognize that even minor differences in disease exposure or health threats could translate into differences in survival or reproductive outcomes (see chapter 8), and we noted some very minor differences in health metrics that may have contributed to slightly lower survival for Big Sur animals compared to Monterey animals. However, the results presented here suggest that there are no overt differences between the sites with respect to patterns of animal health or disease incidence. Samples collected and archived provide future opportunities to evaluate specific 
causes of sea otter pathology, either pathogen or contaminant related (for example, see chapter 9 for an analysis of protozoal infection rates), which may shed a different light on the data collected.

\section{References Cited}

Arnemo, J.M., and Caulkett, N., 2007, Stress, chap 8. of West, G., Heard D., and Caulkett, N., eds., Zoo animal and wildlife immobilization and anesthesia: Ames, Iowa, Blackwell Publishing, p. 103-110.

Bornez, R., Linares. M.B., and Vergara. H., 2009, Haematological, hormonal and biochemical blood parameters in lamb_-Effect of age and blood sampling time. Livestock Science, v. 121, nos. 1-2, p. 200-206.

Calvert, C.A., and Thomason, J.D., 2012, Cardiovascular infections, in Greene, C.E., ed., Infectious diseases of the dog and cat (4th ed.): New York City, NY. Elsevier, p. 912-936.

Evans, E.W., 2011, Proteins, lipids, and carbohydrates, in Latimer, K.S., ed., Duncan \& Prasse's veterinary laboratory medicine-Clinical pathology (5th ed.): Ames, Iowa, Wiley-Blackwell, p. 173209.

Glickman, L.T., Glickman, N.W., Moore, G.E., Goldstein, G.S., and Lewis, H.B., 2009, Evaluation of the risk of endocarditis and other cardiovascular events on the basis of the severity of periodontal disease in dogs: Journal of the American Veterinary Medical Association, v. 234, no. 4, p. 486-494.

Monson, D.H., McCormick, C., and Ballachey, B.F., 2001, Chemical anesthesia of northern sea otters (Enhydra lutris) — Results of past field studies: Journal of Zoo and Wildlife Medicine, v. 32, no. 2, p. 181-189.

Pohlman, L.M., 2010, Basophils, mast cells, and their disorders, in Weiss, D.J., and Wardrop, K.J., eds., Schalm's veterinary hematology (6th ed.); Ames, Iowa, Wiley-Blackwell, p. 290-297.

Price, H.L., and Ohnishi, S.T., 1980, Effects of anesthetics on the heart: Federation Proceedings, v. 39, no. 5, p. 1575-1579.

Rebar, A.H., Lipscomb, T.P., Harris, R.K., and Ballachey, B.E., 1995, Clinical and clinical laboratory correlates in sea otters dying unexpectedly in rehabilitation centers following the Exxon Valdez oil spill: Veterinary Pathology, v. 32, p. 346-350.

Tripathi, N.K., Gregory, C.R., and Latimer, K.S., 2011, Urinary system, in Latimer, K.S., ed., Duncan \& Prasse's veterinary laboratory medicine_Clinical pathology (5th ed.): Ames, Iowa, WileyBlackwell, p. 253-282.

Webb, J.L., and Latimer, K.S., 2011, Leukocytes, in Latimer, K.S., ed., Duncan \& Prasse's veterinary laboratory medicine-Clinical pathology (5th ed.): Ames, Iowa, Wiley-Blackwell: p. 45-82.

Williams, T.D., Rebar, A.H., Teclaw, R.F., and Yoos, P.E., 1992, Influence of age, sex, capture technique, and restraint on hematologic measurements and serum chemistries of wild California sea otters; Veterinary Clinical Pathology, v. 21, no. 4, p. 106-110.

Winer, J.N., and others, 2013, The dental pathology of southern sea otters (Enhydra lutris nereis): Journal of Comparative Pathology, v. 149, nos. 2-3, p. 346-355. 
Table 3. Scoring matrix for sea otter subjective health assessment.

[Parameter: Hgb, hemoglobin; PCV, packed cell volume; WBC, total white blood cell count]

\begin{tabular}{|c|c|c|}
\hline \multirow{2}{*}{ Parameter } & \multicolumn{2}{|c|}{ Scoring range } \\
\cline { 2 - 3 } & Low (normal) & High (abnormal) \\
\hline Gross condition & & 5 \\
\hline Subjective weight & 1 & 10 \\
\hline Physical exam & 1 & \\
\hline Dental exam & & 5 \\
\hline Incisor & 1 & \\
\hline Canine & 1 & 5 \\
\hline Molar/pre-molar & 1 & 5 \\
\hline Blood parameters & & 5 \\
\hline PCV & 1 & 5 \\
\hline Hgb & 1 & 5 \\
\hline WBC & 1 & 10 \\
\hline Differential count & 1 & \\
\hline Serum chemistry panel & 1 & \\
\hline
\end{tabular}


Table 4. Health assessment scores for 144 sea otters screened with respect to 10 parameters and captured at Monterey and Big Sur study sites, central California.

[See table 3 for ranking scale of each parameter. For each individual otter, the otter identification number (Otter ID), date of capture, sex and study site are also shown. Study site: BSR, Big Sur coast; MON, Monterey Peninsula. Sex: F, female; M, male. Subj weight: Subjective weight. Total cond: Total condition. PVC: Packed cell volume. Hgb: Hemoglobin. WBC: Total while blood cell count. Diff count: Blood differential count. Serum chem: Serum chemistry. Total Blood: a score based on the evaluation of the individual components of the blood panel]

\begin{tabular}{|c|c|c|c|c|c|c|c|c|c|c|c|c|c|c|c|c|}
\hline \multirow[b]{2}{*}{$\begin{array}{l}\text { Otter } \\
\text { ID }\end{array}$} & \multirow[b]{2}{*}{$\begin{array}{l}\text { Capture } \\
\text { date }\end{array}$} & \multirow[b]{2}{*}{$\begin{array}{l}\text { Study } \\
\text { site }\end{array}$} & \multirow[b]{2}{*}{ Sex } & \multicolumn{3}{|c|}{$\begin{array}{l}\text { Physical } \\
\text { findings }\end{array}$} & \multirow[b]{2}{*}{ 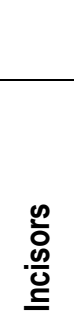 } & \multicolumn{3}{|c|}{ Dental exam } & \multicolumn{6}{|c|}{ Blood-based parameters } \\
\hline & & & & 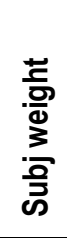 & 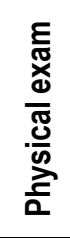 & 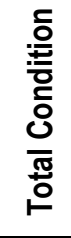 & & 迎 & 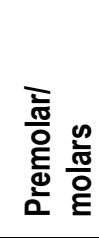 & 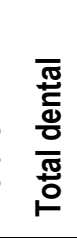 & ऐ্র & 음 & 罗 & $\begin{array}{l}\text { 䓂 } \\
\text { ơ } \\
\text { 告 }\end{array}$ & 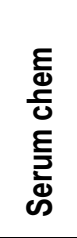 & 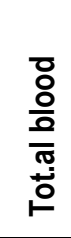 \\
\hline 1170.10 & $12 / 6 / 10$ & MON & M & 3 & 1 & 4 & 1 & 1 & 1 & 3 & 1 & 1 & 1 & 2 & 1 & 6 \\
\hline 945.03 & $6 / 10 / 09$ & MON & M & 1 & 2 & 3 & 2 & 3 & 3 & 8 & 1 & 1 & 2 & 1 & 1 & 6 \\
\hline 985.04 & $6 / 8 / 10$ & MON & $M$ & 2 & 3 & 5 & 3 & 3 & 4 & 10 & 1 & 1 & 1 & 1 & 1 & 5 \\
\hline 998.05 & $2 / 26 / 08$ & MON & M & 1 & 6 & 7 & 1 & 1 & 2 & 4 & 1 & 1 & 1 & 2 & 1 & 6 \\
\hline 1024.06 & $6 / 9 / 09$ & MON & M & 2 & 7 & 9 & 4 & 5 & 5 & 14 & 1 & 1 & 1 & 1 & 1 & 5 \\
\hline 1030.06 & $4 / 23 / 09$ & MON & M & 1 & 3 & 4 & 2 & 2 & 2 & 6 & 1 & 1 & 1 & 2 & 1 & 6 \\
\hline 1030.06 & $12 / 16 / 10$ & MON & M & 1 & 2 & 3 & 2 & 2 & 2 & 6 & 1 & 1 & 1 & 2 & 1 & 6 \\
\hline 1065.08 & $4 / 22 / 09$ & MON & M & 1 & 3 & 4 & 2 & 2 & 2 & 6 & 1 & 1 & 1 & 2 & 1 & 6 \\
\hline 1108.09 & $4 / 22 / 09$ & MON & $M$ & 2 & 4 & 6 & 2 & 2 & 2 & 6 & 1 & 1 & 1 & 2 & 1 & 6 \\
\hline 1108.09 & $6 / 9 / 10$ & MON & M & 1 & 1 & 2 & 2 & 2 & 4 & 8 & 1 & 1 & 1 & 1 & 2 & 6 \\
\hline 1114.09 & $4 / 29 / 09$ & MON & M & 2 & 1 & 3 & 1 & 1 & 1 & 3 & 2 & 2 & 1 & 1 & 1 & 7 \\
\hline 1114.09 & $12 / 15 / 10$ & MON & $M$ & 1 & 1 & 2 & 1 & 1 & 1 & 3 & 1 & 1 & 1 & 2 & 1 & 6 \\
\hline 1116.09 & $5 / 28 / 09$ & MON & M & 1 & 3 & 4 & 1 & 1 & 1 & 3 & 1 & 1 & 1 & 2 & 1 & 6 \\
\hline 1118.09 & $5 / 28 / 09$ & MON & M & 1 & 3 & 4 & 1 & 1 & 1 & 3 & 1 & 1 & 1 & 1 & 2 & 6 \\
\hline 1121.09 & 6/10/09 & MON & M & 1 & 2 & 3 & 2 & 1 & 1 & 4 & 1 & 1 & 1 & 1 & 1 & 5 \\
\hline 1131.08 & $11 / 11 / 08$ & MON & M & 1 & 1 & 2 & 1 & 1 & 1 & 3 & 1 & 1 & 1 & 1 & 1 & 5 \\
\hline 1156.10 & $2 / 2 / 10$ & MON & M & 1 & 1 & 2 & 2 & 1 & 2 & 5 & 1 & 1 & 1 & 1 & 2 & 6 \\
\hline 941.03 & $4 / 22 / 09$ & MON & $F$ & 1 & 1 & 2 & 2 & 2 & 3 & 7 & 1 & 1 & 1 & 1 & 1 & 5 \\
\hline 946.03 & $12 / 2 / 09$ & MON & $F$ & 2 & 2 & 4 & 2 & 1 & 3 & 6 & 1 & 1 & 1 & 1 & 2 & 6 \\
\hline 1000.05 & $6 / 17 / 09$ & MON & $\mathrm{F}$ & 1 & 1 & 2 & 2 & 1 & 1 & 4 & 1 & 1 & 1 & 1 & 1 & 5 \\
\hline 1011.06 & $7 / 28 / 10$ & MON & $F$ & 1 & 2 & 3 & 2 & 1 & 2 & 5 & 1 & 1 & 1 & 1 & 1 & 5 \\
\hline 1016.06 & $4 / 29 / 09$ & MON & $F$ & 2 & 2 & 4 & 3 & 2 & 2 & 7 & 1 & 1 & 1 & 2 & 1 & 6 \\
\hline 1017.06 & $4 / 29 / 09$ & MON & $F$ & 1 & 3 & 4 & 2 & 2 & 2 & 6 & 1 & 1 & 1 & 1 & 1 & 5 \\
\hline 1028.06 & $2 / 6 / 06$ & MON & $F$ & 1 & 1 & 2 & 1 & 1 & 1 & 3 & 1 & 1 & 1 & 1 & 2 & 6 \\
\hline 1029.06 & $8 / 20 / 09$ & MON & $F$ & 1 & 2 & 3 & 2 & 2 & 3 & 7 & 1 & 1 & 1 & 1 & 1 & 5 \\
\hline 1032.07 & $9 / 23 / 09$ & MON & $F$ & 1 & 1 & 2 & 2 & 1 & 2 & 5 & 1 & 1 & 1 & 1 & 1 & 5 \\
\hline 1032.07 & $12 / 15 / 10$ & MON & $F$ & 1 & 2 & 3 & 2 & 2 & 3 & 7 & 1 & 2 & 1 & 1 & 2 & 7 \\
\hline 1037.07 & $4 / 23 / 09$ & MON & $\mathrm{F}$ & 3 & 3 & 6 & 2 & 2 & 2 & 6 & 1 & 1 & 1 & 1 & 1 & 5 \\
\hline
\end{tabular}




\begin{tabular}{|c|c|c|c|c|c|c|c|c|c|c|c|c|c|c|c|c|}
\hline \multirow[b]{2}{*}{$\begin{array}{l}\text { Otter } \\
\text { ID }\end{array}$} & \multirow[b]{2}{*}{$\begin{array}{l}\text { Capture } \\
\text { date }\end{array}$} & \multirow[b]{2}{*}{$\begin{array}{l}\text { Study } \\
\text { site }\end{array}$} & \multirow[b]{2}{*}{ Sex } & \multicolumn{3}{|c|}{$\begin{array}{l}\text { Physical } \\
\text { findings }\end{array}$} & \multicolumn{4}{|c|}{ Dental exam } & \multicolumn{6}{|c|}{ Blood-based parameters } \\
\hline & & & & 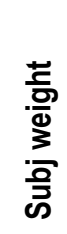 & 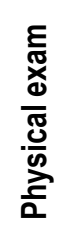 & 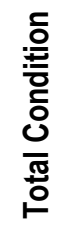 & $\begin{array}{l}\stackrel{0}{\circ} \\
\frac{0}{0} \\
\stackrel{0}{\varrho}\end{array}$ & $\begin{array}{l}\stackrel{\mathscr{E}}{E} \\
\stackrel{5}{\bar{N}}\end{array}$ & 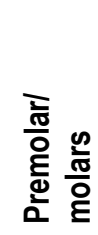 & 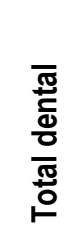 & ্ㅗㅁ & 음 & 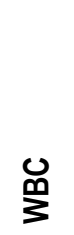 & $\begin{array}{l}\text { 芌 } \\
\text { ठั } \\
\text { 告 }\end{array}$ & 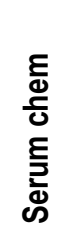 & $\begin{array}{l}\text { 음 } \\
\frac{0}{0} \\
\frac{\pi}{0} \\
\text { 믐 }\end{array}$ \\
\hline 1037.07 & $10 / 27 / 10$ & MON & $F$ & 1 & 2 & 3 & 3 & 2 & 3 & 8 & 1 & 2 & 1 & 2 & 2 & 8 \\
\hline 1038.07 & $7 / 29 / 10$ & MON & $\mathrm{F}$ & 1 & 1 & 2 & 1 & 2 & 1 & 4 & 1 & 1 & 1 & 1 & 1 & 5 \\
\hline 1042.07 & $7 / 29 / 10$ & MON & $\mathrm{F}$ & 1 & 1 & 2 & 2 & 1 & 1 & 4 & 1 & 2 & 1 & 1 & 1 & 6 \\
\hline 1046.07 & $6 / 10 / 09$ & MON & $\mathrm{F}$ & 2 & 4 & 6 & 3 & 3 & 4 & 10 & 1 & 1 & 1 & 2 & 2 & 7 \\
\hline 1049.07 & $9 / 27 / 07$ & MON & $\mathrm{F}$ & 2 & 1 & 3 & 2 & 1 & 1 & 4 & 1 & 1 & 1 & 1 & 1 & 5 \\
\hline 1050.07 & $9 / 27 / 07$ & MON & $\mathrm{F}$ & 2 & 1 & 3 & 1 & 1 & 1 & 3 & 1 & 1 & 1 & 1 & 1 & 5 \\
\hline 1051.07 & 9/27/07 & MON & $\mathrm{F}$ & 2 & 1 & 3 & 1 & 1 & 1 & 3 & 1 & 1 & 1 & 1 & 2 & 6 \\
\hline 1052.07 & 9/27/07 & MON & $F$ & 2 & 1 & 3 & 1 & 1 & 1 & 3 & 1 & 1 & 1 & 1 & 2 & 6 \\
\hline 1053.07 & $7 / 14 / 09$ & MON & $\mathrm{F}$ & 2 & 3 & 5 & 1 & 1 & 1 & 3 & 1 & 1 & 3 & 1 & 3 & 9 \\
\hline 1053.07 & $6 / 7 / 10$ & MON & $\mathrm{F}$ & 2 & 1 & 3 & 2 & 1 & 1 & 4 & 1 & 1 & 1 & 2 & 1 & 6 \\
\hline 1056.08 & $8 / 26 / 09$ & MON & $\mathrm{F}$ & 1 & 1 & 2 & 1 & 1 & 1 & 3 & 1 & 1 & 1 & 1 & 1 & 5 \\
\hline 1056.08 & $12 / 2 / 09$ & MON & $\mathrm{F}$ & 1 & 1 & 2 & 1 & 1 & 1 & 3 & 1 & 1 & 1 & 1 & 1 & 5 \\
\hline 1057.08 & $12 / 16 / 10$ & MON & $\mathrm{F}$ & 1 & 1 & 2 & 1 & 1 & 1 & 3 & 1 & 1 & 1 & 2 & 1 & 6 \\
\hline 1064.08 & $8 / 24 / 09$ & MON & $\mathrm{F}$ & 2 & 1 & 3 & 1 & 1 & 1 & 3 & 1 & 1 & 1 & 1 & 1 & 5 \\
\hline 1109.09 & $4 / 22 / 09$ & MON & $\mathrm{F}$ & 3 & 3 & 6 & 2 & 2 & 3 & 7 & 1 & 1 & 3 & 2 & 1 & 8 \\
\hline 1109.09 & $6 / 8 / 10$ & MON & $F$ & 3 & 3 & 6 & 3 & 2 & 4 & 9 & 1 & 1 & 1 & 1 & 1 & 5 \\
\hline 1110.09 & $4 / 23 / 09$ & MON & $\mathrm{F}$ & 1 & 2 & 3 & 2 & 2 & 2 & 6 & 1 & 1 & 1 & 1 & 1 & 5 \\
\hline 1110.09 & $10 / 27 / 10$ & MON & $\mathrm{F}$ & 1 & 1 & 2 & 2 & 2 & 2 & 6 & 1 & 1 & 1 & 1 & 1 & 5 \\
\hline 1111.09 & $4 / 23 / 09$ & MON & $\mathrm{F}$ & 1 & 1 & 2 & 1 & 1 & 1 & 3 & 1 & 1 & 1 & 1 & 1 & 5 \\
\hline 1113.09 & $4 / 29 / 09$ & MON & $F$ & 1 & 1 & 2 & 2 & 2 & 1 & 5 & 1 & 1 & 1 & 1 & 2 & 6 \\
\hline 1115.09 & $5 / 28 / 09$ & MON & $\mathrm{F}$ & 3 & 3 & 6 & 2 & 2 & 3 & 7 & 2 & 2 & 1 & 1 & 1 & 7 \\
\hline 1117.09 & $5 / 28 / 09$ & MON & $\mathrm{F}$ & 1 & 1 & 2 & 1 & 1 & 1 & 3 & 1 & 1 & 1 & 2 & 1 & 6 \\
\hline 1117.09 & $10 / 28 / 10$ & MON & $\mathrm{F}$ & 3 & 3 & 6 & 1 & 1 & 1 & 3 & 1 & 1 & 3 & 1 & 2 & 8 \\
\hline 1119.09 & 6/9/09 & MON & $\mathrm{F}$ & 2 & 2 & 4 & 2 & 1 & 1 & 4 & 1 & 1 & 1 & 2 & 1 & 6 \\
\hline 1120.09 & $6 / 9 / 09$ & MON & $\mathrm{F}$ & 1 & 2 & 3 & 3 & 3 & 3 & 9 & 1 & 1 & 1 & 1 & 2 & 6 \\
\hline 1122.09 & $6 / 10 / 09$ & MON & $\mathrm{F}$ & 1 & 1 & 2 & 1 & 1 & 1 & 3 & 1 & 1 & 1 & 1 & 1 & 5 \\
\hline 1122.09 & $10 / 7 / 10$ & MON & $F$ & 1 & 1 & 2 & 2 & 1 & 2 & 5 & 1 & 1 & 1 & 1 & 1 & 5 \\
\hline 1123.09 & $6 / 10 / 09$ & MON & $\mathrm{F}$ & 2 & 2 & 4 & 2 & 1 & 3 & 6 & 1 & 1 & 1 & 1 & 1 & 5 \\
\hline 1124.09 & $6 / 16 / 09$ & MON & $\mathrm{F}$ & 2 & 1 & 3 & 1 & 1 & 1 & 3 & 1 & 1 & 2 & 1 & 1 & 6 \\
\hline 1125.09 & $6 / 16 / 09$ & MON & $\mathrm{F}$ & 1 & 1 & 2 & 1 & 1 & 1 & 3 & 1 & 1 & 1 & 1 & 1 & 5 \\
\hline 1126.09 & $6 / 16 / 09$ & MON & $\mathrm{F}$ & 2 & 2 & 4 & 2 & 2 & 3 & 7 & 1 & 1 & 1 & 2 & 2 & 7 \\
\hline 1126.09 & $7 / 29 / 10$ & MON & $\mathrm{F}$ & 2 & 2 & 4 & 2 & 2 & 3 & 7 & 1 & 1 & 1 & 1 & 1 & 5 \\
\hline 1127.09 & $6 / 16 / 09$ & MON & $\mathrm{F}$ & 2 & 2 & 4 & 3 & 2 & 3 & 8 & 1 & 1 & 1 & 1 & 1 & 5 \\
\hline 1127.09 & $6 / 7 / 10$ & MON & $\mathrm{F}$ & 2 & 1 & 3 & 3 & 2 & 3 & 8 & 1 & 1 & 1 & 2 & 1 & 6 \\
\hline 1128.09 & $6 / 17 / 09$ & MON & $\mathrm{F}$ & 1 & 1 & 2 & 1 & 1 & 1 & 3 & 1 & 1 & 1 & 2 & 1 & 6 \\
\hline 1128.09 & $7 / 28 / 10$ & MON & $\mathrm{F}$ & 1 & 1 & 2 & 2 & 3 & 1 & 6 & 1 & 1 & 1 & 2 & 1 & 6 \\
\hline
\end{tabular}




\begin{tabular}{|c|c|c|c|c|c|c|c|c|c|c|c|c|c|c|c|c|}
\hline \multirow[b]{2}{*}{$\begin{array}{l}\text { Otter } \\
\text { ID }\end{array}$} & \multirow[b]{2}{*}{$\begin{array}{l}\text { Capture } \\
\text { date }\end{array}$} & \multirow[b]{2}{*}{$\begin{array}{l}\text { Study } \\
\text { site }\end{array}$} & \multirow[b]{2}{*}{ Sex } & \multicolumn{3}{|c|}{$\begin{array}{l}\text { Physical } \\
\text { findings }\end{array}$} & \multicolumn{4}{|c|}{ Dental exam } & \multicolumn{6}{|c|}{ Blood-based parameters } \\
\hline & & & & 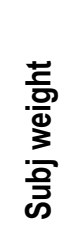 & 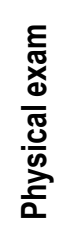 & 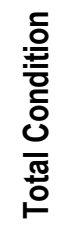 & $\begin{array}{l}\stackrel{0}{\circ} \\
\frac{0}{0} \\
\stackrel{0}{\varrho}\end{array}$ & $\begin{array}{l}\stackrel{\mathscr{E}}{E} \\
\stackrel{5}{\bar{N}}\end{array}$ & 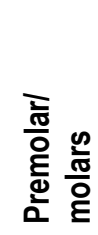 & 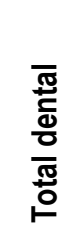 & ্ㅗㅁ & 음 & 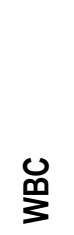 & $\begin{array}{l}\text { 芌 } \\
\text { ठั } \\
\text { 告 }\end{array}$ & 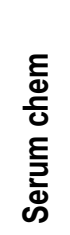 & $\begin{array}{l}\text { 음 } \\
\frac{0}{0} \\
\frac{\pi}{0} \\
\text { 믐 }\end{array}$ \\
\hline 1129.08 & $11 / 11 / 08$ & MON & $\mathrm{F}$ & 1 & 1 & 2 & 1 & 1 & 1 & 3 & 1 & 1 & 1 & 1 & 1 & 5 \\
\hline 1132.09 & $6 / 17 / 09$ & MON & $\mathrm{F}$ & 1 & 1 & 2 & 3 & 3 & 3 & 9 & 1 & 1 & 1 & 1 & 2 & 6 \\
\hline 1133.09 & $8 / 24 / 09$ & MON & $\mathrm{F}$ & 1 & 1 & 2 & 2 & 2 & 1 & 5 & 1 & 1 & 1 & 1 & 1 & 5 \\
\hline 1134.09 & $8 / 24 / 09$ & MON & $\mathrm{F}$ & 1 & 1 & 2 & 2 & 2 & 2 & 6 & 1 & 1 & 1 & 1 & 2 & 6 \\
\hline 1134.09 & $2 / 1 / 10$ & MON & $\mathrm{F}$ & 1 & 1 & 2 & 2 & 1 & 2 & 5 & 1 & 1 & 1 & 1 & 2 & 6 \\
\hline 1153.10 & $2 / 1 / 10$ & MON & $\mathrm{F}$ & 1 & 1 & 2 & 2 & 1 & 2 & 5 & 2 & 1 & 1 & 1 & 2 & 7 \\
\hline 1154.10 & $2 / 1 / 10$ & MON & $\mathrm{F}$ & 1 & 1 & 2 & 2 & 1 & 2 & 5 & 2 & 1 & 1 & 1 & 1 & 6 \\
\hline 1155.10 & $2 / 1 / 10$ & MON & $F$ & 1 & 1 & 2 & 1 & 1 & 1 & 3 & 2 & 1 & 1 & 1 & 1 & 6 \\
\hline 1157.10 & $6 / 7 / 10$ & MON & $\mathrm{F}$ & 3 & 1 & 4 & 2 & 2 & 2 & 6 & 1 & 1 & 1 & 1 & 1 & 5 \\
\hline 1158.10 & $7 / 28 / 10$ & MON & $\mathrm{F}$ & 3 & 3 & 6 & 2 & 2 & 3 & 7 & 1 & 1 & 1 & 2 & 1 & 6 \\
\hline 1130.08 & $11 / 6 / 08$ & MON & $\mathrm{F}$ & 2 & 1 & 3 & 2 & 2 & 2 & 6 & 1 & 1 & 1 & 1 & 3 & 7 \\
\hline 1091.08 & $11 / 6 / 08$ & BSR & $M$ & 3 & 2 & 5 & 2 & 3 & 3 & 8 & 1 & 1 & 1 & 1 & 2 & 6 \\
\hline 1093.08 & $11 / 6 / 08$ & BSR & $M$ & 2 & 2 & 4 & 2 & 1 & 1 & 4 & 1 & 1 & 1 & 1 & 2 & 6 \\
\hline 1094.08 & $11 / 7 / 08$ & BSR & $M$ & 3 & 2 & 5 & 4 & 2 & 2 & 8 & 1 & 1 & 1 & 2 & 2 & 7 \\
\hline 1094.08 & $11 / 3 / 09$ & BSR & $M$ & 2 & 2 & 4 & 3 & 2 & 4 & 9 & 1 & 1 & 1 & 1 & 1 & 5 \\
\hline 1095.08 & $11 / 5 / 08$ & BSR & $M$ & 2 & 1 & 3 & 2 & 1 & 2 & 5 & 1 & 1 & 1 & 1 & 1 & 5 \\
\hline 1096.08 & $11 / 7 / 08$ & BSR & $M$ & 3 & 2 & 5 & 3 & 2 & 4 & 9 & 1 & 1 & 1 & 1 & 1 & 5 \\
\hline 1099.08 & $11 / 7 / 08$ & BSR & $M$ & 3 & 1 & 4 & 2 & 1 & 1 & 4 & 1 & 1 & 1 & 2 & 1 & 6 \\
\hline 1137.09 & $11 / 3 / 09$ & BSR & $M$ & 2 & 2 & 4 & 3 & 3 & 4 & 10 & 1 & 1 & 1 & 1 & 2 & 6 \\
\hline 1143.09 & $11 / 4 / 09$ & BSR & $\mathrm{M}$ & 2 & 1 & 3 & 2 & 2 & 4 & 8 & 1 & 1 & 1 & 1 & 2 & 6 \\
\hline 1147.09 & $11 / 4 / 09$ & BSR & $M$ & 1 & 1 & 2 & 1 & 1 & 1 & 3 & 3 & 2 & 1 & 1 & 1 & 8 \\
\hline 1165.10 & $9 / 25 / 10$ & BSR & $M$ & 1 & 1 & 2 & 2 & 1 & 2 & 5 & 1 & 1 & 1 & 2 & 1 & 6 \\
\hline 1067.08 & $9 / 23 / 10$ & BSR & $\mathrm{F}$ & 2 & 2 & 4 & 2 & 1 & 3 & 6 & 1 & 1 & 1 & 2 & 1 & 6 \\
\hline 1067.08 & $11 / 5 / 08$ & BSR & $\mathrm{F}$ & 2 & 1 & 3 & 2 & 1 & 2 & 5 & 1 & 1 & 1 & 2 & 1 & 6 \\
\hline 1068.08 & $11 / 5 / 08$ & BSR & $\mathrm{F}$ & 2 & 2 & 4 & 1 & 1 & 1 & 3 & 1 & 1 & 1 & 2 & 1 & 6 \\
\hline 1069.08 & $11 / 5 / 08$ & BSR & $\mathrm{F}$ & 2 & 1 & 3 & 1 & 1 & 1 & 3 & 1 & 1 & 1 & 1 & 1 & 5 \\
\hline 1070.08 & $11 / 5 / 08$ & BSR & $F$ & 2 & 1 & 3 & 1 & 2 & 2 & 5 & 1 & 1 & 1 & 1 & 1 & 5 \\
\hline 1071.08 & $11 / 5 / 08$ & BSR & $\mathrm{F}$ & 2 & 2 & 4 & 2 & 2 & 4 & 8 & 1 & 1 & 1 & 1 & 1 & 5 \\
\hline 1072.08 & $11 / 5 / 08$ & BSR & $\mathrm{F}$ & 2 & 1 & 3 & 2 & 1 & 1 & 4 & 1 & 1 & 1 & 1 & 3 & 7 \\
\hline 1073.08 & $11 / 6 / 08$ & BSR & $\mathrm{F}$ & 2 & 1 & 3 & 1 & 2 & 2 & 5 & 1 & 1 & 1 & 2 & 1 & 6 \\
\hline 1074.08 & $11 / 6 / 08$ & BSR & $\mathrm{F}$ & 1 & 2 & 3 & 2 & 2 & 2 & 6 & 1 & 1 & 1 & 2 & 3 & 8 \\
\hline 1074.08 & $9 / 24 / 10$ & BSR & $\mathrm{F}$ & 2 & 2 & 4 & 3 & 3 & 3 & 9 & 1 & 1 & 1 & 2 & 1 & 6 \\
\hline 1075.08 & $11 / 6 / 08$ & BSR & $\mathrm{F}$ & 2 & 1 & 3 & 1 & 1 & 2 & 4 & 1 & 1 & 1 & 2 & 1 & 6 \\
\hline 1076.08 & $11 / 6 / 08$ & BSR & $\mathrm{F}$ & 1 & 1 & 2 & 2 & 2 & 2 & 6 & 1 & 1 & 1 & 2 & 2 & 7 \\
\hline 1077.08 & $11 / 7 / 08$ & BSR & $\mathrm{F}$ & 2 & 1 & 3 & 2 & 2 & 2 & 6 & 1 & 1 & 1 & 2 & 1 & 6 \\
\hline 1078.08 & $11 / 6 / 08$ & BSR & $\mathrm{F}$ & 1 & 3 & 4 & 3 & 3 & 3 & 9 & 1 & 1 & 1 & 2 & 1 & 6 \\
\hline
\end{tabular}




\begin{tabular}{|c|c|c|c|c|c|c|c|c|c|c|c|c|c|c|c|c|}
\hline \multirow[b]{2}{*}{$\begin{array}{l}\text { Otter } \\
\text { ID }\end{array}$} & \multirow[b]{2}{*}{$\begin{array}{l}\text { Capture } \\
\text { date }\end{array}$} & \multirow[b]{2}{*}{$\begin{array}{l}\text { Study } \\
\text { site }\end{array}$} & \multirow[b]{2}{*}{ Sex } & \multicolumn{3}{|c|}{$\begin{array}{l}\text { Physical } \\
\text { findings }\end{array}$} & \multirow[b]{2}{*}{$\begin{array}{l}\stackrel{\mathscr{\nu}}{\circ} \\
\frac{. \mathscr{N}}{0} \\
\underline{=}\end{array}$} & \multicolumn{3}{|c|}{ Dental exam } & \multicolumn{6}{|c|}{ Blood-based parameters } \\
\hline & & & & $\begin{array}{l}\text { 등 } \\
\frac{0}{0} \\
\frac{3}{0} \\
\frac{0}{\bar{D}}\end{array}$ & 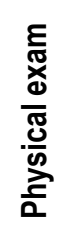 & 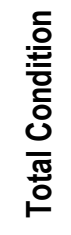 & & 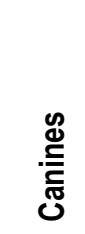 & 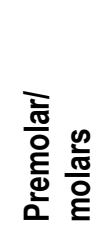 & 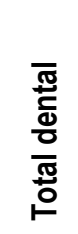 & ঠे & 음 & 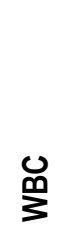 & $\begin{array}{l}\text { 䓂 } \\
\text { 品 } \\
\text { 告 }\end{array}$ & 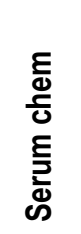 & $\begin{array}{l}\text { 음 } \\
\frac{\text { o }}{0} \\
\frac{\pi}{\sigma} \\
\stackrel{0}{0}\end{array}$ \\
\hline 1079.08 & $11 / 7 / 08$ & BSR & $\mathrm{F}$ & 1 & 1 & 2 & 1 & 1 & 1 & 3 & 1 & 1 & 1 & 1 & 1 & 5 \\
\hline 1080.08 & $11 / 7 / 08$ & BSR & $\mathrm{F}$ & 1 & 1 & 2 & 2 & 1 & 1 & 4 & 1 & 1 & 1 & 1 & 1 & 5 \\
\hline 1081.08 & $11 / 7 / 08$ & BSR & $\mathrm{F}$ & 2 & 2 & 4 & 3 & 3 & 4 & 10 & 1 & 1 & 1 & 1 & 3 & 7 \\
\hline 1082.08 & $11 / 7 / 08$ & BSR & $\mathrm{F}$ & 1 & 2 & 3 & 2 & 2 & 2 & 6 & 1 & 1 & 1 & 2 & 3 & 8 \\
\hline 1083.08 & $11 / 7 / 08$ & BSR & $\mathrm{F}$ & 2 & 2 & 4 & 3 & 2 & 3 & 8 & 1 & 1 & 1 & 2 & 1 & 6 \\
\hline 1084.08 & $11 / 7 / 08$ & BSR & $\mathrm{F}$ & 1 & 1 & 2 & 1 & 2 & 1 & 4 & 1 & 1 & 1 & 2 & 1 & 6 \\
\hline 1085.08 & $11 / 8 / 08$ & BSR & $\mathrm{F}$ & 1 & 1 & 2 & 1 & 1 & 1 & 3 & 1 & 1 & 1 & 1 & 1 & 5 \\
\hline 1086.08 & $11 / 7 / 08$ & BSR & $\mathrm{F}$ & 2 & 3 & 5 & 1 & 1 & 1 & 3 & 1 & 1 & 1 & 2 & 1 & 6 \\
\hline 1087.08 & $11 / 8 / 08$ & BSR & $\mathrm{F}$ & 2 & 7 & 9 & 1 & 1 & 1 & 3 & 1 & 1 & 4 & 1 & 4 & 11 \\
\hline 1088.08 & $11 / 10 / 08$ & BSR & $\mathrm{F}$ & 2 & 1 & 3 & 2 & 2 & 1 & 5 & 1 & 1 & 1 & 1 & 1 & 5 \\
\hline 1089.08 & $11 / 6 / 08$ & BSR & $\mathrm{F}$ & 2 & 1 & 3 & 2 & 2 & 2 & 6 & 1 & 1 & 1 & 2 & 1 & 6 \\
\hline 1090.08 & $11 / 10 / 08$ & BSR & $\mathrm{F}$ & 2 & 4 & 6 & 2 & 2 & 4 & 8 & 1 & 1 & 1 & 2 & 2 & 7 \\
\hline 1092.08 & $11 / 11 / 08$ & BSR & $\mathrm{F}$ & 3 & 4 & 7 & 2 & 2 & 2 & 6 & 4 & 4 & 1 & 2 & 2 & 13 \\
\hline 1097.08 & $11 / 11 / 08$ & BSR & $F$ & 2 & 1 & 3 & 1 & 2 & 1 & 4 & 1 & 1 & 1 & 1 & 1 & 5 \\
\hline 1097.08 & $9 / 24 / 10$ & BSR & $F$ & 2 & 1 & 3 & 3 & 2 & 2 & 7 & 1 & 1 & 1 & 2 & 2 & 7 \\
\hline 1098.08 & $11 / 11 / 08$ & BSR & $\mathrm{F}$ & 3 & 1 & 4 & 2 & 1 & 1 & 4 & 1 & 1 & 1 & 1 & 2 & 6 \\
\hline 1103.08 & $11 / 11 / 08$ & BSR & $\mathrm{F}$ & 3 & 1 & 4 & 3 & 3 & 3 & 9 & 1 & 1 & 1 & 1 & 1 & 5 \\
\hline 1104.08 & $11 / 11 / 08$ & BSR & $\mathrm{F}$ & 2 & 1 & 3 & 2 & 2 & 2 & 6 & 1 & 1 & 1 & 1 & 2 & 6 \\
\hline 1105.08 & $11 / 11 / 08$ & BSR & $\mathrm{F}$ & 2 & 1 & 3 & 1 & 2 & 2 & 5 & 1 & 1 & 1 & 2 & 1 & 6 \\
\hline 1106.08 & $11 / 11 / 08$ & BSR & $\mathrm{F}$ & 2 & 2 & 4 & 3 & 3 & 3 & 9 & 1 & 1 & 1 & 1 & 1 & 5 \\
\hline 1135.09 & $11 / 2 / 09$ & BSR & $\mathrm{F}$ & 2 & 2 & 4 & 1 & 1 & 1 & 3 & 1 & 1 & 1 & 1 & 1 & 5 \\
\hline 1136.09 & $11 / 2 / 09$ & BSR & $\mathrm{F}$ & 3 & 2 & 5 & 1 & 1 & 2 & 4 & 1 & 1 & 1 & 1 & 1 & 5 \\
\hline 1138.09 & $11 / 3 / 09$ & BSR & $\mathrm{F}$ & 1 & 1 & 2 & 1 & 1 & 1 & 3 & 1 & 1 & 1 & 1 & 1 & 5 \\
\hline 1139.09 & $11 / 3 / 09$ & BSR & $F$ & 2 & 1 & 3 & 2 & 1 & 1 & 4 & 1 & 1 & 1 & 1 & 1 & 5 \\
\hline 1140.09 & $11 / 3 / 09$ & BSR & $F$ & 1 & 1 & 2 & 3 & 4 & 3 & 10 & 1 & 1 & 1 & 1 & 4 & 8 \\
\hline 1141.09 & $11 / 3 / 09$ & BSR & $F$ & 1 & 1 & 2 & 2 & 2 & 2 & 6 & 1 & 1 & 1 & 1 & 3 & 7 \\
\hline 1141.09 & $9 / 25 / 10$ & BSR & $\mathrm{F}$ & 1 & 1 & 2 & 2 & 2 & 2 & 6 & 1 & 1 & 1 & 2 & 1 & 6 \\
\hline 1142.09 & $9 / 24 / 10$ & BSR & $F$ & 3 & 1 & 4 & 2 & 1 & 2 & 5 & 1 & 1 & 1 & 2 & 1 & 6 \\
\hline 1144.09 & $11 / 4 / 09$ & BSR & $\mathrm{F}$ & 1 & 1 & 2 & 1 & 1 & 1 & 3 & 1 & 1 & 1 & 2 & 1 & 6 \\
\hline 1145.09 & $11 / 4 / 09$ & BSR & $\mathrm{F}$ & 1 & 1 & 2 & 2 & 2 & 2 & 6 & 1 & 1 & 1 & 2 & 3 & 8 \\
\hline 1146.09 & $11 / 4 / 09$ & BSR & $\mathrm{F}$ & 1 & 1 & 2 & 1 & 1 & 1 & 3 & 1 & 1 & 1 & 1 & 1 & 5 \\
\hline 1148.09 & $11 / 5 / 09$ & BSR & $\mathrm{F}$ & 2 & 1 & 3 & 2 & 1 & 1 & 4 & 1 & 1 & 1 & 2 & 1 & 6 \\
\hline 1150.09 & $11 / 5 / 09$ & BSR & $\mathrm{F}$ & 1 & 1 & 2 & 2 & 2 & 1 & 5 & 1 & 1 & 1 & 1 & 2 & 6 \\
\hline 1151.09 & $11 / 5 / 09$ & BSR & $\mathrm{F}$ & 1 & 1 & 2 & 2 & 2 & 2 & 6 & 1 & 1 & 1 & 2 & 1 & 6 \\
\hline 1159.10 & $9 / 22 / 10$ & BSR & $\mathrm{F}$ & 1 & 1 & 2 & 1 & 1 & 1 & 3 & 1 & 1 & 1 & 2 & 1 & 6 \\
\hline 1160.10 & $9 / 22 / 10$ & BSR & $F$ & 1 & 1 & 2 & 1 & 1 & 1 & 3 & 1 & 1 & 1 & 1 & 3 & 7 \\
\hline
\end{tabular}




\begin{tabular}{|c|c|c|c|c|c|c|c|c|c|c|c|c|c|c|c|c|}
\hline \multirow[b]{2}{*}{$\begin{array}{l}\text { Otter } \\
\text { ID }\end{array}$} & \multirow[b]{2}{*}{$\begin{array}{l}\text { Capture } \\
\text { date }\end{array}$} & \multirow[b]{2}{*}{$\begin{array}{l}\text { Study } \\
\text { site }\end{array}$} & \multirow[b]{2}{*}{ Sex } & \multicolumn{3}{|c|}{$\begin{array}{l}\text { Physical } \\
\text { findings }\end{array}$} & \multicolumn{4}{|c|}{ Dental exam } & \multicolumn{6}{|c|}{ Blood-based parameters } \\
\hline & & & & 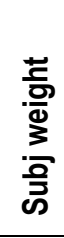 & 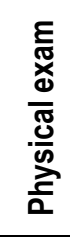 & 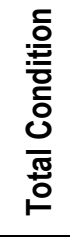 & 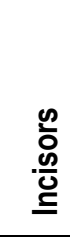 & 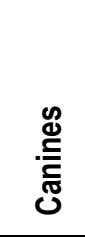 & 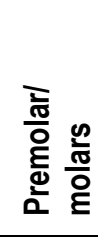 & 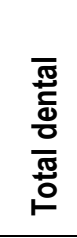 & 님 & 음 & 罗 & $\begin{array}{l}\text { 䓂 } \\
\text { ठ․ } \\
\text { 告 }\end{array}$ & 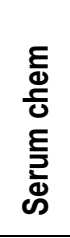 & 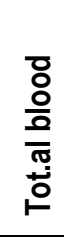 \\
\hline 1161.10 & $9 / 22 / 10$ & BSR & $\mathrm{F}$ & 3 & 1 & 4 & 2 & 3 & 2 & 7 & 1 & 1 & 1 & 2 & 1 & 6 \\
\hline 1162.10 & $9 / 23 / 10$ & BSR & $\mathrm{F}$ & 2 & 1 & 3 & 2 & 1 & 1 & 4 & 1 & 1 & 1 & 2 & 1 & 6 \\
\hline 1163.10 & $9 / 24 / 10$ & BSR & $\mathrm{F}$ & 1 & 1 & 2 & 1 & 1 & 1 & 3 & 1 & 1 & 1 & 1 & 1 & 5 \\
\hline 1164.10 & $9 / 24 / 10$ & BSR & $\mathrm{F}$ & 1 & 1 & 2 & 2 & 2 & 2 & 6 & 1 & 1 & 1 & 1 & 1 & 5 \\
\hline 1166.10 & $9 / 25 / 10$ & BSR & $\mathrm{F}$ & 1 & 2 & 3 & 1 & 1 & 1 & 3 & 1 & 1 & 2 & 1 & 4 & 9 \\
\hline 1167.10 & $9 / 25 / 10$ & BSR & $F$ & 1 & 1 & 2 & 1 & 1 & 2 & 4 & 1 & 1 & 3 & 1 & 1 & 7 \\
\hline 1168.10 & $9 / 27 / 10$ & BSR & $F$ & 1 & 1 & 2 & 3 & 2 & 3 & 8 & 1 & 3 & 1 & 2 & 1 & 8 \\
\hline 1169.10 & $9 / 27 / 10$ & BSR & $\mathrm{F}$ & 1 & 1 & 2 & 2 & 1 & 1 & 4 & 1 & 1 & 1 & 1 & 1 & 5 \\
\hline
\end{tabular}


Table 5. Clinical pathology results for 143 sea otters captured at Monterey and Big Sur study sites, central California.

[Blood samples were collected from each study animal at time of capture and sent to a diagnostic laboratory (IDEXX Laboratories, Westbrook, ME), which conducted tests and provided results on 42 standard blood parameters as shown. Also shown for each individual is the otter identification number (Otter ID [BRD number]), date of capture, sex (F, female; M, male), and study site (BSR, Big Sur coast; MON, Monterey Peninsula). For each otter; Alk Phos, alkaline phosphatase; ALT, alanine aminotransferase; AST, aspartate aminotransferase; CK, creatine kinase; Tot Prot, total protein; Glob, globulin; Tot Bili, total bilirubin; Dir Bili, direct bilirubin; BUN, blood urea nitrogen; Creat, creatinine; Chol, cholesterol; Gluc, glucose; Ca, calcium; Phos, phosphorus; TCO2, total carbon dioxide; Chlor, chloride; K, potassium; Na, sodium; A/G Ratio, albumin:globulin ratio; B/C Ratio, blood urea nitrogen:creatinine ratio]

\begin{tabular}{|c|c|c|c|c|c|c|c|c|c|c|c|c|c|c|c|c|c|c|c|c|c|c|c|c|}
\hline $\begin{array}{l}\text { Otter ID } \\
\text { (BRD } \\
\text { number) }\end{array}$ & $\begin{array}{c}\text { Capture } \\
\text { date }\end{array}$ & $\begin{array}{l}\text { Study } \\
\text { site }\end{array}$ & Sex & 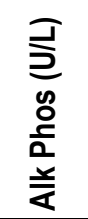 & 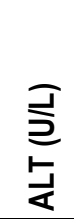 & $\underset{\frac{5}{2}}{\stackrel{D}{2}}$ & 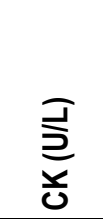 & $\begin{array}{l}\bar{\partial} \\
\frac{1}{0} \\
\text { 응 } \\
\frac{0}{\alpha}\end{array}$ & 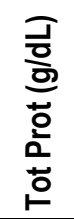 & $\begin{array}{l}\bar{\partial} \\
\frac{7}{0} \\
\text { 응 }\end{array}$ & 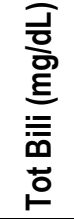 & $\begin{array}{l}\text { 흠 } \\
\text { 흘 } \\
\overline{\overline{0}} \\
. \overline{0}\end{array}$ & 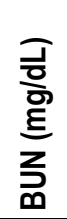 & 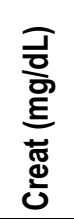 & $\begin{array}{l}\bar{\partial} \\
\text { 흥 } \\
\text { ᄐ) } \\
\overline{\frac{0}{0}}\end{array}$ & 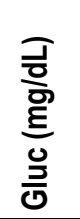 & 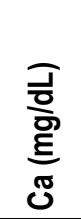 & 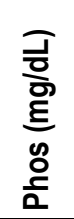 & 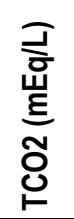 & 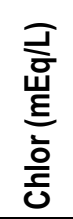 & 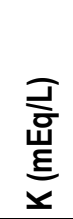 & 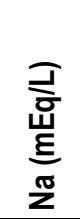 & 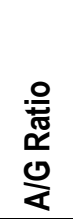 & 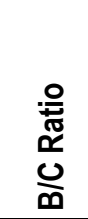 \\
\hline 945.03 & $06 / 11 / 09$ & MON & $M$ & 138 & 111 & 108 & 196 & 2.7 & 6.8 & 4.1 & 0.1 & 0.1 & 65 & 0.5 & 133 & 130 & 8.9 & 3.3 & 20 & 121 & 4.4 & 153 & 0.7 & 130 \\
\hline 985.04 & $06 / 09 / 10$ & MON & $M$ & 91 & 137 & 110 & 189 & 2.6 & 6.9 & 4.3 & 0 & 0 & 64 & 0.4 & 87 & 110 & 8.5 & 2.9 & 30 & 112 & 4.7 & 150 & 0.6 & 160 \\
\hline 998.05 & $02 / 27 / 08$ & MON & $M$ & 110 & 138 & 142 & 192 & 2.6 & 5.9 & 3.3 & 0.1 & 0.1 & 50 & 0.5 & 146 & 109 & 7.9 & 4.3 & 25 & 115 & 4 & 151 & 0.8 & 100 \\
\hline 1024.06 & 06/10/09 & MON & $M$ & 66 & 188 & 188 & 147 & 2.5 & 6.7 & 4.2 & 0.1 & 0.1 & 50 & 0.4 & 126 & 148 & 8.5 & 5.9 & 24 & 116 & 4.9 & 150 & 0.6 & 125 \\
\hline 1030.06 & $04 / 24 / 09$ & MON & M & 74 & 114 & 93 & 166 & 2.7 & 7 & 4.3 & 0.1 & 0.1 & 51 & 0.6 & 135 & 112 & 8.9 & 4.6 & 27 & 113 & 4.4 & 152 & 0.6 & 85 \\
\hline 1030.06 & $12 / 17 / 10$ & MON & $M$ & 105 & 166 & 145 & 169 & 2.6 & 7 & 4.4 & 0 & 0 & 71 & 0.4 & 151 & 146 & 8.2 & 3.7 & 26 & 113 & 4.1 & 149 & 0.6 & 177.5 \\
\hline 1065.08 & $04 / 23 / 09$ & MON & $M$ & 120 & 153 & 118 & 164 & 2.6 & 6.9 & 4.3 & 0 & 0 & 39 & 0.4 & 143 & 103 & 8.8 & 4.7 & 26 & 115 & 3.9 & 151 & 0.6 & 97.5 \\
\hline 1108.09 & $04 / 23 / 09$ & MON & $M$ & 98 & 176 & 158 & 293 & 2.4 & 6.8 & 4.4 & 0.1 & 0.1 & 51 & 0.5 & 128 & 102 & 8.4 & 4.4 & 27 & 111 & 4.5 & 147 & 0.5 & 102 \\
\hline 1108.09 & $06 / 09 / 10$ & MON & M & 131 & 266 & 318 & 207 & 2.5 & 6.6 & 4.1 & 0 & 0 & 75 & 0.4 & 137 & 91 & 9 & 2.9 & 27 & 116 & 4.3 & 151 & 0.6 & 187.5 \\
\hline 1114.09 & 05/01/09 & MON & $M$ & 98 & 180 & 173 & 1016 & 3 & 6.6 & 3.6 & 0.1 & 0.1 & 52 & 0.5 & 95 & 130 & 8.5 & 4.5 & 27 & 119 & 4.1 & 153 & 0.8 & 104 \\
\hline 1114.09 & $04 / 28 / 10$ & MON & $M$ & 90 & 103 & 133 & 253 & 2.7 & 6.5 & 3.8 & 0.1 & 0.1 & 64 & 0.4 & 103 & 112 & 7.8 & 4.3 & 27 & 119 & 4.5 & 154 & 0.7 & 160 \\
\hline 1114.09 & $12 / 16 / 10$ & MON & $M$ & 99 & 194 & 139 & 291 & 2.9 & 6.6 & 3.7 & 0.1 & 0.1 & 71 & 0.4 & 112 & 114 & 8.3 & 4.7 & 27 & 118 & 4.8 & 154 & 0.8 & 177.5 \\
\hline 1116.09 & 05/29/09 & MON & $M$ & 116 & 231 & 250 & 341 & 2.9 & 6.3 & 3.4 & 0.2 & 0.1 & 53 & 0.5 & 162 & 101 & 8.4 & 2.9 & 21 & 118 & 3.6 & 153 & 0.9 & 106 \\
\hline 1118.09 & 05/29/09 & MON & $M$ & 73 & 230 & 506 & 13014 & 2.7 & 6.3 & 3.6 & 0.1 & 0.1 & 43 & 0.4 & 147 & 181 & 8.4 & 4.4 & 27 & 117 & 4.2 & 155 & 0.8 & 107.5 \\
\hline 1121.09 & $06 / 11 / 09$ & MON & $M$ & 58 & 145 & 196 & 1695 & 2.8 & 6.9 & 4.1 & 0.2 & 0.1 & 53 & 0.5 & 135 & 93 & 8.7 & 3.5 & 23 & 120 & 4.1 & 157 & 0.7 & 106 \\
\hline 1131.08 & $11 / 14 / 08$ & MON & $M$ & 221 & 126 & 107 & 764 & 3.1 & 5.2 & 2.1 & 0.1 & 0.1 & 49 & 0.3 & 176 & 179 & 10.1 & 8.6 & 19 & 117 & 4.4 & 151 & 1.5 & 163.3 \\
\hline 1156.10 & $02 / 03 / 10$ & MON & $M$ & 100 & 87 & 98 & 294 & 2.7 & 7 & 4.3 & 0.2 & 0 & 73 & 0.5 & 126 & 102 & 8.3 & 3.6 & 22 & 117 & 4.1 & 153 & 0.6 & 146 \\
\hline 1170.10 & $12 / 17 / 10$ & MON & $M$ & 114 & 279 & 254 & 546 & 2.8 & 6.6 & 3.8 & 0.1 & 0 & 68 & 0.4 & 143 & 149 & 8.4 & 4.9 & 24 & 117 & 3.9 & 152 & 0.7 & 170 \\
\hline 941.03 & $04 / 24 / 09$ & MON & $\mathrm{F}$ & 84 & 68 & 65 & 204 & 2.7 & 6.8 & 4.1 & 0.2 & 0.1 & 38 & 0.4 & 128 & 147 & 8.8 & 4.5 & 28 & 116 & 3.8 & 155 & 0.7 & 95 \\
\hline 946.03 & $12 / 03 / 09$ & MON & $\mathrm{F}$ & 130 & 104 & 74 & 152 & 2.9 & 7.1 & 4.2 & 0.1 & 0 & 82 & 0.4 & 151 & 132 & 8.5 & 3.9 & 25 & 111 & 3.9 & 146 & 0.7 & 205 \\
\hline 1000.05 & 06/18/09 & MON & $\mathrm{F}$ & 103 & 147 & 91 & 217 & 2.9 & 6.3 & 3.4 & 0.2 & 0.1 & 65 & 0.5 & 213 & 138 & 8.9 & 3.5 & 25 & 116 & 3.6 & 152 & 0.9 & 130 \\
\hline
\end{tabular}




\begin{tabular}{|c|c|c|c|c|c|c|c|c|c|c|c|c|c|c|c|c|c|c|c|c|c|c|c|c|}
\hline $\begin{array}{l}\text { Otter ID } \\
\text { (BRD } \\
\text { number) }\end{array}$ & $\begin{array}{c}\text { Capture } \\
\text { date }\end{array}$ & $\begin{array}{l}\text { Study } \\
\text { site }\end{array}$ & Sex & 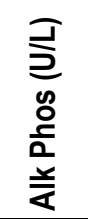 & 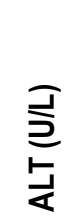 & $\underset{\frac{5}{2}}{\stackrel{Ð}{2}}$ & 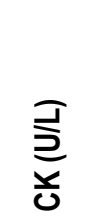 & $\begin{array}{l}\frac{\bar{\sigma}}{2} \\
\text { 흥 } \\
\frac{0}{\bar{\alpha}}\end{array}$ & 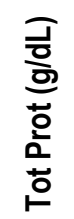 & $\begin{array}{l}\text { 긍 } \\
\text { 응 } \\
\text { 응 }\end{array}$ & 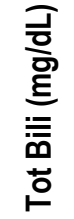 & 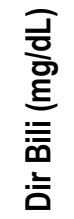 & 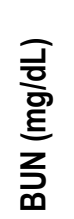 & 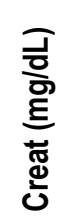 & 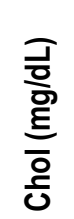 & 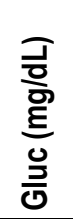 & 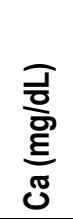 & 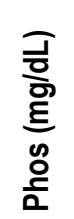 & 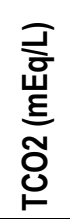 & 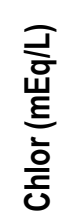 & 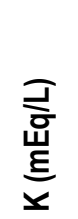 & 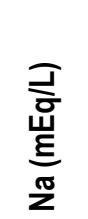 & 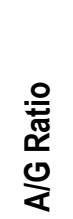 & 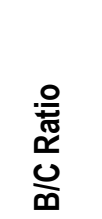 \\
\hline 1011.06 & $07 / 29 / 10$ & $\overline{\mathrm{ION}}$ & $F$ & 109 & 163 & 96 & 153 & 2.9 & 6.6 & 3.7 & 0.2 & 0.1 & 68 & 0.3 & 188 & 106 & 8.2 & 3.7 & 21 & 126 & 4.2 & 158 & 0.8 & 226.7 \\
\hline 1016.06 & 130100 & ON & $\mathrm{F}$ & 103 & 93 & 60 & 125 & 2.8 & 6.6 & 3.8 & 0.2 & 0.1 & 53 & 0.5 & 180 & 131 & & 4.9 & 21 & 118 & 3.7 & 152 & 0.7 & 106 \\
\hline 1017.06 & $04 / 30 / 09$ & MON & $\mathrm{F}$ & 82 & 108 & 81 & 287 & 3 & 7 & 4 & 0.6 & 0.2 & 51 & 0.3 & 128 & 107 & 8.9 & 3.8 & 22 & 117 & 3.6 & 153 & 0.8 & 170 \\
\hline 1029.06 & $8 / 25 / 09$ & ON & $F$ & 93 & 95 & 85 & 197 & 2.8 & 7 & 4.2 & 0.4 & 0.2 & 60 & 0.3 & 115 & 121 & 8.6 & & 25 & 115 & 3.9 & 152 & I. & 200 \\
\hline & 0 & MON & $\mathrm{F}$ & 79 & 73 & 72 & 201 & 3.1 & 6.5 & 3.4 & 0.1 & 0.1 & 66 & 0.4 & 120 & 125 & 8 & 5.5 & 25 & 116 & 4.6 & 152 & .9 & 165 \\
\hline 1032.07 & $12 / 16 / 10$ & MON & $\mathrm{F}$ & 106 & 89 & 68 & 340 & 2.9 & 6.5 & 3.6 & 0.1 & 0.1 & 82 & 0.4 & 115 & 138 & 8.6 & 4.7 & 23 & 112 & 3.9 & 147 & 0.8 & 205 \\
\hline 1037.07 & $04 / 2$ & ON & $F$ & 90 & 118 & 12 & 687 & 2.9 & 6.7 & 3.8 & 0.2 & 0.1 & 67 & 0.5 & 239 & 129 & & 5.1 & 29 & 117 & 3.8 & 160 & & 134 \\
\hline 1037.07 & $10 / 29 / 10$ & MON & $\mathrm{F}$ & 92 & 206 & 11 & 156 & 2.6 & 6.6 & 4 & 0.1 & 0.1 & 82 & 0.4 & 201 & 175 & 8.3 & 4.4 & 27 & 113 & 3.9 & 149 & 0.7 & 205 \\
\hline 1038.07 & $07 / 30$ & ON & $\mathrm{F}$ & 107 & 116 & 90 & 224 & 2.8 & 6.3 & 3.5 & 0.1 & 0.1 & 66 & 0.2 & 187 & 113 & 8.5 & $\mathrm{v}$ & 20 & 118 & 4 & 154 & 0.0 & 330 \\
\hline & & ר & $\mathrm{F}$ & 71 & 97 & & 240 & 2.8 & 6.5 & 3.7 & 0.1 & 0.1 & 48 & 0.3 & 138 & 99 & & 6.3 & 21 & 115 & 4.3 & 152 & & 60 \\
\hline 1042.09 & 105/09 & MON & $\mathrm{F}$ & 79 & 198 & 160 & 191 & 2.8 & 6.9 & 4.1 & 0.1 & 0.1 & 74 & 0.3 & 172 & 119 & 9 & 2.3 & 26 & 116 & 3.7 & 153 & 0.7 & 246.7 \\
\hline 1046.07 & $06 / 11 / 09$ & MON & $\mathrm{F}$ & 79 & 112 & 92 & 107 & 2.6 & 6.8 & 4.2 & 0.1 & 0.1 & 88 & 0.3 & 196 & 120 & 8.7 & 4 & 23 & 122 & 4.3 & 156 & 0.6 & 293.3 \\
\hline 1053.07 & & ON & $\mathrm{F}$ & 91 & 148 & 196 & 684 & 2.7 & 7.3 & 4.6 & 0.1 & 0.1 & 55 & 0.4 & 128 & 134 & 9. & 6.1 & 30 & 111 & 4.5 & 151 & & 137.5 \\
\hline 1053.07 & 06/08/10 & MON & $\mathrm{F}$ & 78 & 123 & 100 & 238 & 2.9 & 6.3 & 3.4 & 0.1 & 0.1 & 53 & 0.3 & 120 & 118 & 9.2 & 4.6 & 25 & 116 & 4.8 & 151 & 0.9 & 176.7 \\
\hline 1056.08 & 0 & ON & $\mathrm{F}$ & 144 & 156 & 14 & 358 & 2.8 & 6.4 & 3.6 & 0.2 & 0.1 & 60 & 0.4 & 109 & 136 & & 4.6 & 25 & 118 & 4.4 & 151 & & 150 \\
\hline & & & $\mathrm{F}$ & 149 & 125 & & & 3 & 6.4 & 3.4 & 0.1 & 0.1 & 70 & 0.4 & 164 & 119 & 9 & 4 & 24 & 113 & 4 & 148 & & 175 \\
\hline 1057.08 & $12 / 17 / 10$ & MON & $\mathrm{F}$ & 91 & 128 & 108 & 298 & 2.8 & 6.2 & 3.4 & 0.1 & 0.1 & 49 & 0.4 & 173 & 89 & 9 & 4.5 & 24 & 114 & 3.7 & 152 & 0.8 & 122.5 \\
\hline 1064.08 & $/ 25 / 09$ & MON & $\mathrm{F}$ & 141 & 178 & 14 & 525 & 2.8 & 6.2 & 3.4 & 0.3 & 0.1 & 61 & 0.3 & 186 & 133 & 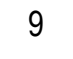 & 6.9 & 27 & 114 & 4.2 & 151 & 0.0 & 203.3 \\
\hline 1109.09 & $/ 23 / 09$ & MON & $\mathrm{F}$ & 52 & 128 & 14 & 635 & 2.1 & 6.9 & 4.8 & 0.1 & 0.1 & 52 & 0.5 & 124 & 116 & 8.7 & 4.6 & 26 & 115 & 4 & 1.5 & 4 & 104 \\
\hline 1109.09 & 06/09/10 & MON & $\mathrm{F}$ & 66 & 94 & 87 & 196 & 2.6 & 7.2 & 4.6 & 0.1 & 0.1 & 39 & 0.4 & 118 & 114 & 8.6 & 4 & 26 & 114 & 4 & 150 & 0.6 & 97.5 \\
\hline & 0 & MON & $\mathrm{F}$ & 99 & 64 & 95 & 150 & 2.7 & 7.2 & 4.5 & 0.1 & 0.1 & 51 & 0.4 & 149 & 122 & 9.1 & 5.2 & 27 & 114 & 4.1 & 153 & 0.6 & 127.5 \\
\hline 1110.09 & $10 / 29 / 10$ & MON & $\mathrm{F}$ & 117 & 127 & 90 & 176 & 2.9 & 6.5 & 3.6 & 0.1 & 0.1 & 55 & 0.3 & 219 & 157 & 8.6 & 4.5 & 26 & 115 & 3.7 & 151 & 0.8 & 183.3 \\
\hline 1111.09 & $04 / 24 / 09$ & MON & $\mathrm{F}$ & 102 & 155 & 159 & 1983 & 3 & 6.4 & 3.4 & 0.1 & 0.1 & 49 & 0.4 & 142 & 124 & 9.6 & 5.1 & 24 & 118 & 3.9 & 155 & 0.9 & 122.5 \\
\hline & $04 / 30 / 09$ & MON & $\mathrm{F}$ & 110 & 132 & 97 & 647 & 2.8 & 6.4 & 3.6 & 0.1 & 0.1 & 76 & 0.4 & 169 & 132 & 8.8 & 6.3 & 22 & 118 & 3.7 & 153 & 0.8 & 190 \\
\hline 1115.09 & $05 / 29 / 09$ & MON & $\mathrm{F}$ & 72 & 87 & 86 & 188 & 2.6 & 7.1 & 4.5 & 0.2 & 0.1 & 53 & 0.4 & 110 & 141 & 8.2 & 5.3 & 23 & 117 & 3.7 & 151 & 0.6 & 132.5 \\
\hline & & & $\begin{array}{l}F \\
F\end{array}$ & $\begin{array}{c}126 \\
93\end{array}$ & & & & & & & & $\begin{array}{l}0.2 \\
0.1\end{array}$ & $\begin{array}{l}42 \\
86\end{array}$ & & $\begin{array}{l}165 \\
153\end{array}$ & $\begin{array}{l}120 \\
126\end{array}$ & $\begin{array}{c}9 \\
8.8\end{array}$ & & & $\begin{array}{l}116 \\
122\end{array}$ & $\begin{array}{l}3.5 \\
3.5\end{array}$ & $\begin{array}{l}152 \\
155\end{array}$ & & $\begin{array}{c}140 \\
286.7\end{array}$ \\
\hline
\end{tabular}




\begin{tabular}{|c|c|c|c|c|c|c|c|c|c|c|c|c|c|c|c|c|c|c|c|c|c|c|c|c|}
\hline $\begin{array}{l}\text { Otter ID } \\
\text { (BRD } \\
\text { number) }\end{array}$ & $\begin{array}{c}\text { Capture } \\
\text { date }\end{array}$ & $\begin{array}{l}\text { Study } \\
\text { site }\end{array}$ & Sex & 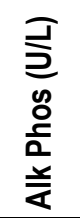 & $\underset{\frac{1}{2}}{\stackrel{2}{2}}$ & 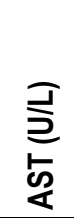 & 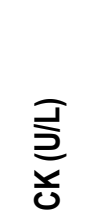 & $\begin{array}{l}\overline{\frac{7}{2}} \\
\frac{0}{0} \\
\frac{0}{4}\end{array}$ & 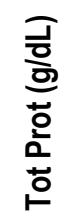 & $\begin{array}{l}\text { 궇 } \\
\text { 응 } \\
\text { 응 }\end{array}$ & 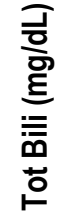 & 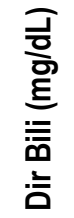 & 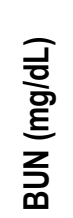 & 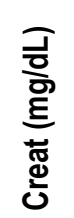 & 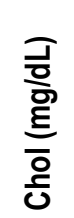 & 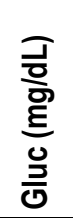 & 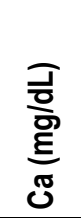 & 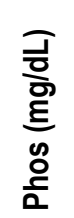 & 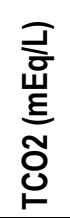 & 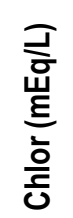 & 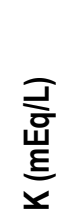 & 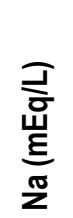 & 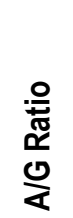 & 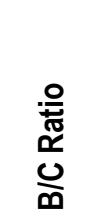 \\
\hline 1119.09 & $06 / 10 / 09$ & MON & $\bar{F}$ & 95 & 180 & 144 & 477 & 2.8 & 6.3 & 3.5 & 0.2 & 0.1 & 49 & 0.4 & 162 & 113 & 8.7 & 5.5 & 23 & 117 & 4.2 & 151 & 0.8 & 122.5 \\
\hline 1120.09 & 06/10/09 & MON & $\mathrm{F}$ & 96 & 121 & 133 & 385 & 2.8 & 7.2 & 4.4 & 0.1 & 0.1 & 79 & 0.4 & 160 & 119 & 12.7 & 4.6 & 25 & 113 & 4.6 & 151 & 0.6 & 197.5 \\
\hline 1122.09 & $06 / 11 / 09$ & MON & $\mathrm{F}$ & 98 & 86 & 75 & 276 & 2.7 & 6.6 & 3.9 & 0.1 & 0.1 & 43 & 0.4 & 150 & 120 & 9.1 & 6.8 & 21 & 120 & 3.8 & 154 & 0.7 & 107.5 \\
\hline 1122.09 & $10 / 08 / 10$ & MON & $F$ & 126 & 117 & 71 & 172 & 2.8 & 6.2 & 3.4 & 0.1 & 0 & 54 & 0.5 & 157 & 127 & 8.8 & 4.7 & 24 & 114 & 4 & 152 & 0.8 & 108 \\
\hline 1123.09 & $06 / 12 / 09$ & MON & $\mathrm{F}$ & 136 & 119 & 130 & 613 & 3.1 & 6.8 & 3.7 & 0.3 & 0.1 & 41 & 0.4 & 190 & 95 & 8.6 & 4.1 & 20 & 115 & 4.1 & 153 & 0.8 & 102.5 \\
\hline 1124.09 & $06 / 17 / 09$ & MON & $F$ & 96 & 206 & 207 & 148 & 3 & 6.5 & 3.5 & 0.2 & 0.1 & 54 & 0.5 & 212 & 127 & 8.8 & 3.6 & 24 & 118 & 3.5 & 156 & 0.9 & 108 \\
\hline 1125.09 & 06/17/09 & MON & $F$ & 109 & 113 & 120 & 320 & 2.8 & 6 & 3.2 & 0.1 & 0.1 & 69 & 0.4 & 127 & 131 & 9 & 4.1 & 27 & 116 & 4.1 & 154 & 0.9 & 172.5 \\
\hline 1126.09 & 06/17/09 & MON & $\mathrm{F}$ & 51 & 125 & 149 & 227 & 2.4 & 7.2 & 4.8 & 0.3 & 0.1 & 33 & 0.5 & 116 & 91 & 8.3 & 3.7 & 21 & 117 & 3.6 & 152 & 0.5 & 66 \\
\hline 1126.09 & $06 / 09 / 10$ & MON & $\mathrm{F}$ & 67 & 119 & 106 & 291 & 2.4 & 7.5 & 5.1 & 0.2 & 0.2 & 33 & 0.4 & 105 & 90 & 8.4 & 4.7 & 26 & 112 & 4.2 & 149 & 0.5 & 82.5 \\
\hline 1126.09 & $07 / 30 / 10$ & MON & $\mathrm{F}$ & 99 & 106 & 84 & 157 & 2.7 & 6.9 & 4.2 & 0.2 & 0.1 & 40 & 0.4 & 112 & 90 & 8.5 & 4.5 & 21 & 116 & 4.1 & 151 & 0.6 & 100 \\
\hline 1127.09 & $06 / 17 / 09$ & MON & $\mathrm{F}$ & 85 & 110 & 84 & 382 & 3 & 6.5 & 3.5 & 0.6 & 0.2 & 55 & 0.4 & 145 & 124 & 8.3 & 4.4 & 27 & 116 & 4 & 154 & 0.9 & 137.5 \\
\hline 1127.09 & $06 / 08 / 10$ & MON & $\mathrm{F}$ & 87 & 170 & 159 & 215 & 2.7 & 6.3 & 3.6 & 0.2 & 0.1 & 60 & 0.3 & 179 & 131 & 8.8 & 4.3 & 26 & 115 & 3.6 & 151 & 0.8 & 200 \\
\hline 1128.09 & 06/18/09 & MON & $F$ & 97 & 116 & 112 & 405 & 2.8 & 6.8 & 4 & 0.2 & 0.1 & 66 & 0.4 & 187 & 112 & 9 & 3.4 & 23 & 117 & 3.5 & 151 & 0.7 & 165 \\
\hline 1128.09 & $07 / 29 / 10$ & MON & $\mathrm{F}$ & 69 & 90 & 84 & 183 & 2.7 & 7.1 & 4.4 & 0.2 & 0.1 & 45 & 0.3 & 146 & 110 & 8.7 & 3.3 & 26 & 116 & 3.4 & 149 & 0.6 & 150 \\
\hline 1129.08 & $11 / 14 / 08$ & MON & $\mathrm{F}$ & 205 & 156 & 178 & 1164 & 0 & 5.5 & 2.5 & 0.2 & 0.1 & 48 & 0.3 & 173 & 143 & 9.9 & 6.2 & 21 & 120 & 4 & 154 & 1.2 & 160 \\
\hline 1132.09 & 06/18/09 & MON & $\mathrm{F}$ & 85 & 143 & 123 & 484 & 2.7 & 6.2 & 3.5 & 0.2 & 0.1 & 65 & 0.4 & 133 & 142 & 8.2 & 6.9 & 24 & 116 & 4.6 & 152 & 0.8 & 162.5 \\
\hline 1133.09 & 08/25/09 & MON & $\mathrm{F}$ & 73 & 129 & 117 & 273 & 2.7 & 6.9 & 4.2 & 0.1 & 0.1 & 75 & 0.4 & 104 & 106 & 9.1 & 5.3 & 25 & 113 & 4.5 & 150 & 0.6 & 187.5 \\
\hline 1134.09 & 08/25/09 & MON & $\mathrm{F}$ & 61 & 68 & 84 & 483 & 2.6 & 7.5 & 4.9 & 0.2 & 0.1 & 47 & 0.5 & 154 & 112 & 8.9 & 4.2 & 25 & 116 & 4 & 150 & 0.5 & 94 \\
\hline 1134.09 & $02 / 02 / 10$ & MON & $\mathrm{F}$ & 78 & 113 & 126 & 422 & 2.7 & 7.6 & 4.9 & 0.1 & 0.1 & 54 & 0.3 & 113 & 119 & 8.7 & 5.4 & 22 & 120 & 4.7 & 155 & 0.6 & 180 \\
\hline 1153.10 & $02 / 02 / 10$ & MON & $\mathrm{F}$ & 124 & 126 & 92 & 326 & 2.8 & 6.2 & 3.4 & 0.3 & 0.1 & 48 & 0.4 & 176 & 144 & 8.8 & 4.1 & 22 & 117 & 4 & 154 & 0.8 & 120 \\
\hline 1154.10 & $02 / 02 / 10$ & MON & $\mathrm{F}$ & 86 & 190 & 124 & 222 & 2.5 & 7 & 4.5 & 0.1 & 0.1 & 66 & 0.3 & 224 & 186 & 8.2 & 3.9 & 20 & 117 & 4.1 & 150 & 0.6 & 220 \\
\hline 1155.10 & $02 / 02 / 10$ & MON & $\mathrm{F}$ & 119 & 167 & 122 & 351 & 2.7 & 6.5 & 3.8 & 0.1 & 0.1 & 55 & 0.3 & 188 & 151 & 8.1 & 4.1 & 19 & 122 & 4.4 & 154 & 0.7 & 183.3 \\
\hline 1157.10 & $06 / 08 / 10$ & MON & $\mathrm{F}$ & 68 & 111 & 97 & 193 & 2.7 & 6.9 & 4.2 & 0.1 & 0.1 & 49 & 0.4 & 142 & 116 & 8.6 & 4.4 & 31 & 111 & 4.3 & 150 & 0.6 & 122.5 \\
\hline 1158.10 & $07 / 29 / 10$ & MON & $\mathrm{F}$ & 84 & 128 & 125 & 418 & 2.7 & 7.4 & 4.7 & 0.2 & 0.1 & 67 & 0.3 & 165 & 133 & 8.8 & 4.5 & 22 & 117 & 3.8 & 152 & 0.6 & 223.3 \\
\hline $\begin{array}{l}1130-08 \\
1066.08\end{array}$ & $\begin{array}{l}11 / 10 / 08 \\
11 / 06 / 08\end{array}$ & $\begin{array}{l}\text { MON } \\
\text { BSR }\end{array}$ & $\begin{array}{l}\mathrm{F} \\
\mathrm{M}\end{array}$ & $\begin{array}{c}129 \\
81\end{array}$ & $\begin{array}{l}276 \\
209\end{array}$ & $\begin{array}{l}237 \\
218\end{array}$ & $\begin{array}{l}598 \\
532\end{array}$ & $\begin{array}{c}3 \\
2.4\end{array}$ & $\begin{array}{l}7 \\
6\end{array}$ & $\begin{array}{c}4 \\
3.6\end{array}$ & $\begin{array}{l}0.2 \\
0.1\end{array}$ & $\begin{array}{l}0.1 \\
0.1\end{array}$ & $\begin{array}{l}108 \\
78\end{array}$ & $\begin{array}{l}0.5 \\
0.8\end{array}$ & $\begin{array}{l}202 \\
135\end{array}$ & $\begin{array}{c}144 \\
91\end{array}$ & $\begin{array}{l}8.3 \\
9.2\end{array}$ & $\begin{array}{c}6.1 \\
8\end{array}$ & $\begin{array}{l}24 \\
26\end{array}$ & $\begin{array}{l}115 \\
115\end{array}$ & $\begin{array}{l}4.1 \\
5.6\end{array}$ & $\begin{array}{l}155 \\
154\end{array}$ & $\begin{array}{l}0.8 \\
0.7\end{array}$ & $\begin{array}{l}216 \\
97.5\end{array}$ \\
\hline 1091.08 & $11 / 10 / 08$ & BSR & $M$ & 89 & 234 & 210 & 504 & 2.5 & 6.4 & 3.9 & 0.1 & 0.1 & 78 & 0.5 & 157 & 135 & 8.2 & 6.3 & 27 & 120 & 4.7 & 154 & 0.6 & 156 \\
\hline
\end{tabular}




\begin{tabular}{|c|c|c|c|c|c|c|c|c|c|c|c|c|c|c|c|c|c|c|c|c|c|c|c|c|}
\hline $\begin{array}{l}\text { Otter ID } \\
\text { (BRD } \\
\text { number) }\end{array}$ & $\begin{array}{l}\text { Capture } \\
\text { date }\end{array}$ & $\begin{array}{l}\text { Study } \\
\text { site }\end{array}$ & Sex & 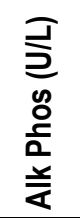 & $\underset{\frac{1}{2}}{\stackrel{2}{2}}$ & 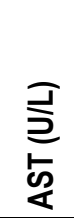 & 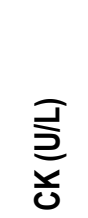 & $\begin{array}{l}\overline{\frac{7}{2}} \\
\frac{0}{0} \\
\frac{0}{4}\end{array}$ & 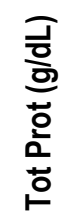 & $\begin{array}{l}\text { 궇 } \\
\text { 응 } \\
\text { 응 }\end{array}$ & 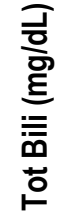 & 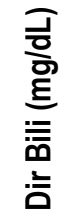 & 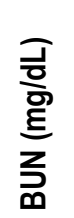 & 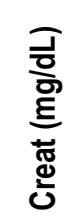 & 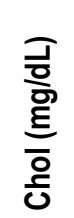 & 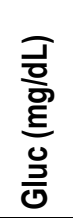 & 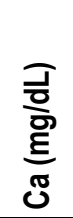 & 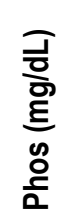 & 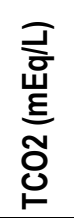 & 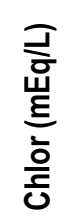 & 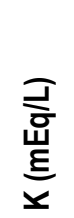 & 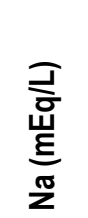 & 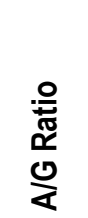 & 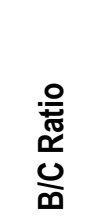 \\
\hline 1093.08 & $11 / 10 / 08$ & BSR & $\bar{M}$ & 64 & 224 & 172 & 884 & 2.4 & 6.9 & 4.5 & 0.1 & 0.1 & 76 & 0.5 & 152 & 133 & 8.3 & 3.8 & 29 & 119 & 4.2 & 153 & 0.5 & 152 \\
\hline 1094.08 & $11 / 10 / 08$ & BSR & M & 65 & 168 & 180 & 743 & 2.2 & 5.9 & 3.7 & 0.1 & 0.1 & 76 & 0.5 & 149 & 90 & 8.1 & 3.5 & 28 & 120 & 4.6 & 154 & 0.6 & 152 \\
\hline 1094.08 & $11 / 04 / 09$ & BSR & $M$ & 107 & 220 & 279 & 102 & 2.7 & 8 & 5.3 & 0.1 & 0.1 & 62 & 0.7 & 177 & 104 & 8.8 & 4.1 & 25 & 113 & 4.2 & 149 & 0.5 & 88.6 \\
\hline 1095.08 & $11 / 06 / 08$ & BSR & $M$ & 64 & 159 & 214 & 2459 & 2.5 & 6.5 & 4 & 0.2 & 0.2 & 63 & 0.5 & 195 & 142 & 8.6 & 5.3 & 23 & 123 & 4.3 & 157 & 0.6 & 126 \\
\hline 1096.08 & $11 / 10 / 08$ & BSR & $M$ & 92 & 186 & 172 & 1034 & 2.7 & 7 & 4.3 & 0.1 & 0.1 & 69 & 0.7 & 141 & 85 & 8.1 & 5.4 & 24 & 115 & 4.8 & 152 & 0.6 & 98.6 \\
\hline 1096.08 & $11 / 05 / 09$ & BSR & $M$ & 126 & 281 & 234 & 309 & 2.5 & 7 & 4.5 & 0.1 & 0.1 & 65 & 0.6 & 177 & 115 & 8.2 & 4.8 & 28 & 116 & 4.6 & 153 & 0.6 & 108.3 \\
\hline 1099.08 & $11 / 10 / 08$ & BSR & $M$ & 60 & 150 & 197 & 963 & 2.6 & 6.6 & 4 & 0.1 & 0.1 & 68 & 0.7 & 159 & 99 & 8.5 & 4.7 & 26 & 116 & 4.7 & 153 & 0.7 & 97.1 \\
\hline 1137.09 & $11 / 04 / 09$ & BSR & $M$ & 107 & 224 & 228 & 246 & 2.5 & 6.6 & 4.1 & 0.1 & 0.1 & 76 & 0.5 & 133 & 143 & 8.3 & 3 & 27 & 116 & 4.1 & 153 & 0.6 & 152 \\
\hline 1143.09 & $11 / 05 / 09$ & BSR & $M$ & 127 & 210 & 244 & 438 & 2.5 & 6.6 & 4.1 & 0.2 & 0.1 & 79 & 0.4 & 167 & 132 & 8.6 & 2 & 27 & 122 & 4.3 & 156 & 0.6 & 197.5 \\
\hline 1147.09 & $11 / 06 / 09$ & BSR & $M$ & 119 & 178 & 153 & 744 & 2.8 & 5.9 & 3.1 & 0.1 & 0.1 & 62 & 0.4 & 174 & 120 & 9.2 & 4.9 & 21 & 123 & 4.1 & 156 & 0.9 & 155 \\
\hline 1165.10 & $09 / 28 / 10$ & BSR & $M$ & 88 & 200 & 183 & 190 & 2.5 & 6.3 & 3.8 & 0.1 & 0.1 & 62 & 0.5 & 135 & 139 & 8.8 & 3.5 & 23 & 98 & 3.7 & 128 & 0.7 & 124 \\
\hline 1067.08 & $09 / 24 / 10$ & BSR & $\mathrm{F}$ & 120 & 170 & 105 & 227 & 2.8 & 6.8 & 4 & 0 & 0 & 63 & 0.3 & 168 & 126 & 9.4 & 3.5 & 25 & 111 & 4.3 & 146 & 0.7 & 210 \\
\hline 1067.08 & $11 / 06 / 08$ & BSR & $F$ & 71 & 166 & 117 & 355 & 2.6 & 6 & 3.4 & 0.2 & 0.1 & 57 & 0.3 & 165 & 133 & 9.1 & 5.9 & 30 & 113 & 4.3 & 151 & 0.8 & 190 \\
\hline 1068.08 & $11 / 06 / 08$ & BSR & $F$ & 106 & 152 & 151 & 1186 & 2.5 & 6.3 & 3.8 & 0.2 & 0.2 & 74 & 0.4 & 190 & 148 & 8.8 & 5.1 & 26 & 119 & 4 & 155 & 0.7 & 185 \\
\hline 1069.08 & $11 / 06 / 08$ & BSR & $F$ & 112 & 296 & 237 & 1137 & 2.6 & 6.5 & 3.9 & 0.2 & 0.2 & 64 & 0.3 & 193 & 165 & 8.4 & 5.7 & 27 & 122 & 3.9 & 158 & 0.7 & 213.3 \\
\hline 1070.08 & $11 / 06 / 08$ & BSR & $\mathrm{F}$ & 99 & 147 & 248 & 2445 & 2.6 & 6.7 & 4.1 & 0.4 & 0.4 & 61 & 0.4 & 194 & 133 & 8.5 & 7.4 & 25 & 119 & 4 & 155 & 0.6 & 152.5 \\
\hline 1071.08 & $11 / 06 / 08$ & BSR & $\mathrm{F}$ & 86 & 269 & 288 & 1089 & 2.8 & 6.4 & 3.6 & 0.1 & 0.1 & 90 & 0.4 & 138 & 153 & 8.4 & 5.9 & 22 & 115 & 4.7 & 150 & 0.8 & 225 \\
\hline 1072.08 & $11 / 06 / 08$ & BSR & $F$ & 93 & 178 & 205 & 526 & 2.5 & 6 & 3.5 & 0.3 & 0.3 & 109 & 0.5 & 173 & 176 & 8.8 & 4.4 & 23 & 120 & 4.6 & 155 & 0.7 & 218 \\
\hline 1073.08 & $11 / 10 / 08$ & BSR & $F$ & 70 & 93 & 86 & 384 & 2.6 & 5.6 & 3 & 0.1 & 0.1 & 62 & 0.3 & 194 & 139 & 8 & 3 & 27 & 119 & 3.7 & 152 & 0.9 & 206.7 \\
\hline 1074.08 & $11 / 10 / 08$ & BSR & $\mathrm{F}$ & 113 & 273 & 313 & 397 & 2.8 & 6.6 & 3.8 & 0.1 & 0.1 & 101 & 0.5 & 222 & 129 & 8.5 & 4.6 & 28 & 114 & 4.4 & 152 & 0.7 & 202 \\
\hline 1074.08 & $09 / 25 / 10$ & BSR & $F$ & 91 & 179 & 157 & 207 & 2.6 & 6.8 & 4.2 & 0 & 0 & 67 & 0.3 & 183 & 117 & 8 & 3.4 & 30 & 114 & 4 & 148 & 0.6 & 223.3 \\
\hline 1075.08 & $11 / 10 / 08$ & BSR & $\mathrm{F}$ & 140 & 159 & 143 & 1101 & 2.7 & 6.9 & 4.2 & 0.2 & 0.1 & 45 & 0.3 & 243 & 131 & 8.8 & 4.9 & 25 & 118 & 3.8 & 154 & 0.6 & 150 \\
\hline 1076.08 & $11 / 10 / 08$ & BSR & $F$ & 112 & 105 & 104 & 209 & 2.4 & 6.3 & 3.9 & 0.1 & 0.1 & 84 & 0.3 & 200 & 92 & 8 & 4.3 & 26 & 119 & 4.2 & 155 & 0.6 & 280 \\
\hline 1077.08 & $11 / 10 / 08$ & BSR & $\mathrm{F}$ & 101 & 117 & 109 & 490 & 2.7 & 6.5 & 3.8 & 0.1 & 0.1 & 75 & 0.04 & 257 & 115 & 8.1 & 5.9 & 23 & 117 & 4.8 & 153 & 0.07 & 187.5 \\
\hline 1078.08 & $11 / 10 / 08$ & BSR & $\mathrm{F}$ & 75 & 80 & 99 & 583 & 2.4 & 6.4 & 4 & 0.1 & 0.1 & 68 & 0.5 & 306 & 169 & 8 & 4.3 & 29 & 118 & 4 & 154 & 0.6 & 136 \\
\hline 1079.08 & $11 / 10 / 08$ & BSR & $F$ & 113 & 103 & 69 & 365 & 3 & 6.4 & 3.4 & 0.2 & 0.1 & 44 & 0.4 & 241 & 132 & 9.5 & 4.1 & 24 & 118 & 3.9 & 153 & 0.9 & 110 \\
\hline
\end{tabular}




\begin{tabular}{|c|c|c|c|c|c|c|c|c|c|c|c|c|c|c|c|c|c|c|c|c|c|c|c|c|}
\hline $\begin{array}{l}\text { Otter ID } \\
\text { (BRD } \\
\text { number) }\end{array}$ & $\begin{array}{l}\text { Capture } \\
\text { date }\end{array}$ & $\begin{array}{l}\text { Study } \\
\text { site }\end{array}$ & Sex & 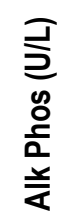 & $\underset{\frac{2}{2}}{\stackrel{2}{2}}$ & 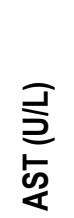 & $\underbrace{\bar{c}}_{\frac{1}{2}}$ & $\begin{array}{l}\text { 긍 } \\
\text { 응 } \\
\frac{0}{2}\end{array}$ & 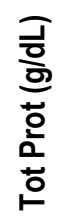 & $\begin{array}{l}\frac{\overline{7}}{} \\
\frac{0}{ㅇ} \\
\frac{0}{\circ} \\
\frac{0}{0}\end{array}$ & 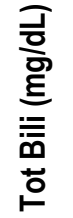 & 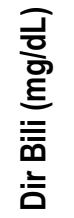 & 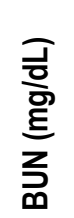 & 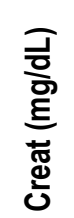 & 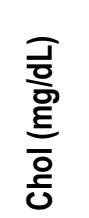 & $\begin{array}{l}\overline{\frac{\partial}{\sigma}} \\
\frac{9}{\mathrm{E}} \\
\frac{\mathrm{o}}{\mathrm{J}}\end{array}$ & 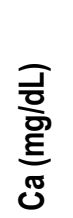 & 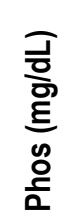 & 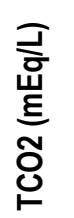 & 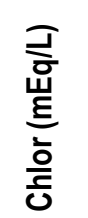 & 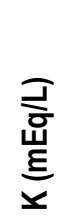 & 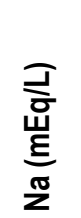 & 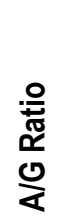 & 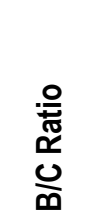 \\
\hline 1080.08 & $11 / 10 / 08$ & \begin{tabular}{|l|l} 
BSR \\
\end{tabular} & $F$ & 110 & 97 & 107 & 670 & 2.7 & 6.6 & 3.9 & $\overline{0.2}$ & 0.1 & 49 & 0.5 & 217 & 129 & 8.5 & 5.8 & 27 & 117 & 4 & 153 & 0.7 & 98 \\
\hline 1081.08 & $11 / 10 / 08$ & BSR & $F$ & 84 & 206 & 307 & 687 & 2.5 & 7.1 & 4.6 & 0.2 & 0.1 & 76 & 0.5 & 194 & 141 & 8.2 & 5.3 & 25 & 119 & 3.9 & 155 & 0.5 & 152 \\
\hline 1082.08 & $11 / 10 / 08$ & BSR & $F$ & 119 & 211 & 154 & 797 & 2.6 & 5.9 & 3.3 & 0.1 & 0.1 & 100 & 0.5 & 174 & 186 & 7.9 & 4.9 & 23 & 119 & 4.3 & 152 & 0.8 & 200 \\
\hline 1083.08 & $11 / 10 / 08$ & BSR & $\mathrm{F}$ & 65 & 94 & 94 & 449 & 2.6 & 6.3 & 3.7 & 0.1 & 0.1 & 58 & 0.4 & 150 & 146 & 7.9 & 4.6 & 25 & 117 & 3.9 & 154 & 0.7 & 145 \\
\hline 1084.08 & $11 / 10 / 08$ & BSR & $F$ & 96 & 121 & 113 & 661 & 2.9 & 6.8 & 3.9 & 0.2 & 0.1 & 64 & 0.5 & 214 & 118 & 8.5 & 4.8 & 26 & 116 & 4.3 & 152 & 0.7 & 128 \\
\hline 1085.08 & $11 / 11 / 08$ & BSR & $F$ & 82 & 104 & 73 & 223 & 2.9 & 6.4 & 3.5 & 0.2 & 0.1 & 65 & 0.5 & 193 & 177 & 8.1 & 5.4 & 24 & 113 & 3.8 & 150 & 0.8 & 130 \\
\hline 1086.08 & $11 / 10 / 08$ & BSR & $F$ & 83 & 149 & 137 & 564 & 2.8 & 7.1 & 4.3 & 0.2 & 0.1 & 58 & 0.4 & 221 & 160 & 8.3 & 5 & 23 & 120 & 4.2 & 153 & 0.7 & 145 \\
\hline 1087.08 & $11 / 11 / 08$ & BSR & $F$ & 71 & 182 & 467 & 6137 & 2 & 6.5 & 4.5 & 0.2 & 0.1 & 153 & 1 & 155 & 85 & 4.8 & 10.2 & 13 & 112 & 4.4 & 145 & 0.4 & 153 \\
\hline 1088.08 & $11 / 12 / 08$ & BSR & $\mathrm{F}$ & 141 & 192 & 165 & 362 & 2.8 & 6.6 & 3.8 & 0.1 & 0.1 & 57 & 0.4 & 215 & 116 & 8.6 & 5.1 & 27 & 114 & 3.7 & 151 & 0.7 & 142.5 \\
\hline 1089.08 & $11 / 10 / 08$ & BSR & $\mathrm{F}$ & 71 & 138 & 130 & 492 & 2.7 & 7 & 4.3 & 0.1 & 0.1 & 72 & 0.5 & 196 & 80 & 8.2 & 4.8 & 24 & 121 & 3.9 & 155 & 0.6 & 144 \\
\hline 1090.08 & $11 / 12 / 08$ & BSR & $\mathrm{F}$ & 110 & 127 & 115 & 306 & 2.6 & 6.7 & 4.1 & 0.1 & 0.1 & 87 & 0.4 & 259 & 96 & 8.6 & 4.2 & 28 & 116 & 4.2 & 153 & 0.6 & 217.5 \\
\hline 1092.08 & $11 / 14 / 08$ & BSR & $\mathrm{F}$ & 80 & 107 & 134 & 131 & 1.9 & 6.8 & 4.9 & 0.1 & 0.1 & 77 & 0.3 & 413 & 136 & 8 & 5.5 & 26 & 116 & 4.2 & 152 & 0.4 & 256.7 \\
\hline 1097.08 & $11 / 14 / 08$ & BSR & $\mathrm{F}$ & 104 & 123 & 153 & 594 & 2.7 & 6.3 & 3.6 & 0.1 & 0.1 & 66 & 0.4 & 187 & 134 & 8.6 & 6.7 & 24 & 116 & 4.8 & 152 & 0.8 & 165 \\
\hline 1097.08 & $09 / 28 / 10$ & BSR & $\mathrm{F}$ & 95 & 115 & 103 & 205 & 2.7 & 6.2 & 3.5 & 0 & 0 & 83 & 0.3 & 186 & 132 & 8.4 & 5 & 21 & 119 & 3.9 & 152 & 0.8 & 276.7 \\
\hline 1098.08 & $11 / 14 / 08$ & BSR & $F$ & 149 & 167 & 165 & 1064 & 2.8 & 6.4 & 3.6 & 0.2 & 0.1 & 82 & 0.3 & 223 & 132 & 9.8 & 4 & 24 & 115 & 4.2 & 150 & 0.8 & 273.3 \\
\hline 1103.08 & $11 / 14 / 08$ & BSR & $F$ & 111 & 239 & 216 & 511 & 2.8 & 6.3 & 3.5 & 0.1 & 0.1 & 66 & 0.5 & 178 & 182 & 8.1 & 4.5 & 21 & 118 & 4.5 & 152 & 0.8 & 132 \\
\hline 1104.08 & $11 / 14 / 08$ & BSR & $F$ & 90 & 102 & 150 & 484 & 2.7 & 6.5 & 3.8 & 0.1 & 0.1 & 82 & 0.4 & 160 & 152 & 8.3 & 7.3 & 24 & 118 & 4.5 & 156 & 0.7 & 205 \\
\hline 1105.08 & $11 / 14 / 08$ & BSR & $\mathrm{F}$ & 107 & 217 & 238 & 1633 & 2.6 & 6.2 & 3.6 & 0.2 & 0.1 & 62 & 0.3 & 158 & 152 & 8.5 & 2.6 & 16 & 123 & 4.2 & 152 & 0.7 & 206.7 \\
\hline 1106.08 & $11 / 14 / 08$ & BSR & $F$ & 125 & 190 & 274 & 877 & 2.7 & 6.4 & 3.7 & 0.1 & 0.1 & 71 & 0.3 & 164 & 137 & 8 & 5.7 & 19 & 121 & 4.6 & 155 & 0.7 & 236.7 \\
\hline 1135.09 & $11 / 04 / 09$ & BSR & $\mathrm{F}$ & 56 & 172 & 135 & 279 & 2.5 & 7 & 4.5 & 0.1 & 0.1 & 67 & 0.4 & 196 & 117 & 8.4 & 3.7 & 28 & 113 & 3.7 & 150 & 0.6 & 167.5 \\
\hline 1136.09 & $11 / 04 / 09$ & BSR & $F$ & 140 & 172 & 114 & 834 & 2.6 & 6.8 & 4.2 & 0.1 & 0.1 & 60 & 0.3 & 246 & 141 & 9.5 & 5 & 24 & 114 & 4.2 & 147 & 0.6 & 200 \\
\hline 1138.09 & $11 / 04 / 09$ & BSR & $F$ & 84 & 150 & 102 & 271 & 2.5 & 6.2 & 3.7 & 0.1 & 0.1 & 61 & 0.4 & 205 & 143 & 8.9 & 3.3 & 25 & 113 & 4 & 149 & 0.7 & 152.5 \\
\hline 1139.09 & $11 / 04 / 09$ & BSR & $\mathrm{F}$ & 109 & 136 & 125 & 845 & 2.8 & 6.9 & 4.1 & 0.2 & 0.1 & 58 & 0.4 & 202 & 115 & 8.9 & 4.1 & 25 & 116 & 4.1 & 152 & 0.7 & 145 \\
\hline 1140.09 & $11 / 04 / 09$ & BSR & $F$ & 125 & 164 & 175 & 337 & 2.6 & 6.5 & 3.9 & 0.2 & 0.1 & 103 & 0.4 & 169 & 125 & 8.4 & 4.6 & 20 & 115 & 4.7 & 150 & 0.7 & 257.5 \\
\hline 1141.09 & $11 / 04 / 09$ & BSR & $F$ & 122 & 158 & 109 & 380 & 2.9 & 7.7 & 4.8 & 0.1 & 0.1 & 70 & 0.5 & 230 & 134 & 8.4 & 2.2 & 22 & 115 & 3.8 & 149 & 0.6 & 140 \\
\hline 1141.09 & 09/28/10 & BSR & $F$ & 147 & 149 & 146 & 118 & 3 & 7.1 & 4.1 & 0.1 & 0.1 & 58 & 0.3 & 193 & 110 & 8.8 & 2.8 & 23 & 115 & 4 & 153 & 0.7 & 193.3 \\
\hline
\end{tabular}




\begin{tabular}{|c|c|c|c|c|c|c|c|c|c|c|c|c|c|c|c|c|c|c|c|c|c|c|c|c|}
\hline $\begin{array}{l}\text { Otter ID } \\
\text { (BRD } \\
\text { number) }\end{array}$ & $\begin{array}{l}\text { Capture } \\
\text { date }\end{array}$ & $\begin{array}{l}\text { Study } \\
\text { site }\end{array}$ & Sex & 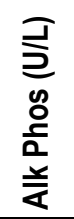 & 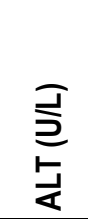 & $\underset{\frac{5}{2}}{\stackrel{Ð}{2}}$ & $\frac{\partial}{2}$ & $\begin{array}{l}\frac{\bar{\partial}}{2} \\
\text { 응 } \\
\frac{0}{\alpha}\end{array}$ & 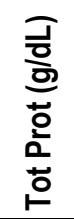 & $\begin{array}{l}\bar{\partial} \\
\frac{7}{6} \\
\text { 응 }\end{array}$ & 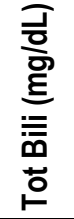 & 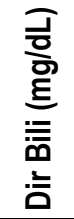 & 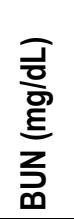 & 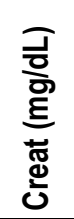 & 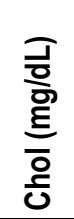 & 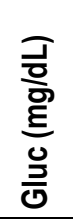 & $\begin{array}{l}\text { 긍 } \\
\text { 일 } \\
\text { 엉 }\end{array}$ & 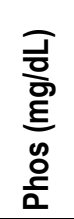 & 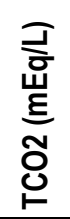 & 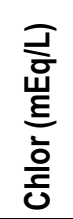 & 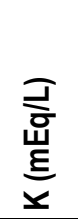 & $\begin{array}{l}\underset{\vec{J}}{\vec{J}} \\
\stackrel{\vec{\xi}}{\underline{z}}\end{array}$ & 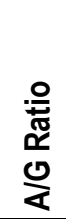 & 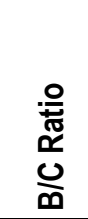 \\
\hline 1142.09 & $09 / 25 / 10$ & BSR & $\bar{F}$ & 73 & 147 & 108 & 199 & 2.9 & 6.7 & 3.8 & 0 & 0 & 57 & 0.3 & 171 & 122 & 9.5 & 5.2 & 28 & 113 & 4.2 & 149 & 0.8 & 190 \\
\hline 1144.09 & /05/09 & SR & $F$ & 122 & 138 & 124 & 316 & 2.9 & 6.9 & 4 & 0.1 & 0.1 & 71 & 0.5 & 178 & 115 & 9.2 & 2.9 & 22 & 116 & 3.9 & 153 & 0.7 & 142 \\
\hline & 1/06/09 & SR & $F$ & 106 & 155 & 108 & 302 & 2.8 & 7.5 & 4.7 & 0.1 & 0.1 & 66 & 0.6 & 238 & 136 & 8.7 & & 24 & 112 & 3.7 & 150 & 6 & 110 \\
\hline 1146.09 & $11 / 06 / 09$ & BSR & $\mathrm{F}$ & 103 & 222 & 150 & 290 & 2.8 & 6 & 3.2 & 0.2 & 0.1 & 54 & 0.3 & 135 & 168 & 9.1 & 5 & 24 & 115 & 3.7 & 152 & 0.9 & 180 \\
\hline 1148.09 & /06/09 & BSR & $\mathrm{F}$ & 88 & 146 & 97 & 248 & 2.7 & 6.9 & 4.2 & 0.1 & 0.1 & 68 & 0.6 & 188 & 173 & 8.8 & 3.3 & 22 & 117 & 3.9 & 153 & 0.6 & 113.3 \\
\hline 1150.09 & $11 / 06 / 09$ & SR & $\mathrm{F}$ & 174 & 189 & 142 & 410 & 2.8 & 7.1 & 4.3 & 0.1 & 0.1 & 92 & 0.4 & 205 & 192 & 8.8 & 4.5 & 20 & 116 & 4.2 & 153 & 0.7 & 230 \\
\hline 1151.09 & $11 / 06 / 09$ & BSR & $\mathrm{F}$ & 115 & 117 & 146 & 1592 & 2.8 & 6.8 & 4 & 0.3 & 0.1 & 73 & 0.4 & 180 & 123 & 8.4 & 4.3 & 21 & 118 & 4.7 & 153 & 0.7 & 182.5 \\
\hline 1159.10 & 9/23/10 & SR & $\mathrm{F}$ & 98 & 119 & 99 & 297 & 2.9 & 6.6 & 3.7 & 0 & 0 & 64 & 0.4 & 186 & 157 & 9.2 & 5.6 & 19 & 114 & 4.3 & 151 & & 160 \\
\hline 1160.10 & 0 & $\mathrm{E}$ & $\mathrm{F}$ & 72 & 167 & 139 & 436 & 2.3 & 6.6 & 4.3 & 0 & 0 & 95 & 0.3 & 159 & 160 & 9.1 & 3.4 & 26 & 113 & 4 & 146 & & 316.7 \\
\hline 1161.10 & $09 / 23 / 10$ & BSR & $\mathrm{F}$ & 93 & 156 & 117 & 406 & 2.9 & 6.6 & 3.7 & 0 & 0 & 63 & 0.4 & 156 & 193 & 9 & 6.5 & 23 & 115 & 4 & 150 & 0.8 & 157.5 \\
\hline 1162.10 & $9 / 24 / 10$ & SR & $\mathrm{F}$ & 106 & 113 & 100 & 234 & 2.6 & 7.2 & 4.6 & 0.1 & 0.1 & 64 & 0.2 & 153 & 100 & 8.7 & 4 & 27 & 112 & 4 & 150 & 0.6 & 320 \\
\hline 1163.10 & $9 / 25 / 10$ & $S R$ & $\mathrm{~F}$ & 268 & 122 & 101 & 449 & 3.3 & 6.1 & 2.8 & 0.1 & 0.1 & 40 & 0.2 & 192 & 140 & 10.1 & 8.5 & 27 & 115 & 4 & 151 & 1.2 & 200 \\
\hline 1164.10 & 09/28/10 & BSR & $\mathrm{F}$ & 123 & 131 & 98 & 560 & 2.7 & 5.9 & 3.2 & 0.1 & 0.1 & 65 & 0.3 & 171 & 192 & 8.9 & 5.9 & 22 & 115 & 4.2 & 147 & 0.8 & 216.7 \\
\hline 1166.10 & 09/28/10 & SR & $\mathrm{F}$ & 119 & 1251 & 1841 & 144 & 2.8 & 6.5 & 3.7 & 0.2 & 0.2 & 63 & 0.4 & 279 & 119 & 8.7 & 3.6 & 21 & 116 & 3.6 & 151 & 0.8 & 157.5 \\
\hline 1167.10 & 09/28/10 & BSR & $\mathrm{F}$ & 141 & 227 & 401 & 218 & 301 & 606 & 305 & 0.2 & 0.1 & 80 & 0.4 & 183 & 100 & 9.4 & 1.9 & 22 & 118 & 3.5 & 155 & 0.9 & 200 \\
\hline 1168.10 & 09/29/10 & BSR & $F$ & 52 & 122 & 113 & 220 & 2.4 & 6.9 & 4.5 & 0.1 & 0.1 & 65 & 0.4 & 146 & 128 & 8.4 & 4.4 & 24 & 114 & 4.2 & 148 & 0.5 & 162.5 \\
\hline
\end{tabular}




\begin{tabular}{|c|c|c|c|c|c|c|c|c|c|c|c|c|c|c|c|c|c|c|c|c|c|c|c|c|}
\hline $\begin{array}{l}\text { Otter ID } \\
\text { (BRD \#) }\end{array}$ & Date & Site & Sex & 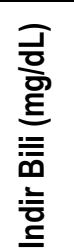 & 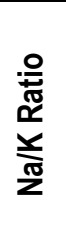 & 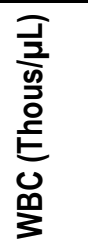 & 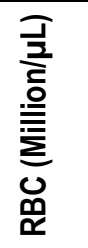 & $\begin{array}{l}\text { 홍 } \\
\text { 흥 } \\
\text { 응 }\end{array}$ & 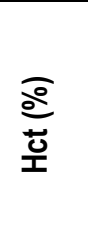 & $\begin{array}{l}\overline{\underline{\underline{L}}} \\
\dot{0}\end{array}$ & 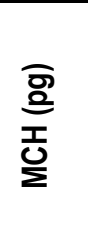 & $\begin{array}{l}\overline{ } \\
\text { 흥 } \\
\text { 원 } \\
\text { 닐 }\end{array}$ & 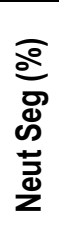 & 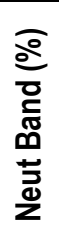 & 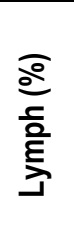 & $\begin{array}{l}\frac{\nwarrow}{\varrho} \\
\stackrel{\circ}{\circ} \\
\frac{0}{2}\end{array}$ & $\frac{\widehat{o}}{\stackrel{0}{0}}$ & 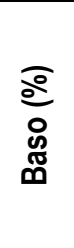 & 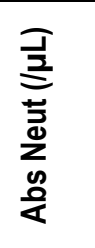 & 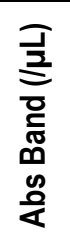 & 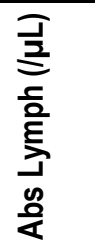 & 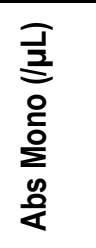 & 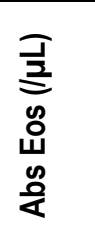 & 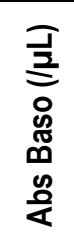 \\
\hline 945.03 & $06 / 11 / 09$ & MON & $M$ & $\overline{0}$ & 35 & 3.7 & 5.06 & 20 & 56.7 & 112 & 39.6 & 35.4 & 44 & 0 & 34 & 7 & 15 & 0 & 1628 & 0 & 1258 & 259 & 555 & 0 \\
\hline 985.04 & 06/09/10 & MON & $M$ & 0 & 32 & 5.4 & 4.86 & 17.7 & 53.5 & 110 & 36.5 & 33.1 & 40 & 0 & 53 & 6 & 1 & 0 & 2160 & 0 & 2862 & 324 & 54 & 0 \\
\hline 998.05 & 02/27/08 & MON & $M$ & 0 & 38 & 5.8 & 5.05 & 20.1 & 57.6 & 114 & 39.9 & 35 & 59 & 0 & 23 & 4 & 14 & 0 & 3422 & 0 & 1334 & 232 & 812 & 0 \\
\hline 1024.06 & 06/10/09 & MON & $M$ & 0 & 31 & 6.5 & 5.17 & 19.7 & 56 & 108 & 38 & 35.1 & 47 & 0 & 40 & 4 & 9 & 0 & 3055 & 0 & 2600 & 260 & 585 & 0 \\
\hline 1030.06 & 04/24/09 & MON & $M$ & 0 & 35 & 9.4 & 4.86 & 19.1 & 53.5 & 110 & 39.2 & 35.7 & 56 & 0 & 16 & 13 & 13 & 2 & 5264 & 0 & 1504 & 1222 & 1222 & 188 \\
\hline 1030.06 & $12 / 17 / 10$ & MON & $M$ & 0 & 36 & 10.1 & 4.64 & 17.5 & 54.2 & 117 & 37.7 & 32.3 & 43 & 0 & 31 & 2 & 24 & 0 & 4343 & 0 & 3131 & 202 & 2424 & 0 \\
\hline 1065.08 & 04/23/09 & MON & $M$ & 0 & 39 & 8.3 & 5 & 20.7 & 54 & 108 & 41.4 & 38.3 & 72 & 0 & 11 & 7 & 10 & 0 & 5976 & 0 & 913 & 581 & 830 & 0 \\
\hline 1108.09 & $4 / 23 / 09$ & MON & M & 0 & 33 & 7.7 & 4.88 & 20.2 & 54.1 & 111 & 41.5 & 37.4 & 64 & 0 & 16 & 6 & 14 & 0 & 4928 & 0 & 1232 & 462 & 1078 & 0 \\
\hline 1108. & $06 / 09$ & MON & M & 0 & 35 & 7.4 & 4.99 & 18.8 & 57.2 & 1 & 37.8 & 32.9 & 68 & & 24 & 8 & 0 & 0 & 5032 & 0 & 1776 & 92 & 0 & 0 \\
\hline 1114 & 0 & & M & 0 & 37 & 5.3 & 3.92 & 16.4 & 47.7 & 12 & 41.7 & 34.3 & 6 & & 32 & 3 & & 0 & & & 1696 & & 265 & 0 \\
\hline 11 & 0 & & M & 0 & 34 & 7. & 4.64 & 18.4 & 54.9 & 11 & 39.7 & 33 & 6 & & 3 & 3 & & 0 & & & 2370 & & & 0 \\
\hline 1114.09 & $12 / 16 / 10$ & MON & M & 0 & 32 & 4.9 & 4.87 & 18.8 & 60.1 & 123 & 38.6 & 31.3 & 53 & 0 & 29 & 3 & 15 & 0 & 2597 & 0 & 1421 & 147 & 735 & 0 \\
\hline 1116.09 & 05/29/09 & MON & M & 0.1 & 43 & 8.3 & 5.26 & 21 & 55.5 & 106 & 39.9 & 37.8 & 35 & 0 & 49 & 3 & 12 & 1 & 2905 & 0 & 4067 & 249 & 996 & 83 \\
\hline 1118.09 & 05/29/09 & MON & M & 0 & 37 & 5 & 5.69 & 22.6 & 59.6 & 105 & 39.7 & 37.9 & 71 & 0 & 21 & 3 & 5 & 0 & 3550 & 0 & 1050 & 150 & 250 & 0 \\
\hline 1121.09 & $06 / 11 / 09$ & MON & M & 0.1 & 38 & 8.4 & 4.84 & 19.2 & 53.9 & 112 & 39.8 & 35.7 & 75 & 0 & 18 & 7 & & 0 & 6300 & 0 & 1512 & 588 & 0 & 0 \\
\hline 1131.08 & $11 / 14 / 08$ & MON & M & 0 & 34 & 6.4 & 4.63 & 16.2 & 49.1 & 106 & 35.1 & 33.1 & 59 & 0 & 29 & 3 & 9 & 0 & 3376 & 0 & 1856 & 192 & 576 & 0 \\
\hline 1156.10 & 02/03/10 & MON & $M$ & 0.2 & 37 & 4 & 4.17 & 19.5 & 47.8 & 115 & 46.7 & 40.8 & 68 & 0 & 21 & 3 & 8 & 0 & 2720 & 0 & 840 & 120 & 320 & 0 \\
\hline 1170.10 & $12 / 17 / 10$ & MON & $M$ & 0.1 & 39 & 6.6 & 4.96 & 17.6 & 55.2 & 111 & 35.4 & 31.8 & 42 & 0 & 37 & 6 & 15 & 0 & 2772 & 0 & 2442 & 396 & 990 & 0 \\
\hline 941.03 & $04 / 24 / 09$ & MON & $\mathrm{F}$ & 0.1 & 41 & 5.4 & 4.9 & 19.9 & 54 & 110 & 40.6 & 36.9 & 48 & 0 & 44 & 2 & 5 & 1 & 2592 & 0 & 2376 & 108 & 270 & 54 \\
\hline 946.03 & $12 / 03 / 09$ & MON & $\mathrm{F}$ & 0.1 & 37 & 8.9 & 5.1 & 20.2 & 57.6 & 113 & 39.7 & 35.2 & 30 & 0 & 60 & 4 & 6 & 0 & 2670 & 0 & 5340 & 356 & 534 & 0 \\
\hline 1000.05 & 06/18/09 & MON & $\mathrm{F}$ & 0.1 & 42 & 5.2 & 4.81 & 19.6 & 52.3 & 109 & 40.8 & 37.6 & 39 & 0 & 49 & 2 & 10 & 0 & 2028 & 0 & 2548 & 104 & 520 & 0 \\
\hline 1011.06 & $07 / 29 / 10$ & MON & $\mathrm{F}$ & 0.1 & 38 & 6.7 & 4.94 & 18.5 & 55.6 & 113 & 37.5 & 33.3 & 55 & 0 & 33 & 2 & 10 & 0 & 3685 & 0 & 2211 & 134 & 670 & 0 \\
\hline 1016.06 & 04/30/09 & MON & $\mathrm{F}$ & 0.1 & 41 & 6.3 & 5.22 & 19.4 & 54.3 & 104 & 37.1 & 35.7 & 43 & 0 & 38 & 1 & 18 & 0 & 2709 & 0 & 2394 & 63 & 1134 & 0 \\
\hline 1017.06 & 04/30/09 & MON & $\mathrm{F}$ & 0.4 & 43 & 7.3 & 4.9 & 19.1 & 54.3 & 111 & 39 & 35.2 & 41 & 0 & 57 & 0 & 2 & 0 & 2993 & 0 & 4161 & 0 & 146 & 0 \\
\hline 1029.06 & 08/25/09 & MON & $\mathrm{F}$ & 0.2 & 39 & 8 & 4.91 & 19.1 & 55.8 & 114 & 38.8 & 34.2 & 36 & 0 & 55 & 2 & 7 & 0 & 2880 & 0 & 4400 & 160 & 560 & 0 \\
\hline 1032.07 & 09/23/09 & MON & $\mathrm{F}$ & 0 & 33 & 7.8 & 4.52 & 18.8 & 50.9 & 113 & 41.6 & 36.9 & 40 & 0 & 46 & 8 & 6 & 0 & 3120 & 0 & 3588 & 624 & 468 & 0 \\
\hline 1032.07 & $12 / 16 / 10$ & MON & $\mathrm{F}$ & 0 & 38 & 6.1 & 4.75 & 16.8 & 53.9 & 114 & 35.4 & 31.2 & 44 & 0 & 41 & 4 & 11 & 0 & 2684 & 0 & 2501 & 244 & 671 & 0 \\
\hline 1037.07 & $04 / 24 / 09$ & MON & $\mathrm{F}$ & 0.1 & 42 & 9.9 & 5.34 & 21.1 & 59 & 111 & 39.5 & 35.7 & 78 & 0 & 13 & 6 & 3 & 0 & 7722 & 0 & 1287 & 594 & 297 & 0 \\
\hline 1037.07 & $10 / 29 / 10$ & MON & $\mathrm{F}$ & 0 & 38 & 9.8 & 4.75 & 16.7 & 52.8 & 111 & 35.1 & 31.6 & 62 & 0 & 18 & 1 & 19 & 0 & 6076 & 0 & 1764 & 98 & 1862 & 0 \\
\hline
\end{tabular}




\begin{tabular}{|c|c|c|c|c|c|c|c|c|c|c|c|c|c|c|c|c|c|c|c|c|c|c|c|c|}
\hline $\begin{array}{l}\text { Otter ID } \\
\text { (BRD \#) }\end{array}$ & Date & Site & Sex & 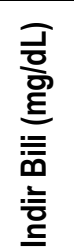 & 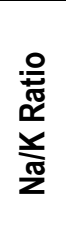 & 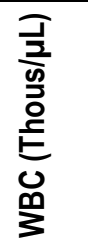 & 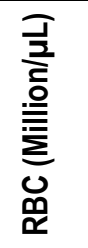 & $\begin{array}{l}\text { 흥 } \\
\text { 응 } \\
\text { 홍 }\end{array}$ & $\frac{\widehat{o}}{\stackrel{0}{0}}$ & $\begin{array}{l}\text { ? } \\
\text { Dे } \\
\sum\end{array}$ & 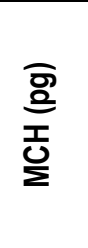 & $\begin{array}{l}\bar{\partial} \\
\text { 흥 } \\
\text { 옹 } \\
\text { 닐 }\end{array}$ & 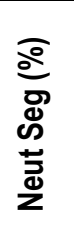 & 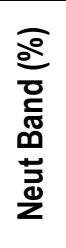 & 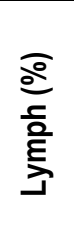 & $\begin{array}{l}\frac{\Xi}{\circ} \\
\stackrel{\circ}{0} \\
\stackrel{0}{2}\end{array}$ & $\frac{\widehat{o}}{\stackrel{0}{o}}$ & $\begin{array}{l}\widetilde{\Xi} \\
\stackrel{\circ}{\circ} \\
\stackrel{g}{\Phi} \\
\infty\end{array}$ & 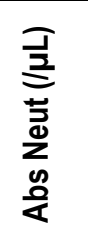 & 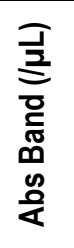 & 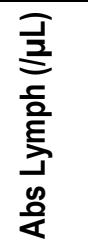 & $\begin{array}{l}\bar{\partial} \\
\frac{1}{5} \\
0 \\
\sum_{0}^{0} \\
\stackrel{0}{0}\end{array}$ & 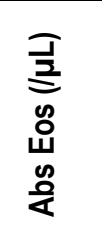 & 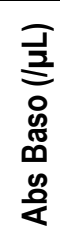 \\
\hline 1038.07 & $07 / 30 / 10$ & MON & $\bar{F}$ & $\overline{0}$ & 39 & 7.6 & 4.8 & 17.7 & 54.9 & 115 & 36.9 & 32.2 & 30 & 0 & 65 & 4 & 1 & 0 & 2280 & 0 & 4940 & 304 & 76 & 0 \\
\hline 1042.07 & $07 / 30 / 10$ & MON & $\mathrm{F}$ & 0 & 35 & 7 & 4.29 & 16.7 & 52.9 & 123 & 38.9 & 31.6 & 66 & 0 & 22 & 7 & 5 & 0 & 4620 & 0 & 1540 & 490 & 350 & 0 \\
\hline 1042.09 & $11 / 05 / 09$ & MON & $\mathrm{F}$ & 0 & 41 & 10.6 & 5.22 & 19.7 & 60.2 & 115 & 37.7 & 32.7 & 59 & 0 & 31 & 2 & 8 & 0 & 6254 & 0 & 3286 & 212 & 848 & 0 \\
\hline 1046.07 & $06 / 11 / 09$ & MON & $\mathrm{F}$ & 0 & 36 & 5.7 & 4.86 & 19.8 & 56.1 & 116 & 40.7 & 35.2 & 50 & 0 & 34 & 3 & 13 & 0 & 2850 & 0 & 1938 & 171 & 741 & 0 \\
\hline 1053.07 & 07/15/09 & MON & $\mathrm{F}$ & 0 & 34 & 12.7 & 4.99 & 19.5 & 52.1 & 104 & 39 & 37.3 & 73 & 0 & 21 & 1 & 5 & 0 & 9271 & 0 & 2667 & 127 & 635 & 0 \\
\hline 1053.07 & $06 / 08 / 10$ & MON & $\mathrm{F}$ & 0 & 31 & 7.7 & 4.95 & 18 & 52.1 & 105 & 36.4 & 34.5 & 41 & 0 & 46 & 2 & 11 & 0 & 3157 & 0 & 3542 & 154 & 847 & 0 \\
\hline 1056.08 & 08/25/09 & MON & $\mathrm{F}$ & 0.1 & 34 & 6.9 & 4.56 & 17.9 & 51.8 & 114 & 39.2 & 34.5 & 40 & 0 & 53 & 5 & 2 & 0 & 2760 & 0 & 3657 & 345 & 138 & 0 \\
\hline 1056.08 & $12 / 03 / 09$ & MON & $\mathrm{F}$ & 0 & 37 & 6.8 & 4.98 & 20.6 & 58.9 & 118 & 41.4 & 35 & 42 & 0 & 50 & 3 & 5 & 0 & 2856 & 0 & 3400 & 204 & 340 & 0 \\
\hline 1057.08 & $12 / 17 / 10$ & MON & $\mathrm{F}$ & 0 & 41 & 10 & 5.23 & 19 & 59.2 & 113 & 36.3 & 32 & 45 & 0 & 27 & 4 & 24 & 0 & 4500 & 0 & 2700 & 400 & 2400 & 0 \\
\hline 1064.08 & 08/25/09 & MON & $\mathrm{F}$ & 0.2 & 36 & 9.1 & 5.15 & 19.2 & 55.3 & 107 & 37.3 & 34.8 & 30 & 0 & 60 & 7 & 3 & 0 & 2730 & 0 & 5460 & 637 & 273 & 0 \\
\hline 1109.09 & $04 / 23 / 09$ & MON & $\mathrm{F}$ & 0 & 38 & 11.8 & 4.53 & 18.3 & 50.2 & 111 & 40.3 & 36.4 & 84 & 0 & 11 & 4 & 1 & 0 & 9912 & 0 & 1298 & 472 & 118 & 0 \\
\hline 1109.09 & $06 / 09 / 10$ & MON & $F$ & 0 & 38 & 6 & 4.97 & 17.8 & 54.4 & 110 & 35.8 & 32.7 & 79 & 0 & 9 & 5 & 7 & 0 & 4740 & 0 & 540 & 300 & 420 & 0 \\
\hline 1110.09 & $04 / 24 / 09$ & MON & $\mathrm{F}$ & 0 & 37 & 7.2 & 4.8 & 18.7 & 52.3 & 109 & 39 & 35.8 & 56 & 0 & 29 & 8 & 7 & 0 & 4032 & 0 & 2088 & 576 & 504 & 0 \\
\hline 1110.09 & $10 / 29 / 10$ & MON & $\mathrm{F}$ & 0 & 41 & 6.9 & 5.4 & 19.1 & 60.9 & 113 & 35.5 & 31.4 & 39 & 0 & 51 & 0 & 10 & 0 & 2691 & 0 & 3519 & 0 & 690 & 0 \\
\hline 1111.09 & $04 / 24 / 09$ & MON & $F$ & 0 & 40 & 9.6 & 5.24 & 19.9 & 55.4 & 106 & 38.1 & 36 & 48 & 0 & 47 & 0 & 5 & 0 & 4608 & 0 & 4512 & 0 & 480 & 0 \\
\hline 1113.09 & 04/30/09 & MON & $\mathrm{F}$ & 0 & 41 & 7.4 & 4.38 & 17.5 & 49.3 & 113 & 40 & 35.6 & 52 & 0 & 38 & 3 & 7 & 0 & 3848 & 0 & 2812 & 222 & 518 & 0 \\
\hline 1115.09 & 05/29/09 & MON & $\mathrm{F}$ & 0.1 & 41 & 7.7 & 4.27 & 16.8 & 45.7 & 107 & 39.3 & 36.7 & 58 & 0 & 33 & 1 & 8 & 0 & 4466 & 0 & 2541 & 77 & 616 & 0 \\
\hline 1117.09 & 05/29/09 & MON & $\mathrm{F}$ & 0 & 43 & 9.2 & 5.16 & 19.9 & 53.1 & 103 & 38.7 & 37.5 & 54 & 0 & 34 & 2 & 10 & 0 & 4968 & 0 & 3128 & 184 & 920 & 0 \\
\hline 1117.09 & $10 / 29 / 10$ & MON & $\mathrm{F}$ & 0 & 44 & 12.2 & 5 & 18.2 & 55.3 & 111 & 36.4 & 32.9 & 72 & 0 & 18 & 6 & 4 & 0 & 8784 & 0 & 2196 & 732 & 488 & 0 \\
\hline 1119.09 & 06/10/09 & MON & $F$ & 0.1 & 36 & 7.1 & 5.39 & 20.2 & 58.7 & 109 & 37.5 & 34.5 & 47 & 0 & 35 & 2 & 16 & 0 & 3337 & 0 & 2485 & 142 & 1136 & 0 \\
\hline 1120.09 & 06/10/09 & MON & $\mathrm{F}$ & 0 & 33 & 7.7 & 5.12 & 19.9 & 57.7 & 113 & 39 & 34.5 & 64 & 0 & 24 & 3 & 9 & 0 & 4928 & 0 & 1848 & 231 & 693 & 0 \\
\hline 1122.09 & $06 / 11 / 09$ & MON & $\mathrm{F}$ & 0 & 41 & 10.7 & 4.59 & 17.1 & 48.9 & 107 & 37.3 & 35 & 52 & 0 & 39 & 2 & 7 & 0 & 5564 & 0 & 4173 & 214 & 749 & 0 \\
\hline 1122.09 & $10 / 08 / 10$ & MON & $F$ & 0.1 & 38 & 7.1 & 5.17 & 17.7 & 56.6 & 110 & 34.3 & 31.3 & 51 & 0 & 36 & 4 & 9 & 0 & 3621 & 0 & 2556 & 284 & 639 & 0 \\
\hline 1123.09 & $06 / 12 / 09$ & MON & $\mathrm{F}$ & 0.2 & 37 & 8.9 & 5.5 & 20.2 & 59.5 & 108 & 36.8 & 34 & 72 & 0 & 18 & 7 & 3 & 0 & 6408 & 0 & 1602 & 623 & 267 & 0 \\
\hline 1124.09 & $06 / 17 / 09$ & MON & $F$ & 0.1 & 45 & 11.3 & 4.7 & 19.2 & 53.1 & 113 & 40.8 & 36.2 & 63 & 0 & 25 & 8 & 4 & 0 & 7119 & 0 & 2825 & 904 & 452 & 0 \\
\hline 1125.09 & 06/17/09 & MON & $\mathrm{F}$ & 0 & 38 & 8 & 5.21 & 19.7 & 55.3 & 106 & 37.8 & 35.5 & 39 & 0 & 53 & 1 & 7 & 0 & 3120 & 0 & 4240 & 80 & 560 & 0 \\
\hline
\end{tabular}




\begin{tabular}{|c|c|c|c|c|c|c|c|c|c|c|c|c|c|c|c|c|c|c|c|c|c|c|c|c|}
\hline $\begin{array}{l}\text { Otter ID } \\
\text { (BRD \#) }\end{array}$ & Date & Site & Sex & 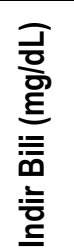 & 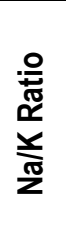 & 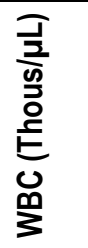 & 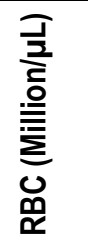 & $\begin{array}{l}\text { 몽 } \\
\text { 응 } \\
\text { 흠 }\end{array}$ & 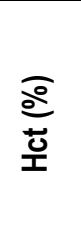 & 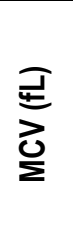 & $\begin{array}{l}\widehat{\widehat{D}} \\
\text { 즐 } \\
\text { 일 }\end{array}$ & 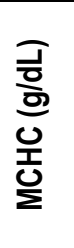 & 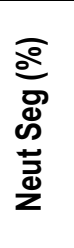 & 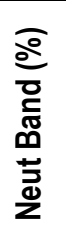 & 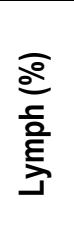 & $\begin{array}{l}\frac{\Im}{\circ} \\
\stackrel{\circ}{0} \\
\stackrel{0}{2}\end{array}$ & $\frac{\widehat{a}}{\frac{0}{0}}$ & $\begin{array}{l}\bar{\Xi} \\
\stackrel{\circ}{\circ} \\
\stackrel{8}{0} \\
\infty\end{array}$ & 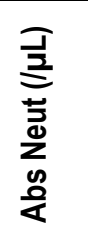 & 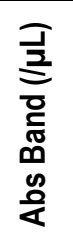 & 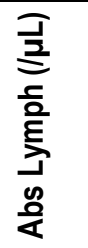 & $\begin{array}{l}\bar{\partial} \\
\frac{1}{2} \\
0 \\
\frac{0}{0} \\
\sum_{0}^{0} \\
\stackrel{0}{\alpha}\end{array}$ & 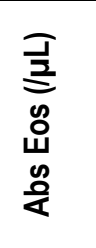 & 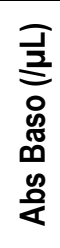 \\
\hline 1126.09 & $06 / 17 / 09$ & $\overline{M O N}$ & $\mathrm{~F}$ & $\overline{0.2}$ & 42 & 6.7 & 5.22 & 20.1 & 55.3 & 106 & 38.4 & 36.3 & 62 & 0 & 23 & 3 & 12 & 0 & 4154 & 0 & 1541 & 201 & 804 & 0 \\
\hline 1126.09 & $06 / 09 / 10$ & MON & $\mathrm{F}$ & 0 & 35 & 8.4 & 5.08 & 17.1 & 51.4 & 101 & 33.8 & 33.4 & 46 & 0 & 32 & 7 & 14 & 1 & 3864 & 0 & 2688 & 588 & 1176 & 84 \\
\hline 1126.09 & 07/30/10 & MON & $\mathrm{F}$ & 0.1 & 37 & 6.5 & 5.24 & 17.5 & 55.5 & 106 & 33.3 & 31.4 & 44 & 0 & 47 & 7 & 2 & 0 & 2860 & 0 & 3055 & 455 & 130 & 0 \\
\hline 1127.09 & 06/17/09 & MON & $\mathrm{F}$ & 0.4 & 39 & 5.4 & 4.97 & 19.2 & 53.1 & 107 & 38.6 & 36.1 & 42 & 0 & 52 & 0 & 6 & 0 & 2268 & 0 & 2808 & 0 & 324 & 0 \\
\hline 1127.09 & 06/08/10 & MON & $\mathrm{F}$ & 0.1 & 42 & 5.4 & 4.84 & 17.4 & 51.3 & 106 & 36 & 34 & 51 & 0 & 30 & 3 & 16 & 0 & 2754 & 0 & 1620 & 162 & 864 & 0 \\
\hline 1128.09 & 06/18/09 & MON & $\mathrm{F}$ & 0.1 & 43 & 7.9 & 4.72 & 19.7 & 52.6 & 111 & 41.6 & 37.4 & 51 & 0 & 34 & 1 & 14 & 0 & 4029 & 0 & 2686 & 79 & 1106 & 0 \\
\hline 1128.09 & 07/29/10 & MON & $\mathrm{F}$ & 0.1 & 44 & 9 & 4.37 & 17 & 50.9 & 116 & 38.9 & 33.4 & 54 & 0 & 35 & 1 & 10 & 0 & 4860 & 0 & 3150 & 90 & 900 & 0 \\
\hline 1129.08 & $11 / 14 / 08$ & MON & $\mathrm{F}$ & 0.1 & 39 & 4.2 & 4.61 & 17 & 49.2 & 107 & 36.8 & 34.5 & 57 & 0 & 37 & 1 & 5 & 0 & 2394 & 0 & 1554 & 42 & 210 & 0 \\
\hline 1132.09 & 06/18/09 & MON & $\mathrm{F}$ & 0.1 & 33 & 6.1 & 4.97 & 18.2 & 50.7 & 102 & 36.6 & 35.8 & 58 & 0 & 31 & 8 & 3 & 0 & 3538 & 0 & 1891 & 488 & 183 & 0 \\
\hline 1133.09 & 08/25/09 & MON & $\mathrm{F}$ & 0 & 33 & 10.5 & 5.16 & 19.8 & 56.6 & 110 & 38.3 & 34.9 & 47 & 0 & 39 & 6 & 8 & 0 & 4935 & 0 & 4095 & 630 & 840 & 0 \\
\hline 1134.09 & 08/25/09 & MON & $\mathrm{F}$ & 0.1 & 38 & 8.4 & 5.23 & 19.3 & 55.8 & 107 & 36.9 & 34.6 & 58 & 0 & 38 & 2 & 2 & 0 & 4872 & 0 & 3192 & 168 & 168 & 0 \\
\hline 1134.09 & $02 / 02 / 10$ & MON & $\mathrm{F}$ & 0 & 33 & 7.9 & 4.03 & 17.4 & 45.3 & 112 & 43 & 38.3 & 72 & 0 & 23 & 2 & 3 & 0 & 5688 & 0 & 1817 & 158 & 237 & 0 \\
\hline 1153.10 & $02 / 02 / 10$ & MON & $\mathrm{F}$ & 0.2 & 39 & 7.8 & 4.04 & 19.8 & 48.4 & 120 & 49 & 40.9 & 40 & 0 & 53 & 2 & 5 & 0 & 3120 & 0 & 4134 & 156 & 390 & 0 \\
\hline 1154.10 & $02 / 02 / 10$ & MON & $\mathrm{F}$ & 0 & 37 & 6.4 & 4.33 & 17.9 & 46.6 & 108 & 41.2 & 38.3 & 58 & 0 & 33 & 7 & 2 & 0 & 3712 & 0 & 2112 & 448 & 128 & 0 \\
\hline 1155.10 & $02 / 02 / 10$ & MON & $\mathrm{F}$ & 0 & 35 & 7.3 & 4.28 & 19.1 & 50.4 & 118 & 44.6 & 37.9 & 48 & 0 & 42 & 6 & 4 & 0 & 3504 & 0 & 3066 & 438 & 292 & 0 \\
\hline 1157.10 & $06 / 08 / 10$ & MON & $\mathrm{F}$ & 0 & 35 & 6.7 & 4.97 & 18.4 & 53.4 & 108 & 37 & 34.4 & 52 & 0 & 28 & 3 & 17 & 0 & 3484 & 0 & 1876 & 201 & 1139 & 0 \\
\hline 1158.10 & $07 / 29 / 10$ & MON & $F$ & 0.1 & 40 & 5.6 & 4.81 & 17.5 & 53.9 & 112 & 36.4 & 32.5 & 66 & 0 & 24 & 2 & 8 & 0 & 3696 & 0 & 1344 & 112 & 448 & 0 \\
\hline $1130-08$ & $11 / 10 / 08$ & MON & $\mathrm{F}$ & 0.1 & 38 & 4.9 & 4.86 & 19.2 & 57.2 & 118 & 39.4 & 33.6 & 51 & 0 & 32 & 8 & 9 & 0 & 2499 & 0 & 1568 & 392 & 441 & 0 \\
\hline 1066.08 & $11 / 06 / 08$ & BSR & M & 0 & 28 & 7.4 & 5.06 & 18.8 & 56.3 & 111 & 37.1 & 33.4 & 61 & 0 & 29 & 0 & 9 & 1 & 4514 & 0 & 2146 & 0 & 666 & 74 \\
\hline 1091.08 & $11 / 10 / 08$ & BSR & M & 0 & 33 & 5.3 & 5.14 & 18.7 & 54.9 & 107 & 36.4 & 34.1 & 66 & 0 & 19 & 4 & 10 & 1 & 3498 & 0 & 1007 & 212 & 530 & 53 \\
\hline 1093.08 & $11 / 10 / 08$ & BSR & M & 0 & 36 & 5 & 5.17 & 20.3 & 58.9 & 114 & 39.3 & 34.4 & 60 & 0 & 26 & 4 & 10 & 0 & 3550 & 0 & 950 & 500 & 355 & 0 \\
\hline 1094.08 & $11 / 10 / 08$ & BSR & M & 0 & 33 & 7.5 & 4.45 & 18.3 & 54.6 & 123 & 41.1 & 33.5 & 58 & 0 & 15 & 2 & 25 & 0 & 4350 & 0 & 1125 & 150 & 1875 & 0 \\
\hline 1094.08 & $11 / 04 / 09$ & BSR & $M$ & 0 & 35 & 6.6 & 5.02 & 18.9 & 61.4 & 122 & 37.6 & 30.8 & 64 & 0 & 23 & 6 & 7 & 0 & 4224 & 0 & 1518 & 396 & 462 & 0 \\
\hline 1095.08 & $11 / 06 / 08$ & BSR & $M$ & 0 & 37 & 8.3 & 4.97 & 18.7 & 54.9 & 110 & 37.6 & 34.1 & 72 & 0 & 20 & 2 & 6 & 0 & 5976 & 0 & 1660 & 166 & 498 & 0 \\
\hline 1096.08 & $11 / 10 / 08$ & BSR & M & 0 & 32 & 4.7 & 5.25 & 19.9 & 58.9 & 112 & 37.8 & 33.7 & 66 & 0 & 19 & 2 & 13 & 0 & 3102 & 0 & 893 & 94 & 611 & 0 \\
\hline 1096.08 & $11 / 05 / 09$ & BSR & $M$ & 0 & 33 & 5.5 & 5.58 & 21.2 & 62.7 & 112 & 38 & 33.8 & 56 & 0 & 33 & 2 & 8 & 1 & 3080 & 0 & 1815 & 110 & 440 & 55 \\
\hline
\end{tabular}




\begin{tabular}{|c|c|c|c|c|c|c|c|c|c|c|c|c|c|c|c|c|c|c|c|c|c|c|c|c|}
\hline $\begin{array}{l}\text { Otter ID } \\
\text { (BRD \#) }\end{array}$ & Date & Site & Sex & 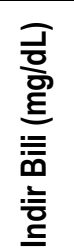 & 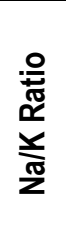 & 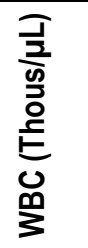 & 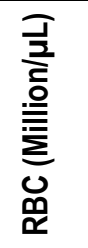 & $\begin{array}{l}\text { 흥 } \\
\text { 응 } \\
\text { 홍 }\end{array}$ & $\frac{\widehat{o}}{\stackrel{0}{0}}$ & $\begin{array}{l}\text { ? } \\
\text { Dे } \\
\sum\end{array}$ & 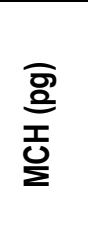 & $\begin{array}{l}\bar{\partial} \\
\text { 흥 } \\
\text { 옹 } \\
\text { 닐 }\end{array}$ & 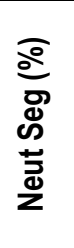 & 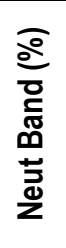 & 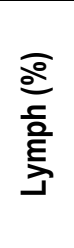 & $\begin{array}{l}\frac{\Xi}{\circ} \\
\stackrel{\circ}{0} \\
\stackrel{0}{2}\end{array}$ & $\frac{\widehat{o}}{\stackrel{0}{o}}$ & $\begin{array}{l}\overparen{\Xi} \\
\stackrel{8}{\circ} \\
\stackrel{8}{0}\end{array}$ & 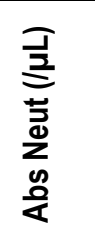 & 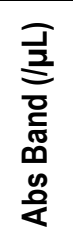 & 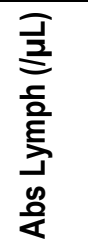 & $\begin{array}{l}\bar{\partial} \\
\frac{1}{5} \\
0 \\
\sum_{0}^{0} \\
\stackrel{0}{0}\end{array}$ & 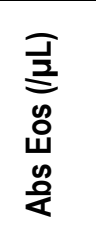 & 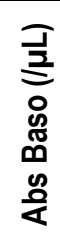 \\
\hline 1099.08 & $11 / 10 / 08$ & $\overline{B S R}$ & $\bar{M}$ & $\overline{0}$ & 33 & 7.1 & 5.25 & 20.3 & 60.3 & 115 & 38.6 & 33.6 & 60 & 0 & 27 & 2 & 11 & 0 & 5734 & 0 & 2162 & 470 & 1034 & 0 \\
\hline 1137.09 & $11 / 04 / 09$ & BSR & M & 0 & 37 & 4.7 & 4.99 & 20.8 & 65.6 & 132 & 41.8 & 31.8 & 56 & 0 & 27 & 5 & 12 & 0 & 2632 & 0 & 1269 & 235 & 564 & 0 \\
\hline 1143.09 & $11 / 05 / 09$ & BSR & M & 0.1 & 36 & 4.5 & 4.99 & 19.8 & 59.7 & 120 & 39.7 & 33.2 & 57 & 0 & 35 & 1 & 6 & 1 & 2565 & 0 & 1575 & 45 & 270 & 45 \\
\hline 1147.09 & $11 / 06 / 09$ & BSR & M & 0 & 38 & 6.9 & 5.12 & 14.2 & 46.6 & 91 & 27.8 & 30.6 & 60 & 0 & 26 & 9 & 5 & 0 & 4140 & 0 & 1794 & 621 & 345 & 0 \\
\hline 1165.10 & 09/28/10 & BSR & M & 0 & 35 & 7.3 & 5.68 & 19.8 & 62.7 & 110 & 34.9 & 31.6 & 50 & 0 & 28 & 3 & 19 & 0 & 3650 & 0 & 2044 & 219 & 1387 & 0 \\
\hline 1067.08 & $09 / 24 / 10$ & BSR & $\mathrm{F}$ & 0 & 34 & 9.9 & 5.17 & 19.7 & 58.1 & 113 & 38.2 & 33.9 & 44 & 0 & 36 & 2 & 18 & 0 & 4356 & 0 & 3564 & 198 & 1782 & 0 \\
\hline 1067.08 & $11 / 06 / 08$ & BSR & $\mathrm{F}$ & 0.1 & 35 & 9.7 & 5.45 & 19.7 & 58.2 & 107 & 36.1 & 33.9 & 64 & 2 & 26 & 3 & 5 & 0 & 6208 & 194 & 2522 & 291 & 485 & 0 \\
\hline 1068.08 & $11 / 06 / 08$ & BSR & $\mathrm{F}$ & 0 & 39 & 9.3 & 5.54 & 19.3 & 58 & 105 & 34.8 & 33.2 & 62 & 0 & 28 & 1 & 9 & 0 & 5766 & 0 & 2604 & 93 & 837 & 0 \\
\hline 1069.08 & $11 / 06 / 08$ & BSR & $\mathrm{F}$ & 0 & 41 & 11.1 & 4.35 & 18.1 & 53.5 & 123 & 41.5 & 33.7 & 61 & 0 & 28 & 7 & 4 & 0 & 6771 & 0 & 3108 & 777 & 444 & 0 \\
\hline 1070.08 & $11 / 06 / 08$ & BSR & $\mathrm{F}$ & 0 & 39 & 5.6 & 5.1 & 19.1 & 59.7 & 117 & 37.5 & 32 & 72 & 0 & 24 & 3 & 1 & 0 & 4032 & 0 & 1344 & 168 & 56 & 0 \\
\hline 1071.08 & $11 / 06 / 08$ & BSR & $\mathrm{F}$ & 0 & 32 & 5.8 & 5.11 & 19.6 & 58.9 & 115 & 38.4 & 33.3 & 53 & 0 & 45 & 2 & 0 & 0 & 3074 & 0 & 2610 & 116 & 0 & 0 \\
\hline 1072.08 & $11 / 06 / 08$ & BSR & $F$ & 0 & 34 & 6.7 & 5.03 & 19.4 & 57.8 & 115 & 38.6 & 33.6 & 70 & 0 & 24 & 2 & 4 & 0 & 4690 & 0 & 1608 & 134 & 268 & 0 \\
\hline 1073.08 & $11 / 10 / 08$ & BSR & $\mathrm{F}$ & 0 & 41 & 4.7 & 5.36 & 19.6 & 58.2 & 109 & 36.6 & 33.7 & 37 & 0 & 38 & 3 & 21 & 1 & 2538 & 0 & 2021 & 141 & 0 & 0 \\
\hline 1074.08 & $11 / 10 / 08$ & BSR & $\mathrm{F}$ & 0 & 35 & 6.2 & 4.73 & 19.1 & 55.4 & 117 & 40.4 & 34.5 & 44 & 0 & 25 & 2 & 29 & 0 & 2728 & 0 & 1550 & 124 & 1798 & 0 \\
\hline 1074.08 & $09 / 25 / 10$ & BSR & $F$ & 0 & 37 & 6.2 & 4.77 & 18.5 & 54.8 & 115 & 38.8 & 33.8 & 60 & 0 & 17 & 3 & 20 & 0 & 3720 & 0 & 1054 & 186 & 1240 & 0 \\
\hline 1075.08 & $11 / 10 / 08$ & BSR & $\mathrm{F}$ & 0.1 & 41 & 4.9 & 5.04 & 19.8 & 57.6 & 114 & 39.2 & 34.3 & 42 & 0 & 41 & 2 & 15 & 0 & 2058 & 0 & 2009 & 98 & 735 & 0 \\
\hline 1076.08 & $11 / 10 / 08$ & BSR & $\mathrm{F}$ & 0 & 37 & 8.9 & 4.98 & 19.4 & 57.5 & 116 & 38.9 & 33.7 & 19 & 0 & 39 & 3 & 39 & 0 & 1691 & 0 & 3471 & 267 & 3471 & 0 \\
\hline 1077.08 & $11 / 10 / 08$ & BSR & $F$ & 0 & 32 & 9.3 & 5.16 & 19.9 & 58.1 & 112 & 38.5 & 34.2 & 40 & 0 & 20 & 4 & 35 & 1 & 3720 & 0 & 1860 & 372 & 3255 & 93 \\
\hline 1078.08 & $11 / 10 / 08$ & BSR & $\mathrm{F}$ & 0 & 39 & 6.7 & 4.94 & 19 & 55.5 & 112 & 38.4 & 34.2 & 41 & 0 & 27 & 1 & 30 & 1 & 2747 & 0 & 1809 & 67 & 2010 & 67 \\
\hline 1079.08 & $11 / 10 / 08$ & BSR & $F$ & 0.1 & 39 & 4.3 & 5.13 & 19.3 & 59.1 & 115 & 37.7 & 32.7 & 49 & 0 & 36 & 4 & 11 & 0 & 2107 & 0 & 1548 & 172 & 473 & 0 \\
\hline 1080.08 & $11 / 10 / 08$ & BSR & $\mathrm{F}$ & 0.1 & 38 & 47 & 5.5 & 20.4 & 61.1 & 111 & 37.1 & 33.4 & 40 & 0 & 45 & 4 & 11 & 0 & 1880 & 0 & 2115 & 188 & 517 & 0 \\
\hline 1081.08 & $11 / 10 / 08$ & BSR & $\mathrm{F}$ & 0.1 & 40 & 5.6 & 4.33 & 18.3 & 54.4 & 126 & 42.3 & 33.7 & 72 & 0 & 15 & 3 & 10 & 0 & 4032 & 0 & 840 & 168 & 560 & 0 \\
\hline 1082.08 & $11 / 10 / 08$ & BSR & $F$ & 0 & 35 & 6.1 & 4.74 & 18.2 & 54.9 & 116 & 38.4 & 33.1 & 47 & 0 & 30 & 5 & 18 & 0 & 2867 & 0 & 1830 & 305 & 1098 & 0 \\
\hline 1083.08 & $11 / 10 / 08$ & BSR & $\mathrm{F}$ & 0 & 39 & 7.1 & 4.81 & 18.7 & 57.1 & 119 & 38.9 & 32.8 & 45 & 0 & 34 & 4 & 17 & 0 & 3195 & 0 & 2414 & 284 & 1207 & 0 \\
\hline 1084.08 & $11 / 10 / 08$ & BSR & $F$ & 0.1 & 35 & 7.3 & 5.36 & 20.7 & 60.3 & 113 & 38.6 & 34.3 & 38 & 0 & 40 & 3 & 19 & 0 & 2774 & 0 & 2920 & 219 & 1387 & 0 \\
\hline 1085.08 & $11 / 11 / 08$ & BSR & $\mathrm{F}$ & 0.1 & 39 & 6.3 & 4.78 & 18.5 & 56.1 & 118 & 38.7 & 32.9 & 49 & 0 & 40 & 2 & 9 & 0 & 3087 & 0 & 2520 & 126 & 567 & 0 \\
\hline
\end{tabular}




\begin{tabular}{|c|c|c|c|c|c|c|c|c|c|c|c|c|c|c|c|c|c|c|c|c|c|c|c|c|}
\hline $\begin{array}{l}\text { Otter ID } \\
\text { (BRD \#) }\end{array}$ & Date & Site & Sex & 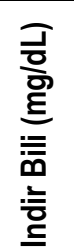 & 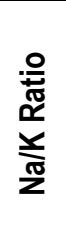 & 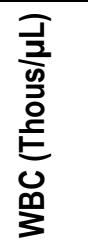 & 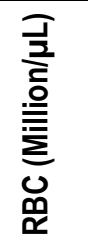 & $\begin{array}{l}\text { 흥 } \\
\text { 응 } \\
\text { 홍 }\end{array}$ & 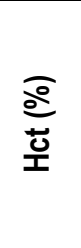 & $\begin{array}{l}\text { ? } \\
\text { Dे } \\
\sum\end{array}$ & $\begin{array}{l}\widehat{\text { D্ }} \\
\text { 정 }\end{array}$ & 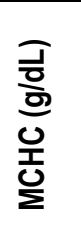 & 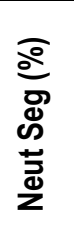 & 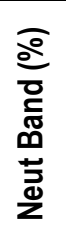 & 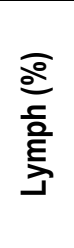 & $\begin{array}{l}\frac{\Xi}{\circ} \\
\stackrel{\circ}{0} \\
\stackrel{0}{2}\end{array}$ & $\frac{\widehat{o}}{\stackrel{0}{o}}$ & $\begin{array}{l}\overparen{\Xi} \\
\stackrel{8}{\circ} \\
\stackrel{8}{0}\end{array}$ & 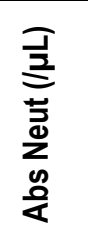 & 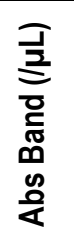 & 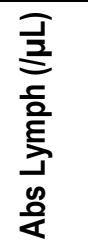 & 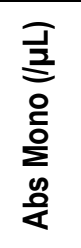 & 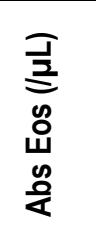 & 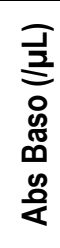 \\
\hline 1086.08 & $11 / 10 / 08$ & $\overline{B S R}$ & $\bar{F}$ & $\overline{0.1}$ & 36 & 9.4 & 4.89 & 19.9 & 58.7 & 120 & 40.7 & 33.9 & 61 & 0 & 23 & 5 & 11 & 0 & 5734 & 0 & 2162 & 470 & 1034 & 0 \\
\hline 1087.08 & $11 / 11 / 08$ & BSR & $\mathrm{F}$ & 0.1 & 33 & 0.5 & 5.63 & 22.7 & 62.3 & 111 & 40.3 & 36.4 & 35 & 0 & 42 & 5 & 18 & 0 & 175 & 0 & 210 & 25 & 90 & 0 \\
\hline 1088.08 & $11 / 12 / 08$ & BSR & $\mathrm{F}$ & 0 & 41 & 8.1 & 4.46 & 19.2 & 53.7 & 120 & 43 & 35.7 & 33 & 0 & 59 & 0 & 8 & 0 & 2673 & 0 & 4779 & 0 & 648 & 0 \\
\hline 1089.08 & $11 / 10 / 08$ & BSR & $\mathrm{F}$ & 0 & 40 & 5.5 & 4.65 & 19.2 & 55.8 & 120 & 41.2 & 34.3 & 47 & 0 & 31 & 3 & 19 & 0 & 2585 & 0 & 1705 & 165 & 1045 & 0 \\
\hline 1090.08 & $11 / 12 / 08$ & BSR & $\mathrm{F}$ & 0 & 36 & 6.4 & 5.05 & 19.7 & 57.7 & 114 & 38.9 & 34.1 & 37 & 0 & 38 & 5 & 20 & 0 & 2368 & 0 & 2432 & 320 & 1280 & 0 \\
\hline 1092.08 & $11 / 14 / 08$ & BSR & $F$ & 0 & 36 & 7.7 & 3.19 & 13.2 & 39.9 & 125 & 41.2 & 33 & 65 & 0 & 18 & 5 & 12 & 0 & 5005 & 0 & 1386 & 385 & 924 & 0 \\
\hline 1097.08 & $11 / 14 / 08$ & BSR & $\mathrm{F}$ & 0 & 32 & 6.4 & 4.67 & 19.2 & 54.3 & 116 & 41.1 & 35.3 & 52 & 0 & 39 & 1 & 8 & 0 & 3328 & 0 & 2496 & 64 & 512 & 0 \\
\hline 1097.08 & $09 / 28 / 10$ & BSR & $\mathrm{F}$ & 0 & 39 & 6.1 & 4.58 & 17.7 & 54.1 & 118 & 38.7 & 32.8 & 57 & 0 & 26 & 1 & 16 & 0 & 3477 & 0 & 1586 & 61 & 976 & 0 \\
\hline 1098.08 & $11 / 14 / 08$ & BSR & $\mathrm{F}$ & 0.1 & 36 & 8.1 & 5.13 & 19.6 & 56.9 & 111 & 38.1 & 34.4 & 41 & 0 & 49 & 1 & 9 & 0 & 3321 & 0 & 3969 & 81 & 729 & 0 \\
\hline 1103.08 & $11 / 14 / 08$ & BSR & $F$ & 0 & 34 & 4.9 & 5 & 19.1 & 56.8 & 114 & 38.2 & 33.6 & 69 & 0 & 21 & 0 & 10 & 0 & 3381 & 0 & 1029 & 0 & 490 & 0 \\
\hline 1104.08 & $11 / 14 / 08$ & BSR & $\mathrm{F}$ & 0 & 35 & 8.7 & 4.71 & 19.4 & 55.9 & 119 & 41.1 & 34.6 & 54 & 0 & 36 & 1 & 9 & 0 & 4698 & 0 & 3132 & 87 & 783 & 0 \\
\hline 1105.08 & $11 / 14 / 08$ & BSR & $F$ & 0.1 & 36 & 6.2 & 4.79 & 19 & 56 & 117 & 39.7 & 34 & 69 & 0 & 15 & 1 & 15 & 0 & 4278 & 0 & 930 & 62 & 930 & 0 \\
\hline 1106.08 & $11 / 14 / 08$ & BSR & $\mathrm{F}$ & 0 & 34 & 5.8 & 4.84 & 18.9 & 55.5 & 115 & 39 & 34 & 49 & 0 & 38 & 0 & 13 & 0 & 2842 & 0 & 2204 & 0 & 754 & 0 \\
\hline 1135.09 & $11 / 04 / 09$ & BSR & $\mathrm{F}$ & 0 & 41 & 7.1 & 5.56 & 20.8 & 66 & 119 & 37.5 & 31.6 & 71 & 0 & 16 & 6 & 7 & 0 & 5041 & 0 & 1136 & 426 & 497 & 0 \\
\hline 1136.09 & $11 / 04 / 09$ & BSR & $F$ & 0 & 35 & 6.7 & 5.25 & 19.7 & 61.7 & 118 & 37.5 & 31.9 & 27 & 0 & 62 & 6 & 5 & 0 & 1809 & 0 & 4154 & 402 & 335 & 0 \\
\hline 1138.09 & $11 / 04 / 09$ & BSR & $\mathrm{F}$ & 0 & 37 & 7.4 & 5.2 & 19 & 60.5 & 116 & 36.6 & 31.4 & 29 & 0 & 62 & 1 & 8 & 0 & 2146 & 0 & 4588 & 74 & 592 & 0 \\
\hline 1139.09 & $11 / 04 / 09$ & BSR & $\mathrm{F}$ & 0.1 & 37 & 8.1 & 5.12 & 20.3 & 64.5 & 126 & 39.7 & 31.5 & 48 & 0 & 41 & 4 & 7 & 0 & 3888 & 0 & 3321 & 324 & 567 & 0 \\
\hline 1140.09 & $11 / 04 / 09$ & BSR & $F$ & 0.1 & 32 & 6.3 & 4.9 & 19.2 & 60.2 & 123 & 39.1 & 31.8 & 43 & 0 & 47 & 2 & 8 & 0 & 2709 & 0 & 2961 & 126 & 504 & 0 \\
\hline 1141.09 & $11 / 04 / 09$ & BSR & $\mathrm{F}$ & 0 & 39 & 6.6 & 5.41 & 20.2 & 65.1 & 120 & 37.3 & 31 & 58 & 0 & 33 & 1 & 7 & 1 & 3828 & 0 & 2178 & 66 & 462 & 66 \\
\hline 1141.09 & 09/28/10 & BSR & $F$ & 0 & 38 & 7.8 & 5.34 & 19.8 & 60.8 & 114 & 37.1 & 32.6 & 53 & 0 & 29 & 4 & 14 & 0 & 4134 & 0 & 2262 & 312 & 1092 & 0 \\
\hline 1142.09 & $09 / 25 / 10$ & BSR & $\mathrm{F}$ & 0 & 35 & 9 & 5.19 & 18.8 & 55.7 & 107 & 36.2 & 33.8 & 53 & 0 & 31 & 3 & 13 & 0 & 4770 & 0 & 2790 & 270 & 1170 & 0 \\
\hline 1144.09 & $11 / 05 / 09$ & BSR & $\mathrm{F}$ & 0 & 39 & 9.9 & 5.67 & 21.9 & 66.3 & 117 & 38.6 & 33 & 20 & 0 & 61 & 5 & 12 & 0 & 1980 & 0 & 6039 & 495 & 1188 & 0 \\
\hline 1145.09 & $11 / 06 / 09$ & BSR & $F$ & 0 & 41 & 6.7 & 5.75 & 20.4 & 63.5 & 110 & 35.4 & 32 & 51 & 0 & 26 & 3 & 20 & 0 & 3417 & 0 & 1742 & 201 & 1340 & 0 \\
\hline 1146.09 & $11 / 06 / 09$ & BSR & $\mathrm{F}$ & 0.1 & 41 & 5.4 & 5.6 & 20 & 63 & 113 & 35.8 & 31.8 & 45 & 0 & 41 & 5 & 9 & 0 & 2430 & 0 & 2214 & 270 & 486 & 0 \\
\hline 1148.09 & $11 / 06 / 09$ & BSR & $F$ & 0 & 39 & 8.2 & 5.13 & 20.3 & 63.5 & 124 & 39.5 & 31.9 & 56 & 0 & 22 & 4 & 18 & 0 & 4592 & 0 & 1804 & 328 & 1476 & 0 \\
\hline 1150.09 & $11 / 06 / 09$ & BSR & $\mathrm{F}$ & 0 & 36 & 7 & 4.99 & 20.2 & 63 & 126 & 40.4 & 32 & 42 & 0 & 39 & 8 & 11 & 0 & 2940 & 0 & 2730 & 560 & 770 & 0 \\
\hline
\end{tabular}




\begin{tabular}{|c|c|c|c|c|c|c|c|c|c|c|c|c|c|c|c|c|c|c|c|c|c|c|c|c|}
\hline $\begin{array}{l}\text { Otter ID } \\
\text { (BRD \#) }\end{array}$ & Date & Site & Sex & 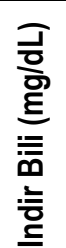 & 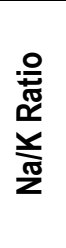 & 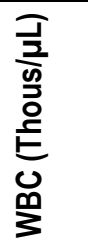 & 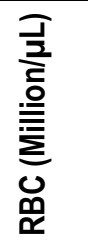 & $\begin{array}{l}\text { 홓 } \\
\text { 응 } \\
\text { 음 }\end{array}$ & 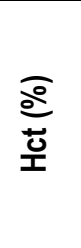 & $\frac{\bar{D}}{\partial}$ & $\begin{array}{l}\text { 을 } \\
\text { 플 }\end{array}$ & $\begin{array}{l}\overline{ } \\
\text { 흥 } \\
\text { 온 } \\
\text { 닐 }\end{array}$ & 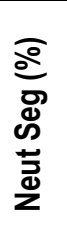 & 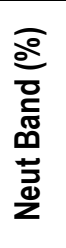 & 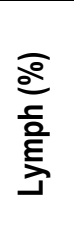 & $\begin{array}{l}\frac{\Xi}{\circ} \\
\stackrel{\circ}{0} \\
\frac{0}{2}\end{array}$ & $\begin{array}{l}\widehat{\frac{o}{o}} \\
\stackrel{\infty}{\infty}\end{array}$ & $\begin{array}{l}\overparen{\varrho} \\
\stackrel{0}{\circ} \\
\stackrel{0}{0} \\
\infty\end{array}$ & 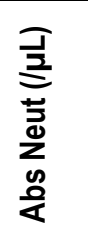 & 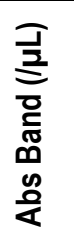 & 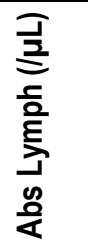 & 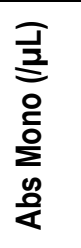 & 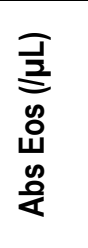 & 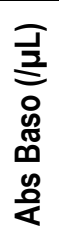 \\
\hline 1151.09 & $11 / 06 / 09$ & BSR & $F$ & $\overline{0.2}$ & 33 & 5.9 & 5.48 & 20.9 & 66.4 & 121 & 38.2 & 31.5 & 31 & 0 & 55 & 0 & 14 & 0 & 1829 & 0 & 3245 & 0 & 826 & 0 \\
\hline 1159.10 & $09 / 23 / 10$ & BSR & $\mathrm{F}$ & 0 & 35 & 7.8 & 5.66 & 20.6 & 63.6 & 112 & 36.4 & 32.4 & 30 & 0 & 48 & 3 & 19 & 0 & 2340 & 0 & 3744 & 234 & 1482 & 0 \\
\hline 1160.10 & $09 / 23 / 10$ & BSR & $\mathrm{F}$ & 0 & 37 & 7 & 4.89 & 17.4 & 52.1 & 106 & 35.5 & 33.4 & 58 & 0 & 29 & 3 & 10 & 0 & 4060 & 0 & 2030 & 210 & 700 & 0 \\
\hline 1161.10 & $09 / 23 / 10$ & BSR & $\mathrm{F}$ & 0 & 38 & 9 & 5.09 & 18.4 & 55.8 & 110 & 36.2 & 33 & 50 & 0 & 29 & 3 & 18 & 0 & 4500 & 0 & 2610 & 270 & 1620 & 0 \\
\hline 1162.10 & $09 / 24 / 10$ & BSR & $\mathrm{F}$ & 0 & 38 & 8.8 & 4.54 & 18.7 & 54.4 & 120 & 41.2 & 34.4 & 40 & 0 & 32 & 8 & 20 & 0 & 3520 & 0 & 2816 & 704 & 1760 & 0 \\
\hline 1163.10 & $09 / 25 / 10$ & BSR & $\mathrm{F}$ & 0 & 38 & 6.3 & 4.93 & 16.5 & 50.7 & 103 & 33.4 & 32.5 & 57 & 0 & 35 & 6 & 2 & 0 & 3591 & 0 & 2205 & 378 & 126 & 0 \\
\hline 1164.10 & $09 / 28 / 10$ & BSR & $\mathrm{F}$ & 0 & 35 & 5.4 & 4.68 & 17.8 & 53.8 & 115 & 37.9 & 33 & 61 & 0 & 24 & 3 & 12 & 0 & 3294 & 0 & 1296 & 162 & 648 & 0 \\
\hline 1166.10 & $09 / 28 / 10$ & BSR & $\mathrm{F}$ & 0 & 42 & 10.7 & 5.16 & 18.9 & 58.1 & 113 & 36.6 & 32.5 & 75 & 0 & 14 & 4 & 7 & 0 & 8025 & 0 & 1498 & 428 & 749 & 0 \\
\hline 1167.10 & $09 / 28 / 10$ & BSR & $\mathrm{F}$ & 0.1 & 44 & 13.1 & 4.83 & 18.9 & 56.6 & 117 & 39 & 33.3 & 68 & 0 & 28 & 1 & 3 & 0 & 8908 & 0 & 3668 & 131 & 393 & 0 \\
\hline 1168.10 & 09/29/10 & BSR & $\mathrm{F}$ & 0 & 35 & 8.1 & 4.41 & 16.6 & 54.8 & 124 & 37.7 & 30.3 & 38 & 0 & 26 & 0 & 36 & 0 & 3078 & 0 & 2106 & 0 & 2916 & 0 \\
\hline
\end{tabular}




\title{
Chapter 3. Gene Transcription-Immune and Detoxification Function in California Sea Otters
}

\author{
A.K. Miles ${ }^{1}$, L. Bowen 1 , M. Tim Tinker ${ }^{1,2}$, and M. Murray ${ }^{3}$
}

\section{Introduction}

The southern sea otter (Enhydra lutris nereis), which occurs only in California, is classified as "depleted" under the Federal Marine Mammal Protection Act (16 U.S.C. $\S \S 1361$ et seq.) and "threatened" under the Federal Endangered Species Act (16 U.S.C. $§ 1531$ et seq.). Historical abundance of southern sea otters was estimated at about 14,500 prior to the fur trade, but now includes only about 3,000 animals in California, distributed between Half Moon Bay (San Mateo County) and Point Conception (Santa Barbara County). Since 1995, the rate of recovery of the southern sea otter has been particularly sluggish, leading to questions about population health and viability.

Suggested causes for the lack of recovery in sea otter numbers are complex and have been attributed to various ecological or human-influenced possibilities. Anthropogenic pressures such as oil spills usually are noted for their sudden dramatic impact on marine and coastal species. The acute effects of these disasters are evaluated by mortality estimations, clinical evaluation, and necropsy examinations (Lipscomb and others, 1993; Monson and others, 2000). The long-term or chronic effects of incidental or periodic events associated with anthropogenic insult to the environment are more difficult to assess. Studies often are restricted to demographic modeling, estimations of reproductive efficiency, or time-differential, age-specific survival rates, yet few biological markers are available that identify sub-lethal pathology attributable to direct anthropogenic influence. Identifying variation in these sub-lethal stressors can provide insights into the physiological challenges faced by individual animals, and ultimately into the role of such stressors in affecting animal health and long-term survival.

Applying contemporary gene transcript analysis to identify genomic response to environmental stress or disease has the potential to transform studies of sea otter ecology and diagnostics (Burczynski and others, 2000; Bartosiewicz and others, 2001; Bourlat and others, 2001). Advanced technologies, based on and developed from biomedical models of human physiology and disease, aid researchers with cutting-edge diagnostic tools for domestic and wildlife veterinary applications (Burczynski and others, 2000; Bartosiewicz and others, 2001; Bowen and others, 2007, 2012; Sitt and others, 2008; Miles and others, 2012). Gene-based diagnostics of sea otters afford the opportunity for minimally invasive assessments of physiologic state in response to intrinsic and extrinsic factors, not only in individuals or populations but potentially at landscape scales (Acevedo-Whitehouse and Duffus, 2009).

Gene transcript analysis affords the opportunity to detect the earliest observable signs of physiological perturbation, as gene transcripts typically are evident prior to clinical manifestations of environmental stressors (McLoughlin and others, 2006). Therefore, clinical application of quantitative gene transcript analysis technology will provide an invaluable addition to current approaches for monitoring potential ecosystem and individual health impairment (McLoughlin and others, 2006). The genes used in our analysis are fundamental to mediation of detoxification and

\footnotetext{
${ }^{1}$ U.S. Geological Survey.

${ }^{2}$ University of California, Santa Cruz.

${ }^{3}$ Monterey Bay Aquarium.
} 
immune function (Schwartz and others, 2004a, 2004b), cellular injury (Ghanem and others, 2006), signal transduction (Burchiel and Luster, 2004), xenobiotic metabolism (Schwartz and others, 2004a, 2004b), or tumorigenesis (Ramesh and others, 2004) (table 6). Measurement of differential transcription of a selected suite of genes potentially can provide an early warning of compromised health and related environmental stressors in free-ranging animals. The suite of genes examined in this study identified potential effects on sea otters in Prince William Sound, Alaska, 20 years after the Exxon Valdez oil spill (Miles and others, 2012). We describe transcript profiles of these genes from populations of southern sea otters ranging from Monterey to Santa Barbara, California, with the particular goal of comparing two focal study sites (Big Sur and Monterey) and placing them within the context of variation from other sites throughout California.

\section{Methods}

\section{Free-Ranging Target Otters}

A total of 180 sea otters were captured in California (43 at Santa Barbara, 2012-2013; 57 at San Luis Obispo, 2012; 51 at Big Sur, 2008-2010; and 29 at Monterey, 2009-2010). Sea otters were captured by scuba divers using Wilson traps (Wendell and others, 1996) and were brought immediately to a shipboard station for processing. These sea otters (as well as reference captive sea otters) were anesthetized with fentanyl citrate and midazolam hydrochloride (Monson and others, 2001) prior to processing. See chapter 1 for more details on capture and sampling methodology.

\section{Captive Reference Otters}

Blood samples from 17 captive sea otters were obtained from the Monterey Bay Aquarium (Monterey, California), Shedd Aquarium (Chicago, Illinois), Oregon Coast Aquarium (Newport, Oregon), and the Vancouver Aquarium (Vancouver, Canada) in 2008, 2009, and 2010, and included both northern and southern subspecies (Bowen and others, 2012). These animals were identified as clinically normal by staff veterinarians at these aquaria during the time interval of blood collection.

\section{Blood Collection and Ribonucleic Acid Extraction}

A 2.5-mL sample from each sea otter was drawn directly into a PAXgene ${ }^{\mathrm{TM}}$ blood ribonucleic acid (RNA) collection tube (PreAnalytiX, Switzerland) from either the jugular or popliteal veins and then frozen at $-20{ }^{\circ} \mathrm{C}$ until extraction of ribonucleic acid (RNA; Bowen and others, 2012). Rapid RNA degradation and induced transcription of certain genes after blood draws has led to the development of methodologies for preserving the RNA transcription profile immediately after blood is drawn. The PAXgen ${ }^{\mathrm{TM}}$ tube contains a blend of RNA stabilizing reagents that protect RNA molecules from degradation by ribonucleases (RNases) and prevents further induction of gene transcription. Without this stabilization, copy numbers of individual messenger RNA (mRNA) species in whole blood can change more than 1,000-fold during storage and transport. The RNA from blood in PAXgene ${ }^{\mathrm{TM}}$ tubes was isolated according to manufacturer standard protocols, which included an on-column deoxyribonuclease (DNase) treatment to remove contaminating genomic DNA (gDNA; silica-based microspin technology), and the extracted RNA was stored at $-80{ }^{\circ} \mathrm{C}$ until analysis. All RNA was checked for quality on a nanodrop 2000 and achieved A260/A280 ratios of about 2.0 and A260/A230 ratios of less than 1.0. 


\section{Complementary Deoxyribonucleic Acid Creation}

A standard complementary DNA (cDNA) synthesis was performed on $2 \mu \mathrm{g}$ of RNA template from each animal. Reaction conditions included 4 units reverse transcriptase (Omniscript ${ }^{\circledR}$, Qiagen, Valencia, California), 1 micromolar $(\mu \mathrm{M})$ random hexamers, 0.5 millimolar $(\mathrm{mM})$ each deoxyribonucleotide triphosphate (dNTP), and 10 units RNase inhibitor, in Reverse Transcriptase buffer (Qiagen, Valencia, CA). Reactions were incubated for 60 minutes at $37^{\circ} \mathrm{C}$, followed by an enzyme inactivation step of 5 minutes at $93{ }^{\circ} \mathrm{C}$, and then stored at $-20^{\circ} \mathrm{C}$ until further analysis.

\section{Real-Time Polymerase Chain Reaction}

Real-time polymerase chain reaction (PCR) systems for the individual, sea otter-specific reference or housekeeping gene (S9) and genes of interest were run in separate wells (Bowen and others, 2012; Miles and others, 2012; table 6). Briefly, $1 \mu \mathrm{L}$ of cDNA was added to a mix containing $12.5 \mu \mathrm{L}$ of QuantiTect SYBR Green ${ }^{\circledR}$ Master Mix $\left[5 \mathrm{mM} \mathrm{Mg}^{2+}\right.$ ] (Qiagen, Valencia, California), $0.5 \mu \mathrm{L}$ each of forward and reverse sequence specific primers, $0.5 \mu \mathrm{L}$ of Uracil-NGlycosylase (Invitrogen, Carlsbad, California), and $10.0 \mu \mathrm{L}$ of RNase-free water; total reaction mixture was $25 \mu \mathrm{L}$. The reaction mixture cDNA samples for each gene of interest and the S9 gene were loaded into 96 well plates in duplicate and sealed with optical sealing tape (Applied Biosystems, Foster City, California). Reaction mixtures containing water, but no cDNA, were used as negative controls; thus, about 3-4 individual sea otter samples were run per plate.

Amplifications were conducted on a 7300 Real-time Thermal Cycler (Applied Biosystems, Foster City, California). Reaction conditions were as follows: (1) $50{ }^{\circ} \mathrm{C}$ for 2 minutes, (2) $95{ }^{\circ} \mathrm{C}$ for 15 minutes, (3) 40 cycles of $94{ }^{\circ} \mathrm{C}$ for 30 seconds, (4) $58^{\circ} \mathrm{C}$ for 30 seconds, (5) $72{ }^{\circ} \mathrm{C}$ for 31 seconds, and (6) an extended elongation phase at $72{ }^{\circ} \mathrm{C}$ for 10 minutes. Reaction specificity was monitored by melting curve analysis using a final data acquisition phase of 60 cycles of $65^{\circ} \mathrm{C}$ for 30 seconds and verified by direct sequencing of randomly selected amplicons (Bowen and others, 2007). Cycle threshold crossing values $\left(\mathrm{C}_{\mathrm{T}}\right)$ for the genes of interest were normalized to the $\mathrm{S} 9$ housekeeping gene.

\section{Statistical Analysis}

Analysis of quantitative PCR (qPCR) data was conducted using normalized values; that is, housekeeping gene threshold crossing (in qPCR, the point at which amplification is exponential) subtracted from the gene of interest threshold crossing for each animal (McLoughlin and others, 2006).

We used nonparametric statistical analyses because the cycle threshold $\left(\mathrm{C}_{\mathrm{T}}\right)$ measure of gene transcription provided by qPCR may have a highly skewed, non-normal distribution (McLoughlin and others, 2006). We conducted multivariate, multi-dimensional scaling (MDS) analysis in conjunction with cluster analysis for statistical and graphical representation of individual sea otters clustered by similarity in transcription and not by predefined groups such as location (Primer E, version 6, Plymouth, United Kingdom). Statistical comparisons of individuals by clusters were made using SIMPROF, which is a similarity profile permutation test for significance among theoretically unstructured clusters of samples. We used ANOSIM (Primer E) one-way nonparametric, multivariate analysis to test for differences in gene transcription among locations; that is, sea otters captured at or near the coastlines of Monterey Bay, Big Sur, San Luis Obispo County, and Santa Barbara County, and the captive sea otters. We used two-way ANOSIM to examine within locations by sex and between or among capture years. Capture years varied by location (for example, sea otters were captured only in 2012 at the San Luis Obispo region); thus, analysis of all locations by years was not possible. Sea otters captured were biased towards adult 
animals (79 percent), precluding any reliable characterization of juvenile gene transcription patterns, so age differences were not evaluated. Statistical significance was based on $p$ values less than or equal to 0.05 , and in the case of the ANOSIM tests, relative to the $R$ statistic value. We further classified clusters in groups based on the number of genes markedly (that is, 3 or more $C_{T}$ value) higher or lower than background values established for captive sea otters deemed clinically healthy ("captives") (Bowen and others, 2012). Hereinafter, "high" or "low" followed by a gene identifier is used only to report those cases where gene transcription values were "markedly" different.

Additionally, we used gene transcript profiling based on per-gene and per-otter response correlation, using normalized qPCR data obtained from each individual otter, which were subjected to hierarchical clustering with complete linkage disequilibrium (Genesis, Graz, Switzerland); this was used to generate a heatmap profile of gene expression and physiologic health status.

\section{Results}

\section{Transcription Pattern Analysis}

Transcription patterns were established for 180 sea otters from the central California coast. Sea otter gene transcription patterns differed among locations (ANOSIM, $p<0.001$, Global $R=$ 0.21 , with 0 permuted statistics $>$ Global $R$ ). Pairwise tests indicated that values for sea otters from the San Luis Obispo area differed significantly $(p<0.001)$ from the background captive values as well as all other areas examined in California; these differences primarily were influenced by San Luis Obispo area sea otters that had markedly lower transcription of HDCMB21P (HDC), thyroid hormone receptor beta (THRB), interleukin 10 (IL-10), and Mx1 than those from the other locations (table 7).

Sea otters from areas of Monterey Bay, Big Sur, or Santa Barbara did not differ from the captives $(p>0.14)$. However, transcription patterns of sea otters from Santa Barbara differed from those from Big Sur and Monterey $(p<0.001)$, which also differed significantly from one another $(p<0.002)$.

Santa Barbara area sea otters had markedly higher levels of the serotonin transport gene (5HTT) and also low HDC and IL-10 relative to sea otters from the other locations, and Monterey and Big Sur sea otters differed primarily in transcription of cyclooxygenase-2 (COX2) and complement cytolysis inhibitor (CYT). The MDS analysis of gene transcription $\mathrm{C}_{\mathrm{T}}$ values depicts separations of which the denser mass of clusters included the captives, with the remaining otters diffusely clustered in smaller groups or single-animal outliers (fig. 7). The captives were the only defined group that clustered tightly. The three-dimensional MDS depiction indicated a better fit (that is, Stress $=0.10$ compared to 0.14 for two dimensions), and visual separation within the densest mass. SIMPROF analysis indicated highly significant $(p=0.001-0.007 ; 79$ percent of the clusters) or significant ( $p=0.01-0.05)$ separations among 33 clusters that included outliers.

Forty-one ( 23 percent of total) sea otters comprising 4 clusters had transcription patterns similar to captives; differences among these clusters comprised mean or outlier $\mathrm{C}_{\mathrm{T}}$ values of targeted genes within $3 \mathrm{C}_{\mathrm{T}}$ of clinically normal captives. Most (27) of these animals were from the area near Big Sur, and the remainder were from areas at Monterey Bay (11) or north of Santa Barbara (3).

Qualifying the clusters into groups based on number of genes with markedly different $\mathrm{C}_{\mathrm{T}}$ than the captives, 6 clusters comprising 19 (11 percent of total) sea otters separated into 2 subgroups, one with both elevated and low gene $\mathrm{C}_{\mathrm{T}}$ values and the other with elevated $\mathrm{C}_{\mathrm{T}}$ values relative to the captives. These were the only sea otters sampled that had transcription patterns that indicated reactive immune or defensive responses. A cluster including five sea otters from Santa 
Barbara had high 5HTT but low HDC and IL-18, whereas one from Big Sur had high IL18 and low CYT and AHR. The remaining Monterey Bay area sea otter (ID 1117) in this group had the most concerning transcription pattern of any sea otter sampled in this study: high COX2, THR, IL10, DRB, Mx1, and low HDC. The other subgroup had 1 San Luis Obispo sea otter with high IL18 and Mx1, 7 Monterey Bay area sea otters with high CYT, and 1 Big Sur and 3 Santa Barbara sea otters with high Mx1 and 5HTT.

All the remaining clusters comprised average transcript values that were markedly lower than those established for captives. Most of the sea otters that comprised this low transcription set were from the southern one-half of the sea otter range in California; that is, San Luis Obispo area (63 percent) and Santa Barbara (25 percent, mostly animals that were captured in 2013), with 6 percent each from the northern sites of Big Sur and Monterey.

In the 4-6 gene groups, 18 sea otters had 6 genes, 13 otters had 5 genes, and 20 otters had 4 genes of markedly lower transcription than those patterns determined for captives. Within these groupings, which 6, 5, or 4 genes were markedly lower varied among the 51 (28 percent of total) sea otters. Within the 6-gene group, HSP 70, IL-10, Mx1, HDC, and COX2 were most evident, and then THR, AHR, IL-18, or CCR3 composed the sixth gene. Transcription of CYT, 5HTT, and $\mathrm{CaM}$ was similar to $\mathrm{C}_{\mathrm{T}}$ values of the captives. Within the 5-gene group, low transcription of HSP70, IL-18, and Mx1 were prominent, followed by HDC, COX2, and IL10, and then CYT, CCR3, and 5HTT. In this group, only AHR and CaM were similar to the captives. Within the 4gene group, low transcription of HDC, HSP70, IL-10, and Mx1 was evident for most (18 of 20) of these otters, whereas 2 otters had an altogether different set of 4 genes of low transcription. Transcriptions of THR, DRB, CCR3, 5HTT, and CaM were similar to those of the captives.

The next grouping of 69 sea otters ( 38 percent of total) comprised 12 sea otters with 3 genes, 39 sea otters with 2 genes, or 18 otters with 1 gene markedly lower in transcription compared to captives. Sea otters from this set were more evenly spread across the range, comprising 24 from San Luis Obispo, 19 from Santa Barbara, 16 from Big Sur, and 9 from Monterey Bay areas. Within the 3-gene group, most had low transcription of COX2, MX1, and either HDC or HSP70. In the 2-gene group, 72 percent of the sea otters had low transcription of IL18 but the second gene was HDC, COX2, HSP70, IL10, or MX1. Within the 1-gene group, 15 sea otters had low transcription of HDC and the remaining 3 had low transcription of IL10.

\section{Within Site-Sex by Year Transcription Differences}

Multivariate analysis of transcription patterns of sex by years indicated no difference at Monterey Bay between 2009 and 2010 ( $\mathrm{p}>0.53$ ). At Big Sur, transcription patterns among years differed $(2008,2009,2010 ; p<0.04)$ attributed to the difference between 2008 and $2010(p<$ $0.001)$, but did not differ by sex $(\mathrm{p}>0.38)$. Although sexes were skewed toward more females captured, no marked or notable differences in transcription were apparent among the suite of genes. Transcription of HDC, CYT, AHR, and THRB decreased from 2008 to 2010 at Big Sur, whereas HSP70, IL-18, and CCR3 increased although values were not markedly different from captive background levels.

Variations in transcription patterns at the Santa Barbara area were most evident, where both sex $(p<0.04)$ and years $(p<0.001)$ differed (table 7). Transcription of COX2 in female sea otters in 2012 was similar to captive levels but low in males in 2012 and in females in 2013. Transcription of genes THRB and 5HTT was markedly higher than captive values in both sexes in 2012, as was CCR3 in females, but in 2013 these values were similar to background values. We found markedly (relative to background) low transcription of IL-10 and MX-1 in 2013 compared to 2012. 


\section{Gene-Specific Patterns}

Cluster analyses did not indicate apparent transcription patterns in individual genes. Because of natural oil seeps near Santa Barbara, we examined elevated (relative to background captive levels) transcription of AHR and THRB among the sampled sea otters. Twenty-six sea otters had high ( $\geq 2 \mathrm{C}_{\mathrm{T}}$ value difference) to markedly high AHR transcription. These animals were from areas of Monterey Bay (7), Big Sur (8), and Santa Barbara (8), with a few (3) from San Luis Obispo. Similarly, 26 sea otters had elevated transcription of THRB, and in this instance, 17 of these animals were from Santa Barbara, 6 from Big Sur, and 3 from Monterey Bay. We also noted that IL-10 was elevated in 26 sea otters (10 in Big Sur, 8 in Santa Barbara, 7 in Monterey Bay, and 1 in San Luis Obispo). Only 19 percent (5) of these sea otters had elevated transcription of all three genes, and 1 additional animal had two of these genes with elevated transcription.

\section{Heatmap Pattern Analysis}

Hierarchical cluster analysis was conducted using individual sea otter transcription data (fig. 8). Profile responses were successful in identifying transcriptional differences between capture locations and years, and yielded varying sized clusters of sea otters consisting of as much as 100 percent of otters from single populations and time points. The largest clusters comprised San Luis Obispo and Santa Barbara otters in 2013. Sea otters from Monterey Bay (2009), Big Sur (both years), and Santa Barbara (2012), and captives also dominated clusters.

\section{Discussion}

\section{Transcription Pattern Analysis}

Transcription patterns analyzed using MDS, ANOSIM, gene-specific patterns, and hierarchical cluster-generated heatmap yielded corroborating results. Most sea otters, particularly those from San Luis Obispo, had patterns of inexplicably low gene transcription in relation to all other study groups. The occurrence of low transcription has been observed in past analyses but the preponderance among California sea otters system-wide was noteworthy. We confirmed that the low transcription values were not a result of a laboratory artifact. We analyzed all samples in duplicate, and if any duplicate samples were $>1 \mathrm{C}_{\mathrm{T}}$ difference, they were re-analyzed. All samples were run with an internal reference standard (that is, housekeeping gene, S9). If the internal reference was greater than $3 \mathrm{C}_{\mathrm{T}}$ values from the average internal reference value for control populations, the entire panel was re-run in duplicate; this was always identified to be a problem of insufficient RNA and those samples were omitted from analysis (that is, $<1$ percent of the samples). Finally, California samples were run with Alaska samples and re-run in different combinations to check for "plate" or "batch" effects, and no such effects were encountered.

Abnormally low levels of select cytokine transcripts may be interpreted as indicative of abnormally low levels of leukocyte activity in that animal; such suppressed activity has been described for $\mathrm{T}$ helper lymphocytes derived from humans with chronic viral infections (Stott and McBain, 2012). Alternatively, low transcription may be the result of unbalanced resource allocation. For example, immune defenses exist to impede infections, but other ecological demands (for example, nutrition, weather, predation) can supersede this, causing immune defenses to be compromised (Martin and others, 2011). It is optimal to increase the allocation to immune defense as reserves increase (Houston and others, 2007); however, the costs associated with mounting an immune response create a delicate balance between a protective immune phenotype (transcription pattern in this case) and a potential misallocation of resources. Tradeoffs among components of the vertebrate immune system itself are common and occur when one part of the immune system is up-regulated while another part is down-regulated; activation of one branch of 
immune function may effectively disable an opposing branch (Pedersen and Babayan, 2011). Failure to acknowledge or measure these trade-offs may lead to erroneous conclusions. What we may deem to be abnormally low transcription may simply be due to a lack of breadth in our transcript panel. Our panel was more specific to organic exposure and included other indicators such as microbial or inflammatory defenses, and was by no means an exhaustive evaluation of genes stimulated by potential environmental stressors in the areas examined.

\section{Within-Site Year by Sex Transcription Differences}

Within-site transcript level differences were most notable at Big Sur and Santa Barbara. Differences in transcript level generally were minimal between sexes, but a there was a distinct separation of transcript level among years for Big Sur sea otters. The Basin and Chalk wildfires in the Big Sur region of central California in 2008 occurred just prior to a scheduled study of sea otters in the adjacent nearshore marine environment. This timing provided an opportunity to assess the potential effects of wildfire on the immunological response of sea otters just downslope from where the fire burned. The Basin fire burned about 66,500 ha between June 21 and late July, whereas the Chalk Fire consumed an additional 6,240 ha between ignition on September 27 and containment by the end of October. For the two wildfires combined, 45 percent of the burned area lay within the drainage basin that discharges into the territory of the Big Sur sea otter population. The fires burned with mixed severity, with partial to complete consumption of the heavy vegetation within the perimeter.

These fires had two potential indirect impacts on the sea otter population that may manifest in a genetic analysis of toxicological effects. First, during and immediately after the wildfires, prevailing nighttime wind patterns consistently carried ash and debris from the smoke column offshore and onto the surface waters (Western Regional Climate Center, 2012). This ash was observed intermixed in the surface water column by capture crews during the November 2008 sampling bout. Second, there are seven minor drainages that carry runoff into the adjacent nearshore environment from the burned area. During November 1-3, the Big Sur Remote Automated Weather Station (RAWS) measured 2.6 in. of rain (Western Regional Climate Center, 2012), the first appreciable rainfall in more than 6 months. This storm event subsequently produced a runoff spike in the Big Sur River that exceeded $200 \mathrm{ft}^{3} / \mathrm{s}$, compared to the $10-15 \mathrm{ft}^{3} / \mathrm{s}$ discharge of the river in the summer and early autumn months. Following this event, the Big Sur area had a slightly drier than normal winter, with $35 \mathrm{in.} \mathrm{(91} \mathrm{percent} \mathrm{of} \mathrm{normal)} \mathrm{precipitation} \mathrm{for} \mathrm{the}$ water year ending in October 2009 (Western Regional Climate Center, 2012). Gene responses were distinctly different between Big Sur temporal groups, identifying detoxification of PAHs (upregulation of AHR, $p<0.001$ ) and associated malignant transformation (up-regulation of HDC, $p$ $=0.05$ ) as the primary responses of sea otters to fire in 2008 compared to those captured in 2009 and 2010 (Bowen and others, 2015). Down-current effects of exposure to PAHs were evidenced in the 2008 sea otters by immune suppression (down-regulation of IL-10, $p<0.0011$; downregulation of Il-18, $p<0.001$; down-regulation of DRB, $p=0.01$ ). Gene transcription patterns in the 2008 sea otters generally were indicative of molecular reactions to organic exposure, malignant transformation, and decreased ability to respond to pathogens that may be consistent with shortterm hydrocarbon exposure. Transcript patterns of Big Sur sea otters in 2009 and 2010 were indicative of a return towards baseline normal.

The separation of Santa Barbara sea otter transcript levels by year and sex was surprising and unexpected. Santa Barbara sea otters in 2012 generally had transcription patterns that may have been related to hydrocarbon exposure, that is, elevated THRB and to some degree, AHR, a pattern unique to this area. This may indicate periodic exposure to the natural oil seeps that occur throughout Santa Barbara channel. Additionally, 5HTT was elevated in 2012, a marker of low 
stress phenotype. In contrast, low transcription in 2013 Santa Barbara sea otters was similar to that seen in otters from the San Luis Obispo area.

Overall, with the exception of low transcription levels of HDC, THRB, IL-10, and Mx1 in San Luis Obispo sea otters (table 7), any observed gene expression levels that differed from background values seemed more unique to individuals rather than influenced by the area in which sea otters resided; that is, these abnormal individuals occurred with approximately equal frequencies at all sites. Consistent and significant elevation of gene transcription was not apparent in any areas of the central California coast (with the exception of Big Sur immediately after the fires), including suspect areas such as the urbanized region encompassing Monterey Bay. We suggest that these patterns indicated immune responses representative of those of healthy captive sea otters, and thus indicated that environmental conditions experienced by sea otters in central California generally fall within the "normal" range, with occasional pulses of challenging conditions occurring throughout the range associated with both natural events (for example, wild fires, oil seeps) or human-caused stressors (for example, organic pollutants or terrestrial pathogens). This conclusion is based on the observation that most sea otters sampled were either similar in gene transcription to captive background levels or had expression of only 1-3 genes markedly different from background. However, consistent patterns of low transcription at the San Luis Obispo study site require further investigation.

Until now, it has only been feasible to study small numbers of genes, usually stemming from a candidate gene approach. However, recent advances in deep sequencing technology allow for the clarification of an unprecedented breadth of gene pathways. Through the deep sequencing of transcriptomes, broad scale identification of gene transcription patterns can provide mechanistic understanding of protective immune phenotypes and immune proxies of health; causal links between molecular patterns and individual and population health become possible (Pedersen and Babayan, 2011). We propose experimentation to use deep sequencing to identify those genes or suites of genes in the California sea otters that may be drawing resources responsible for the low transcription profile at San Luis Obispo in our current gene panel. Until such experimentation is realized, the cause cannot be substantiated.

\section{References Cited}

Acevedo-Whitehouse, K., and Duffus, L.J., 2009, Effects of environmental change on wildlife health: Philosophical Transactions of the Royal Society B, v. 364, p. 3429-3438.

Bartosiewicz, M., Penn, S., and Buckpitt, A., 2001, Applications of gene arrays in environmental toxicology-Fingerprints of gene regulation associated with cadmium chloride, benzo(a)pyrene, and trichloroethylene: Environmental Health Perspectives, v. 109, p. 71-74.

Beineke, A., Siebert, U., Muller, G., and Baumgartner, W., 2007, Increased blood interleukin-10 mRNA levels in diseased free-ranging harbor porpoises (Phocoena phocoena): Veterinary Immunology and Immunopathology, v. 115, p. 100-106.

Bommer, U.A., and Thiele, B.J., 2004, The translationally controlled tumour protein (TCTP): International Journal of Biochemistry and Cell Biology, v. 36, p. 379-385.

Bourlat, Sarah J., and others, 2013, Genomics in marine monitoring-New opportunities for assessing marine health status: Marine Pollution Bulletin, v. 74, no. 1, p. 19-31.

Bowen, L., Aldridge, B., Miles, A.K., and Stott, J.L., 2006, Expressed MHC class II genes in sea otters (Enhydra lutris) from geographically disparate populations: Tissue Antigens, v. 67, p. 402-408.

Bowen, L., Miles, A.K., Kolden, C.A., Saarinen, J.A., Bodkin, J.L., Murray, M., and Tinker, T., 2015, Effects of wildfire on sea otter (Enhydra lutris) immune function: Marine Mammal Science, v. 31, p. 191-210. 
Bowen, L., Miles, A.K., Murray, M., Haulena, M., and others, 2012, Gene transcription in sea otters (Enhydra lutris) - Development of a diagnostic tool for sea otter and ecosystem health: Molecular Ecology Ressources, v. 12, p. 67-74.

Bowen, L., Schwartz, J., Aldridge, B., Riva, F., Miles, A.K., Mohr, F.C., and Stott, J.L., 2007, Differential gene expression induced by exposure of captive mink to fuel oil-A model for the sea otter: EcoHealth, v. 4, p. 298-309.Burchiel, S.W., and Luster, M.I., 2004, Signaling by environmental polycyclic aromatic hydrocarbons in human lymphocytes: Clinical Immunology, v. 98 , p. 2-10.

Burczynski, M.E., McMillian, M., Ciervo, J., Li, L., Parker, J.B., Dunn, R.T. II, and others, 2000, Toxicogenomics-based discrimination of toxic mechanism in HepG2 human hepatoma cells. Toxicological Science, v. 58, p. 399-415.

Chen, Z., Wang, H., Matsumura, K., and Qian, P., 2012, Expression of calmodulin and myosin light chain kinase during larval settlement of the barnacle Balanus amphitrite: PLoS ONE, v. 7, no. 2 , p. e31337.

De Maio, A., 1999, Heat shock proteins-Facts, thoughts, and dreams: Shock, v. 11, p. 1-12.

Dong, L., Ma, Q., and Whitlock, J.P., Jr., 1997, Down-regulation of major histocompatibility complex Q1b gene expression by 2,3, 7,8-tetrachlorodibenzo-p-dioxin: Journal of Biological Chemistry, v. 272, p. 29614-29619.

Ghanem, M.M., Batelli, L.A., Mercer, R.R., Scabilloni, J.F., Kashon, M.L., Ma, J.Y., and others, 2006, Apoptosis and bax expression are increased by coal dust in the polycyclic aromatic hydrocarbon-exposed lung: Environmental Health Perspectives, v. 114, p. 1367-1373.

Goldsby, R.A., Kindt, T.J., Osborne, B.A., and Kuby, J., 2003, Immunology (5th ed.): New York, W.H. Freeman and Company, 603 p.

Gurish, M.F., Humbles, A., Tao, H., Finkelstein, S., Boyce, J.A., Gerard, C., Friend, D.S., and Austen, K.F., 2002, CCR3 is required for tissue eosinophilia and larval cytotoxicity after infection with Trichinella spiralis: The Journal of Immunology, v. 168, p. 5730-5736.

Harris, S.G., Padilla, J., Koumas, L., Ray, D., and Phipps, R.P., 2002, Prostaglandins as modulators of immunity: Trends in Immunology, v. 23, p. 144-150.

Houston, A.I., McNamara, J.M., Barta, Z., and Klasing, K.C., 2007, The effect of energy reserves and food availability on optimal immune defence: Proceedings of the Royal Society BBiological Sciences, v. 274, no. 1627, p. 2835-2842.

Iwama, G.K., Mathilakath, M.V., Forsyth, R.B., and Ackerman, P.A., 1999, Heat shock proteins and physiological stress in fish: American Zoologist, v. 39, p. 901-909.

Jenne, D.E., and Tschopp, J., 1989, Molecular structure and functional characterization of a human complement cytolysis inhibitor found in blood and seminal plasma-Identity to sulfated glycoprotein 2, a constituent of rat testis fluid: Proceedings of the National Academy of Sciences of the United States of America, v. 86, p. 7123-7127.

Jennings, K.A., Loder, M.K., and Sheward, W.J., 2006, Increased expression of the 5-HT transporter confers a low-anxiety phenotype linked to decreased 5-HT transmission: Journal of Neuroscience, v. 30, p. 8955-8964.

Kibenge, M.J.T., Munir, K., and Kibenge, F.S.B., 2005, Constitutive expression of Atlantic salmon Mx1 protein in CHSE-214 cells confers resistance to infectious salmon Anaemia virus: Virology Journal, v. 2, p. 75.

Kringel, H., Iburg, T., Dawson, H., Aasted, B., and Roepstorff, A., 2006, A time course study of immunological responses in Trichuris suis infected pigs demonstrates induction of a local type 2 response associated with worm burden: International Journal for Parasitology, v. 36, p. 915-924.

Krumm, B., Meng, X., Li, Y., Xiang, Y., and Deng J., 2008, Structural basis for antagonism of human interleukin 18 by pox-virus interleukin 18-binding protein: Proceedings of the National Academy of Sciences of the United States of America, v. 105, p. 20711-20715. 
Li, S., Xie, L., Zhang, C., Zhang, Y., Gu, M., and others, 2004, Cloning and expression of a pivotal calcium metabolism regulator-Calmodulin involved in shell formation from pearl oyster (Pinctada fucata): Comparative Biochemistry and Physiology Part B-Biochemistry and Molecular Biology, v. 138, p. 235-243.

Lipscomb, T.P., Harris, R.K., Moeller, R.B., Pletcher, J.M., Haebler, R.J., and Ballachey, B.E. 1993, Histopatholgic lesions in sea otters exposed to crude oil: Veterinary Pathology, v. 30, p. $1-11$.

Ma, Q., Geng, Y., Xu, W., Wu, Y., and others, 2010, The role of translationally controlled tumor protein in tumor growth and metastasis of colon adenocarcinoma cells: Journal of Proteome Research, v. 9, p. 40-49.

Martin, L.B., Hopkins, W.A., Mydlarz, L.D., and Rohr, J.R., 2011, The effects of anthropogenic global changes on immune functions and disease resistance: Annals of the New York Academy of Sciences, v. 1195, p. 129-148.

McLoughlin, K., Turteltaub, K., Bankaitis-Davis, D., Gerren, R., and others, 2006, Limited dynamic range of immune response gene expression observed in healthy blood donors using RTPCR: Molecular Medicine, v. 12, p. 185-195.

Miles, A.K, Bowen, L., Ballachey, B., Bodkin, J.L., Murray, M., Keister, R.A., Stott, J.L., 2012, Variations of transcript profiles between sea otters (Enhydra lutris) from Prince William Sound, Alaska, and clinically normal reference otters: Marine Ecology Progress Series, v. 451, p. 201-212

Monson, D.H., Doak, D.F., Ballachey, B.E., Johnson, A., and Bodkin, J.L., 2000, Long-term impact of the Exxon Valdez oil spill on sea otters, assessed through age-dependent mortality patterns: Proceedings of the National Academy of Sciences of the United States of America, v. 97, p. 6562-6567.

Monson, D.H., McCormick, C., and Ballachey, B., 2001, Chemical anesthesia of northern sea otters (Enhydra lutris) — Results of past field studies: Journal of Zoo and Wildlife Medicine, v. 32, p. $181-189$.

Oesch-Bartlomowicz, B., and Oesch, F., 2005, Phosphorylation of cytochromes P450-First discovery of a posttranslational modification of a drug-metabolizing enzyme: Biochemical and Biophysical Research Communications, v. 338, p. 446-449.

Pedersen, A.B., and Babayan, S.A., 2011, Wild immunology: Molecular Ecology, v. 20, no. 5, p. 872-880.

Quintana, F.J., Basso, A.S., Iglesias, A.H., Korn, T., Farez, M.F., Bettelli, E., Caccamo, M., Oukka, M., and Weiner, H.L., 2008, Control of T(reg) and T(H)17 cell differentiation by the aryl hydrocarbon receptor: Nature, v. 453, p. 6-7.

Raisuddin, S., Kwok, K.W.H., Leung, K.M.Y., Schlenk, D., and Lee, J., 2007, The copepod Tigriopus - A promising marine model organism for ecotoxicology and environmental genomics: Aquatic Toxicology, v. 83, p. 161-173.

Ramesh, A., Walker, S.A., Hood, D.B., Guillen, M.D., Schneider, K., and Weyand, E.H., 2004, Bioavailability and risk assessment of orally ingested polycyclic aromatic hydrocarbons: International Journal of Toxicology, v. 23, p. 301-333.

Rigopoulou, E.I., Abbott, W.G., Haigh, P., and Naoumov, N.V., 2005, Blocking of interleukin-10 receptor-A novel approach to stimulate T-helper cell type 1 responses to hepatitis Cvirus: Clinical Immunology, v. 117, p. 57-64.

Schwartz, J.A., Aldridge, B.M., Lasley, B.L., Snyder, P.W., Stott, J.L., and Mohr, F.C., 2004a, Chronic fuel oil toxicity in American mink (Mustela vison)—Systemic and hematological effects of ingestion of a low-concentration of bunker $\mathrm{C}$ fuel oil: Toxicology and Applied Pharmacology, v. 200, p. 146-158. 
Schwartz, J.A., Aldridge, B.M., Stott, J.L., and Mohr, F.C, 2004b, Immunophenotypic and functional effects of bunker $\mathrm{C}$ fuel oil on the immune system of American mink (Mustela vison): Veterinary Immunology and Immunopathology, v. 101, p. 179-190.

Sitt, T., Bowen, L., Blanchard, M.T., Smith, B.R., Gershwin, L.J., Byrne, B.A., and Stott, J.L., 2008, Quantitation of leukocyte gene expression in cetaceans: Developmental and Comparative Immunology, v. 32, p. 1253-1259.

Squire, L.R., and others, 2008, Fundamental Neuroscience, 3rd ed.: Amsterdam, Elsevier /Academic Press, 143 p.

Stott, J.L., and McBain, J.F., Longitudinal monitoring of immune System parameters of cetaceans and application to their health management, chap. 63 of Miller, E., and Fowler, M., eds., Fowler's zoo and wild animal medicine-Current therapy, p. 482-489.

Tabuchi, M., Veldhoen, N., Dangerfield, N., Jeffries, S., Helbing, C.C., and Ross, P.S., 2006, PCB-related alteration of thyroid hormones and thyroid hormone receptor gene expression in free-ranging harbor seals (Phoca vitulina): Environmental Health Perspectives, v. 114, p. 1024-1031.

Tanabe, S., Iwata, H., and Tatsukawa, R., 1994, Global contamination by persistent organochlorines and their ecotoxicological impact on marine mammals: Science of the Total Environment, v. 154, p. 163-177.

Tsai, M.J., and O'Malley, B.W., 1994, Molecular mechanisms of action of steroid/thyroid receptor superfamily members: Annual Review of Biochemistry, v. 63, p. 451-486.

Tsan, M.F., and Gao, B., 2004, Cytokine function of heat shock proteins: American Journal of Physiology-Cell Physiology, v. 286, p. C739-C744.

Tumpey, T.M., Szretter, K.J., Van Hoeven, N., Katz, J.M., and others, 2007, The Mx1 gene protects mice against the pandemic 1918 and highly lethal human H5N1 influenza viruses: Journal of Virology, v. 81, p. 10818-10821.

Tuynder, M., Fiucci, G., Prieur, S., Lespagnol, A., and others, 2004, Translationally controlled tumor protein is a target of tumor reversion: Proceedings of the National Academy of Sciences of the United States of America, v.101, p. 15364-15369.

Veldhoen, M., Hirota, K., Westendorf, A.M., Buer, J., Dumoutier, L., Renauld, J.C., and Stockinger, B., 2008, The aryl hydrocarbon receptor links TH17-cell-mediated autoimmunity to environmental toxins: Nature, v. 453, p. 106-109.

Wegner, K.M., Kalbe, M., Rauch, G, Kurtz, J., Schaschl, H., and Reusch, T.B.H., 2006, Genetic variation in MHC class II expression and interactions with MHC sequence polymorphism in three-spined sticklebacks: Molecular Ecology, v. 15, p. 1153-1164.

Wendell, F., Pattison, C., Harris, M., (1996) Sea Otter, Enhydra lutris, containment management: field studies and feasibility assessment. Calif Fish Game, Administrative Report 96-5. 10 p.

Western Regional Climate Center, 2012, Data from West wide drought tracker: Western Regional Climate Center web page, accessed December 23, 2012, at https://wrcc.dri.edu/.

Zheng, S., Song, Y., Qiu, X., Sun, T., Ackland, M.L., and Zhang, W., 2008, Annetocin and TCTP expressions in the earthworm Eisenia fetida exposed to PAHs in artificial soil: Ecotoxicology and Environmental Safety, v. 71, p. 566-573. 
Table 6. Documented function of 13 genes identified in sea otters.

\begin{tabular}{|c|c|}
\hline Gene & Gene function \\
\hline HDC & $\begin{array}{l}\text { The HDCMB21P gene codes for a translationally controlled tumor protein (TCTP) implicated in } \\
\text { cell growth, cell cycle progression, malignant transformation, tumor progression, and in the } \\
\text { protection of cells against various stress conditions and apoptosis (Bommer and Thiele, 2004; } \\
\text { Tuynder and others, 2004; Ma and others, 2010). Up-regulation of HDC is indicative of the } \\
\text { development or existence of cancer. Environmental triggers may be responsible for population- } \\
\text { based, up-regulation of HDC. HDC transcription is known to increase with exposure to } \\
\text { carcinogenic compounds such as polycyclic aromatic hydrocarbons (Bowen and others, 2007; } \\
\text { Raisuddin and others, 2007; Zheng and others, 2008). }\end{array}$ \\
\hline $\mathrm{COX} 2$ & $\begin{array}{l}\text { Cyclooxygenase-2 (COX2) catalyzes the production of prostaglandins that are responsible for } \\
\text { promoting inflammation (Goldsby and others, 2003). Cox } 2 \text { is responsible for the conversion of } \\
\text { arachidonic acid to prostaglandin } \mathrm{H} 2 \text {, a lipoprotein critical to the promotion of inflammation } \\
\text { (Harris and others, 2002). Up-regulation of COX2 is indicative of cellular or tissue damage and } \\
\text { an associated inflammatory response. }\end{array}$ \\
\hline CYT & $\begin{array}{l}\text { The complement cytolysis inhibitor (CYT) protects against cell death (Jenne and Tschopp, } \\
\text { 1989). Up-regulation of CYT is indicative of cell or tissue death. }\end{array}$ \\
\hline AHR & $\begin{array}{l}\text { The arylhydrocarbon receptor (AHR) responds to classes of environmental toxicants including } \\
\text { polycyclic aromatic hydrocarbons, polyhalogenated hydrocarbons, dibenzofurans, and dioxin } \\
\text { (Oesch-Bartlomowicz and Oesch, 2005). Depending on the ligand, AHR signaling can modulate } \\
\left.\text { T-regulatory ( } \mathrm{T}_{\mathrm{REG}} \text { ) (immune-suppressive) or T-helper type } 17 \text { ( } \mathrm{T}_{\mathrm{H}} 17\right) \text { (pro-inflammatory) } \\
\text { immunologic activity (Quintana and others, 2008; Veldhoen and others, 2008). }\end{array}$ \\
\hline THR & $\begin{array}{l}\text { The thyroid hormone receptor (THR) beta can be used as a mechanistically based means of } \\
\text { characterizing the thyroid-toxic potential of complex contaminant mixtures (Tabuchi and others, } \\
\text { 2006). Thus, increases in THR transcription may indicate exposure to organic compounds } \\
\text { including polychlorinated biphenyls (PCBs) and associated potential health effects such as } \\
\text { developmental abnormalities and neurotoxicity (Tabuchi and others, 2006). Hormone-activated } \\
\text { transcription factors bind deoxyribonucleic acid (DNA) in the absence of hormone, usually } \\
\text { leading to transcriptional repression (Tsai and O'Malley, 1994). }\end{array}$ \\
\hline HSP 70 & $\begin{array}{l}\text { The heat shock protein } 70 \text { (HSP 70) is produced in response to thermal or other stress (Iwama } \\
\text { and others, 1999; Tsan and Gao, 2004). In addition to being expressed in response to a wide } \\
\text { array of stressors (including hyperthermia, oxygen radicals, heavy metals, and ethanol), heat } \\
\text { shock proteins act as molecular chaperones (De Maio, 1999). For example, heat shock proteins } \\
\text { aid in the transport of the AHR/toxin complex in the initiation of detoxification (Tanabe and } \\
\text { others, 1994). }\end{array}$ \\
\hline IL-18 & $\begin{array}{l}\text { Interleukin-18 (IL-18) is a pro-inflammatory cytokine (Goldsby and others, 2003). IL-18 plays } \\
\text { an important role in inflammation and host defense against microbes (Krumm and others, 2008). }\end{array}$ \\
\hline IL-10 & $\begin{array}{l}\text { Interleukin-10 (IL-10) is an anti-inflammatory cytokine (Goldsby and others, 2003). Levels of } \\
\text { IL-10 have been correlated with relative health of free-ranging harbor porpoises; for example, } \\
\text { increased amounts of IL-10 correlated with chronic disease, whereas the cytokine was relatively } \\
\text { reduced in apparently fit animals experiencing acute disease (Beineke and others, 2007). } \\
\text { Association of IL-10 transcription with chronic disease also has been documented in humans } \\
\text { (Rigopoulou and others, 2005). }\end{array}$ \\
\hline DRB & $\begin{array}{l}\text { A component of the major histocompatibility complex, the DRB class II gene, is responsible for } \\
\text { the binding and presentation of processed antigen to } \mathrm{T}_{\mathrm{H}} \text { lymphocytes, thereby facilitating the } \\
\text { initiation of an immune response (Goldsby and others, 2003; Bowen and others, 2006). Up- } \\
\text { regulation of MHC genes has been positively correlated with parasite load (Wegner and others, } \\
\text { 2006), whereas down-regulation of MHC has been associated with contaminant exposure (Dong } \\
\text { and others, 1997). }\end{array}$ \\
\hline
\end{tabular}




\begin{tabular}{|c|l|}
\hline Gene & \multicolumn{1}{c|}{ Gene function } \\
\hline Mx1 & $\begin{array}{l}\text { The Mx1 gene responds to viral infection (Tumpey and others, 2007). Vertebrates have an early } \\
\text { strong innate immune response against viral infection, characterized by the induction and } \\
\text { secretion of cytokines that mediate an antiviral state, leading to the up-regulation of the Mx1 } \\
\text { gene (Kibenge and others, 2005). }\end{array}$ \\
\hline CCR3 & $\begin{array}{l}\text { The chemokine receptor 3 (CCR3) binds at least seven different chemokines and is expressed on } \\
\text { eosinophils, mast cells (MC), and a subset of Th cells (Th2) that generate cytokines implicated in } \\
\text { mucosal immune responses (Gurish and others, 2002; Kringel and others, 2006). Up-regulation } \\
\text { of CCR3 occurs in the presence of parasites (Gurish and others, 2002; Kringel and others, 2006). }\end{array}$ \\
\hline 5HTT & $\begin{array}{l}\text { The serotonin transport gene codes for an integral membrane protein that transports the } \\
\text { neurotransmitter serotonin from synaptic spaces into presynaptic neurons. This transport of } \\
\text { serotonin by the SERT protein terminates the action of serotonin and recycles it in a sodium- } \\
\text { dependent manner (Jennings and others, 2006; Squire and others, 2008). Increased transcription } \\
\text { of 5HTT confers a low anxiety phenotype (Jennings and others, 2006). }\end{array}$ \\
\hline CaM & $\begin{array}{l}\text { Calmodulin (CaM) is a small acidic Ca }{ }^{2+} \text {-binding protein, with a structure and function that is } \\
\text { highly conserved in all eukaryotes. CaM activates various Ca }{ }^{2+} \text {-dependent enzyme reactions, } \\
\text { thereby modulating a wide range of cellular events, including metabolism control, muscle } \\
\text { contraction, exocytosis of hormones and neurotransmitters, and cell division and differentiation } \\
\text { (Chen and others, 2012). CaM also has been reported to be a pivotal calcium metabolism } \\
\text { regulator in the shell formation (Li and others, 2004). }\end{array}$ \\
\hline
\end{tabular}


Table 7. Geometric mean, cycle threshold $\left(\mathrm{C}_{\mathrm{T}}\right)$ of 13 genes identified in free-ranging sea otters sampled in Monterey, Big Sur, San Luis Obispo, and Santa Barbara, and in clinically normal captive reference animals sampled, 2008-13.

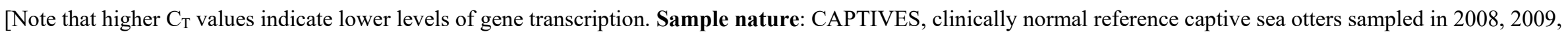

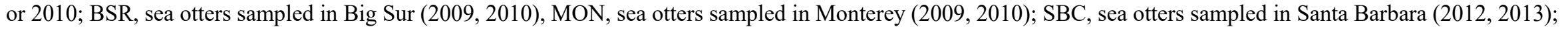

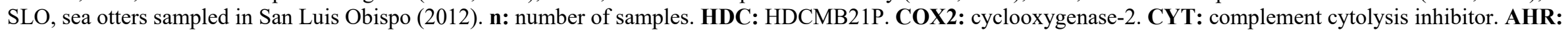
aryl hydrocarbon receptor THRB: thyroid hormone receptor beta. HSP70: heat shock protein 70. IL-18: interleukin 18. IL-10: interleukin 10. DRB: major histocompatibility complex DRB gene CCR3: chemokine receptor 3 5HTT: serotonin transport gene CaM: calmodulin]

\begin{tabular}{|c|c|c|c|c|c|c|c|c|c|c|c|c|c|c|c|}
\hline $\begin{array}{l}\text { Sample } \\
\text { nature }\end{array}$ & $\begin{array}{c}\text { Sample totals and } \\
\text { breakdown }\end{array}$ & $\mathrm{n}$ & HDC & cox2 & CYT & AHR & THRB & HSP70 & IL-18 & IL-10 & DRB & Mx1 & CCR3 & $5 \mathrm{HTT}$ & CaM \\
\hline \multirow[t]{3}{*}{ CAPTIVES } & Total & 17 & 5.90 & 6.78 & 2.41 & 11.01 & 13.30 & 9.62 & -1.11 & 13.71 & -0.78 & 10.99 & 4.59 & 10.97 & -1.06 \\
\hline & Male & 7 & 6.92 & 7.81 & 2.77 & 11.00 & 13.94 & 9.35 & -1.90 & 13.90 & 1.20 & 11.25 & 4.44 & 10.70 & -1.43 \\
\hline & Female & 10 & 5.28 & 6.14 & 2.19 & 11.01 & 12.87 & 9.82 & -1.10 & 13.57 & -0.58 & 10.81 & 4.69 & 11.17 & -0.97 \\
\hline MON & Total & 30 & 6.62 & 5.72 & 1.92 & 10.07 & 13.59 & 10.99 & 1.33 & 13.77 & 0.70 & 12.31 & 4.83 & 11.56 & 0.29 \\
\hline \multirow[t]{5}{*}{ BSR } & Total & 51 & 6.64 & 8.18 & 3.60 & 10.73 & 13.48 & 10.57 & 1.84 & 13.69 & 0.61 & 11.90 & 4.81 & 12.00 & -0.21 \\
\hline & & & & & & & & & & & & & & & \\
\hline & 2008 & 27 & 5.93 & 8.54 & 3.09 & 10.01 & 12.93 & 10.87 & 2.35 & 14.16 & 0.43 & 11.13 & 5.38 & 12.05 & -0.22 \\
\hline & 2009 & 12 & 6.56 & 7.96 & 3.16 & 11.56 & 13.04 & 10.73 & 1.13 & 12.20 & -0.72 & 12.91 & 4.78 & 11.74 & -0.18 \\
\hline & 2010 & 12 & 8.68 & 7.62 & 5.82 & 11.62 & 15.29 & 9.77 & 1.75 & 14.24 & 1.14 & 12.74 & 3.75 & 12.15 & -0.21 \\
\hline \multirow[t]{4}{*}{ SLO } & Total & 56 & 9.23 & 8.75 & 2.38 & 11.04 & 16.65 & 12.82 & 1.53 & 18.44 & 0.29 & 14.95 & 5.38 & 11.98 & 0.34 \\
\hline & & & & & & & & & & & & & & & \\
\hline & Male & 11 & 9.35 & 8.44 & 2.34 & 10.96 & 16.20 & 11.05 & 1.79 & 18.02 & -0.47 & 14.58 & 5.73 & 11.23 & -0.27 \\
\hline & Female & 45 & 9.19 & 8.83 & 2.41 & 11.06 & 16.76 & 13.29 & 1.44 & 18.55 & 0.31 & 15.04 & 5.29 & 12.17 & 0.37 \\
\hline \multirow[t]{6}{*}{ SBC } & Total & 43 & 8.46 & 8.38 & 1.98 & 10.48 & 12.69 & 11.49 & 1.41 & 15.07 & 0.39 & 12.06 & 4.06 & 9.63 & 0.27 \\
\hline & Male & 22 & 8.64 & 8.88 & 1.91 & 10.74 & 12.18 & 11.76 & 1.30 & 14.56 & 0.35 & 12.18 & 4.35 & 9.52 & 0.29 \\
\hline & Female & 21 & 8.25 & 7.81 & 2.05 & 10.13 & 12.82 & 11.06 & 1.52 & 15.45 & -0.44 & 11.79 & 3.70 & 9.60 & 0.25 \\
\hline & 2012 & 20 & 8.70 & 7.89 & 2.49 & 10.01 & 10.73 & 10.32 & 1.16 & 12.59 & 0.36 & 10.54 & 3.39 & 8.16 & 0.24 \\
\hline & 2013 & 23 & 8.27 & 8.84 & 1.62 & 10.92 & 14.67 & 12.61 & 1.66 & 17.61 & 0.42 & 13.56 & 4.75 & 11.11 & -0.30 \\
\hline & & & & & & & & & & & & & & & \\
\hline
\end{tabular}




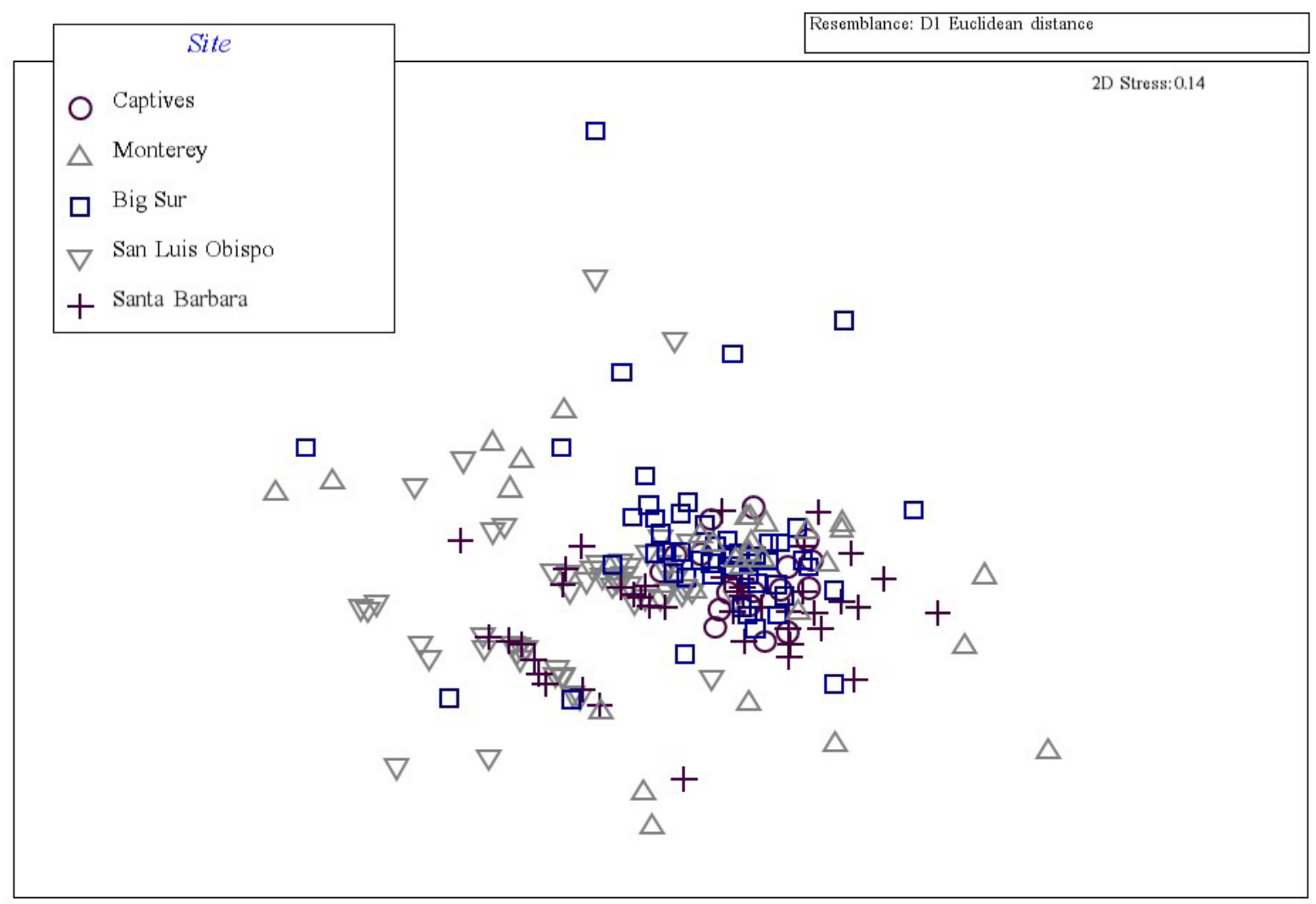

Figure 7. Graph showing multivariate, multi-dimensional scaling (MDS) analysis of gene transcription profiles of sea otters captured at four areas of the central California coast, 2008-13, in comparison to captive healthy sea otters. Substantial clustering is apparent about the tightly ordered captive group, as are numerous outliers in threedimensional space. 


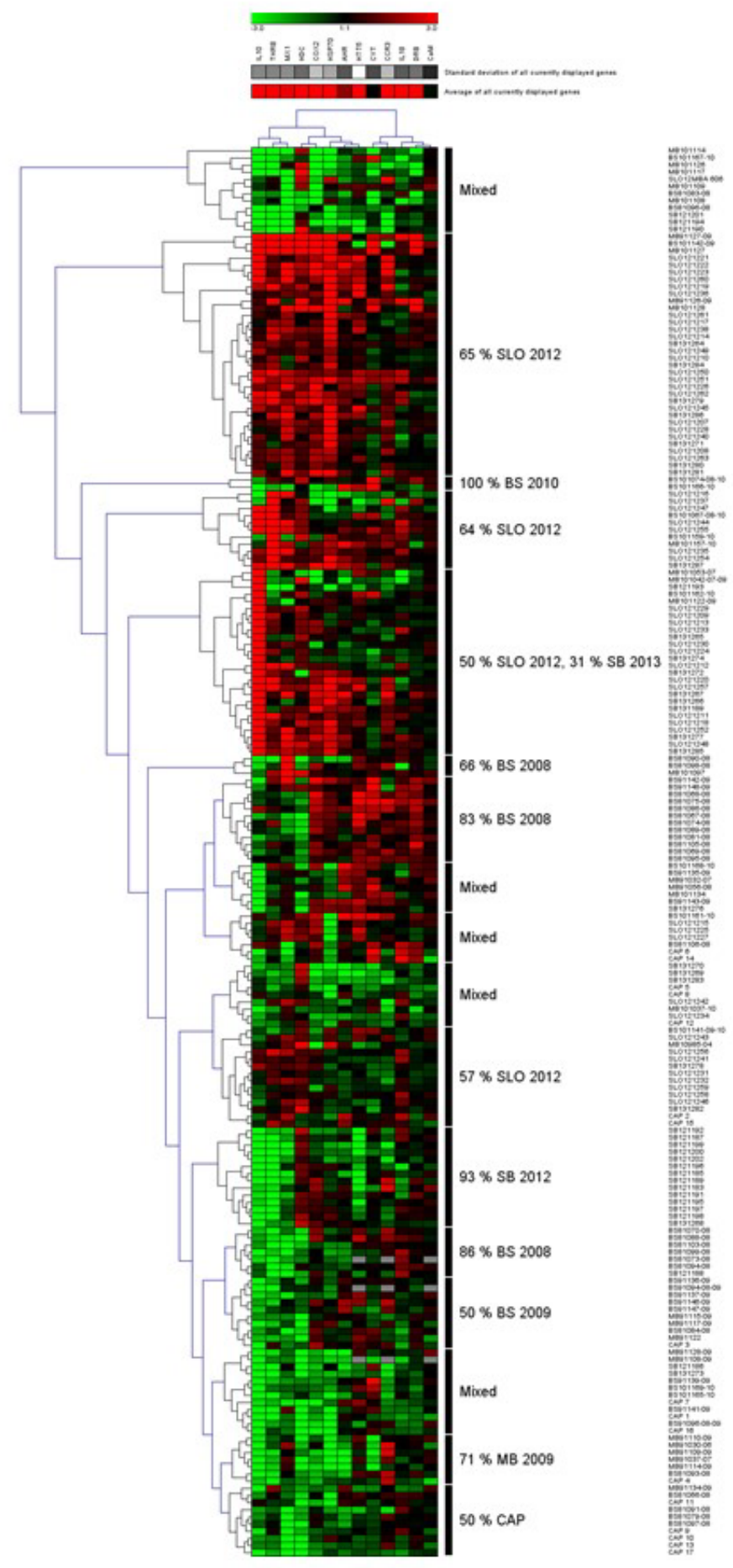

Figure 8. Gene expression profile showing transcription matrix of 13 target genes in clinically normal reference captive sea otters (CAP), and sea otters captured in Monterey Bay (MB), Big Sur (BS), San Luis Obispo (SLO), and Santa Barbara (SB), central California. Matrix shows hierarchical clustering with complete linkage disequilibrium; Genesis, Graz, Switzerland. Green indicates higher relative transcription levels and red indicates lower relative transcription levels. 


\title{
Chapter 4. Movement Behavior and Home Range Use of Sea Otters at Big Sur and Monterey
}

\author{
M. Tim Tinker ${ }^{1,2}$, L. Maxine Tarjan², Emily Golson³, Gena Bentall ${ }^{1,4}$, and Nicole LaRoche ${ }^{2}$
}

\section{Introduction}

A comprehensive understanding of the risks to sea otter population recovery associated with specific environmental or anthropogenic stressors requires detailed information on movement behavior and home-range use by individuals in the population. Data on movements and spatial use patterns also are important for characterizing population structure; that is, the spatial scale at which individuals within the population interact with each other, with their prey, and with their environment. Predator populations in which individuals are highly mobile are considered "spatially unstructured", or wellmixed, such that individuals are likely to interact with any other individual, and will have similar exposures to various threats, irrespective of their geographic location at a given point in time. Conversely, predator populations in which individuals have small home ranges and restricted movements are "spatially structured", such that individuals only interact with other nearby individuals, and their risk of exposure to various threats is highly dependent on their geographic location. Thus, an understanding of movement behavior and population structure is necessary to understand the degree to which sub-populations are limited by resource abundance or external threats at local rather than regional scales.

In studies of tagged or otherwise marked individuals, there are numerous accepted approaches for measuring and describing movement behavior. At the most basic level, relocating tagged individuals at some regular time interval and measuring the net distance they have moved away from their location at the previous interval (their "net linear displacement", NLD), provides the fundamental data required for virtually all spatial models of population growth, disease dynamics, or demographic connectivity among habitat patches. For example, diffusion models and other so-called "Eulerian" spatial models can be parameterized from the NLD values of many individuals (from which one can estimate dispersal distance probability distributions) and used to estimate the rate at which populations will recover, invade, or otherwise spread into new habitat (Hastings, 1996; Kot and others, 1996; Borger and others, 2008). More advanced models require not only the average NLD per unit time, but also information on the frequency distribution of this statistic, and how it varies between different classes of individuals within the population (Neubert and others, 1995; Shigesada and others, 1995). The magnitude and distribution of NLD values also can provide insight into the degree of population structure.

\footnotetext{
${ }^{1}$ U.S. Geological Survey.

${ }^{2}$ University of California, Santa Cruz.

${ }^{3}$ Moss Landing Marine Laboratory, California State University, Monterey Bay.

${ }^{4}$ Monterey Bay Aquarium.
} 
In contrast to population-level Eulerian models, "Lagrangian" models, such as random-walk models, focus on individual-level movement behavior over short, discrete time steps (Borger and others, 2008). Measurement of sequential step lengths and turning angles provides the data needed to parameterize correlated random walk (CRW) models, which allow for a more mechanistic understanding of animal dispersal (Turchin, 1998). For example, the degree to which observed distributions of NLD values conform to expected NLD distributions calculated from CRW models can provide insight into whether individual movements are "biased" (Kareiva and Shigesada, 1983). Movements will be biased if individuals are avoiding certain habitat features, or are "attracted" to other locations such as the center of their individual home range.

Another approach to describing the distribution of animals in space and time is the statistical analysis of individual home ranges. Seton (1909) recognized that animals restrict their movements to particular areas over time, and Burt (1943) termed this area a home range, defined as "that area traversed by an individual in its normal activities of food gathering, mating, and caring for the young." Geographical definition of animal home ranges is necessary to answer critical questions in studies of habitat selection (Thomas and Taylor, 2006; Borger and others, 2008), mating systems (Hedmark and others, 2007; Vanpé and others, 2009), and carrying capacity (Mitchell and Powell, 2012), and in formulating expectations about the habitat and species with which an individual will interact.

According to an optimality approach to animal space-use, home ranges should be a product of animals maximizing benefits—resources contained within an area—while minimizing costs of travel and resource acquisition (Mitchell and Powell 2012). Home ranges, therefore, are predicted to be finite in size because the cost of obtaining resources increases with travel distance. The benefits and costs of occupying a home range depend on resource availability and distribution. If resources are abundant, individuals can occupy a small range, but if resources are scarce or widely distributed, home ranges must be large to meet energetic demands and other requirements.

Sea otter habitats across California differ in the availability of resources, such as kelp and invertebrate prey. Sea otter home ranges often encompass persistent patches of kelp canopy, which is preferred habitat for resting and feeding. As a keystone predator of kelp forest ecosystems, sea otters are capable of depleting preferred prey types following establishment in an area (Estes and others, 1978). As the duration of sea otter occupancy differs across the California range, some sites are expected to be more food-limited than others. Previous work contrasted feeding by sea otters at San Nicholas Island, a site rich in invertebrate prey, and central California, an area with a longer-standing sea otter population that is resource-limited (Tinker, Bentall, and Estes, 2008). The difference in prey availability was indicated in foraging success (measured as energy recovery rates while feeding), with sea otters recovering energy at a rate two times higher at San Nicolas Island than in central California, and spending only one-half as much time foraging. Differences in search and handling times of prey across California sites are expected to impact sea otter home-range size.

The bathymetry of the habitat also is expected to influence home-range shape and space-use in sea otters. Because of physiological limits in diving capabilities, the extent of the continental shelf has a large impact on the offshore availability of benthic prey. Among the study sites for this project, Monterey Bay has a more extensive continental shelf than Big Sur. Sea otters, therefore, are capable of using resources farther offshore in Monterey. The shape of home ranges likely is influenced by these differences between habitats.

In addition to the probable effects of habitat type on home-range characteristics, different resource requirements between the sexes are likely to influence animal space-use. Previous studies of the social structure of sea otters show that males range more widely than females and have larger home ranges, except when they are engaged in defense of a small reproductive territory (Loughlin, 1980; Ribic, 1982; Jameson, 1989). 
The objectives of this study are to examine the expected differences in home-range size between males and females, and to determine if home-range characteristics vary between the two main study sites. We hypothesize that:

1. Home-range size will vary depending on sex, with territorial males having the smallest home ranges, non-territorial males the largest home ranges, and females having intermediate home ranges;

2. Sea otters in resource-limited areas will have larger home ranges than individuals in resource-rich areas; and

3. Home range-area in Big Sur will be more dependent on the coastal extent of the home range than in Monterey.

We will accomplish these objectives by quantifying home-range characteristics using a new algorithm of home-range estimation.

\section{Methods}

\section{Movement Behavior}

Individual tagged otters at both study sites were resighted at intervals of 3-7 days each week using radio telemetry, and their positions were recorded in a geographic information system- (GIS-) compliant database following standardized methods (see chapter 1). Because the number of observations per week varied between individual otters, we selected (randomly) one location per week per otter as the fundamental unit of observation. The geographic coordinates for each data point (in units of decimal degrees and California 1983 Teale Albers projection) were then translated to a onedimensional coastal axis: specifically, linear interpolation was used to assign each resight location the value of the closest point along the "As The Otter Swims" (ATOS) line, defined by 500-m intervals along the 10-m isobath (Tinker and others, 2006). We expressed the ATOS values in decimal units rather than rounding to the nearest integer ATOS value (for example, a resight location that was onethird of the way between ATOS point 367 and 368 was assigned a value of 367.33).

The annual dispersal distance, or net linear displacement (NLD), for each otter was calculated as the absolute difference in decimal ATOS values between the location of the otter on an arbitrary resight date and its location at a second arbitrary resight date from 350 to 380 days after the first date. For each otter, we randomly selected (with replacement) 100 starting and ending resight dates to obtain a tribution of representative annual NLD values for each otter. We used these data to fit probability density functions using maximum likelihood estimation techniques. Previous studies have used various probability distributions to describe sea otter dispersal distances, including exponential and leptokurtic distributions (Krkosek and others, 2007; Tinker, Doak, and Estes, 2008). In this case, visual examination of frequency histograms of individual NLD values indicated somewhat leptokurtic or "fattailed" distributions, characterized by many small values but a few very large values. Accordingly we selected the Weibull probability distribution to fit to empirical NLD observations. The Weibull density function is more flexible than the exponential distribution, and is described by a scale parameter $(a)$ that specifies the magnitude of the mean expected value, and a shape parameter $(b)$ that determines the variance and skew:

$$
f(x \mid a, b)=\frac{b}{a}\left(\frac{x}{a}\right)^{b-1} e^{-(x / a)^{b}}
$$


When $b<1$ the resulting distribution is more leptokurtic than the exponential and has higher variance, but when $b=1$ the Weibull converges to the exponential distribution (fig. 9). We fit Weibull distributions to NLD data for 6 groups of sea otters: (1) males, (2) adult females, and (3) sub-adult females from Big Sur; and (4) males, (5) adult females, and (6) sub-adult females from Monterey. We divided females into two age classes because previous analyses have shown that juvenile/sub-adult animals (individuals $<4$ years of age) may have different movement behavior than adults (individuals $\geq$ 4 years of age); however, we did not classify males by age class because of sample-size limitations. For each group, we calculated the mean and 95-percent confidence limits for the $a$ and $b$ parameters, and we evaluated whether NLD distributions differed between groups based on non-overlap of 95-percent confidence intervals. We then conducted variance component analysis to determine the degree to which variation in annual NLD values was explained by (1) site differences, (2) age/sex differences, (3) among-individual differences, and (4) within-individual (year-to-year) differences. For this latter analysis, we analyzed NLD data using a random-effects general linear model and restricted maximum likelihood estimation of variance components.

We next analyzed sea otter movements using a CRW model, to determine whether/how movements were biased. For this analysis we computed distributions of step-lengths and turning angles for each otter from contiguous sequences of weekly resight values. The step length for week $i\left(l_{i}\right)$ was calculated as the absolute difference between decimal ATOS value at week $i$ and the value at week $i-1$. The turning angle $\left(\theta_{i}\right)$ was assumed to be $0^{\circ}$ if the otter had moved "up- coast" (decreasing ATOS values) from its location at week $i-1$, and $180^{\circ}$ if the otter had moved "down-coast" (increasing ATOS values). The expected net linear displacement $\left(N L D_{E}\right)$ for an otter over a specified period of time, $T$, was calculated as:

$$
N L D_{E}(T)=\left[n(T) \cdot m_{2}+2 m_{1}^{2}\left(\frac{\Psi}{1-\Psi}\right)\left(n-\frac{1-\Psi^{(n(T)-1)} / 2}{1-\Psi}\right)\right]^{0.5},
$$

where

$n(T) \quad$ is the number of weekly intervals in period

$T, m_{1} \quad$ is the arithmetic mean of recorded step lengths,

$m_{2} \quad$ is the arithmetic mean of squared step lengths, and

$\Psi \quad$ is the arithmetic mean of the cosine of recorded turning angles.

We calculated $N L D_{E}$ for values of $T$ ranging from 4 weeks to 1 year, and used bootstrap re-sampling to account for measurement variance (1,000 random samples of step lengths and turning angles were selected with replacement for each otter for each value of $T$ ). We next computed observed net linear displacement $\left(N L D_{O}\right)$ for each otter, for each value of $T$, using the methods described above for calculating annual dispersal distance. Movement bias was then calculated as $N L D_{O}-N L D_{E}$ and plotted as a function of time for four groups of animals: (1) adult females, (2) sub-adult females, (3) males whose resights were restricted to one area of the coast (and thus had a unimodal spatial distribution), and (4) males whose resights were clustered in two or more widely separated areas of the coast (and thus had a multimodal spatial distribution). 


\section{Home-Range Analysis}

Statistical methods to estimate individual home ranges use observed resighting locations to estimate the future probability of an individual occurring at any point in space, and to delineate a boundary encompassing some cumulative probability of occurrence. A notable limitation of existing methods is their inability to incorporate information about underlying environmental features that influence animal space-use. Because of this limitation, existing methods often perform well in encompassing areas that are used, but perform poorly at identifying unused areas. This limitation is particularly apparent for sea otters (fig. 10), whose space-use is restricted by the complex coastal boundary and water depth.

Recognizing the inadequacy of existing home-range methods for describing sea otter homerange behavior, we developed a new method of home-range estimation, which we refer to hereinafter as "Permissible home-range estimation", or PHRE. This method better represents limiting habitat characteristics than existing home-range methods, and is described in detail elsewhere (Tarjan and Tinker, 2016). In brief, the PHRE method is based on a transformation of resight locations to indicate relevant landscape features; specifically, each resight location was assigned the value of the closest point along the one-dimensional ATOS line (the decimal ATOS value), and the appropriate depth value was assigned through interpolation to the $10-\mathrm{m}$, high-resolution, multi-beam sonar bathymetric data collected by the California Department of Fish and Wildlife (CDFW Marine Region GIS Laboratory). Depth values were log-transformed to ensure normality and to produce a variable that varied from minus infinity to infinity $(-\infty$ to $\infty)$. Because home-range boundaries could change over the lifetime of an animal, 2 years of data were used for each home-range estimate.

A bivariate kernel probability density function was fit to the decimal ATOS and $\log (\operatorname{depth})$ variables for each individual, and this function was then back-transformed to geographic space to define a permissible home-range kernel density surface (Tarjan and Tinker, 2016). The output of the algorithm includes a GIS layer of the kernel density grid (ESRI ArcMap ${ }^{\mathrm{TM}}$ Shapefile), as well as a polygon delineating the 90-percent kernel home-range boundaries (fig. 11) and a vector of descriptive statistics, for use in comparing home-range characteristics. Specifically, we computed the following home-range statistics for each individual:

1. Total area (in square kilometers);

2. Number of distinct centers of use (COU, defined as home-range polygons separated by more than 2 kilometers);

3. Maximum distance between COU polygons;

4. "Coastline extent", or cumulative distance (in kilometers) of coastline contained within all home-range polygons; and

5. "Range span", or distance (in kilometers) between the northernmost and southernmost points of the home range.

Among-individual distributions of home-range area, maximum distance between centers of use, coastline extent, and range span were highly right-skewed, and so were log-transformed for normality (Kolmogorov-Smirnov test, $\mathrm{p}>0.05$ ). Examination of the log-transformed distribution of coastline extent for males indicated a highly bimodal distribution with a clear break at value 2 (fig. 12), and this was confirmed by a significant Hartigans "dip" test for unimodality (Dip Stat $=0.104, \mathrm{P}=0.0075$; Hartigan and Hartigan,1985). Accordingly, we classified males into one of two categories: (1) those with log-transformed coastline extent values of less than 2 were classified as residents (M1), and (2) those with log-transformed coastline extent values of greater than 2 were classified as transients (M2). Linear models were created and analyses of variance (ANOVAs) were used to test for effects of site (Big Sur compared to Monterey) and sex (F compared to M1 compared to M2) on all home-range 
characteristics, as well as the interactions between the main effects. Analyses of the maximum distance between centers of use were limited to individuals with home ranges with more than one center of use. Data on the number of centers of use were nonparametric, so the effects of sex and site were evaluated separately using Mann-Whitney $U$ tests, and a $\chi^{2}$ contingency test was used to evaluate the hypothesis that sex, site, and number of centers of use were independent. Finally, we used a general linear model (GLM) to test for a functional relation between home-range area and coastline extent, assuming a loglinear relation.

\section{Results}

The frequency distributions of observed values of sea otter annual dispersal (net linear displacement or "NLD") were well fit by Weibull probability density functions (fig. 13). Male otters at both sites had more strongly leptokurtic distributions than females, with a long right tail to the distribution corresponding to "extreme" long-distance movements (fig. 13A-B). In contrast, NLD distributions of females were less skewed, with almost all annual dispersal values ranging from 0 to 20 km (fig. 13C-F). The sex-based difference in distribution skew was indicated by significantly lower "b" parameters for males relative to females (fig. 14), and, thus, a higher probability of making longdistance movements of over $20 \mathrm{~km}$ (table 8). Sub-adult females at Big Sur had slightly lower mean NLD values than adult females, whereas at Monterey, sub-adult females had slightly more skewed NLD distributions and greater mean NLD values (fig. 14). Overall, annual dispersal distances were similar between sites, but individuals at both sites were highly variable with respect to NLD, with amongindividual and within-individual differences contributing more to variance than age and sex-class differences (fig. 15).

The CRW model tended to over-estimate NLD values for male and female sea otters, and the negative bias in observed NLD values $\left(\mathrm{NLD}_{\mathrm{O}}-\mathrm{NLD}_{\mathrm{E}}\right)$ increased over time (fig. 16). Increasing negative bias in NLDo compared to NLDE is indicative of a centralizing tendency in sea otter movements, such that individuals are more likely to turn towards a "focal attractor" (for example, a home-range "center of use") the farther they are away from that point. Sub-adult females showed greater variation in movement bias than adult females, and males with multi-modal spatial distributions also were more variable than adult females (fig. 16), indicating the occasional longer-distance movements of these animals (fig. 13).

\section{Home-Range Analysis}

Permissible home-range estimation outperformed other available methods - kernel density estimation (KDE; Silverman, 1986; Worton, 1989) and local convex hull analysis (LoCoH, Getz and Wilmers 2004) — for this species. Home-range estimates were biologically accurate, largely excluding terrestrial areas and water beyond the depth range that is accessible to sea otters (compare figs. 10 and $11)$.

Average (plus or minus standard deviation) home-range characteristics (grouped by site and sex) are presented in table 9. The number of distinct home-range centers of use (COUs) did not differ between sites (fig. 17A), either for females $\left(\chi^{2}=3.02, \mathrm{P}=0.389\right)$ or males $\left(\chi^{2}=0.72, \mathrm{P}=0.868\right)$. However, sex was a significant predictor of the number of COUs (Mann-Whitney U-test statistic $U=2155, \mathrm{P}<$ 0.01), with 80-percent or more of females having just one COU, while less than 60-percent of males at both sites had one COU. The number of COUs also differed between resident males and transient males $\left(\chi^{2}=36.68, \mathrm{P}<0.0001\right)$, with more than 90-percent of resident males having one COU but 75 -percent of transient males having two or more COU (fig. 17B). 
Male sea otters had a greater maximum distance between COUs than did females (ANOVA Fstatistic with $1 / 78$ degrees of freedom $\left.\mathrm{F}_{1,78}=18.29, \mathrm{P}<0.001\right)$, but there were no differences between sites $\left(\mathrm{F}_{1,78}=2.24, \mathrm{P}=0.14\right)$ or interactions between sex and site $\left(\mathrm{F}_{1,78}=0.06, \mathrm{P}=0.81\right)$. Similar patterns were noted for other home-range statistics (fig. 18). The total area (in square kilometers) of home-range polygons did not differ between sites $\left(\mathrm{F}_{1,2}=0.39, \mathrm{P}=0.53\right)$, and there was no interaction between sex and site $\left(\mathrm{F}_{2,136}=0.89, \mathrm{P}=0.45\right)$, but females had a larger home range than resident males and a smaller home range than transient males $\left(\mathrm{F}_{1,2}=14.17, \mathrm{P}<0.001\right)$. The coastline extent of home ranges did not differ between sites $\left(\mathrm{F}_{1,2}=0.30, \mathrm{P}=0.58\right)$, and there was no interaction between sex and site $\left(\mathrm{F}_{2,136}=\right.$ $0.46, \mathrm{P}=0.64)$, but the coastline extent of female home ranges was longer than that of resident males and shorter than that of transient males $\left(\mathrm{F}_{1,2}=26.83, \mathrm{P}<0.001\right)$. The total length of coastline spanned by otter home ranges did not differ between sites $\left(\mathrm{F}_{1,2}=0.57, \mathrm{P}=0.45\right)$, and there was no interaction between sex and site $\left(\mathrm{F}_{2,136}=0.28, \mathrm{P}=0.76\right)$. Female movements spanned a longer stretch of coastline than those of resident males and a shorter stretch of coastline than transient males $\left(\mathrm{F}_{1,2}=25.51\right.$, $\mathrm{P}<0.001)$. Finally, coastline extent increased more rapidly as a function of home-range area in Big Sur as compared to Monterey Bay; power functions fit to the data were highly significant and their slopes differed significantly (fig. 19; $\mathrm{F}_{2,136}=8.1408, \mathrm{P}<0.001$ ). This difference indicates the narrower continental shelf and, thus, the more elongated home ranges in Big Sur as compared to Monterey. To achieve a proportional increase in home-range area, sea otters in Monterey can simply use more offshore habitat, but in Big Sur the only option for increasing home-range area is to extend use north of south along the coast, which necessitates moving along a much longer stretch of coastline than in Monterey.

\section{Discussion}

Sea otter movement behavior in the current studies generally was consistent with that observed in previous studies of sea otter movements (Ralls and others, 1996; Tinker, Doak, and Estes, 2008), in that female movements were more restricted than male movements, and sub-adult females were more likely to make occasional long-distance movements than adult females (at least in Monterey; table 8). This analysis confirms the previously-reported observation that adult female sea otters show strong site fidelity, rarely dispersing more than $20 \mathrm{~km}$ from their current location within a 1-year period (table 8), and, thus, are most at risk from (and good indicators of) local environmental or anthropogenic stressors. The best-fit Weibull distribution for adult females at Monterey had a smaller scale parameter and smaller shape parameter than the distribution for Big Sur females, meaning that the average dispersal distance of Monterey females was smaller than that of Big Sur females, yet there was a higher probability of Monterey females making a long distance movement of $>20 \mathrm{~km}$. It is unclear why this difference exists; the longer average NLD of Big Sur females may simply indicate the fact that the narrow/steep slope of the continental shelf at Big Sur means that females have to cover more coastline to have access to the same quantity of prey resources, but the occasional long-distance movements of Monterey females are more difficult to explain.

In contrast to females, the distribution of annual NLD values for males was strongly leptokurtic (fig. 13), and with a long right tail that was better described by a Weibull distribution than by the more traditional exponential distribution. Although the shape of male NLD distributions was identical at both sites (as measured by the shape parameter), the scale parameter was significantly greater at Big Sur, indicating the longer average annual dispersal distances of males in this region (fig. 14). This difference may be explained by the fact that males at Big Sur had greater distances to travel to find abundant food resources than did Monterey males, given that Big Sur is farthest from the north and south ends of the range where sea otter densities are lower and per-capita food abundance is greater. However, although site- and sex-based differences in movement behavior do exist, most of the variability in NLD was 
explained by individual variation, suggesting that variable life history and foraging strategies of sea otters contribute more to variation in annual movements than do environmental differences between Big Sur and Monterey. Understanding what factors explain this variation will be a productive area for further research.

For both females and males, the CRW analysis indicated a strong centralizing bias to sea otter movements (fig. 19). This bias indicates that sea otters tend to limit their movements to well-defined and relatively compact home ranges, within which they move extensively but outside of which they are unlikely to move, particularly in the case of adult females. There is some reason to believe that this trait may be "hard wired" into sea otters, as female sea otters in Prince William Sound and southeast Alaska also have been reported to have restricted annual movements (Garshelis and Garshelis 1984, Bodkin and others, 2004). Nevertheless, an important consequence of this trait is that demographic processes in the population will be strongly spatially structured, because reproductive-age females are the demographically "relevant" component of the population. The more mobile males do contribute to genetic connectivity, but because they do not directly contribute to intrinsic population growth (unless fertilization of estrous females were a limiting factor, which has never been reported), their movements will have no effect on demographic connectivity. This spatial structure of the southern sea otter population has profound conservation implications; for example, it means that the factors that limit female survival and thus control population growth will tend to operate locally, not regionally. This is particularly likely to be the case for per-capita prey abundance because the benthic invertebrates that sea otters feed on are sessile and their abundance varies enormously over short distances owing to smallscale variation in recruitment and post-settlement processes (Gaines and others, 1985; Broitman and others, 2008). Together, these observations point towards a need to consider density-dependent processes and population status at scales of tens of kilometers, rather than at regional scales or at the scale of the entire population.

\section{Home-Range Estimation}

Permissible home-range estimation allowed for statistical descriptions of home-range use that were more consistent with sea otter biology than previous methods. In particular, the resulting homerange polygons did not include terrestrial areas and encompassed water depths that are accessible by diving sea otters (fig. 11). Accurate estimates of home-range size and location have been previously unavailable for this species, so this methodological advance will be a major contribution to studies of sea otter ecology, with potential application to research on exposure to anthropogenic disturbance, encounter rates with pathogens, and access to resources. This method also will be applicable to ecological studies of other species whose home ranges are restricted by complex boundaries or across environmental gradients, such as water depth, primary productivity, or temperature. Increased accuracy in defining home ranges will allow researchers and resource managers to better understand habitat use requirements and ultimately to improve conservation efforts for a variety of species.

Evidence based on body condition and foraging rates suggests that sea otters in Big Sur are slightly more resource-limited than sea otters in Monterey Bay (see chapters 6 and 7). However, despite this small difference in apparent resource abundance we noted no significant differences in home-range areas between sites (although the average home-range area was largest for males in Monterey), although there was much individual-level variability in this statistic. The similarity of home range-area across sites likely is due to the relative similarity between sites in terms of habitat characteristics and population density. Although some difference in resource availability may exist between Monterey Bay and Big Sur, it may not be drastic enough to heavily influence sea otter home-range use, and, in fact, results presented elsewhere in this report generally suggest that both sites are at or near carrying 
capacity. We suggest that an analysis including study sites that are significantly less than carrying capacity, such as the Santa Barbara Channel and San Nicolas Island, will be a fruitful next step.

Although absolute home-range size did not differ between sites, there were subtle differences in space use of sea otters at the two sites that likely were related to differences in the distribution of resources and coastal bathymetry. In support of our hypothesis, we found that the coastline extent of home ranges increased with home-range area much more rapidly in Big Sur than in Monterey Bay. This pattern supports the fact that sea otters in Big Sur are only able to increase the area of their home range by extending it farther along the coastline, owing to the narrow continental shelf at this site. In contrast, the wide continental shelf at Monterey Bay means that sea otters are able to access offshore resources, and, thus, can increase the area of their home range by extending it offshore.

These differences in habitat and home-range shape have energetic implications for sea otters. The energy required to access all resources within a home-range area depends on the shape of that area. A circular home range allows sea otters to access all their resources by swimming a relatively short distance. An elliptical home range means that a sea otter needs to swim the full length of the ellipse to access all the resources, thus expending more energy for travel. The greater energy requirement for long, narrow elliptical home ranges likely restricts the maximum home-range area that can be maintained. Indeed, the maximum home-range area in Big Sur $\left(20.11 \mathrm{~km}^{2}\right)$ is less than one-half the maximum home-range area in Monterey Bay $\left(45.22 \mathrm{~km}^{2}\right.$ ). Habitat bathymetry and the spatial distribution of resources, therefore, can have a profound effect on the maximum home-range area that is attainable, and ultimately will affect the equilibrium population density in a given area.

Home-range statistics were similar between females and males overall; however, sex-based comparisons were complicated by the existence of two distinct strategies of home-range behavior shown by males. At both sites, some males ("M1", resident males) had strong site fidelity, using just one homerange center that encompassed a small area and spanned a short stretch of coast. A second group of males ("M2", transient males) were far more mobile, frequently moving among multiple home-range centers distributed along the California coast, which together encompassed a larger area and spanned a longer stretch of coast. These distinct home-range strategies are apparently related to reproductive strategy; the M1 males in this study were all "territorial", maintaining reproductive territories in kelpdominated habitats where females tend to congregate for all or most of the year. In contrast, M2 males did not generally maintain breeding territories (or only did so for parts of the year), and instead moved regularly between male-dominated regions (generally soft-sediment, non-kelp habitats), with occasional opportunistic visits to female-dominated areas. These alternative reproductive tactics seem to be associated with substantially different patterns of home-range use. Female home-range characteristics tended to fall midway between the home-range metrics of the two male strategies.

Space-use patterns are important for population health because they can affect interactions between individuals and spatially-explicit stressors. Although transient males tend to encounter many different individuals in the population, females and resident males are restricted to interactions with other local females. Moreover, the probability of encountering localized anthropogenic disturbances, pollution, or other features of the environment depends on the ranging capabilities of the individual; highly mobile transient males can potentially encounter a wider range of stressors as they move throughout central California, whereas resident males and females may be subject to greater intensity of local disturbances within their home range, if they exist. Differences in home-range use and movement patterns across sexes and mating tactics, therefore, could be considered when identifying threats to this species. 


\section{References Cited}

Bodkin, J.L., Esslinger, G.G., and Monson, D.H., 2004, Foraging depths of sea otters and implications to coastal marine communities: Marine Mammal Science, v. 20, p. 305-321.

Borger, L., Dalziel, B.D. and Fryxell. J.M. 2008. Are there general mechanisms of animal home range behaviour?-A review and prospects for future research: Ecology Letters, v. 11, p. 637-650.

Broitman, B.R., Blanchette, C.A., Menge, B.A., Lubchenco, Krenz, J.C., Foley, M., Raimondi, P.T., Lohse, D., and Gaines, S.D., 2008, Spatial and temporal patterns of invertebrate recruitment along the West Coast of the United States: Ecological Monographs, v. 78, p. 403-421.

Burt, W.H., 1943, Territoriality and home range concepts as applied to mammals: Journal of Mammalogy, v. 24, p. 346-352.

Gaines, S., Brown, S., and Roughgarden J., 1985, Spatial variation in larval concentrations as a cause of spatial variation in settlement for the barnacle, Balanus glandula: Oecologia, v. 67, p. 267-272.

Garshelis, D.L., and Garshelis, J.A., 1984, Movements and management of sea otters [Enhydra lutris] in Alaska [USA]: Journal of Wildlife Management, v. 48, p. 665-678.

Getz, W.M., and Wilmers, C.C., 2004, A local nearest-neighbor convex-hull construction of home ranges and utilization distributions: Ecography, v. 27, p. 489-505.

Hartigan, J.A., and Hartigan, P.M., 1985, The dip test of unimodality: Annals of Statistics, v. 13, p. 7084.

Hastings, A., 1996, Models of spatial spread-A synthesis: Biological Conservation, v. 78, p. 143-148.

Hedmark, E., Persson, J., Segerstro, P., Landa, A., and Ellegren, H., 2007, Paternity and mating system in wolverines Gulo gulo: Wildlife Biology, v. 13, p. 13-30.

Jameson, R.J., 1989, Movements, home range, and territories of male sea otters off central California: Marine Mammal Science, v. 5, p. 159-172.

Kareiva, P.M., and Shigesada, N., 1983, Analyzing insect movement as a correlated random walk: Oecologia, v. 56, p. 234-238.

Kot, M., Lewis, M.A., and Van Den Driessche, P., 1996, Dispersal data and the spread of invading organisms: Ecology, v. 7, p. 2027-2042.

Krkosek, M., Lauzon-Guay, J.S., and Lewis, M.A., 2007, Relating dispersal and range expansion of California sea otters: Theoretical Population Biology, v. 71, p. 401-407.

Loughlin, T.R., 1980, Home range and territoriality of sea otters near Monterey, California: Journal of Wildlife Management, v. 44, p. 576-582.

Mitchell, M.S., and Powell, R., 2012, Foraging optimally for home ranges: Journal of Mammalogy, v. 93, p. 917-928.

Neubert, M.G., Kot, M., and Lewis, M.A., 1995, Dispersal and pattern-formation in a discrete-time predator-prey model: Theoretical Population Biology, v. 48, p. 7-43.

Ralls, K., Eagle T.C., and Siniff, D.B., 1996, Movement and spatial use patterns of California sea otters: Canadian Journal of Zoology, v. 74, p. 1841-1849.

Ribic, C.A., 1982, Autumn movement and home range of sea otters in California: The Journal of Wildlife Management, v. 46, 795-801.

Seton, E.T., 1909, Life-histories of northern animals-An account of the mammals of Manitoba: New York, Scribner, $1267 \mathrm{p}$.

Shigesada, N., Kawasaki, K., and Takeda., Y., 1995, Modeling stratified diffusion in biological invasions: American Naturalist, v. 146, p. 229-251.

Silverman, B.W., 1986, Density estimation for statistics and data analysis: London, Chapman and Hall, $176 \mathrm{p}$. 
Tarjan, L.M., and Tinker, M.T., 2016, Permissible Home Range Estimation (PHRE) in Restricted Habitats: A New Algorithm and an Evaluation for Sea Otters: PLoS ONE v. 11(3): e0150547. https://doi.org/10.1371/journal.pone.0150547

Thomas, D.L., and Taylor, E.J., 2006, Study designs and tests for comparing resource use and availability II: Journal of Wildlife Management, v. 70, p. 324-336.

Tinker, M.T., Bentall, G., and Estes, J.A., 2008, Food limitation leads to behavioral diversification and dietary specialization in sea otters: Proceedings of the National Academy of Sciences of the United States of America, v. 105, p. 560-565.

Tinker, M.T., Doak, D.F., and Estes., J.A. 2008, Using demography and movement behavior to predict range expansion of the southern sea otter: Ecological Applications, v. 18, p. 1781-1794.

Tinker, M.T., Doak, D.F., Estes, J.A., Hatfield, B.B., Staedler, M.M., and Bodkin, J.L., 2006, Incorporating diverse data and realistic complexity into demographic estimation procedures for sea otters: Ecological Applications, v. 16, p. 2293-2312.

Turchin, P., 1998, Quantitative analysis of movement-Measuring and modeling population redistribution in animals and plants: Sunderland, Massachusetts, Sinauer Associates, Inc., 396 p.

Vanpé, C., Morellet, N., Kjellander, P., Goulard, M., Liberg, O., and Hewison, A.J.M., 2009, Access to mates in a territorial ungulate is determined by the size of a male's territory, but not by its habitat quality: The Journal of Animal Ecology, v. 78, p. 42-51.

Worton, B.J., 1989, Kernel methods for estimating the utilization of distribution in home-range studies: Ecology, v. 70, p. 164-168. 
Table 8. Results of an analysis of annual net linear displacement for sea otters, grouped by sex and age-class, at Big Sur and Monterey, central California.

[Scale (a) and shape (b) parameter estimates are shown for Weibull distributions fit to each dataset (mean estimates and lower $\left[\mathrm{CI}_{\mathrm{L} 95}\right]$ and upper [ $\left.\mathrm{CI}_{\mathrm{U} 95}\right]$ 95-percent confidence limits are presented). Mean movement distances for each group (Mean move) and the cumulative probabilities of moving more than 20 kilometers from the starting location within a 1-year period (Probability move $>20 \mathrm{~km}$ ) also are shown, as calculated from the appropriate cumulative distribution functions]

\begin{tabular}{|c|c|c|c|c|c|c|c|c|c|}
\hline Site & Sex/age class & $\begin{array}{c}a \\
\text { (scale) }\end{array}$ & 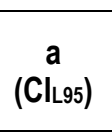 & $\begin{array}{c}a^{a} \\
\text { (Clug5) }\end{array}$ & $\begin{array}{c}\text { b } \\
\text { (shape) }\end{array}$ & $\begin{array}{c}\mathbf{b} \\
\left(\mathrm{Cl}_{\llcorner .95}\right)\end{array}$ & $\begin{array}{c}\mathbf{b} \\
\text { (Clug5) }\end{array}$ & $\begin{array}{l}\text { Mean } \\
\text { move }\end{array}$ & $\begin{array}{c}\text { Probability } \\
\text { move } \\
>20 \mathrm{~km}\end{array}$ \\
\hline \multirow[t]{3}{*}{ Big Sur } & Male, all ages & 3.848 & 3.175 & 4.663 & 0.408 & 0.386 & 0.432 & 13.156 & 0.141 \\
\hline & Female, adult & 4.141 & 3.902 & 4.395 & 0.756 & 0.731 & 0.782 & 4.883 & 0.037 \\
\hline & Female, sub-adult & 3.706 & 3.357 & 4.090 & 0.737 & 0.696 & 0.780 & 4.384 & 0.031 \\
\hline \multirow[t]{3}{*}{ Monterey } & Male, all ages & 1.591 & 1.424 & 1.779 & 0.398 & 0.386 & 0.411 & 5.935 & 0.065 \\
\hline & Female, adult & 3.265 & 3.127 & 3.408 & 0.637 & 0.625 & 0.649 & 4.197 & 0.042 \\
\hline & Female, sub-adult & 3.748 & 3.330 & 4.218 & 0.662 & 0.626 & 0.700 & 5.182 & 0.048 \\
\hline
\end{tabular}

Table 9. Home-range characteristics across sexes and Big Sur coast and Monterey Peninsula study sites, central California.

[Data are shown as averages plus or minus standard deviation, and are summarized for females (F), all males (M), resident males (M1), and transient males (M2) at both study sites. n/a, not applicable]

\begin{tabular}{lccccccc}
\hline Study site & Sex & $\begin{array}{c}\text { Sample } \\
\text { size } \\
\text { (n) }\end{array}$ & $\begin{array}{c}\text { Area } \\
\text { (square } \\
\text { kilometers) }\end{array}$ & $\begin{array}{c}\text { Number of } \\
\text { centers of } \\
\text { use (COUs) }\end{array}$ & $\begin{array}{c}\text { Maximum } \\
\text { distance } \\
\text { between COUs } \\
\text { (kilometers) }\end{array}$ & $\begin{array}{c}\text { Coastline } \\
\text { extent } \\
\text { (kilometers) }\end{array}$ & $\begin{array}{c}\text { Range span } \\
\text { (kilometers) }\end{array}$ \\
\hline Big Sur coast & $\mathrm{F}$ & 29 & $7.82 \pm 3.84$ & $1.21 \pm 0.41$ & $5.04 \pm 3.78$ & $14.72 \pm 6.99$ & $18.12 \pm 10.44$ \\
& $\mathrm{M}$ & 7 & $6.46 \pm 3.66$ & $1.57 \pm 0.79$ & $40.22 \pm 40.97$ & $10.54 \pm 6.75$ & $49.42 \pm 65.67$ \\
& $\mathrm{M} 1$ & 3 & $2.74 \pm 1.61$ & $1.00 \pm 0.01$ & $\mathrm{n} / \mathrm{a}$ & $3.94 \pm 1.52$ & $3.95 \pm 1.52$ \\
Monterey & $\mathrm{M} 2$ & 4 & $9.25 \pm 0.89$ & $2.00 \pm 0.82$ & $40.22 \pm 40.97$ & $15.48 \pm 3.67$ & $83.53 \pm 70.74$ \\
Peninsula & $\mathrm{F}$ & 83 & $7.48 \pm 7.89$ & $1.23 \pm 0.63$ & $7.01 \pm 12.90$ & $12.34 \pm 7.76$ & $19.06 \pm 27.68$ \\
& & & & & & & \\
& $\mathrm{M}$ & 23 & $10.11 \pm 12.60$ & $1.83 \pm 1.27$ & $18.51 \pm 17.21$ & $10.81 \pm 8.88$ & $30.59 \pm 39.70$ \\
& M1 & 11 & $2.07 \pm 1.78$ & $1.09 \pm 0.30$ & $\mathrm{n} / \mathrm{a}$ & $3.53 \pm 0.96$ & $7.76 \pm 14.75$ \\
& M2 & 12 & $17.49 \pm 13.81$ & $2.50 \pm 1.45$ & $18.51 \pm 17.21$ & $17.49 \pm 7.43$ & $51.52 \pm 44.23$ \\
\hline
\end{tabular}



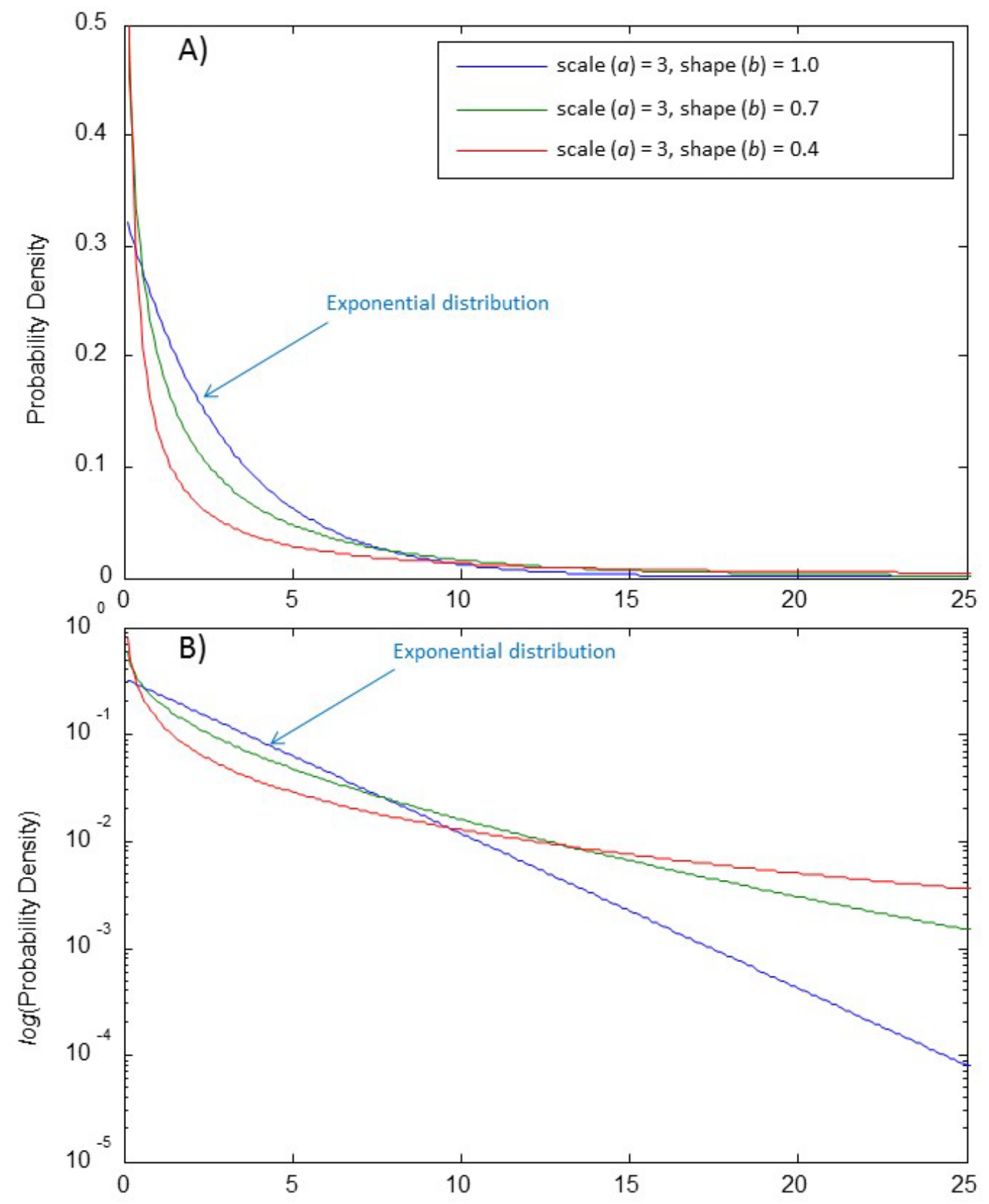

Distance moved in 1 year (annual net linear displacement, $\mathrm{km}$ )

Figure 9. Graphs of (A) Weibull probability density functions used to model sea otter dispersal distance probabilities showing the effect of variation in the shape parameter $(b)$ on the degree of skew or leptokurtosis of the distribution, and (B) same as (A) but plotted on log-transformed $y$-axis to show how low values of $b$ are associated with higher probabilities of very short or very long dispersal distances. Note that when $b=1$, Weibull is equal to the exponential probability density function. 
A)

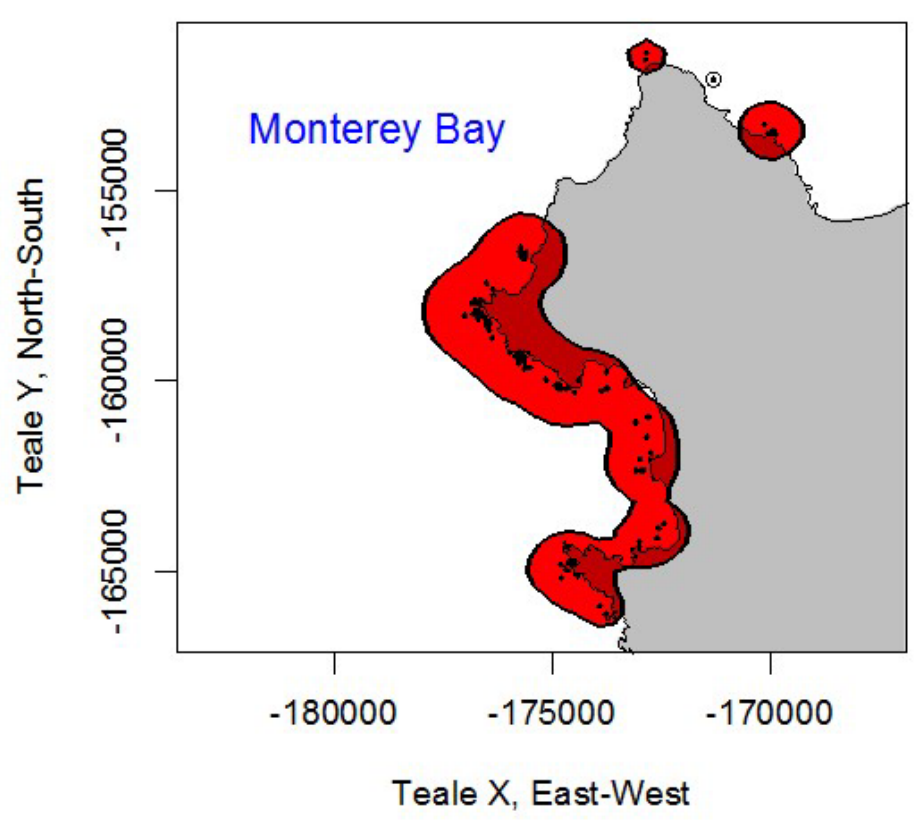

B)

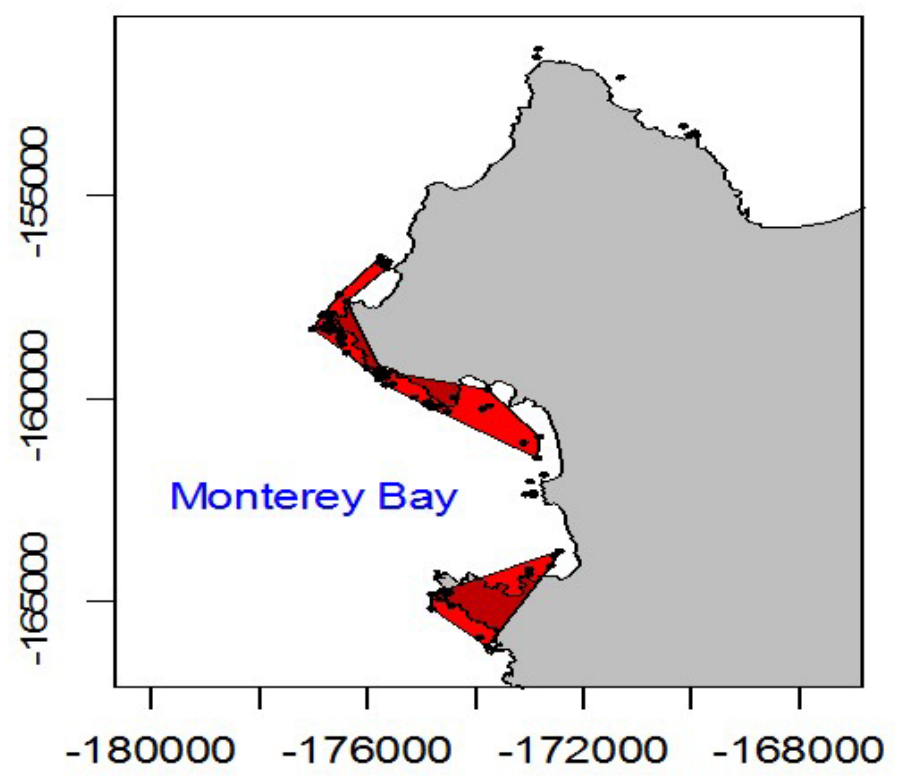

Figure 10. Schematics showing home-range estimate (red shaded areas on Monterey Bay) for sea otter 1317 (A) using kernel density estimation (smoothing parameter $h=475 \mathrm{~m}, 90$-percent kernel) in geographic coordinate space (Monterey Peninsula, California), and (B) using local convex hull analysis ( $\mathrm{LoCoH}, 90$-percent isopleth). In (A) points represent the resights of sea otter 1317 (female). This method incorrectly defines terrestrial areas (gray) as part of the sea otter home range. This method also over-estimates the depth usage of sea otters. Decreasing the smoothing parameter only serves to increase the number of satellite centers of use without significantly improving the problem of including terrestrial areas in the home range. In (B), points represent the resights of sea otter 1317. Although $\mathrm{LoCoH}$ analysis is designed to recognize boundaries within a habitat, the coastline is too complex for accurate representation, and terrestrial habitat (gray) is still included in the home-range estimate. In comparison to kernel density analysis, this method more accurately represents the offshore depth usage of sea otters. 

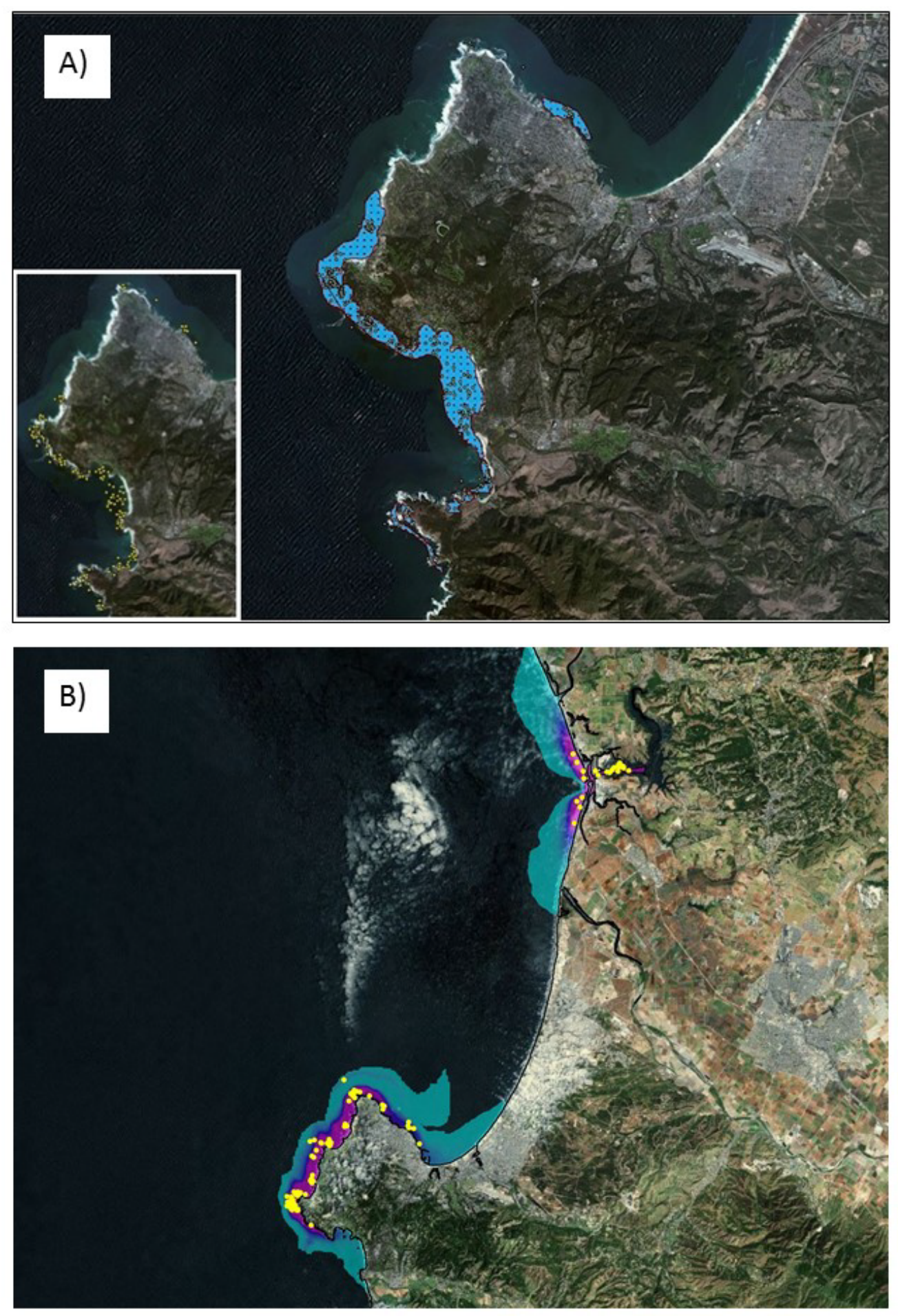

Figure 11. Geographic information system images showing sea otter resights and the results of the "permissible home range estimation" for two sea otters at the Monterey study site, central California. A, Daily resight locations (inset, yellow dots) for a female sea otter, and the associated 90-percent home-range polygon (blue shaded area on coastline). B, Daily resight locations (yellow dots) for a male sea otter, and the associated kernel density surface, shaded from high probability of occurrence (purple area closest to shoreline) to low probability of occurrence (light blue area seaward from purple area). Note the bimodal home range with two widely separated centers of use. 


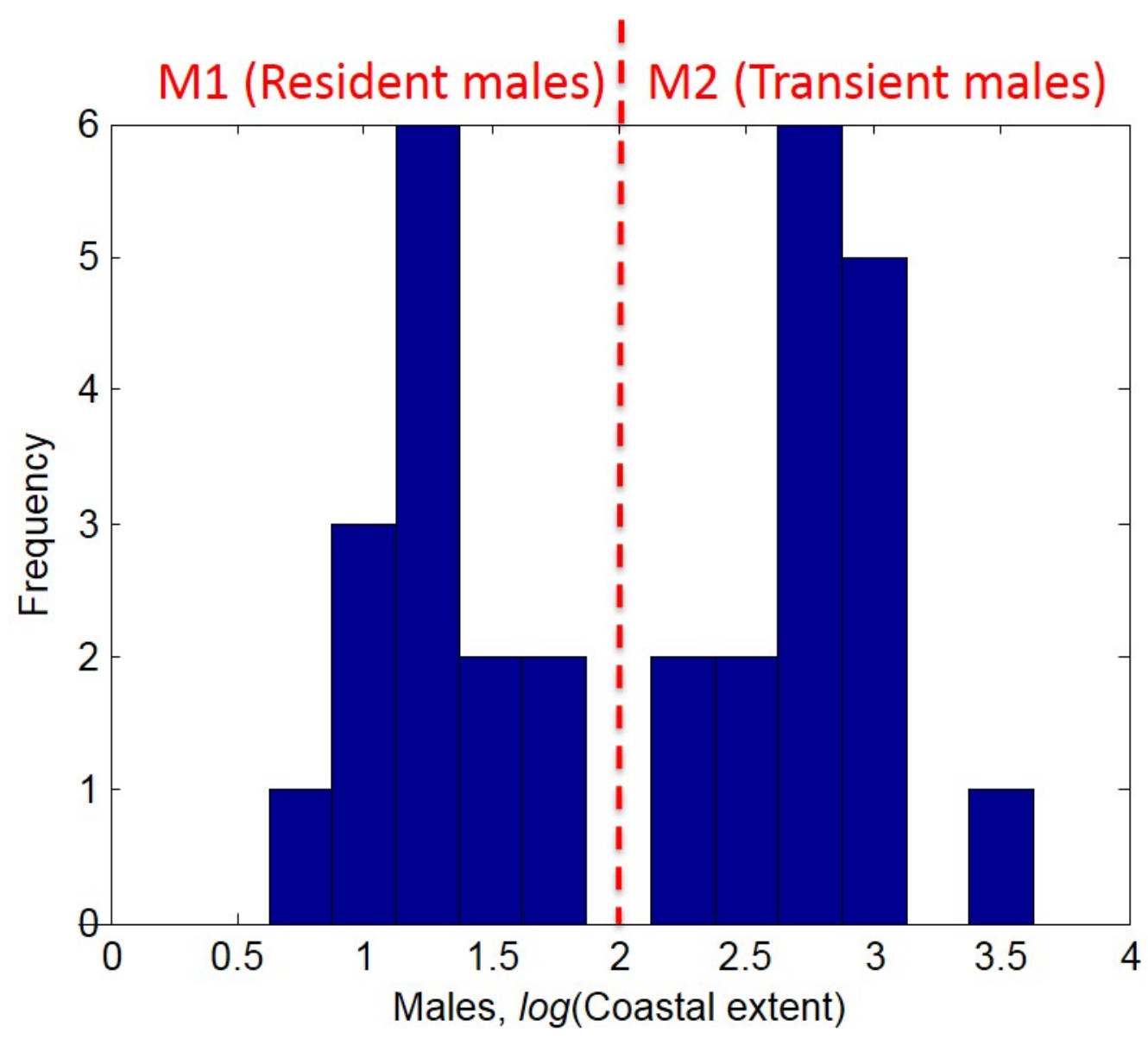

Figure 12. Frequency histogram showing log-transformed values of "home-range coastal extent" (the number of kilometers of coastline contained within home-range polygons) for male otters monitored in this study. The bimodal distribution is clearly evident, with an intermodal break-point at value 2; individuals to the left of this point were classified as M1 males, and individuals to the right of this point were classified as M2 males. 

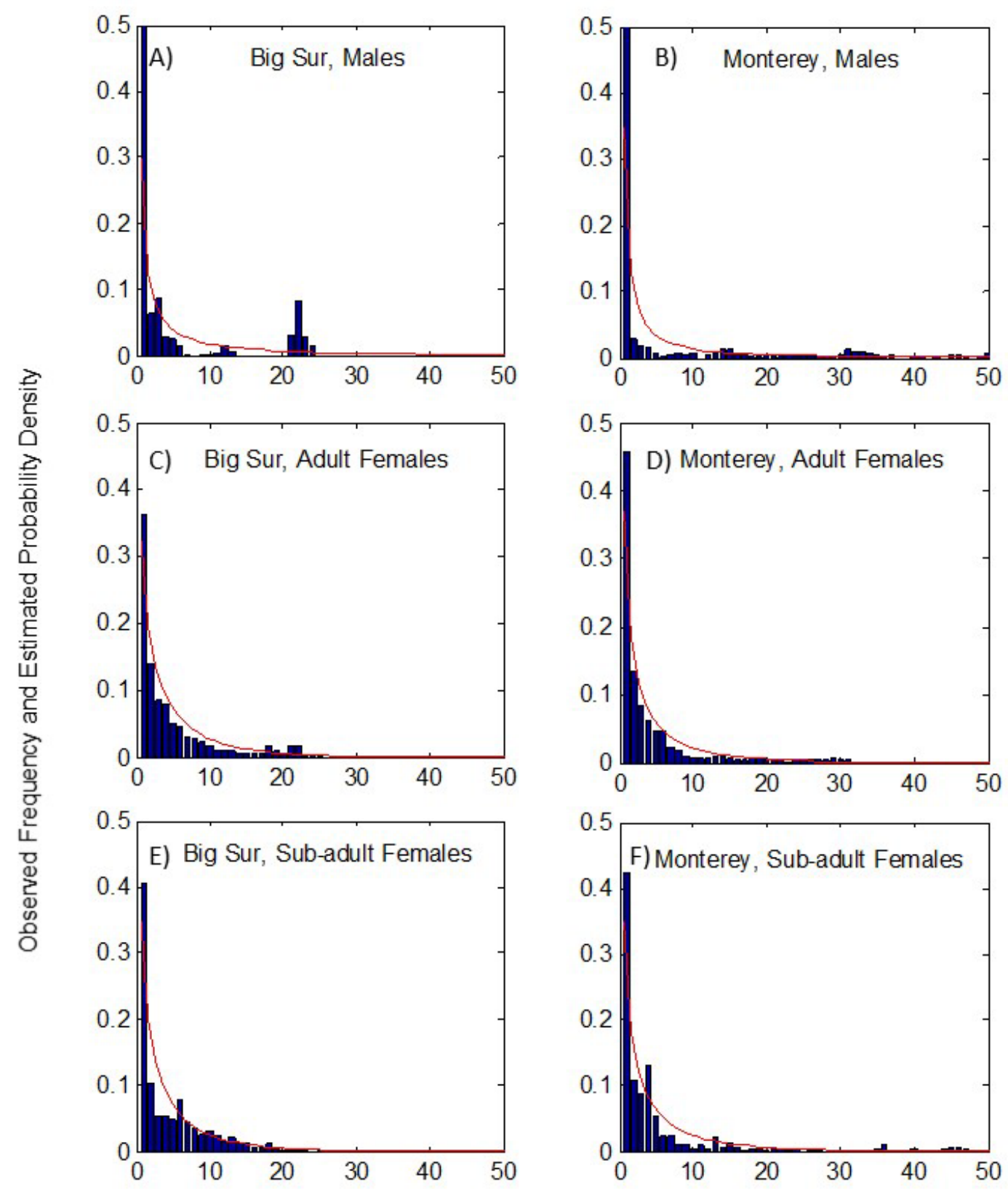

Distance moved in 1 year (annual net linear displacement, km)

Figure 13. Frequency histograms showing annual net linear displacement values, and associated Weibull probability density functions (fit using maximum likelihood estimates), for sea otters in this study, at Monterey and Big Sur, central California. Data are for male sea otters at (A) Big Sur and (B) Monterey, adult female sea otters at (C) Big Sur and (D) Monterey, and sub-adult female sea otters at (E) Big Sur and (F) Monterey. 


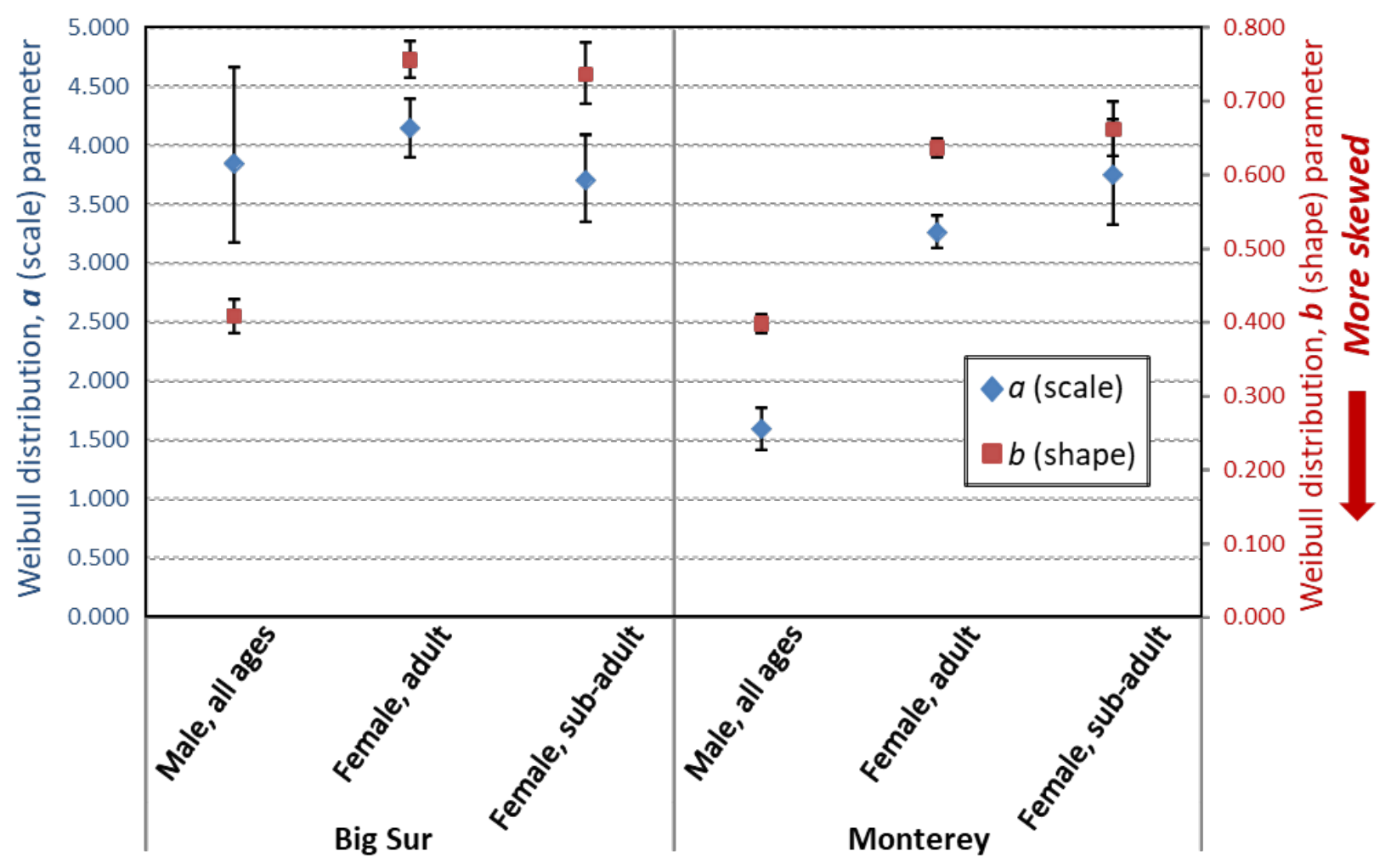

Figure 14. Boxplots showing maximum likelihood estimates for the two parameters of the Weibull probability functions fit to sea otter net linear displacement data (see fig. 13). Parameter a determines the scale (or mean expected value), and parameter $b$ determines the shape of the distribution, with lower values creating more leptokurtic distributions (see fig. 9). Mean parameter estimates and 95-percent confidence intervals (error bars) are shown for males, adult females, and sub-adult females at the Big Sur coast and Monterey Peninsula study sites, central California. 


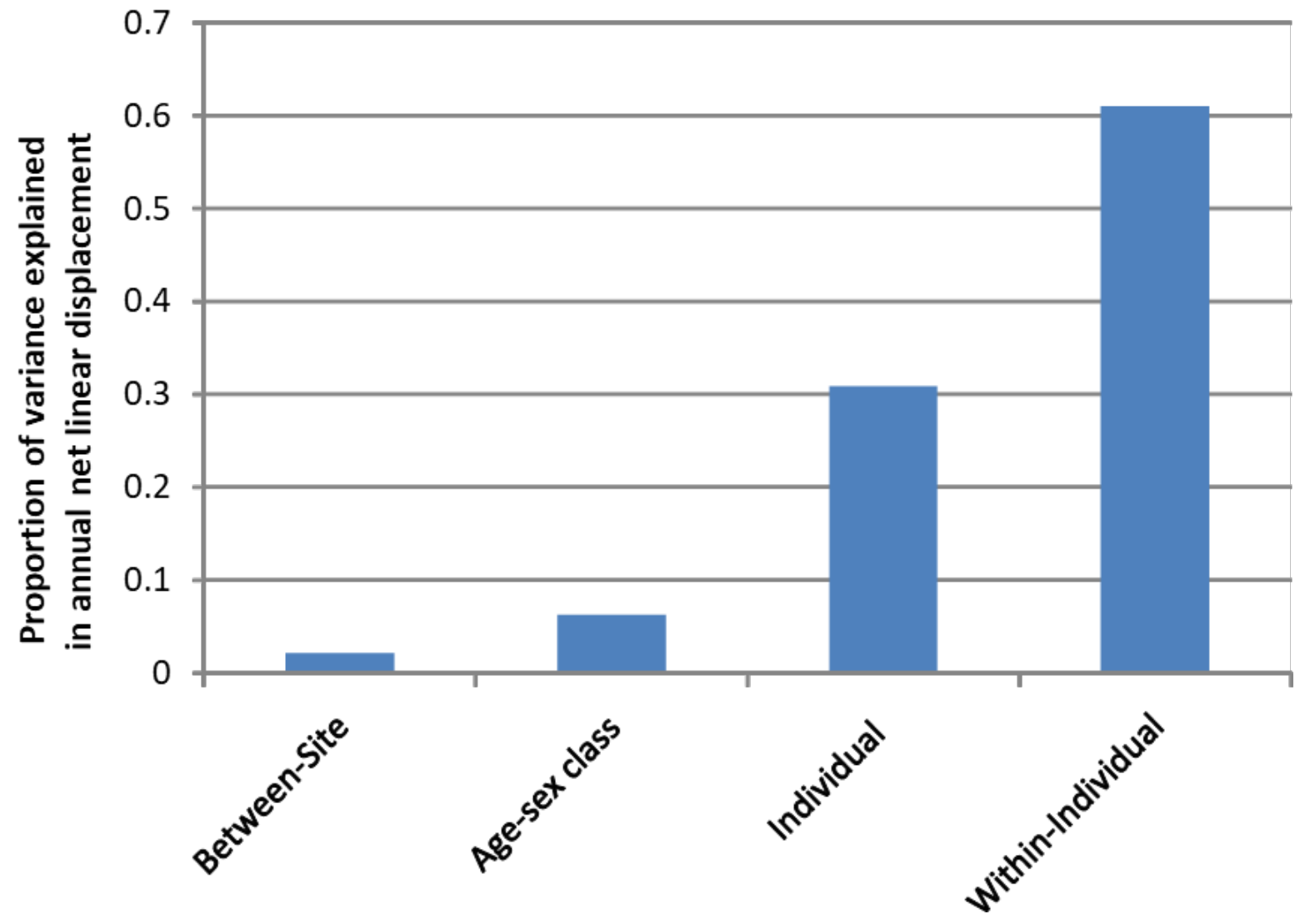

Figure 15. Graph showing results of a variance components analysis of sea otters showing the proportion of variation in annual net linear displacement values explained by four effects-between-site differences, age/sex class differences, among-individual differences, and within-individual variation. 
A) Adult females

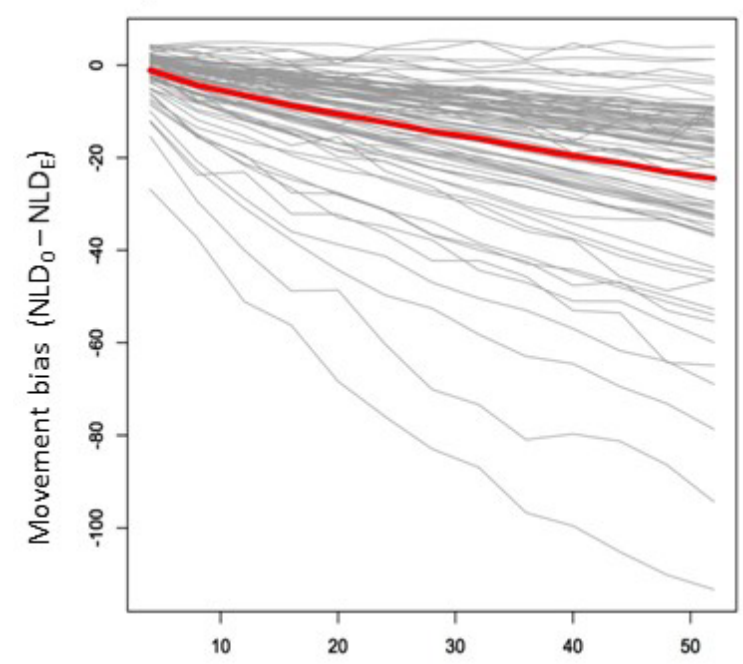

C) Males, unimodal distribution

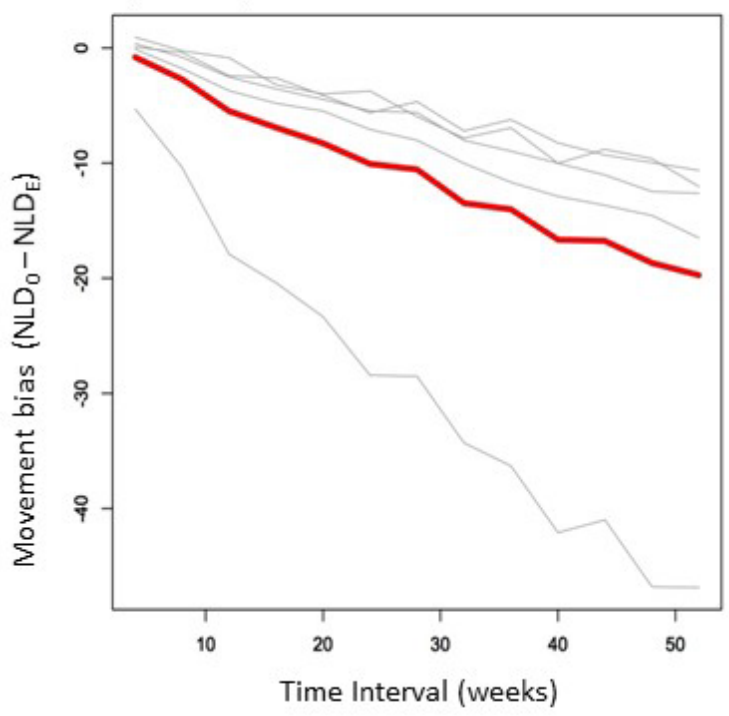

B) Sub-adult females

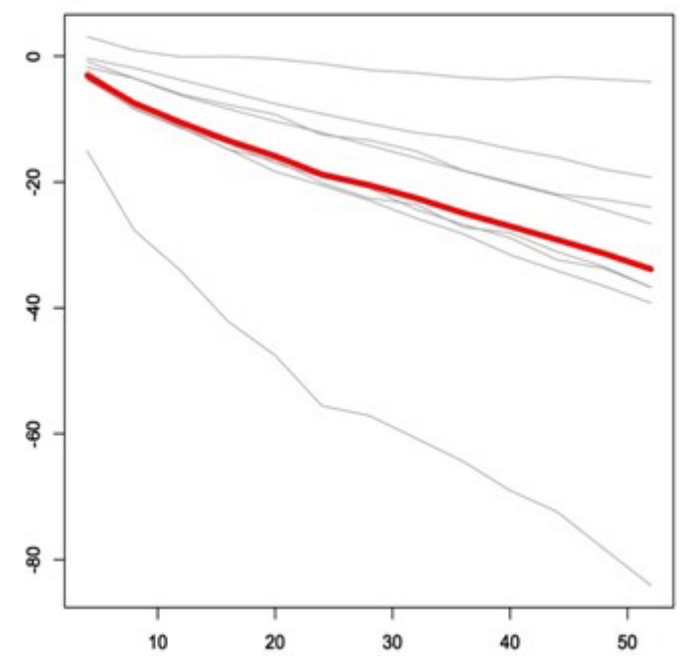

D) Males, multimodal distribution

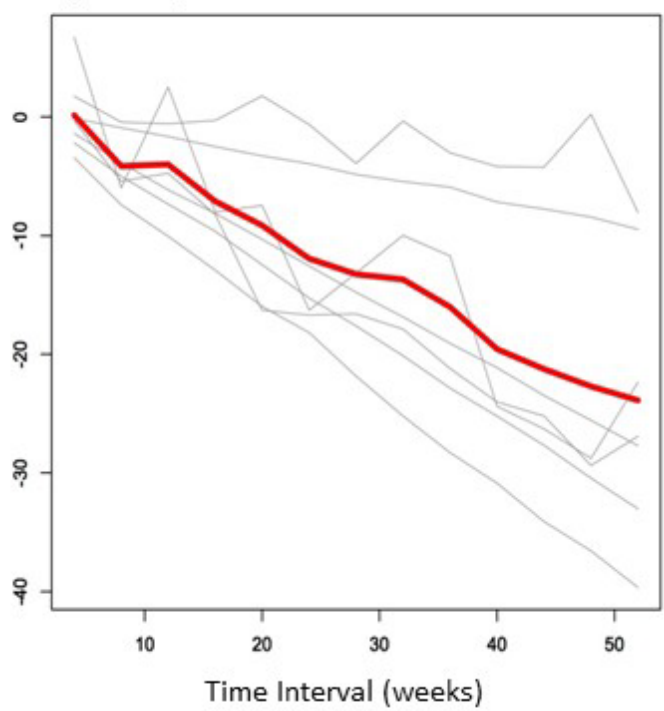

Figure 16. Graphs showing difference between observed net linear displacement (NLDo) and expected net linear displacement ( $N L D_{E}$, as calculated from correlated random walk models using methods described in the body text) for male and female sea otters plotted as a function of time intervals over which NLD was estimated and measured, ranging from 4 weeks to 1 year. Thin gray lines show the NLDo minus NLDE values for individual animals, and thick red lines show averages for all individuals in the study group. Data are plotted for four groups of animals-(A) adult females, (B) sub-adult females, (C) males with unimodal home-range distributions (resident males), and (D) males with multimodal home-range distributions (transient males). 


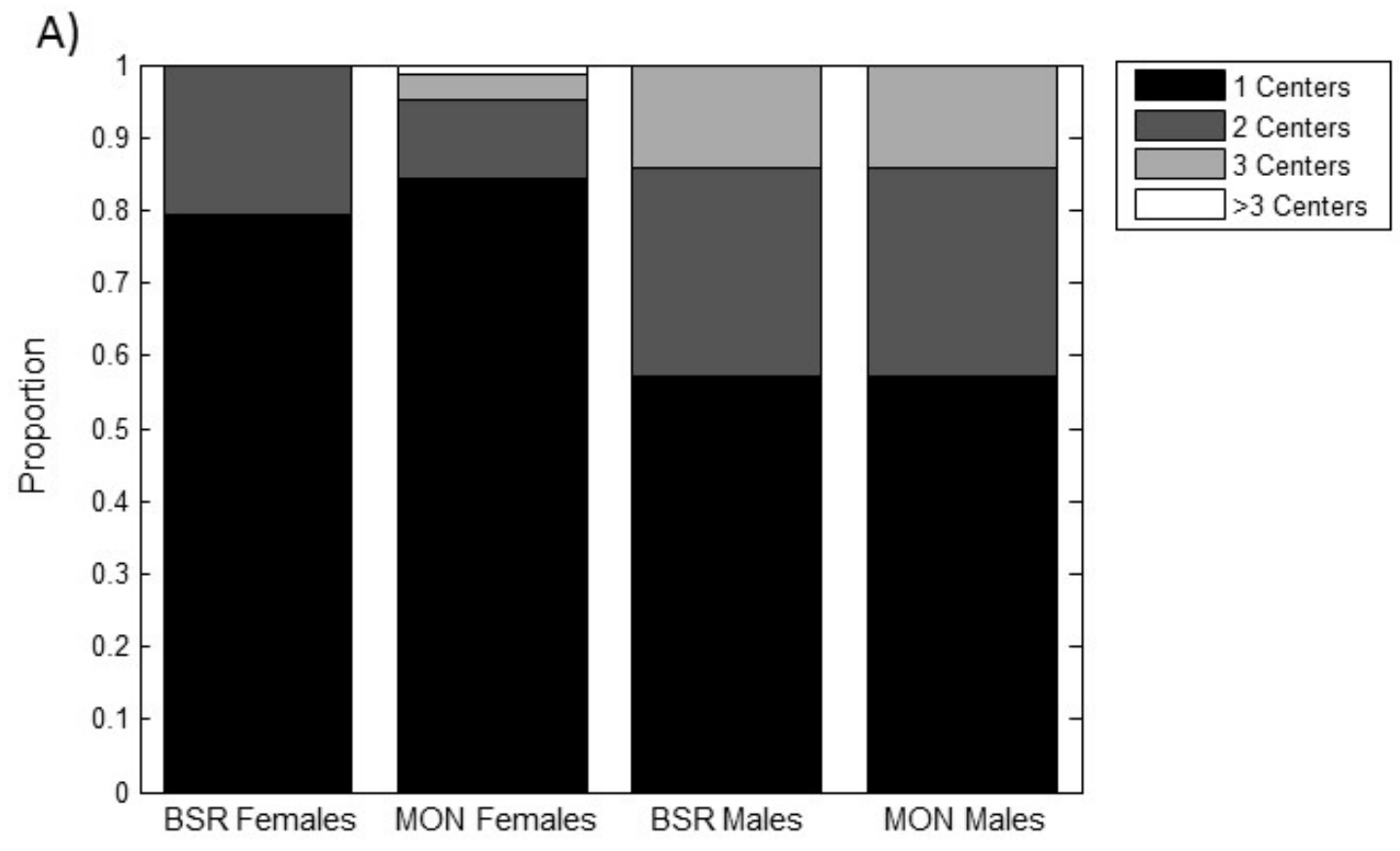

B)

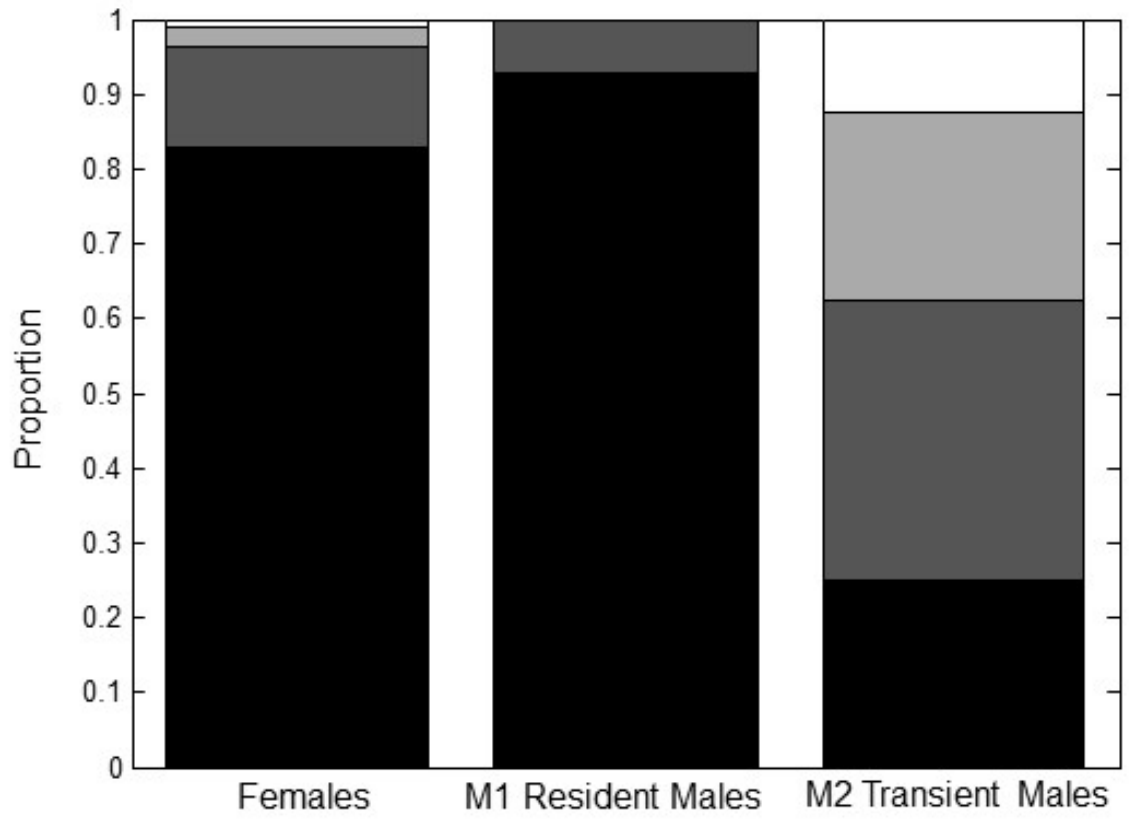

Figure 17. Graphs showing (A) proportion of sea otters with different numbers of centers of use, compared between study sites and sexes (MON, Monterey Peninsula; BSR, Big Sur coast), and (B) comparison of number of centers of use between females, M1 males, and M2 males, central California. 
A)

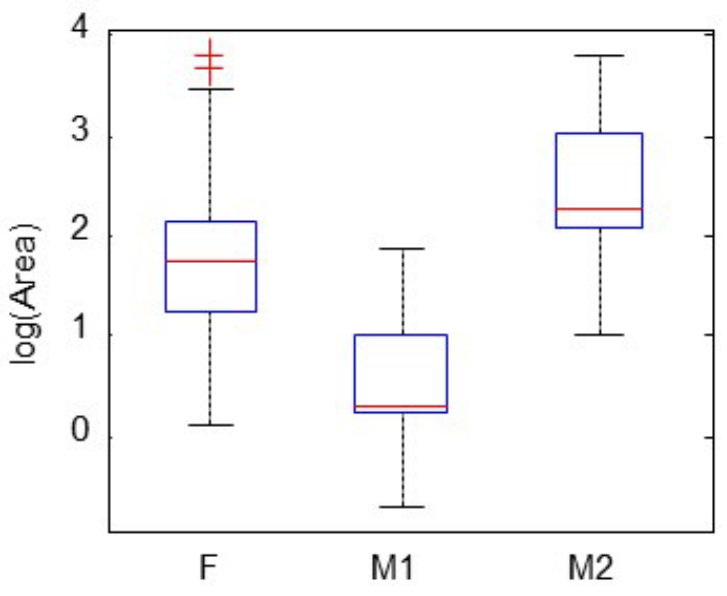

C)

Coastline Extent

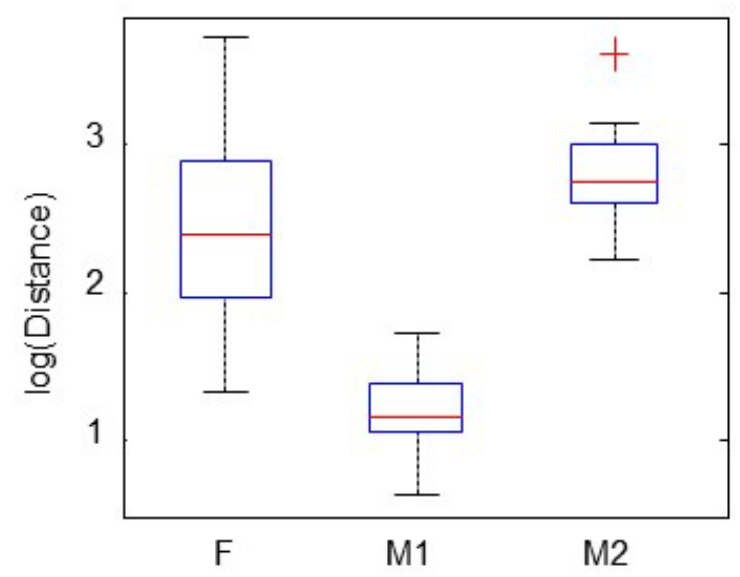

B) Dist b/t $\mathrm{COU}$

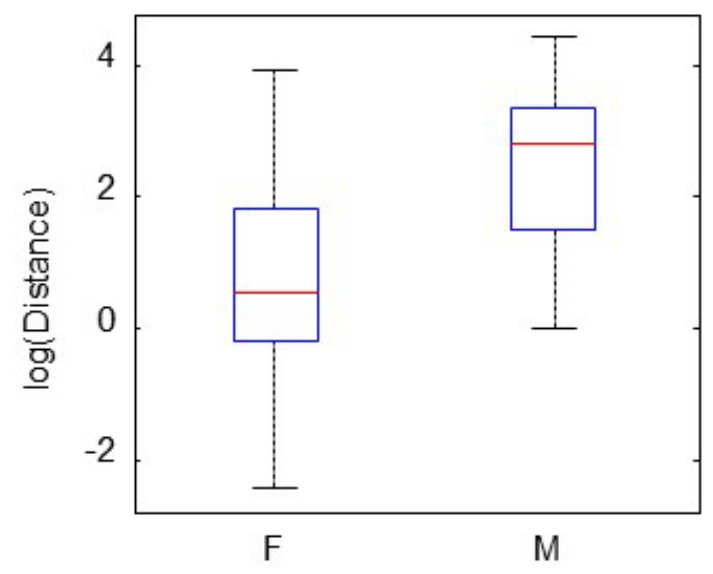

D)

\section{Range Span}

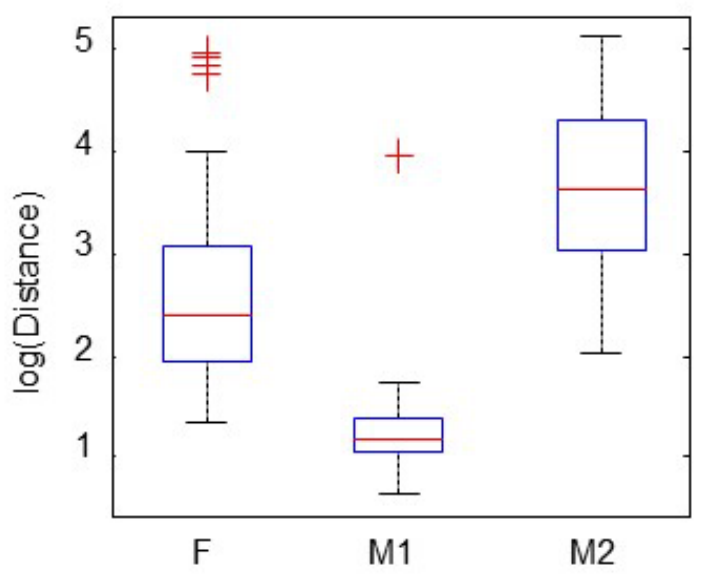

Figure 18. Boxplots comparing sea otter females, M1 males (residents), and M2 males (transients) in terms of four home-range statistics-(A) home-range area, $(B)$ distribution between centers of use, (C) coastline extent, and (D) range span, in central California. In all cases, distributions indicate log-transformed values. Boxes encompass the interquartile range (IQR), whiskers encompass the full distribution excluding outliers (from the lower quartile minus 1.5X IQR, to the upper quartile plus 1.5X IQR). Means of each home statistic differed significantly between groups. 


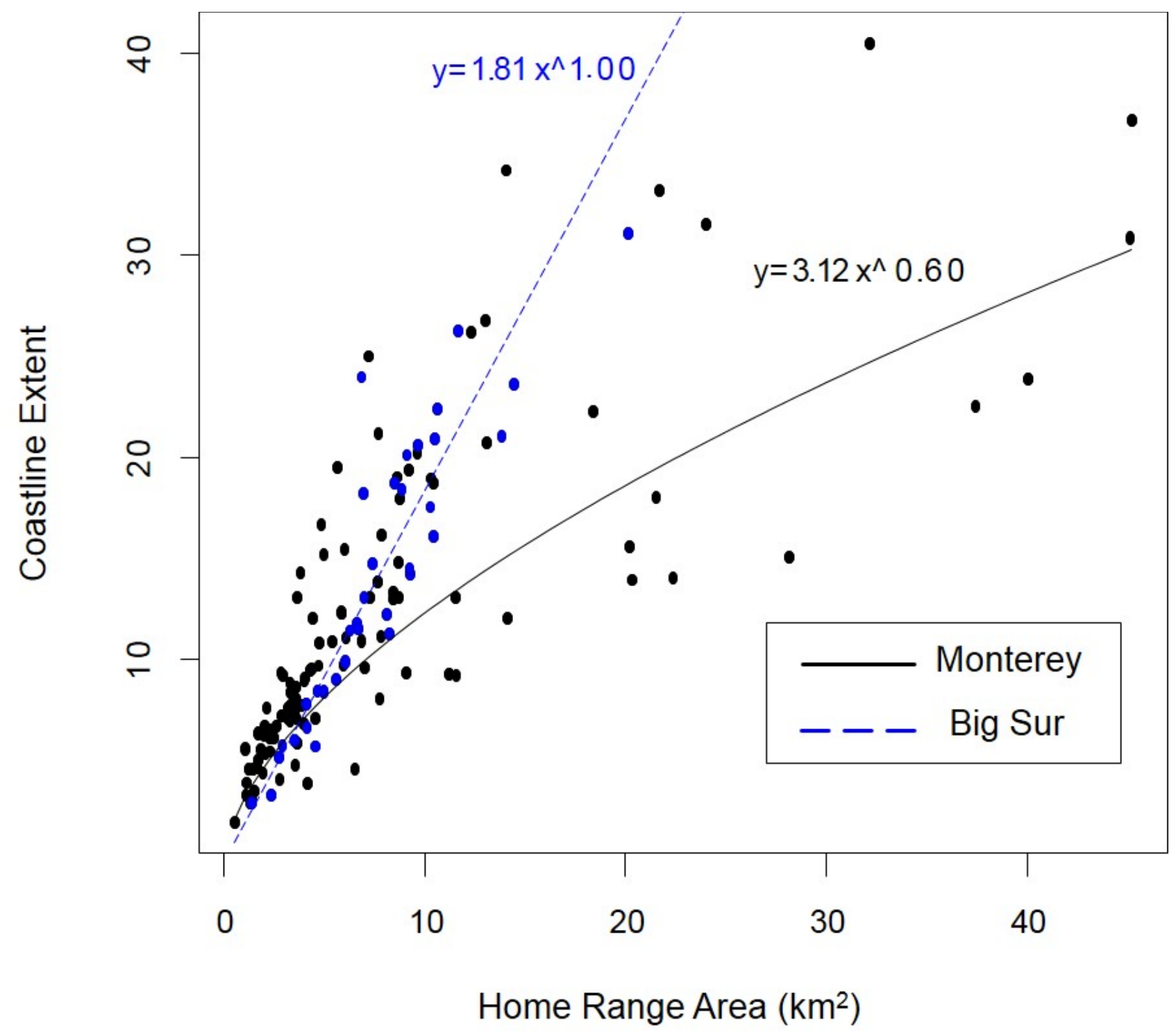

Figure 19. Graph showing coastline extent (in kilometers) plotted as a function of home-range area (in square kilometers) for otters in Monterey Bay and Big Sur, central California. Power functions were fit to the data, and their slopes differed significantly (degrees of freedom $(\mathrm{df})=1, F=8.1408, P<0.01$ ), indicating that coastline extent increased more rapidly with home-range area for sea otters at Big Sur as compared to Monterey. 


\title{
Chapter 5. Dive Behavior and Time-Activity Budgets
}

\author{
M. Tim Tinker ${ }^{1,2}$, Nicole M. Thometz ${ }^{2,3}$, Michelle M. Staedler ${ }^{4}$, Teri Nicholson ${ }^{4}$, and Joseph A. Tomoleoni ${ }^{1}$
}

\section{Introduction}

Sea otters have the highest mass-specific metabolic requirements of any marine mammal. Because of their lack of a blubber layer, they have negligible energetic reserves (Kenyon, 1969; Morrison and others, 1974; Yeates and others, 2007); thus, foraging behavior and diving capacities are critical components of food acquisition and survival. As might be expected for an energy-limited apex predator, sea otter foraging activity and diet selection are strongly dependent on population density and per-capita food abundance (Garshelis and others, 1986; Watt and others, 2000; Gelatt and others, 2002; Tinker and others, 2008), and density-dependent variation in foraging success is thought to be a primary determinant of equilibrium density (Estes, 1990; Bodkin and others, 2000). Although there is extensive published information on variation in foraging behavior and prey selection, much less is known about the details of diving behavior in sea otters. An investigation by Bodkin and others (2004) of diving behavior of northern sea otters (Enhydra lutris kenyoni) in Port Althorp, Alaska, provided the first description of sea otter dive attributes, average dive depths, and differences in diving behavior between males and females. Foraging dives in Port Althorp typically occurred in depths of 2-30 m; however, individuals occasionally made deeper dives of as much as $100 \mathrm{~m}$ in depth. Females and males showed differences in foraging dive depths, with females diving $<20 \mathrm{~m}$ on 85 percent of their dives and males diving $>45 \mathrm{~m}$ on 50-percent of their dives (Bodkin and others, 2004). A summary of dive behavior in southern sea otters in central California showed similar patterns (Tinker and others, 2006). Sex-based differences in dive behavior are not surprising, given the larger size of males (Leith, 1989; Burns, 1999). Less is known about the effects of other factors that could influence the dive behavior of sea otters, including age, reproductive status, population density, prey distribution, benthic habitat quality, and bathymetric characteristics of the local area.

Sea otters can adjust their diving behavior in response to decreased food abundance, making dives of greater depth or duration to find and retrieve prey. They also can increase the proportion of their daily activity budget spent feeding (thus decreasing the time spent resting or in other activities). Comparisons of sea otter time-activity budgets have been made from Alaska to California, and have consistently shown that at higher population densities and lower per-capita food abundance, the percentage of time spent feeding can increase from 20 percent to as much as 50 percent (Estes and others, 1982; Garshelis and others, 1986; Gelatt and others, 2002; Bodkin and others, 2007; Tinker and others, 2008). Different methodologies have been used to measure time-activity budgets, including scan sampling (Estes and others, 1986), radio telemetry (Loughlin, 1980; Ribic, 1982; Ralls and Siniff, 1990), and time depth recorders or TDRs (Bodkin and others, 2007), but all have shown similar results. The relation between activity budgets and resource abundance provides a useful tool for assessments of population status with respect to carrying capacity. However, other factors contribute to variation to time-activity budgets, including sex and age-class differences, and seasonal variation in prey abundance (Gelatt and others, 2002). Reproductive status in females also may affect foraging activity (Osterrieder

\footnotetext{
${ }^{1}$ U.S. Geological Survey.

${ }^{2}$ University of California, Santa Cruz.

${ }^{3}$ University of San Francisco.

${ }^{4}$ Monterey Bay Aquarium.
} 
and Davis, 2011; Staedler, 2011; Esslinger and others, 2014). These sources of variation can complicate inter-population contrasts, and so should be incorporated in analyses of activity budgets used to assess population status.

Another component of sea otter behavior that may vary in response to resource abundance is haul-out behavior. In a resource-limited environment, hauling out may enhance long-term individual reproductive success and survival by decreasing thermal flux and, thus, overall energy requirements (Costa and Kooyman, 1984; Yeates and others, 2007). The challenge has been to develop a reliable method of measuring sea otter haul-out behavior in the wild, which is not biased by daylight-dependent and line-of-sight observations (Harrold and Hardin, 1986; Maldini and others, 2012). By applying logistic regression modeling to a predictable body-core-temperature feature of hauled-out sea otters, we developed a reliable and non-biased method to predict this behavior from continuous diving-depth and temperature data recovered from TDR records (Nicholson and others, 2018). We reasoned that investigating haul-out behavior of sea otters in Big Sur and Monterey might provide further insights into behavioral responses of sea otters to limited prey resources at key life history stages, and implications for sea otter reproductive success and survival.

We measured dive behavior of sea otters in Monterey and Big Sur using archival TDRs as part of the comparative study of sea otter ecology at these two sites. The data contained in this chapter also were included in Thometz and others (2016) as part of a range-wide examination of southern sea otter dive behavior. For the Big Sur-Monterey Study, our objectives were to

1. Describe diving behavior and basic dive attributes (for example, mean and maximum dive depths and durations, bottom time, post dive intervals) of sea otters at the two study sites;

2. Identify sources of variation in diving attributes and behavior, including differences in location, age, sex, reproductive status and diet specialization;

3. Using established methods, calculate time-activity budgets from TDR records to assess relative food resource abundance at the two study sites;

4. Evaluate other potential sources of variation in activity budgets, including age, sex, reproductive status and diet specialization; and

5. Examine factors that explain variation in haul-out behavior.

Results of these analyses should shed light on the role of habitat differences and food resource abundance on the behavior and limitations of sea otter populations in central California.

\section{Methods}

\section{Dive Behavior}

Archival data from TDRs were collected from September 2007 to December 2011. Archived data ranged from 66 days to 1,094 days, dependent on individual otter survival and the battery life of the TDR, for a total of 377,496 hours of recorded data (table 10). Data from 39 study animals from Big Sur ( 2 males and 9 females) and Monterey ( 6 males and 22 females) were used for diving behavior analyses (see chapter 1 for detailed methods of sea otter capture techniques, TDR implantation methods, and tracking methods). In addition to adult $(\mathrm{n}=34)$ study animals, a small set of sub-adult $(\mathrm{n}=5)$ animals, independent otters under 3 years of age, were included in the analysis.

Because TDRs record diving behavior for an average of 1-2 years, many females in the study gave birth to (and raised until weaning) one or more pups during the period of TDR data logging. This allowed for examination of the effects of reproductive status on diving behavior. Specifically, females were categorized into one of four reproductive status categories based on the presence/age of their pup: (1) female with no pup (NP), (2) female with very small pup (VP, pup $\leq 3$ weeks old), (3) female with 
small pup (SP, pup $>3$ and $\leq 10$ weeks old), and (4) female with large pup (LP, pup $>10$ weeks old). Datasets for each female were divided into the appropriate category based on their reproductive status (NP-LP) on each day of TDR data logging, and we treated each combination of individual/reproductive status as an independent record for statistical analyses. These records, together with data records from males (MA) and sub-adult females (SA), resulted in six demographic classes that were used for statistical comparisons.

Raw TDR data were downloaded from the TDR instruments and pre-processed using the software "Instrument Helper" (version 3.0; Wildlife Computers, Redmond Washington) to correct depth readings for drift from the zero mark, and to compile the depth readings (at 2-second intervals) into distinct dives and contiguous surface intervals. For each identified dive, a series of six descriptive parameters were calculated (all depths in meters and all times in seconds): (1) maximum dive depth $(D E P),(2)$ duration of the sub-surface dive interval $(D T),(3)$ duration of time at spent at the bottom of the dive $(B T,=90$-percent of maximum depth and thus not necessarily on the ocean floor), (4) duration of the post-dive surface interval ( $P D I$, the number seconds elapsed until the next dive), (5) descent rate (DRT, vertical swim-speed from surface to bottom), and (6) ascent rate ( $A R T$, vertical swim-speed from bottom to surface). These parameters were used to classify dives as feeding or non-feeding dives (that is, dives conducted during grooming, traveling, or social behavior), following previously published methods (Bodkin and others, 2004, 2007). Briefly, a logistic classification function was used to classify each recorded dive based on a combination of the six dive parameters:

$$
\log \left(\frac{P}{1-P}\right)=\beta_{1}+\beta_{2}(D T)+\beta_{3}(B T / D T)+\beta_{4}(A R T)+\beta_{5}(D T)(A R T)+\beta_{6}(B T / D T)(D R T)
$$

where $\beta_{l}$ is a vector of parameters fit by maximum likelihood, and all dives with $\mathrm{P}>0.5$ were classified as feeding dives. Equation 3 was initially fit to a sub-sample of 5,000 "confirmed" dives (dives made by study animals that an observer was able to visually confirm as either $1=$ feeding or $2=$ non-feeding dive), and then validated by application to a second sub-sample of 1,000 confirmed dives, to ensure a classification accuracy of $>99$-percent. Best-fit values for $\beta_{l}$ were:

$$
[-5.115,0.258,0.487,-0.210,0.063,3.543]
$$

We applied equation 3 to each TDR record to classify all dives, and then subdivided the entire TDR record into contiguous "bouts" of similar activity states (inactive/resting $=\mathrm{R}$, feeding $=\mathrm{F}$, and "active other" = AO).

The net result of these pre-processing steps was a sample of 86,441 feeding bouts comprising $3,857,794$ foraging dives. Because individual dives within a feeding bout tended to be auto-correlated with respect to some dive parameters, and because some parameters of interest were measured across dives, we used feeding bouts as the fundamental statistical unit for analysis. For each distinct feeding bout we calculated a series of 12 statistics: (1) number of dives per bout, (2) bout duration, (3) mean dive depth, (4) mean dive duration (DT), (5) mean post-dive interval (PDI), (6) variance in post-dive interval (variance PDI), (7) mean bottom time (BT), (8) mean ratio of bottom time to dive time (BT/DT), (9) maximum dive depth, (10) maximum dive duration, (11) mean descent rate, and (12) mean ascent rate. Mean values of each bout statistic were then computed for each individual, and to ensure independence, we used individual animals as the level of replication for further statistical tests. 
Differences in dive attributes were first evaluated by location (fixed effect, two levels - Big Sur, Monterey) and by demographic class (fixed effect, six levels-NP, VP, SP, LP, Male (M), SA), using a three-way PERMANOVA, in which the interaction between location and class was evaluated as a third factor. Although some individuals only occurred within one demographic class, other individuals were represented in two or more classes during the course of data recording; for simplicity, we treat these as independent records. All dive attributes were normalized before analyses, Bray-Curtis similarity and 999 permutations were used. We then used a two-way PERMANOVA to test for differences between dive attributes by location and by class without including the interaction term and a one-way PERMANOVA to test for the influence of only the interaction term (location*class). Pairwise comparisons for both the two-way PERMANOVA and one-way PERMANOVA were made $a$ posteriori. When significant differences occurred, similarity percentages (SIMPER) analyses were used to identify the contribution of each factor to the observed differences. All PERMANOVA analyses and comparisons were made using PRIMER 6 and PERMANOVA+ software (Anderson and others, 2008).

To determine whether dive behavior varied as a function of prey selection and diet composition, a Multivariate Analysis of Variance (MANOVA) was used to test for differences in dive attributes between otters assigned to five different diet specializations (see chapter 6 for details). We used Wilks lambda to evaluate the significance of the multi-variate model, and in the case of significance we used univariate F-tests to evaluate diet-based differences in individual dive parameters.

\section{Haul-Out Behavior}

Because of sample size limitations for males and sub-adult age classes, we limited our analysis of haul-out behavior to data from reproductive females from Big Sur ( $n=6,5$ adult and 1 sub-adult TDR days $>324)$ and Monterey $(n=18$ AD, TDR days $>328)$. We reasoned that reproductive-age females presumably experience the greatest nutritional stress, especially during late-lactation when their foraging effort is greatest (Staedler 2011), and also are the demographic group of greatest interest in terms of understanding factors affecting population growth. To further improve statistical power for among-group comparisons at Monterey, we added data from another 15 adult females captured in Monterey prior to the current study (U.S. Geological Survey and Monterey Bay Aquarium, unpub. data, 2006-07).

To detect occurrence of haul out behavior from TDR records, we used a logistic classification function similar in form to equation 3, that was based on measurable attributes of the temperature trace during prolonged ( $>3.5$ hours) non-diving (that is, resting) intervals:

$$
\log \left(\frac{P}{1-P}\right)=\alpha+\beta\left(\left(T_{s t}-T_{\text {min }}\right)+\left(T_{\text {max }}-T_{\text {min }}\right)\right),
$$

where
$P \quad$ is the probability that the otter is hauled out,
$T_{s t} \quad$ is the body temperature at the start of the resting period,
$T_{\max } \quad$ is the maximum body temperature occurring during the resting period,
$T_{\min } \quad$ is the minimum body temperature occurring during the resting period prior to the maximum temperature, and $\alpha$ and $\beta$ are parameters fit by maximum likelihood.

Equation 4 was fit to a sample of "training data" (temperature traces from known haul-out and in-water resting periods), then validated by visual confirmation of positive (hauled-out) and negative (resting in water) predicted outcomes (Nicholson and others, 2018). After application of this model to data from 
the current study, we further validated model predictions with field observations of each study animal, and confirmed that the model performed at an 85 percent or greater prediction success rate for each individual. To improve parsimony, we rejected any model-detected haul-out bouts occurring when ambient air temperatures were $>20^{\circ} \mathrm{C}$, as these conditions were most likely to lead to misclassification of at-sea resting bouts as haul-out bouts. We also rejected bouts characterized by short $(<1 \mathrm{~h})$ but substantial temperature anomalies or fluctuations: although a few of these intervals may have been incorrectly rejected, our goal was to identify and estimate the prevalence of prolonged and uninterrupted intervals ashore. For each day of each TDR record we then calculated the percentage of time spent hauled-out.

To evaluate factors important for predicting variation in haul out behavior, we used general linear models (GLMs) to test for effects of study site (BSR compared to MON), average individual diving depth, season, and reproductive status on the frequency of haul-out behavior. To describe seasonal effects, we defined two seasons (winter and summer) based on relative differences in mean monthly air temperature measured by National Oceanic and Atmospheric Administration buoys off Monterey and Cape San Martin. (We note that seasonal differences must be interpreted with caution because the $>20^{\circ} \mathrm{C}$ filter applied to detected haul out bouts will lead to more excluded bouts in summer than in winter). For female reproductive status, we used the same classifications identified in section, "Dive Behavior" (NP, VP, SP and LP), but also added an "estrous" category for females that were visually confirmed to be engaged in mating activity (estrous generally occurs shortly after weaning a pup). We used maximum likelihood methods to fit various models with different combinations of main effects, and used an information theoretic approach to select the best-supported model or models with the smallest Akaike information criterion (AIC) values (Burnham and Anderson, 2002).

\section{Time-Activity Budgets}

For each 24-hour period of each TDR record, we calculated a time-activity budget by summing the cumulative time spent in each of three activity states: (1) F = feeding, (2) AO = active-other, and (3) $\mathrm{R}=$ inactive/resting (see Bodkin and others, 2007, for details). A typical 24-hour period from a TDR record with dive behavior and activity classifications is shown in figure 20. Time-activity budgets were then summarized for all otters, with each otter classified by location and sex, and for females we also calculated activity budgets for each of four reproductive states (NP, VP, SP and LP). We used two-way ANOVA to test for variation in percentage of time spent feeding due to sex and study site (MON compared to BSR), as well as interactions between these main effects. We then used single factor ANOVA to test for differences between study sites with respect to percentage of time spent feeding for females in different reproductive states (NP, VP, SP and LP). Pooling all female otters from both sites, we used mixed-effects ANOVA to test for differences in percent time feeding between reproductive states, with individual otters treated as a random effect. Finally, we used single-factor ANOVA to test for differences in percentage of time spent feeding between otters assigned to five different diet specializations (see chapter 6).

\section{Results}

\section{Dive Behavior}

Attributes of feeding dives and foraging bouts generally were similar at Big Sur and Monterey, with average dive depths of about $9 \mathrm{~m}$ and average dive durations of about 1 minute at both sites. Ninety-nine percent (99-percent) of feeding dives occurred at depths shallower than $37 \mathrm{~m}$ in Big Sur and $39 \mathrm{~m}$ in Monterey; the deepest recorded dive $(87 \mathrm{~m})$ occurred at Big Sur, whereas the longest recorded 
dive (472 seconds) occurred at Monterey. A complete summary of dive attributes at each site is provided in table 11. Based on a complete model with interaction, dive attributes differed significantly by class (three-way PERMANOVA: $\left.\mathrm{F}_{5,73}=3.95, \mathrm{P}=0.001\right)$, but not by location $\left(\mathrm{F}_{1,73}=1.25, \mathrm{P}=0.258\right)$ or by their interaction $\left(\mathrm{F}_{5,73}=0.50, \mathrm{P}=0.947\right)$. Using a reduced model without interaction term, the influence of demographic class remained highly significant (two-way PERMANOVA: F5,78 $=4.73, \mathrm{P}=$ $0.001)$ and the influence of location was marginally significant $\left(\mathrm{F}_{1,78}=2.58, \mathrm{P}=0.055\right)$. Pairwise tests were run for all demographic classes and nearly all were significantly different $(\mathrm{P}<0.05)$ from one another (table 12). Pairs that were marginally significant $(\mathrm{P}<0.1)$ included MA and SA $(\mathrm{t}=1.54, \mathrm{P}=$ $0.059)$, VP and SP $(\mathrm{t}=1.53, \mathrm{P}=0.074)$, and SP and SA $(\mathrm{t}=1.67, \mathrm{P}=0.065)$. Groups that were not significantly different were $\mathrm{NP}$ and $\mathrm{SA}(\mathrm{t}=0.92, \mathrm{P}=0.48)$ and $\mathrm{LP}$ and $\mathrm{SA}(\mathrm{t}=1.24, \mathrm{P}=0.21)$.

The SIMPER analysis highlighted the dive attributes that contributed the most (cumulative contribution $>30$-percent) to the differences noted between each demographic class (table 12). Maximum dive depth (fig. 21) and maximum dive duration (fig. 22) contributed most to the differences noted between males (MA) and all female classes (NP-LP). Number of dives per bout also contributed greatly to the differences between MA and LP (males made fewer dives per bout). Variance in PDI, ascent rate, and maximum dive duration were the most important dive attributes between NP and VP (females without pups made longer dives and had less variable surface intervals than females with very small pups), whereas descent rate, maximum dive duration and ascent rate were the most important attributes distinguishing NP from SP (females with small pups descended more rapidly, had longer dive times, and ascended more rapidly than females without a pup). Between NP and LP, the greatest contributing attributes to observed differences were number of dives per bout, bottom time, and mean dive duration (females with large pups had more dives per bout and had longer dives because they spent more time at bottom). The most influential variables between VP and LP were variance PDI, number of dives per bout, and mean bout duration; however, between VP and SA, differences were driven by maximum dive depth, maximum dive duration, and variance PDI (table 12).

To test differences between individuals of the same reproductive state in different locations (table 13), a one-way PERMANOVA was run with only the location*class interaction term. The interaction term was significant (one-way PERMANOVA: $F_{11,73}=2.5, P=0.001$ ) and pairwise comparisons were made. Only NP individuals differed significantly between locations $(\mathrm{t}=1.76, \mathrm{P}=$ 0.03 ), and all other pairwise comparisons between individuals of the same class at each location were non-significant. The SIMPER analysis indicated that the dive attributes that contributed the most (cumulative contribution > 30-percent) to the difference between NP individuals in Big Sur and Monterey were mean bout duration, maximum dive duration, and number of dives per bout. In particular, females with no pup in Big Sur had longer maximum dive durations, longer foraging bouts, and more dives per bout than those in Monterey.

Dive parameters differed significantly among otters with different diet specialization $\left(\lambda_{U}=0.0008, F_{12,22}=2.31, P<0.0001\right)$. In particular, diet specialists differed with respect to dive depth $\left(F_{1,33}=5.94, P=0.020\right)$, dive duration $\left(F_{1,33}=6.65, P=0.014\right)$, bottom time $\left(F_{1,33}=6.59, P=0.015\right)$, and ascent rate $\left(\mathrm{F}_{1,33}=4.75, \mathrm{P}=0.037\right)$. As shown in figure 23 , sea otters that specialized on Cancer crabs (type 1) and abalone (type 2) had lower ascent rates and greater dive durations, depths and bottom times compared to sea otters that specialized on mussels (type 3), turban snails (type 4), and clams (type 5).

\section{Haul-Out Behavior}

The model that best fit data on haul-out frequency included effects of study site and mean diving depth. Females in Monterey hauled out more frequently than Big Sur females (4.1-percent \pm 1.0 compared to 0.4-percent \pm 0.07 ). All Big Sur females spent a minimal amount of time hauled out. By contrast, Monterey females had great variability with respect to hauling out. Some (5) females rarely 
hauled out, others (8) hauled out frequently during parts of the year, and 2 females hauled out throughout most or all of the year. These differences may be partially explained by mean diving depth (fig. 24A); deeper divers ( $>10 \mathrm{~m}$ ) hauled-out rarely if ever. We limited further analyses to sea otters from Monterey to clarify other effects more clearly after removing the effect of study site. The bestsupported model included effects of reproductive status and season, with an additional random effect for individual otter (indicating that much of the variation was related to differences among individuals that were not explained by season or status). Haul-out behavior was strongly influenced by reproductive status - specifically, the estrous period when females in our sample hauled-out most frequently (16.3percent \pm 1.2 standard error [SE], fig. 24B). Haul-out behavior did not vary significantly among the remaining categories of reproductive status. Season had a small effect: females tended to haul-out slightly more during winter than summer (9.4 -percent $\pm 6.6 \mathrm{SE}$ vs. $6.5 \pm$ percent $0.9 \mathrm{SE})$, although we note that exclusion of haul-out bouts with $>20{ }^{\circ} \mathrm{C}$ ambient temperatures may have contributed to this difference. Reproductive status had the greatest relative effect on haul-out behavior (45.3-percent), followed by individual otter effects (26.2-percent), and seasonal differences (4.8-percent).

\section{Time-Activity Budgets}

Percentage of time spent foraging differed significantly between the two sites (fig. 25A) when all classes were pooled $\left(\mathrm{F}_{1,35}=9.08, \mathrm{P}=0.005\right)$, with sea otters at $\mathrm{BSR}$ spending more time feeding than otters at MON (45.49 \pm 5.07 compared to $41.48 \pm 4.68$, respectively; see table 14 for details on the relation between sex and age class on behavior). Percentage of time in active-other behaviors also differed between sites $\left(\mathrm{F}_{1,35}=9.164, \mathrm{P}=0.005\right)$, with $\mathrm{BSR}$ otters spending less time in non-feeding activities than MON otters (5.76 \pm 2.44 vs. $9.02 \pm 2.62$, respectively), but there were no differences in inactive/resting behavior $\left(\mathrm{F}_{1,35}=1.667, \mathrm{P}=0.205\right)$. The effect of sex on percentage of time spent feeding was not significant $\left(\mathrm{F}_{1,35}=1.577, \mathrm{P}=0.217\right)$, although the marginally significant interaction between sex and site $\left(\mathrm{F}_{1,35}=3.457, \mathrm{P}=0.071\right)$ indicated an especially high percentage of time spent feeding (50.83-percent) by BSR males (table 14).

Limited sample sizes for sub-adult animals (BSR $n=1$, MON n=4) precluded testing for differences in behavior between sites and age classes. Pooling the data for both sites, we noted no significant difference in time spent foraging between age classes $(\mathrm{F}=0.357, \mathrm{P}=0.555)$.

When we parsed data by reproductive status, we determined that percentage of time spent feeding differed between study sites for females without pups $\left(\mathrm{F}_{1,29}=8.76, \mathrm{P}=0.007\right)$, such that females from BSR spent more time feeding than MON females (table 15). However, there were no significant between-site differences in activity budgets for females with pups, irrespective of pup stage. Pooling data for both sites, a mixed-effects model indicated a strongly significant effect of female reproductive status on percentage of time spent feeding $\left(\mathrm{F}_{3,50}=127.4, \mathrm{P}=<0.0001\right)$ : specifically, the percent time feeding by females increased significantly $(\mathrm{P}<0.05)$ as pups grew from VP to SP, and from SP to LP stages (table 16, fig. 25B).

We did not find any differences in activity budget between otters with different diet specializations $\left(\mathrm{F}_{4,30}=0.766, \mathrm{P}=0.556\right)$.

\section{Discussion}

Sea otters in Big Sur and Monterey seem to have similar diving behavior overall. This finding is not surprising, considering that both locations have fairly similar benthic habitat types, and support high-density sea otter populations where intra-specific competition for prey may be fairly high. Typical dive times were about 1 minute with average dive depths of about $9 \mathrm{~m}$, although these parameters were highly variable (table 11). Our data differ from data presented by Bodkin and others (2004) in that we 
did not observe a clear bimodal foraging depth pattern. Sea otters in Port Althorp, Alaska, foraged mainly at depths of 2 to 30 meters (84 percent of all dives); however, many individuals, particularly males, showed a bimodal foraging depth distribution with a secondary modal feeding depth occurring at 30-60 m (Bodkin and others, 2004). At Big Sur and Monterey, sea otters foraged mostly at depths of 3$15 \mathrm{~m}$ and had progressively fewer foraging dives in deeper water, although the two Big Sur males had a barely discernible secondary modal depth at 40-60 m. Males dove to the greatest depths in our study (fig. 21), reaching maximum depths of $87 \mathrm{~m}$ in Big Sur and $77 \mathrm{~m}$ in Monterey. The difference in maximum dive depths between the two locations likely is a result of the differing bathymetry of each location (the coastal shelf drops off steeply at Big Sur, but is broad and shallow at Monterey), and not a result of any physiological differences in diving ability.

In contrast to the similarity in diving behavior across sites, we determined that differences in age, sex, reproductive status and diet were associated with substantial differences in diving behavior. For example, sub-adults had more dives per bout $(60.0 \pm 10.5)$ and longer mean bout durations $(132.1 \pm 18.8 \mathrm{~min}$ ) than their adult female counterparts (with the exception of those having large pups, as discussed later in this section). Additionally, sub-adults had the second-longest maximum dive durations $(273.8 \pm 32.7 \mathrm{~s})$ and spent the second-longest amount of time foraging per day (47.6 \pm 2.5 -percent). These results suggest that sub-adult sea otters in central California show a greater foraging effort than do adults (table 12). Sub-adult animals likely are physiologically limited and behaviorally naive in comparison to adults (Burns, 1999; Noren and others, 2002; Richmond, Burns, and Rea, 2006) and these factors may ultimately require the increased foraging effort by sub-adult animals.

Adult females showed striking variation in diving behavior that corresponded with changes in their reproductive status. Females with very small pups substantially decreased their number of dives per bout, bout durations, maximum dive depths, and maximum dive durations in comparison to females with no pup (table 12). The changes seem to be adaptive behavior adjustments for pup care, indicating the high level of maternal attention a neonate sea otter pup needs to survive. Female sea otters with very small pups also showed greater post dive intervals and greater variance in these post dive intervals, likely due to the necessity of tending to their pup between dives. Decreased maximum dive depths for females with very small pups and small pups (fig. 21) also probably are an adaptive adjustment to improve pup survival outcomes, but imply a substantial decrease in potential foraging areas available to these females, with potential implications for foraging success.

As a pup matures from a highly dependent neonate to a large pup making diving and foraging attempts alongside its mother, females substantially alter their diving and foraging behavior yet again. The energetic toll of rearing young is high in sea otters (Thometz and others, 2014); in response to these high energetic demands, females with large pups have greater numbers of dives per bout and longer bout durations than those without large pups, and also decrease the interval between dives (indicating less time spent with the pup and more time diving for food). Females must not only acquire enough food for themselves, but they also must share an increasingly large amount of their prey items with their pups, which decreases the caloric intake of the mom, necessitating behavioral modifications that greatly increase the overall amount of time they spend feeding when a large pup is present. Despite all these behavioral modifications, females nearing the end of lactation are in poor body condition owing to depletion of body reserves over the 6-month pup dependency period, resulting in increased risk of mortality (see chapters 7, 8 and 10).

Another behavioral strategy that females may use to mitigate costs of reproduction is haul-out behavior, although opportunities for hauling out may vary seasonally and spatially. We noted significant differences in hauling out between our two study sites. At Monterey, many of the tagged females hauled out regularly, especially in response to estrous or mating, but at Big Sur, females rarely hauled out. Our sample size from Big Sur $(n=6)$ may have been too low to reliably estimate haul-out behavior at the 
population level; however, during field observation, the behavior was only noted three times during the entire 3-year study period. One possible explanation for this difference is the differing geographic features and coastline topologies at the two sites. Monterey Peninsula is a winding and complex coastline with significant intertidal habitats that are protected from prevailing northwest winds and swell, providing ample haul-out opportunities. By contrast, the Big Sur coastline is relatively linear and highly exposed, with minimal inter-tidal habitats, and so may simply provide fewer haul-out opportunities. Because hauling out may act to reduce thermally induced metabolic requirements during periods of nutritional stress, females from Monterey may thus experience a geographic advantage by having more haul-out opportunities, enabling individuals to reduce energy costs and potentially to enhance their survival and long-term reproductive success (see chapter 8 ). This advantage may be especially critical for females that have recently weaned a pup and are entering estrous in poor body condition with minimal energy reserves. Indeed, the incidence of mortality associated with "endlactation syndrome" was slightly lower among Monterey females than among Big Sur females (see chapter 10).

In addition to evaluating differences in haul-out and dive behavior, the TDR data allowed us to examine variation in time-activity budgets. The percentage of time spent feeding by sea otters at both sites was over 40 percent - quite high compared to growing populations where prey resources are abundant (Jolly, 1997; Bentall, 2005; Bodkin and others, 2007) but similar to populations thought to be at carrying capacity where prey resource abundance limits further growth (Estes and others, 1982; Garshelis and others, 1986; Gelatt and others, 2002; Tinker and others, 2008). We determined that sea otters at the Big Sur study site spent slightly more time feeding than otters at Monterey (fig. 25A), suggesting that prey resources may be slightly more depleted at the Big Sur site. This difference was particularly apparent for Big Sur males, which is consistent with the finding that males at Big Sur also showed much lower rate of energy gain while feeding (chapter 6) and poorer body condition (chapter 7) than males at Monterey. As expected based on previous analyses (Osterrieder and Davis, 2011; Staedler, 2011), female reproductive status was a major determinant of percentage of time spent feeding, which dropped to 24 percent for females with very small pups but increased to almost 50 percent for females with large pups (fig. 25B).

Understanding sea otter dive behavior is not as simple as describing differences between males and females; habitat type and bathymetry, population density, available resources, age, reproductive status, and diet specialization also play important roles in dictating diving behavior. For example, the differences in dive attributes we noted between sea otters that used different prey types (fig. 23) were consistent with earlier reports that diet specialization is indicated by variation in dive behavior (Tinker and others, 2007). Such behavioral differences are likely to reinforce diet specialization, as individuals become channeled into different behavioral strategies. Overall, the dive behavior and time-activity budgets of sea otters at Big Sur and Monterey are indicative of populations that are strongly influenced by resource limitation, and show patterns of variation that suggest adaptive responses to low prey availability coupled with high energy demands at critical life history stages. These patterns are likely to be indicated in survival and pup weaning success (see chapter 8), and ultimately in population recovery.

\section{References Cited}

Anderson, M.J., Gorley, R.N., and Clarke, K.R., 2008, PERMANOVA+ for PRIMER — Guide to software and statistical methods: Plymouth, United Kingdom, PRIMER-E Ltd., 214 p.

Bentall, G.B., 2005, Morphological and behavioral correlates of population status in the southern sea otter-A comparative study between central California and San Nicolas Island: Santa Cruz, University of California, Master's thesis. 
Bodkin, J.L., Burdin, A.M., and Ryazanov, D.A., 2000, Age- and sex-specific mortality and population structure in sea otters: Marine Mammal Science, v. 16, p. 201-219.

Bodkin, J.L., Esslinger, G.G., and Monson, D.H., 2004, Foraging depths of sea otters and implications to coastal marine communities: Marine Mammal Science, v. 20, p. 305-321.

Bodkin, J.L., Monson, D.H., and Esslinger, G.G., 2007, Activity budgets derived from time-depth recorders in a diving mammal: Journal of Wildlife Management, v. 71, p. 2034-2044.

Burnham, K.P., and Anderson, D.R., 2002, Model selection and inference-A practical informationtheoretic approach (2d. ed.): New York, Springer-Verlag, 488 p.

Burns, J.M., 1999, The development of diving behavior in juvenile Weddell seals-Pushing physiological limits in order to survive: Canadian Journal of Zoology, v. 77, p. 737-747.

Costa, D.P., and Kooyman, G.L., 1984, Contribution of specific dynamic action to heat balance and thermoregulation in the sea otter Enhydra lutris: Physiological Zoology, v. 57, p. 199-203.

Esslinger, G.G., Bodkin, J.L., Breton, A.R., Burns, J.M., and Monson, D.H., 2014, Temporal patterns in the foraging behavior of sea otters in Alaska: The Journal of Wildlife Management, v. 78, no. 4, p. 689-700.

Estes, J.A., 1990, Growth and equilibrium in sea otter populations: Journal of Animal Ecology, v. 59, p. 385-402.

Estes, J.A., Jameson, R.J. and Rhode E.B.. 1982. Activity and prey selection in the sea otter - influence of population status on community structure. American Naturalist 120:242-258.

Estes, J.A., Underwood, K.E., and Karmann, M.J., 1986, Activity-time budgets of sea otters in California [USA]: Journal of Wildlife Management, v. 50, p. 626-636.

Garshelis, D.L., Garshelis, J.A., and Kimker, A.T., 1986, Sea otter time budgets and prey relationships in Alaska: Journal of Wildlife Management, v. 50, p. 637-647.

Gelatt, T.S., Siniff, D.B., and Estes, J.A., 2002, Activity patterns and time budgets of the declining sea otter population at Amchitka Island, Alaska: Journal of Wildlife Management, v. 66, p. 29-39.

Harrold, C., and Hardin, D., 1986, Prey consumption on land by the California sea otter Enhydra-lutris: Marine Mammal Science, v. 2, p. 309-313.

Jolly, J.M., 1997, Foraging ecology of the sea otter, Enhydra lutris, in a soft-sediment community: Santa Cruz, University of California, Master's dissertation.

Kenyon, K.W., 1969, The sea otter in the eastern Pacific Ocean: North American Fauna, v. 68, p. 1352.

Leith, D.E., 1989, Adaptations to deep breath-hold diving respiratory and circulatory mechanics: Undersea Biomedical Research, v. 16, p. 345-354.

Loughlin, T.R., 1980, Radio telemetric determination of the 24-hour feeding activities of sea otters, Enhydra lutris, in Amlaner, C J., and MacDonald, D.W., eds., A handbook on biotelemetry and radio tracking: Oxford, United Kingdom, Permagon Press, p. 717-724.

Maldini, D., Scoles, R., Eby, R., Cotter, M., and Rankin, R.W., 2012, Patterns of sea otter haul-out behavior in a California tidal estuary in relation to environmental variables: Northwestern Naturalist, v. 93, p. 67-78.

Morrison, P., Rosenmann, M., and Estes, J.A., 1974, Metabolism and thermoregulation in the sea otter: Physiological Zoology, v. 47, p. 218-229.

Nicholson, T. E., K. A. Mayer, M. M. Staedler, J. A. Fujii, M. J. Murray, A. B. Johnson, M. T. Tinker, and K. S. Van Houtan. 2018. Gaps in kelp cover may threaten the recovery of California sea otters. Ecography, https://doi.org/10.1111/ecog.03561

Noren, S.R., Lacave, G., Wells, R.S., and Williams, T.M., 2002, The development of blood oxygen stores in bottlenose dolphins (Tursiops truncates): implications for diving capacity: Journal of Zoology, v. 258, p. 105-113. 
Osterrieder, S.K., and Davis, R.W., 2011, Sea otter female and pup activity budgets, Prince William Sound, Alaska: Journal of the Marine Biological Association of the UK, v. 91, p. 883-892.

Ralls, K., and Siniff, D.B., 1990, Time budgets and activity patterns in California sea otters: Journal of Wildlife Management, v. 54, p. 251-259.

Ribic, C.A., 1982, Autumn activity of sea otters in California: Journal of Mammalogy, v. 63, p. $702-$ 706.

Richmond, J.P., Burns, J.M., and Rea, L.D., 2006, Ontogeny of total body oxygen stores and aerobic dive potential in Steller sea lions (Eumetopias jubatus): Journal of Comparative Physiology B, v. 176, p.535-545.

Staedler, M.M., 2011, Individual variation in maternal care and provisioning in the southern sea otter (Enhydra lutris nereis) — Causes and consequences of diet specialization in a top predator; Santa Cruz, University of California, Master's thesis.

Thometz, N.M., Staedler, M.M., Tomoleoni, J.A., Bodkin, J.L., Bentall, G.B., and Tinker, M.T., 2016: Trade-offs between energy maximization and parental care in a central place forager, the sea otter: Behavioral Ecology, v. 27, no. 5, p. 1552-1566.

Thometz, N.M., Tinker, M.T., Staedler, M.M., Mayer, K.A., and Williams, T.M., 2014, Energetic demands of immature sea otters from birth to weaning-Implications for maternal costs, reproductive behavior and population-level trends: Journal of Experimental Biology, v. 217, p. 2053-2061.

Tinker, M.T., Bentall, G., and Estes, J.A., 2008, Food limitation leads to behavioral diversification and dietary specialization in sea otters: Proceedings of the National Academy of Sciences of the United States of America, v. 105, p. 560-565.

Tinker, M.T., Costa, D.P., Estes, J.A., and Wieringa, N., 2007, Individual dietary specialization and dive behaviour in the California sea otter-Using archival time-depth data to detect alternative foraging strategies: Deep Sea Research II, v. 54, p. 330-342.

Tinker, M.T., Estes, J.A., Ralls, K., Williams, T.M., Jessup, D., and Costa, D.P., 2006, Population dynamics and biology of the California sea otter (Enhydra lutris nereis) at the southern end of its range: Santa Barbara, University of California, Coastal Research Center, Marine Science Institute, MMS OCS Study 2006-007, MMS Cooperative Agreement Number 14-35-0001-31063.

Watt, J., Siniff, D.B., and Estes, J.A., 2000, Inter-decadal patterns of population and dietary change in sea otters at Amchitka Island, Alaska: Oecologia (Berlin), v. 124, p. 289-298.

Yeates, L.C., Williams, T.M., and Fink, T.L., 2007, Diving and foraging energetics of the smallest marine mammal, the sea otter (Enhydra lutris): Journal of Experimental Biology, v. 210, p. 19601970. 
Table 10. Summary information on time-depth recorder deployments for study animals in the current study.

[Study site: BSR, Big Sur coast; MON, Monterey Peninsula. Sex: F, Female; M, Male. Pup birth? and Pup success?: N, No; Y, Yes; N/A, not applicable]

\begin{tabular}{|c|c|c|c|c|c|c|c|c|c|}
\hline $\begin{array}{l}\text { Study } \\
\text { site }\end{array}$ & Otter ID & Sex & Age & $\begin{array}{l}\text { Age } \\
\text { class }\end{array}$ & Implant date & $\begin{array}{l}\text { Time-depth recorder } \\
\text { (TDR) stop date }\end{array}$ & $\begin{array}{l}\text { Total } \\
\text { TDR } \\
\text { days } \\
\end{array}$ & $\begin{array}{c}\text { Pup } \\
\text { birth } \\
?\end{array}$ & $\begin{array}{c}\text { Pup } \\
\text { success } \\
?\end{array}$ \\
\hline BSR & $5-093$ & $\mathrm{~F}$ & 8.0 & A & 30-Mar-09 & 13-Sep-09 & 167 & $\mathrm{Y}$ & $\mathrm{N}$ \\
\hline BSR & $5-283$ & F & 10.0 & A & 22-Mar-09 & 16-Jun-09 & 86 & Y & $\mathrm{N}$ \\
\hline BSR & $6-043$ & F & 9.0 & A & 12-Nov-08 & 9-Jun-09 & 209 & $\mathrm{Y}$ & Y \\
\hline BSR & $6-209$ & $\mathrm{~F}$ & 6.0 & A & 30-Nov-08 & 5-Feb-10 & 432 & Y & $\mathrm{N}$ \\
\hline BSR & $6-370$ & M & 8.0 & A & 10-Nov-08 & 3-Nov-09 & 358 & $\mathrm{~N} / \mathrm{A}$ & N/A \\
\hline BSR & $6-409$ & M & 9.0 & A & 9-Nov-08 & 4-Nov-09 & 360 & $\mathrm{~N} / \mathrm{A}$ & N/A \\
\hline BSR & $6-436$ & $\mathrm{~F}$ & 5.0 & A & 11-Nov-08 & 24-Sep-10 & 682 & $\mathrm{Y}$ & $\mathrm{Y}$ \\
\hline BSR & $7-660$ & F & 9.0 & A & 11-Nov-08 & 10-Nov-11 & 1,094 & Y & $\mathrm{Y}$ \\
\hline BSR & $6-067$ & F & 4.0 & $\mathrm{~A}$ & 3-Nov-09 & 9-Nov-11 & 736 & $\mathrm{~N}$ & $\mathrm{~N} / \mathrm{A}$ \\
\hline BSR & $6-553$ & F & 2.5 & SA & 4-Nov-09 & 24-Sep-10 & 324 & $\mathrm{~N}$ & $\mathrm{~N} / \mathrm{A}$ \\
\hline BSR & $6-514$ & F & 4.0 & $\mathrm{~A}$ & 3-Nov-09 & 4-May-11 & 547 & Y & $\mathrm{N}$ \\
\hline MON & $7-649$ & $\mathrm{~F}$ & 4.0 & A & 28-Sep-07 & 6-Oct-08 & 374 & $\mathrm{Y}$ & $\mathrm{Y}$ \\
\hline MON & $7-609$ & F & 4.0 & A & 26-Sep-07 & 19-Aug-08 & 328 & $\mathrm{~N}$ & N/A \\
\hline MON & $7-828$ & F & 6.0 & A & 27-Sep-07 & $10-$ Sep-08 & 349 & $\mathrm{Y}$ & $\mathrm{N}$ \\
\hline MON & $4-257$ & $\mathrm{~F}$ & 5 & A & 28-Sep-07 & 10-Sep-08 & 348 & $\mathrm{Y}$ & $\mathrm{N}$ \\
\hline MON & $5-117$ & $\mathrm{~F}$ & 5.5 & A & 1-Feb-10 & 23-Jun-10 & 142 & $\mathrm{Y}$ & $\mathrm{Y}$ \\
\hline MON & $6-765$ & F & 8.0 & A & 7-Jun-10 & 16-Jun-11 & 374 & $\mathrm{Y}$ & $\mathrm{Y}$ \\
\hline MON & Jack & M & 6.5 & A & 2-Feb-10 & 9-Jan-11 & 341 & N/A & N/A \\
\hline MON & ORWH & M & 10.0 & A & 8-Jun-10 & 5-Feb-11 & 242 & N/A & $\mathrm{N} / \mathrm{A}$ \\
\hline MON & $5-217$ & $\mathrm{~F}$ & 6.0 & A & 2-Feb-10 & 12-Jun-10 & 130 & $\mathrm{~N}$ & N/A \\
\hline MON & $5-349$ & F & 7.0 & A & 22-Apr-09 & 8-Jun-10 & 412 & $\mathrm{Y}$ & $\mathrm{Y}$ \\
\hline MON & $7-722$ & F & 5.0 & A & 30-Apr-09 & $24-O c t-10$ & 542 & $\mathrm{Y}$ & Y \\
\hline MON & $6-381$ & $\mathrm{~F}$ & 6.0 & A & 16-Jun-09 & 15-Aug-10 & 425 & Y & $\mathrm{Y}$ \\
\hline MON & $1030-06$ & M & 9.0 & A & 23-Apr-09 & 25 -Aug-10 & 489 & N/A & N/A \\
\hline MON & $1037-07$ & $\mathrm{~F}$ & 7.0 & A & 23-Apr-09 & 22-Jun-10 & 425 & $\mathrm{Y}$ & $\mathrm{Y}$ \\
\hline MON & $7-633$ & $\mathrm{~F}$ & 5.5 & A & 17-Jun-09 & 28-Jul-10 & 406 & $\mathrm{~N}$ & N/A \\
\hline MON & $5-296$ & $\mathrm{~F}$ & 5.5 & $\mathrm{~A}$ & 23-Apr-09 & 27-Oct-10 & 552 & $\mathrm{Y}$ & $\mathrm{Y}$ \\
\hline MON & $6-485$ & M & 6.0 & $\mathrm{~A}$ & 30-Apr-09 & 27-Apr-10 & 362 & N/A & N/A \\
\hline MON & $6-493$ & $\mathrm{~F}$ & 4.0 & $\mathrm{~A}$ & 11-Jun-09 & 4-Sep-11 & 815 & $\mathrm{Y}$ & $\mathrm{Y}$ \\
\hline MON & $7-747$ & M & 10.0 & A & 22-Apr-09 & 8-Jun-10 & 412 & N/A & N/A \\
\hline MON & $5-069$ & $\mathrm{~F}$ & 2.5 & SA & 28-May-09 & 28 -Oct-10 & 518 & $\mathrm{~N}$ & N/A \\
\hline MON & $6-268$ & F & 7.0 & $\mathrm{~A}$ & 17-Jun-09 & 7-Jun-10 & 355 & Y & $\mathrm{Y}$ \\
\hline MON & Marigold & $\mathrm{F}$ & 1.1 & SA & 24-Aug-09 & 29-Oct-09 & 66 & $\mathrm{~N}$ & $\mathrm{~N} / \mathrm{A}$ \\
\hline MON & George & M & 11.0 & AA & 9-Jun-09 & 31-Mar-10 & 295 & N/A & $\mathrm{N} / \mathrm{A}$ \\
\hline MON & $6-131$ & $\mathrm{~F}$ & 9.0 & $\mathrm{~A}$ & 16-Jun-09 & 6-Jun-10 & 355 & $\mathrm{Y}$ & $\mathrm{N}$ \\
\hline MON & Lola & F & 3.1 & $\mathrm{~A}$ & 16-Dec-10 & 2-Sep-11 & 260 & $\mathrm{~N}$ & $\mathrm{~N} / \mathrm{A}$ \\
\hline MON & Blanca & F & 2.3 & SA & 3-Dec-09 & 26-Jun-11 & 570 & Y & $\mathrm{N}$ \\
\hline MON & $1000-05$ & F & 8.0 & $\mathrm{~A}$ & 17-Jun-09 & 3-Oct-10 & 473 & Y & $\mathrm{Y}$ \\
\hline MON & $1038-07$ & $\mathrm{~F}$ & 5.0 & A & 29-Jul-10 & 7-Aug-11 & 374 & $\mathrm{Y}$ & $\mathrm{Y}$ \\
\hline
\end{tabular}


Table 11. Dive attributes of southern sea otters from Big Sur coast and Monterey Peninsula study sites, central California.

[Statistics shown represent arithmetic means for all individuals in each study site (plus or minus standard errors), with the exception of the last three columns that show the maximum recorded dive depth, the $99^{\text {th }}$ percentile of dive depths (that is, the depth above which 99 percent of all dives occurred) and the maximum recorded dive duration at each site. Study site: BSR, Big Sur coast; MON, Monterey Peninsula. n: Number of otters in given site PDI: Mean post-dive interval. Variance PDI: Variance in post-dive interval. BD/DT: Mean ratio of bottom time to dive time]

\begin{tabular}{|c|c|c|c|c|c|c|c|c|c|c|c|c|c|c|c|c|}
\hline $\begin{array}{l}\text { Study } \\
\text { site }\end{array}$ & $\mathrm{n}$ & $\begin{array}{c}\text { Dives per } \\
\text { bout } \\
\text { (number) }\end{array}$ & $\begin{array}{c}\text { Bout } \\
\text { duration } \\
\text { (minutes) }\end{array}$ & $\begin{array}{c}\text { Dive } \\
\text { depth } \\
\text { (meters) }\end{array}$ & $\begin{array}{c}\text { Dive } \\
\text { duration } \\
\text { (seconds) }\end{array}$ & $\begin{array}{c}\text { PDI } \\
\text { (seconds) }\end{array}$ & $\begin{array}{l}\text { Variance } \\
\text { PDI } \\
\text { (seconds) }\end{array}$ & $\mathrm{BT} / \mathrm{DT}$ & $\begin{array}{l}\text { Bottom } \\
\text { time } \\
\text { (seconds) }\end{array}$ & $\begin{array}{l}\text { Maximum } \\
\text { depth } \\
\text { per bout } \\
\text { (meters) }\end{array}$ & $\begin{array}{l}\text { Maximum } \\
\text { duration } \\
\text { per bout } \\
\text { (minutes) }\end{array}$ & $\begin{array}{l}\text { Descent } \\
\text { rate } \\
\text { (meters } \\
\text { per } \\
\text { second) }\end{array}$ & $\begin{array}{l}\text { Ascent } \\
\text { rate } \\
\text { (meters } \\
\text { per } \\
\text { second) }\end{array}$ & $\begin{array}{l}\text { Maximum } \\
\text { depth } \\
\text { record } \\
\text { (meters) }\end{array}$ & $\begin{array}{l}\text { Depth } \\
\text { 99th } \\
\text { percentile } \\
\text { (meters) }\end{array}$ & $\begin{array}{l}\text { Maximum } \\
\text { dive } \\
\text { duration } \\
\text { (seconds) }\end{array}$ \\
\hline & 11 & $51.9(4.7)$ & & & & & & & & & & $0.76(0.04)$ & $0.84(0.07)$ & 87 & & 370 \\
\hline MON & 28 & $42.0(2.7)$ & $102.9(4.9)$ & $9.2(0.8)$ & $62.3(3.0)$ & $99.4(2.8)$ & $91.5(2.8)$ & $0.61(0.01)$ & $39.3(2.2)$ & $43.9(1.8)$ & $237.8(8.5)$ & $0.75(0.02)$ & $0.82(0.04)$ & 77 & 39 & 472 \\
\hline
\end{tabular}

Table 12. Dive attributes of southern sea otters in six classes, with data pooled from individuals for analysis from Big Sur coast and Monterey Peninsula study sites, central California.

[Values presented are means (plus or minus standard error). Class: NP, female with no pup; VP, female with very small pup; SP, female with small pup; LP, female with large pup; MA, male; SA, subadult n: Number of otters in given class PDI: Mean post-dive interval. Variance PDI: Variance in post-dive interval. BD/DT: Mean ratio of bottom dive time]

\begin{tabular}{|c|c|c|c|c|c|c|c|c|c|c|c|c|c|}
\hline Class & $\mathrm{n}$ & $\begin{array}{c}\text { Dives per } \\
\text { bout } \\
\text { (number) }\end{array}$ & $\begin{array}{c}\text { Bout } \\
\text { duration } \\
\text { (minutes) }\end{array}$ & $\begin{array}{c}\text { Dive } \\
\text { depth } \\
\text { (meters) }\end{array}$ & $\begin{array}{c}\text { Dive } \\
\text { duration } \\
\text { (seconds) }\end{array}$ & $\begin{array}{c}\text { PDI } \\
\text { (seconds) }\end{array}$ & $\begin{array}{l}\text { Variance } \\
\text { PDI } \\
\text { (seconds) }\end{array}$ & BT/DT & $\begin{array}{l}\text { Bottom } \\
\text { time } \\
\text { (seconds) }\end{array}$ & $\begin{array}{l}\text { Maximum } \\
\text { depth } \\
\text { per bout } \\
\text { (meters) }\end{array}$ & $\begin{array}{l}\text { Maximum } \\
\text { duration } \\
\text { per bout } \\
\text { (minutes) }\end{array}$ & $\begin{array}{l}\text { Descent } \\
\text { rate } \\
\text { (meters per } \\
\text { seconds) }\end{array}$ & $\begin{array}{c}\text { Ascent } \\
\text { rate } \\
\text { (meters per } \\
\text { second) }\end{array}$ \\
\hline NP & 25 & $54.5(4.0)$ & $129.3(7.2)$ & $7.9(1.2)$ & $61.3(4.4)$ & $86.8(6.5)$ & $82.2(4.2)$ & $0.58(0.02)$ & $37.2(3.2)$ & $40.3(2.6)$ & $258.9(12.5)$ & $0.69(0.03)$ & $0.69(0.06)$ \\
\hline VP & 17 & $24.9(5.0)$ & $74.8(8.9)$ & $9.7(1.5)$ & $61.6(5.5)$ & $122.6(8.1)$ & $118.2(5.2)$ & $0.60(0.02)$ & $38.6(4.0)$ & $26.7(3.2)$ & $178.9(15.6)$ & $0.79(0.04)$ & $1.01(0.07)$ \\
\hline SP & 15 & $43.2(5.1)$ & $114.4(9.2)$ & $10.0(1.5)$ & $67.7(5.6)$ & $99.1(8.3)$ & $93.9(5.3)$ & $0.61(0.02)$ & $43.5(4.1)$ & $29.8(3.3)$ & $177.4(16.0)$ & $0.80(0.04)$ & $0.94(0.07)$ \\
\hline LP & 15 & $61.1(5.1)$ & $144.2(9.2)$ & $9.1(1.5)$ & $65.9(5.6)$ & $76.2(8.3)$ & $72.4(5.3)$ & $0.61(0.02)$ & $42.2(4.1)$ & $39.4(3.3)$ & $199.9(16.0)$ & $0.73(0.04)$ & $0.81(0.07)$ \\
\hline MA & 8 & $38.0(7.6)$ & $115.9(13.7)$ & $11.2(2.3)$ & $71.0(8.4)$ & $114.4(12.4)$ & $97.1(7.9)$ & $0.58(0.03)$ & $44.5(6.1)$ & $72.4(4.9)$ & $277.0(23.9)$ & $0.81(0.07)$ & $0.86(0.11)$ \\
\hline SA & 5 & $60.0(10.5)$ & $132.1(18.8)$ & $7.2(3.1)$ & $59.5(11.5)$ & 83.1(17.0) & $85.3(10.8)$ & $0.58(0.04)$ & $34.9(8.3)$ & $52.9(6.7)$ & $273.8(32.7)$ & $0.70(0.09)$ & $0.67(0.15)$ \\
\hline
\end{tabular}


Table 13. Dive attributes of southern sea otters in six classes determined for Big Sur coast and Monterey Peninsula study sites, central California.

[Values presented are means (plus or minus standard deviation ) Study site: BSR, Big Sur coast; MON, Monterey Peninsula. Class: NP, female with no pup; VP, female with very small pup; SP, female with small pup; LP. female with large pup; MA, male; SA, subadult. n: Number of otters in given class PDI: Mean post-dive interval. Variance PDI: Variance in post-dive interval. BD/DT: Mean ratio of bottom time to dive time]

\begin{tabular}{|c|c|c|c|c|c|c|c|c|c|c|c|c|c|c|}
\hline $\begin{array}{l}\text { Study } \\
\text { site }\end{array}$ & Class & $\mathrm{n}$ & $\begin{array}{l}\text { Dives per } \\
\text { bout } \\
\text { (number) }\end{array}$ & $\begin{array}{c}\text { Bout } \\
\text { duration } \\
\text { (minutes) }\end{array}$ & $\begin{array}{l}\text { Dive depth } \\
\text { (meters) }\end{array}$ & $\begin{array}{c}\text { Dive } \\
\text { duration } \\
\text { (seconds) }\end{array}$ & $\begin{array}{c}\text { PDI } \\
\text { (seconds) }\end{array}$ & $\begin{array}{c}\text { Variance PDI } \\
\text { (seconds) }\end{array}$ & BT/DT & $\begin{array}{l}\text { Bottom time } \\
\text { (seconds) }\end{array}$ & $\begin{array}{l}\text { Maximum } \\
\text { depth } \\
\text { (meters) }\end{array}$ & $\begin{array}{l}\text { Maximum } \\
\text { duration } \\
\text { (minutes) }\end{array}$ & $\begin{array}{l}\text { Descent rate } \\
\text { (meters per } \\
\text { second) }\end{array}$ & $\begin{array}{c}\text { Ascent } \\
\text { rate } \\
\text { (meters per } \\
\text { second) }\end{array}$ \\
\hline BSR & NP & 8 & $65.1(6.6)$ & $155.6(11.9)$ & $8.7(2.0)$ & $66.6(7.3)$ & $77.6(10.8)$ & $78.4(6.9)$ & $0.57(0.02)$ & $40.1(5.3)$ & $36.8(4.3)$ & $240.3(20.7)$ & $0.72(0.06)$ & $0.75(0.10)$ \\
\hline BSR & VP & 5 & $25.2(8.34)$ & $81.2(15.0)$ & $9.8(2.5)$ & $64.9(9.2)$ & $121.2(13.6)$ & $121.1(8.7)$ & $0.59(0.03)$ & $40.4(6.7)$ & $27.4(5.4)$ & $180.4(26.2)$ & $0.82(0.07)$ & $1.04(0.12)$ \\
\hline BSR & SP & 5 & $49.5(8.4)$ & $131.0(15.0)$ & $8.8(2.5)$ & $67.6(9.2)$ & $92.2(13.6)$ & $94.4(8.7)$ & $0.60(0.03)$ & $42.7(6.7)$ & $30.4(5.4)$ & $187.2(26.2)$ & $0.78(0.07)$ & $0.90(0.12)$ \\
\hline BSR & LP & 5 & $67.7(8.4)$ & $159.4(15.0)$ & $8.2(2.5)$ & $64.3(9.2)$ & $71.6(13.6)$ & $72.2(8.7)$ & $0.59(0.03)$ & $39.7(6.7)$ & $38.6(5.4)$ & $189.2(26.2)$ & $0.75(0.07$ & $0.80(0.12)$ \\
\hline BSR & MA & 2 & $44.9(13.2)$ & $143.7(23.7)$ & $13.0(3.9)$ & 79.2(14.6) & $121.9(21.5)$ & $96.3(13.7)$ & $0.59(0.05)$ & $50.2(10.5)$ & $80.5(8.5)$ & $270.0(41.4)$ & $0.85(0.11)$ & $0.92(0.19)$ \\
\hline BSR & SA & 1 & $59.3(18.7)$ & $133.3(33.6)$ & $6.4(5.5)$ & $57.5(20.6)$ & $83.3(30.4)$ & $86.4(19.4)$ & $0.56(0.07)$ & $32.9(14.9)$ & $46.0(12.1)$ & $238.0(58.5)$ & $0.66(0.16)$ & $0.64(0.27)$ \\
\hline MON & NP & 17 & $44.0(4.5)$ & $103.0(8.1)$ & 7.1(1.3) & $56.1(5.0)$ & $96.1(7.4)$ & $86.0(4.5)$ & $0.59(0.02)$ & $34.3(3.6)$ & $43.9(2.9)$ & $277.6(14.2)$ & $0.66(0.04)$ & $0.64(0.07)$ \\
\hline MON & VP & 12 & $24.6(5.4)$ & $68.4(9.7)$ & $9.7(1.6)$ & $58.2(6.0)$ & $123.9(8.8)$ & $115.2(5.6)$ & $0.61(0.02)$ & $36.7(4.3)$ & $26.0(3.5)$ & $177.3(16.9)$ & $0.76(0.05)$ & $0.97(0.08)$ \\
\hline MON & SP & 10 & $37.0(5.9)$ & $97.8(10.6)$ & $11.2(1.7)$ & $67.7(6.5)$ & $105.9(9.6)$ & $93.4(6.1)$ & $0.63(0.02)$ & $44.3(4.7)$ & $29.2(3.8)$ & $167.6(18.5)$ & $0.82(0.05)$ & $0.99(0.09)$ \\
\hline MON & LP & 10 & $54.6(5.9)$ & $129.0(10.6)$ & $9.9(1.7)$ & $67.5(6.5)$ & $80.8(9.6)$ & $72.6(6.1)$ & $0.64(0.02)$ & $44.8(4.7)$ & $40.2(3.8)$ & $210.6(18.5)$ & $0.72(0.05)$ & $0.81(0.09)$ \\
\hline MON & MA & 8 & $31.2(7.6)$ & $88.0(13.7)$ & $9.5(2.3)$ & $62.8(8.4)$ & $106.9(12.4)$ & $97.9(7.91)$ & $0.57(0.03)$ & $38.9(6.1)$ & $64.3(4.9)$ & $284.0(23.9)$ & $0.78(0.07)$ & $0.80(0.11)$ \\
\hline MON & SA & 4 & $60.6(9.4)$ & $131.0(16.8)$ & $8.0(2.8)$ & $61.5(10.3)$ & $83.0(15.2)$ & $84.1(9.7)$ & $0.59(0.03)$ & $36.9(7.4)$ & $59.8(6.0)$ & $309.5(29.2)$ & $0.74(0.08)$ & $0.70(0.13)$ \\
\hline
\end{tabular}


Table 14. Comparison of mean percentages of time spent in three activity states (foraging, active-other, and inactive) for males, females, and sub-adult females at Big Sur coast and Monterey Peninsula study sites, central California.

[BSR, Big Sur coast study site; MON, Monterey Peninsula study site; F, females; M, males; SA, subadult females; $\mathrm{SD} \pm$, plus or minus standard deviation; n, number of individuals; “.”, value not calculated because of sample size]

\begin{tabular}{|c|c|c|c|c|c|c|c|c|c|c|c|c|}
\hline \multirow[t]{2}{*}{ Behavior } & \multicolumn{2}{|c|}{$\begin{array}{c}\text { BSR M } \\
(n=2)\end{array}$} & \multicolumn{2}{|c|}{$\begin{array}{c}\text { BSR F } \\
(n=8)\end{array}$} & \multicolumn{2}{|c|}{$\begin{array}{c}\begin{array}{c}\text { BSR SA } \\
(n=1)\end{array}\end{array}$} & \multicolumn{2}{|c|}{$\begin{array}{c}\text { MON M } \\
(n=6)\end{array}$} & \multicolumn{2}{|c|}{$\begin{array}{c}\text { MON F } \\
(n=12)\end{array}$} & \multicolumn{2}{|c|}{$\begin{array}{c}\text { MON SA } \\
(n=4)\end{array}$} \\
\hline & Mean & SD \pm & Mean & SD士 & Mean & $S D \pm$ & Mean & SD \pm & Mean & SD \pm & Mean & $S D \pm$ \\
\hline Foraging & 50.83 & 0.75 & 44.14 & 5.12 & 44.70 & & 40.47 & 4.36 & 41.76 & 3.98 & 47.54 & 4.29 \\
\hline Active-other & 5.57 & 0.11 & 5.71 & 2.90 & 6.44 & & 9.75 & 2.29 & 8.54 & 2.84 & 8.46 & 2.41 \\
\hline Inactive & 45.39 & 0.69 & 50.13 & 4.30 & 48.86 & & 49.78 & 5.97 & 43.99 & 4.42 & 44.00 & 2.54 \\
\hline
\end{tabular}


Table 15. Summary of mean percentages of time spent in three activity states (foraging, active-other, and inactive) for female sea otters, grouped by Big Sur coast and Monterey Peninsula study sites, age class, and reproductive state, in central California.

[Values in BOLD ITALICS indicate a significant difference between sites. Study site, age class, and reproductive state: BSR, Big Sur coast study site; MON, Monterey Peninsula study site; A, adult; SA, sub-adult; NP, no pup; VP, very small pup; SP, small pup, LP, large pub. n, number of otters within category; $\mathbf{S D} \pm$, plus or minus standard deviation; “.”, value not calculated because of sample size]

\begin{tabular}{cccccccc}
\hline $\begin{array}{c}\text { Study site, age } \\
\text { class and } \\
\text { reproductive } \\
\text { state }\end{array}$ & $\mathbf{n}$ & \multicolumn{2}{c}{ Foraging } & \multicolumn{2}{c}{ Active-other } & \multicolumn{2}{c}{ Inactive/Resting } \\
\hline BSR, A, NP & 8 & Mean & SD \pm & Mean & SD \pm & Mean & SD \pm \\
BSR, A, VP & 6 & 26.58 & 6.45 & 7.20 & 4.84 & 66.21 & 4.02 \\
BSR, A, SP & 4 & 39.95 & 8.65 & 8.42 & 6.40 & 51.63 & 2.27 \\
BSR, A, LP & 4 & 48.14 & 7.72 & 7.69 & 5.06 & 44.16 & 2.73 \\
& & & & & & & \\
BSR, SA, NP & 1 & 44.95 &. & 6.47 &. & 48.58 &. \\
BSR, SA, VP & 1 & 13.15 &. & 4.50 &. & 82.35 &. \\
BSR, SA, SP & 0 &. &. &. &. &. &. \\
BSR, SA, LP & 0 &. &. &. &. &. &. \\
& & & & & & & \\
MON, A, NP & 18 & 39.06 & 3.85 & 9.09 & 2.65 & 51.85 & 4.04 \\
MON, A, VP & 14 & 23.92 & 7.14 & 7.89 & 3.50 & 68.18 & 8.82 \\
MON, A, SP & 11 & 39.34 & 3.78 & 7.78 & 3.41 & 52.89 & 3.47 \\
MON, A, LP & 11 & 50.12 & 4.24 & 8.92 & 3.94 & 40.95 & 5.75 \\
& & & & & & & \\
MON, SA, NP & 4 & 47.89 & 4.39 & 8.49 &. & 43.62 &. \\
MON, SA, VP & 1 & 27.38 & 2.44 & 6.32 &. & 66.30 &. \\
MON, SA, SP & 1 & 45.46 & 2.46 & 6.58 &. & 47.96 &. \\
MON, SA, LP & 0 &. &. &. &. &. &. \\
\hline
\end{tabular}

Table 16. Percentage of time and standard deviation for foraging, active-other, and inactive (resting) behaviors for all female sea otters in the study, grouped by reproductive status.

[Reproductive status: NP, no pup; SP, small pup; LP, large pup; VP, very small pup. Symbol: \pm , plus or minus standard deviation]

\begin{tabular}{cccc}
\hline Reproductive status & Foraging & Active-other & Inactive \\
\hline NP & $41.07 \pm 5.04$ & $7.96 \pm 2.82$ & $50.34 \pm 4.61$ \\
VP & $24.32 \pm 6.87$ & $7.48 \pm 3.63$ & $68.20 \pm 7.74$ \\
SP & $39.87 \pm 5.01$ & $7.86 \pm 3.89$ & $52.26 \pm 3.17$ \\
LP & $48.14 \pm 7.27$ & $8.43 \pm 3.55$ & $43.43 \pm 5.08$ \\
\hline
\end{tabular}




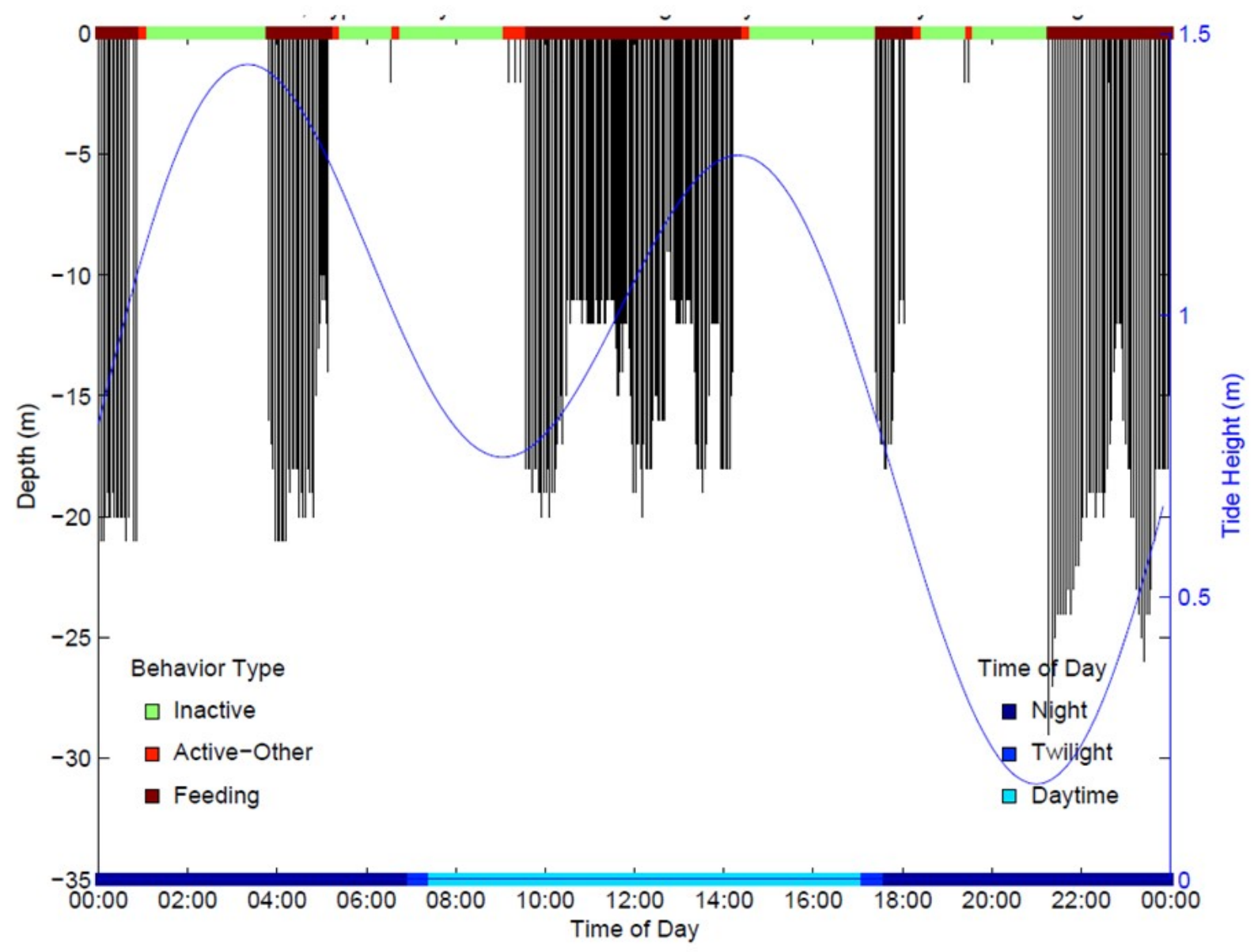

Figure 20. Time-depth recorder trace showing dive behavior over a 24-hour period for a typical sea otter. Lefthand vertical axis shows depth (in meters [m]) of the otter at each point in time (black lines), and horizontal axis shows time (in hours), with dark blue shading representing nighttime hours and light blue shading representing daytime hours. Tidal height (in $\mathrm{m}$ ) is indicated by the curved blue line (right-hand vertical axis). Activity state of the otter at each point in time is shown as a color-coded strip along the top horizontal axis. Foraging bouts (the five blocks of foraging activity, representing contiguous periods of feeding dives) are distributed throughout the 24-hour period, both day and night. 


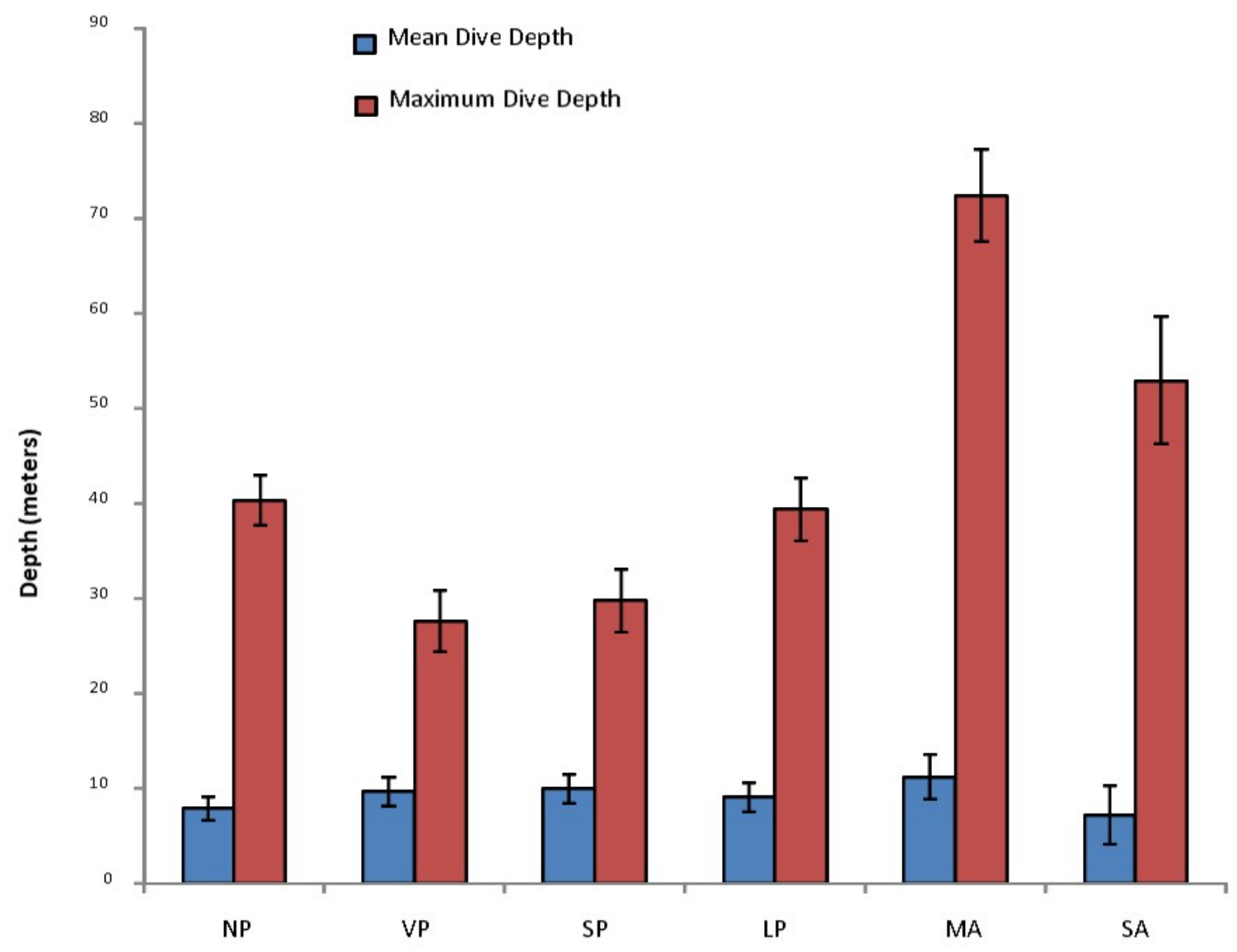

Figure 21. Graph showing mean and maximum dive depths for southern sea otters in six classes-NP, female with no pup; VP, female with very small pup; SP, female with small pup; LP. female with large pup; MA, male; SA, subadult. Data are pooled from Monterey and Big Sur locations, central California. Error bars display plus or minus standard error. 


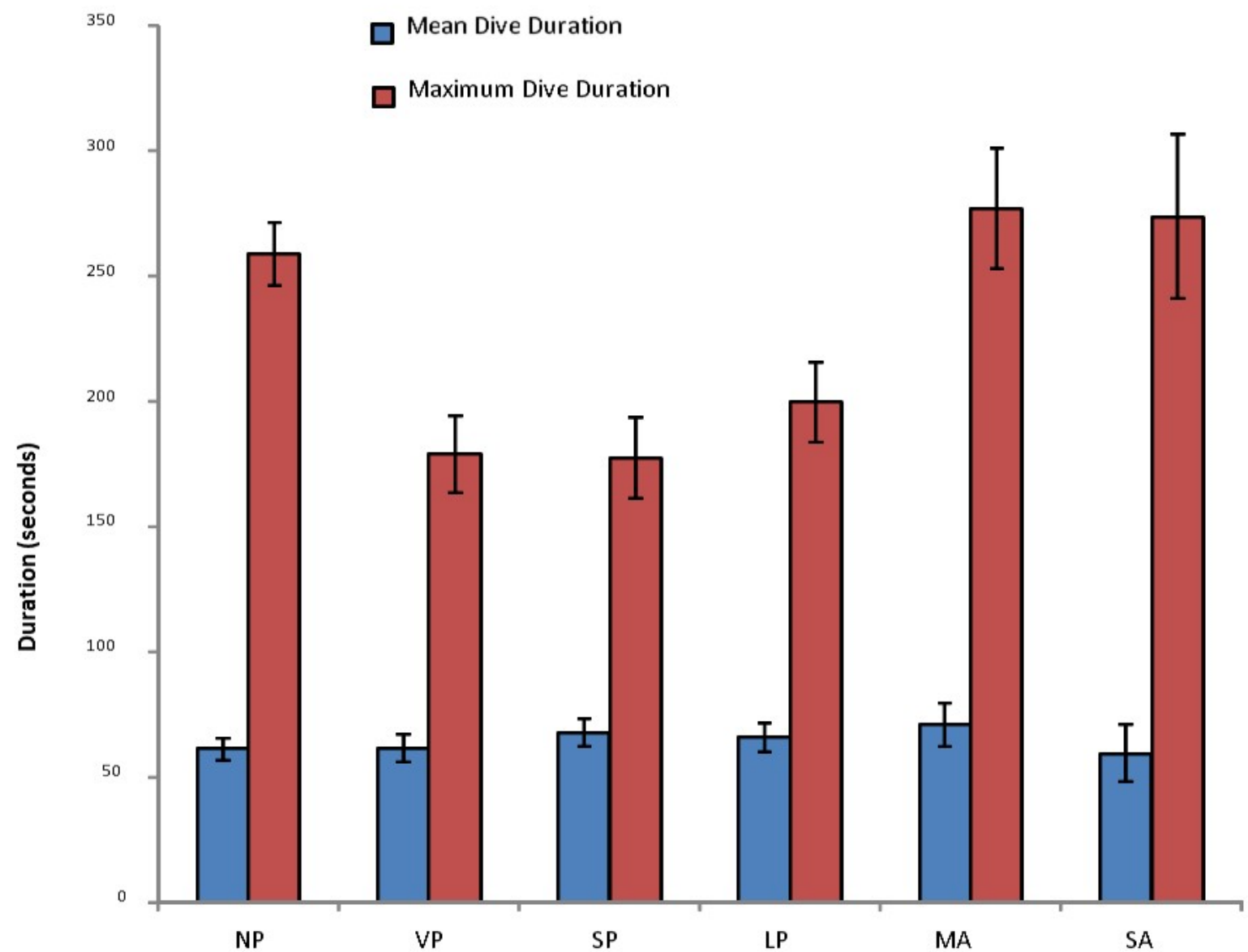

Figure 22. Graphs showing mean and maximum dive durations for southern sea otters in six classes-NP, female with no pup; VP, female with very small pup; SP, female with small pup; LP. female with large pup; MA, male; SA, subadult. Data are pooled from both Monterey and Big Sur locations, central California. Error bars display plus or minus standard error. 

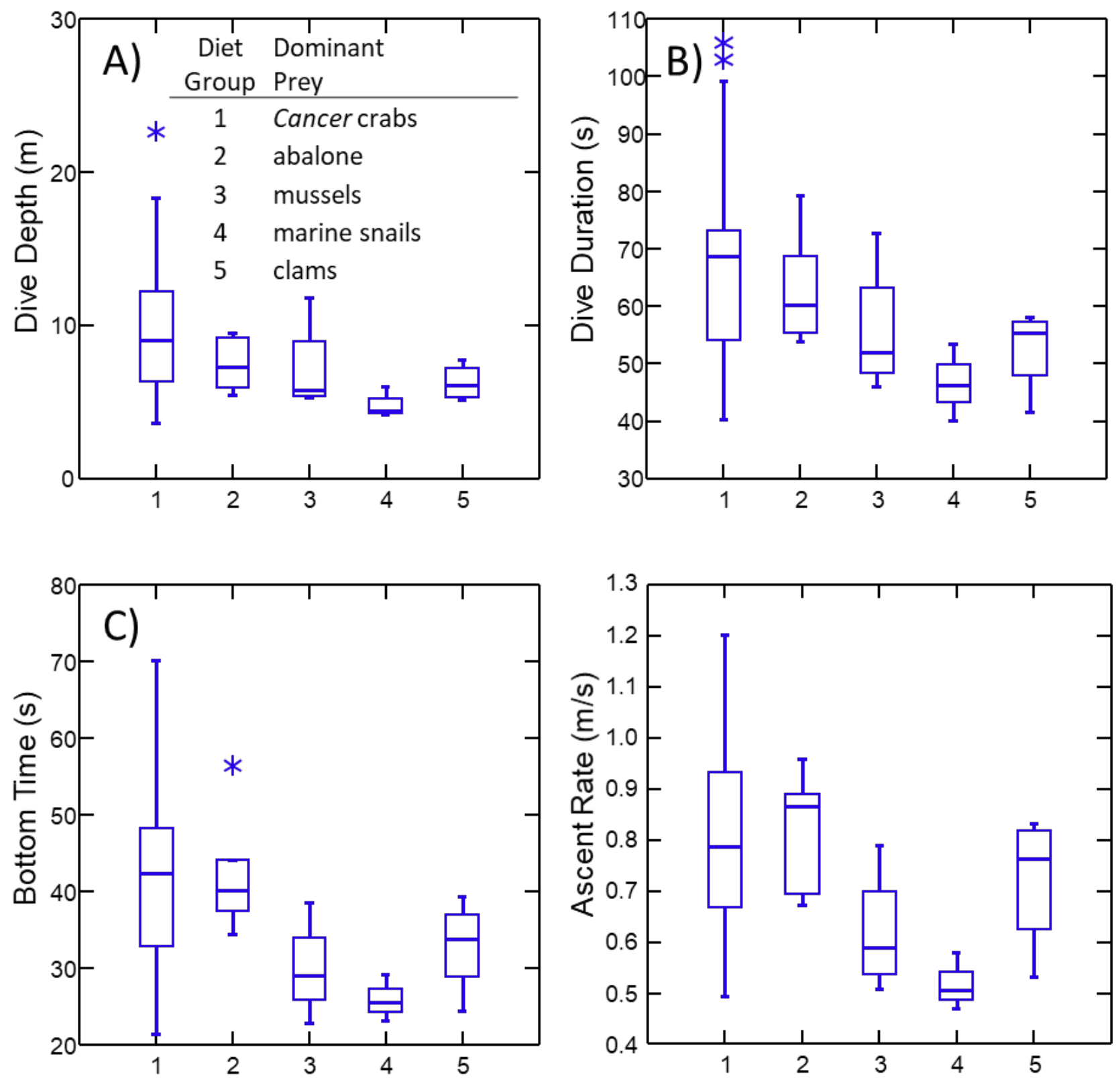

Diet Specialist Type

Diet Specialist Type

Figure 23. Box plots showing distributions of four southern sea otter dive attributes that varied as a function of individual diet specialization (diet groups 1-5) - (A) mean dive depth, (B) mean dive duration, (C) mean dive bottom time, and (D) mean ascent rate. Data are pooled from Monterey and Big Sur locations, central California pooled. Boxes encompass the interquartile range (IQR), whiskers encompass the full distribution excluding outliers (from the lower quartile minus 1.5X IQR, to the upper quartile plus 1.5X IQR). 
A)

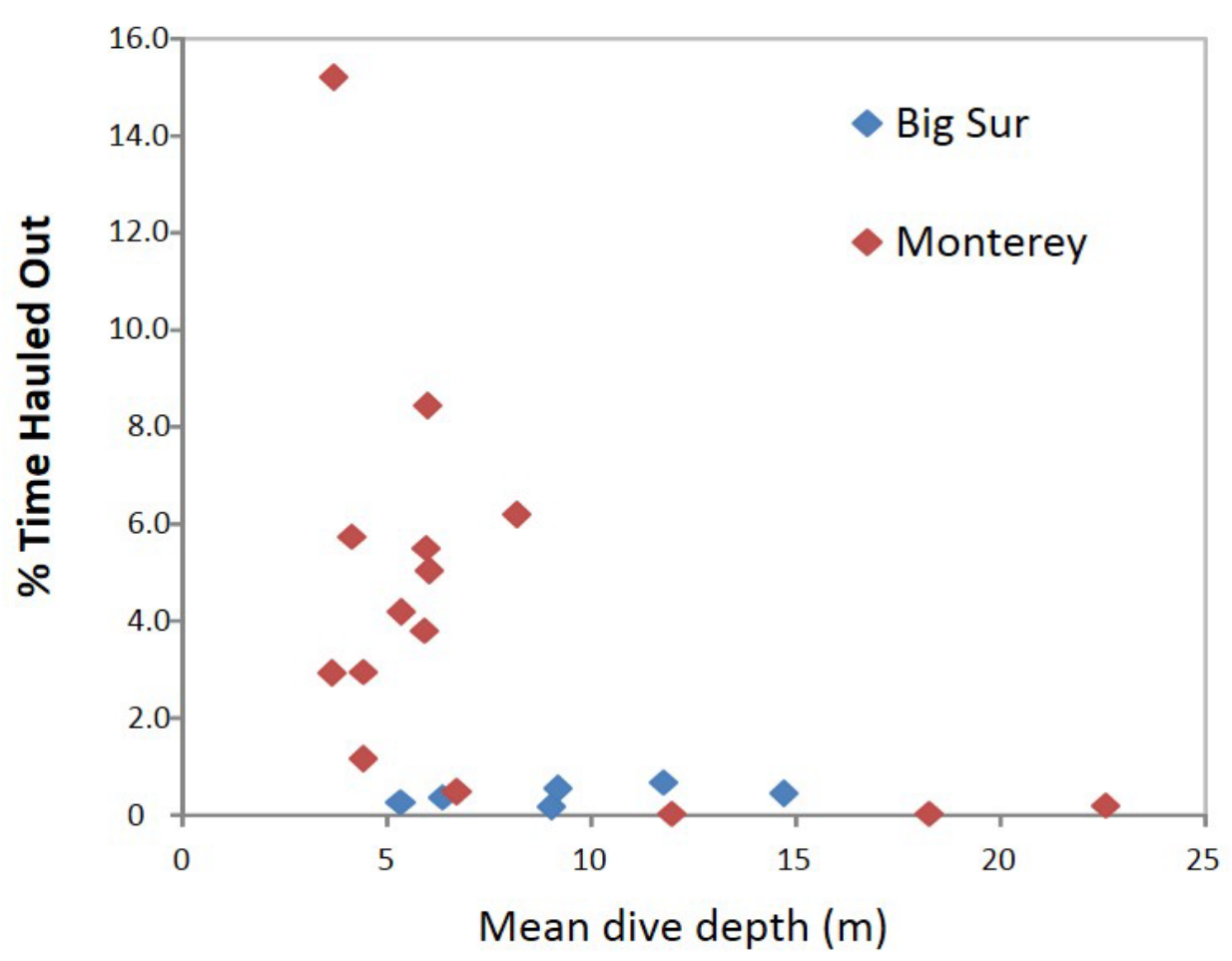

B)

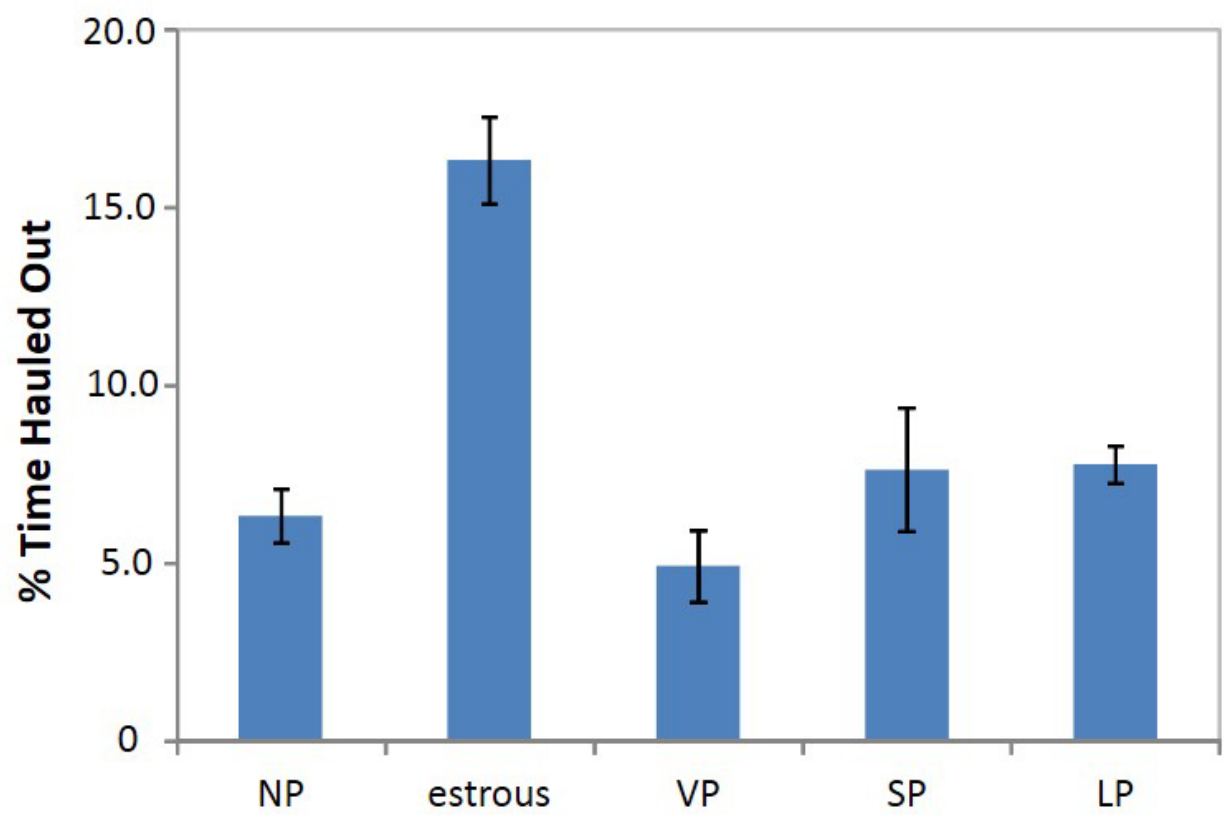

Figure 24. Graphic representations of variation in the percentage of time female southern sea otters spent hauled out from Monterey Peninsula study site, central California. A, Scatterplot showing variation in haul-out frequency as a function of mean dive depth (in meters [m]). B, Graph showing mean haul-out frequency for females in different reproductive states-NP, female with no pup; estrous; VP, female with very small pup; SP, female with small pup; LP. female with large pup (in Monterey Peninsula study site because Big Sur coast study site females rarely haul out). Error bars display plus or minus standard error. 


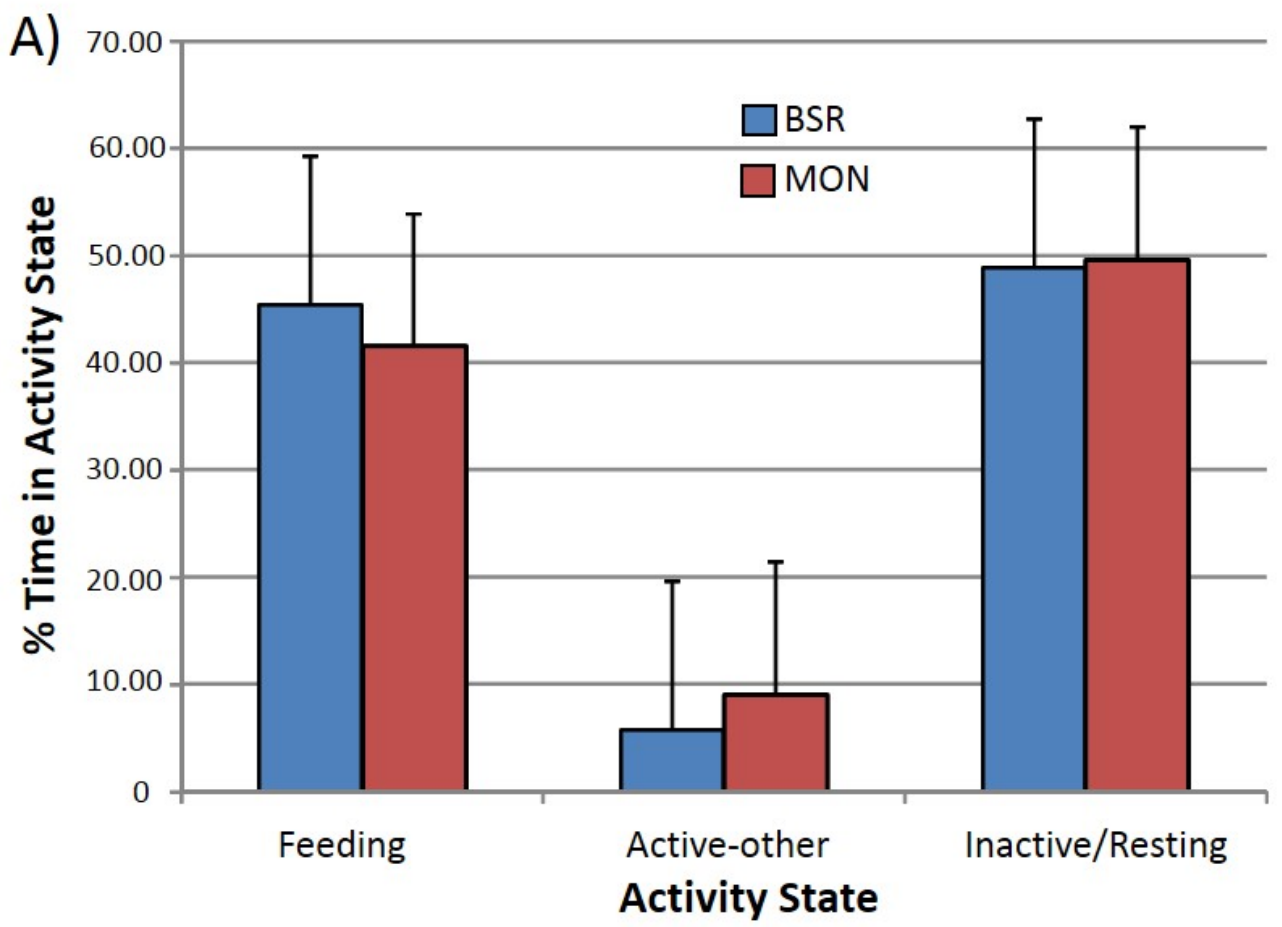

B)

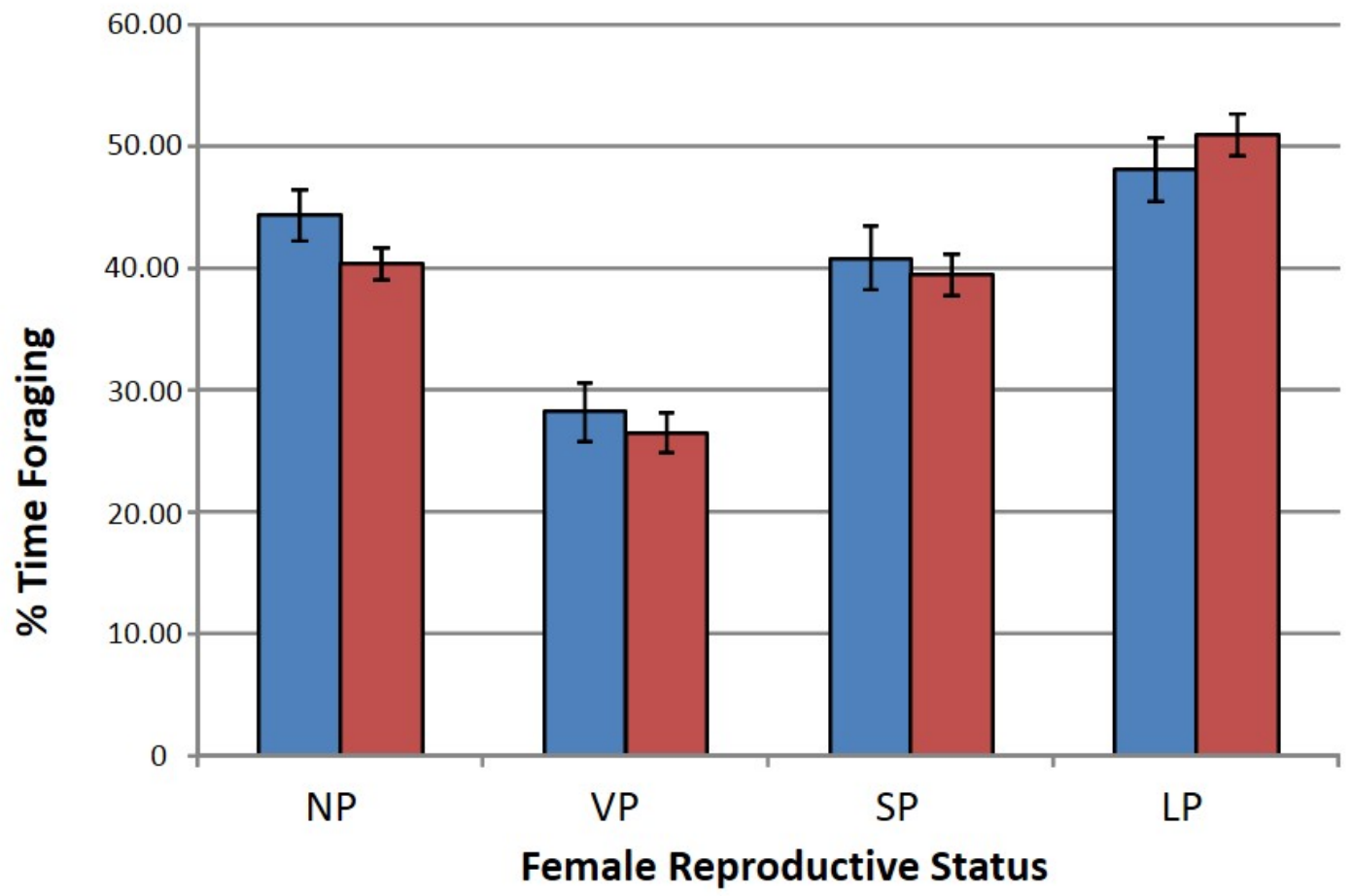

Figure 25. Graphs showing time-activity budgets, as estimated from time-depth recorder data, for female southern sea otters, central California. A, Percentage of time spent in feeding, active-other, and inactive behaviors for sea otters from Big Sur coast (BSR) and Monterey Peninsula (MON) study sites. B, Percentage of time spent feeding for otters from BSR and MON study sites in four different reproductive states-NP, no pup; VP, very small pup; SP, small pup; LP, Large pup. Error bars display plus or minus standard error. 


\title{
Chapter 6. Foraging Ecology and Tool Use
}

\author{
M. Tim Tinker ${ }^{1,2}$, Jessica Fujii ${ }^{3}$, Seth Newsome ${ }^{4}$, Gena Bentall ${ }^{1,2}$, Michelle Staedler ${ }^{2}$, and Joseph A. Tomoleoni ${ }^{1}$
}

\section{Introduction}

Foraging ecology of sea otters has been well-studied (for example, Calkins, 1978; Estes and others, 1981; Kvitek and others, 1993; Doroff and Degange, 1994; Ralls and others, 1995; Watt and others, 2000; Estes and others, 2003; Laidre and Jameson, 2006; Tinker and others, 2007; Newsome and others, 2009), and information on sea otter diets and foraging behavior has been used to infer the status of populations with respect to prey resource abundance (for example, Garshelis and others, 1986; Dean and others, 2002; Monson and Bowen, 2015) and to quantify sea otter impacts on prey populations (for example, Hines and Pearse, 1982; Ostfeld, 1982; Kvitek and Oliver, 1988; Estes and Duggins, 1995; Watson and Estes, 2001; Hughes and others, 2013). Studies of sea otter foraging ecology benefit from unique properties of sea otter behavior; specifically, sea otters dive to the sea bottom to capture their prey but then return to the surface to handle and consume it while lying on their backs, usually within sight of shore, making it possible for a trained observer with a high-powered telescope to directly record all aspects of diet composition and feeding activity from tagged individuals (see chapter 1, section, "Methods"). The resulting datasets on individual diet and foraging behavior are unique among marine mammals; represent one of the most comprehensive data sources on foraging ecology for any wild carnivore; and have allowed scientists to investigate how diet and predator-prey interactions contribute to individual health, fitness, population growth and even disease exposure (Johnson and others, 2009).

In the current study, we recorded and analyzed foraging behavior and diet of tagged sea otters at Big Sur and Monterey to achieve four main objectives:

1. Determine whether sea otter diet composition was roughly similar at the two sites, or if there were differences in prey-use that could potentially contribute to variation in health or disease exposure risk;

2. Measure the rate of energy intake of feeding sea otters at each site to assess how the sites compared with respect to foraging success (and, by inference, with respect to resource abundance);

3. Contrast foraging success of sea otters at Big Sur and Monterey with that of sea otters at other sites throughout California and around the North Pacific, including Washington, British Columbia, Alaska, and Russia; and

4. Measure and compare the degree of diet diversity (at the population level) and diet specialization (at the individual level) between Big Sur and Monterey.

These latter metrics have been determined to provide insights into per-capita food abundance, with population-level diets becoming more diverse and individual-level diets becoming more specialized when prey resource competition is high and per-capita food abundance is low (Tinker and others, 2008, 2012).

\footnotetext{
${ }^{1}$ U.S. Geological Survey.

${ }^{2}$ University of California, Santa Cruz.

${ }^{3}$ Monterey Bay Aquarium.

${ }^{4}$ University of New Mexico, Albuquerque.
} 
We augmented observational data with stable isotope analysis of vibrissae sampled from study animals, which provided further insight into diet composition and diversity at the population and individual levels (Newsome and others, 2009). Briefly, analysis of ratios of stable isotopes of carbon $\left(\delta^{13} \mathrm{C}\right)$ and nitrogen $\left(\delta^{15} \mathrm{~N}\right)$ stored in inert tissues such as bone or vibrissae can be used to evaluate diet of predators, assuming that stable isotope ratios of potential prey species are also known and are sufficiently distinct (Bearhop and others, 2004). The distribution of stable isotope ratio values of individual predators on the Cartesian axes of $\delta^{13} \mathrm{C}$ compared to $\delta^{15} \mathrm{~N}$ provides a characterization of dietary niche of the predator population, the so-called "isotopic niche space" (Newsome and others, 2012). Because vibrissae provide a longitudinal record of individual diets over about a 1-year period, it is possible to evaluate temporal variation in diet at the individual level (within-individual variation) and compare this to between-individual variation, thereby providing information about the degree of individual-level specialization (Newsome and others, 2009, 2012).

Tool use is another aspect of sea otter foraging behavior that often has been noted but has not been well studied. The first well-described record of sea otter tool use was made in California by Fisher (1939), with subsequent reports by Hall and Schaller (1964), Kenyon (1969), Houk and Giebel (1974), and Riedman and Estes (1990). Foraging sea otters will acquire a rock, empty shell, or other hard object from the ocean floor and use it to crack open their prey as either a hammer or an anvil. This behavior is an unusually conspicuous and well-developed example of tool use for a non-primate mammal, and is thought to facilitate handling of well-armored invertebrate prey (Fisher, 1939; Riedman and Estes, 1990; Shumaker and others, 2011). We reasoned that an examination of tool use variation across populations, and the ecological drivers that maintain these behaviors, potentially would provide useful insights into the role of prey resource abundance in shaping behavior.

\section{Methods}

We measured sea otter diet and foraging behavior following standardized field methods used in previous observational studies, as described in chapter 1 of this report and documented in various publications (for example, Tinker and others, 2008, 2012). To obtain quantitative measures of the occurrence of various prey species in each individual diet, field counts of prey capture frequency and prey shell diameter were converted to estimates of consumed biomass and caloric content using speciesspecific power functions for converting prey diameter to wet edible biomass and kilocalories per gram (Oftedal and others, 2007). These data were then analyzed using a Monte-Carlo procedure to estimate the diet composition of each individual (in terms of the proportion of consumed biomass contributed by each prey taxa) and rate of energy gain, or kilocalories consumed per minute of feeding (Tinker and others, 2008). The Monte-Carlo procedure explicitly incorporates sampling uncertainty and adjusts for many recognized biases associated with direct observations of sea otter foraging (Tinker and others, 2012). A detailed description of the algorithm is presented elsewhere (Tinker and others, 2012).

To account for inconsistencies in taxonomic resolution of prey capture observations, all prey items were subsequently classified into 24 distinct functional groups of taxonomically and (or) morphologically similar species (table 17), hereinafter referred to as "prey types". We limited all further analyses to those tagged sea otters for which a minimum of 10 foraging bouts and 300 feeding dives were recorded, resulting in a sample of 101 individuals $(n=36$ individuals from Big Sur and $n=65$ individuals from Monterey).

To compare diet composition between sites, we graphically compared the relative contribution of each prey type to the population-level diet (the "average" diet of sea otters at each site). We then used multivariate analysis of variance (MANOVA) to contrast the relative prevalence of key prey types in male and female diets at both sites, with sex and study site treated as fixed effects and individual otters treated as experimental units. For this analysis we limited analysis to those prey types comprising over 
5-percent of diets overall. In cases where main effects were found to be significant (using Wilks's lambda, $\lambda_{U}$ ), we conducted post-hoc, pairwise comparisons of individual prey variables across treatment levels.

We analyzed the mean rate of energy gain for foraging otters at Big Sur and Monterey using analysis of variance (ANOVA), with sex and study site treated as fixed effects and individual as a random effect. We also evaluated how the rate of energy gain at Big Sur and Monterey compared to other sea otter populations for which similar analyses have been conducted (U.S. Geological Survey, unpub. data, 2000-15). For this analysis, we classified each sea otter population in one of three categories (based on their history, current abundance, annual growth rates, and other relevant published data for each site): (1) recently established and rapidly growing populations, where food resource abundance is presumably not limiting to population growth; (2) long-established, stable or slowly increasing populations where resource abundance is thought to be limiting further growth; or (3) populations that have decreased for reasons unrelated to food abundance (for example, disease or predation) and where per-capita food resources seem to be effectively unlimited. We graphically compared the mean rate of energy gain across sites to assess which of these three categories Big Sur and Monterey populations were most similar to with respect to rate of energy gain.

We measured the dietary diversity $\left(H_{i}\right)$ of each individual otter, $i$, using the Shannon Weiner index:

$$
H_{i}=-\sum_{j}^{J} p_{i j} \log \left(p_{i j}\right)
$$

where $p_{i j}$ is the proportion of the diet of individual $i$ that is made up of prey type $j$. We used ANOVA to compare $H_{i}$ between study sites. The arithmetic mean value of $H_{i}$ at each site was used to estimate the "within-individual component" of variation in dietary niche, or WIC. Diet diversity at the population level, or the total niche width (TNW), was calculated for each site as:

$$
T N W=-\sum_{j}^{J} q_{j} \log \left(q_{j}\right),
$$

where $q_{j}$ is the proportion of the population-level that is made up of prey type $j$. To assess the degree to which individual otters had specialized diets at each site, we calculated the WIC/TNW ratio at each site, values of which will be significantly less than 1 when there is individual diet specialization (Bolnick and others, 2002). We also calculated the degree to which individual diets overlapped with the populationlevel average diet, a metric referred to as the index of proportional similarity $(P S)$ :

$$
P S=\frac{1}{N} \sum_{i}^{N}\left(1-0.5 \sum_{j}^{J}\left|p_{i j}-q_{j}\right|\right),
$$

where $N$ is the total number of individuals in the sample and $p_{i j}$ and $q_{j}$ are as described earlier in this paragraph. As with WIC/TNW, values of $P S$ significantly less than 1 indicate individual dietary specialization within the population (Bolnick and others, 2002). To account for the differing sample sizes at Monterey compared to Big Sur, we used bootstrap resampling to estimate WIC/TNW and PS for each site, with mean values, standard errors, and 95-percent confidence limits calculated from 10,000 randomly drawn (with replacement) samples of 36 individuals for each site. 
Assuming that significant levels of individual diet specialization were detected at each site, we used hierarchical cluster analysis to classify otters into groups of animals with similar diet specializations, following previously-published methods (Tinker, 2004; Tinker and others, 2007, 2008). For this analysis, we treated individual sea otters from both sites as experimental units, and key prey types (those prey types comprising more than 5 percent of diets overall) as variables. Euclidean distances were used as diet similarity measures, and clusters were identified using the Lance-Williams "flex beta" clustering algorithm with beta $=-.25$ (Lance and Williams, 1967; Scheibler and Schneider, 1985). We selected the optimal number of clusters based on profile plots of root mean square standard deviation (RMSSTD) and the pseudo-F value (the optimal number of clusters is expected to produce a local minima of the RMSSTD and a maximal value of the pseudo-F value). We then used discriminant analysis (DA) to evaluate effectiveness of the clustering classification; specifically, we used Wilks's lambda $\left(\lambda_{U}\right)$ to test for differences among diet groups with respect to the discriminant functions computed from the underlying prey frequency variables, and we assessed the percentage of individuals that could be correctly assigned to diet groups based on the discriminant classification functions (the raw classification matrix and the jackknife resampled classification matrix are both presented). We plotted a bar graph of the mean frequency of each prey type in the diets of individual otters belonging to each of the identified groups to characterize each of these groupings (or "specialist types") based on the dominant prey type or types. We also examined whether there were significant differences between the two study sites in terms of the proportion of otters belonging to each diet group, constructing a contingency table and using Pearson chi square $\left(\chi^{2}\right)$ and Goodman-Kruskal's lambda $\left(\lambda_{G-K)}\right.$ to test for independence between study site and diet group.

\section{Stable-Isotope Analysis}

Vibrissae from sea otters at Monterey and Big Sur were collected at time of capture (as described in chapter 1) and sectioned to produce 20 evenly-spaced sub-samples per animal that indicated prey consumption at 20 periods over a 1-year period (Tyrell and others, 2013). To characterize the potential isotopic niche space represented by invertebrate prey species, invertebrates were sampled in 2004, 2006, and 2008 at two study sites - San Simeon/Cambria and Monterey Bay (Oftedal and others, 2007). We analyzed 21 species of invertebrates that comprise $>90$-percent of prey consumed by sea otters in the respective study sites (Estes and others, 2003; Tinker and others, 2008, 2012). Based on functional similarities, the 21 species were condensed into eight general prey types for the central California mainland coast (table 17). Isotopic data for invertebrates collected from San Simeon/Cambria and Monterey Bay were similar for $\delta^{13} \mathrm{C}$ and $\delta^{15} \mathrm{~N}$, and, thus, were combined. Purple sea urchins (Strongylocentrotus purpuratus) are more abundant in kelp forest communities on the central California mainland coast (Oftedal and others, 2007) and contribute more to sea otter diets than red sea urchins $(S$. franciscanus), but the two species were combined in table 17.

Prey specimens were rinsed of sediment and (or) detritus, weighed, and measured using digital calipers. Inedible portions of prey (for example, the spines and tests of sea urchins, carapace of large crabs and lobsters, snail and abalone shells) were removed prior to lyophilization. The dried edible portion was homogenized by grinding to a coarse powder in a Wiley mill. For reasons discussed elsewhere (Newsome and others, 2010), we did not lipid-extract any of the prey samples. About $0.5 \mathrm{mg}$ of the homogenized tissue sample was sealed into tin boats for isotopic analysis. Carbon $\left(\delta^{13} \mathrm{C}\right)$ and nitrogen $\left(\delta^{15} \mathrm{~N}\right)$ isotope values were determined using a Carlo Erba elemental analyzer (NC 2500; Carlo Erba, Milan, Italy) interfaced with a Thermo-Finnigan Delta Plus XL mass spectrometer at the Carnegie Institution of Washington (Washington, D.C.). Isotopic results are expressed as $\delta$ values, $\delta^{13} \mathrm{C}$ or $\delta^{15} \mathrm{~N}=$ $1000 *$ [(Rsample $\left.\left.-\mathrm{R}_{\text {standard }} / \mathrm{R}_{\text {standard }}\right)-1\right]$, where $\mathrm{R}_{\text {sample }}$ and $\mathrm{R}_{\text {standard }}$ are the $13 \mathrm{C} / 12 \mathrm{C}$ or $15 \mathrm{~N} / 14 \mathrm{~N}$ ratios of 
the sample and standard, respectively. The standards are Vienna-Pee Dee Belemnite limestone (V-PDB) for carbon and atmospheric N2 for nitrogen. The units are expressed as parts per thousand, or per mil (\%). The within-run standard deviation of an acetanilide standard was less than or equal to $(\leq) 0.2 \%$ for both $\delta^{13} \mathrm{C}$ and $\delta^{15} \mathrm{~N}$ values. We also measured the carbon-to-nitrogen $([\mathrm{C}] /[\mathrm{N}])$ ratios of each sample; mean (plus or minus standard deviation) ratios for each prey type are presented in table 18 .

For $\delta^{13} \mathrm{C}$ and $\delta^{15} \mathrm{~N}$ analysis, vibrissae were rinsed once with a 2- to- 1 ratio chloroform-methanol solution to remove surface contaminants. Cleaned vibrissae were then sub-sampled into approximately 0.5-mg segments using nail clippers. Dried vibrissae segments (about $0.5 \mathrm{mg}$ ) were sealed into tin capsules and $\delta^{13} \mathrm{C}$ and $\delta^{15} \mathrm{~N}$ values were determined using the mass spectrometer system. As a control for the quality of keratin, we measured the $[\mathrm{C}] /[\mathrm{N}]$ ratios of each sample. Atomic $[\mathrm{C}] /[\mathrm{N}]$ ratios of all keratin samples were 3.3-3.5 and 3.2-3.4, respectively, well within the range that characterized unaltered protein (Ambrose, 1990). To correct measured sea otter vibrissae isotope values for trophic discrimination ( $\Delta$ diet-keratin), we used trophic discrimination factors (TDFs) of $2.5 \%$ and $3.0 \%$ for $\delta^{13} \mathrm{C}$ and $\delta^{15} \mathrm{~N}$, respectively (Newsome and others, 2010). Consumer TDFs are known to vary depending on the quality of prey consumed, growth rate, physiological condition, and (or) excretion pathways (Vanderklift and Ponsard, 2003; Caut and others, 2009). Our previous work on sea otters at San Nicolas Island, California showed that TDFs, although variable, were not biased by diet type, and the mean values used were appropriate for most animals (Newsome and others, 2010).

We compared isotopic niche diversity of sea otters at Big Sur and Monterey by graphical comparison of the dispersion of individual $\delta^{13} \mathrm{C}$ and $\delta^{15} \mathrm{~N}$ values from each population. A convex hull polygon that encompassed all individuals was computed for each study site, and the area covered by each convex hull was calculated as a measure of population-level niche diversity. A second measure of total niche width in isotopic space $\left(\mathrm{TNW}_{I}\right)$ was calculated as the summed variance of $\delta^{13} \mathrm{C}$ and $\delta^{15} \mathrm{~N}$

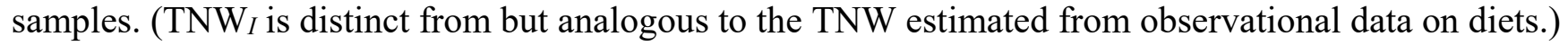
We used variance component analysis (using "Restricted Maximum Likelihood" or REML methods) to estimate the proportion of total variation due to between-individual differences $\left(\mathrm{BIC}_{I}\right)$ and withinindividual variation $\left(\mathrm{WIC}_{I}\right)$. The degree of individual niche-space specialization was then calculated as the $\mathrm{WIC}_{I} / \mathrm{TNW}_{I}$ ratio, with lower values indicating more extreme individual specialization. A Bayesian stable isotope mixing model "MixSIR" (Moore and Semmens, 2008) was used to estimate proportional contributions of eight major prey categories to sea otter diets at Monterey and Big Sur (table 17). We then used the Bayesian posterior distributions of estimated prey contributions to compute two diet space metrics: (1) the index $\varepsilon I$, which represents population-level diet specialization (values of $\varepsilon I$ can vary between $0=$ ultra-generalist and $1=$ ultra-specialist); and (2) the index $S_{I}$, which represents individuallevel diet specialization or individual-population diet similarity (values of $S_{I}$ can vary between $0=u l t r a-$ specialist and $1=$ ultra-generalist). $S_{I}$ is distinct from but analogous to the $P S$ index calculated from observational diet data. A detailed description of the analytical methods used to calculate diet space metrics is presented elsewhere (Newsome and others, 2012).

\section{Tool-Use Analysis}

To examine tool use frequency variation between Monterey and Big Sur populations and among individuals, we calculated the mean percentage of dives with observed tool use per forage bout at the population and individual level and compared with single factor ANOVA. We also developed a set of generalized linear mixed effects models (GLMMs) with binomial distribution and logit link functions, and used variance components analysis (calculated using Restricted Maximum Likelihood method) to examine the relative contributions of various factors to the likelihood that a tool would be used on a given dive. Factors evaluated for inclusion in the model included study site (Monterey Peninsula 
compared to Big Sur coast), age class (adult or sub-adult), sex (male or female), foraging habitat (kelp forest, rocky intertidal, rocky subtidal open water, soft-sediment subtidal), type of prey captured, and the diet specialization of an individual (see table 19). Forage bouts were nested within individuals and treated as a random effect. For these analyses, prey types identified in table 18 were further grouped into nine categories, with all soft-bodied prey items grouped together, as we did not expect sea otters to require tools for such prey. Dives where abalone were captured were excluded from these analyses because of the difficulty in identifying the use of tools associated with this prey item (Houk and Geibel, 1974). Interactions between prey type and diet specialist type were included to explore the hypothesis that individuals would use tools differently for some prey items depending on their overall diet specialization. Wald's Test was used to identify significant terms with non-significant terms dropped in subsequent models. The best supported model was selected using corrected Akaike information criterion (AICc; Burnham and Anderson, 2002). We further explored the interaction between prey type and diet specialization by comparing the probability of tool use by prey type for each diet specialization. Using Tukey's Honestly Significantly Different (HSD) analysis, we made pairwise comparisons for each diet type and prey type. All data manipulation and statistical analyses were completed using R.2.13.2 (R Development Core Team, 2010).

\section{Results}

Diets of sea otters at both study sites were highly diverse, and generally included a similar suite of benthic invertebrate prey types (fig. 26). However, there were significant differences in diets between study sites $\left(\lambda_{U}=0.820, \mathrm{~F}_{8,91}=2.49, \mathrm{P}=0.0172\right)$ and between males and females $\left(\lambda_{U}=0.814, \mathrm{~F}_{8,91}=2.60\right.$, $\mathrm{P}=0.0132)$. Sea otters at Big Sur consumed a higher proportion of abalone $\left(\mathrm{F}_{1,98}=7.19, \mathrm{P}=0.0086\right)$, and a lower proportion of clams $\left(\mathrm{F}_{1,98}=6.97, \mathrm{P}=0.0097\right)$ and Cancer crabs $\left(\mathrm{F}_{1,98}=5.84, \mathrm{P}=0.0175\right)$ than did sea otters at Monterey (fig. 26). Males tended to consume more clams and worms than females $\left(\mathrm{F}_{1,98}=4.73\right.$, $\mathrm{P}=0.0321$ for clams; $\mathrm{F}_{1,98}=8.62, \mathrm{P}=0.0041$ for worms $)$ and fewer kelp crabs $\left(\mathrm{F}_{1,98}=6.45, \mathrm{P}=0.0127\right)$.

In addition to site- and sex-based differences in diet composition, there also was significant variation in the rate of energy gain while feeding. Sea otters at Big Sur had a lower rate of energy gain overall than did sea otters at Monterey $\left(\mathrm{F}_{1,97}=4.20, \mathrm{P}=0.0431\right.$; fig. $\left.27 \mathrm{~A}\right)$; however, there was a significant site-by-sex interaction $\left(\mathrm{F}_{1,97}=5.74, \mathrm{P}=0.0185\right)$ such that Big Sur males had a lower rate of energy gain than did Big Sur females, whereas Monterey males had a similar or higher rate of energy gain than did Monterey females (fig. 27B). Placed in a broader context, the rate of energy gain by sea otters at both sites was near the lower end of the range of values measured across 16 sea otter populations between California and Russia, and were most similar to values measured from longestablished, stable, or slowly increasing populations where resource abundance is thought to be limiting further growth (fig. 28).

Individual-level diet diversity of sea otters at Big Sur $\left(H_{i}=1.09 \pm 0.096\right)$ was significantly lower $\left(\mathrm{F}_{1,97}=5.09, \mathrm{P}=0.0264\right)$ than that of otters at Monterey $\left(H_{i}=1.35 \pm 0.064\right)$, and female diet diversity tended to be higher than that of males $\left(\mathrm{F}_{1,97}=11.96, \mathrm{P}=0.0008\right)$ in both study sites. Although diet diversity was relatively low at the individual level, population-level dietary niche was very broad because of individual diet specialization. Individual specialization occurs when individual diets compose a small proportion of the population-level dietary niche and are thus dissimilar from the population average diet, as indicated by low values of WIC/TNW and PS indices. These indices were low at both sites, with values at $\mathrm{Big} \operatorname{Sur}(\mathrm{WIC} / \mathrm{TNW}=0.56 \pm 0.035, P S=0.44 \pm 0.033, \mathrm{CI} 95=0.38-0.51$ ) somewhat lower although not statistically different from values at Monterey (WIC/TNW $=0.62 \pm 0.026$, $P S=0.48 \pm 0.024, \mathrm{CI}_{95}=0.43-0.53$ ). Cluster analysis indicated that individual otters could be grouped into one of five different diet specialization types (fig. 29). An examination of these groups using 
discriminant analysis (DA) showed clear dietary differences $\left(\lambda_{U}=0.0014, \mathrm{~F}_{19,4,96}=17.63, \mathrm{P}<0.0001\right)$, and otters were correctly assigned to diet groups 97-percent of the time using a canonical discriminant function, with a jackknife classification accuracy of 90-percent (table 20). The relative contribution of various prey types to the diets of otters in each specialist group is shown in figure 30, and further details about each group are provided in table 19. Otters that were classified into group 1 had the most diverse diets, with Cancer crabs being the most common prey, whereas otters from the remaining four groups tended to have more specialized diets that were dominated by just one or two prey types (fig. 30, table 19). Contingency table analysis indicated significant interaction between study site and diet group $\left(\chi^{2}=15.05, \mathrm{df}=4, \mathrm{P}=0.005, \lambda_{G-K}=0.0972, \mathrm{P}=0.0305\right)$ : otters with type- 2 diets (abalone specialists) were more common at Big Sur than Monterey, whereas otters with type-5 diets (clam specialists) were more common at Monterey.

\section{Stable-Isotope Analysis}

Invertebrate species common in the diet of southern sea otters spanned multiple habitats, trophic levels, and ecologically defined functional groups, resulting in a broad range of values in both $\delta^{13} \mathrm{C}$ and $\delta^{15} \mathrm{~N}$ (table 17). Variation in mean isotope values of individuals within a prey type was relatively small in comparison to variation among prey types (fig. 31), and standard deviations for $\delta^{13} \mathrm{C}$ and $\delta^{15} \mathrm{~N}$ were $\leq 1 \%$ for most prey types (table 17). The combination of high isotopic variation among prey types but low variation within prey types created a large isotopic prey space that individual sea otters potentially occupy.

The distribution of individual $\delta^{13} \mathrm{C}$ and $\delta^{15} \mathrm{~N}$ values for sea otters at Big Sur and Monterey together occupied a large proportion of bivariate isotopic niche space (fig. 31), suggesting diverse diets at both study sites. Although population-level niche diversity was almost identical at each site based on convex hull analysis (fig. 32A) and $T I N W_{I}$ (fig. 32B), there was very little overlap in the mean isotope values of individual sea otters from Big Sur and Monterey (fig. 31), suggesting significant dietary differences between sites. Most individuals from Big Sur had lower mean vibrissae $\delta^{15} \mathrm{~N}$ values in comparison to their counterparts from Monterey, and MixSIR analyses indicated that sea otters from Big Sur likely consumed a higher proportion of abalone and mussels, whereas sea otters from Monterey likely consumed a higher proportion of clams and Cancer crabs (table 17). Sea otters at both study sites showed a high degree of individual niche specialization, although $\mathrm{WIC}_{I} / \mathrm{TINW}_{I}$ ratios were slightly lower at Big Sur than they were at Monterey (fig. 32C), indicating slightly more extreme specialization at Big Sur. The distribution of diet space indices (incorporating individual differences and parameter uncertainty from the MixSIR posterior distributions) showed distinct patterns of variation at both sites (fig. 33). Population-level niche specialization $\left(\varepsilon_{I}\right)$ was slightly greater at Big Sur than at Monterey $(0.63 \pm 0.18$ vs. $0.45 \pm 0.13$, respectively). There was a bimodal distribution of individual-population diet similarity at both sites $\left(S_{I}\right)$, although this pattern was more pronounced at Big Sur (fig. 33). This pattern indicates that some individuals at both sites had extremely specialized diets.

\section{Tool-Use Analysis}

The mean (plus or minus standard error) frequency of tool use did not vary significantly between Big Sur (11.94 \pm 4.33 -percent of dives) and Monterey (14.11 \pm 2.61 -percent; $\chi 2=116, p=0.663)$. Otters from both sites had a frequency of tool use ranging from 0 to $>90$-percent of their foraging dives (fig. 34). Much of the variation in tool use frequency was explained by individual diet specialization, with snail specialists being the only individuals who used tools in >50-percent of their dives (fig. 35). Variance component analysis indicated that the prey type captured on a given feeding dive explained 33.43-percent of the variance in tool use probability, whereas individual diet specialization explained a 
further 30.71-percent (after accounting for prey type), with the remaining variation explained by sex (3.73-percent), feeding habitat (0.16-percent), and unexplained between- and within-individual variation (31.97-percent). Study site and individual age each represented less than 0.0001-percent of the variance. The best-fit GLMM model (AICc =5091.7) included prey type and diet specialization as fixed effects, as well as their interaction, and forage bouts nested within individuals as a random effect (table 21). Using Wald's Test, all included factors contributed significantly to the model fit (table 22).

Pairwise comparisons indicated that snails and other bivalves (including scallops and cockles) were the prey groups most likely to be consumed with the use of a tool, regardless of individual diet specialization (fig. 36). Snail specialists had the highest probability of tool use when consuming their core prey (Probability $=99.8$-percent, $\mathrm{p}<0.001$ ), but also were more likely to use tools across most other prey types than were otters from all other diet specialization groups. The probability of tool use while consuming mussels, Cancer crabs, kelp crabs, and other crabs was not significantly different from the probability of tool use while consuming soft-bodied prey, irrespective of diet specialization (fig. 36).

\section{Discussion}

Diets of sea otters at Big Sur and Monterey (fig. 26, table 18) included a broad range of benthic subtidal and intertidal invertebrates, consistent with previously published data on sea otter diets in central California (Estes and others, 1981; Hines and Pearse, 1982; Ostfeld, 1982; Ralls and others, 1995; Jolly, 1997; Estes and others, 2003; Tinker and others, 2008; Tinker and others, 2012). There were two differences in diet composition between sites, one easily explained and the other more interesting. The greater prevalence of clams in the diets of Monterey sea otters is not surprising given the greater abundance of soft-sediment benthic habitat around the Monterey Peninsula and southern Monterey Bay, which supports abundant infaunal clams (Hallenbeck and others, 2012). The higher prevalence of abalone in diets of Big Sur sea otters was more surprising, as sea otters have been present at high densities at Big Sur far longer than they have at Monterey. Given the well-described depleting effects of sea otter predation on abalone (Lowry and Pearse, 1973; Hines and Pearse, 1982), it might be expected that abalone depletion would be greater at Big Sur than Monterey. The fact that sea otters continue to rely heavily on abalone at Big Sur, despite more than a one-half century of continuous sea otter presence in this area, suggests that abalone populations in central California are able to sustain this level of predation, possibly because the positive indirect effects of sea otter predation (that is, decreases in urchin competitors and increased kelp abundance) may equal or exceed the negative direct consumptive effects (Raimondi and others, 2015).

In addition to dietary differences between sites, there also were dietary differences between males and females, with males consuming more clams and worms and fewer kelp crabs. This pattern likely indicates a greater reliance on soft-sediment habitat for feeding by males. In central California, soft sediment habitats are thought to support less diverse, less energetically profitable prey communities than rocky, kelp-dominated habitats (Kvitek and Oliver, 1988). Sub-adult and non-territorial males may be driven to using these sub-optimal foraging habitats owing to intra-specific competition in kelpdominated habitats (or owing to active exclusion from those areas by territorial males), a pattern that is consistent with the lower rate of energy gain by males at the BSR study site. However, this scenario is apparently inconsistent with the fact that males at Monterey actually had higher rates of energy gain than their female counterparts (fig. 27). This inconsistency may be explained in part by differences in coastal bathymetry of the two study sites, In Monterey, males have ready access to the broad and shallow continental shelf of Monterey Bay, and the upwelling-driven productivity it supports. In contrast, Big Sur males have to travel great distances to access these same resources. Thus, Big Sur males may be more geographically "blocked in" than males at Monterey. Another reason for this 
inconsistent pattern is that the Monterey sample contained (by chance) a higher ratio of territorial males to non-territorial males, and territorial males may have access to better foraging habitat.

Overall, rate of energy gain for foraging sea otters was slightly lower at Big Sur than at Monterey (fig. 27), suggesting that food resource abundance may be more limiting for sea otters at Big Sur. However, when compared to a wide array of sea otter populations from around the North Pacific, foraging success for sea otters at Big Sur and Monterey appeared to fall within the range of populations thought to be resource-limited (fig. 28), with otters at Big Sur just slightly more impacted than otters in other regions. This pattern was entirely consistent with patterns of individual diet specialization, a phenomenon that has been found to be related to the degree of resource competition (Tinker and others, 2008, 2012). Both Big Sur and Monterey sea otters had substantial degrees of individual diet specialization; however Big Sur otters had slightly more specialized diets. Despite this difference, the same types of diet specialization occurred at both sites, in largely similar frequencies (although there were more abalone specialists at Big Sur and fewer clam specialists; fig. 30). Together, these patterns suggest that (1) Big Sur sea otters may be slightly more impacted by resource competition than Monterey sea otters, although both populations are at the lower end of the scale of resource abundance when compared to other populations; and (2) diet specialization in sea otters, a behavioral response to resource limitation, tends to occur in a consistent fashion from site to site, perhaps driven by highly conserved patterns of prey profitability and the constraints of learning on prey-handling skills (Tinker and others, 2009). It also has been suggested that frequency-dependent dynamics (rare specializations become more profitable) may also be a factor that helps maintain multiple co-occurring diet specializations within a population with approximately consistent ratios (Estes and others, 2003).

\section{Stable-Isotope Analysis}

Patterns of niche variation inferred from stable isotope analysis were consistent with and supported patterns inferred from the observational data. At the population level, both sites were characterized by similarly diverse diets, occupying a large proportion of available niche space; however, these niche space distributions differed between sites. Dietary differences inferred from isotope analysis closely matched differences inferred from observational data, with abalone and mussels more prevalent at Big Sur and clams and Cancer crabs more prevalent at Monterey (compare table 17 with fig. 26). This consistency between the two methods increases our confidence in both methods (because the datasets are entirely independent and, thus, unlikely to produce similar biases) and confirms previously published conclusions that stable isotopes provide a useful method of measuring diets in sea otters (Newsome and others, 2009, 2010). The stable-isotope analysis also resulted in estimates of individual diet specialization that were consistent with the observational data, indicating that sea otters at both sites showed substantial specialization, but this pattern was slightly more pronounced at Big Sur than at Monterey (fig. 32). The density plots of population-level dietary niche specialization ( $\varepsilon I$ ) compared to dietary similarity $\left(S_{I}\right)$ indicated that the degree of individual specialization within the population may be multimodal, with some otters showing extreme specialization and dissimilarity from the population average. These patterns have direct implications for sea otter health, as certain prey types are more likely to expose sea otters to disease-causing pathogens and environmental toxins (Johnson and others, 2009; Miller and others, 2010), and thus diet specialization can lead to greater variation in disease outcomes. 


\section{Tool-Use Analysis}

We explored ecological and behavioral factors that may influence tool-use variation among individuals. Tool use in sea otters has been repeatedly documented across populations but very little is known about individual variation. We determined that tool-use frequency was largely dependent on prevalence of difficult-to-access prey in an individual diet. Neither Big Sur nor Monterey populations used tools at a high frequency, suggesting that this behavior is not universally required for successful foraging.

The most significant predictor of tool use was the type of prey being consumed. Average tool use frequency most likely did not vary between Monterey and Big Sur because sea otter diets there were relatively similar (fig. 26). The prey class most likely to be associated with tool use was snails (fig. 35). Although marine snails are relatively easy to capture, they have heavily calcified exoskeletons without readily accessible meat. The most common snail species consumed were turban snails (Chlorostoma spp., formerly Tegula spp.). These small $(2-5-\mathrm{cm})$ snails have thick, compact shells and small openings. Turban snails have a low per-capita energy return, and yet individual sea otters that specialized on snails are just as likely to reach their daily caloric demands as those in other specialist groups (Tinker, 2004; Oftedal and others, 2007). Tool use likely is a key factor in making snails energetically profitable to sea otters.

The existence of individual diet specialization at Big Sur and Monterey provided an opportunity to explore the importance of prey type (environmental factors), feeding behaviors (learned behavioral factors), and their interaction in relation to tool use. Variation in tool-use frequency among individuals in a population is most likely attributed to individual diet and the necessity of learning how to use tools to consume core prey items. Although the rate of tool-use varied by prey type within each specialist group (fig. 35), snail specialists were more likely than other specialists to use tools on almost all other hard-shelled prey types (fig. 36).

Many individuals who specialized on prey items that were not associated with tool use (that is, mussels and urchins) still used tools on occasion. The ability for individuals to "casually" use tools differs from sponging in bottlenose dolphins (Mann and others, 2008) and larvae fishing by New Caledonian crows (Rutz and others, 2010). Unlike with these other tool-using species, tool use by sea otters may have a lower cost of learning. It is still unclear if the casual tool users are responding to a particularly difficult prey item, or if some other factor drives their occasional tool use. If there is a very low cost associated with learning how to use tools, it is likely that the behavior is not more common because the benefit also is relatively low.

Age was excluded as a contributing factor to tool-use occurrence in our models. We would expect to see individuals use tools more frequently as they became more proficient with age, as seen in chimpanzees and New Caledonian crows (Lonsdorf, 2006; Holzhaider and others, 2010). In this case, a lack of any significant relation is likely an artifact of our study samples. We would predict snail specialists to show the greatest age effect because tool use plays a large role in their foraging strategy. However, only two of our snail specialists were sub-adults at the time of capture. More foraging observations from juveniles (just post-weaning) to sub adults likely would be necessary to see an age effect, as this is the time period when individuals must quickly learn foraging strategies to survive.

As the first quantitative study of sea otter tool use, this study determined that individuals vary in their frequency of tool use in response to their encounter rates with prey that are difficult to access. As with many tool-using species, ecological factors (such as the physical characteristics of prey) play a critical role in explaining complex behaviors (Collins and McGrew, 1987; Patterson and Mann, 2011). Future studies would benefit from quantifying the cost and benefits of learning to use tools, as well as from further exploration to how individuals learn to use tools. Such studies, although challenging, would be possible by combining both observational studies and captive animal experiments. 


\section{References Cited}

Ambrose, S.H., 1990, Preparation and characterization of bone and tooth collagen for isotopic analysis: Journal of Archaelogical Science, v. 17 p. 431-451.

Bearhop, S., Adams, C.E., Waldron, S,. Fuller, R.A., and Macleod, H., 2004, Determining trophic niche width-A novel approach using stable isotope analysis: Journal of Animal Ecology, v. 73, p. 10071012.

Bolnick, D.I., Yang, L.H., Fordyce, J.A,. Davis, J.M., and Svanbäck, R., 2002, Measuring individuallevel resource specialization: Ecology, v. 83, p. 2936-2941.

Burnham, K.P., and Anderson, D.R., 2002, Model Selection and Multimodel Inference: A Practical Information-Theoretic Approach: Springer, New York, NY, 488 p.

Calkins, D.G., 1978, Feeding behavior and major prey species of the sea otter, Enhydra lutris, in Montague Strait, Prince William Sound, Alaska: Fishery Bulletin, v. 76, p. 125-131.

Caut, S., Angulo, E., and Courchamp, F., 2009, Variation in discrimination factors $(\Delta 15 \mathrm{~N}$ and $\Delta 13 \mathrm{C})-$ The effect of diet isotopic values and applications for diet reconstruction: Journal of Applied Ecology, v. 46, p. 443-453.

Collins, D.A., and McGrew, W.C., 1987, Termite fauna related to differences in tool-use between groups of chimpanzees (Pan troglodytes): Primates, v. 28, p. 457-471.

Dean, T.A., Bodkin, J.L., Fukuyama, A.K., Jewett, S.C., Monson, D.H., O'Clair, C.E., and VanBlaricom. G.R., 2002, Food limitation and the recovery of sea otters following the 'Exxon Valdez' oil spill: Marine Ecology Progress Series, v. 241, p. 255-270.

Doroff, A.M., and Degange, A.R., 1994, Sea otter, Enhydra lutris, prey composition and foraging success in the northern Kodiak Archipelago: National Marine Fisheries Service Fishery Bulletin 92, no. 4, p.704-710.

Estes, J.A., and Duggins. D.O., 1995, Sea otters and kelp forests in Alaska-Generality and variation in a community ecological paradigm: Ecological Monographs, v. 65, p. 75-100.

Estes, J.A., Jameson, R.J., and Johnson, A.M., 1981, Food selection and some foraging tactics of sea otters, in Chapman J.A., and Pursley, D., eds., Worldwide Furbearer Conference ProceedingsVolume 1, August 3-11, 1980, Frostburg, Maryland: Baltimore, University of Maryland Press, p. 606-641.

Estes, J.A., Riedman, M L., Staedler M.M., Tinker, M.T., and Lyon B.E., 2003, Individual variation in prey selection by sea otters-Patterns, causes and implications: Journal of Animal Ecology, v. 72, p. $144-155$.

Fisher, E.M., 1939, Habits of the southern sea otter: Journal of Mammology, v. 20, p. 21 - 36.

Garshelis, D.L., Garshelis, J.A., and Kimker, A.T., 1986, Sea otter time budgets and prey relationships in Alaska: Journal of Wildlife Management, v. 50, p. 637-647.

Hall, K.R.L., and Schaller, G.B., 1964, Tool-Using Behavior of the California Sea Otter: Journal of Mammalogy, v. 45, p. 287-298, https://doi.org/10.2307/1376994.

Hallenbeck, T.R., Kvitek, R.G., and Lindholm, J., 2012, Rippled scour depressions add ecologically significant heterogeneity to soft-bottom habitats on the continental shelf: Marine Ecology Progress Series, v. 468, p. 119-133.

Hines, A.H., and Pearse, J.S., 1982, Abalones, shells, and sea otters-Dynamics of prey populations in central California: Ecology, v. 63, p. 1547-1560.

Holzhaider, J.C., Hunt, G.R., and Gray, R.D., 2010, The development of pandanus tool manufacture in wild New Caledonian crows: Behaviour, v. 147, p. 553-586.

Houk, J.L., and Geibel, J.J., 1974, Observation of underwater tool use in sea otter, Enhydra lutris Linnaeus: California Fish and Game, v. 60, p. 207-208. 
Hughes, B.B., Eby, R., Van Dyke, E., Tinker, M.T., Marks, C.I., Johnson, K.S., and Wasson K., 2013, Recovery of a top predator mediates negative eutrophic effects on seagrass: Proceedings of the National Academy of Sciences of the United States of America, v. 110, p. 15313-15318.

Johnson, C.K., Tinker, M.T., Estes, J.A., Conrad, P.A., Staedler, M., Miller, M.A., Jessup, D.A., and Mazet. J.A.K., 2009, Prey choice and habitat use drive sea otter pathogen exposure in a resourcelimited coastal system: Proceedings of the National Academy of Sciences of the United States of America, v. 106, p. 2242-2247.

Jolly, J.M., 1997, Foraging ecology of the sea otter, Enhydra lutris, in a soft-sediment community. Santa Cruz, University of California, Master's dissertation.

Kenyon, K.W., 1969, The Sea Otter in the Eastern Pacific Ocean: North American Fauna, v. 68, p. 1 352. https://doi.org/10.3996/nafa.68.0001.

Kvitek, R.G., Bowlby, C.E., and Staedler, M., 1993, Diet and foraging behavior of sea otters in southeast Alaska: Marine Mammal Science, v. 9, p. 168-181.

Kvitek, R.G., and Oliver, J.S. ,1988, Sea otter foraging habits and effects on prey populations and communities in soft-bottom environments, in Vanblaricom, G.R., and Estes, J.A., eds., The community ecology of sea otters: New York, Springer-Verlag, p. 22-47.

Laidre, K.L., and Jameson, R.J., 2006, Foraging patterns and prey selection in an increasing and expanding sea otter population: Journal of Mammalogy, v 87, p. 799-807.

Lance, G.N., and Williams, W.T., 1967, A general theory of classification sorting strategies-I, Hierarchical system: Computer Journal, v. 9, p. 373-380.

Lonsdorf, E.V., 2006, What is the role of mothers in the acquisition of termite-fishing behaviors in wild chimpanzees (Pan troglodytes schweinfurthii)?: Animal Cognition, v. 9, p. 36-46.

Lowry, L.F. and Pearse, J.S., 1973, Abalones and sea urchins in an area inhabited by sea otters: Marine Biology, v. 23, p. 213-219.

Mann, J., Sargeant, B.L., Watson-Capps, J.J., Gibson, Q.A., Heithaus, M.R., Connor, R.C., and Patterson, E., 2008, Why do dolphins carry sponges?: PloS One, v. 3, no. 12, https://doi.org/10.1371/journal.pone.0003868.

Miller, M.A., Kudela, R.M., Mekebri, A., Crane, D., Oates, S.C., Tinker, M.T., Staedler, M., Miller, W.A., Toy-Choutka S., Dominik, C. Hardin, D., Langlois, G., Murray, M., Ward, K., and Jessup, D.A., 2010, Evidence for a novel marine harmful algal bloom-Cyanotoxin (microcystin) transfer from land to sea otters: PLoS One, v. 5, p. e12576.

Monson, D.H., and Bowen., L. 2015, Evaluating the status of individuals and populations-Advantages of multiple approaches and time scales, in Larson, S.E., Bodkin, J.L., and VanBlaricom, G.R., eds., Sea otter conservation (1st ed.): London, Academic Press, p. 122-152.

Moore, J.W., and Semmens, B.X., 2008, Incorporating uncertainty and prior information into stable isotope mixing models: Ecology Letters, v. 11, p. 470-480.

Newsome, S.D., Bentall, G.B., Tinker, M.T., Oftedal, O.T., Ralls, K., Estes, J.A., and Fogel, M.L., 2010 , Variation in $\delta 13 \mathrm{C}$ and $\delta 15 \mathrm{~N}$ diet-Vibrissae trophic discrimination factors in a wild population of California sea otters: Ecological Applications, v .20, p. 1744-1752.

Newsome, S.D., Tinker, M.T., Monson, D.H., Oftedal, O.T., Ralls, K., Staedler, M.M., Fogel, M.L., and Estes, J.A., 2009, Using stable isotopes to investigate individual diet specialization in California sea otters (Enhydra lutris nereis): Ecology, v. 90, p. 961-974.

Newsome, S.D., Yeakel, J.D., Wheatley, P.V., and Tinker, M.T., 2012, Tools for quantifying isotopic niche space and dietary variation at the individual and population level: Journal of Mammalogy, v. 93, p. 329-341. 
Oftedal, O.T., Ralls, K., Tinker, M.T., and Green, A., 2007, Nutritional constraints on the southern sea otter in the Monterey Bay National Marine Sanctuary, and a comparison to sea otter populations at San Nicolas Island, California and Glacier Bay, Alaska: Joint Final Report to the Monterey Bay National Marine Sanctuary and the Marine Mammal Commission, 23 p.

Ostfeld, R.S., 1982, Foraging strategies and prey switching in the California sea otter: Oecologia, v. 53, p. $170-178$.

Patterson, E.M., and Mann, J., 2011, The ecological conditions that favor tool use and innovation in wild bottlenose dolphins (Tursiops sp.): PloS One, v. 6, no. 8, https://doi.org/10.1371/journal.pone.0022243.

R Core Team, 2010, R: A language and environment for statistical computing: R Foundation for Statistical Computing, Vienna, Austria. http://www.R-project.org/.

Raimondi, P., Jurgens, L.J., and Tinker, M.T., 2015, Evaluating potential conservation conflicts between two listed species-Sea otters and black abalone: Ecology, v. 96, p. 3102-3108.

Ralls, K., Hatfield, B.B., and Siniff, D.B., 1995, Foraging patterns of California sea otters as indicated by telemetry: Canadian Journal of Zoology, v. 73, p. 523-531.

Riedman, M.L., and Estes, J.A., 1990. The Sea Otter (Enhydra lutris): Behavior, Ecology, and Natural History: United States Fish and Wildlife Service, Biological Report, 90(14): 1-126. ISSN 08951926, Journal of Mammalogy, v. 76, p. 263-264, https://doi.org/10.2307/1382334.

Rutz, C., Bluff, L.A., Reed, N., Troscianko, J., Newton, J., Inger, R., Kacelnik, A., and Bearhop, S., 2010, The ecological significance of tool use in New Caledonian crows: Science, v. 329, p. 15231526.

Scheibler, D., and Schneider, W., 1985, Monte Carlo tests of the accuracy of cluster analysis algorithms - A comparison of hierarchical and nonhierarchical methods: Multivariate Behavioral Research, v. 20, p. 283-304.

Schumaker, R.W., Walkup, K.R. and Beck, B.B., 2011, Animal Tool Behaviour: The Use and Manufacture of Tools by Animals: The John Hopkins University Press, Baltimore.

Tinker, M.T., 2004, Sources of variation in the foraging behavior and demography of the sea otter, Enhydra lutris: Santa Cruz, University of California, Ph.D dissertation.

Tinker, M.T., Bentall, G., and Estes, J.A., 2008, Food limitation leads to behavioral diversification and dietary specialization in sea otters: Proceedings of the National Academy of Sciences of the United States of America, v. 105, p. 560-565.

Tinker, M.T., Costa, D.P., Estes, J.A., and Wieringa, N., 2007. Individual dietary specialization and dive behaviour in the California sea otter-Using archival time-depth data to detect alternative foraging strategies: Deep Sea Research II, v. 54, p. 330-342.

Tinker, M.T., Guimarães, P.R., Novak, M., Marquitti, F.M.D., Bodkin, J.L., Staedler, M., Bentall, G., and Estes, J.A., 2012, Structure and mechanism of diet specialisation-Testing models of individual variation in resource use with sea otters: Ecology Letters, v. 15, p. 475-483.

Tinker, M.T., Mangel, M., and Estes, J.A., 2009, Learning to be different-Acquired skills, social learning, frequency dependence, and environmental variation can cause behaviourally mediated foraging specializations: Evolutionary Ecology Research, v. 11, p. 841-869.

Tyrell, L.P., Newsome, S.D., Fogel, M.L., Viens, M., Bowden, R., and Murray, M.J., 2013, Vibrissae growth rates and trophic discrimination factors in captive southern sea otters (Enhydra lutris nereis): Journal of Mammalogy, v. 94, p. 331-338.

Vanderklift, M., and Ponsard, S., 2003, Sources of variation in consumer-diet $\delta 15 \mathrm{~N}$ enrichment-A meta-analysis: Oecologia, v. 136, p. 169-182.

Watson, J., and Estes, J.A., 2011, Stability, resilience, and phase shifts in rocky subtidal communities along the west coast of Vancouver Island, Canada: Ecological Monographs, v. 81, no. 2, p. 215-239. 
Watt, J., Siniff, D.B., and Estes, J.A., 2000, Inter-decadal patterns of population and dietary change in sea otters at Amchitka Island, Alaska: Oecologia (Berlin), v. 124, p. 289-298. 
Table 17. Stable Isotope characterization of sea otter prey types used in the analysis of isotopic niche space.

[For each prey category the data fields shown are the most common species, number of samples used in stable isotope samples (n), mean $\delta^{13} \mathrm{C}$ value $\left(\delta^{13} \mathrm{C}\right)$, standard deviation of $\delta^{13} \mathrm{C}$ values (SD directly to right of $\delta^{13} \mathrm{C}$ ), mean $\delta^{15} \mathrm{~N}$ value $\left(\delta^{15} \mathrm{~N}\right)$, standard deviation of $\delta^{15} \mathrm{~N}$ values (SD directly to right of $\left.\delta^{15} \mathrm{~N}\right)$, the carbon-to-nitrogen ratio $(([\mathrm{C}] /[\mathrm{N}]$, plus or minus standard deviation in parentheses), and the estimated percentage contribution of each prey type (based on Bayesian mixture model analysis; see body text) to the diets of sea otters at the Monterey Peninsula (MON) and Big Sur coast (BSR) study sites (plus or minus standard deviations)]

\begin{tabular}{|c|c|c|c|c|c|c|c|c|}
\hline Prey type & Species & $\mathrm{n}$ & $\delta^{13} \mathrm{C}$ & SD & $\delta^{15 N}$ & SD & {$[\mathrm{C}] /[\mathrm{N}]$} & $\begin{array}{c}\text { Estimated percentage } \\
\text { of diet } \\
\text { MON / BSR }\end{array}$ \\
\hline Cancer crabs & $\begin{array}{l}\text { Cancer antennarius, } C . \\
\text { magister, } C . \text { productus }\end{array}$ & 34 & -15.6 & 0.8 & 14.1 & 0.8 & $4.1(0.3)$ & $25.5(1.2) / 20.2(1.2)$ \\
\hline Abalone & $\begin{array}{l}\text { Haliotis cracherodii, } \\
\text { H. rufescens }\end{array}$ & 22 & -15.5 & 0.9 & 9.5 & 0.9 & $3.8(0.3)$ & $11.0(1.3) / 24.9(2.0)$ \\
\hline Sea urchins & $\begin{array}{l}\text { Strongylocentrotus } \\
\text { purpuratus, S. franciscanus }\end{array}$ & 16 & -17.0 & 1.1 & 9.4 & 0.4 & $4.7(0.8)$ & $10.5(0.6) / 5.5(0.4)$ \\
\hline Clams & $\begin{array}{l}\text { Tresus nuttalli, Protothaca } \\
\text { staminea, Saxidomus nuttalli, } \\
\text { Macoma nasuta }\end{array}$ & 56 & -15.5 & 1.0 & 11.4 & 0.7 & $4.1(0.5)$ & $9.7(1.1) / 1.4(0.2)$ \\
\hline $\begin{array}{l}\text { Northern } \\
\text { kelp crabs }\end{array}$ & Pugettia producta & 27 & -13.3 & 1.1 & 11.6 & 0.8 & $4.8(0.6)$ & $8.7(0.5) / 9.0(0.6)$ \\
\hline $\begin{array}{l}\text { California } \\
\text { mussels }\end{array}$ & Mytilus californianus & 18 & -17.5 & 0.9 & 9.2 & 0.5 & $4.0(0.4)$ & $8.3(0.4) / 19.9(0.8)$ \\
\hline Tegula snails & $\begin{array}{c}\text { Tegula funebralis, T. pulligo, } \\
\text { T. brunnea, T. montereyi }\end{array}$ & 24 & -14.3 & 0.9 & 10.6 & 0.7 & $4.5(0.4)$ & $7.6(0.5) / 2.5(0.2)$ \\
\hline $\begin{array}{l}\text { Fat } \\
\text { innkeeper } \\
\text { worms }\end{array}$ & Urechis caupo & 16 & -15.6 & 0.7 & 11.7 & 0.6 & $4.3(0.4)$ & $4.7(0.2) / 8.7(0.6)$ \\
\hline
\end{tabular}


Table 18. List of more than 75 prey species (or higher taxa) consumed by sea otters over the course of the study.

[Because it was often difficult to distinguish taxonomically and/or morphologically similar species from a distance, all prey were grouped into 24 functional groups (referred to as "prey types" in the body text)]

\begin{tabular}{|c|c|c|}
\hline Functional group & Prey common name & Scientific name \\
\hline \multirow[t]{2}{*}{ Urchin } & red urchin & $\begin{array}{l}\text { Strongylocentrotus (Mesocentrotus) } \\
\text { franciscanus }\end{array}$ \\
\hline & purple urchin & Strongylocentrotus purpuratus \\
\hline \multirow[t]{4}{*}{ Cancer crab } & Pacific rock crab & Cancer antennarius \\
\hline & dungeness crab & Cancer (Metacarcinus) magister \\
\hline & red rock crab & Cancer productus \\
\hline & Cancer crab, unidentified & Cancer sp. \\
\hline \multirow[t]{2}{*}{ Kelp crab } & northern kelp crab & Pugettia producta \\
\hline & graceful kelp crab & Pugettia gracilis \\
\hline \multirow[t]{2}{*}{ Sand crab } & spiny mole crab & Blepharipoda occidentalis \\
\hline & Pacific sand crab & Emerita analoga \\
\hline \multirow[t]{2}{*}{ Crab other } & decorator/masking crab & Loxorhynchus crispatus \\
\hline & unidentified crab & \\
\hline \multirow[t]{4}{*}{ Mussel } & horse mussel & Modiolus \\
\hline & California mussel & Mytilus californianus \\
\hline & bay mussel & Mytilus trossulus \\
\hline & mussel, unidentified & \\
\hline \multirow[t]{18}{*}{ Clam } & Nuttall's cockle & Clinocardium nuttallii \\
\hline & giant rock scallop & Crassadoma gigantea \\
\hline & sunset clam & Gari californica \\
\hline & Macoma clam & Macoma spp. \\
\hline & surf clam & Mactromeris spp. \\
\hline & softshell clam & Mya arenaria \\
\hline & geoduck clam & Panopea generosa \\
\hline & scallop, unidentified & Pectinidae spp. or Serripes spp. \\
\hline & rock jingle & Pododesmus macroschisma \\
\hline & littleneck clam & Prototheca (Leukoma) staminea \\
\hline & Washington clam & Saxidomus nuttalli \\
\hline & razor clam & Siliqua patula \\
\hline & jackknife clam & Tagelus californianus \\
\hline & tellin clam & Tellina spp. \\
\hline & Pismo clam & Tivela stultorum \\
\hline & gaper clam & Tresus nuttallii \\
\hline & rough paddock & Zirfaea pilsbryi \\
\hline & clam, unidentified & \\
\hline \multirow[t]{4}{*}{ Marine snail } & top snail & Calliostoma spp. \\
\hline & red turban snail & Lithopoma (Pomaulax) gibberosus \\
\hline & Nassa snail & Nassarius fossatus \\
\hline & moon snail & Polinices sp. \\
\hline
\end{tabular}




\begin{tabular}{|c|c|c|}
\hline Functional group & Prey common name & Scientific name \\
\hline & brown turban snail & Tegula (Chlorostoma) brunnea \\
\hline & Monterey turban snail & Tegula (Chlorostoma) montereyi \\
\hline & turban snail, unidentified & Turbinidae \\
\hline & snail, unidentified & \\
\hline \multirow[t]{3}{*}{ Abalone } & black abalone & Haliotis cracherodii \\
\hline & red abalone & Haliotis rufescens \\
\hline & abalone, unidentified & \\
\hline \multirow[t]{6}{*}{ Sea star } & blood star & Henricia sp. \\
\hline & brittle star & Ophiuroidea \\
\hline & bat star & Patiria miniata \\
\hline & ochre star & Pisaster ochraceus \\
\hline & sunflower star & Pycnopodia helianthoides \\
\hline & sea star, unidentified & \\
\hline \multirow[t]{5}{*}{ Worm } & pile worm & Nereis sp. \\
\hline & polychaete, unidentified & Polychaeta \\
\hline & peanut worm & Sipunculus nudus \\
\hline & fat innkeeper worm & Urechis caupo \\
\hline & worm, unidentified & \\
\hline \multirow[t]{6}{*}{ Chiton } & gumboot chiton & Cryptochiton stelleri \\
\hline & lined chiton & Tonicella sp. \\
\hline & Katy chiton & Katharina tunicata \\
\hline & chiton, unidentified & Polyplacophora sp. \\
\hline & mossy chiton & Mopalia sp. \\
\hline & Stenoplax chiton & Stenoplax fallax \\
\hline Limpet & owl limpet & Lottia gigantea \\
\hline Tunicate & stalked tunicate & Styela sp. \\
\hline Cucumber & red sea cucumber & Cucumaria sp. \\
\hline Sponge & orange puffball sponge & Tethya californiana \\
\hline \multirow[t]{3}{*}{ Small crustacean } & acorn barnacle & Balanus sp. \\
\hline & Isopod & Idotea sp. \\
\hline & gooseneck barnacle & Pollicipes polymerus \\
\hline Sand dollar & sand dollar & Dendraster excentricus \\
\hline Octopus & Octopus & Octopus sp. \\
\hline Squid & market squid & Loligo (Doryteuthis) opalescens \\
\hline Lobster & spiny lobster & Panulirus interruptus \\
\hline \multirow[t]{2}{*}{ Other kelp invert } & coralline algae & Corallina sp., Clathromorphum sp. \\
\hline & Nudibranch & Opisthobranchia \\
\hline Fish & kelp greenling & Hexagrammos decagrammus \\
\hline Fish egg mass & greenling egg mass & Hexagrammos sp. \\
\hline
\end{tabular}


Table 19. Summary of the five sea otter diet groups identified using hierarchical cluster analysis.

[All sea otters at the Big Sur coast (BSR) and Monterey Peninsula (MON) study sites were classified into one of the five groups based on their diet composition (see body text for details). For each group, the table summarizes the predominant prey type or types consumed by otters belonging to that group, their dietary diversity, and the number (n) of tagged otters from each group at each study site and overall. n/a, not applicable]

\begin{tabular}{|c|c|c|c|c|c|c|}
\hline $\begin{array}{l}\text { Diet } \\
\text { group }\end{array}$ & Dominant prey & Secondary prey & Diversity & $\mathrm{n}, \mathrm{BSR}$ & $\mathrm{n}, \mathrm{MON}$ & $\mathrm{n}$, total \\
\hline 1 & Cancer crabs & $\begin{array}{l}\text { Urchins, } \\
\text { miscellaneous }\end{array}$ & 1.49 & 16 & 34 & 50 \\
\hline 2 & Abalone & $\mathrm{n} / \mathrm{a}$ & 1.05 & 10 & 3 & 13 \\
\hline 3 & Mussels & Urchins & 1.32 & 7 & 11 & 18 \\
\hline 4 & Marine snails & Kelp crabs & 1.20 & 3 & 9 & 12 \\
\hline 5 & Clams & Cancer crabs & 1.29 & 0 & 8 & 8 \\
\hline
\end{tabular}


Table 20. Results of discriminant analysis of individual sea otter diets used to evaluate the efficacy of diet-type groupings identified using hierarchical cluster analysis.

[Top matrix shows the percentage of sea otters that were classified correctly into one of the five diet groups using the discriminant classification function computed from the relative prevalence of each of 24 prey types in the diet of each otter. The bottom matrix shows the percentage of sea otters that were classified correctly using a cross validation or 'jack-knife' classification, whereby all cases but one were used to develop a new discriminant classification function that was applied to the remaining case, and this process was repeated with each case omitted in turn. See table 19 for diet group details]

\section{Classification matrix (Cases in row categories classified into columns)}

\begin{tabular}{ccccccc}
\hline $\begin{array}{c}\text { Diet } \\
\text { group }\end{array}$ & $\mathbf{1}$ & $\mathbf{2}$ & $\mathbf{3}$ & $\mathbf{4}$ & $\mathbf{5}$ & $\begin{array}{c}\text { Percentage } \\
\text { of sea } \\
\text { otters } \\
\text { classified } \\
\text { correctly }\end{array}$ \\
\hline \hline $\mathbf{1}$ & 11 & 1 & 0 & 0 & 0 & 92 \\
\hline $\mathbf{2}$ & 0 & 48 & 1 & 0 & 1 & 96 \\
\hline $\mathbf{3}$ & 0 & 0 & 18 & 0 & 0 & 100 \\
\hline $\mathbf{4}$ & 0 & 0 & 0 & 8 & 0 & 100 \\
\hline $\mathbf{5}$ & 0 & 0 & 0 & 0 & 13 & 100 \\
\hline Total & 11 & 49 & 19 & 8 & 14 & 97 \\
\hline \hline
\end{tabular}

Jackknifed classification matrix

\begin{tabular}{ccccccc}
\hline $\begin{array}{c}\text { Diet } \\
\text { group }\end{array}$ & $\mathbf{1}$ & $\mathbf{2}$ & $\mathbf{3}$ & $\mathbf{4}$ & $\mathbf{5}$ & $\begin{array}{c}\text { Percentage } \\
\text { of sea } \\
\text { otters } \\
\text { classified } \\
\text { correctly }\end{array}$ \\
\hline \hline $\mathbf{1}$ & 11 & 1 & 0 & 0 & 0 & 92 \\
\hline $\mathbf{2}$ & 1 & 44 & 1 & 2 & 2 & 88 \\
\hline $\mathbf{3}$ & 0 & 3 & 15 & 0 & 0 & 83 \\
\hline $\mathbf{4}$ & 0 & 0 & 0 & 8 & 0 & 100 \\
\hline $\mathbf{5}$ & 0 & 0 & 0 & 0 & 13 & 100 \\
\hline Total & 12 & 48 & 16 & 10 & 15 & 90 \\
\hline \hline
\end{tabular}

Table 21. Generalized linear mixed effects models (GLMM) showing the relation between the probabilities of occurrence of tool use and various factors, including site (population ), prey type, sex, age, habitat, and diet specialization.

[Individual otters and forage bouts nested within individuals were both treated as random effects for these models. AICc, Akaike information criterion, corrected]

\begin{tabular}{lcc}
\hline \multicolumn{1}{c}{ Model } & $\begin{array}{c}\text { Degrees of } \\
\text { freedom }\end{array}$ & AlCc \\
\hline Site + Diet Specialization+ Prey Type+ Age + Sex + Habitat & 22 & 5111.8 \\
Site + Diet Specialization* Prey Type+ Age + Sex + Habitat & 46 & 5090.2 \\
Diet Specialization*Prey Type & 38 & 5091.7 \\
\hline
\end{tabular}


Table 22. Wald Test results for best-supported generalized linear mixed effects model explaining tool use probability as a function of diet specialization of the sea otter and prey type on a given dive.

\begin{tabular}{lccc}
\hline \multicolumn{1}{c}{ Factor } & Wald's Test $\mathbf{X}^{2}$ & Degrees of freedom & $\boldsymbol{p}$ value \\
\hline Diet & 61.0 & 4 & $1.8 \times 10^{-12}$ \\
Prey & 244.3 & 8 & 0.0 \\
Diet*Prey & 59.9 & 32 & 0.002 \\
\hline
\end{tabular}

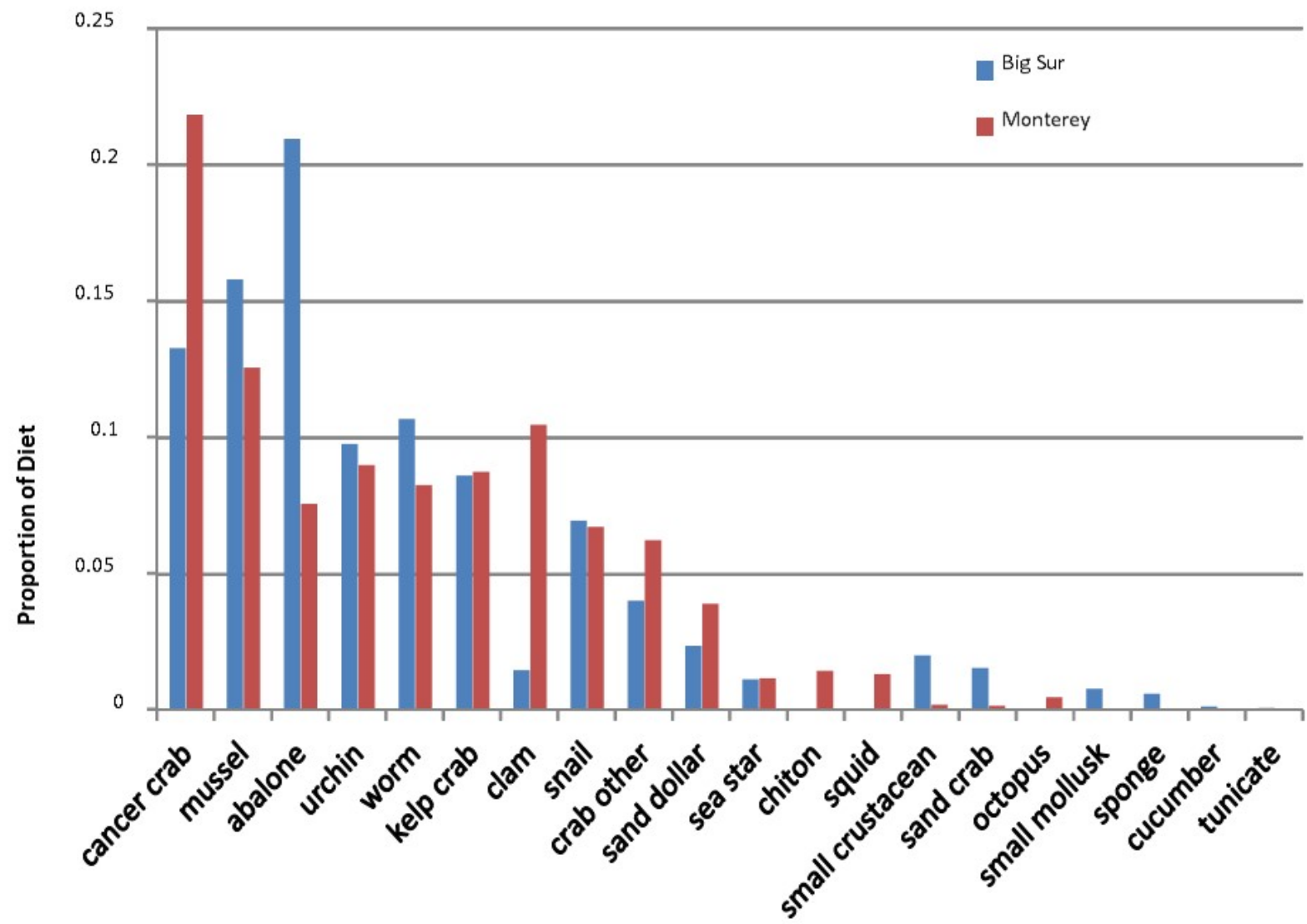

Figure 26. Histogram showing contribution of 20 prey types to the diets of sea otters at Big Sur coast and Monterey Peninsula study sites, central California. See table 18 for species included in each prey-type category. 
A)

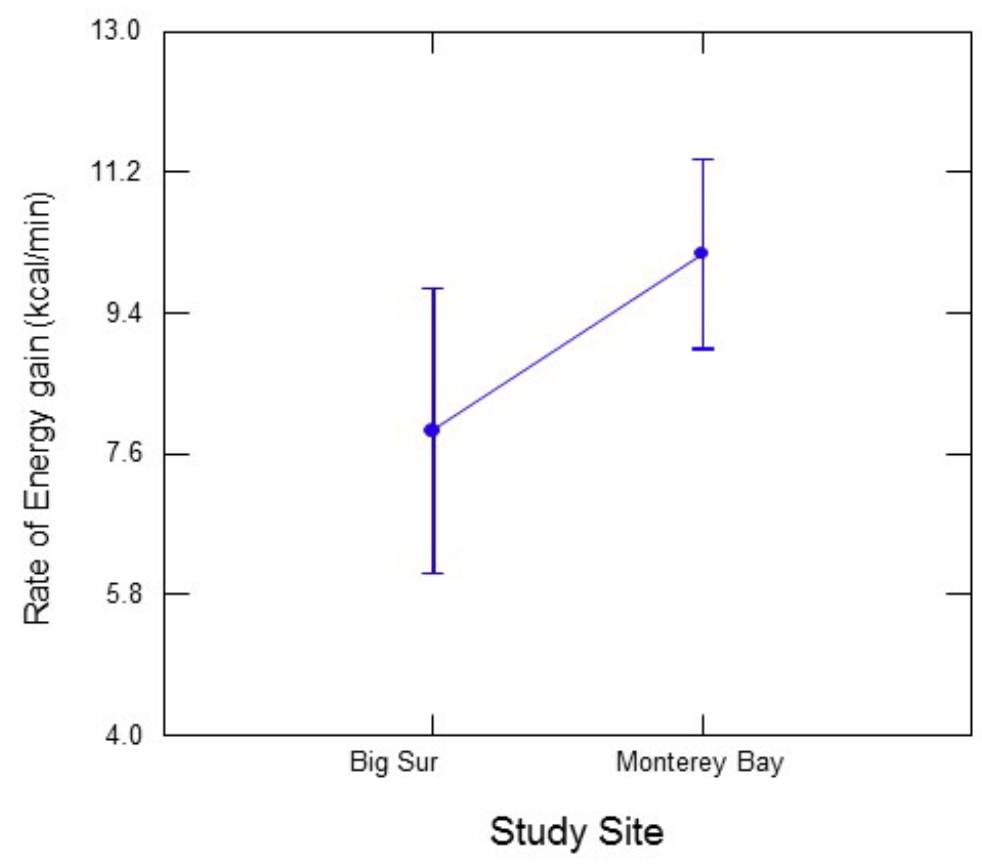

B)

Big Sur

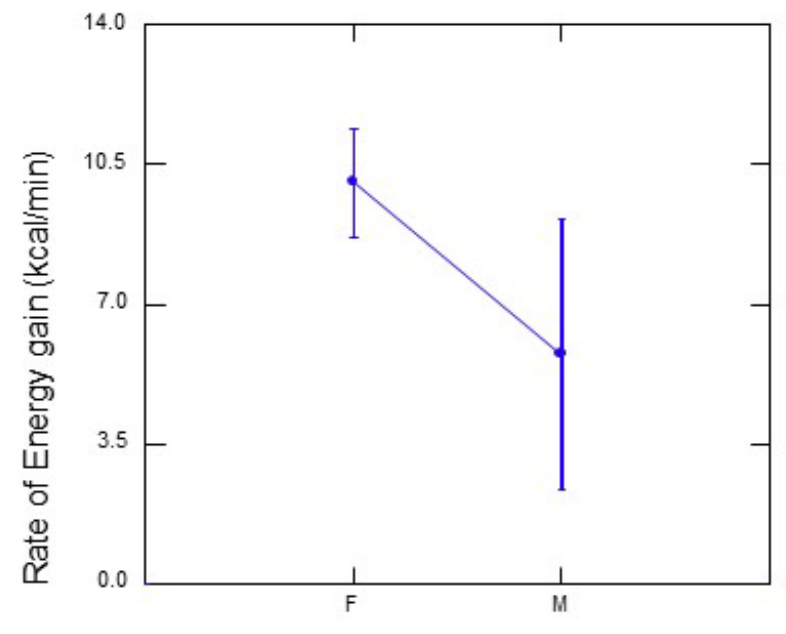

Sex
Monterey Bay

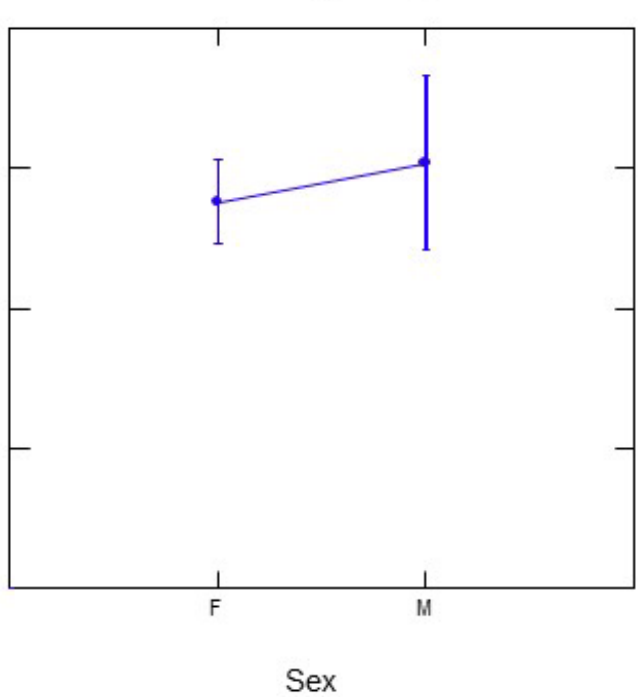

Figure 27. Graphs showing comparisons of the estimated rate of energy gain of sea otters while feeding (in kilocalories per minute [kcal/min]), corresponding to results of two-way analysis of variance with interaction effect (see body text for details). Error bars represent 95-percent confidence intervals around estimates. A, Mean rates for all animals at Big Sur coast compared to Monterey Peninsula study sites. B, Interaction between sex and study site; males have a lower rate than females at Big Sur, but a higher rate than females at Monterey. 


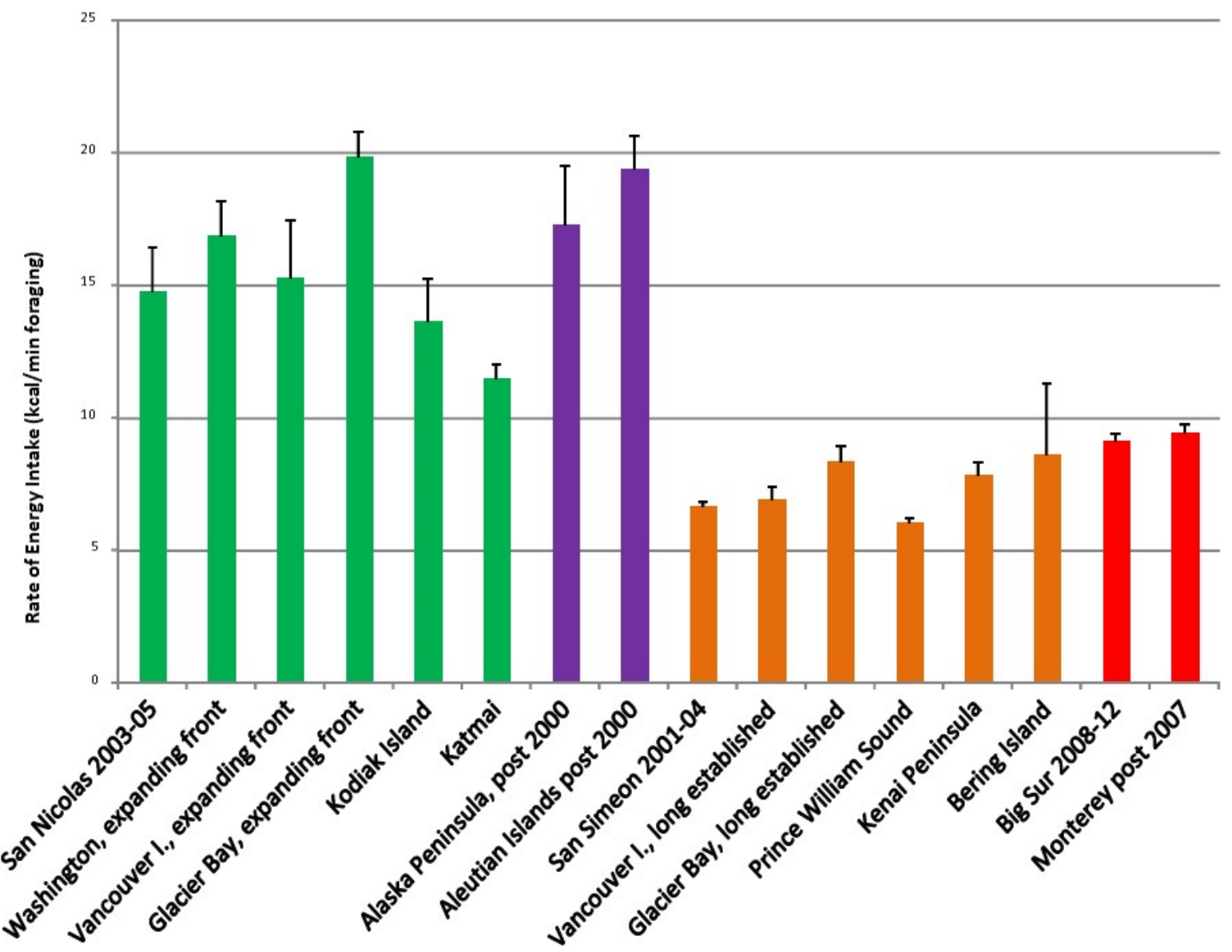

Figure 28. Graph showing comparison of the mean rate of energy gain for sea otters in 16 sea otter populations from across their range in the North Pacific, based on field studies conducted using standardized methodologies. Populations are color-coded into four categories-Green (bars 1-6 from left side of graph), recently established and rapidly growing populations, where food resource abundance is presumably not limiting to population growth; orange (bars 9-14 from left side of graph), long-established, stable or slowly increasing populations where resource abundance is thought to be limiting further growth; purple (bars 7-8 from left side of graph), populations that have decreased for reasons un-related to food abundance (for example, disease or predation) and where percapita food resources are abundant; red (rightmost two bars), the Big Sur coast and Monterey Peninsula study sites. Error bars represent 95-percent confidence intervals around estimates. 
A)

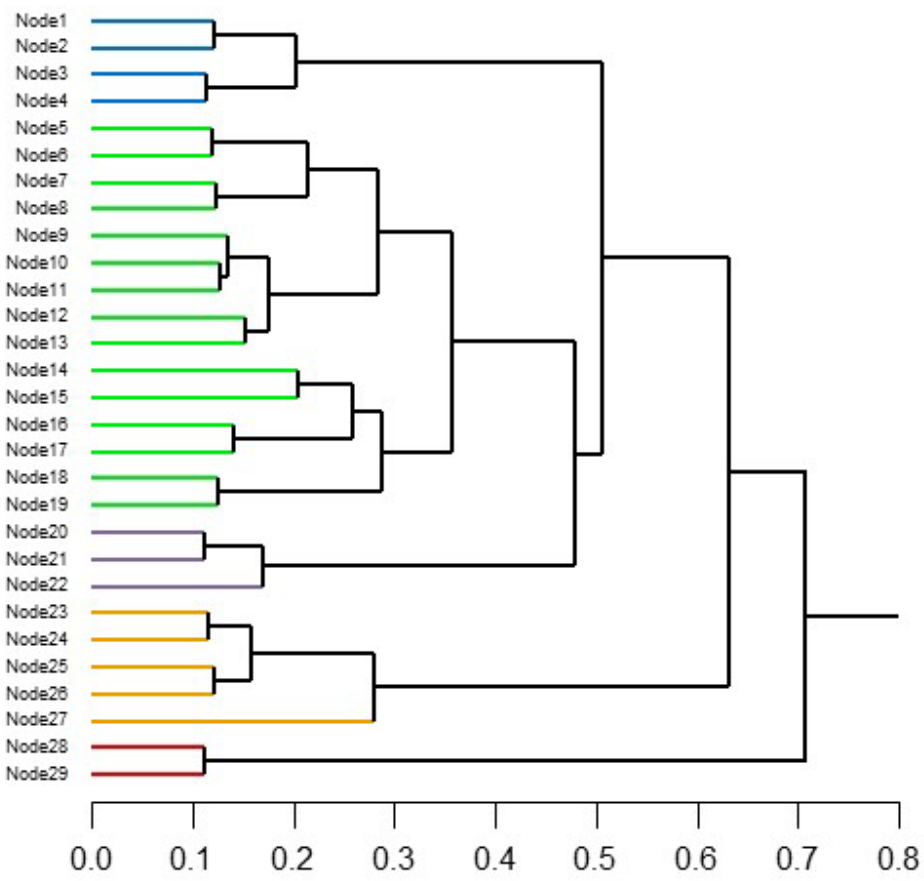

B)

Inter-cluster distances
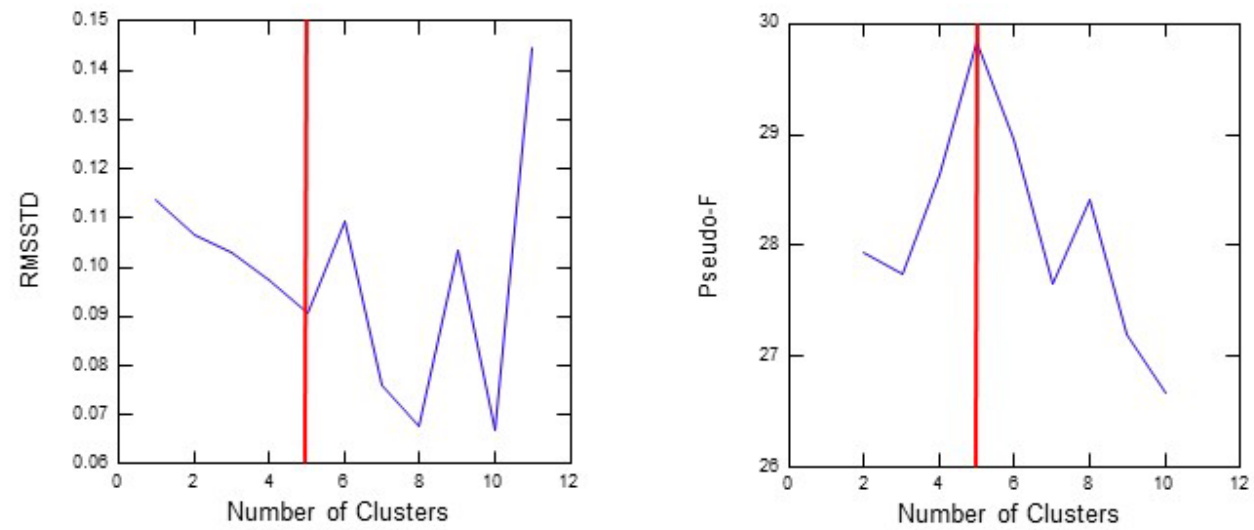

Figure 29. Graphical representations of results of a hierarchical cluster analysis of sea otter diet composition. For this analysis, individual otters were considered cases and the relative frequency of the 11 most common prey types were the variables. A, Dendrogram or "Cluster Tree", with branches "pruned" at Euclidean Distance of 0.1 to simplify presentation, such that each terminal node represents 3-10 individual otters. Terminal nodes of five distinct clusters are uniquely color-coded. B, Cluster validity graphs used to select the optimal number of distinct clusters, based on a local minima of the root mean square standard deviation (RMSSTD, on left) and a local maxima of the pseudo- $F$ value (on right). 


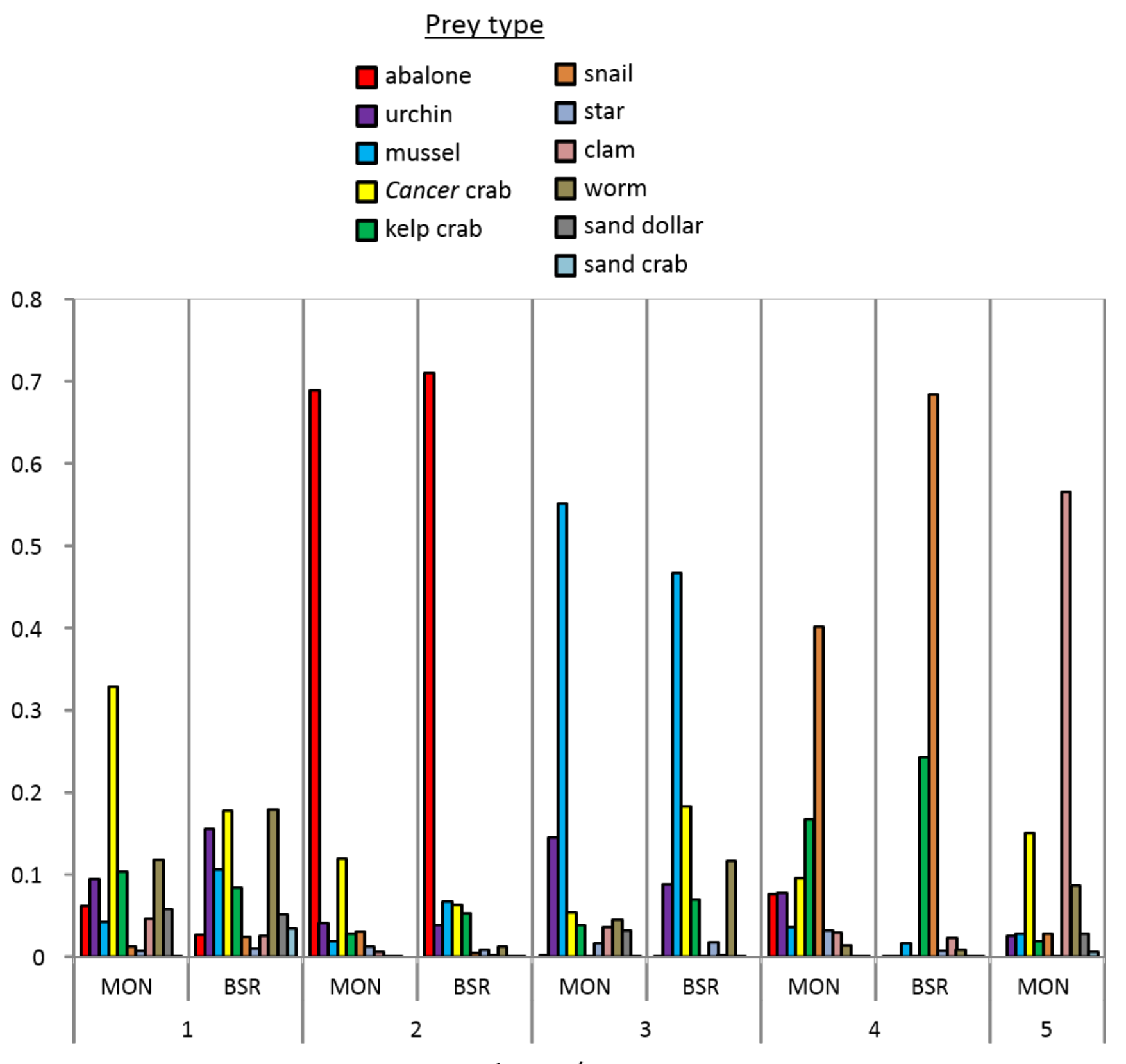

Study Site/Diet Group

Figure 30. Histogram showing contribution of 11 key prey types to the diets of sea otters belonging to each of 5 diet-type groups (as identified by cluster analysis) at Big Sur coast and Monterey Peninsula study sites, central California. See table 17 for species included in each prey-type category. 


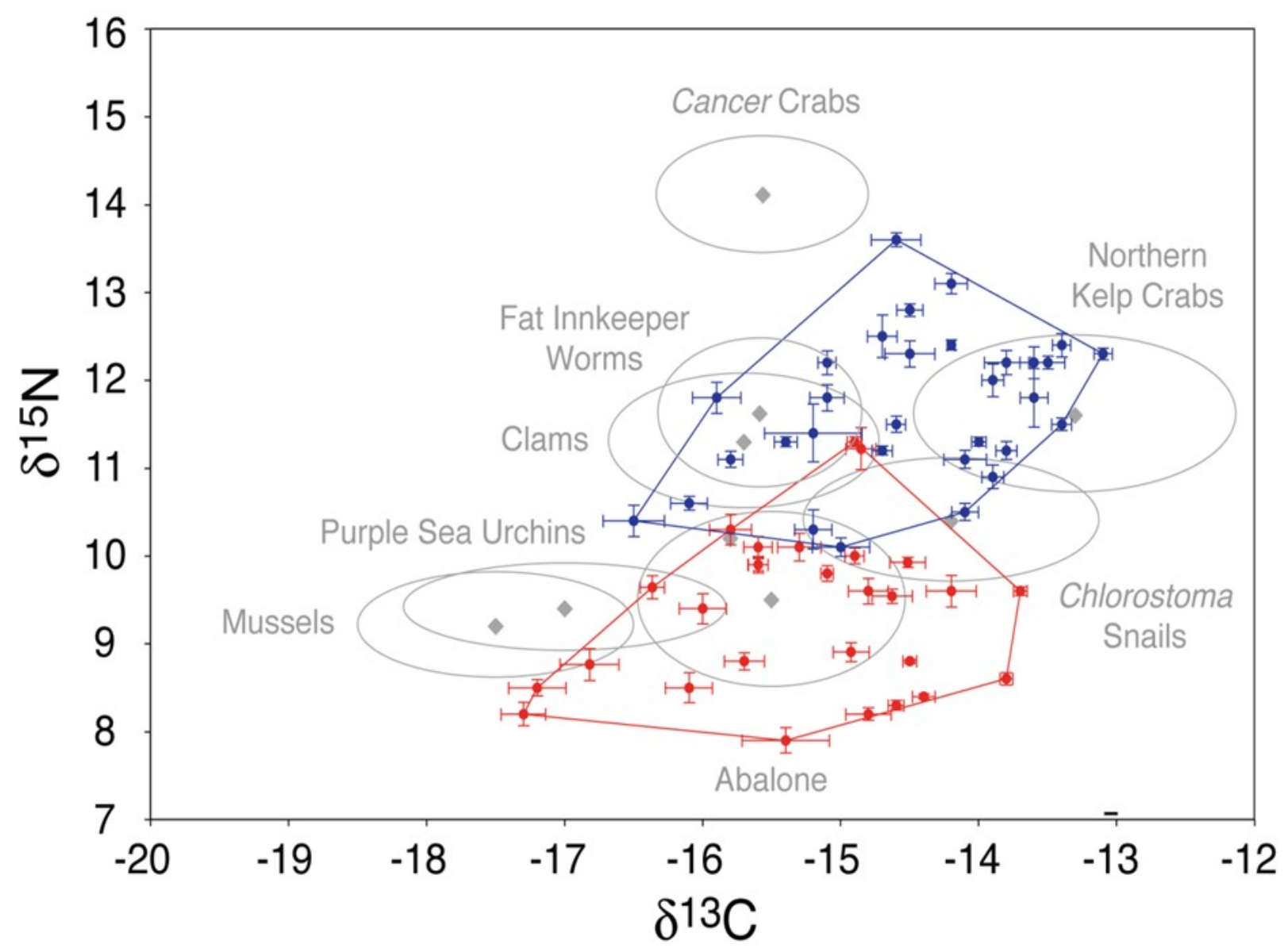

Figure 31. Biplots showing comparison of $\delta^{13} \mathrm{C}$ to $\delta^{15} \mathrm{~N}$ for California sea otter populations and their potential prey. Values are for sea otter vibrissae (Monterey Peninsula study site, blue circles, and Big Sur coast study site, red circles) and common prey (gray diamonds). Error bars associated with mean vibrissae isotope values denote standard error; ellipses associated with mean prey isotope values represent standard deviation. See table 20 for sample sizes and mean isotope values of prey types. See section, "Methods", for an explanation of the trophic discrimination factors applied to each population. 
A)

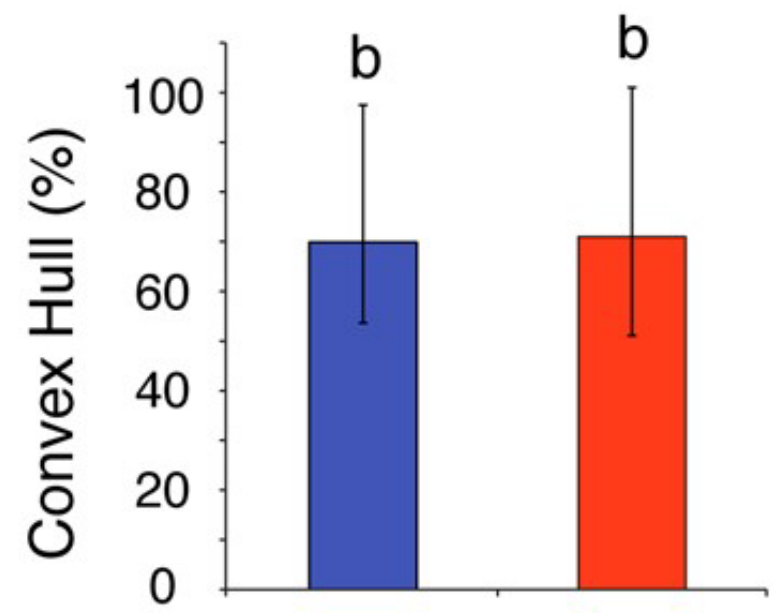

B)

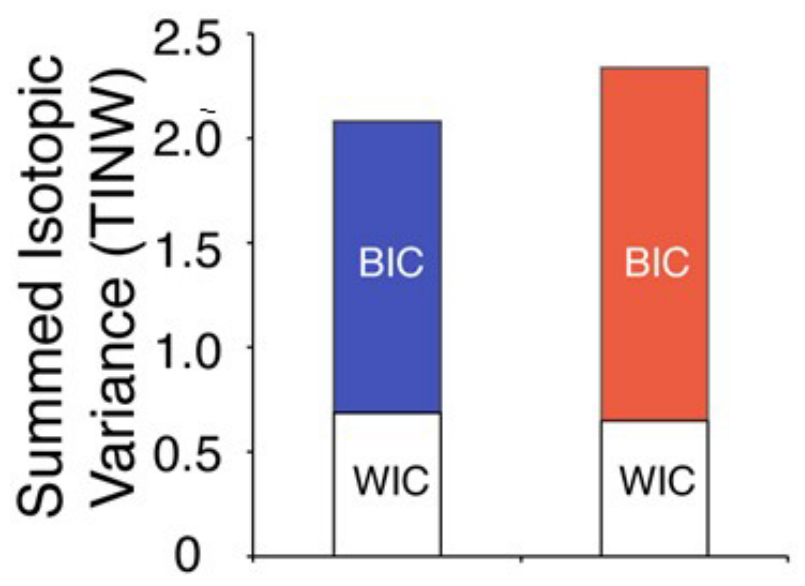

C)

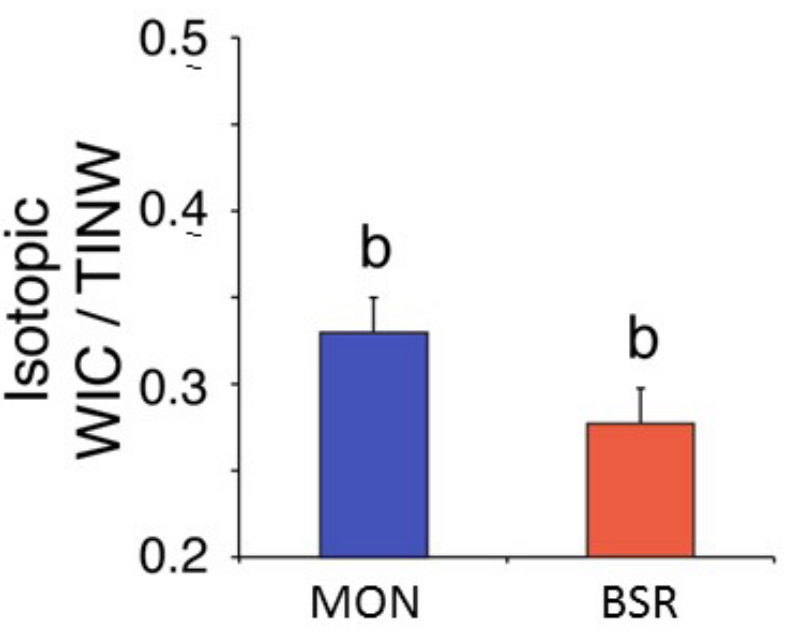

Figure 32. Graphs showing comparison of isotopic niche variation statistics at Big Sur coast (BSR) and Monterey Peninsula (MON) study sites, central California. A, Total isotopic niche space occupied by sea otters at each site, as measured by convex hull area. B, Summed variance of stable isotope values (Total Isotopic Niche Width, TINWI) at each site. C, Individual niche-space specialization, calculated as the Within-individual Variance Component $\left(\mathrm{WIC}_{\mathrm{C}}\right)$ /Total Isotopic Niche Width (TINW/) ratio (lower values indicate more extreme individual specialization). Error bars represent 95-percent confidence intervals. 

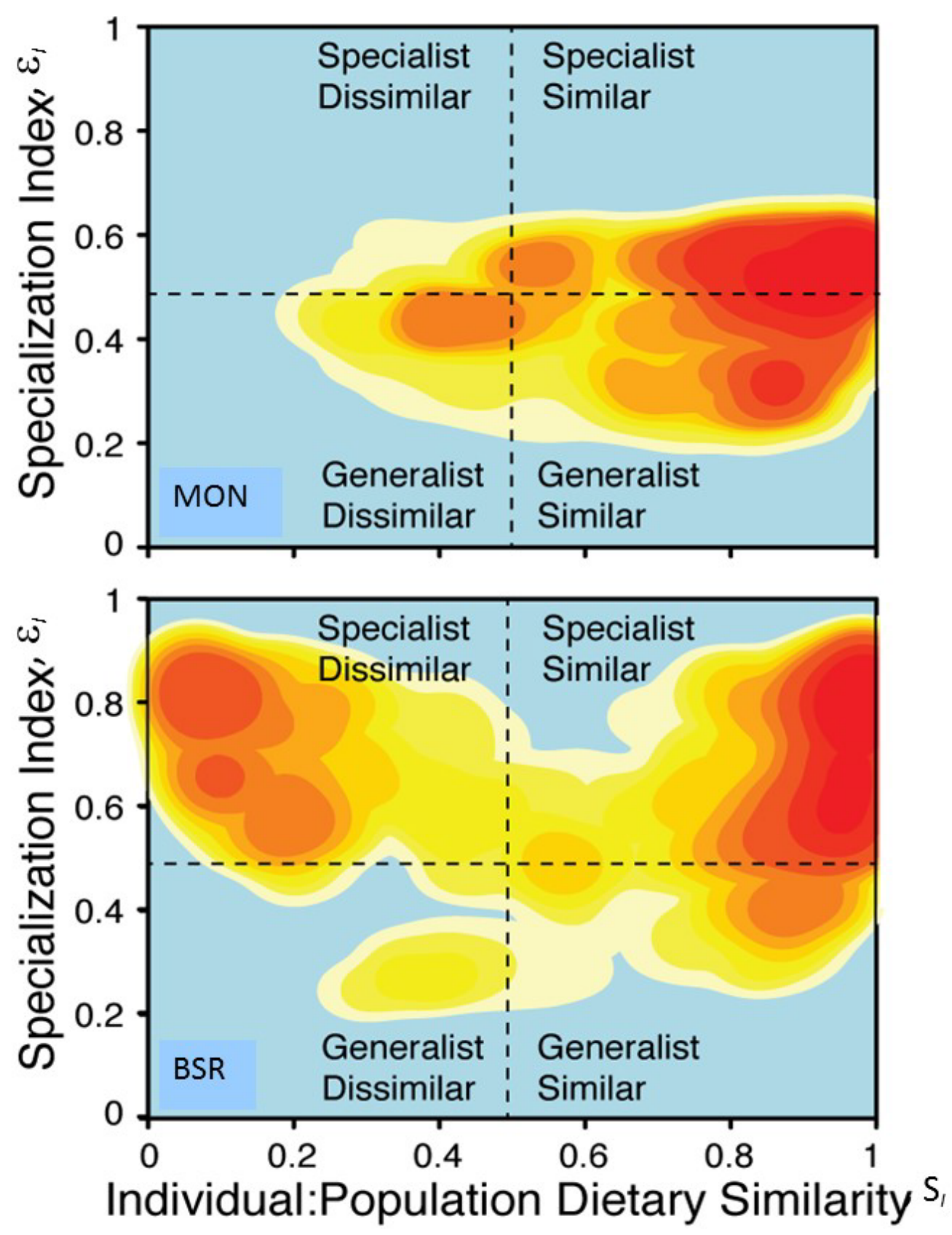

Figure 33. Density plots showing population-level dietary niche specialization (El) compared to individualpopulation dietary similarity $\left(S_{I}\right)$, based on stable isotope analysis, for sea otters at Monterey Peninsula (MON; top plot) and Big Sur coast (BSR; bottom plot) study sites, central California. Density plots can be subdivided into four quadrants - (1) Individuals that are niche specialists with diets dissimilar to the population (Specialist/Dissimilar), (2) niche specialists with diets similar to the population (Specialist/Similar), (3) niche generalists with diets dissimilar to the population (Generalist/Dissimilar), and (4) niche generalists with diets similar to the population (Generalist/Similar). Probability contour regions were set at 10-percent intervals; red (darker polygon shapes) represents high density, and light yellow (lighter polygon shapes) represents low density. 


\section{Big Sur}

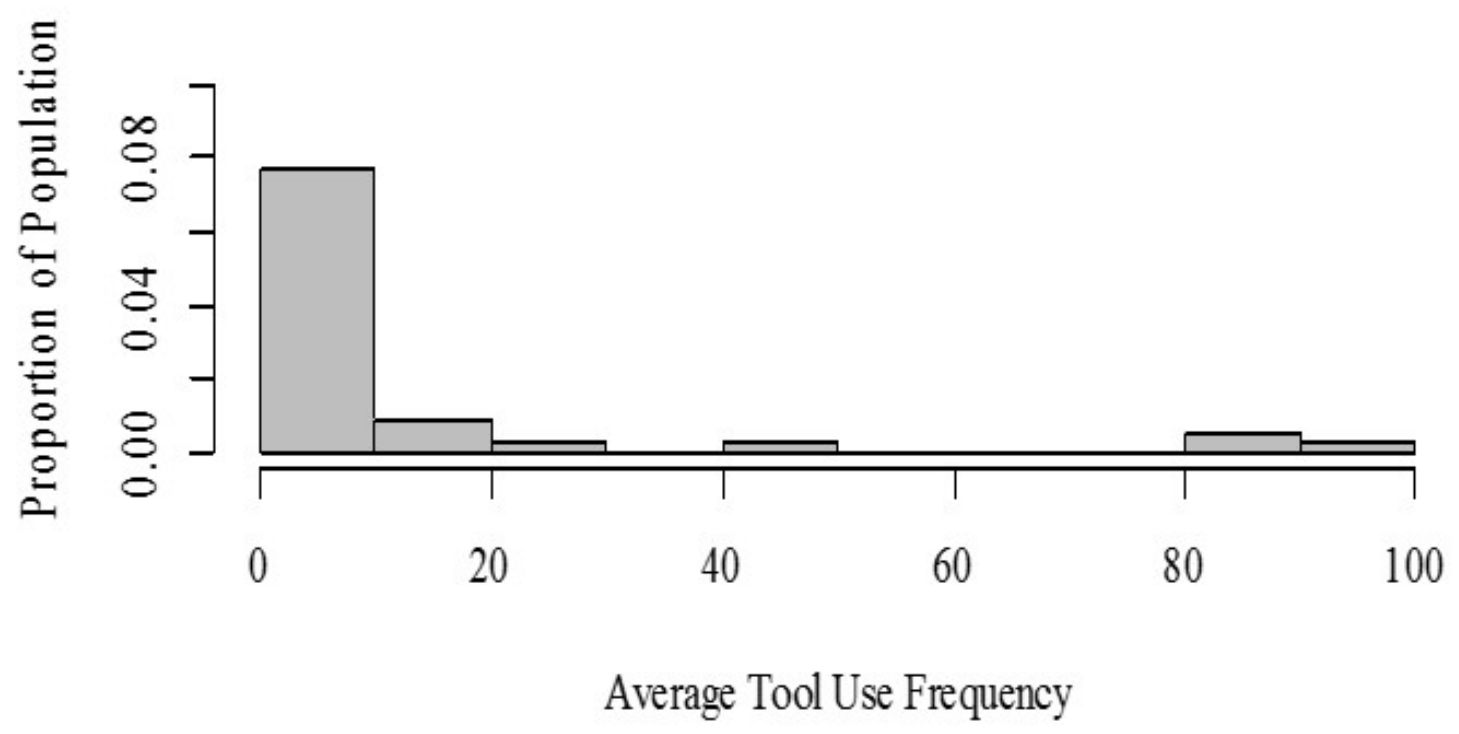

\section{Monterey}

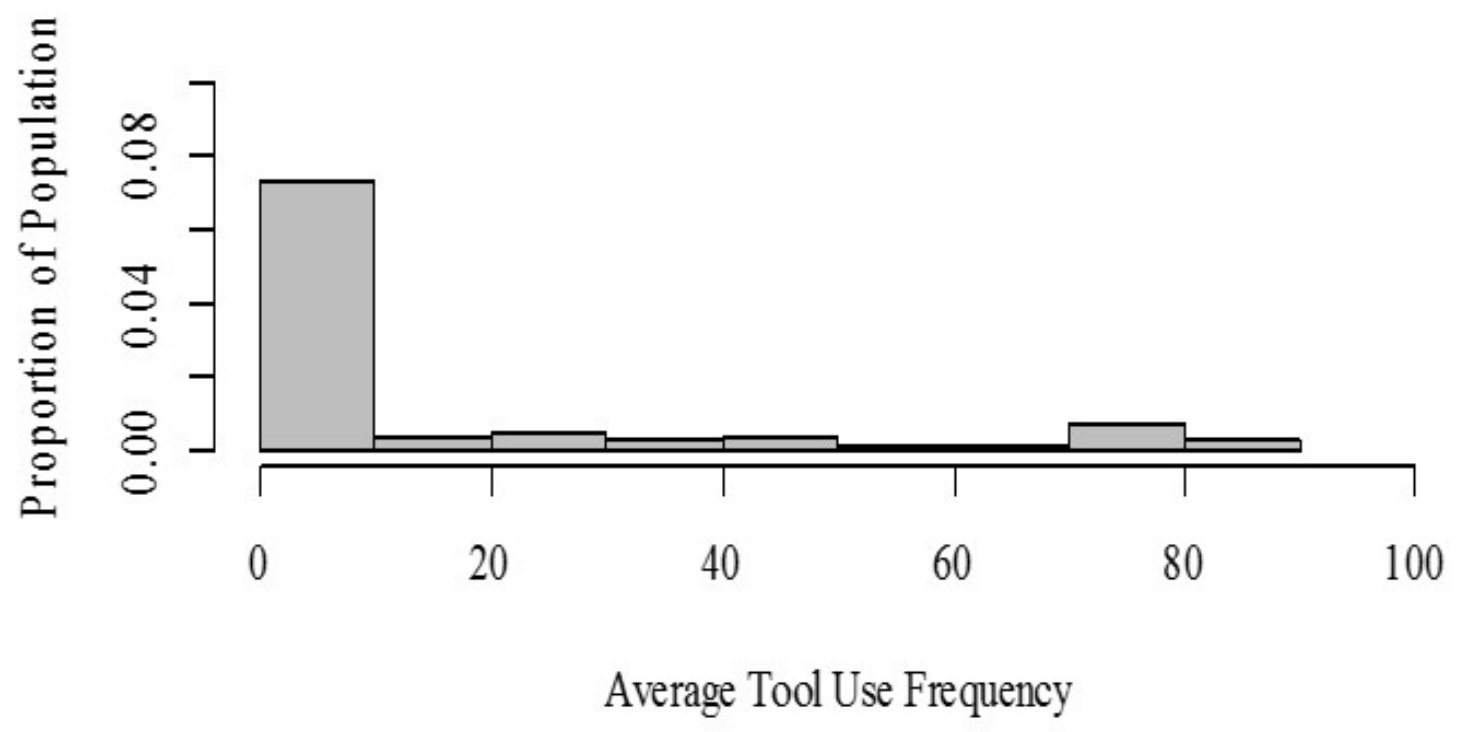

Figure 34. Graphs showing comparison of tool use frequency variation among individuals from Big Sur coast (top graph) and Monterey Peninsula (bottom graph) study sites, central California. At both locations, a small proportion of the sample population used tools at a very high frequency, whereas most individuals used tools very infrequently. Numbers on $x$-axis are percentages. 


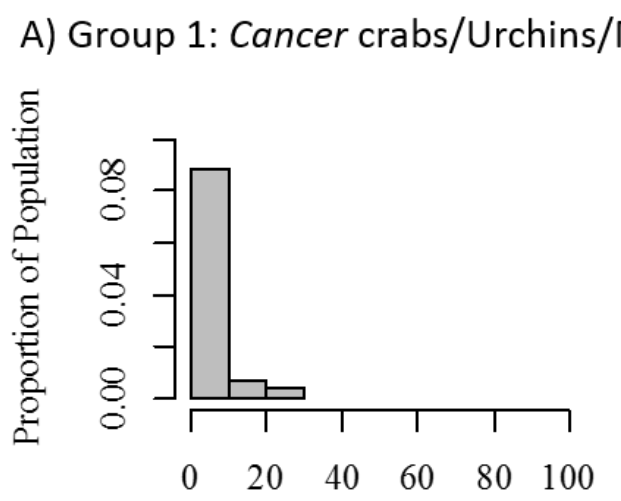

B) Group 3: Mussels \& Urchins

C) Group 5: Clams/Crabs/Worms

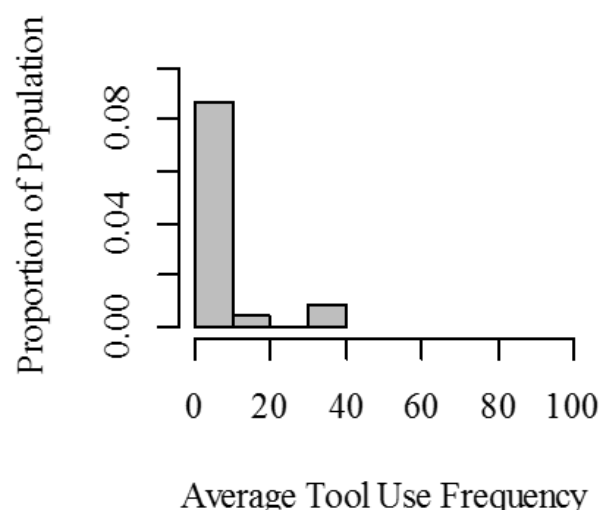

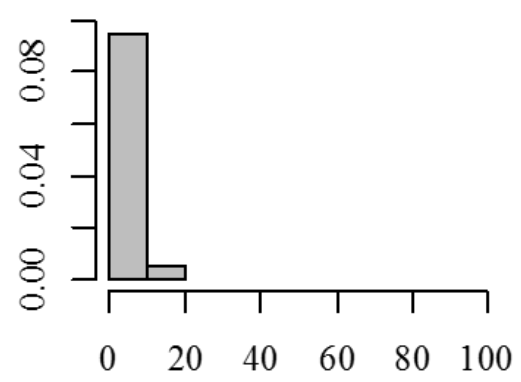

D) Group 4: Snails

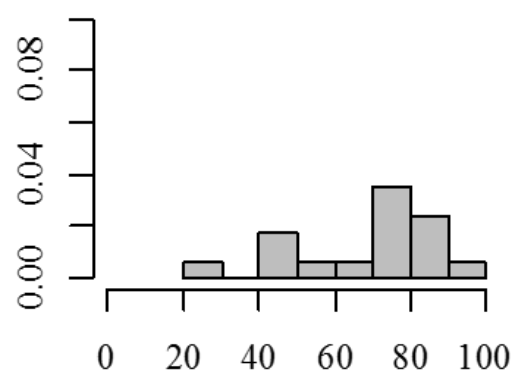

Average Tool Use Frequency

Figure 35. Histograms showing individual tool use frequency by diet specialization group (see body text, table 19, and fig. 30). Proportion of the population (y-axis) refers to the percentage of the overall population (including nontool users) at both study sites. Snail specialists were the only individuals who used tools on more than 50 percent of their foraging dives. Numbers on $x$-axis are percentages. 


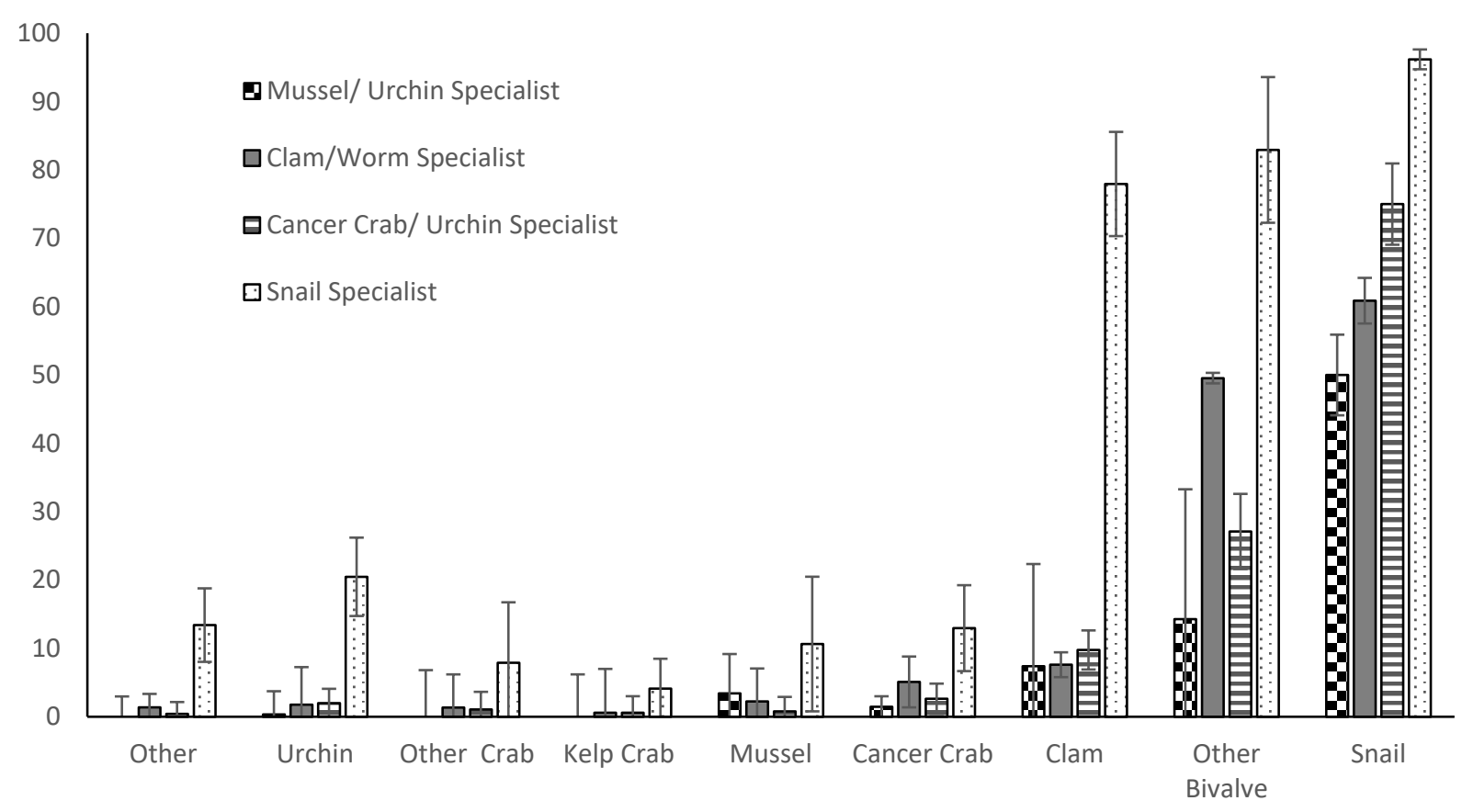

Figure 36. Graph showing average frequency of tool use on different types of prey by sea otters belonging to four different diet specialization groups-checkered, group 3 (mussels/urchins); solid gray, group 5 (clams/crabs/worms); lined, group 1 (Cancer crabs/urchins/miscellaneous); dotted, group 4 (snail specialists). 


\title{
Chapter 7. Variation in Body Condition in California Sea Otters
}

\author{
M. Tim Tinker ${ }^{1,2}$
}

\section{Introduction}

For populations of apex carnivores whose maximum densities are ultimately limited by abundance of their prey - that is, populations in which density-dependent changes in survival are associated with per-capita decreases in food resources - a cause-and-effect relation is expected between relative food abundance and one or more aspects of individual health and condition, and a lessened condition will ultimately result in decreased survival or fecundity. As a result, assessments of relative body condition of individuals within a population can provide insights into the status of that population with respect to carrying capacity (Monson and Bowen, 2015). Although simple in concept, identifying an appropriate metric with which to assess body condition can be challenging. For the purpose of this study, we are limiting our definition of body condition to those physiological traits affected by nutritional stress (for example, relative amount of body fat or muscle mass). Evaluating body mass relative to body length is a widely used method of measuring relative body condition (Schulte-Hostedde and others, 2005), with the most common approach being to estimate residuals from a regression of body mass (or log body mass) compared to some measure of structural size, such as body length. This type of index may be useful for assessing relative availability of food resources over the near-term, but it is subject to many statistical and biological limitations that can sometimes lead to spurious results (Green, 2001). Another approach to assessing the nutritional status of individual animals over longer time periods is to evaluate age-specific length or mass, and (or) the rate of body growth in juveniles (Fowler, 1990). This can be done by measuring absolute body length or body mass after statistically controlling for age, which is accomplished by fitting some sort of asymptotic growth function (Stewart and others, 2005).

Numerous different indices have been used in past analyses of sea otter body condition, including mass-to-length ratio, relative length at age, and relative size at age (that is, residuals from length or mass compared to age growth curves), and residuals from a log-linear function of body mass regressed against body length (Bodkin and others, 2002; Rotterman and Monnett, 2002; Laidre and others, 2006; Tinker and others, 2008; Monson 2009). Different indices have different advantages and disadvantages. For example, mass-to-length ratio is very easy to compute but is confounded by age and length differences; relative length at age provides a good age-independent measure of structural size but does not provide insight into short-term variation in muscle and fat stores associated with food abundance; residuals from a log-linear regression of body mass compared to body length can provide insight into short-term variation in body fat stores, but among-population comparisons are potentially confounded by differences in average structural size among sites. The use of many indices generally may be most useful, as different indices may indicate different components of body condition that vary at different scales. Nutritional condition in sea otters clearly has been found to be related to resource abundance and population status in northern populations (Bodkin and others, 2002; Rotterman and Monnett, 2002; Laidre and others, 2006; Monson, 2009). Here, we compare indices of body condition among different sub-populations of southern sea otter to determine (1) if there is variation in body condition among sites in California; (2) if variation in body condition is related to variation in foraging

\footnotetext{
${ }^{1}$ U.S. Geological Survey.

${ }^{2}$ University of California, Santa Cruz.
} 
success, and(3) if there is support for the hypothesis that sea otters at some locations are affected by decreased per-capita resource abundance.

\section{Methods}

We measured body mass, total length, and tail length of each otter captured at BSR and MON study sites, as described in chapter 1, sections, "Introduction" and "Methods". We estimated age of each animal at time of capture based on patterns of tooth wear and on degree of fur "grizzle" around the head and neck. These "field age estimates" were validated for a sub-sample of animals for which "tooth age estimates" (based on cementum analysis of extracted pre-molars) also were available. Age estimates using the two techniques were determined to be strongly correlated $(\mathrm{R}=0.71)$; however, to avoid any potential age bias and to maintain consistency, we used field age estimates for all subsequent analyses (because tooth age estimates were not available for all study animals). We combined data from the current study with data collected previously at four other study sites - CAM, Cambria/San Simeon (2001-04), PTC, Point Conception (2001-04), SNI, San Nicolas Island (2003-05), and MB1, Monterey Bay Area (2000-04). (Note that MON and MB1 samples were spatially similar but temporally distinct, and so were treated as independent study units.) Our total sample size for analysis of body condition was 440 sea otters, ranging in estimated age from 6 months to 18 years.

We compared sea otter body condition among sites by comparing relative body mass and total length after controlling for age effects (thus, the condition of each study animal was assessed relative to the "average" mass and length of all otters of that age). To accomplish this, we used maximum likelihood methods to fit sex-specific Von Bertalanffy growth models (Ratkowsky, 1986) to the lengthcompared to-age and mass-compared to-age data for all otters from all 6 study sites:

$$
\begin{gathered}
L_{x, s}=c_{s, 1}+a_{s, 1}\left(1-e^{-k_{s, 1}\left(x-b_{s, 1}\right)}\right), \text { and } \\
M_{x, s}=c_{s, 2}+a_{s, 2}\left(1-e^{-k_{s, 2}\left(x-b_{s, 2}\right)}\right),
\end{gathered}
$$

where

$$
\begin{aligned}
x & \text { is the estimated age of each animal, } \\
M & \text { is total body mass, } \\
L & \text { is total body length, } \\
s & \text { is sex (1= female, } 2=\text { male), and } \\
c, a, k \text { and } b & \text { are the Von Bertalanffy growth function parameters. }
\end{aligned}
$$

$L$ represents total length (nose to tail) rather than "length corrected for tail length" $\left(L^{c}\right)$, as has been recommended elsewhere (Monson 2009), because we did not have tail length measurements for sea otters at three of the six study sites. To determine if variation in $L$ provided a reliable estimate of variation in $L^{c}$, we plotted a regression of $L$ compared to $L^{c}$ for otters from BSR, MON and PTC, and determined that there was a strong linear relation between these two measures (coefficient of determination $\left[\mathrm{R}^{2}\right]=0.95$ ), with no indication of site-specific differences in residual distributions (fig. 37). We, therefore, concluded that total length $(L)$ provided a reliable index for assessing variation in structural size, as long as among-site comparisons were limited to California. We used bootstrap resampling to account for sample-size differences among sites; specifically, for both males and females, we randomly drew 20 otters (with replacement) from each study site, fit equations 8 and 9, and then 
repeated this 1,000 times and used the mean expected values to define the "average" length or mass at age for each sex.

We calculated two indices of relative condition for each animal as the residual values from the mean growth curves calculated from equations 8 and 9. Residuals from the length compared to age function represent an index of relative structural size after controlling for age; this structural size index (SSI) provides insights into long-term patterns of food abundance at a given site, as these long-term conditions determine the growth rates and asymptotic skeletal size of otters during the first 5-7 years of life. Residuals from the mass compared to age function provide a more comprehensive body condition index (BCI), indicating relative structural size, as well as dynamic body stores (muscle and fat biomass) that can increase or decrease in response to short-term variation in food abundance, animal health or reproductive status. These two indices are expected to be strongly correlated, as otters that have greater structural size at a given age clearly have the potential to reach larger body mass; however, short-term variation in conditions can lead to some variation in mass for an otter of a given age and body length. Accordingly, we also computed residuals from a linear regression of BCI compared to SSI, which represent a "dynamic condition index" (DCI), to assess short-term variation in conditions at each study site.

We graphically compared the SSI, BCI, and DCI among sites using boxplots, and used analysis of variance (ANOVA) to test for statistically significant differences in all three indices among sites. We conducted separate analyses for males and females because data on movements and habitat use patterns suggested that males and females may experience local resource depletion in different ways and, thus, have distinct patterns of variation in relative body condition. Finally, we used an inverse logit transformation of $\mathrm{BCI}$ to create a rescaled body condition index $(\mathrm{BCI})$ for each sex at each study site that varied between 0 and 1 :

$$
B C I^{\prime}=e^{B C I} /\left(1+e^{B C I}\right)
$$

We used bootstrap resampling to account for sampling error and sample size differences among sites in calculating BCI'. To evaluate the role of prey resource abundance on body condition, we used linear regression analysis to test for a relation between BCI' and foraging performance of study animals of each sex at each study site (specifically, the estimated rate of energy gain while feeding; see chapter 6).

\section{Results}

Growth curves fit to length-at-age data showed that sea otter structural size tends to reach asymptotic values at about 4-6 years of age (fig. 38), with males achieving a greater structural size (average asymptotic length $=127 \mathrm{~cm}$ ) than females (average asymptotic length $=118 \mathrm{~cm}$ ). Mass-at-age data also indicated an asymptote at about 4-6 years for females (fig. 39A), but for males the asymptotic values were not reached until about 8 years of age (fig. 39B), suggesting that males may continue to increase in muscle mass for some years after reaching maximum structural size, perhaps increasing their chances at successfully defending a reproductive territory.

Structural size varied significantly among study sites for both males and females (table 23). Female otters at BSR had lower SSI values than did female otters from all five other study sites, whereas female otters at MON had higher SSI values than females from BSR but lower SSI values than SNI (fig. 40A). Male otters at BSR had significantly lower SSI values than males from the SNI study site, but this was not the case for males at the MON study site (fig. 40B). 
The overall BCI also varied significantly among study sites for both males and females (table 24), with patterns of variation generally matching those of the SSI. Female otters at BSR had lower BCI values than did female otters from the PTC and SNI study sites, but did not differ statistically from females at MON, MB1 or CAM, whereas females from MON had lower BCI values than females from SNI but higher BCI values than females from CAM (fig. 41A). Male otters at BSR had lower BCI values than did male otters from MON, PTC and SNI study sites, but did not differ statistically from males at MB1 or CAM, whereas males from MON had higher BCI values than males from BSR but did not differ statistically from males at the other study sites (fig. 41B).

The dynamic condition index (DCI) varied significantly among sites (table 25); however, the patterns of variation were very different from the SSI and BCI indices. The values of DCI for females were higher at the CAM study site than all other sites, but there were no other statistically significant differences (fig. 42A). Males at BSR had higher DCI values than did males at MON and SNI, while males at MON had lower DCI values than males at BSR, but did not differ significantly from males at any other study site (fig. 42B).

There was a strong positive relation across sites between rescaled body condition index (BCI') and estimated rate of energy gain while feeding $\left(\mathrm{R}^{2}=0.7482\right.$, linear model $\mathrm{F}$ statistic $=23.77, \mathrm{P}=$ 0.001), and this trend was consistent for both sexes (fig. 43).

\section{Discussion}

Sea otters in California showed considerable variation in relative body condition, suggesting that their population status with respect to carrying capacity varied considerably from site to site. Differences in structural size (figs. 38, 40) indicated that sea otters at Big Sur and (to a lesser extent) Monterey in the early 2000s had particularly low length at age, indicating slower growth rates among juveniles and lower asymptotic sizes of adults for both males and females compared to the current Monterey Peninsula study site. In contrast, otters at San Nicolas Island and Point Conception, the most recently colonized sites where population densities were lower than at the other sites, had significantly greater length at age than Big Sur animals. Otters from these two sites also showed the greatest mass at age (BCI, figs. 39 and 41), whereas otters from Big Sur and Cambria had the lowest mass at age. Both females and males from the current sample at Monterey tended to have slightly better body condition than did otters from Monterey in the earlier sample, highlighting the fact that temporal variation in body condition does occur even at high-density sites, potentially in response to annual variation in prey abundance, environmental stressors (for example, disease prevalence), and other factors.

The dynamic condition index showed very different patterns, which were largely inconsistent with the other two indices. To some extent, these patterns may indicate short-term variation in dynamic body reserves that are not necessarily consistent with longer-term patterns; for example, the fact that females at the CAM study site had high DCI but low BCI and SSI could indicate a temporal trend towards higher prey abundance at that time (such that animals that had experienced poor conditions during growth and development were experiencing more abundant food resources at time of sampling). However, such dynamic trends can be hard to interpret because sea otter fat and muscle mass can change so substantially over short periods of time, and also can indicate factors such as reproductive status or disease exposure. Compared to other study sites, a higher proportion of females at CAM also possibly were in early-term pregnancy, although we excluded data from palpably pregnant females for this analysis (as pregnancy is associated by an increase in mass of 2-5 kg), we recognize that early-term pregnancies can go undetected, but can still lead to subtle increases in body mass.

Overall, our results suggest that the BCI (relative mass at age) provides the most reliable index of body condition for comparing populations in California, as it captures age-specific differences in structural size and dynamic body reserves. The strong relation between BCI' and foraging success (fig. 
43) shows that the per-capita abundance of key prey resources translates directly into differences in body condition, and these in turn can translate into differential survival and reproductive success (see chapter 8; Monson and others, 2000). Based on our comparison among sites, it seems that both Big Sur and Monterey have relatively low food abundance compared to sites with lower otter densities (for example, PCI and SNI), but at the time of this study, this resource limitation seems to be stronger at the BSR study site.

\section{References Cited}

Bodkin, J.L., Ballachey, B.E., Dean, T.A., Fukuyama, A.K., Jewett, S.C., McDonald, L., Monson, D.H., O'Clair, C.E., and VanBlaricom, G.R., 2002, Sea otter population status and the process of recovery from the 1989 'Exxon Valdez' oil spill: Marine Ecology Progress Series, v. 241, p. 237-253.

Fowler, C.W., 1990, Density dependence in northern fur seals (Callorhinus Ursinus): Marine Mammal Science, v. 6, p. 171-195.

Green, A.J., 2001, Mass/length residuals-Measures of body condition or generators of spurious results?: Ecology, v. 82, p. 1473-1483.

Laidre, K.L., Estes, J.A., Tinker, M.T., Bodkin, J.L., Monson, D.H. and Schneider, K.B., 2006, Patterns of growth and body condition in sea otters from the Aleutian archipelago before and after the recent population decline: Journal of Animal Ecology, v. 75, p. 978-989.

Monson, D.H., and Bowen, L., 2015, Evaluating the status of individuals and populations-Advantages of multiple approaches and time scales, in Larson, S.E., Bodkin, J.L., and VanBlaricom, G.R., eds., Sea otter conservation (1st ed.): London, Academic Press, p. 122-152.

Monson, D.H., 2009, Sea otters (Enhydra lutris) and Steller sea lions (Eumetopias jubatus) in the North Pacific_-Evaluating mortality patterns and assessing population status at multiple time scales: Santa Cruz, University of California, PhD dissertation, $223 \mathrm{p}$.

Monson, D.H., Estes, J.A., Bodkin, J.L., and Siniff, D.B., 2000, Life history plasticity and population regulation in sea otters: Oikos, v. 90, p. 457-468.

Ratkowsky, D.A., 1986, Statistical properties of alternative parameterizations of the von Bertalanffy growth curve: Canadian Journal of Fisheries and Aquatic Sciences, v. 43, p. 742-747.

Rotterman, L.M., and Monnett, C., 2002, Length-mass and total body length of adult female sea otters in Prince William Sound before and after the Exxon Valdez oil spill: Marine Mammal Science, v. 18, p. 977-993.

Schulte-Hostedde, A.I., Zinner, B., Millar, J.S., and Hickling, G.J., 2005, Restitution of mass-size residuals - Validating body condition indices: Ecology, v. 86, p. 155-163.

Stewart, K., Bowyer, R.T., Dick, B., Johnson, B., and Kie, J.G., 2005, Density-dependent effects on physical condition and reproduction in North American elk-An experimental test: Oecologia, v. 143, p. 85-93.

Tinker, M.T., Bentall, G., and Estes J.A., 2008, Food limitation leads to behavioral diversification and dietary specialization in sea otters: Proceedings of the National Academy of Sciences of the United States of America, v. 105, p. 560-565. 
Table 23. Analysis of variance results for among-site contrasts of sea otter structural size index at six California study sites.

[SS, Sum of Squares; df, degrees of freedom; MS, Mean Square; F, test statistic; Prob $>$ F, probability effect is significantly different from 0]

$\underline{\text { Females }}$

Source SS df $\quad$ MS $\quad F \quad$ Prob $>F$

$\begin{array}{lrrrrr}\text { Groups } & 1064.93 & 5 & 212.985 & 18.58 & 3.40629 \mathrm{e}-16 \\ \text { Error } & 3645.66 & 318 & 11.464 & & \\ \text { Total } & 4710.59 & 323 & & & \end{array}$

$\underline{\text { Males }}$

\begin{tabular}{lrrrrr} 
Source & SS & df & MS & F & Prob>F \\
\hdashline Groups & 207.98 & 5 & 41.5954 & 2.48 & 0.0363 \\
Error & 1830.00 & 109 & 16.7890 & & \\
Total & 2037.98 & 114 & & &
\end{tabular}

Table 24. Analysis of variance results for among-site contrasts of sea otter body condition index at six California study sites.

[SS, Sum of Squares; df, degrees of freedom; MS, Mean Square; F, test statistic; Prob $>$ F, probability effect is significantly different from 0]

$\underline{\text { Females }}$

Source SS df MS F Prob $>F$

Groups $\quad 222.05 \quad 5 \quad 44.4103 \quad 8.35 \quad 1.95914 \mathrm{e}-07$

Error $\quad 1691.11 \quad 318 \quad 5.3180$

Total $1913.17 \quad 323$

$\underline{\text { Males }}$

Source SS df MS F Prob $>F$

Groups $\quad 289.79 \quad 5 \quad 57.9576 \quad 5.89 \quad 7.40264 \mathrm{e}-05$

$\begin{array}{llll}\text { Error } & 1072.57 & 109 & 9.8401\end{array}$

Total $1362.36 \quad 114$ 
Table 25. Analysis of variance results for among-site contrasts of sea otter dynamic condition index, at six California study sites.

[SS, Sum of Squares; df, degrees of freedom; MS, Mean Square; F, test statistic; Prob $>$ F, probability effect is significantly different from 0]

\section{Females}

Source SS df MS F Prob $>$ F

Groups $\quad 141.62 \quad 5 \quad 28.3237 \quad 6.91 \quad 3.87694 \mathrm{e}-06$

$\begin{array}{llll}\text { Error } & 1304.17 & 318 & 4.1012\end{array}$

Total $1445.79 \quad 323$

$\underline{\text { Males }}$

\begin{tabular}{lrrrrr}
\hline Source & SS & df & MS & F & Prob $>$ F \\
\hdashline Groups & 130.789 & 5 & 26.1577 & 4.79 & 0.0005 \\
Error & 595.180 & 109 & 5.4604 & & \\
Total & 725.969 & 114 & & &
\end{tabular}




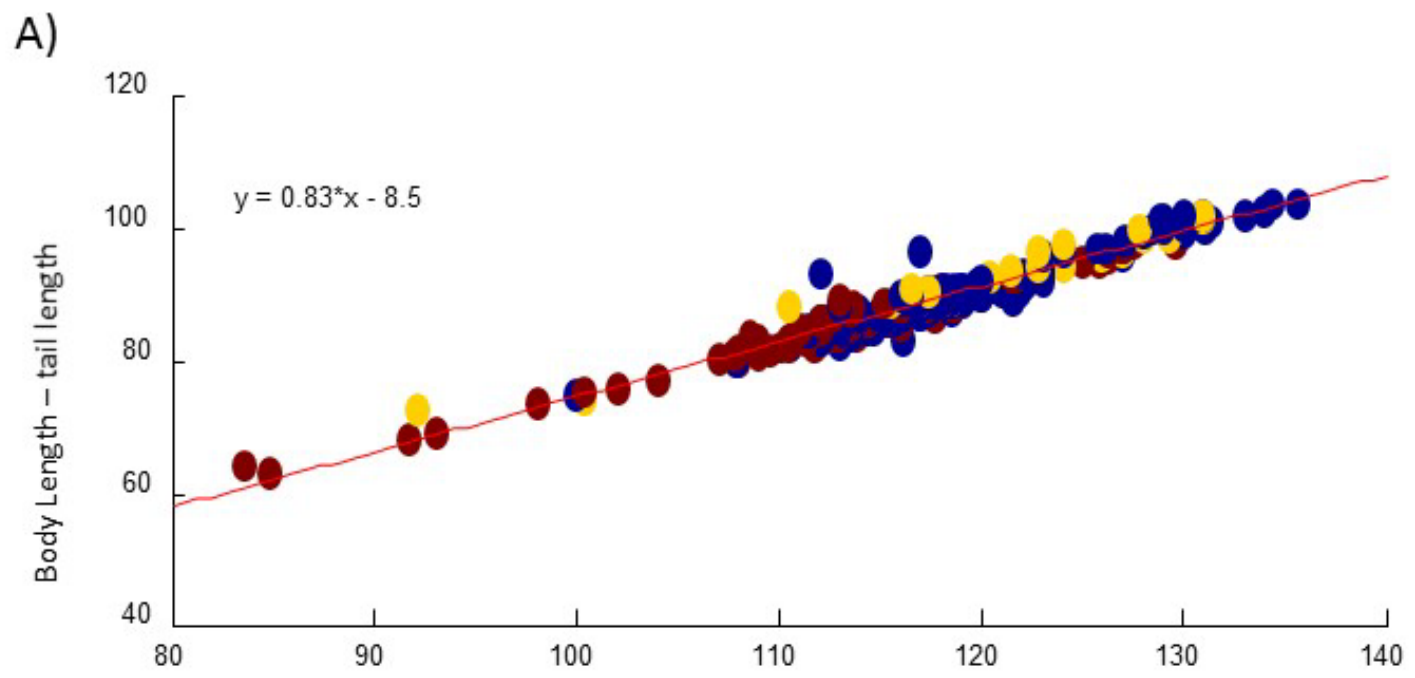

B)

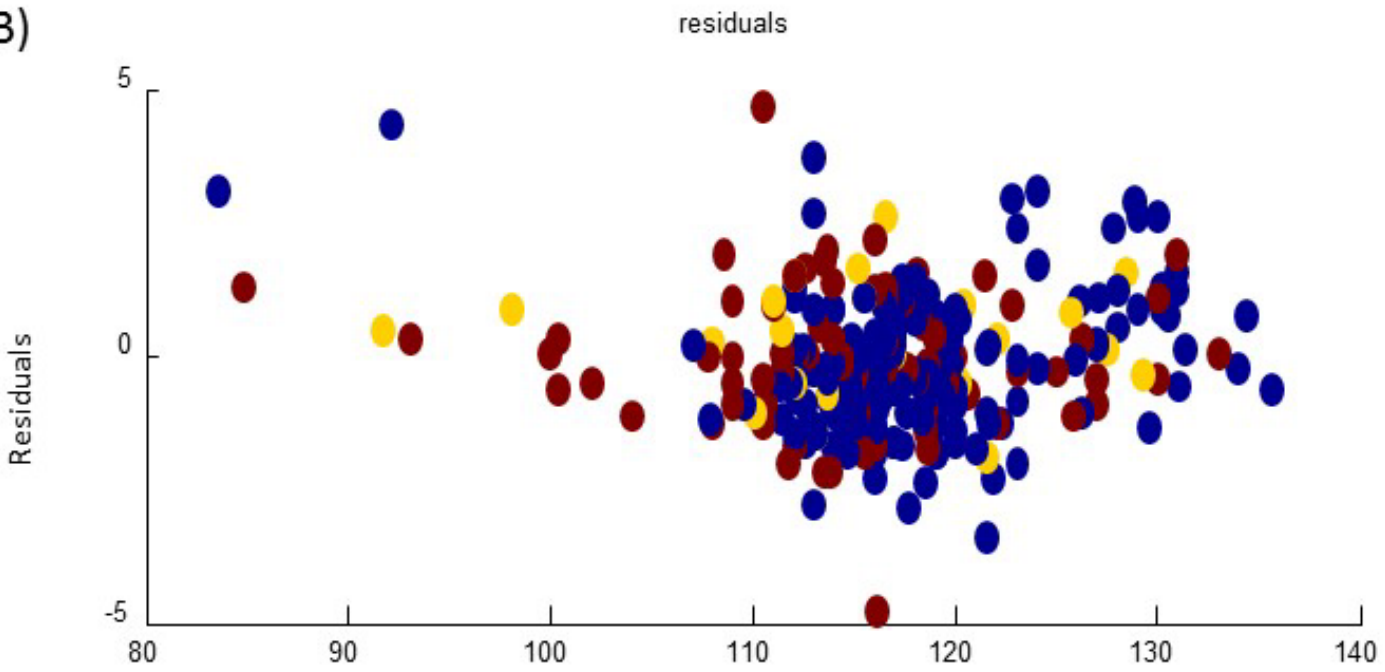

Body Length

Figure 37. Graphs showing linear regression (A) and residuals from the linear regression (B) of sea otter body length minus tail length compared to body length including tail, for sea otters at three different California study sites (individual animals are shown color-coded by study site: blue $=$ MON, red $=B S R$, yellow $=$ PTC). 
A)

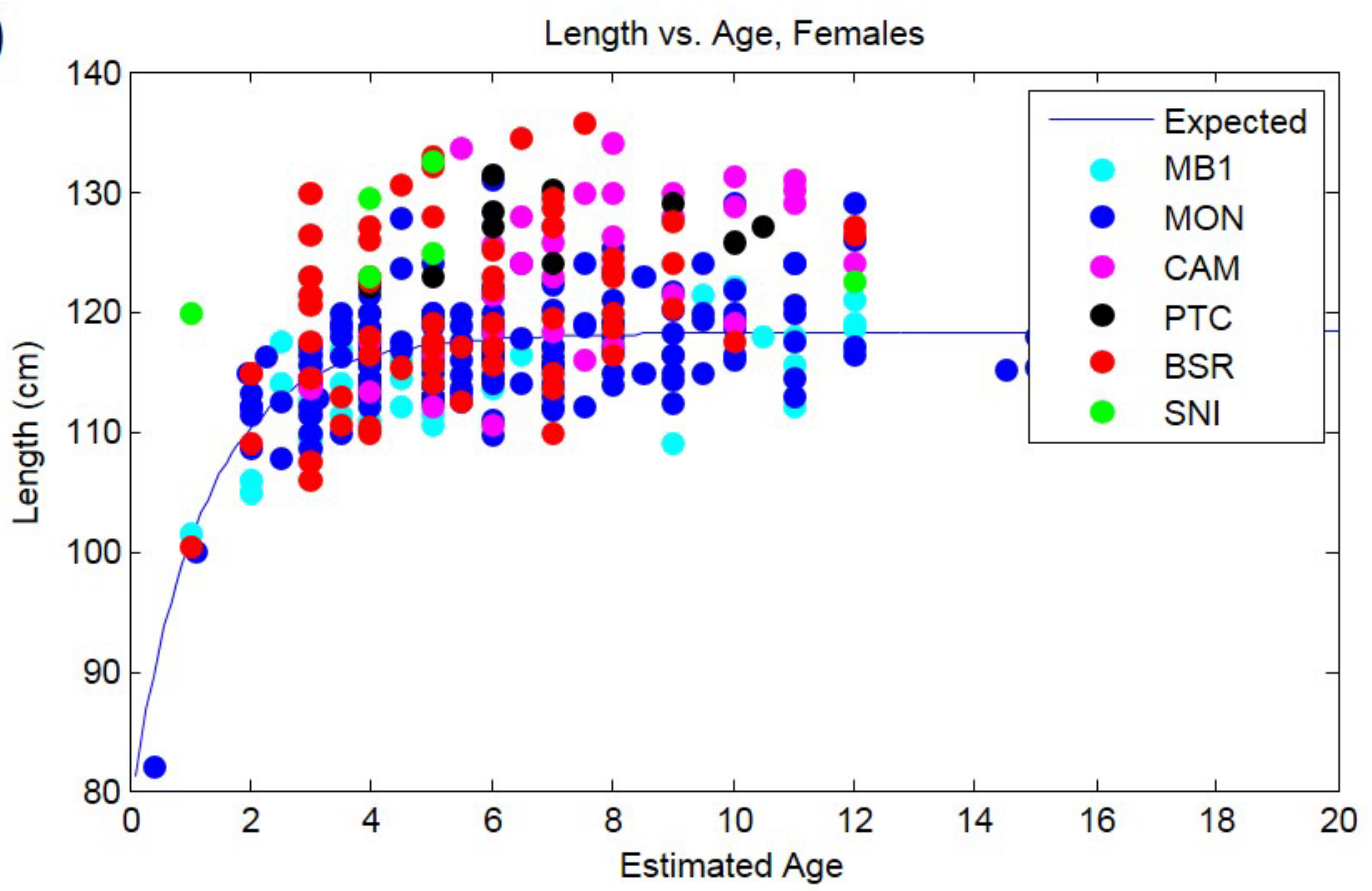

B)

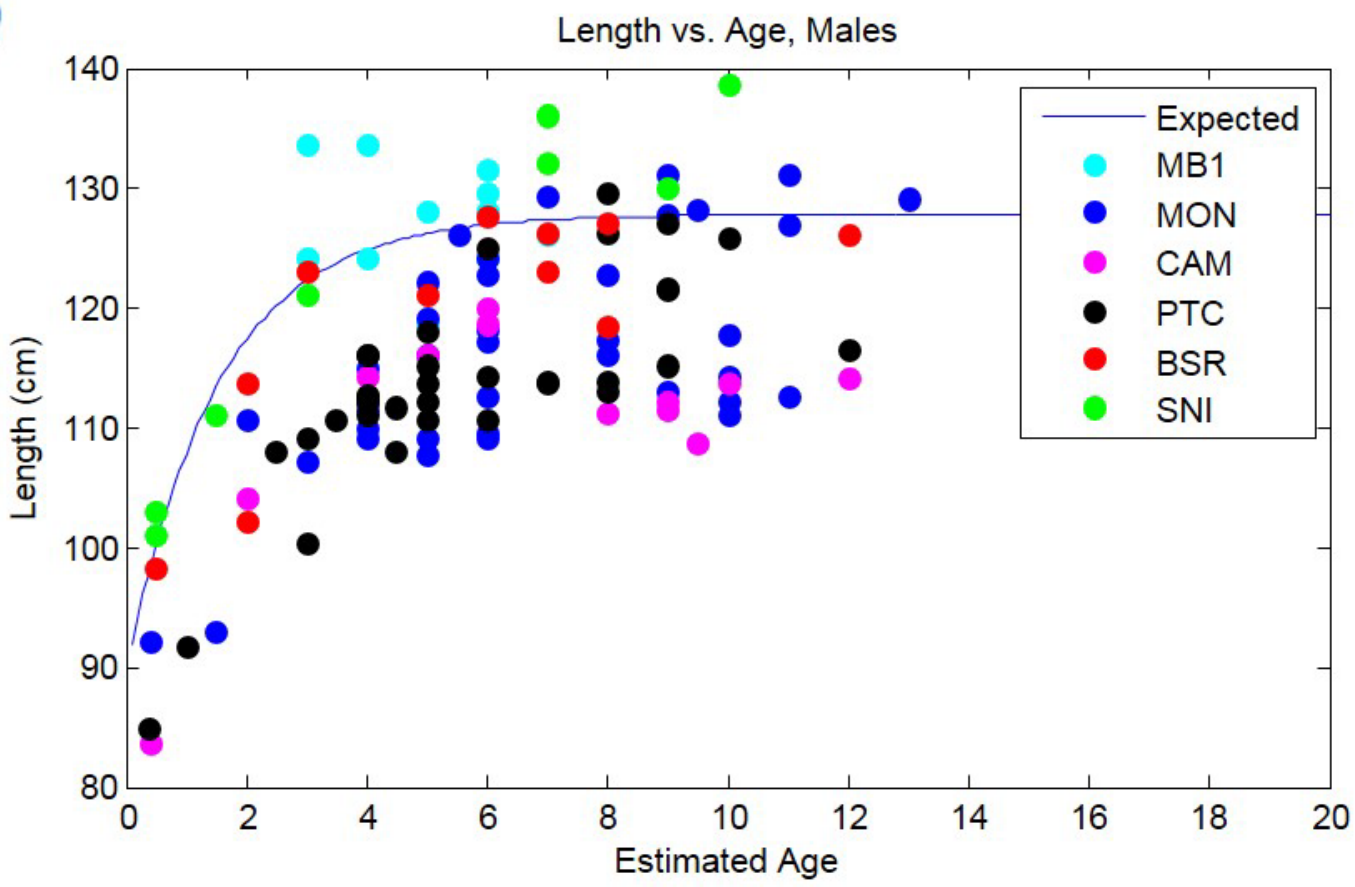

Figure 38. Scatterplots showing body length (in centimeters [cm]) compared to estimated age for sea otters at six different study sites in California. A, Length compared to age data for females. B, Length compared to age data for males. Best-fit Von Bertlanffy growth curves are plotted as blue lines. MB1, Monterey Bay (2000-04); MON, Monterey Peninsula (2008-11); CAM, Cambria/San Simeon (2001-04); PTC, Point Conception (2001-04); BSR, Big Sur coast (2008-11); SNI, San Nicolas Island (2003-05). 
A)

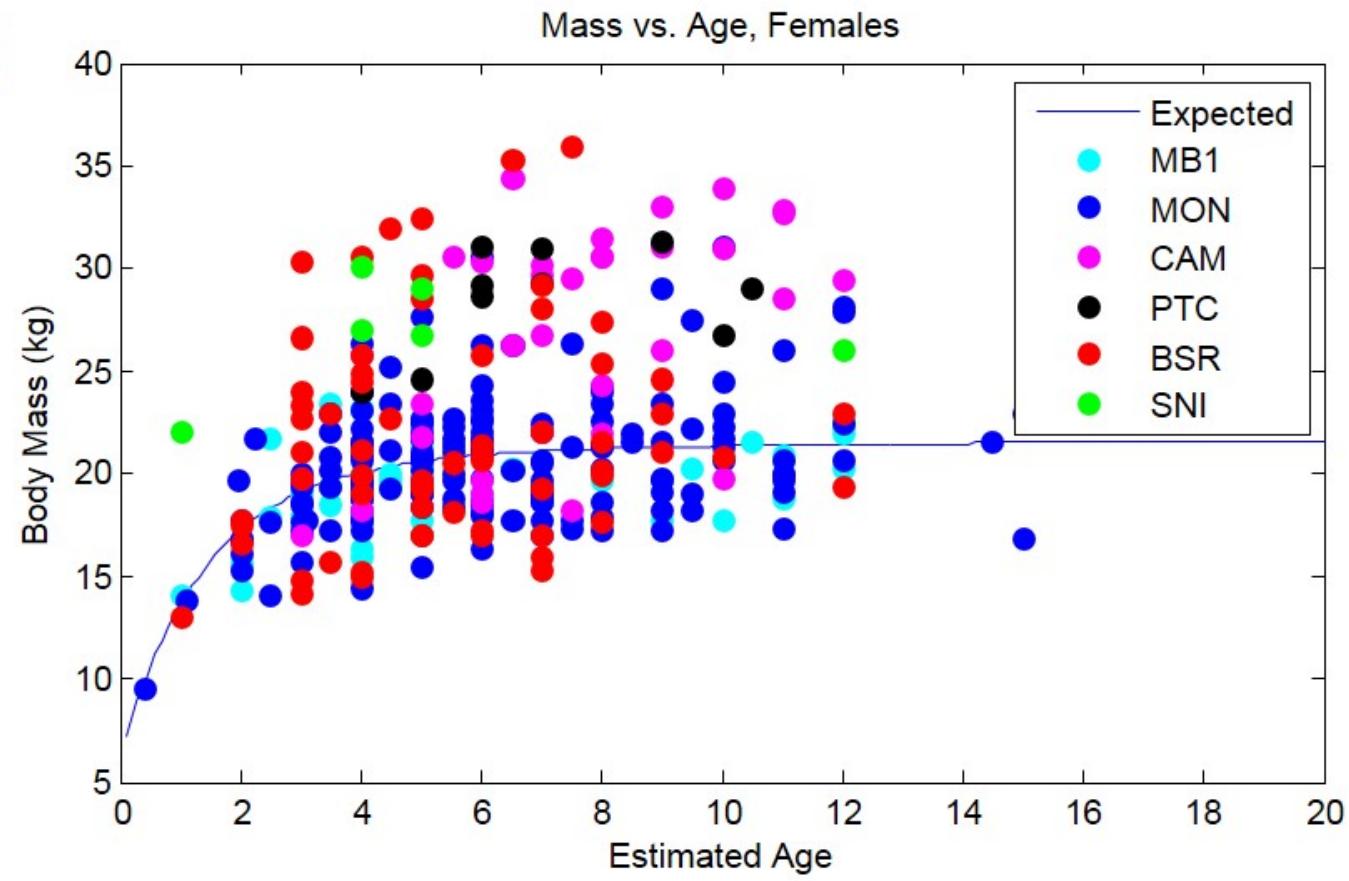

B)

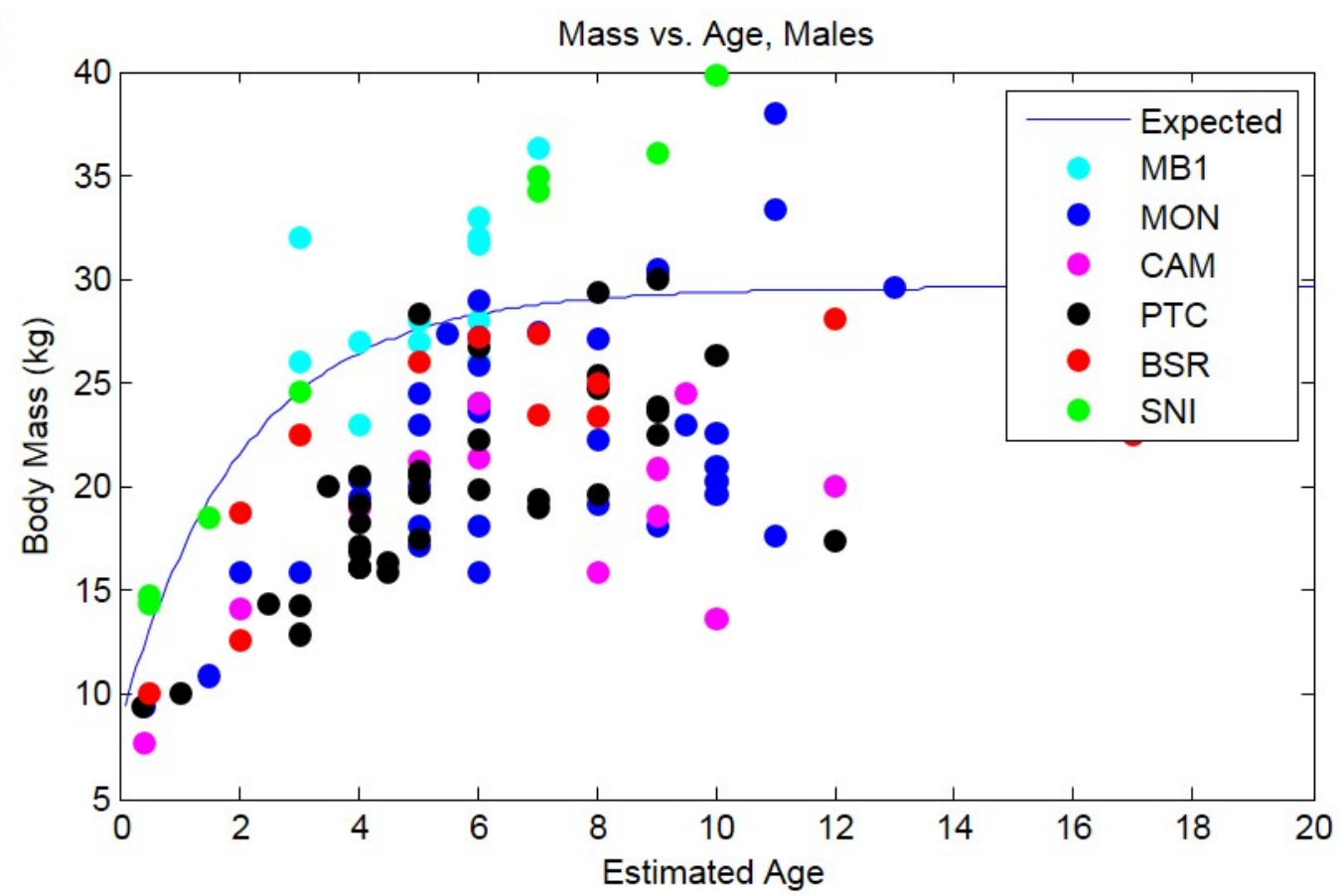

Figure 39. Scatterplots showing body mass (in kilograms [kg]) compared to estimated age for sea otters at six different study sites in California. A, Mass compared to age data for females. B, Mass compared to age data for males. Best-fit Von Bertlanffy growth curves are plotted as blue lines. MB1, Monterey Bay (2000-04); MON, Monterey Peninsula (2008-11); CAM, Cambria/San Simeon (2001-04); PTC, Point Conception (2001-04); BSR, Big Sur coast (2008-11); SNI, San Nicolas Island (2003-05). 
A)

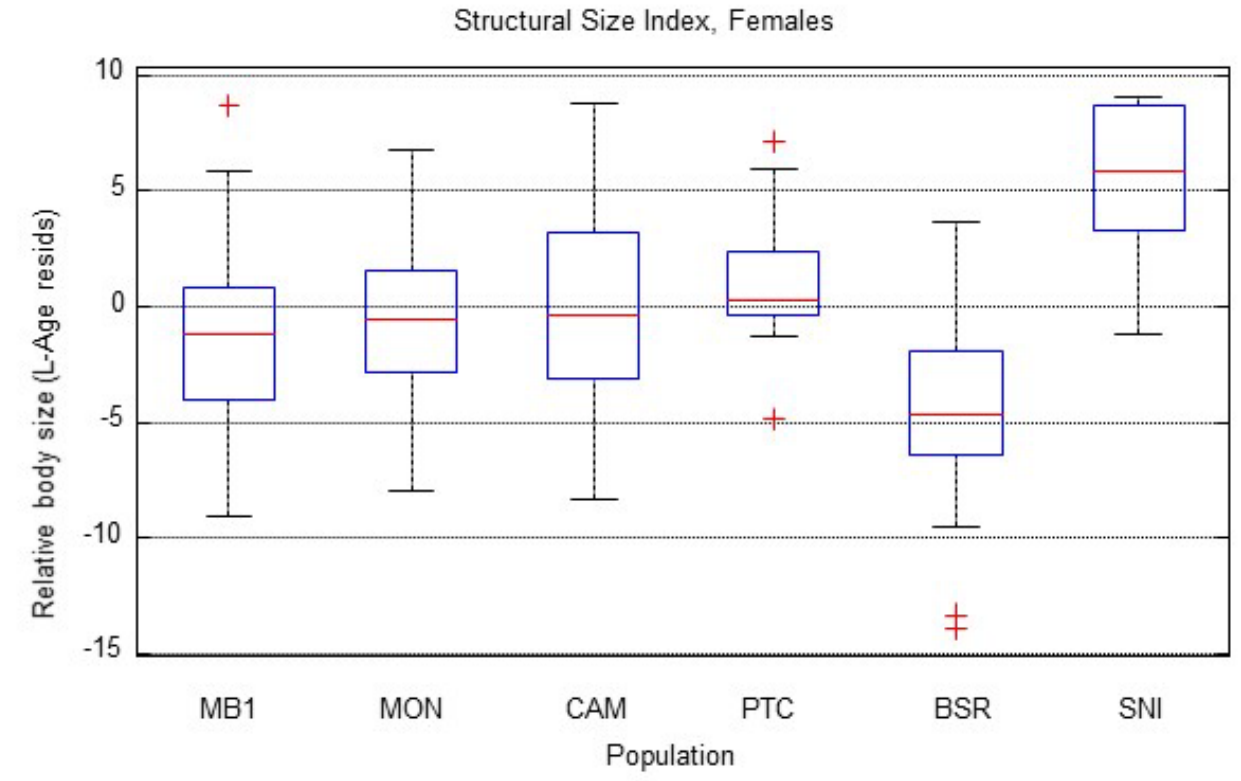

Structural Size Index, Males

B)

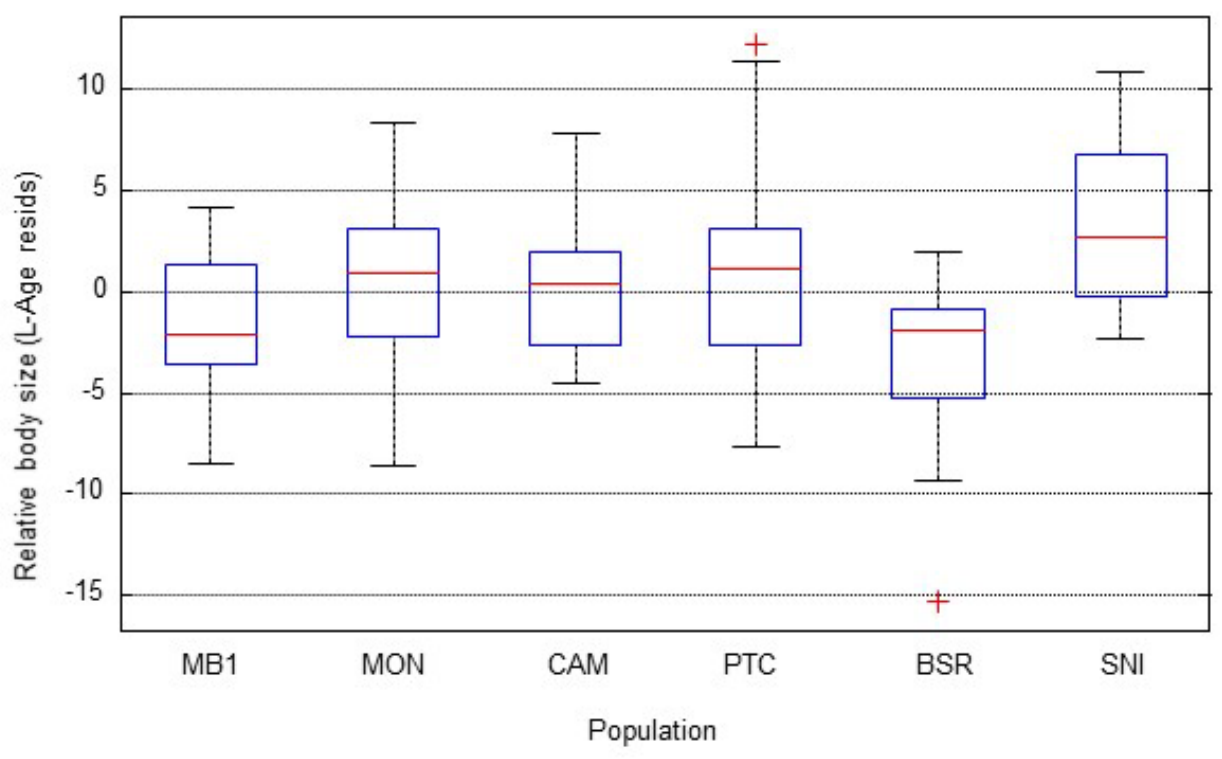

Figure 40. Boxplots showing residuals from length compared to age growth curves (see figure 38) plotted for sea otters at six different study sites in California. Values represent an index of structural size (SSI). A, SSI values for females. B, SSI values for males. Boxes encompass the interquartile range (IQR), whiskers encompass the full distribution excluding outliers (from the lower quartile minus 1.5X IQR, to the upper quartile plus 1.5X IQR). 
Body Condition Index, Females

A)

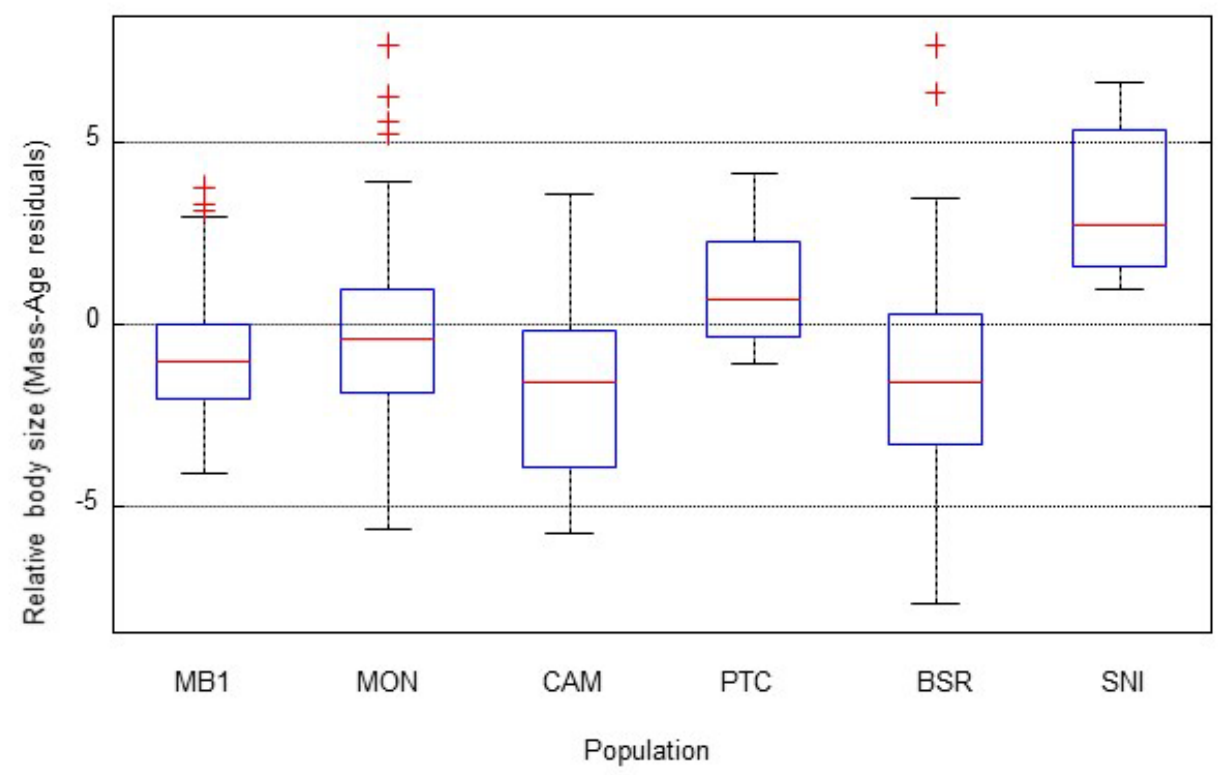

B)

Body Condition Index, Males

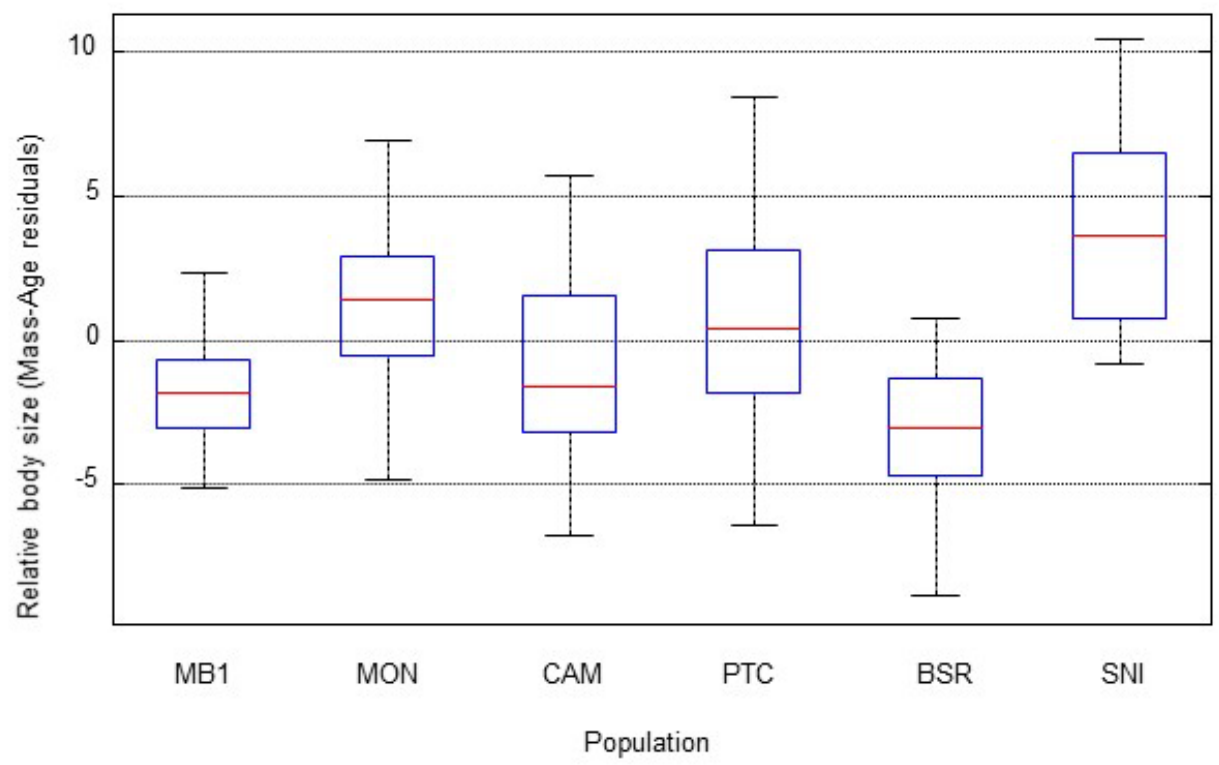

Figure 41. Boxplots showing residuals from mass compared to age growth curves (see figure 39) plotted for sea otters at six different study sites in California. Values represent an index of overall body condition (BCl). $\mathrm{A}, \mathrm{BCl}$ values for females. $\mathrm{B}, \mathrm{BCl}$ values for males. Boxes encompass the interquartile range (IQR), whiskers encompass the full distribution excluding outliers (from the lower quartile minus 1.5X IQR, to the upper quartile plus 1.5X IQR). 
A)

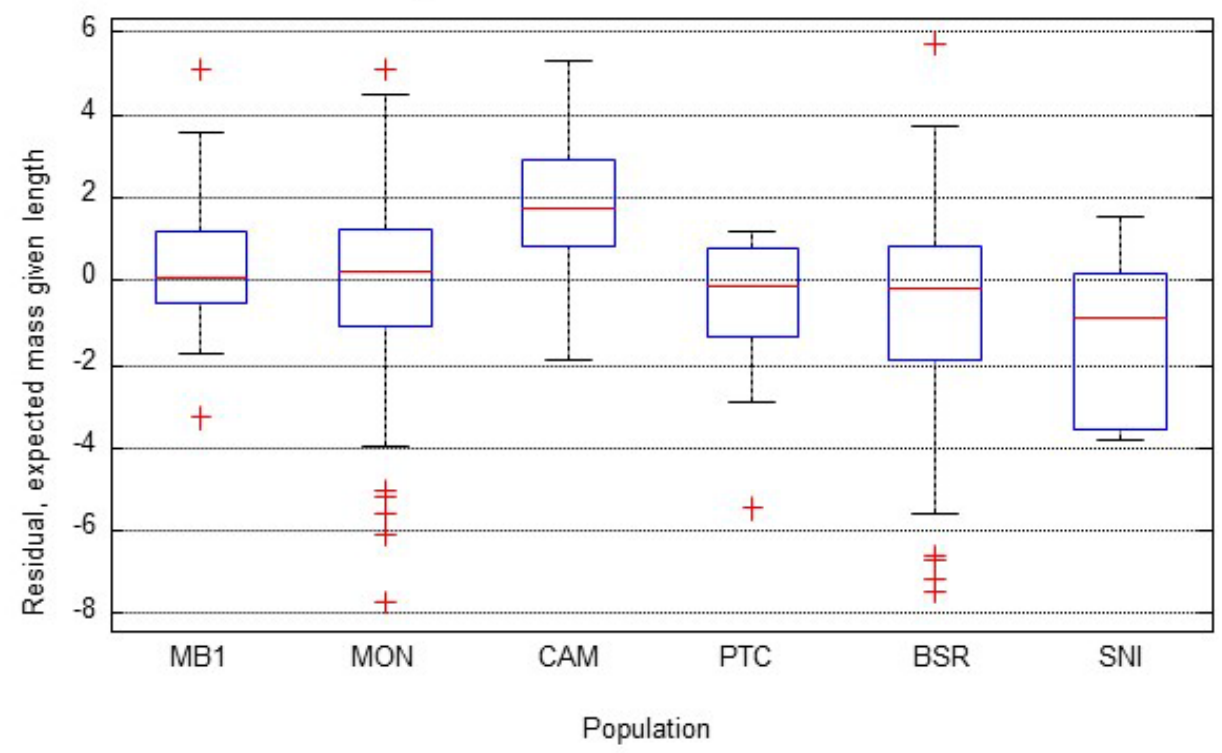

B)

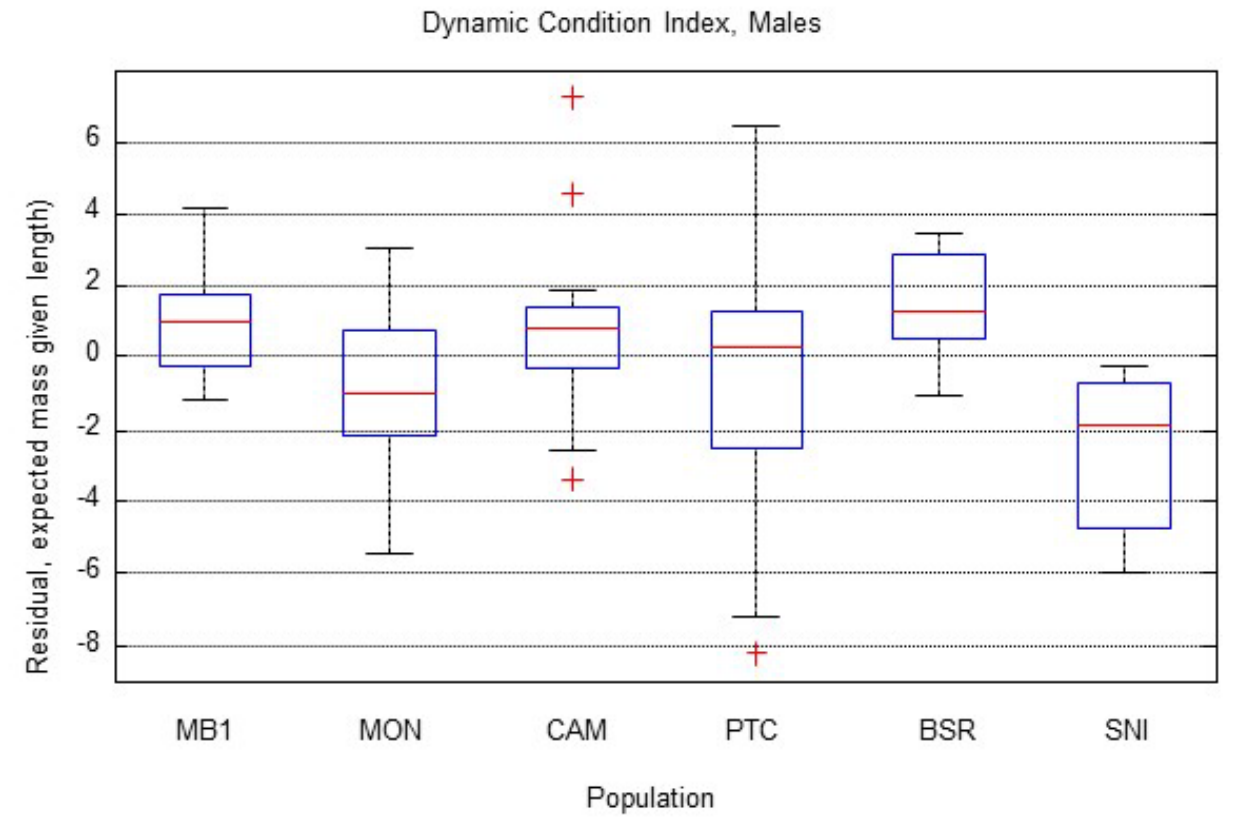

Figure 42. Boxplots showing residuals from a regression of $\mathrm{BCl}$ compared to $\mathrm{SSI}$ values (that is, deviations from the expected mass-at-age compared to Length-at-age relationship) for sea otters at six different study sites in California. Values represent an index of dynamic body condition (DCl). A, DCl values for females. $\mathrm{B}, \mathrm{DCl}$ values for males. Boxes encompass the interquartile range (IQR), whiskers encompass the full distribution excluding outliers (from the lower quartile minus 1.5X IQR, to the upper quartile plus 1.5X IQR). 


\section{Body Condition vs. Foraging Success}

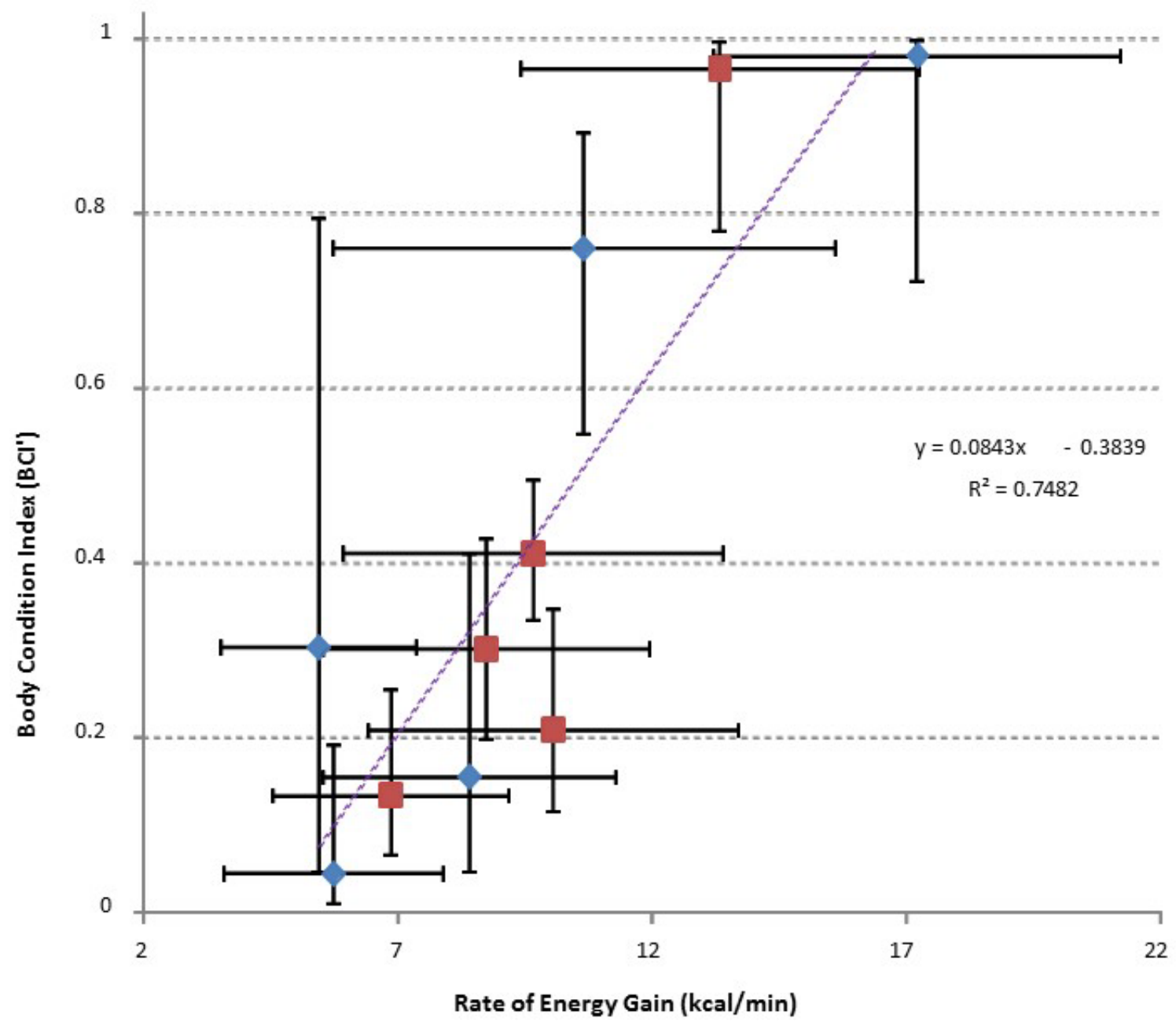

Figure 43. Regression plot showing average logit-transformed body condition index (BCl') compared to average estimated rate of energy gain (in kilocalories per minute [kcal/min]) for male and female sea otters at five sites in California. A sixth site, Point Conception, could not be plotted because there were insufficient foraging data to estimate rate of energy gain. Error bars represent 95-percent confidence intervals. 


\title{
Chapter 8. Survival and Reproduction
}

\author{
M. Tim Tinker ${ }^{1,2}$, Michelle Staedler ${ }^{3}$, Gena Bentall ${ }^{1,3}$, and Joseph A. Tomoleoni ${ }^{1}$
}

\section{Introduction}

One of the central components of the comparative study of sea otter populations at the Big Sur coast (BSR) and Monterey Peninsula (MON) study sites is the evaluation of survival rates at each site. Survival analyses often are at the heart of radio-tagging wildlife studies, providing biologists with an understanding of demographic drivers of population change, and insights into population viability and the degree to which various putative risk factors of concern to managers (such as anthropogenic or natural stressors) translate into demographically significant effects (Caughley, 1966; Pollock and others, 1990; Beissinger and McCullough, 2002). By measuring and comparing survival rates of different age and sex classes, as well as reproductive success, it is possible to gain insights into key factors that may be limiting recovery of a population, by taking advantage of distinctive demographic patterns associated with various risk factors. For example, broadly occurring stressors such as anthropogenic or naturally occurring toxins without clear point sources (for example, widespread algal bloom events) might be expected to affect all age/sex classes; in contrast, acute point sources of mortality generally are spatially (and [or] temporally) distinct, whereas prey-mediated threats will disproportionately affect the age/sex classes that use that prey type (for example, parasites occurring in small/shallow prey such as sand crabs are most likely to affect the juvenile sea otters that regularly consume those prey; Mayer and others, 2003).

In many species, density-dependent mortality also tends to be focused on certain age-sex classes. In sea otters, density-dependent variation in mortality and reproduction is somewhat unusual as compared to patterns typical of many other mammals; specifically, birth rates are largely invariant, whereas survival of pups and certain older age classes tend to vary as a function of resource abundance (Monson and others, 2000; Tinker and others, 2006; Chinn and others, 2016). This pattern seems to indicate a bet-hedging strategy, whereby reproductive-age females tend to produce pups each year irrespective of body condition, but successful weaning of those pups tends to be strongly dependent on the condition of the mother and, thus, food availability (Monson and others, 2000), indicating the high energetic costs of pup rearing after birth (Thometz and others, 2014). Density-dependent mortality of some older animals also is variable among age/sex classes; in northern areas, this mortality tends to be focused primarily on juveniles and aged adults in late winter (Bodkin and others, 2000), whereas in California, it seems that reproductive-age females may be especially vulnerable near the end of lactation, when they are experiencing the greatest energetic demands due to pup provisioning and lactation (Chinn and others, 2016). In either case, comparative studies aimed at evaluating effects of density-independent mortality factors (including anthropogenic stressors) must also control for any confounding effects of density-dependent mortality.

\footnotetext{
${ }^{1}$ U.S. Geological Survey.

${ }^{2}$ University of California, Santa Cruz.

${ }^{3}$ Monterey Bay Aquarium.
} 
In the current study, we compared age- and sex-specific survival to help distinguish between alternate hypotheses about factors limiting sea otter population growth in central California. If anthropogenic stressors are a primary driver of population trends, we expect that survival generally would be higher at a "pristine" site (BSR) and lower at a highly impacted site (MON). On the other hand, if population trends are more strongly driven by density-dependent processes associated with resource abundance, we might expect survival rates to be lower at BSR where food resources are seemingly more limiting and body condition is poorer (see chapters 6 and 7). Age- and sex-based differences in survival, as well as differences in reproductive success, also may provide important clues. For example, if point-source factors such as terrestrially derived toxins and parasites are predominant, then we might expect wide-ranging animals (such as adult males) to be most impacted, as these animals have the greatest potential exposure to multiple watersheds (Johnson and others, 2009). Conversely, if density-dependent mortality factors were more important than point-source factors mortality factors, we might expect to see decreases in female weaning success and survival of animals in poor nutritional condition, as well as higher mortality risk for females near the end-of-lactation period (Chinn and others, 2016).

We evaluated vital rates of radio-tagged animals over the monitoring period, allowing us to compare survival and reproductive success between BSR and MON. We also broadened our analyses to include similar data from previous monitoring studies in California conducted by our group (Tinker and others, 2006), as this allowed for more generalized evaluation of patterns. We used a rigorous and flexible Bayesian hierarchical survival model to evaluate sources of variation in survival and reproduction (weaning success), including age/sex differences, reproductive status, relative body condition (a proxy for density-dependent nutritional stress), and extrinsic site-dependent factors independent of these other intrinsic variables. We used our results to evaluate support for two competing hypotheses: (1) Anthropogenic sources of mortality are the most important limiting factors, in which case we expect to see lowest survival at sites and among age/sex classes most exposed to anthropogenic influences; and 2) density-dependent factors are the most limiting factors, in which case we expect to see lowest survival at the longest-occupied and highest-density sites (such as BSR) and among demographic classes most susceptible to resource limitation. We also examine detailed patterns of variation in survival to clarify the importance of specific risk factors.

\section{Methods}

Our analyses were aimed at measuring variation in survival and reproductive success caused by differences in age, sex, reproductive status and mean body condition, as well as site-specific risk factors independent of these intrinsic factors (for example, anthropogenic stressors). We used an instantaneous hazards model for this analysis, treating survival as a continuous process observed at discreet intervals, with the likelihood of survival of an individual animal over a particular time interval given by its cumulative probability of avoiding mortality risks, or hazards, over that interval (Heisey and Patterson, 2006). In essence, we can think of the instantaneous "hazards" at any point in time, $h(t)$, as an approximation of the conditional mortality probability over a short interval. Modeling instantaneous hazards (compared to modeling survival directly) has many biological and mathematical advantages, including the fact that instantaneous hazards are independent of time scale, and lead to simple multiplicative models (so called "proportional hazards models") where the relative levels of mortality risk associated with particular covariates can be estimated as hazard ratios (Heisey and Patterson, 2006; Heisey and others, 2007; Halstead and others, 2012). We used a non-parametric Kaplan-Meier approach (Sinha and Dey, 1997) to estimate instantaneous proportional hazards from staggered-entry monitoring data, and then used these to estimate the contribution of various fixed and random effects to survival rates. 
We used the telemetry-based resighting data from 245 radio-tagged sea otters at six study sites: (1) BSR, Big Sur coast (current study); (2) MON, Monterey Peninsula (current study); (3) CAM, Cambria/San Simeon (2001-04); (4) PTC, Point Conception (2001-04); (5) SNI, San Nicolas Island (2003-05); and (6) MB1, Monterey Bay Area (2000-04 (MON and MB1 samples were spatially similar but temporally distinct, and so were treated as independent study sites). These six sites varied geographically and in terms of relative density, with SNI and PTC representing recently established, low-density populations where prey resources were believed to be abundant during the period of study (Tinker, Bentall, and Estes, 2008), whereas the remaining four sites represented high-density, longestablished sea otter populations. Levels of anthropogenic influence also varied among sites, with BSR, SNI and PTC expected to have low impacts from human activities, and CAM, MB1 and MON expected to have higher impacts. Our exclusion of highly compromised animals from radio-tagging (that is, animals not expected to survive the surgery; see chapters 1 and 2) may introduce a slight bias towards higher-than-otherwise survival rates, as we may have inadvertently filtered out animals likely to die in the first month after tagging. However, because this represented a very small number of animals relative to the entire sample, and these exclusions occurred at all study sites, our sample of animals at each site is representative of the local populations and, thus, any biases are likely to be small.

Our model incorporated fixed effects of age, sex, reproductive status, and mean body condition, as well as random site effects, also referred to as shared frailties (Banerjee and others, 2003; Halstead and others, 2012). Because independent hazards are multiplicative in nature (as with many timedependent biological processes), it is more convenient to formulate hazard models in terms of $\log$ (hazards), which are additive and, thus, lend themselves to the fitting of linear models using maximum likelihood or Bayesian methods. Specifically, over a short time interval $(t=r$ to $t=r+1)$, the cumulative effects of all hazards for an individual of age $i, \operatorname{sex} j$, at site $k$, having covariate values $X_{c}$, and reproductive status $e$ can be estimated by the "log unit cumulative hazard" $\left(\gamma_{r}\right)$, which represents the additive effects of various $\log$ (hazards) within that time interval:

$$
\gamma_{r, i, j, c, e, k}=\gamma_{0}+\rho_{r}+\delta_{i, j}+\beta_{1} X_{c}+\beta_{2} Y_{e}+Z_{k}
$$

where

$\gamma_{0} \quad$ represents the baseline log hazard rate,

$\rho_{r} \quad$ represents the effect of time-varying hazards (that is, the hazard ratio associated with conditions specific to time interval $r$, relative to the baseline mean hazard rate),

$\delta_{i, j} \quad$ represents the effect of age-varying hazards (that is, the hazard ratio associated with animals of age $i$ and sex $j$ relative to the baseline mean hazard rate),

$\beta \quad$ represents a vector of parameter values each corresponding to the fixed-effects of a particular covariate $\left(X_{c}\right.$ and $\left.Y_{e}\right)$, and

$Z_{k} \quad$ represents any additional random effects (or shared frailties) associated with study site $k$ that were not accounted for by any of the fixed-effects already included in the model.

Although residual site differences were treated as random effects in our model, they nonetheless correspond to key hypotheses of our study; if anthropogenic factors are important, then we expect high levels of variance among sites, with differences corresponding to relative levels of anthropogenic influence. Conversely, if density-dependent factors predominate, then we would expect to see little 
residual variance among sites, with most of the variation in survival explained by the fixed effects (age, sex, reproductive status and relative body condition).

The time-varying and age-varying hazards (in equation $11, \rho$ and $\delta$, respectively) were both treated as continuously varying effects, estimated using non-parametric conditional auto-regressive (CAR) methods (Sinha and Dey, 1997; Banerjee and others, 2003). Additional covariates evaluated in equation 11 included body condition and reproductive status. To evaluate the effect of body condition $\left(X_{c}\right)$ on survival, we used individual-specific values for body condition index (BCI, calculated as described in chapter 7) when these data were available, but for otters where a BCI value was not available (for example, for females that were pregnant at time of capture), the otter was assigned the appropriate mean sex- and site-specific body condition index. A negative value of $\beta_{1}$ would correspond to increased hazards for animals with lower-than-average BCI values, and decreased hazards for animals with higher-than-average BCI values. To evaluate the effect of reproductive status $\left(Y_{e}\right)$ on survival, female otters were assigned a score of $0-1$ at each time interval: $0=$ female with no pup or a dependent pup of 1-5 months old, and $1=$ "end-lactation period" status (females with dependent pups $>5$ months old or having weaned a pup within the last 30 days).

In addition to the full model shown in equation 11, we also evaluated simpler models with fewer effects, and used model deviance information criterion (DIC) values to select the most parsimonious model (Spiegelhalter and others, 2002). Because equation 11 is expressed in terms of the log of the cumulative hazards, the baseline instantaneous hazard rate is estimated by $\exp \left(\gamma_{0}\right)$, whereas all other parameters represent the log of a hazard ratio, whereby a parameter value of 0 corresponds to a ratio of 1 (no significant effect), a value of $<0$ corresponds to a ratio of $<1$ or a decrease in hazard rates relative to baseline values, and a value of $>0$ corresponds to a ratio of $>1$ or an increase in hazard rates relative to baseline values.

Although log hazards are convenient for statistical estimation, the metric we are really interested in is the conditional survival probability for a given otter over a specified time period. That is, given that an individual is alive at time $r$, what is the likelihood it will survive until time $T$ ? This probability can be calculated from instantaneous hazards as:

$$
S(T \mid r)=\exp \left[-\left\{\int_{r}^{r+1} h(u) d u+\int_{r+1}^{r+2} h(u) d u+\ldots+\int_{T-1}^{T} h(u) d u\right\}\right]
$$

where each of the integral terms on the right-hand side of equation 12 represents the "unit cumulative hazard" over a short time interval, and can be approximated by a point-estimate of the instantaneous hazard rate:

$$
\int_{r}^{r+1} h(u) d u \cong h(r)=\exp \left(\gamma_{r}\right)
$$

Thus the conditional survival can be calculated from a summation of log unit cumulative hazard values $\left(\gamma_{r}\right.$, as calculated in equation 11) over the time period of interest:

$$
S(T \mid r)=\exp \left[-\sum_{t=r+1}^{T} \exp \left(\gamma_{t}\right)\right]
$$


Bayesian Markov Chain Monte Carlo (MCMC) fitting algorithms (implemented using the MATLAB and JAGS “Just Another Gibbs Sampler" programming environments) were used to fit equations 11 and 14 to interval-censored survival/mortality data from radio-tagged sea otters. Bayesian model fitting was conducted following standard procedures; all parameters were initiated with uninformative parameters, and 10,000 burn-in MCMC iterations were conducted to allow model convergence, before saving 10,000 iterations (thinned by a factor of 3) for evaluation of posterior distributions. We graphically evaluated the traces of three independently initiated MCMC chains and examined $\hat{\mathrm{r}}$ values to ensure model convergence and stability (Brooks and others, 2011).

The Kaplan-Meier analysis of individual survival histories consists of identifying the leftcensored and right-censored entry points for each monitoring record (the date first tagged and the date last observed), as well as the fate at the end of the monitoring period (died, still alive, or disappeared and, thus, unknown fate). Our model was evaluated at time-step intervals of 1 week. Survival outcomes for each otter, over each observed time interval, were represented as random Bernoulli trials with probabilities determined by equation 14, and these comprise the binomial likelihoods maximized by the MCMC algorithm (Heisey and others, 2007).

We present results of the model fitting in terms of posterior distributions of log hazard ratios and the derived annual survival probabilities. The inclusion of terms in the final model was determined by model comparisons using DIC values (Spiegelhalter and others, 2002), and we evaluated the significance of model parameters by determining whether the 95-percent credible intervals (CI95, which correspond loosely to the 95-percent confidence intervals for statistics computed using non-Bayesian methods) excluded 0 , because a value of 0 corresponds to a hazard ratio of 1 (no significant effect). We tested for variation in survival among sites due to "random effects" (differences associated with environmental, ecological, or anthropogenic influences) by evaluating the degree of overlap in the 95percent credible intervals of $Z_{i}$. Because there could still be realized net differences in survival between sites, even in the absence of differences due to random effects (for example, differences caused by differing distributions of covariate values between sites), we also calculated the 95-percent credible intervals for pairwise contrasts of age- and sex-specific survival rates among sites. To evaluate the overall effect of density-dependent variation in body condition on survival rates, we plotted the estimated site-specific adult survival rates as a function of site-specific body condition index values (with separate plots for males and females), and fit a nonlinear power function of the form $y=p 1 \cdot x^{p 2}$. A positive relation between body condition and survival rate would be represented by positive values of parameters $p 1$ and $p 2$ (with the 95-percent confidence interval [CI] for each parameter excluding 0 ).

\section{Reproduction}

We used longitudinal records of observational data from radio-tagged individual females to estimate per-capita birth rates (number of pups born per year per female) and pup survival rates (also called weaning success rates). To estimate birth rates we used the algorithm of Eberhart and Schneider (1994):

$$
\bar{b}=\frac{1}{K} \sum_{k=1}^{K} b_{k}\left(\frac{365}{N_{k}}\right),
$$

where
$K$
is the total number of females monitored for at least 365 days,
$b_{k} \quad$ is the number of observed births observed for female $k$, and
$N_{k} \quad$ is the number of days female $k$ was monitored. 
Because we determined that a significant number of pup births were missed when the pups died soon after birth, we restricted our analysis of birth rates to those females having TDR implants $(\mathrm{n}=25)$, as the TDR records allowed us to reliably detect all birth events (Tinker, Bodkin, and others, 2008). We estimated overall mean birth rate and the 95-percent confidence interval using bootstrap resampling, and also compared individual birth rates between BSR and MON using one-way ANOVA.

To estimate pup survival rates, we used Bayesian MCMC algorithms to fit an instantaneous proportional hazards model similar to the model used to analyze adult survival rates, but modified to estimate the instantaneous hazard rate over a 20-week pup dependency period (we assumed that pups that survived to 20 weeks were successfully weaned; Siniff and Ralls, 1991). This approach allowed us to measure variation in mortality risk over the course of the pup dependency period, and also to include risk covariates thought to affect pup survival probability (specifically, the age of the mother, body condition, and site-specific random effects). In this model, pup age was substituted for time, and expressed in units of days since the birth of the pup ( $t=0$ at the instant of birth). We estimated $\log$ (unit cumulative hazards) over the interval $t=d$ to $t=d+1$ as:

$$
\gamma_{d, a, c, k}=\theta_{d}+\varphi_{a}+\beta_{3} X_{c}+Z_{k},
$$

where

$\theta_{d} \quad$ represents baseline hazards assumed to vary with pup age,

$\varphi_{a} \quad$ represents the effect of the age of the mother (that is, the hazard ratio associated with having a mother of $a$ years of age, scaled relative to the baseline hazard rate for a 3year-old mother),

$\beta_{3} \quad$ is the parameter corresponding to the effect of the body condition of the mother, and $\left(X_{c}\right)$, and $Z_{k} \quad$ represents any additional random effects (or shared frailties) associated with study site $k$ that were not accounted for by the fixed-effects included in the model.

The body condition of the mother was assessed based on her relative mass-at-age (BCI, calculated as described in chapter 7), averaged across all capture events for that female. A negative value of $\beta$ would correspond to increased hazards for mothers with lower-than-average BCI values, and decreased hazards for mothers with higher-than-average BCI values. The age-varying hazards $(\theta$ and $\varphi)$ were treated as continuously varying effects, estimated using non-parametric conditional auto-regressive (CAR) methods (Sinha and Dey, 1997; Banerjee and others, 2003).

The pup survival model was evaluated at time-step intervals of 1 day, and survival outcomes for each pup, over each time step, were represented as random Bernoulli trials with conditional probabilities given by:

$$
S(T \mid d)=\exp \left[-\sum_{t=d+1}^{T} \exp \left(\gamma_{t}\right)\right]
$$

Methods of Bayesian model fitting, evaluation, and statistical assessment of parameter significance were identical to the procedures or analysis of independent otter survival. We used data from the BSR, MON, MB1, CAM and SNI study sites. We plotted the mean weaning success rate as a continuous function of the age and body condition of the mother, and also compared average weaning success rates across sites. 


\section{Results}

\section{Survival}

All model runs converged within 10,000 iterations, with well-conditioned posterior sample traces and $\hat{r}$ values less than 1.1. Evaluation of DIC values indicated a lack of support for inclusion of the continuous time-varying effect $\left(\rho_{r}\right)$, but all other parameters were included in the best-supported model. A summary of parameter estimates for the $\log$ (hazard) model (equation 11) is provided in table 26.

Hazards associated with age and sex differences (CAR parameters $\delta_{i, j}$ ) varied, which resulted in "inverted-U" type survival curves for males and females that are typical for sea otters and other large mammals (fig. 44). The other covariates included in the model, body condition (relative mass-at-age) and reproductive status (for females), both had significant impacts on hazard rates (table 26, fig. 45). The lower the mean body condition index, the greater the hazard rate, such that a decrease in body condition from "average" to "very poor" (for example, CAM females) was associated with a 1.9× increase in hazard rate ( $\left.\mathrm{CI}_{95} 1.2-3.1\right)$, whereas an increase in body condition from average to very good (for example, SNI females) was associated with a two-thirds decrease in hazard rate (CI95 0.13-0.77). This effect was evident as a positive relation between body condition and survival rates across all study sites for females and males (fig. 46). For all study sites except SNI and PTC there also was a 3.6× increase in hazard rate (CI95 1.9-6.3) for females when they were in "end-lactation" status.

After accounting for all fixed-effect covariates (age, sex, body condition, and reproductive status), there was no statistically significant variation in hazard rates (based on overlap of 95-percent credible intervals) associated with random effects among study sites (table 26), although MB1 tended towards slightly lower hazard rates whereas CAM tended towards slightly higher hazard rates (fig. 47). When the full model was evaluated at each site, survival differed significantly between sites (figs. 48 and 49), but these were primarily driven by the differences in mean body condition and the proportion of end-lactation status females at each study site. Survival rates of adult females at Big Sur tended to be slightly lower than adult females at Monterey in the current study, a difference that was marginally significant (the 90-percent credible interval, CI, did not include zero, although the $95 \%$ CI did include 0 ; fig. 48B), and this pattern was even more evident for adult males (95\% CI for the difference between survival rates did not include 0; fig. 49B). A summary of adult survival rate estimates for females and males is provided in table 27 , and table 28 shows the mean annual age-specific survival rate estimates for females and males at each site. 


\section{Reproduction}

Based on the number of pups born to TDR-implanted females $(n=25)$ over the course of their monitoring period (1-3 years), the mean birth rate for adult females at both sites was 0.97 (95-percent $\mathrm{CI}=0.77-1.19)$. There was no significant difference in female birth rates between Big Sur and Monterey $(\mathrm{F}=0.97, \mathrm{df}=24, \mathrm{P}=0.335)$.

Our analysis of pup mortality patterns was based on the observed survival outcomes for 413 pups born to study animals between 2001 and 2012 at five study sites. The probability of pup mortality varied as a function of pup age, as has been previously reported (Riedman and others, 1994; Monson and others, 2000; Tinker and others, 2006), although the CAR model we used allowed for a more detailed examination of the nature of this variation (fig. 50). The instantaneous hazard rate was highest immediately after birth (when many new-born pups are abandoned), decreased sharply over the next week but then increased again from 3 to 6 weeks of age, and then decreased to low values for the period between 6 and 14 weeks of age. After about 14 weeks of age, the hazard rate again began to increase continuously until the time of weaning (which generally occurs between 20 and 25 weeks of age).

The degree of mortality risk experienced by pups was affected by two factors: (1) the age of the mother of the pup, and (2) the relative body condition of the mother of the pup. The pup hazard ratio dropped significantly for pups with mothers that were 8 years or older (fig. 51), whereas pups whose mothers were in good body condition (, for example, SNI females, mean BCI $=3.95$ ) had a 30-percent decrease in hazards $\left(\mathrm{CI}_{95}=2\right.$ - 49) relative to pups whose mothers were in average condition (fig. 52). As a result of these effects, average weaning success rates increased from 0.64 for 3 -year-old mothers to 0.71 for 10 -year-old mothers (fig. 53A), and likewise increased from 0.59 for mothers in poor condition $(\mathrm{BCI}=-0.3)$ to 0.73 for mothers in good condition $(\mathrm{BCI}=0.3$; fig. 52B). There were no significant differences in pup hazard rates associated with random effects among sites; however, variation in mean female body condition among sites (chapter 7) resulted in some differences in realized weaning success. The weaning success rate for females at Big Sur was slightly lower than for females at Monterey (mean difference $=-.01$ ), although this difference was only marginally significant (fig. 54). Age-specific weaning success rates for females at Big Sur and Monterey are provided in table 29. 


\section{Discussion}

The analysis of survival and reproduction rates of tagged study animals monitored in the current study and previously conducted telemetry studies in California revealed interesting patterns and provide key insights into the factors affecting population growth in central California. The Bayesian-based proportional hazards analysis produced estimates of age-specific survival that were consistent with previously published sea otter vital rates (Tinker and others, 2006), but allowed for a greater flexibility in examining the contributions of various fixed and random effects to variation in survival. This was important, as it allowed us to evaluate predictions associated with various hypotheses about factors limiting sea otter population growth in central California. In particular, if anthropogenic stressors were a strong driver of population trends in central California, we would expect higher survival at the "pristine" site (BSR) and lower survival at the highly impacted site (MON); additionally, we would expect variation in hazard rates among sites that would correspond to the magnitude of environmental or anthropogenic stressors at these and other sites. The first prediction was not supported by the data: that is, survival rates generally were lower at BSR than at MBA (figs. 48 and 49). The second prediction was not strongly supported by the data either, although there was some suggestion of variation among sites (marginally significant) beyond that explained by body condition and reproductive status (fig. 47). Specifically, otters at the MB1 site had slightly lower hazard rates whereas otters at CAM had slightly higher hazard rates. This is interesting in light of earlier epidemiological analyses of study animals at these two sites, which indicated higher Toxoplasma gondii infection rates among sea otters at CAM than at MB1 (Johnson and others, 2009), and also elevated Sarcocystus neurona infection rates at CAM (Miller and others, 2010), suggesting the possibility that parasite exposure may have been contributing to differing survival rates at these sites in the early 2000s (Miller and others, 2002). However, no significant differences were found between BSR and MON with respect to site-specific hazard rates in the current study (beyond those associated with body condition and reproductive status), a pattern consistent with the similarity in parasite infection rates between these two areas (chapter 9).

An alternative scenario to the one described in the paragraph above is that population trends are driven largely by density-dependent processes associated with resource abundance. Under this scenario, we would expect survival rates to be lower where food resources were more limiting and body condition (defined as relative body mass after controlling for sex and age differences) was poorer. This prediction was well supported by the data, with a significant positive relation between survival rates and mean body condition index across all sites, for both males and females (fig. 46). The inclusion of body condition as a covariate in the model explained much of the variation in survival rates (fig. 45A). Moreover, the elevated mortality risk associated with the end-lactation period for females in high density populations (fig. 45B) also is suggestive of the contribution of nutritional/energetic stress to elevated mortality and, thus, lower population growth. Females incur high energetic costs associated with pup rearing and provisioning (Thometz and others, 2014), leading to increased foraging effort during this period (chapter 5) and leaving them highly susceptible to any number of stressors they encounter when they come into estrous after weaning, such as parasite infections or aggression by males (chapter 10). These results highlight important factors that affect survival probabilities, a key step in identifying the ultimate drivers of population growth, but they do not shed light on specific proximate mechanisms of mortality. Detailed analysis of cause-of-death in individual animals is required for this latter step, and the interaction between poor body condition associated with end-lactation period and exposure to environmental stressors is clearly evident in the necropsy results reported in chapter 10. 
Patterns of variation in pup survival were largely consistent with the results noted for adult survival, in that female body condition was an important predictor of weaning success (fig. 53). The age of the mother also was important, likely indicative of the fact that more experienced females were more likely to successfully navigate the challenges of pup rearing (Riedman and others, 1994; Monson and others, 2000; Tinker and others, 2006). Variation in the magnitude of the hazard rate over the course of the pup dependency period (fig. 50) was consistent with expectations of life history theory (Monson and others, 2000). Specifically, females are expected to make a decision about whether to go through with pup rearing or to abandon the pup based on their own body condition at parturition; this corresponds to the high hazard rate in the weeks immediately after pup birth. Once they have committed to pup rearing, the hazard rate is expected to drop, but as females near the end of the pup dependency, the energetic load of pup provisioning may push some females beyond their metabolic and behavioral capacity to respond, resulting in a secondary increase in the hazard rates. These predicted patterns are well borne out by our results (fig. 50).

Combining all our estimates of birth rates and survival rates of pups and independent otters, we calculated the expected population growth rates at Big Sur and Monterey using a projection matrix (following methods described in Tinker and others, 2006), and compared these rates with the observed trends at each site from 2006 to 2010, based on census data (https://www.sciencebase.gov/catalog/item/5a32d390e4b08e6a89d88583 ). At Big Sur, the growth rate predicted by a matrix model parameterized with vital rate estimates from our analysis of tagged otters was -3.0-percent, very similar to the observed trend of -2.5-percent. At Monterey, the predicted growth rate was 4.5-percent, somewhat higher than the observed trend of 2.4-percent (although well within the range of parameter uncertainty). Overall, our results successfully predict the direction and general magnitude of population trends at the two sites over the study period, suggesting that the measured rates of reproduction and survival of study animals were representative of the vital rates of other otters in the population.

Variation in sea otter survival rates across study sites in California points to the role of densitydependent resource limitation in driving population trends in central California. Our results do not preclude the possibility that anthropogenic influences may contribute to overall mortality in less obvious ways; for example, environmental stressors introduced by human activities may increase the net health impacts experienced by females during end-lactation. However, the spatial patterns in survival reported here suggest that any such synergistic effects between anthropogenic stressors and resource-abundance are not evident in terms of geographic proximity to areas of high human impact, and, thus, likely are not tied to point-source inputs of pollution. To the contrary, age-specific hazards for both adults and pups actually were highest at the "low human impact" Big Sur site. Certain types of anthropogenic influences are indeed spatially diffuse; for example, terrestrial inputs of nitrogen-based fertilizers and other organic pollutants have been found to lead to increased frequency and intensity of toxic algal blooms, including those resulting in domoic acid intoxication (Mos, 2001), and these blooms occur across a broad geographic range and are not limited to areas immediately adjacent to the nutrient inputs (Lefebvre and others, 2002). These types of widespread and non-spatially explicit risk factors are beyond the scope of our current study to evaluate and would need to be investigated by other approaches, such as comparisons between California and other regions. In the current study, although we cannot rule out the possibility of widespread anthropogenic impacts on survival, our results point towards a clear pattern of density-dependent population regulation. Specifically, the strong effects of lowered body condition on hazard rates suggest that population abundance in areas of the highest sea otter densities (including both BSR and MON) likely is approaching the environmental carrying capacity, and, thus, continued recovery of the population will depend on range expansion and population growth in areas of lower density. 


\section{References Cited}

Banerjee, S., Wall, M.M., and Carlin, B.P., 2003, Frailty modeling for spatially correlated survival data, with application to infant mortality in Minnesota: Biostatistics, v. 4, p. 123-142.

Beissinger, S.R. and McCullough, D.R., eds., 2002, Population viability analysis: Chicago, University of Chicago Press, 593 p.

Bodkin, J.L., Burdin, A.M., and Ryazanov, D.A., 2000, Age- and sex-specific mortality and population structure in sea otters: Marine Mammal Science, v. 16, p. 201-219.

Brooks, S., Gelman, A., Jones, G., and Meng, X.-L., 2011, Handbook of Markov Chain Monte Carlo: Boca Raton, Florida, Taylor and Francis, 619 p.

Caughley, G., 1966, Mortality patterns in mammals: Ecology, v. 47, p. 906-918.

Chinn, S.M., Miller, M.A., Tinker, M.T., Staedler, M.M., Batac, F.I., Dodd, E.M., and Henkel, L.A., 2016, The high cost of motherhood-End-lactation syndrome in southern sea otters (Enhydra lutris nereis) on the central California, USA, coast: Journal of Wildlife Diseases, v. 52, no. 2, p. 307-315.

Halstead, B.J., Wylie, G.D., Coates, P.S., Valcarcel, P., and Casazza, M.L., 2012, Bayesian shared frailty models for regional inference about wildlife survival: Animal Conservation, v. 15, p. 117-124.

Heisey, D.M., and Patterson, B.R., 2006, A review of methods to estimate cause-specific mortality in presence of competing risks: Journal of Wildlife Management, v. 70, p. 1544-1555.

Heisey, D.M., Shaffer, T.L., and White. G.C., 2007, The ABCs of nest survival-Theory and application from a biostatistical perspective: Studies in Avian Biology, v. 34, p. 13-33.

Johnson, C.K., Tinker, M.T., Estes, J.A., Conrad, P.A., Staedler, M., Miller, M.A., Jessup, D.A., and Mazet, J.A.K., 2009, Prey choice and habitat use drive sea otter pathogen exposure in a resourcelimited coastal system: Proceedings of the National Academy of Sciences of the United States of America, v. 106, p. 2242-2247.

Lefebvre, K.A., Bargu, S., Kieckhefer, T., and Silver, M.W. ,2002, From sanddabs to blue whales-The pervasiveness of domoic acid: Toxicon, v. 40, p. 971-977.

Mayer, K.A., Dailey, M.D., and Miller, M.A., 2003, Helminth parasites of the southern sea otter Enhydra lutris nereis in central California-Abundance, distribution and pathology: Diseases of Aquatic Organisms, v. 53, p. 77-88.

Miller, M.A., Conrad, P.A., Harris, M., Hatfield, B., Langlois, G., Jessup, D.A., Magargal, S.L., Packham, A.E., Toy-Choutka, S., Melli, A.C., Murray, M.A., Gulland, F.M., and Grigg, M.E., 2010, A protozoal-associated epizootic impacting marine wildlife-Mass-mortality of southern sea otters (Enhydra lutris nereis) due to Sarcocystis neurona infection: Veterinary Parasitology, v. 172, p. 183194.

Miller, M.A., Gardner, I.A., Kreuder, C., Paradies, D.M., Worcester, K.R., Jessup, D.A., Dodd, E., Harris, M.D., Ames, J.A., Packham, A.E., and Conrad, P.A., 2002, Coastal freshwater runoff is a risk factor for Toxoplasma gondii infection of southern sea otters (Enhydra lutris nereis): International Journal for Parasitology, v. 32, p. 997-1006.

Monson, D.H., Estes, J.A., Bodkin, J.L., and Siniff, D.B., 2000, Life history plasticity and population regulation in sea otters: Oikos, v. 90, p. 457-468.

Mos, L., 2001, Domoic acid-A fascinating marine toxin: Environmental Toxicology and Pharmacology, v. 9, p. 79-85.

Pollock, K.H., Nichols, J.D., Brownie, C., and Hines, J.E., 1990, Statistical inference for capturerecapture experiments: Wildlife Monographs, v. 107, p. 1-98.

Riedman, M.L., Estes, J.A., Staedler, M.M., Giles, A.A., and Carlson, D.R., 1994, Breeding patterns and reproductive success of California sea otters: Journal of Wildlife Management, v. 58, p. 391-399. Sinha, D., and Dey, D.K., 1997, Semiparametric Bayesian analysis of survival data: Journal of the American Statistical Association, v. 92, p. 1195-1212. 
Siniff, D.B., and Ralls, K., 1991, Reproduction, survival and tag loss in California sea otters: Marine Mammal Science, v. 7, p. 211-229.

Spiegelhalter, D.J., Best, N.G., Carlin, B.P., and Van Der Linde, A., 2002, Bayesian measures of model complexity and fit: Journal of the Royal Statistical Society-Series B (Statistical Methodology), v. 64, p. 583-639.

Thometz, N.M., Tinker, M.T., Staedler, M.M., Mayer, K.A., and Williams, T.M., 2014, Energetic demands of immature sea otters from birth to weaning-Implications for maternal costs, reproductive behavior and population-level trends: Journal of Experimental Biology, v. 217, p. 2053-2061.

Tinker, M.T., Bentall, G., and Estes, J.A., 2008, Food limitation leads to behavioral diversification and dietary specialization in sea otters: Proceedings of the National Academy of Sciences of the United States of America, v. 105, p. 560-565.

Tinker, M.T., Bodkin, J., Staedler, M.M., Esslinger, G., and Monson, D.H., 2008, Using TDR records to detect life history events in sea otters: Third International Biologging Science Symposium, September 1-5, 2008, Pacific Grove, California.

Tinker, M.T., Doak, D.F., Estes, J.A., Hatfield, B.B., Staedler, M.M., and Bodkin, J.L., 2006, Incorporating diverse data and realistic complexity into demographic estimation procedures for sea otters: Ecological Applications, v. 16, p. 2293-2312. 
Table 26. Parameter estimates for $\log$ (hazard) function from Bayesian survival analysis.

[See body text, equation 8 . Note that $\exp \left(\gamma_{0}\right)$ represents $\log ($ baseline instantaneous hazards), whereas all other parameters represent $\log$ (hazard ratios). Std. dev: Standard deviation. CI_95_L: 95-percent confidence interval (low). CI_95_H: 95percent confidence interval (high)]

\begin{tabular}{|c|c|c|c|c|c|}
\hline Parameter & Description & Mean & Std. dev & Cl_95_L & Cl_95_H \\
\hline$\gamma_{0}$ & Baseline $\log$ hazards ( $=5$-year-old female) & -3.238 & 1.982 & -7.251 & 0.209 \\
\hline$\beta(1)$ & Body condition effect & -0.327 & 0.133 & -0.600 & -0.078 \\
\hline$\beta(2)$ & Reproductive status effect (end lactation) & 1.268 & 0.299 & 0.661 & 1.837 \\
\hline $\mathrm{Z}(1)$ & Random site effect, Big Sur, 08-12 & -0.006 & 0.321 & -0.702 & 0.655 \\
\hline$Z(2)$ & Random site effect, Monterey, 08-12 & -0.113 & 0.294 & -0.751 & 0.439 \\
\hline $\mathrm{Z}(3)$ & Random site effect, Monterey 01-04 & -0.443 & 0.346 & -1.195 & 0.048 \\
\hline $\mathrm{Z}(4)$ & Random site effect, Cambria, 01-04 & 0.324 & 0.323 & -0.205 & 1.030 \\
\hline $\mathrm{Z}(5)$ & Random site effect, Pt. Conception 01-04 & 0.145 & 0.366 & -0.500 & 0.981 \\
\hline $\mathrm{Z}(6)$ & Random site effect, San Nicolas I. 03-05 & 0.070 & 0.490 & -0.862 & 1.156 \\
\hline$\delta(1,1)$ & Age-specific hazards, females age 1 & -0.187 & 2.139 & -4.077 & 4.056 \\
\hline$\delta(2,1)$ & Age-specific hazards, females age 2 & -1.259 & 2.019 & -4.823 & 2.753 \\
\hline$\delta(3,1)$ & Age-specific hazards, females age 3 & -2.055 & 1.988 & -5.525 & 1.845 \\
\hline$\delta(4,1)$ & Age-specific hazards, females age 4 & -2.468 & 1.976 & -5.921 & 1.393 \\
\hline$\delta(5,1)$ & Age-specific hazards, females age 5 & -2.492 & 1.962 & -5.913 & 1.369 \\
\hline$\delta(6,1)$ & Age-specific hazards, females age 6 & -2.248 & 1.954 & -5.667 & 1.650 \\
\hline$\delta(7,1)$ & Age-specific hazards, females age 7 & -1.755 & 1.948 & -5.142 & 2.151 \\
\hline$\delta(8,1)$ & Age-specific hazards, females age 8 & -1.537 & 1.942 & -4.909 & 2.386 \\
\hline$\delta(9,1)$ & Age-specific hazards, females age 9 & -1.286 & 1.940 & -4.650 & 2.617 \\
\hline$\delta(10,1)$ & Age-specific hazards, females age 10 & -0.960 & 1.936 & -4.293 & 2.945 \\
\hline$\delta(11,1)$ & Age-specific hazards, females age 11 & -0.931 & 1.937 & -4.286 & 2.941 \\
\hline$\delta(12,1)$ & Age-specific hazards, females age 12 & -0.698 & 1.939 & -4.051 & 3.188 \\
\hline$\delta(13,1)$ & Age-specific hazards, females age 13 & -0.486 & 1.939 & -3.856 & 3.372 \\
\hline$\delta(14,1)$ & Age-specific hazards, females age 14 & -0.247 & 1.949 & -3.655 & 3.592 \\
\hline$\delta(15,1)$ & Age-specific hazards, females age 15 & -0.029 & 1.975 & -3.598 & 3.675 \\
\hline$\delta(16,1)$ & Age-specific hazards, females age 16 & 0.119 & 2.055 & -3.703 & 3.869 \\
\hline$\delta(17,1)$ & Age-specific hazards, females age 17 & 0.048 & 2.225 & -4.347 & 3.991 \\
\hline$\delta(18,1)$ & Age-specific hazards, females age 18 & -0.097 & 2.570 & -5.475 & 4.390 \\
\hline$\delta(1,2)$ & Age-specific hazards, males age 1 & 0.084 & 2.049 & -3.545 & 4.148 \\
\hline$\delta(2,2)$ & Age-specific hazards, males age 2 & -0.748 & 2.003 & -4.319 & 3.178 \\
\hline$\delta(3,2)$ & Age-specific hazards, males age 3 & -1.265 & 2.039 & -4.979 & 2.678 \\
\hline$\delta(4,2)$ & Age-specific hazards, males age 4 & -1.614 & 2.081 & -5.455 & 2.267 \\
\hline$\delta(5,2)$ & Age-specific hazards, males age 5 & -1.561 & 2.031 & -5.275 & 2.264 \\
\hline$\delta(6,2)$ & Age-specific hazards, males age 6 & -1.206 & 1.962 & -4.726 & 2.720 \\
\hline$\delta(7,2)$ & Age-specific hazards, males age 7 & -0.900 & 1.936 & -4.322 & 3.027 \\
\hline$\delta(8,2)$ & Age-specific hazards, males age 8 & -1.019 & 1.941 & -4.427 & 2.959 \\
\hline$\delta(9,2)$ & Age-specific hazards, males age 9 & -1.243 & 1.958 & -4.672 & 2.759 \\
\hline$\delta(10,2)$ & Age-specific hazards, males age 10 & -1.458 & 1.978 & -4.925 & 2.500 \\
\hline$\delta(11,2)$ & Age-specific hazards, males age 11 & -1.554 & 1.989 & -5.057 & 2.365 \\
\hline$\delta(12,2)$ & Age-specific hazards, males age 12 & -1.439 & 1.981 & -4.943 & 2.529 \\
\hline
\end{tabular}




\begin{tabular}{cccccc}
\hline Parameter & Description & Mean & Std. dev & Cl_95_L & Cl_95_H \\
\hline$\delta(13,2)$ & Age-specific hazards, males age 13 & -1.211 & 1.975 & -4.733 & 2.805 \\
$\delta(14,2)$ & Age-specific hazards, males age 14 & -1.113 & 1.989 & -4.675 & 2.947 \\
$\delta(15,2)$ & Age-specific hazards, males age 15 & -1.176 & 2.037 & -4.849 & 3.009 \\
$\delta(16,2)$ & Age-specific hazards, males age 16 & -1.426 & 2.169 & -5.394 & 2.955 \\
$\delta(17,2)$ & Age-specific hazards, males age 17 & -1.743 & 2.473 & -6.687 & 3.044 \\
$\delta(18,2)$ & Age-specific hazards, males age 18 & -2.059 & 3.044 & -8.699 & 3.466 \\
\hline
\end{tabular}

Table 27. Adult (5-year-old) annual survival rate estimates for females (top) and males (bottom) at 6 study sites.

[Std. dev: Standard deviation. CI_95_L: 95-percent confidence interval (low). CI_95_H: 95-percent confidence interval (high). n/a, not applicable]

\begin{tabular}{llcccc}
\hline Sex & \multicolumn{1}{c}{ Study site } & Mean & Std. dev & Cl_95_L & Cl_95_H \\
\hline \multirow{2}{*}{ Females } & Big Sur, 08-12 & 0.925 & 0.028 & 0.863 & 0.969 \\
& Monterey, 08-12 & 0.950 & 0.018 & 0.909 & 0.980 \\
& Monterey 01-04 & 0.957 & 0.019 & 0.913 & 0.984 \\
& Cambria, 01-04 & 0.878 & 0.044 & 0.781 & 0.948 \\
& Point Conception 01-04 & $\mathrm{n} / \mathrm{a}$ & $\mathrm{n} / \mathrm{a}$ & $\mathrm{n} / \mathrm{a}$ & $\mathrm{n} / \mathrm{a}$ \\
& San Nicolas I. 03-05 & 0.983 & 0.012 & 0.951 & 0.997 \\
Males & & & & & \\
& Big Sur, 08-12 & 0.736 & 0.130 & 0.450 & 0.935 \\
& Monterey, 08-12 & 0.932 & 0.036 & 0.849 & 0.984 \\
& Monterey 01-04 & 0.877 & 0.069 & 0.714 & 0.974 \\
& Cambria, 01-04 & 0.818 & 0.081 & 0.639 & 0.950 \\
& Point Conception 01-04 & 0.900 & 0.048 & 0.793 & 0.974 \\
& San Nicolas I. 03-05 & 0.962 & 0.029 & 0.886 & 0.995 \\
\hline
\end{tabular}


Table 28. Mean annual age-specific sea otter survival rate estimates for females (top part of table) and males (bottom part of table) at six California study sites.

[n/a, not applicable]

\begin{tabular}{|c|c|c|c|c|c|c|c|}
\hline \multirow[b]{2}{*}{ Sex } & \multirow[b]{2}{*}{ Age } & \multicolumn{6}{|c|}{ Study area } \\
\hline & & 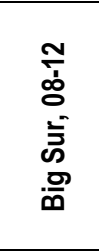 & 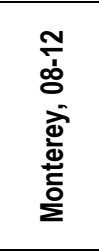 & 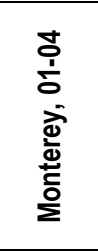 & 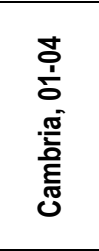 & 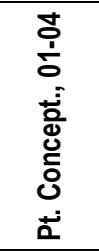 & 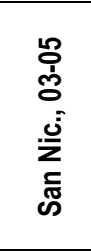 \\
\hline \multirow[t]{18}{*}{ Female } & 1 & 0.682 & 0.779 & 0.810 & 0.636 & $\mathrm{n} / \mathrm{a}$ & 0.929 \\
\hline & 2 & 0.793 & 0.859 & 0.880 & 0.680 & $\mathrm{n} / \mathrm{a}$ & 0.956 \\
\hline & 3 & 0.894 & 0.929 & 0.940 & 0.830 & $\mathrm{n} / \mathrm{a}$ & 0.979 \\
\hline & 4 & 0.928 & 0.953 & 0.960 & 0.884 & $\mathrm{n} / \mathrm{a}$ & 0.986 \\
\hline & 5 & 0.930 & 0.954 & 0.961 & 0.886 & $\mathrm{n} / \mathrm{a}$ & 0.986 \\
\hline & 6 & 0.911 & 0.941 & 0.950 & 0.857 & $\mathrm{n} / \mathrm{a}$ & 0.982 \\
\hline & 7 & 0.859 & 0.906 & 0.920 & 0.777 & $\mathrm{n} / \mathrm{a}$ & 0.971 \\
\hline & 8 & 0.828 & 0.884 & 0.901 & 0.731 & $\mathrm{n} / \mathrm{a}$ & 0.964 \\
\hline & 9 & 0.784 & 0.853 & 0.875 & 0.668 & $\mathrm{n} / \mathrm{a}$ & 0.954 \\
\hline & 10 & 0.715 & 0.803 & 0.831 & 0.572 & $\mathrm{n} / \mathrm{a}$ & 0.937 \\
\hline & 11 & 0.707 & 0.798 & 0.827 & 0.563 & $\mathrm{n} / \mathrm{a}$ & 0.936 \\
\hline & 12 & 0.646 & 0.752 & 0.786 & 0.484 & $\mathrm{n} / \mathrm{a}$ & 0.919 \\
\hline & 13 & 0.583 & 0.703 & 0.743 & 0.408 & $\mathrm{n} / \mathrm{a}$ & 0.901 \\
\hline & 14 & 0.504 & 0.639 & 0.686 & 0.320 & $\mathrm{n} / \mathrm{a}$ & 0.877 \\
\hline & 15 & 0.426 & 0.573 & 0.625 & 0.243 & $\mathrm{n} / \mathrm{a}$ & 0.849 \\
\hline & 16 & 0.372 & 0.524 & 0.580 & 0.194 & $\mathrm{n} / \mathrm{a}$ & 0.827 \\
\hline & 17 & 0.398 & 0.548 & 0.602 & 0.217 & $\mathrm{n} / \mathrm{a}$ & 0.838 \\
\hline & 18 & 0.451 & 0.594 & 0.645 & 0.267 & $\mathrm{n} / \mathrm{a}$ & 0.858 \\
\hline \multirow[t]{18}{*}{ Male } & 1 & 0.635 & 0.749 & 0.627 & 0.628 & 0.645 & 0.870 \\
\hline & 2 & 0.639 & 0.872 & 0.779 & 0.667 & 0.812 & 0.936 \\
\hline & 3 & 0.697 & 0.922 & 0.862 & 0.786 & 0.883 & 0.961 \\
\hline & 4 & 0.775 & 0.944 & 0.900 & 0.843 & 0.916 & 0.973 \\
\hline & 5 & 0.764 & 0.941 & 0.895 & 0.836 & 0.912 & 0.971 \\
\hline & 6 & 0.681 & 0.917 & 0.854 & 0.774 & 0.877 & 0.959 \\
\hline & 7 & 0.594 & 0.889 & 0.807 & 0.706 & 0.837 & 0.945 \\
\hline & 8 & 0.630 & 0.901 & 0.827 & 0.734 & 0.853 & 0.951 \\
\hline & 9 & 0.691 & 0.920 & 0.859 & 0.781 & 0.881 & 0.960 \\
\hline & 10 & 0.742 & 0.935 & 0.885 & 0.820 & 0.903 & 0.968 \\
\hline & 11 & 0.763 & 0.941 & 0.895 & 0.835 & 0.911 & 0.971 \\
\hline & 12 & 0.738 & 0.934 & 0.882 & 0.816 & 0.901 & 0.967 \\
\hline & 13 & 0.683 & 0.918 & 0.855 & 0.775 & 0.877 & 0.959 \\
\hline & 14 & 0.656 & 0.909 & 0.841 & 0.755 & 0.866 & 0.955 \\
\hline & 15 & 0.674 & 0.915 & 0.850 & 0.768 & 0.873 & 0.958 \\
\hline & 16 & 0.735 & 0.933 & 0.881 & 0.814 & 0.900 & 0.967 \\
\hline & 17 & 0.799 & 0.951 & 0.912 & 0.861 & 0.926 & 0.976 \\
\hline & 18 & 0.849 & 0.964 & 0.935 & 0.897 & 0.946 & 0.982 \\
\hline
\end{tabular}


Table 29. Weaning success rates for female sea otters at Big Sur and Monterey study sites.

\begin{tabular}{cll}
\hline $\begin{array}{c}\text { Female } \\
\text { age }\end{array}$ & $\begin{array}{c}\text { Big Sur } \\
\text { (08-12) }\end{array}$ & $\begin{array}{c}\text { Monterey } \\
\text { (08-12) }\end{array}$ \\
\hline 1 & 0 & 0 \\
2 & 0 & 0 \\
3 & 0.624 & 0.636 \\
4 & 0.615 & 0.627 \\
5 & 0.620 & 0.632 \\
6 & 0.652 & 0.663 \\
7 & 0.668 & 0.679 \\
8 & 0.648 & 0.660 \\
9 & 0.640 & 0.652 \\
10 & 0.684 & 0.694 \\
11 & 0.730 & 0.740 \\
12 & 0.758 & 0.767 \\
13 & 0.781 & 0.789 \\
14 & 0.798 & 0.805 \\
15 & 0.804 & 0.810 \\
\hline
\end{tabular}



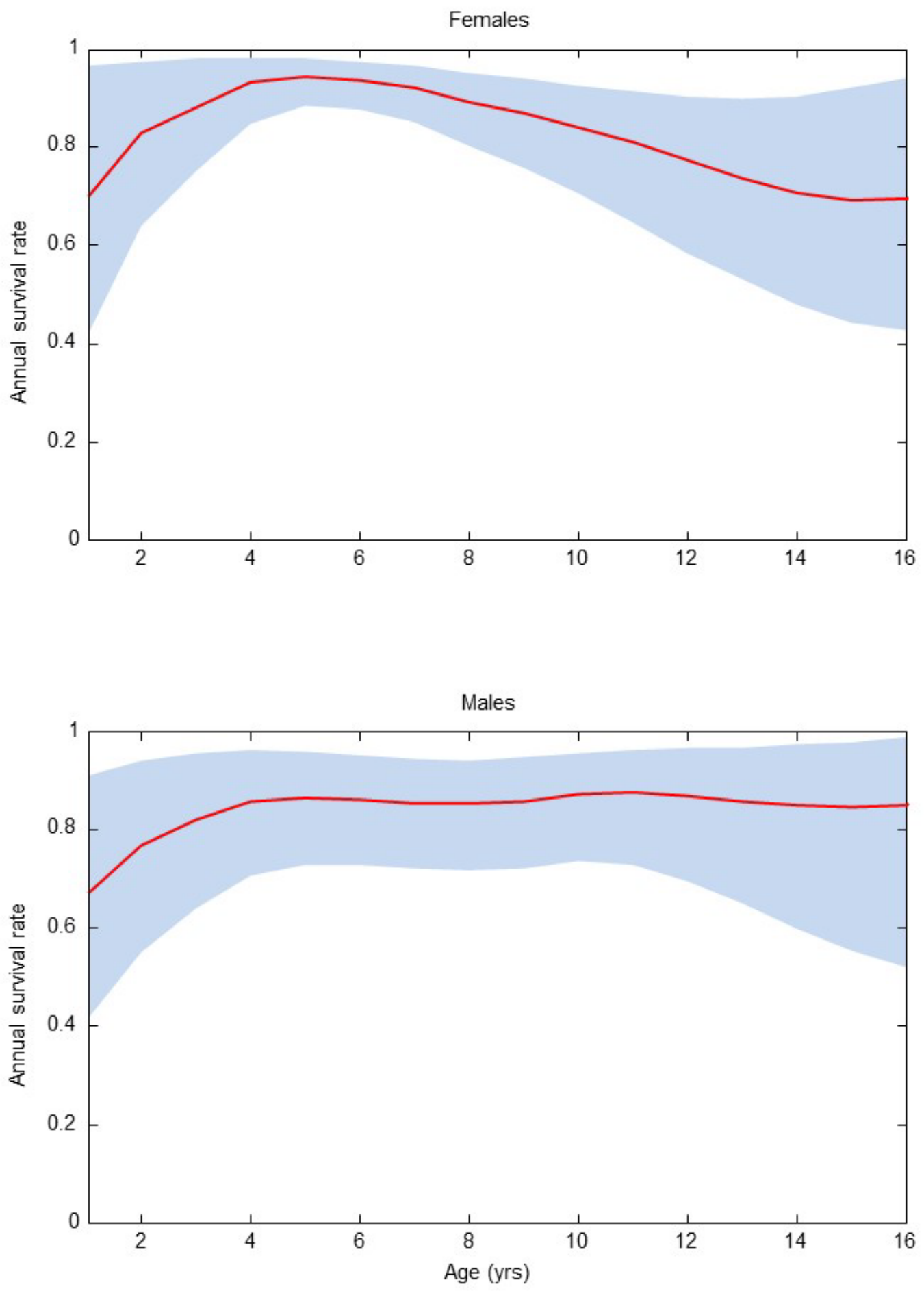

Figure 44. Graph showing average age-specific annual survival rates for female (top graph) and male (bottom graph) sea otters across all study sites, with mean estimated values indicated by the solid line and 95 -percent credible intervals $\left(\mathrm{Cl}_{95}\right)$ indicated by the shaded areas. 

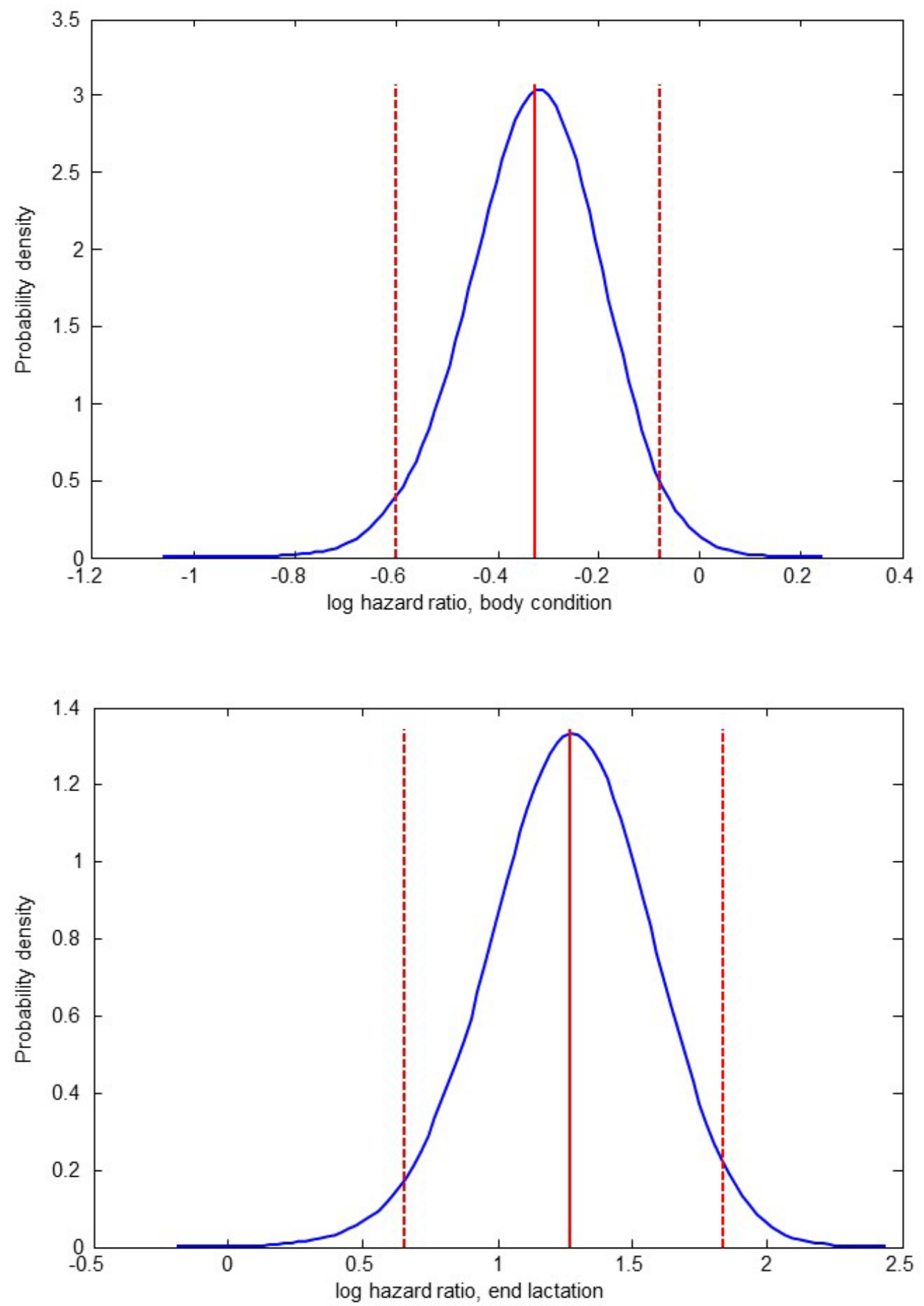

Figure 45. Graphs showing Bayesian posterior distributions for model estimates of $\beta_{1}$, the effect of sea otter body condition (top graph), and $\beta_{2}$, the effect of reproductive status (bottom graph). In both cases the parameters represent the log of the hazard ratios, so a value of "0" corresponds to a ratio of 1 (no change in hazard rates associated with the effect), whereas values significantly greater or less than 0 represent a change in hazard rate associated with the effect. Solid vertical red line indicates the mean of the posterior distribution, dashed vertical red lines indicate the $95 \%$ quantiles (the region between the dashed vertical lines is defined as the $95 \%$ credible interval, or $\mathrm{Cl}$ ). 
Survival vs. Condition, Female
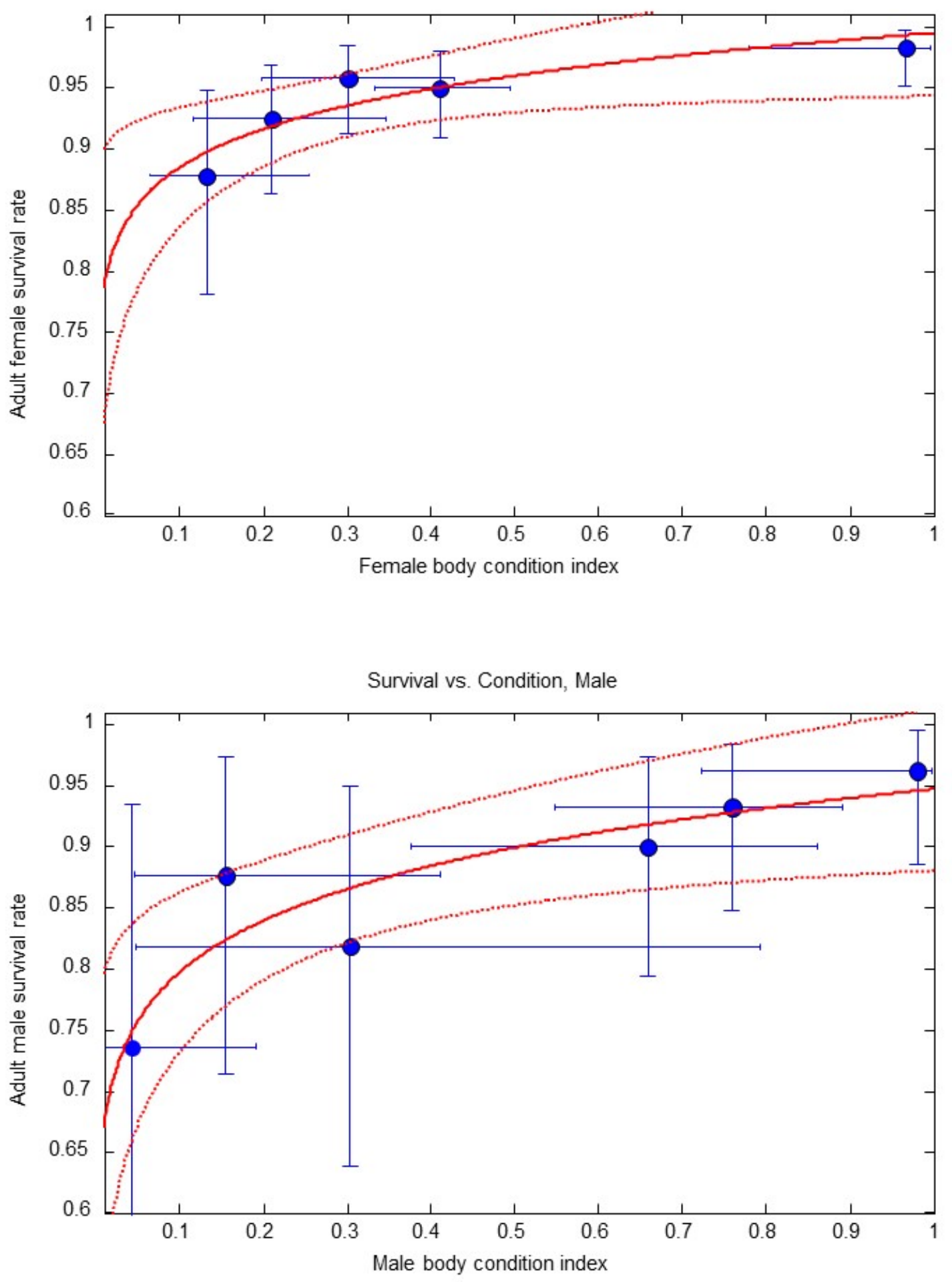

Figure 46. Graphs showing relationship between southern sea otter body condition ( $\mathrm{BCl}$ ', relative mass at age) and survival rates for females (top graph) and males (bottom graph), as estimated from data collected at six study sites throughout California. (Note that no females were present at Point Conception site, so only five female data points are available.) Fitted power functions are shown with 95-percent confidence intervals; there are significant, positive relationships between survival and body condition across study sites for both females and males. 


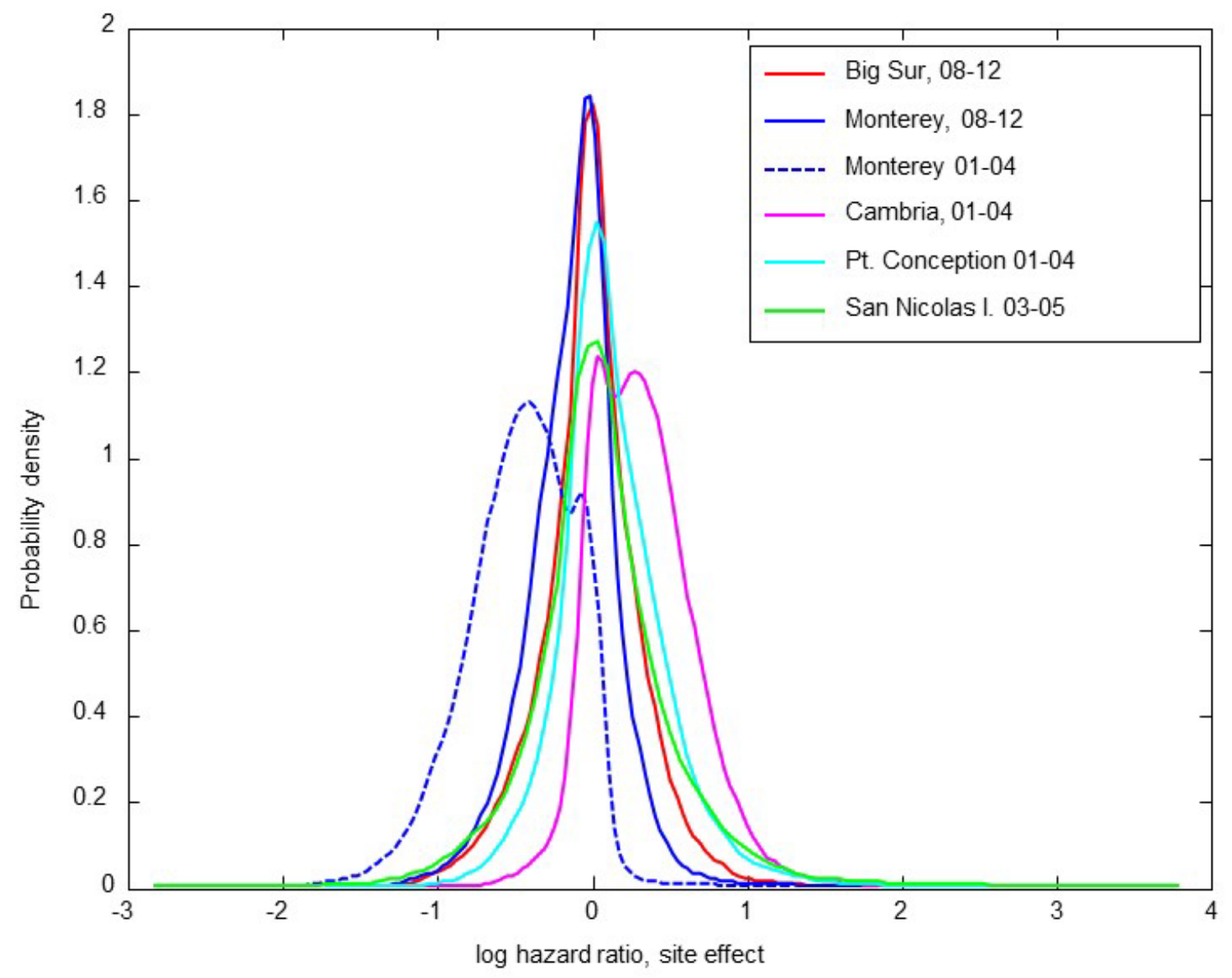

Figure 47. Graph showing Bayesian posterior distributions for model estimates of random differences in sea otter survival at six study sites throughout California. Random effect parameters represent the log of the hazard ratios for otters at each site after controlling for differences due to fixed effects included in the model. Value of " 0 " corresponds to a ratio of 1 (no change in hazard rates relative to the overall mean value), whereas values significantly greater or less than 0 represent increases or decreases in hazards associated with that site. 

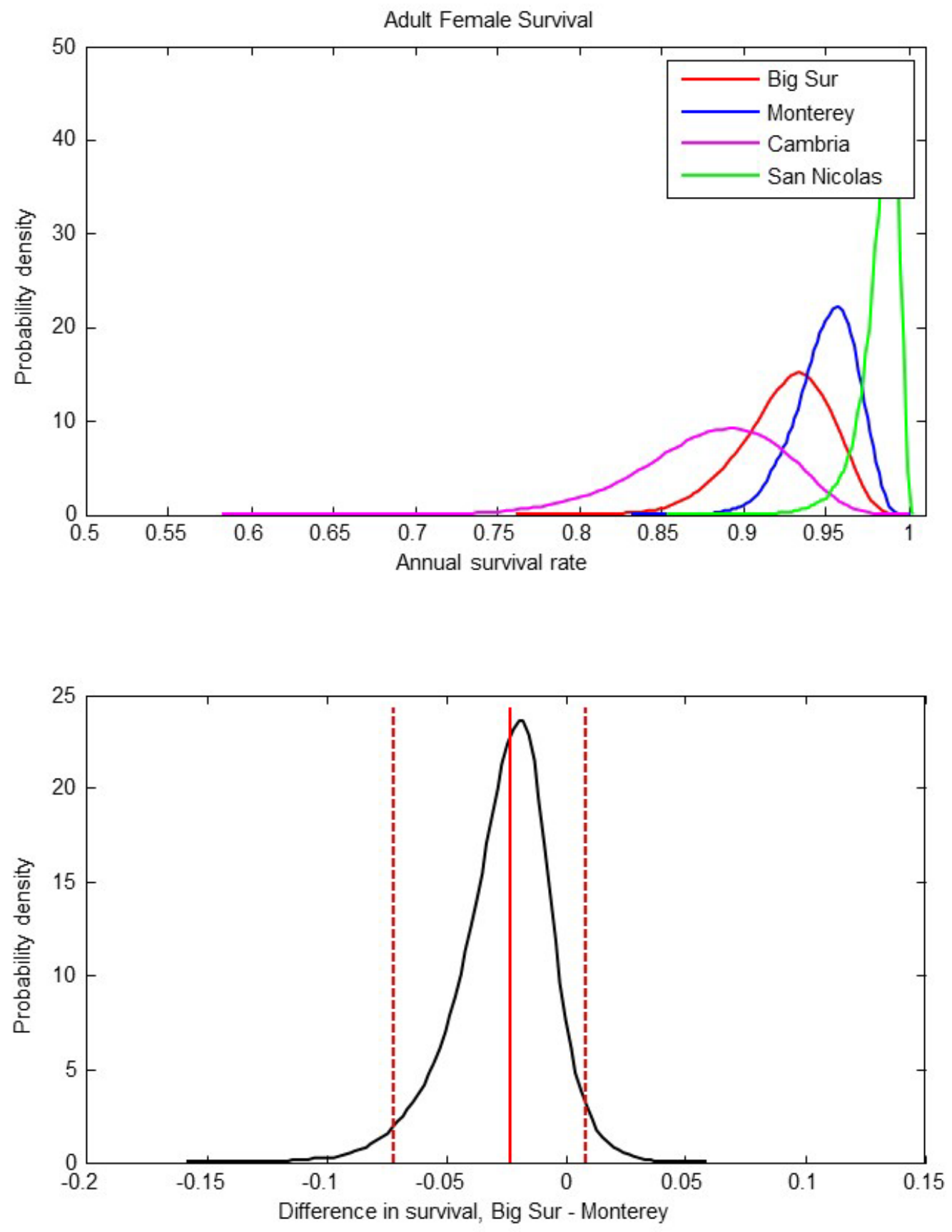

Figure 48. Graphs showing Bayesian posterior distributions for model estimates of adult (5-year old) female sea otter survival at four study sites in California (top graph), and posterior distribution for estimated difference in survival rates between adult females at Big Sur and Monterey (bottom graph; a negative value indicates lower survival at Big Sur relative to Monterey). In the lower panel, solid vertical red line indicates the mean of the posterior distribution, dashed vertical red lines indicate the $95 \%$ quantiles (the region between the dashed vertical lines is defined as the $95 \%$ credible interval, or $\mathrm{Cl}$ ). 

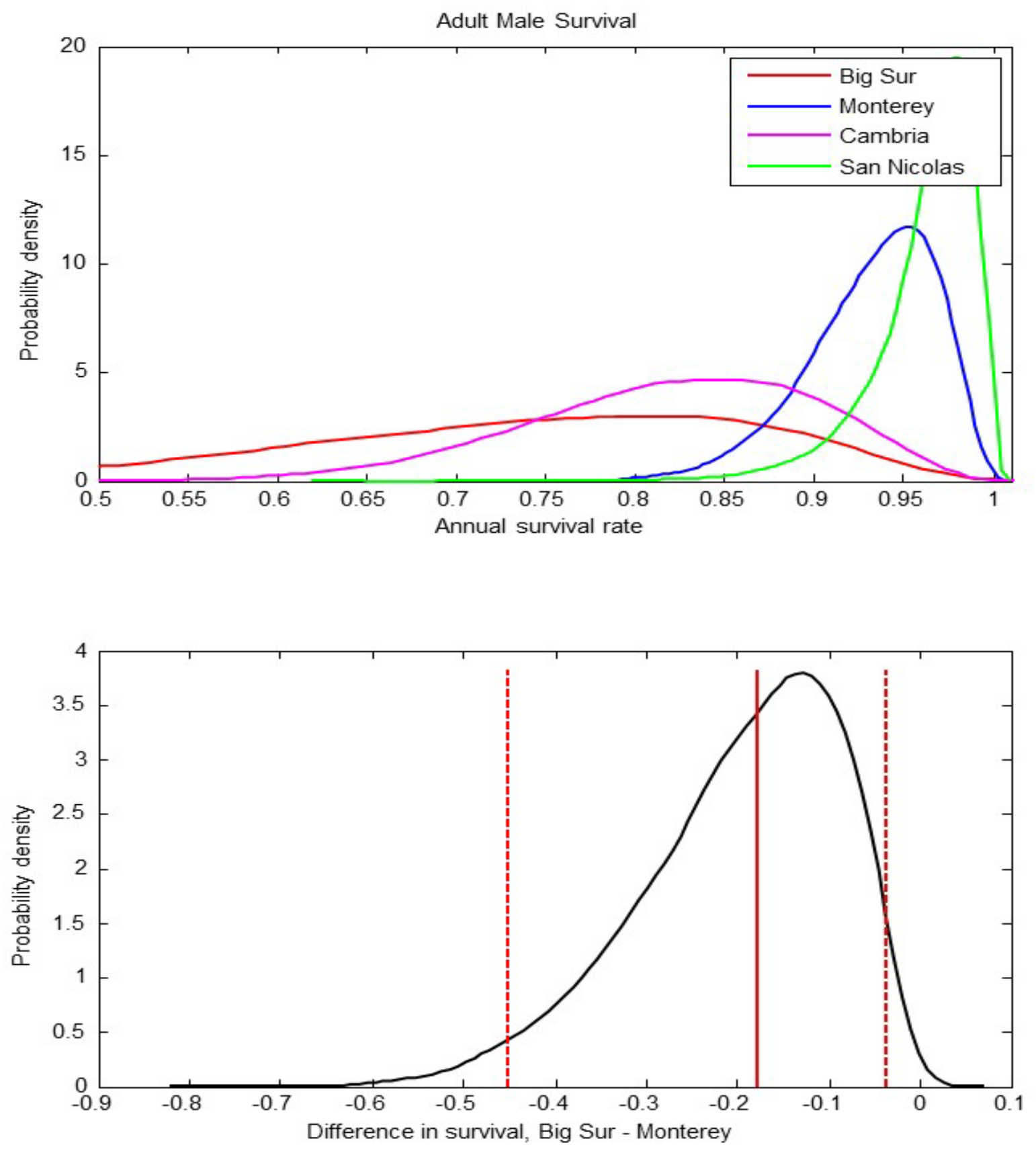

Figure 49. Graphs showing Bayesian posterior distributions for model estimates of adult (5-year old) male sea otter survival at four study sites in California (top graph), and posterior distribution for estimated difference in survival rates between adult males at Big Sur and Monterey (bottom graph; a negative value indicates lower survival at Big Sur relative to Monterey). In the lower panel, solid vertical red line indicates the mean of the posterior distribution, dashed vertical red lines indicate the $95 \%$ quantiles (the region between the dashed vertical lines is defined as the $95 \%$ credible interval, or $\mathrm{Cl}$ ). 


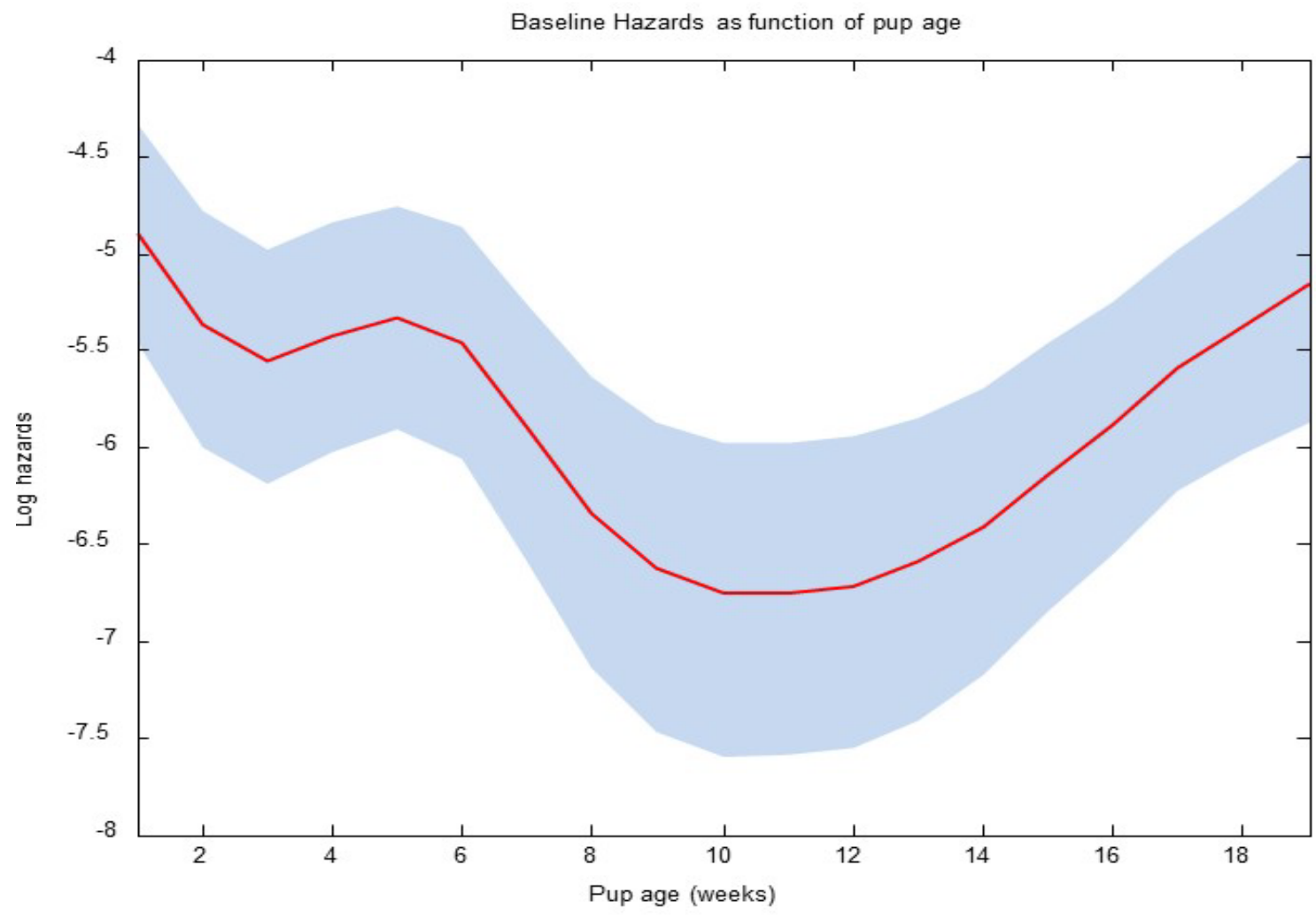

Figure 50. Graph showing log hazard rates for sea otter pups as a function of pup age (measured in weeks since birth). Lower log hazard rates indicate higher survival probability. Mean estimated values are indicated by the solid line, and 95-percent credible intervals $\left(\mathrm{Cl}_{95}\right)$ are indicated by the shaded areas. 


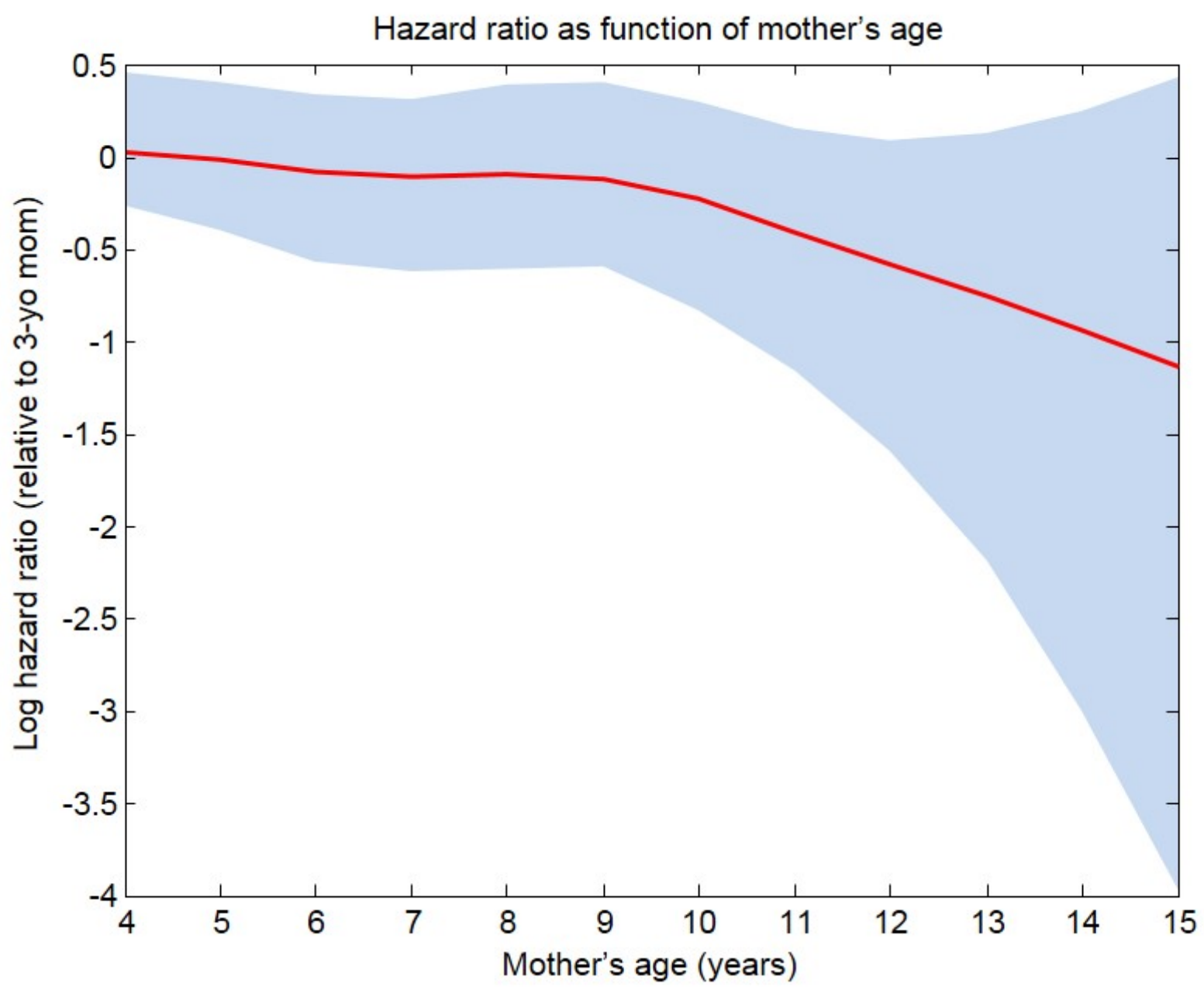

Figure 51. Graph showing log hazard ratio for sea otter pups as a function of age of mother age, with a value of 0 indicating a hazard ratio of 1 relative to a 3-year-old female (baseline value). Negative log hazard ratios indicate higher survival probability relative to a 3-year-old. Mean estimated values are indicated by the solid line and 95percent credible intervals (Cl95) are indicated by the shaded areas. 


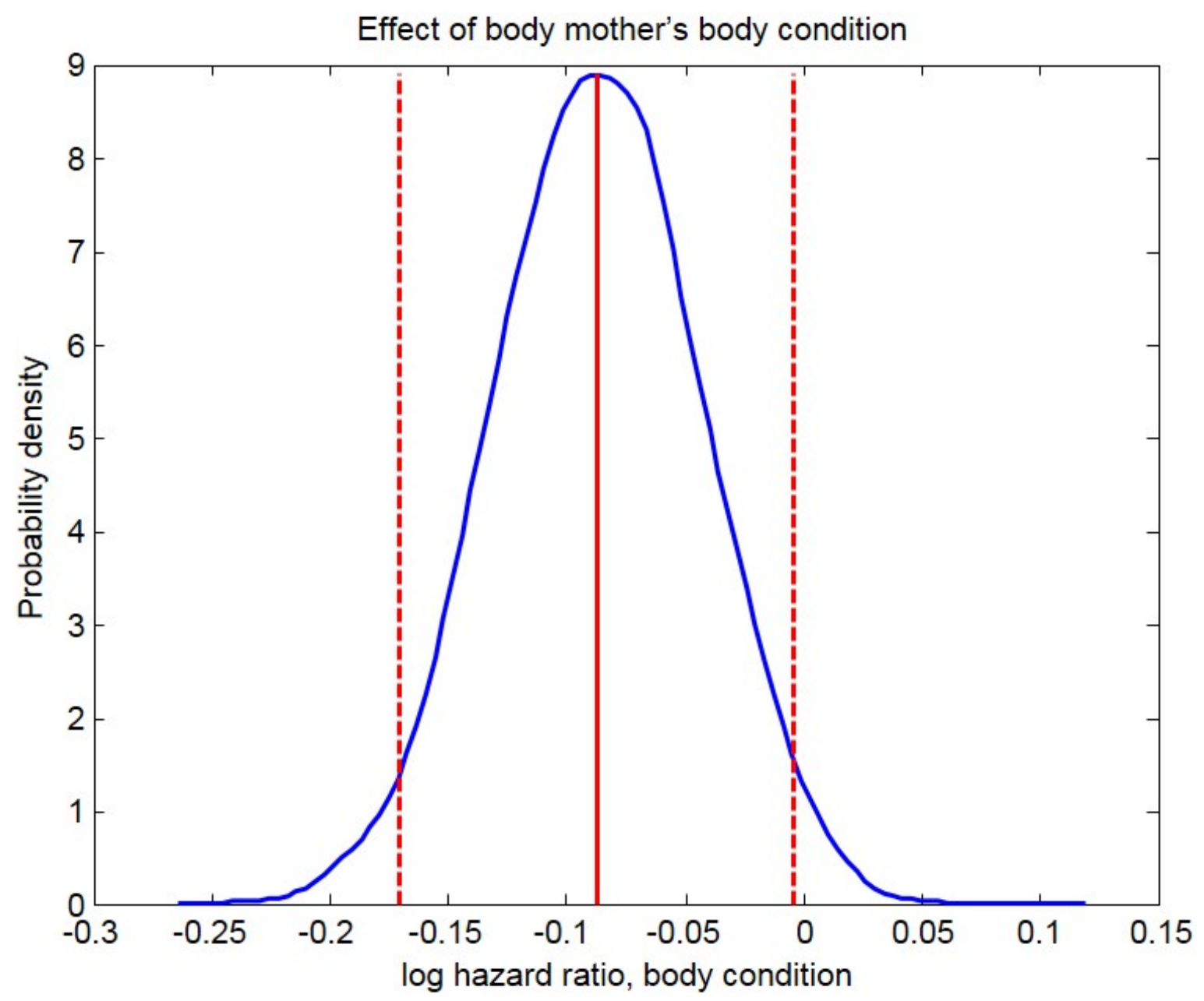

Figure 52. Graph showing Bayesian posterior distribution for model estimates of $\beta_{3}$, the effect of the body condition of the sea otter mother on pup survival. The parameter represents the log of the hazard ratios, so a value of " 0 " corresponds to a ratio of 1 (no change in hazard rates relative to a female in "average" condition), whereas a value significantly less than 0 represents a decrease in hazard rate associated with mothers in higher-than-average condition (or an increase in hazards for mothers in poor condition). Solid vertical red line indicates the mean of the posterior distribution, dashed vertical red lines indicate the $95 \%$ quantiles (the region between the dashed vertical lines is defined as the $95 \%$ credible interval, or $\mathrm{Cl}$ ). 

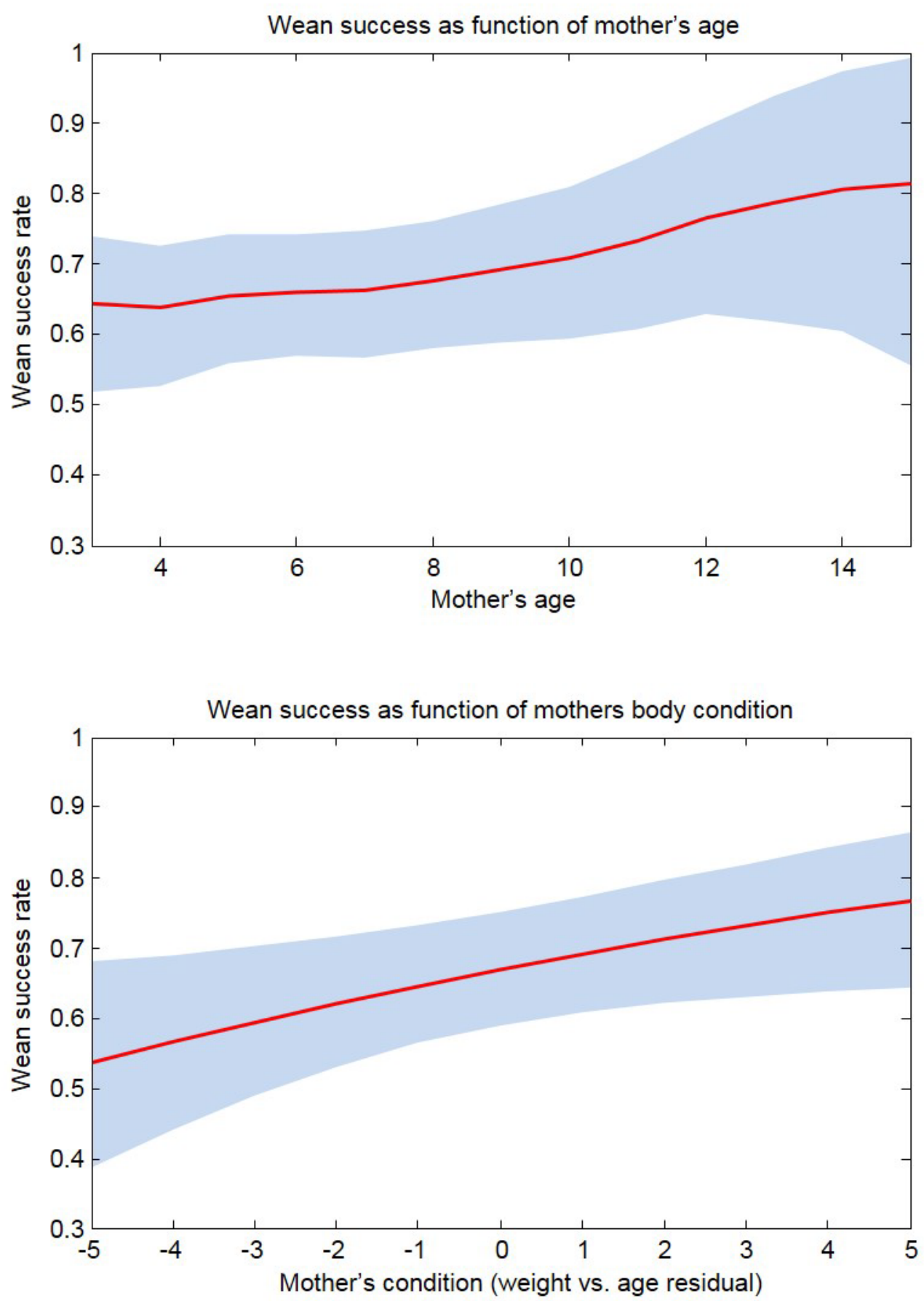

Figure 53. Graphs showing weaning success rates (sea otter pup survival) plotted as a function of age of mother (top graph) and body condition of mothers (body condition index, relative mass at age). Mean estimated values are indicated by the solid line, and 95-percent credible intervals (Cl95) are indicated by the shaded areas. 

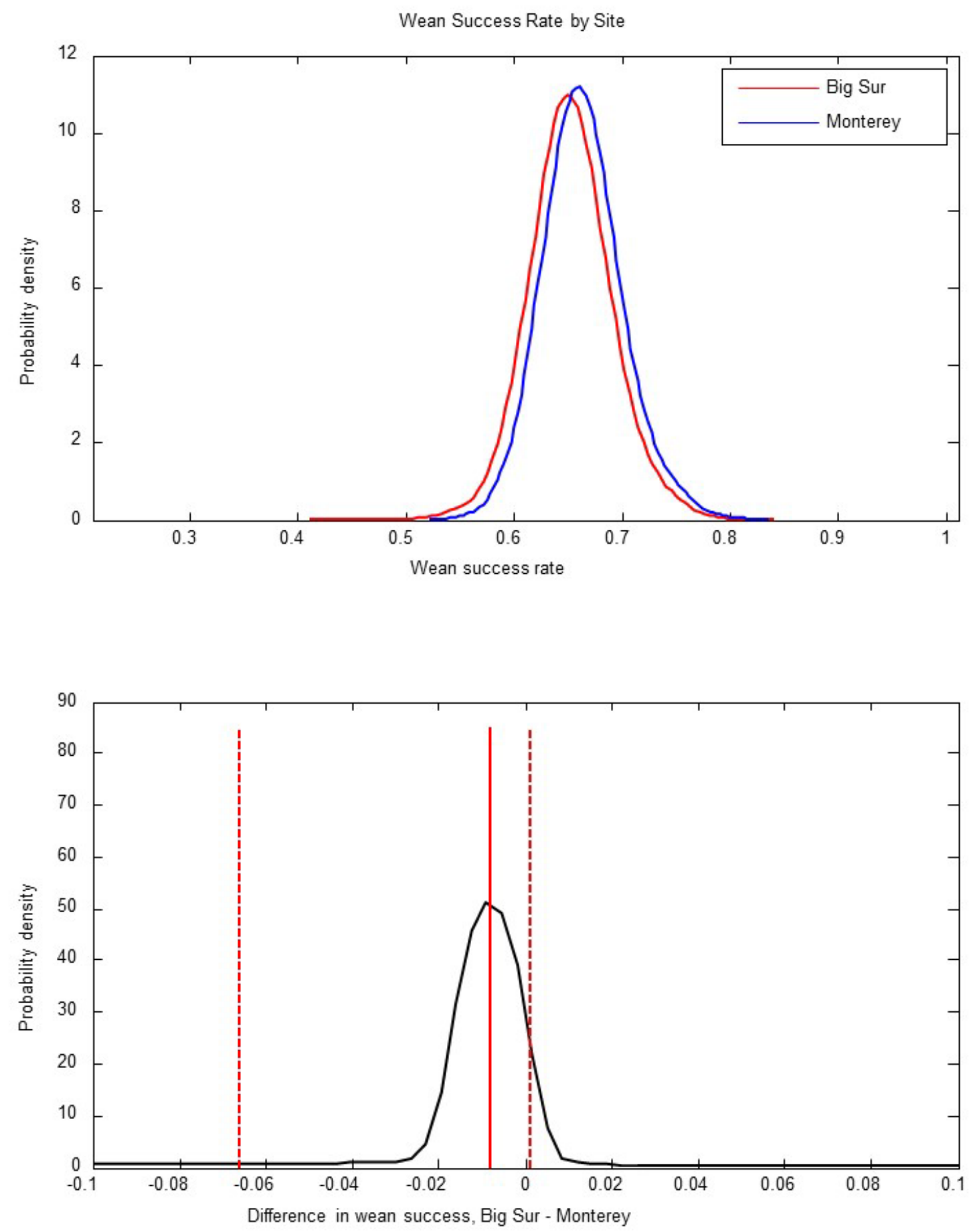

Figure 54. Graphs showing Bayesian posterior distributions for model estimates of weaning success for an adult (5-year old) female sea otter at the Big Sur and Monterey study sites (top graph), and posterior distribution for estimated difference in weaning success rates between Big Sur and Monterey study sites (bottom graph; a negative value indicates lower pup survival at Big Sur relative to Monterey). In the lower panel, solid vertical red line indicates the mean of the posterior distribution, dashed vertical red lines indicate the $95 \%$ quantiles (the region between the dashed vertical lines is defined as the $95 \%$ credible interval, or $\mathrm{Cl}$ ). 


\title{
Chapter 9. Epidemiological Analysis of Protozoal Infections in Sea Otters at Big Sur and Monterey
}

\author{
Tristan Burgess ${ }^{1}$, M. Tim Tinker2,3, Christine Johnson'1, Holly MacCormick³, Ann Melli', and \\ Patricia A. Conrad 1
}

\section{Introduction}

Protozoal disease, including disease caused by the parasite Toxoplasma gondii (Miller and others, 2004), is a common cause of mortality in southern sea otters (Kreuder and others, 2003). Along with the closely related parasite Sarcocystis neurona, T. gondii is considered an important component of the land-to-sea transfer of infectious parasites that affect sea otter health (Miller and others, 2007; Conrad and others, 2009). The basis for concluding that $T$. gondii and $S$. neurona originate from land is that the only known definitive hosts are opossums for $S$. neurona parasites (Miller and others, 2010), and members of the felid family, including domestic cats, bobcats, and mountain lions for T. gondii parasites (Miller and others, 2002a). Understanding factors that cause variation in the infection rates of sea otters with $T$. gondii is important because (1) information on the mechanisms of infection can lead to improved management policy and conservation actions; (2) we can use $T$. gondii infections as a model to understand the role of land-to-sea pollution on sea otter health (Conrad and others, 2005; Jessup and others, 2007; VanWormer, Conrad, and others, 2013; VanWormer and others, 2014); and (3) sea otters represent a useful sentinel species on the basis of their high trophic level, spatial philopatry and tractability for study, and thus, information gleaned from epidemiological analysis of sea otter health and disease can inform more broadly on nearshore marine ecosystem health.

Past research has shown that many environmental, behavioral, and demographic variables can lead to variation in sea otter infection risk for $T$. gondii. Coastal freshwater runoff is one factor that has been shown to increase risk of exposure to T. gondii (Miller and others, 2002a). Other reported risk factors include sea otter age, sex, mobility, and prey choice (Johnson and others, 2009). The role of diet as a risk factor for infection is an interesting feature of the sea otter study system, and indicates two key facts: (1) many disease-causing parasites that affect sea otters are acquired through prey consumption and not by contact with conspecifics, and (2) sea otters in central California have very high degrees of individual specialization in diet, such that individuals living in the same location at the same time use different subsets of the available prey species (Estes and others, 2003; Tinker and others, 2008, 2012), and, thus, might have differential risk of infection. During the course of the current study, extensive data on sea otter habitat use, movements, and foraging ecology were collected, allowing us to test their potential contributions to variation in $T$. gondii infections. One issue of particular interest was whether or diet specialization on snails represented a risk factor, as this dietary pattern was found to be associated with higher risk in a previous study (Johnson and others, 2009).

\footnotetext{
${ }^{1}$ University of California, Davis.

${ }^{2}$ U.S. Geological Survey.

${ }^{3}$ University of California, Santa Cruz.
} 
Using results from serological analysis of blood samples collected from all study animals, we measured and compared patterns of T. gondii infection in sea otters from Big Sur and Monterey. We used standard epidemiological techniques to test for putative risk factors for infection with $T$. gondii, including geographic location (comparison between more developed the Monterey site and the less developed Big Sur site), age, sex, home-range size, mobility, and diet composition. Our goal was to test whether $T$. gondii, a parasite shed primarily by wild and domestic felids, is more common among sea otters living in areas adjacent to human population centers and areas heavily impacted by runoff or sewage than among those living in a more pristine area.

\section{Methods}

\section{Study Population}

Samples from all 135 study animals (chapter 1, table 1) captured between November 2008 and October 2010 were included in serological analysis, along with samples from an additional 25 sea otters captured and sampled in Monterey between January 2006 and October 2008, for a total of 159 individuals. Data on diet composition and home-range use were included where the minimum hours of foraging budget or resights (chapter 6) had been recorded. Animals were resighted 3-7 times per week for the duration of the study.

\section{Serum Collection and Analysis}

Whole blood was collected from otters captured as described in chapter 1. Blood samples were allowed to clot and centrifuged at $1,500 \times g$ for 10 minutes and stored at $-70^{\circ} \mathrm{C}$ until testing. Serum was drawn off and tested for $T$. gondii using an indirect fluorescent antibody test. This test has been standardized by serial dilution and a cutoff of 1:320 or greater has been determined to be optimal for detection of T.gondii infection in southern sea otters of known infection status (Miller and others, 2002b).

\section{Risk Factors}

Putative risk factors that were evaluated for association with $T$. gondii infection status included study site (Big Sur compared to Monterey, with the comparison assumed to be a proxy for proximity to human influences), age, length, weight, and sex. In addition to these variables, we assessed potential dietary risk factors including diet type (individuals were assigned to one of five dietary groupings using hierarchical cluster analysis of species-specific prey consumption rates; see chapter 6), diet diversity (Shannon Weiner index; see chapter 6) and snail consumption (as a percentage of biomass consumed). Home-range area (in square kilometers), the number of centers of use and the net linear displacement (in kilometers) were assessed as indices of individual mobility (see chapter 4).

\section{Univariate Analysis}

Chi-square tests (categorical variables), Fisher's exact tests (for small sample sizes, when expected counts in contingency tables were less than 5) and t-tests (for continuous variables) were used to assess the association of $T$. gondii antibody status and potential risk factors. $P$ values less than 0.05 were considered significant, and odds ratios and 95-percent confidence intervals were calculated for categorical variables. Analyses were conducted using R version 2.15 (R Core Development Team). 


\section{Logistic Regression Analysis}

Logistic regression models were used to examine effects of individual animal variables (age, length, weight, sex), diet type, and the movement/habitat use metrics on $T$. gondii infection status. Variables with $\mathrm{P}<0.25$ in the univariate analysis were initially used to build a multivariate model in a modified forward stepwise fashion. The addition of other excluded variables was assessed after the initial model selection stage by adding these to the model and checking for improved model parsimony. Models were selected on the basis of reduced AIC and likelihood ratio tests. A first-order, site-sex interaction term was added specifically to test the hypothesis that $T$. gondii infection risk might vary between sites for females, but not for males because the greater site fidelity of females (see chapter 4) makes them more reliable indicators of local environmental influences. Collinearity of predictors was assessed using linear regression. Collinear predictors were assessed by autoregression, and residuals of the autoregression model were used to assess whether residual predictive value remained. Odds ratios and 95-percent confidence intervals were calculated to measure the strength of the association between each risk factor and $T$. gondii serological status. Analyses were conducted using R version 2.15 (R Core Development Team).

\section{Results}

Univariate tests (tables 30 and 31) indicated that sex, age, and body size (length and weight) were all significant predictors of $T$ gondii infection, whereas diet type was marginally significant and site effects were not significant. Body length $(\mathrm{P}=0.0013)$ and body weight $(\mathrm{P}=0.0045)$ were significantly associated with $T$. gondii infection status, but these two variables were strongly correlated $\left(\mathrm{P}<2.2 \times 10^{-16}\right)$ and both were associated with age $\left(\mathrm{P}=1.32 \times 10^{-11} ; \mathrm{P}=7.34 \times 10^{-9}\right)$. Sequential autoregression of length (Wald $\mathrm{P}<0.001)$ and weight $(\mathrm{P}=0.009)$ against age determined that the residuals were significant predictors of infection status while simultaneously accounting for age, but neither approach yielded a superior model (based on AIC) to that including length alone, so only length was used in further models.

Diet class was a significant predictor of $T$. gondii exposure risk, but not all classes differed significantly. The best fitting model compared diet classes 2 and 4 to a reference category combining classes 1, 3 and 5. Class 2 (abalone and Cancer crab) did not differ significantly from the reference but model fit (based on AIC) was improved by the retention of this category. Class 4 (primarily marine snails and some kelp crab) was associated with a significant increase $(\mathrm{P}=0.024)$ in $T$. gondii infection risk. Infection risk did not differ significantly between sites in the univariate analysis $(\mathrm{P}=0.327)$, but when stratified by site, female otters had significantly lower T. gondii exposure than males at Monterey Bay $(\mathrm{P}=0.005)$. Prevalence of $T$. gondii infection did not differ by sex among animals sampled at Big $\operatorname{Sur}(\mathrm{P}=0.6805)$. For this reason an interaction term of $\mathrm{sex}^{*}$ site was included in the final multivariate model (table 32).

The multivariate model (table 33) shows the relative contributions of the various risk factors while adjusting for differences between sample populations. The final model identified increasing length $(\mathrm{P}=0.0291)$ and membership of the snail specialist dietary grouping $(\mathrm{P}=0.0165)$ as significant risk factors. Additionally, the site-by-sex interaction was significant $(\mathrm{P}=0.0381$ for females and 0.366 for males) in this model, and the inclusion of this variable improved model fit based on AIC. This term indicates that association of site with observed $T$. gondii antibody prevalence differed significantly between sexes. After adjusting for differences in all significant variables identified, the odds ratio (95percent CI) for $T$. gondii seropositivity for females at Monterey, compared with those at Big Sur, was $0.05(0-0.61)$, whereas the same ratio for males was $0.68(0.03-13.83)$. 


\section{Discussion}

Our small scale study of otters sampled at these two study sites from 2006 to 2010 confirmed findings of other studies of otters sampled previously across the larger California landscape (Miller and others, 2002a; Johnson and others, 2009), showing that there is substantial variation in T. gondii infection rates in sea otters in central California, and that much of this variation can be explained by differences associated with location, age, sex, and diet.

The correlation of increasing $T$. gondii infection with increasing age is consistent with a constant infection risk over time as, once infected, animals remain infected and likely seropositive for some time post-infection (although the duration of seroprevalence is not well understood for sea otters and likely varies among individuals). The cumulative likelihood of exposure to the parasite, therefore, should increase with age. However, if infection with the parasite carries some mortality risk, we might expect a "survivor bias" to emerge over time that would act to decrease the realized prevalence among older animals. A survivor bias occurs when infected individuals are more likely to drop out of the population due to disease, leaving a higher proportion of uninfected adults. The combination of these two opposing trends might be expected to lead to a nonlinear, declining (or asymptotic) age effect. This may explain the finding that body length was a better predictor of infection risk than was age estimate. Body length is an increasing, asymptotic function of age (see chapter 7), and may in some cases be a superior predictor of age than estimation based on tooth wear, especially in younger animals. Length, therefore, might be a better predictor of $T$. gondii infection than age because of its asymptotic nature, if infection rates are also an asymptotic function of age.

As with a previous analysis of $T$. gondii infection risks (Johnson and others, 2009), individual diet specialization was determined to be a significant predictor of seroprevalence. In our analyses, three of five diet specialist types showed no difference in infection risks, but two diet types were associated with increased risk. Otters that specialized on marine snails were 18.1 (95-percent CI 1.81-180.48) times more likely to be infected with $T$. gondii than were otters specializing in other prey types, consistent with previously published findings (Johnson and others, 2009). Our findings add further support to the conclusions of Johnson and others (2009) about the association between marine snail consumption and the risk of infection with $T$. gondii. This is especially significant considering that the study animals used in the current analysis are independent from the sample of animals used in the earlier analysis, and represent an entirely different area of the coast (Big Sur). The close agreement in findings between the two studies suggests that the processes underlying the snail- $T$. gondii association are relatively consistent over time and space. Ongoing research is aimed at clarifying the mechanism underlying this association.

Sea otter mobility was not a significant predictor of infection risk among the otters sampled in this study, at least as measured by the three variables we used in the analyses (total home-range area, the number of distinct centers of use, and net linear displacement). We had expected that otters with larger home range might have greater likelihood of passing through an area where environmental loading of $T$. gondii oocysts was higher, and, thus, have a higher risk of infection, a pattern reported by Johnson and others (2009) for S. neurona infection risk as measured by movement (in kilometers) over a 90-day period. Our failure to find such a pattern in the current study suggests lack of a consistent relation between movement and infection risk, but could also be indicative of the difference in measures used to show otter mobility, a lack of statistical power, or the smaller spatial scale at which this current study was conducted. Greater mobility also might have been too highly correlated with sex (males having greater mobility, as has been previously observed) in this small-scale study to evaluate this effect fully in a multivariable analysis.

The most unexpected finding from our analyses of otters sampled was the unusually low seroprevalence among females at Monterey (7.8-percent) during 2006-10 compared with otters sampled 
previously in this study area. The data reported by Johnson and others (2009) show T. gondii seroprevalence among live-captured sea otters at Monterey averaging 30.8-percent (range 20-43.5percent) over the years 1998-2003 or 29.9-percent (range 22.2-47.1-percent) for females only. No such temporal disparity is apparent for the Big Sur study site, for which we report a seroprevalence of 18.5percent (17-percent among females), which does not differ significantly from the seroprevalence of 25percent (13-percent among females) recorded in 2003. This comparison underscores the importance of caution in interpreting comparisons between a single pair of study sites over a short time period. Whether the observed decrease at Monterey is due to demographic changes (population turnover), decreased exposure (altered rainfall patterns, improved stormwater and sewage management, and [or] changes in the abundance, age structure, or health status of domestic and feral cats) is not known.

This low prevalence in female otters in Monterey from 2006 to 2010 translated to a significant difference in $T$. gondii prevalence among female otters at our two study sites. After adjusting for other effects such as body length and diet type, females from the Monterey study site had a $22 \times$ lower prevalence than did females in Big Sur. This result was surprising, and in direct contrast to the pattern we expected given previous studies (for example, Miller and others, 2008) that suggest that proximity to watersheds with anthropogenic influences (especially high abundance of domestic cats) is the greatest risk factor for $T$. gondii infections. Any interpretation of this result must be made cautiously, given the fact that our comparison is based on data from just two study sites over a 3-year period. Nonetheless, a few points are worth making to help clarify the significance of these unexpected findings. Sea otters are thought to become infected with $T$. gondii after consuming prey that contain oocysts (the infective stage of the parasite) that have been shed by a felid, transported from the land to the sea through runoff, and incorporated in the marine food web. All steps in this chain must be evaluated and localized to specific watersheds and coastal segments to fully understand the impact of anthropogenic activities on otters living in the Monterey and Big Sur nearshore environment. Furthermore, the scale at which disease exposure risk driven by these processes varies in sea otter habitat has not been explicitly assessed. The zone of influence of a given terrestrial outflow source on the surrounding marine environment presumably depends on the quantity and timing of discharge as well as oceanographic processes, bathymetry, and biotic features of the environment immediately offshore. The degree to which these processes have locally attributable effects in determining the fate of $T$. gondii oocysts in runoff from land is the subject of ongoing investigations.

There are many differences between the Monterey and Big Sur study sites, and although we have no way of knowing which (if any) of these contributed to the different infection rates that we measured, we highlight a few key differences that could form the basis of future investigations. Monterey has a higher density of domestic and feral cats compared to Big Sur, whereas Big Sur has fewer domestic cats than Monterey but a higher density of bobcats and mountain lions that also carry the $T$. gondii parasite (Miller and others, 2008; VanWormer, Fritz, and others, 2013). Hydrological processes differ greatly between the two sites as well; the Monterey area has many storm drains that drain urban areas with impervious surfaces, as well many major rivers (for example, the Carmel and Salinas Rivers) that drain large agriculturally dominated watersheds; conversely, Big Sur has many small, unpopulated or sparsely populated watersheds with very steep terrain, such that particles deposited on land can be transported quickly to the marine environment during rain events. The differences in bathymetry and substrate between the two study sites differ in important ways. The steep bathymetry and rocky subtidal habitat at Big Sur mean that kelp beds are very close to shore (CDFW Marine Region, GIS Unit; Hallenbeck and others, 2012), most notably at watershed pour points, and so sea otters feeding and resting in these kelp beds are near putative inputs of $T$. gondii oocysts. In contrast, the shallow bathymetry of the Monterey study site means that kelp beds are farther from shore, and there are no kelp beds close to major watershed pour points. If $T$. gondii transmission is strongly influenced by aggregation in kelp biofilms 
and subsequent concentration by marine snails (Shapiro and others, 2014), these physical/environmental differences in habitat and kelp distribution may contribute to the differing risk of otter infection along the California coastline. Future studies and experiments could be designed to directly test some of these proposed mechanisms.

In conclusion, our intensive study of otters at Big Sur and Monterey suggest that within this study area, risk factors such as individual age and prey use patterns were associated with $T$. gondii infection. Sea otter exposure to $T$. gondii at the Monterey study site was lower during this study period than it has been in the past, leading to a large disparity in prevalence between study sites. Our finding of 18.5-percent $T$. gondii prevalence among otters living off the Big Sur coast suggest wild felids could be a source of infectious oocysts to the marine environment in more pristine areas (Miller and others, 2008; VanWormer, Fritz, and others, 2013), but further research would be required to determine this with any certainty. Overall, these results underscore the temporal variability and stochastic nature of disease processes in sea otter populations, and the need for enhanced understanding of land-sea pathogen pollution in the context of specific mechanisms of exposure and infection that incorporate both environmental characteristics of the habitat and ecological interactions of the parasites and hosts.

\section{References Cited}

Conrad, P.A., Miller, M.A., Kreuder, C., James, E.R., Mazet, J., Dabritz, H., Jessup, D.A., Gulland, F., and Grigg, M.E., 2005, Transmission of toxoplasma-Clues from the study of sea otters as sentinels of Toxoplasma gondii flow into the marine environment: International Journal for Parasitology, v. 35, p. 1155-1168.

Conrad, P.A., VanWormer, E., Shapiro, K., Miller, M., Kreuder-Johnson, C., Tinker, T., Grigg, M., Largier, J., Carpenter, T., and Mazet, J.K., 2009, Tracking Toxoplasma gondii from land to sea: American Journal of Tropical Medicine and Hygiene, v. 81, p. 198.

Estes, J.A., Riedman, M.L., Staedler, M.M., Tinker, M.T., and Lyon, B.E., 2003, Individual variation in prey selection by sea otters-Patterns, causes and implications: Journal of Animal Ecology, v. 72, p. 144-155.

Hallenbeck, T.R., Kvitek, R.G., and Lindholm, J., 2012, Rippled scour depressions add ecologically significant heterogeneity to soft-bottom habitats on the continental shelf: Marine Ecology Progress Series, v. 468, p. 119-133.

Jessup, D.A., Miller, M.A., Kreuder-Johnson, C., Conrad, P.A., Tinker, M.T., Estes, J., and Mazet, J.A.K., 2007, Sea otters in a dirty ocean: Journal of the American Veterinary Medical Association, v. 231, p. 1648-1652.

Johnson, C.K., Tinker, M.T., Estes, J.A., Conrad, P.A., Staedler, M., Miller, M.A., Jessup, D.A., and Mazet, J.A.K., 2009, Prey choice and habitat use drive sea otter pathogen exposure in a resourcelimited coastal system: Proceedings of the National Academy of Sciences of the United States of America, v. 106, p. 2242-2247.

Kreuder, C., Miller, M.A., Jessup, D.A., Lowenstine, L.J., Harris, M.D., Ames, J.A., Carpenter, T.E., Conrad, P.A., and Mazet, J.A.K., 2003, Patterns of mortality in southern sea otters (Enhydra lutris nereis) from 1998-2001: Journal of Wildlife Diseases, v. 39, p. 495-509.

Miller, M.A., Conrad, P.A., Harris, M., Hatfield, B., Langlois, G., Jessup, D.A., Magargal, S.L., Packham, A.E., Toy-Choutka, S., Melli, A.C., Murray, M.A., Gulland, F.M., and Grigg, M.E., 2010, A protozoal-associated epizootic impacting marine wildlife-Mass-mortality of southern sea otters (Enhydra lutris nereis) due to Sarcocystis neurona infection: Veterinary Parasitology, v. 172, p. 183194. 
Miller, M.A., Gardner, I.A., Kreuder, C., Paradies, D.M., Worcester, K.R., Jessup, D.A., Dodd, E., Harris, M.D., Ames, J.A., Packham, A.E., and Conrad, P.A., 2002a, Coastal freshwater runoff is a risk factor for Toxoplasma gondii infection of southern sea otters (Enhydra lutris nereis): International Journal for Parasitology, v. 32, p. 997-1006.

Miller, M.A., Gardner, I.A., Packham, A., Mazet, J K., Hanni, K.D., Jessup, D., Estes, J., Jameson, R., Dodd, E., Barr, B.C., Lowenstine, L.J., Gulland, F.M., and Conrad, P.A., 2002b, Evaluation of an indirect fluorescent antibody test (IFAT) for demonstration of antibodies to Toxoplasma gondii in the sea otter (Enhydra lutris): Journal of Parasitology, v. 88, p. 594-599.

Miller, M.A., Grigg, M.E., Kreuder, C., James, E.R., Melli, A.C., Crosbie, P.R., Jessup, D.A., Boothroyd, J.C., Brownstein, D., and Conrad, P.A., 2004, An unusual genotype of Toxoplasma gondii is common in California sea otters (Enhydra lutris nereis) and is a cause of mortality: International Journal for Parasitology, v. 34, p. 275-284.

Miller, M.A., Grigg, M.E., Miller, W.A., Dabritz, H.A., James, E.R., Melli, A.C., Packham, A.E., Jessup, D., and Conrad, P.A., 2007, Toxoplasma gondii and Sarcocystis neurona infections of Pacific coastal sea otters in California, USA - Evidence for land-sea transfer of biological pathogens: Journal of Eukaryotic Microbiology, v. 54, p. 48S-49S.

Miller, M.A., Miller, W.A., Conrad, P.A., James, E.R., Melli, A.C., Leutenegger, C.M., Dabritz, H.A., Packham, A.E., Paradies, D., Harris, M., Ames, J., Jessup, D.A., Worcester, K., and Grigg, M.E., 2008, Type X Toxoplasma gondii in a wild mussel and terrestrial carnivores from coastal CaliforniaNew linkages between terrestrial mammals, runoff and toxoplasmosis of sea otters: International Journal for Parasitology, v. 38, p. 1319-1328.

Shapiro, K., Krusor, C., Mazzillo, F.M.M., Conrad, P.A., Largier, J.L., Mazet, J.A.K., Silver, M.W., 2014, Aquatic polymers can drive pathogen transmission in coastal ecosystem: Proceedings of the Royal Society B-Biological Sciences, v. 281, no. 1795, doi:10.1098/rspb.2014.1287.

Tinker, M.T., Bentall, G., and Estes, J.A., 2008, Food limitation leads to behavioral diversification and dietary specialization in sea otters: Proceedings of the National Academy of Sciences of the United States of America, v. 105, p. 560-565.

Tinker, M.T., Guimarães, P.R., Novak, M., Marquitti, F.M.D., Bodkin, J.L., Staedler, M., Bentall, G., and Estes, J.A., 2012, Structure and mechanism of diet specialisation-Testing models of individual variation in resource use with sea otters: Ecology Letters, v. 15, p. 475-483.

VanWormer, E., Conrad, P.A., Miller, M.A., Melli, A.C., Carpenter, T.E., and Mazet, J.A.K., 2013, Toxoplasma gondii, source to sea-Higher contribution of domestic felids to terrestrial parasite loading despite lower infection prevalence: EcoHealth, v. 10, no. 3, p. 277-289.

VanWormer, E., Fritz, H.M., Shapiro, K., Mazet, J.A.K., and Conrad, P.A., 2013, Molecules to modeling-Toxoplasma gondii oocysts at the human-animal-environment interface: Comparative Immunology, Microbiology and Infectious Diseases, v. 36, p. 217-231.

VanWormer E., Miller, M.A., Conrad, P.A., Grigg, M.E., Rejmanek, D., Carpenter, T.E., and Mazet, J.A.K., 2014, Using molecular epidemiology to track Toxoplasma gondii from terrestrial carnivores to marine hosts-Implications for public health and conservation: PLoS Neglected Tropical Diseases, $v$. 8 , no. 5, p. e2852. 
Table 30. Univariate analysis of putative categorical risk factors for Toxoplasma gondii antibody status in 159 sea otters sampled 2007-10 at Monterey and Big Sur, central California.

[ $p$ values are chi-square $p$ values for site and Fisher's exact test for sex and diet specialization. Antibody levels were determined by indirect fluorescent test (as described in chapter 9, section "Methods") with a positive cut-off titer of greater than or equal to $1: 320 . \mathbf{9 5 \%} \mathbf{C I}$ : 95 -percent confidence interval]

\begin{tabular}{llllll}
\hline Risk factor & Group & Seroprevalence & Odds ratio & $95 \% \mathrm{Cl}$ & $\mathbf{p}$ value \\
\hline Sex & Female & $0.12(\mathrm{n}=130)$ & 1 & - & \\
\multirow{3}{*}{ Site } & Male & $0.32(\mathrm{n}=28)$ & 3.63 & $1.39-9.47$ & 0.0162 \\
& Monterey & $0.13(\mathrm{n}=94)$ & 1 & - & \\
Diet & Big Sur & $0.18(\mathrm{n}=65)$ & 1.55 & $0.65-3.7$ & 0.4467 \\
& Type 1 & $0.06(\mathrm{n}=48)$ & 1.00 & - & 0.1065 \\
& Type 2 & $0.25(\mathrm{n}=12)$ & 5.00 & $0.87-28.86$ & \\
& Type 3 & $0.06(\mathrm{n}=16)$ & 1.00 & $0.1-10.35$ & \\
& Type 4 & $0.25(\mathrm{n}=12)$ & 5.00 & $0.87-28.86$ & \\
& Type 5 & $0.29(\mathrm{n}=7)$ & 6.00 & $0.8-44.95$ & \\
& Unknown & $19(\mathrm{n}=64)$ & 3.46 & $0.92-13.04$ & \\
\hline
\end{tabular}

Table 31. Associations of continuous variables with Toxoplasma gondii positive antibody status in 159 sea otters sampled 2007-10 at Monterey and Big Sur, central California.

[Welch two-sample $t$-test. Antibody levels were determined by indirect fluorescent test (as described in chapter 9, section

"Methods") with a positive cut-off titer of greater than or equal to 1:320. df: Degrees of freedom. $\boldsymbol{p}$ value: probability value]

\begin{tabular}{llll}
\hline Risk factor & $\boldsymbol{t}$ & df & $\boldsymbol{p}$ value \\
\hline Age & -2.775 & 35.67 & 0.0087 \\
Body length & -3.56 & 29.83 & 0.0013 \\
Body weight & -3.086 & 28.04 & 0.0045 \\
\hline
\end{tabular}


Table 32. Ordinary logistic regression models predicting Toxoplasma gondii positive antibody status on the basis of body length, site, sex, diet type, and percentage of snails in the diet by biomass.

[For this analysis, the five diet specialization types were collapsed to just three: type 2 , type 4 , and type $1 / 3 / 5$. Antibody levels determined by indirect fluorescent test (as described in chapter 9, section "Methods") with a positive cut-off titer of greater than or equal to 1:320. df: Degrees of freedom. AIC: Akaike information criterion]

\begin{tabular}{lll}
\hline Model & df & AIC \\
\hline length+diet+sex+sex*site & 81 & 48.78 \\
length+site & 85 & 49.02 \\
length+diet+site+Snail & 82 & 51.07 \\
length+diet+site+Snail+sex & 81 & 53.01 \\
length+diet+site & 83 & 57.44 \\
length+site+Snail & 84 & 62.08 \\
\hline
\end{tabular}

Table 33. Multivariable logistic regression model of risk factors for Toxoplasma gondii serum positive antibody status.

[Final model does not contain snail consumption as a proportion of biomass (although the variable slightly improved model fit), as it was not a significant predictor of antibody status, and inclusion of this variable precluded useful estimation of the other variables due to inadequate power. Antibody levels were determined by indirect fluorescent test (as described in chapter 9, section "Methods") with a positive cut-off titer of greater than or equal to 1:320. The adjusted odds-ratio, 95\% confidence interval (CI) and probability of type-I error (p-value) are shown for each level of the categorical risk factors, except for the reference levels (marked as "REF") that the other levels were compared to, for which the odds ratio is, by definition, 1, and there are no p-values (indicated by "_")]

\begin{tabular}{lllll}
\hline Risk factor & & Adjusted odds ratio & $95 \% \mathrm{Cl}$ & $\mathrm{p}$ value \\
\hline Length & centimeters & 1.44 & $1.05-1.97$ & 0.0291 \\
Diet & All other diet types & 1 & REF & - \\
& Abalone specialists & 5.86 & $0.63-54.19$ & 0.2642 \\
& Snail specialists & 18.14 & $1.82-180.48$ & 0.0165 \\
Sex & Female & 1 & REF & - \\
& Male & 0.06 & $0-4.12$ & 0.9527 \\
\multirow{3}{*}{ Sex:Site } & Female: Monterey compared to Big & 0.05 & $0-0.61$ & 0.0381 \\
& $\quad$ Sur & & $0.03-13.83$ & 0.366 \\
& Male: Monterey compared to. Big & 0.68 & & \\
\hline
\end{tabular}




\title{
Chapter 10. Preliminary Findings from Necropsy of Tagged Sea Otters from the Monterey-Big Sur Study
}

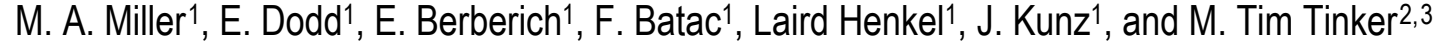

\section{Background}

All VHF transmitter-implanted otters from the Monterey and Big Sur coastal regions that stranded, died, and had the carcass recovered, plus any VHF transmitter-implanted animals that were euthanized during the course of the study (July 2008 through March 2012), were submitted to the California Department of Fish and Wildlife Marine Wildlife Veterinary Care and Research Center (CDFW-MWVCRC) for postmortem examination. For animals recovered in an advanced state of postmortem decomposition, necropsy typically consisted of gross examination with photographs of any potential lesions. In some cases, tissues were collected for microscopic examination, and postmortem radiographs were performed to rule out gunshot as a cause of death, or to confirm and characterize bone lesions. For animals received in fresh or moderate postmortem condition, the examination process was more extensive, consisting of gross necropsy, microscopic examination of all major tissues, bacterial and (or) fungal culture, and biochemical analysis of feces, urine, liver or other samples for the presence of marine and freshwater biotoxins as funding permitted. Subsamples of many tissues, urine, serum, bile, pericardial fluid, and cerebrospinal fluid also were cryoarchived at $-80^{\circ} \mathrm{C}$ to facilitate future diagnostic testing. Postmortem radiographs were performed for some cases, as directed by case history and presentation. Tissues examined on the microscope included multiple lymph nodes, spleen, liver, pancreas, kidney, adrenal gland, bladder, reproductive tract, ovary, testis, epididymis, lung, heart, aorta, peripheral nerves and ganglia, multiple skeletal muscles, diaphragm, tongue, tonsil, soft palate, esophagus, thyroid and parathyroid glands, thymus, omentum, pituitary gland, trigeminal nerve and ganglion, cerebrum, cerebellum and brainstem. Microscopic examination of hematoxylin and eosinstained tissues was completed by veterinary pathologists, and final reports were prepared that took into consideration information from gross and microscopic examination, bacterial culture, biochemical analysis, and any available antemortem tests. The goal of these analyses basically was to document general patterns in the cause of death of study animals in the Big Sur-Monterey study, and to identify any unexpected or prevalent risk factors. Issues of particular interest would be causes of death associated with anthropogenic risk factors, and whether these causes differed among study sites.

\footnotetext{
${ }^{1}$ California Department of Fish and Wildlife.

${ }^{2}$ U.S. Geological Survey.

${ }^{3}$ University of California, Santa Cruz.
} 


\section{Summary of Lesions, Test Results, and Diagnoses}

In addition to detailed necropsy reports, two summary tables were prepared to compare broader disease and mortality patterns among sea otters that were tagged in the Monterey and Big Sur coastal regions. The stranding location, stranding date, and overall demographical information is summarized in table 34 for 17 tagged sea otters from the Monterey and Big Sur sample populations that were submitted for necropsy as of March 2012. Nearly all were female (82-percent), and 94-percent were adults or aged adults. Slightly more than one-half (53-percent) were examined in fresh postmortem condition, 18percent were moderately decomposed, and 29-percent were severely decomposed or mummified. Roughly equal proportions of necropsied otters were examined from Monterey Bay (53-percent, $n=9$ ) and the Big Sur coast (47-percent, $n=8$ ). All but one animal were recovered from the area where they had originally been tagged (that is, Monterey or Big Sur).

For 16 otters with known nutritional condition (minus one mummified animal), 31-percent died with fair-to-abundant adipose stores, whereas 69-percent had scant or no fat at the time of necropsy. Roughly equal proportions of sea otters with fair-to-abundant adipose stores were recovered from the Monterey Bay (3) and Big Sur (2) areas, and one of the three Monterey Bay animals had originally been captured in Big Sur. Sixteen animals had intact gastrointestinal tracts; of these, 12 had scant or no food in the gastrointestinal tract, and the remainder contained moderate or abundant digesta. Fragments of crab carapace, especially large (Cancer) crabs, were apparent in digesta at necropsy, along with mussel shell fragments and various invertebrate parts (table 34). Three of the 17 otters were alive when recovered; one otter died post-stranding and the other two were humanely euthanized owing to a poor clinical prognosis. For 10 adult female sea otters that were fresh enough for accurate determination, 9 had moderate or severe nose wounds at necropsy. Of nine adult females that were fresh enough for determination, four were lactating, and three additional females had prominent mammary glands suggestive of recent pregnancy. Estimates of the number of completed pregnancies per adult female, as determined by counts of grossly apparent corpora albicans on both ovaries (not including follicles or corpora lutea indicative of current, pre-implantation cycling or pregnancy), averaged 2.59 (range 0-5). Both adult males were producing sperm at the time of death, although a low number of spermatozoa were observed in the testicle and epididymis of sea otter 5738-10 (an aged adult) on histology.

\section{Congenital Malformations and Conditions with Undetermined Cause}

Numerous physical conditions noted during necropsy of tagged southern sea otters could represent normal variation or congenital malformations. Because the southern sea otter population has experienced a significant bottleneck over the past century, it is important to monitor these conditions. All physical abnormalities that were observed during the current study appeared to be mild and incidental. These conditions were found in the heart, kidneys and reproductive tract (table 34), and included cardiac valve hemal cysts $(n=2)$, para-ovarian cysts $(n=3)$ and renal cortical cysts $(n=1)$. Most defects appeared to be incidental, and are fairly common in southern sea otters (Melissa Miller, unpub. data 2018). However, one adult female had unilateral ovarian agenesis, a condition that could affect fertility (fig. 55).

\section{Antemortem and Postmortem Bacterial Culture}

The results of aerobic and anaerobic bacterial culture are summarized in table 35 . Bacterial data were available for 12 of the 17 tagged sea otters. All bacterial isolation and identification were performed at the University of California Davis Veterinary Medical Teaching Hospital. In table 35, samples where the "Collection Date" is indicated in bold text were obtained during capture, whereas all other isolates denoted in black text were collected during necropsy. Swabs of potential lesions were 
submitted for aerobic +/- anaerobic bacterial culture using non-selective media. Fecal samples were submitted for assessment using a "Fecal Pathogen Protocol" that was developed during a prior study of southern sea otter enteric bacterial flora (Miller and others, 2010). Opportunistic bacterial pathogens detected in live otter feces included Streptococcus infantarius ss coli $(\mathrm{n}=1)$, Vibrio alginolyticus $(\mathrm{n}=$ $2)$, and Vibrio parahaemolyticus $(\mathrm{n}=2)$. Only once were the same bacterial pathogens detected in feces of live otters, and feces or tissues of the same animal postmortem.

Localized or systemic bacterial infection was a common primary or contributing cause of death for stranded otters (table 36). Underlying causes of these infections included shark bite, mating wounds, anthropogenic trauma, and, possibly, dental disease or enterocolitis. The spectrum of bacteria isolated from the tagged otters is similar to isolates obtained from random-source stranded sea otters (Kreuder and others, 2003). The bacterial isolates encompass a range of weak opportunistic pathogens (for example, non-hemolytic E. coli), moderate pathogens (for example, Streptococcus phocae), and primary pathogens (for example, Erysipelothrix rhusiopathiae). Mixed infections of aerobic and anaerobic bacteria were common in bite wounds, abscesses, and associated lymph nodes.

\section{Antemortem and Postmortem Tests for Biotoxins}

The results of antemortem and postmortem tests for the presence of freshwater or marine biotoxins are summarized in table 37, and include saxitoxin (STX), domoic acid (DA), microcystin $(\mathrm{MC})$, nodularin (NO), anatoxin-a (AN), and okadaic acid (OA) in samples from tagged sea otters. Two assays were used for biotoxin detection: (1) Enzyme-Linked Immunosorbent Assay (ELISA), a relatively inexpensive test that indirectly measures toxin activity; and (2) liquid chromatography/mass spectrometry (LC/MS), a sensitive, specific, and expensive assay that measures biotoxin content. Both ELISA and LC/MS assays were used for DA detection, only ELISA was used for STX detection, and only LC/MS was used to detect all other toxins. Of 14 necropsied otters with samples available for testing for DA, 8 out of 14 (57-percent) tested positive for DA above established minimum detection limits (MDLs) in one or more samples, and 2 animals (14-percent) had urine DA concentrations $>200$ parts per billion, which could be suggestive of DA intoxication. Several DA-negative animals only had serum available for testing. Because of the short systemic half-life of DA and the relative insensitivity of serum as a screening sample, it is possible that additional otters would have tested DA-positive if urine or gastrointestinal content were available for analysis.

Tissues from two of the six LC/MS-tested sea otters were low-positive for the freshwater cyanotoxin $\mathrm{MC}$, and this toxin could have been a contributing factor in the death of the animals. Microcystin testing should be performed for additional animals as funding permits, based on available samples. Because conventional diagnostic tests only detect free (unbound) microcystins, and because these toxins bind covalently (for example, strongly) to tissue receptors, a high potential exists for falsenegative tests. For these reasons and because repeated testing of the same sample matrices have yielded variable results, additional testing for $\mathrm{MC}$ in sea otters has been suspended until improved and validated diagnostic tests become available.

Sea otter gastrointestinal content or feces tested low-positive for STX for five otters. The significance of this finding is not well understood, but merits investigation. Like DA, STX is widely distributed throughout the California coastal ecosystem. Characterizing associations with STX detection and sea otter illness or death can be difficult when toxin is present at low concentrations, and because of the mode of action of this group of toxins, lesions are absent or non-specific. Tests for OA and assays for the cyanotoxins AN and NO were negative in all cases. 


\section{Significant Findings from Gross Necropsy, Histopathology, and Diagnostic Testing}

A summary of findings from gross necropsy, histopathology, and diagnostic testing for each tagged otter is provided in table 38, including subjective weighting of the relative severity of each finding. These findings are further distilled in primary and contributing causes of death in table 36 . As reported in prior studies (Kreuder and others, 2003), it is common for southern sea otters to have multiple concurrent or interrelated disease processes at the time of death, as table 38 shows. With the exception of markedly autolyzed animals where detailed lesion characterization was not possible, nearly all the tagged otters had $>1$ concurrent disease process that was at least moderately severe. As shown in table 38, discovery of three or more independent pathological processes impacting otter survival at necropsy is common, and some conditions (that is, protozoal encephalitis, microcystin, and domoic acid toxicity) have been reported to have connections with anthropogenic pollution.

In addition to the two animals mentioned in section, "Antemortem and Postmortem Tests for Biotoxins", with possible DA intoxication, sea otters with chronic and (or) recurrent DA exposure may develop progressive heart muscle damage and cardiac failure (cardiomyopathy syndrome). Three of 14 tagged sea otters with sufficient detail for accurate determination had gross and (or) microscopic lesions of mild-to-severe cardiomyopathy syndrome. Other suspected causes of cardiac muscle damage in sea otters include infection by protozoal parasites and viruses, and possibly exposure to other environmental biotoxins in addition to DA.

Localized and (or) systemic bacterial infection, spanning a range of opportunistic and primary pathogens, also was common among examined animals. Some of these bacteria inhabit marine or estuarine environments, and others may be derived from freshwater or marine fecal contamination. Pathogenic bacteria can concentrate in tissues of filter-feeding invertebrates, including species that are consumed by sea otters and humans, such as mussels and clams. For 11 sea otters with sufficient detail for accurate determination, 91-percent had localized and (or) systemic bacterial infections at necropsy, and the infections were considered severe for 64-percent of examined animals.

The low proportion of sea otters with significant infections by acanthocephalan parasites and protozoa in this study partly indicates sample bias; for both groups of pathogens, risk of clinically severe disease is highest in immature animals, whereas nearly all otters in the current study (16 out of 17) were adults or aged adults. This low prevalence also could indicate sample bias, dietary preferences, cyclic or long-term trends in parasite prevalence, and other factors that could not be measured.

Trauma-associated death was observed for eight sea otters in the current study. Of the 13 animals that were fresh enough for accurate determination, trauma of any cause was found in 8 cases, including confirmed or suspected shark bite $(\mathrm{n}=4)$, confirmed or suspected boat strike $(\mathrm{n}=2)$, and infection secondary to recent surgery $(n=2)$. Fight-associated trauma also was found in a sea otter that died with confirmed shark bite.

Emaciation was a consistent finding at necropsy, which is not surprising, given the presence of chronic disease in most stranded animals. Of the 17 animals where nutritional condition could be accurately assessed at necropsy, 3 were in good to excellent nutritional condition at the time of death, 3 were in poor or fair condition, and 10 were thin or emaciated (6 out of 8 animals at Big Sur and 4 out of 9 animals at Monterey; table 34). Nearly all the thin or emaciated sea otters had severe, concurrent, subacute, or chronic disease processes at necropsy (table 38).

The current study provides important insight into influences of sea otter biology, the reproductive cycle, intraspecific interactions, and nutritional stress on the health of the southern sea otter population. One important finding that resulted from this Coastal Conservancy-supported research is an improved understanding of the importance of end-lactation syndrome (ELS) as an obstacle to southern sea otter population recovery. As a condition that is intricately associated with the high energetic requirements of sea otter reproduction, ELS is a condition that affects only adult females. Factors 
associated with ELS as a primary or contributing cause of death include the high energetic costs of pregnancy and pup care, nutritional stress, concurrent disease, and aggression by male sea otters during estrus. For 12 adult females that were sufficiently fresh for accurate determination, caloric stress due to pregnancy and postpartum pup care was a possible contributing factor for the death of 7 animals, and ELS was a primary cause of death for 2 animals. This result is especially concerning because survival of reproductive-age female sea otters is critical to achieve population recovery.

The primary and contributing causes of death for each otter are summarized in table 36 . These determinations were made after carefully considering all available data for each case, including the clinical history, as well as results from gross necropsy, histopathology, and available diagnostic tests. The primary and top two contributing causes of death were determined for 15 of the 17 tagged sea otters. Advanced autolysis prohibited identification of antemortem lesions for the remaining two animals. Prominent primary causes of death were presumptive or confirmed shark bite $(\mathrm{n}=4)$, bacterial septicemia $(n=3)$, and ELS $(n=4)$. Three out of 4 otters with ELS as the primary cause of death originated from the Big Sur coastline, whereas shark bite cases were divided between Monterey $(n=2)$ and Big Sur $(n=2)$. Both suspected boat strike cases were recovered from Monterey Bay, including Moss Landing Harbor, a high-risk area for boat strikes due to the close proximity of large numbers of sea otters to boats.

The most common secondary or contributing causes of death included probable or possible domoic acid intoxication $(n=4)$, gastric ulcers and melena $(n=7)$, and acanthocephalan peritonitis and ELS (2 each). Three additional cases of bacterial infection were also noted as either a primary process of sequela.

When pooled across the primary and top two contributing causes of death, key causes of sea otter death across 15 tagged, necropsied otters with sufficient tissue detail for lesion identification included bacterial infection as a primary cause of death or sequela $(n=7)$, gastric ulcers and melena $(n$ $=7)$, ELS $(n=6)$, possible or probable domoic acid intoxication $(n=4)$, shark bite $(n=4)$, and presumed boat strike $(n=2)$. Necropsied otters with ELS, cardiomyopathy and shark bite as primary or contributing causes of death were evenly divided between BSR and MON; two additional otters with mating trauma as a contributing cause of death were recovered from BSR. Three of the four possible or probable DA intoxication cases were recovered along the MON coast. Both otters with acanthocephalan peritonitis were recovered from MON. Selected images of findings during gross necropsy of the tagged sea otters are included here (figs. 55-70).

Our research has helped focus attention on ELS as an important target for improving southern sea otter population recovery. Knowledge gained through the current study should help catalyze scientific study of ELS. An improved understanding of possible links between poor population recovery, nutritional stress, and ELS in the central part of the sea otter range helped lead to abolishment of the "no otter zone", allowing expansion of the sea otter population into historical range in southern California and, hopefully, achieving population recovery over the coming years.

Because our sample size is very small in this preliminary review of tagged, necropsied sea otters, inference of population-level trends should be done with caution. Reassessment of disease patterns will be completed in the future when a larger sample of tagged animals has been examined by veterinary pathologists. Additionally, comparing data from detailed postmortem examinations with information gleaned from ongoing field monitoring efforts, antemortem tests and foraging data will be critical. Factors that limit the precision of lesion interpretation include postmortem scavenging and autolysis; these obstacles commonly are encountered during prospective observational studies of wide-ranging, tagged, wild marine animals such as sea otters. An additional obstacle is the limited ability to test all animals for all potential pathogens and toxins owing to cost constraints and sample availability. Despite these unavoidable obstacles, sustained multi-institutional collaboration has enabled scientists to 
complete a landmark study. The Monterey Bay-Big Sur study is the first of its kind to use a "life history" approach to examining in detail the intersection between sea otter biology and environment, and the ecology of disease. By synergizing the efforts of sea otter biologists, veterinarians, and pathologists, experts in statistical modeling, toxicology, and other scientific disciplines, we have learned much about the complex interplay between sea otters and their environment. As monitoring of tagged otters and postmortem examinations continue over the next few years, additional insight provided by this work will continue to guide sea otter conservation efforts.

\section{References Cited}

Kreuder, C., Miller, M.A., Jessup, D.A., Lowenstine, L.J., Harris, M.D., Ames, J.A., Carpenter, T.E., Conrad, P.A., and Mazet, J.A., 2003, Patterns of mortality in southern sea otters (Enhydra lutris nereis) from 1998-2001: Journal of Wildlife Diseases, v. 39, no. 3, p. 495-509.

Miller, M.A., Byrne, B.A., Jang, S.S., Dodd, E.M., Dorfmeier, E., Harris, M.D., Ames, J., Paradies, D., Worcester, K., Jessup, D.A., and Miller, W.A., 2010, Enteric bacterial pathogen detection in southern sea otters (Enhydra lutris nereis) is associated with coastal urbanization and freshwater runoff: Veterinary Research, v. 41, no. 1, 13 p. 
Table 34. Stranding location, stranding date, and overall demographical information for tagged, necropsied sea otters from Monterey Peninsula and Big Sur coast study sites, central California.

[Abund: Abundant. Carcass condition: Mod Decomp, moderate decomposition; Adv decomp, advanced decomposition; Mumm/frag, mummified/fragmented. Parasites: C, Corynosoma; P, Profilicollis acanthocephalans observed in the intestines. Med: Medium. Mod: Moderate. n/a: not applicable. Unk: unknown]

\begin{tabular}{|c|c|c|c|c|c|c|c|c|c|c|c|c|c|c|c|c|c|c|c|c|c|c|}
\hline 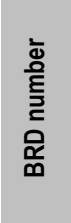 & 总 & ॐ & 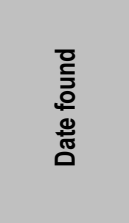 & 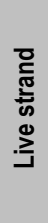 & 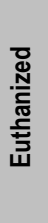 & 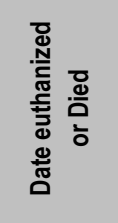 & \& & 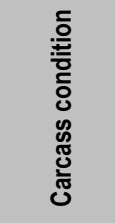 & 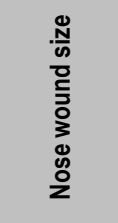 & 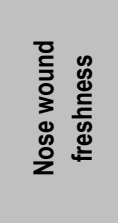 & 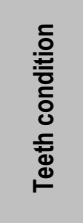 & 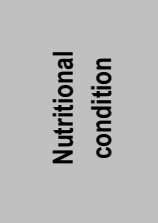 & 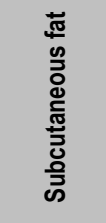 & 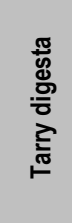 & $\begin{array}{l}\text { 亏亏 } \\
\text { 은 } \\
\text { 인 }\end{array}$ & बेँ & 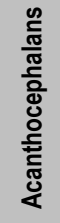 & 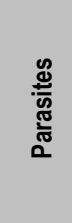 & 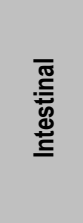 & 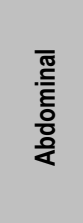 & 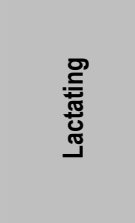 & 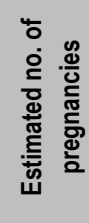 \\
\hline 1090 & BSR & $\mathrm{F}$ & $11 / 25 / 08$ & $\mathrm{~N}$ & $\mathrm{~N}$ & Unk & AA & $\begin{array}{c}\text { Mod } \\
\text { decomp }\end{array}$ & Medium & White & Poor & Emaciated & Fair & No & None & $\mathrm{n} / \mathrm{a}$ & $\mathrm{Y}$ & $\mathrm{C}$ & Mild & None & Not noted & 5 \\
\hline 1106 & BSR & $\mathrm{F}$ & $8 / 24 / 09$ & $\mathrm{~N}$ & $\mathrm{~N}$ & Unk & A & $\begin{array}{c}\text { Adv } \\
\text { decomp }\end{array}$ & Decomp & Decomp & Unk & Emaciated & None & Yes & None & $\mathrm{n} / \mathrm{a}$ & $\mathrm{Y}$ & $\mathrm{C}$ & Mild & None & Y (Scant) & 2 \\
\hline 1069 & BSR & $\mathrm{F}$ & 9/14/09 & $\mathrm{N}$ & $\mathrm{N}$ & Unk & A & Fresh & Large & Pink & Good & Emaciated & None & Yes & None & $\mathrm{n} / \mathrm{a}$ & $\mathrm{Y}$ & $\mathrm{C}$ & Mild & None & $\begin{array}{l}\text { Y (Lots, } \\
\& \text { prom. } \\
\text { mammary } \\
\text { glands) }\end{array}$ & $3-3.5$ \\
\hline 1074 & BSR & $\mathrm{F}$ & $5 / 31 / 11$ & $\mathrm{~N}$ & $\mathrm{~N}$ & Unk & AA & Fresh & Large & Pink & Poor & Emaciated & None & Yes & None & $\mathrm{n} / \mathrm{a}$ & $\mathrm{Y}$ & $\mathrm{C}$ & Mild & None & $\begin{array}{l}\text { No, but } \\
\text { mammary } \\
\text { tissue } \\
\text { prominent }\end{array}$ & 1 \\
\hline 1139 & BSR & $\mathrm{F}$ & $9 / 16 / 11$ & $\mathrm{~N}$ & $\mathrm{~N}$ & Unk & A & Fresh & Large & Pink & Good & Emaciated & None & Yes & Little & $\begin{array}{c}\text { Cancer } \\
\text { crab }\end{array}$ & $\mathrm{Y}$ & $\mathrm{C}$ & Mod & None & $\begin{array}{l}\text { Yes (milk } \\
\text { collected) }\end{array}$ & 2 \\
\hline 1089 & BSR & $\mathrm{F}$ & $11 / 8 / 11$ & $\mathrm{~N}$ & $\mathrm{~N}$ & Unk & A & Fresh & Large & Pink & Fair & Emaciated & None & Yes & Med. & $\begin{array}{c}\text { Mostly } \\
\text { urchins, } \\
\text { crab, } \\
\text { snail }\end{array}$ & $\mathrm{Y}$ & $\mathrm{C}>\mathrm{P}$ & Mod & None & $\mathrm{N}$ & 3 \\
\hline 1093 & BSR & M & $11 / 8 / 08$ & $\mathrm{Y}$ & $\mathrm{N}$ & $11 / 8 / 08$ & A & Fresh & None & None & Good & Fair to good & Fair & No & None & $\mathrm{n} / \mathrm{a}$ & $\mathrm{Y}$ & $\mathrm{C}$ & Mild & None & $\mathrm{n} / \mathrm{a}$ & $\mathrm{n} / \mathrm{a}$ \\
\hline 1094 & BSR & M & $11 / 23 / 09$ & $\mathrm{~N}$ & $\mathrm{~N}$ & Unk & A & $\begin{array}{c}\text { Adv } \\
\text { decomp } \\
\end{array}$ & Small & White & Fair & Excellent & Abund & No & Little & $\begin{array}{c}\text { Inkeeper, } \\
\text { crab }\end{array}$ & $\mathrm{Y}$ & $\mathrm{C}=\mathrm{P}$ & Mild & None & $\mathrm{n} / \mathrm{a}$ & $\mathrm{n} / \mathrm{a}$ \\
\hline 1033 & MON & $\mathrm{F}$ & 7/7/09 & $\mathrm{N}$ & $\mathrm{N}$ & Unk & A & $\begin{array}{c}\text { Mumm/ } \\
\text { frag }\end{array}$ & Decomp & Decomp & Good & Unknown & Unk & Unk & Unk & $\mathrm{n} / \mathrm{a}$ & $\mathrm{U}$ & Unk & Unk & Unk & Unk & Unk \\
\hline 1064 & MON & $\mathrm{F}$ & 11/19/09 & $\mathrm{N}$ & $\mathrm{N}$ & Unk & SA & $\begin{array}{c}\text { Adv } \\
\text { decomp }\end{array}$ & Decomp & Decomp & Unk & Emaciated & Scant & Yes & Med. & $\begin{array}{l}\text { Crabs } \\
\text { and } \\
\text { algae }\end{array}$ & $\mathrm{Y}$ & $\mathrm{C}$ & Mild & None & $\mathrm{n} / \mathrm{a}$ & $\mathrm{n} / \mathrm{a}$ \\
\hline 1051 & MON & $\mathrm{F}$ & $3 / 22 / 10$ & $\mathrm{~N}$ & $\mathrm{~N}$ & Unk & A & $\begin{array}{c}\text { Mod } \\
\text { decomp }\end{array}$ & Small & White & Good & Excellent & Abund & Unk & Unk & $\begin{array}{l}\text { sea star, } \\
\text { snail, } \\
\text { Cancer } \\
\text { crab }\end{array}$ & $\mathrm{Y}$ & $\mathrm{C}$ & Mod & Unk & $\begin{array}{l}\text { No, but } \\
\text { prominent } \\
\text { mammary } \\
\text { glands }\end{array}$ & Unk \\
\hline
\end{tabular}




\begin{tabular}{|c|c|c|c|c|c|c|c|c|c|c|c|c|c|c|c|c|c|c|c|c|c|c|}
\hline 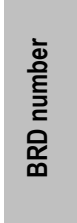 & 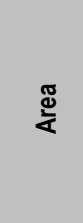 & ふో & 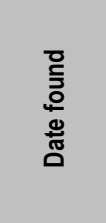 & 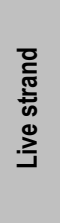 & 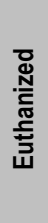 & 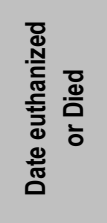 & 胥 & 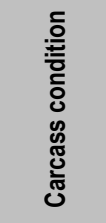 & 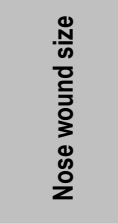 & 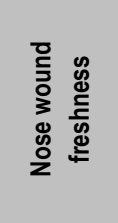 & 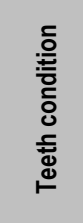 & 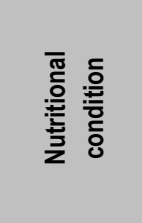 & 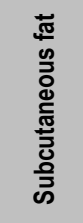 & 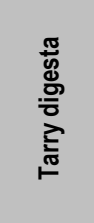 & 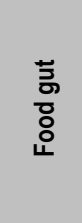 & बेँ & 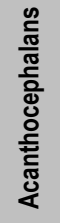 & 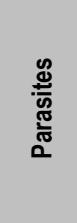 & 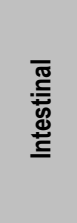 & 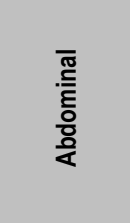 & 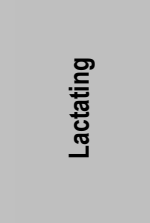 & 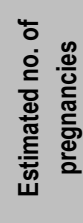 \\
\hline 1155 & MON & $\mathrm{F}$ & $6 / 12 / 10$ & $\mathrm{~N}$ & $\mathrm{~N}$ & Unk & A & Fresh & Medium & Red & Good & Emaciated & None & Yes & None & $\begin{array}{c}\text { sea } \\
\text { water } \\
\text { and } \\
\text { melena }\end{array}$ & $\mathrm{Y}$ & $\mathrm{C}>\mathrm{P}$ & Mod & None & No & 2.5 \\
\hline 1042 & MON & $\mathrm{F}$ & $5 / 12 / 11$ & $\mathrm{~N}$ & $\mathrm{~N}$ & Unk & A & $\begin{array}{c}\text { Adv } \\
\text { decomp }\end{array}$ & Decomp & Decomp & Fair & Emaciated & None & Yes & Med. & $\begin{array}{l}\text { mussel, } \\
\text { crab }\end{array}$ & $\mathrm{N}$ & & No & None & Y (Scant) & $1+$ \\
\hline 1037 & MON & $\mathrm{F}$ & $6 / 18 / 11$ & $\mathrm{~N}$ & $\mathrm{~N}$ & Unk & AA & $\begin{array}{c}\text { Mod } \\
\text { decomp }\end{array}$ & Large & Pink & Fair & Poor & Scant & No & Full & $\begin{array}{c}\text { Cancer } \\
\text { crab, } \\
\text { octopus, } \\
\text { kelp crab }\end{array}$ & Y & $\mathrm{C}$ & Mod & None & $\begin{array}{l}\text { No, but } \\
\text { mammary } \\
\text { tissue } \\
\text { prominent }\end{array}$ & 5 \\
\hline 1016 & MON & $\mathrm{F}$ & $10 / 5 / 11$ & $\mathrm{Y}$ & $\mathrm{Y}$ & $10 / 5 / 11$ & A & Fresh & Large & Red & Fair & Emaciated & Scant & Slight & None & $\mathrm{n} / \mathrm{a}$ & Y & $\mathrm{P}>\mathrm{C}$ & Mod & Moderate & $\begin{array}{l}\text { Yes } \\
\text { (Scant } \\
\text { milk, } \\
\text { small, flat, } \\
\text { atrophied } \\
\text { mammary } \\
\text { glands) }\end{array}$ & $3.5+$ \\
\hline 1109 & MON & $\mathrm{F}$ & $3 / 11 / 12$ & $\mathrm{~N}$ & $\mathrm{~N}$ & Unk & A & Fresh & Large & White & Fair & Fair & Fair & No & None & $\mathrm{n} / \mathrm{a}$ & Y & $\mathrm{P}>\mathrm{C}$ & Mild & None & $\mathrm{N}$ & 5 \\
\hline 1024 & MON & M & $3 / 29 / 10$ & $\mathrm{Y}$ & Y & $3 / 29 / 10$ & AA & Fresh & Medium & Red & Poor & Poor & Scant & Slight & Little & $\begin{array}{l}\text { Clams, } \\
\text { other un- } \\
\text { ID soft } \\
\text { tissue }\end{array}$ & $\mathrm{Y}$ & $\mathrm{C}$ & Mild & None & $\mathrm{n} / \mathrm{a}$ & $\mathrm{n} / \mathrm{a}$ \\
\hline
\end{tabular}


Table 35. Aerobic and anaerobic bacterial culture findings for tagged, necropsied sea otters from Monterey Peninsula and Big Sur coast study sites, central California.

[Samples where "Collection Date" is denoted in bold text are antemortem bacterial cultures (that is, samples collected during capture), while those in standard text indicate postmortem bacterial cultures. "+" indicates growth of bacterium in the sample, and "-“ indicates no growth]

\begin{tabular}{|c|c|c|c|c|c|c|c|c|c|c|c|c|c|c|c|c|c|c|c|}
\hline $\begin{array}{c}\text { BRD } \\
\text { number }\end{array}$ & Area & Sex & $\begin{array}{c}\text { Necropsy } \\
\text { date }\end{array}$ & $\begin{array}{c}\text { Collection } \\
\text { date }\end{array}$ & Culture site & 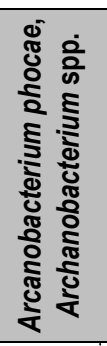 & 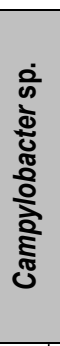 & 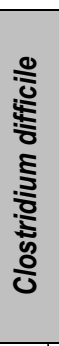 & 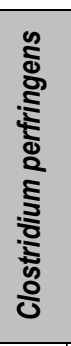 & 广̄ & 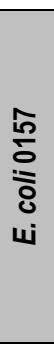 & 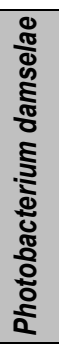 & 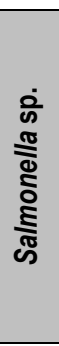 & 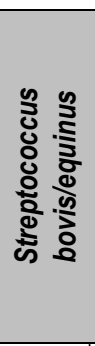 & 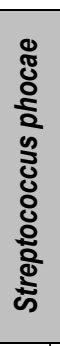 & 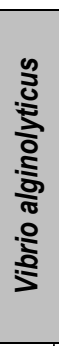 & 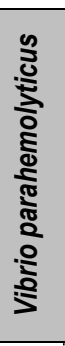 & $\begin{array}{l}\text { के } \\
\circ \\
\frac{0}{3} \\
\frac{3}{5}\end{array}$ & 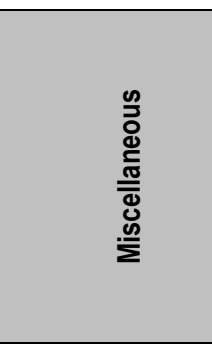 \\
\hline \multirow[t]{7}{*}{1090} & BSR & $\mathrm{F}$ & $11 / 27 / 08$ & 11/10/08 & Feces & & - & - & - & & - & & - & - & & & & - & \\
\hline & & & & $\begin{array}{l}11 / 27 / 08 \\
11 / 27 / 08\end{array}$ & $\begin{array}{l}\text { Feces } \\
\text { Incision }\end{array}$ & & - & - & - & & - & + & & - & & & & - & $\begin{array}{l}\text { Salmonella } \\
\text { saintpaul } \\
\text { Proteus sp., } \\
\text { Shewanella } \\
\text { putrefaciens }\end{array}$ \\
\hline & & & & $11 / 27 / 08$ & Liver & & & & & & & & & & & & & & \\
\hline & & & & $11 / 27 / 08$ & Lymph node - inguinal & & & & & & & + & & & & & & & \\
\hline & & & & $11 / 27 / 08$ & Muscle & & & & & + & & & & & & & & & \\
\hline & & & & $11 / 27 / 08$ & Spleen & & & & & & & & & & & & & & \\
\hline & & & & $11 / 27 / 08$ & Transmitter & & & & & & & + & & & & & & & \\
\hline 1106 & BSR & $\mathrm{F}$ & $8 / 27 / 09$ & 11/11/08 & Feces & & - & - & - & & - & & - & - & & & & & Vibrio cholerae \\
\hline \multirow[t]{2}{*}{1069} & BSR & $\mathrm{F}$ & $9 / 16 / 09$ & $11 / 5 / 08$ & Feces & & - & - & - & & - & & - & - & & & & - & \\
\hline & & & & $\begin{array}{l}9 / 16 / 09 \\
9 / 16 / 09 \\
9 / 16 / 09 \\
\end{array}$ & $\begin{array}{l}\text { Abscess } \\
\text { Lymph node - } \\
\text { retropharyngeal } \\
\text { Wound }\end{array}$ & $\begin{array}{l}+ \\
+ \\
\end{array}$ & & & & & & & & & $\begin{array}{l}+ \\
+ \\
\end{array}$ & $\begin{array}{l}+ \\
+ \\
+ \\
+\end{array}$ & & & $\begin{array}{l}\text { Bacilli } \\
\text { Nonenteric } \\
\quad \text { Bacteria } \\
\text { Shewanella } \\
\text { putrefaciens }\end{array}$ \\
\hline \multirow[t]{4}{*}{1074} & BSR & $\mathrm{F}$ & $6 / 1 / 11$ & $11 / 6 / 08$ & Feces & & - & - & - & & - & & - & - & & & & - & \\
\hline & & & & $9 / 24 / 10$ & Feces & & - & - & - & & - & & - & - & & + & & & \\
\hline & & & & $6 / 1 / 11$ & Heart blood & & & & & + & & & & & & & & & NFG 3 \\
\hline & & & & $6 / 1 / 11$ & Liver & & & & & & & & & & & & & & \\
\hline
\end{tabular}




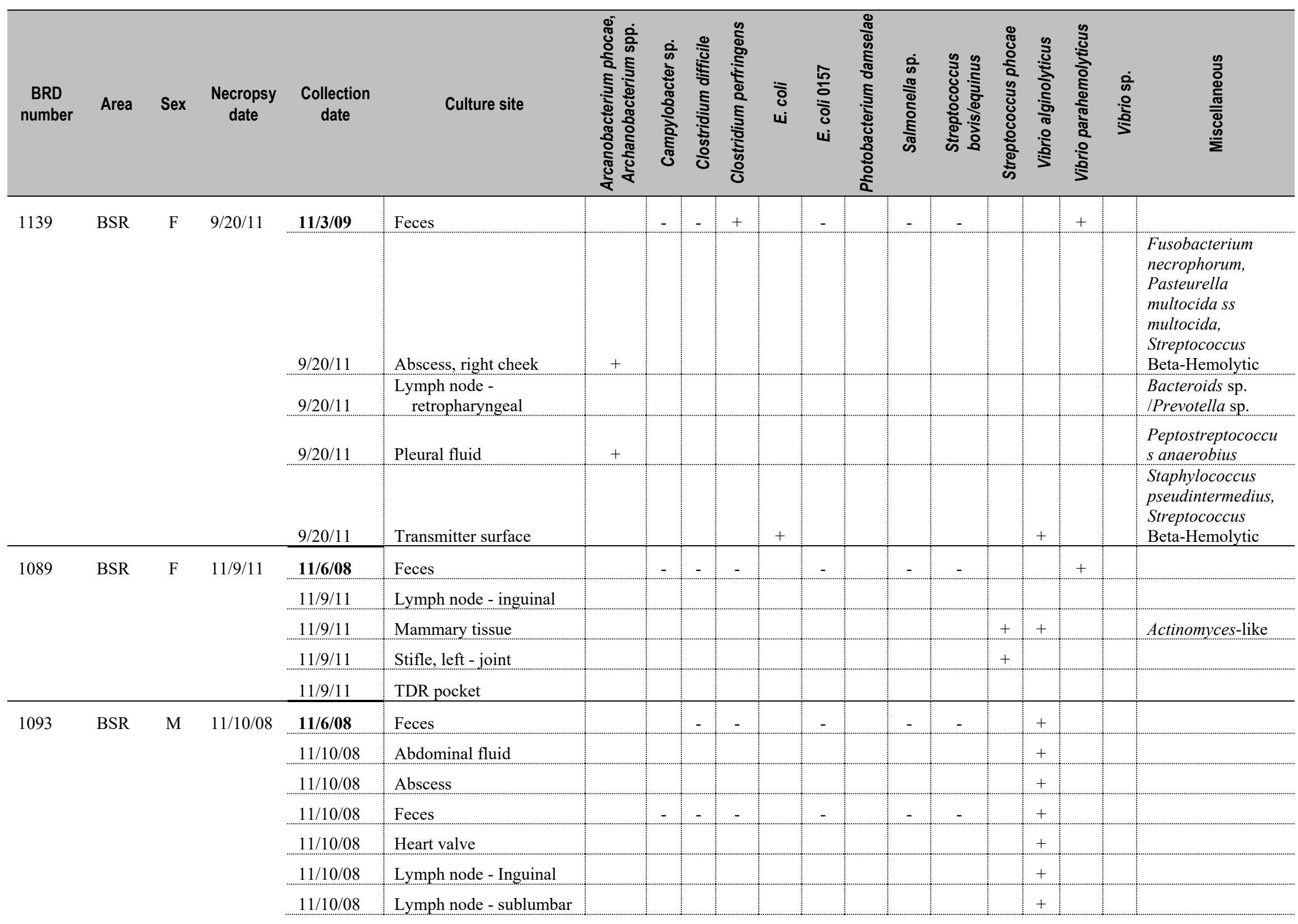




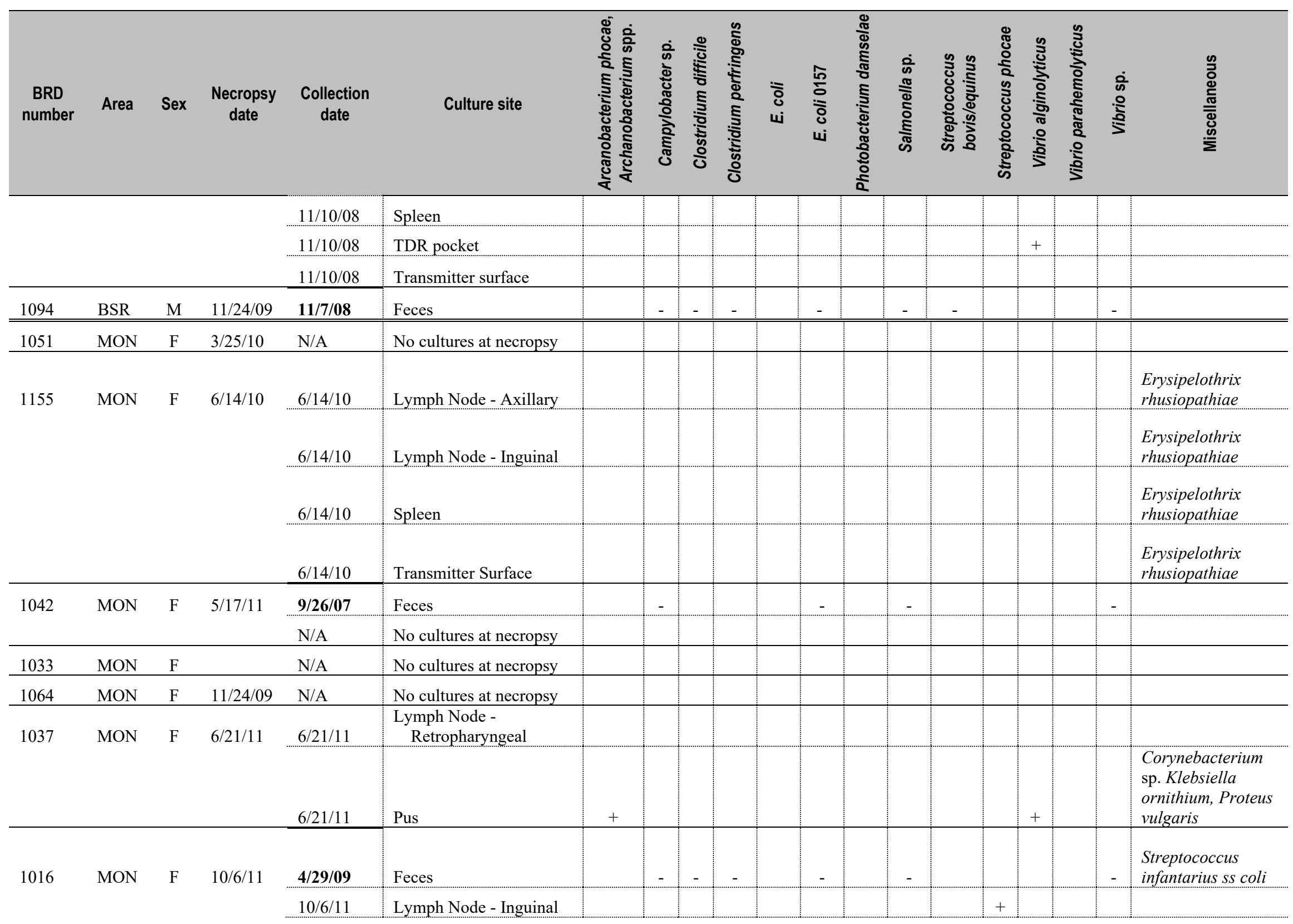




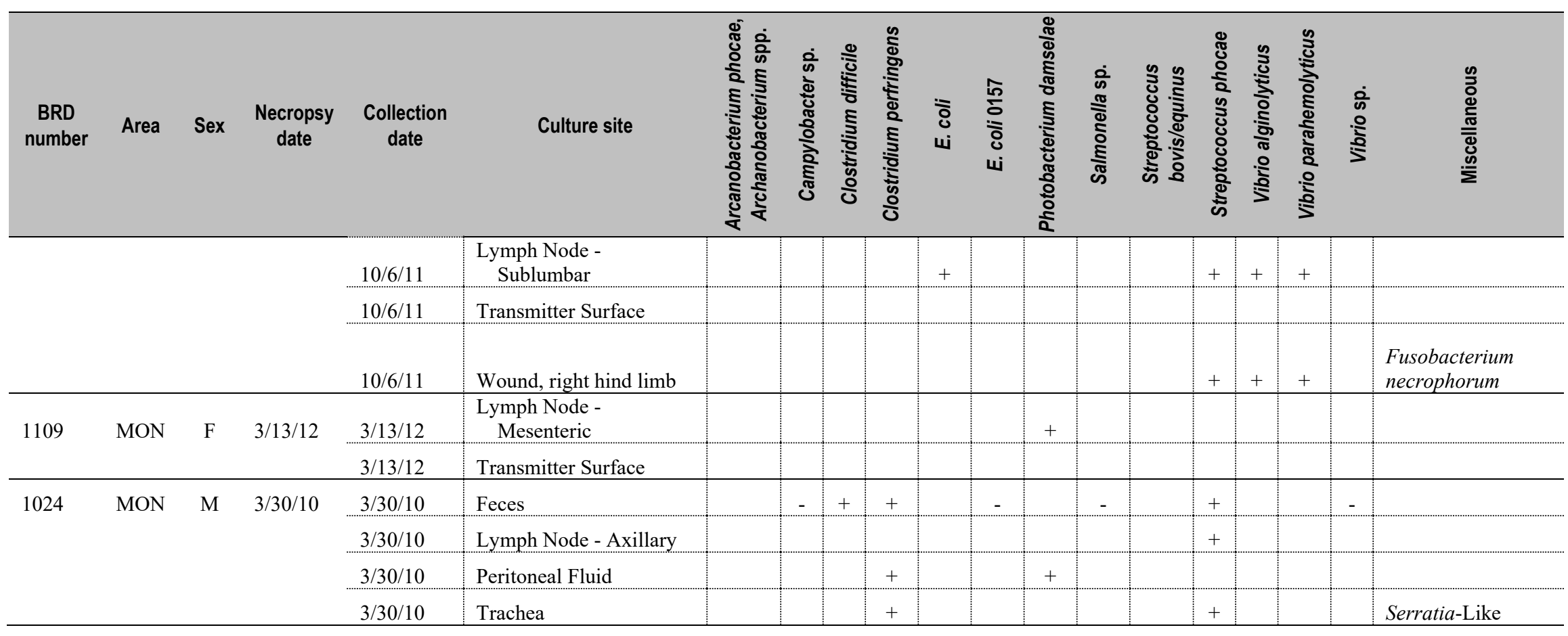


Table 36. Overview of implant history, and primary and contributing causes of death for tagged, necropsied sea otters from Monterey Peninsula and Big Sur coast study sites, central California.

[TDR, time-depth recorder; VHF, very high frequency radio transmitters]

\begin{tabular}{|c|c|c|c|c|c|c|}
\hline $\begin{array}{c}\text { BRD } \\
\text { number }\end{array}$ & Area & Sex & $\begin{array}{l}\text { Instruments recovered and } \\
\text { associated findings }\end{array}$ & $\begin{array}{c}\text { Primary cause of } \\
\text { death }\end{array}$ & Contributing number 1 & Contributing number 2 \\
\hline 1090 & BSR & $\mathrm{F}$ & $\begin{array}{l}\text { TDR and VHF present and free- } \\
\text { floating (no entrapments) }\end{array}$ & $\begin{array}{l}\text { Surgery-associated } \\
\text { bacterial infection } \\
\text { and sepsis } \\
\end{array}$ & Skeletal muscle necrosis & Dental abscess/tooth wear \\
\hline 1106 & BSR & $\mathrm{F}$ & $\begin{array}{c}\text { VHF free-floating, TDR } \\
\text { entrapped and twisted in omental } \\
\text { stalk }\end{array}$ & $\begin{array}{l}\text { End lactation } \\
\text { syndrome }\end{array}$ & Gastric ulcers and melena & None \\
\hline 1069 & BSR & $\mathrm{F}$ & $\begin{array}{l}\text { VHF free-floating, TDR } \\
\text { entrapped in omental bursa }\end{array}$ & $\begin{array}{l}\text { End lactation } \\
\text { syndrome }\end{array}$ & $\begin{array}{l}\text { Infected nose wound } \\
\text { leading to bacterial sepsis }\end{array}$ & Gastric ulcers and melena \\
\hline 1074 & BSR & $\mathrm{F}$ & $\begin{array}{l}\text { No TDR. VHF entrapped in } \\
\text { omental bursa }\end{array}$ & $\begin{array}{l}\text { Nose } \\
\text { wound } / \text { mating } \\
\text { trauma }\end{array}$ & Emaciation/starvation & Gastric ulcers and melena \\
\hline 1139 & BSR & $\mathrm{F}$ & $\begin{array}{l}\text { TDR and VHF present and free- } \\
\text { floating (no entrapments) }\end{array}$ & $\begin{array}{l}\text { End lactation } \\
\text { syndrome }\end{array}$ & $\begin{array}{l}\text { Infected nose wound } \\
\text { leading to bacterial sepsis }\end{array}$ & Gastric ulcers and melena \\
\hline 1089 & BSR & $\mathrm{F}$ & $\begin{array}{c}\text { VHF free-floating (no } \\
\text { entrapments). TDR had been } \\
\text { surgically-placed in the falciform } \\
\text { ligament and appeared } \\
\text { unremarkable }\end{array}$ & $\begin{array}{l}\text { Shark bite, } \\
\text { presumptive, } \\
\text { subacute }\end{array}$ & $\begin{array}{l}\text { Dilated cardiomyopathy } \\
\text { (DCM) }\end{array}$ & $\begin{array}{l}\text { Possible domoic acid } \\
\text { intoxication }\end{array}$ \\
\hline 1093 & BSR & $\mathrm{M}$ & $\begin{array}{c}\text { VHF free-floating (no } \\
\text { entrapments). TDR had been } \\
\text { surgically-placed in the falciform } \\
\text { ligament and appeared } \\
\text { unremarkable }\end{array}$ & $\begin{array}{l}\text { Post-surgical } \\
\text { wound infection } \\
\text { leading to bacterial } \\
\text { septicemia }\end{array}$ & $\begin{array}{l}\text { Coat very wet over dorsal } \\
\text { lumbosacral region }\end{array}$ & $\begin{array}{l}\text { Skeletal muscle necrosis } \\
\text { (rhabdomyolysis) }\end{array}$ \\
\hline 1094 & BSR & M & $\begin{array}{l}\text { No TDR. VHF present and free- } \\
\text { floating (no entrapments) }\end{array}$ & $\begin{array}{l}\text { Shark bite, } \\
\text { confirmed, acute }\end{array}$ & Fight trauma & None \\
\hline 1033 & MON & $\mathrm{F}$ & $\begin{array}{c}\text { Unknown (advanced } \\
\text { decomposition) }\end{array}$ & Unknown & Unknown & Unknown \\
\hline
\end{tabular}




\begin{tabular}{|c|c|c|c|c|c|c|}
\hline $\begin{array}{l}\text { BRD } \\
\text { number }\end{array}$ & Area & Sex & $\begin{array}{l}\text { Instruments recovered and } \\
\text { associated findings }\end{array}$ & $\begin{array}{c}\text { Primary cause of } \\
\text { death }\end{array}$ & Contributing number 1 & Contributing number 2 \\
\hline 1064 & MON & $\mathrm{F}$ & $\begin{array}{c}\text { Unknown (advanced } \\
\text { decomposition) }\end{array}$ & Unknown & Unknown & Emaciation/starvation \\
\hline 1051 & $\mathrm{MON}$ & $\mathrm{F}$ & $\begin{array}{l}\text { TDR present but recovered some } \\
\text { distance from traumatized } \\
\text { carcass on the beach. VHF } \\
\text { present and free-floating (no } \\
\text { entrapments) }\end{array}$ & $\begin{array}{l}\text { Shark bite, } \\
\text { presumptive }\end{array}$ & $\begin{array}{l}\text { Possible domoic acid } \\
\text { Intoxication }\end{array}$ & None \\
\hline 1155 & $\mathrm{MON}$ & $\mathrm{F}$ & $\begin{array}{l}\text { TDR and VHF present and } \\
\text { loosely wrapped in omentum but } \\
\text { not entrapped. Transmitter bump } \\
\text { mark on right kidney }\end{array}$ & $\begin{array}{l}\text { End lactation } \\
\text { syndrome }\end{array}$ & Bacterial septicemia & Gastric ulcers and melena \\
\hline 1042 & $\mathrm{MON}$ & $\mathrm{F}$ & $\begin{array}{l}\text { No TDR. VHF present and free- } \\
\text { floating (no entrapments) }\end{array}$ & $\begin{array}{l}\text { Intestinal } \\
\text { perforation and } \\
\text { peritoneal scarring, } \\
\text { leading to colon } \\
\text { impaction } \\
\end{array}$ & Emaciation/starvation & Gastric ulcers and melena \\
\hline 1037 & MON & $\mathrm{F}$ & $\begin{array}{l}\text { TDR free-floating (no } \\
\text { entrapments). VHF entrapped in } \\
\text { omental bursa }\end{array}$ & Cardiomyopathy & $\begin{array}{l}\text { Possible domoic acid } \\
\text { intoxication }\end{array}$ & End lactation syndrome \\
\hline 1016 & MON & $\mathrm{F}$ & $\begin{array}{l}\text { No TDR. VHF present and } \\
\text { loosely wrapped in fold of } \\
\text { mesentery, but not entrapped }\end{array}$ & $\begin{array}{l}\text { Boat strike with } \\
\text { secondary } \\
\text { bacterial } \\
\text { infection }\end{array}$ & Acanthocephalan peritonitis & End lactation syndrome \\
\hline 1109 & MON & $\mathrm{F}$ & $\begin{array}{l}\text { No TDR. VHF present and free- } \\
\text { floating (no entrapments) }\end{array}$ & $\begin{array}{l}\text { Shark bite, } \\
\text { presumptive, acute }\end{array}$ & Acanthocephalan peritonitis & Segmental enteritis \\
\hline 1024 & $\mathrm{MON}$ & M & $\begin{array}{l}\text { VHF free-floating. TDR } \\
\text { entrapped in omental bursa }\end{array}$ & $\begin{array}{l}\text { Prior blunt trauma } \\
\text { (Possible boat } \\
\text { strike) with chronic } \\
\text { jaw fracture plus } \\
\text { localized and } \\
\text { systemic bacterial } \\
\text { infection }\end{array}$ & $\begin{array}{l}\text { Possible domoic acid } \\
\text { intoxication }\end{array}$ & Gastric ulcers and melena \\
\hline
\end{tabular}


Table 37. Findings from antemortem and postmortem tests for domoic acid and cyanotoxins for tagged, necropsied sea otters from Monterey Peninsula and Big Sur coast study sites, central California.

[ "-“ indicates that DA and cyanotoxins were not detected. "+" indicates detection of less than 100 parts per billion (ppb). "++" indicates detection of 100-200 ppb. "+++" indicates detection greater than $200 \mathrm{ppb}$. "+/-“" indicates detection below minimum detection limit]

\begin{tabular}{|c|c|c|c|c|c|c|c|c|c|c|c|}
\hline $\begin{array}{c}\text { BRD } \\
\text { number }\end{array}$ & Area & Sex & $\begin{array}{c}\text { Necropsy } \\
\text { date }\end{array}$ & $\begin{array}{c}\text { Sample } \\
\text { date }\end{array}$ & Tissue type & Saxitoxin & Domoic acid & Microcystin & Nodularin & Anatoxin-A & $\begin{array}{c}\text { Okadaic } \\
\text { acid }\end{array}$ \\
\hline \multirow[t]{5}{*}{1106} & BSR & $\mathrm{F}$ & $8 / 27 / 09$ & $11 / 11 / 08$ & Serum & & - & & & & \\
\hline & & & & $11 / 5 / 08$ & Feces & $+/-$ & - & & & & \\
\hline & & & & $11 / 5 / 08$ & Serum & - & - & & & & \\
\hline & & & & $9 / 16 / 09$ & Milk & - & + & & & & \\
\hline & & & & $9 / 16 / 09$ & Serum & - & - & & & & \\
\hline \multirow[t]{3}{*}{1074} & BSR & $\mathrm{F}$ & $6 / 1 / 11$ & $11 / 6 / 08$ & Serum & - & - & & & & \\
\hline & & & & $9 / 24 / 10$ & Serum & - & - & & & & \\
\hline & & & & $6 / 1 / 11$ & Liver & & - & - & - & - & - \\
\hline \multirow[t]{2}{*}{1139} & BSR & $\mathrm{F}$ & $9 / 20 / 11$ & $9 / 20 / 11$ & Liver & & + & - & - & - & - \\
\hline & & & & $9 / 20 / 11$ & Urine & & +++ & - & - & - & - \\
\hline \multirow[t]{3}{*}{1089} & BSR & $\mathrm{F}$ & $11 / 9 / 11$ & $11 / 6 / 08$ & Serum & - & + & & & & \\
\hline & & & & $11 / 9 / 11$ & Liver & & + & - & - & - & - \\
\hline & & & & $11 / 9 / 11$ & Urine & & +++ & - & - & - & - \\
\hline \multirow[t]{6}{*}{1093} & BSR & M & $11 / 10 / 08$ & $11 / 6 / 08$ & Feces & + & + & & & & \\
\hline & & & & $11 / 6 / 08$ & Serum & - & - & & & & \\
\hline & & & & $11 / 6 / 08$ & Serum & & - & - & - & - & - \\
\hline & & & & $11 / 10 / 08$ & Liver & & - & + & & & - \\
\hline & & & & $11 / 10 / 08$ & Feces & - & - & & & & \\
\hline & & & & $11 / 10 / 08$ & Urine & - & - & & & & \\
\hline 1094 & BSR & $\mathrm{M}$ & $11 / 24 / 09$ & $11 / 7 / 08$ & Serum & - & - & & & & \\
\hline 1051 & MON & $\mathrm{F}$ & $3 / 25 / 10$ & $3 / 25 / 10$ & $\begin{array}{l}\text { Stomach } \\
\text { Contents }\end{array}$ & $+/-$ & + & & & & \\
\hline 1155 & MON & $\mathrm{F}$ & $6 / 14 / 10$ & $2 / 1 / 10$ & Serum & + & + & & & & \\
\hline
\end{tabular}




\begin{tabular}{|c|c|c|c|c|c|c|c|c|c|c|c|}
\hline $\begin{array}{c}\text { BRD } \\
\text { number }\end{array}$ & Area & Sex & $\begin{array}{c}\text { Necropsy } \\
\text { date }\end{array}$ & $\begin{array}{c}\text { Sample } \\
\text { date }\end{array}$ & Tissue type & Saxitoxin & Domoic acid & Microcystin & Nodularin & Anatoxin-A & $\begin{array}{c}\text { Okadaic } \\
\text { acid }\end{array}$ \\
\hline & & & & $6 / 14 / 10$ & Serum & - & - & & & & \\
\hline & & & & $6 / 14 / 10$ & Urine & - & + & & & & \\
\hline 1042 & MON & $\mathrm{F}$ & $5 / 17 / 11$ & $7 / 29 / 10$ & Serum & - & - & & & & \\
\hline \multirow[t]{5}{*}{1037} & MON & $\mathrm{F}$ & $6 / 21 / 11$ & $4 / 23 / 09$ & Serum & - & - & & & & \\
\hline & & & & $6 / 21 / 11$ & Colon contents & & + & - & - & - & - \\
\hline & & & & $6 / 21 / 11$ & Liver & & - & - & - & - & - \\
\hline & & & & $6 / 21 / 11$ & $\begin{array}{l}\text { Serum } \\
\text { Stomach }\end{array}$ & & - & - & - & - & - \\
\hline & & & & $6 / 21 / 11$ & contents & & - & - & - & - & - \\
\hline 1016 & $\mathrm{MON}$ & $\mathrm{F}$ & $10 / 6 / 11$ & $4 / 29 / 09$ & Serum & - & - & & & & \\
\hline \multirow[t]{2}{*}{1109} & MON & $\mathrm{F}$ & $3 / 13 / 12$ & $4 / 22 / 09$ & Serum & - & - & & & & \\
\hline & & & & $6 / 8 / 10$ & Serum & - & - & & & & \\
\hline \multirow{4}{*}{1024} & & & & $3 / 30 / 10$ & Serum & - & - & & & & \\
\hline & & & & $3 / 30 / 10$ & $\begin{array}{l}\text { Stomach } \\
\text { contents }\end{array}$ & $+/-$ & + & & & & \\
\hline & & & & $3 / 30 / 10$ & Urine & - & + & & & & \\
\hline & & & & $3 / 30 / 10$ & Liver & & & $+/-$ & & & \\
\hline
\end{tabular}


Table 38. Findings from gross necropsy, histopathology, and diagnostic testing (including subjective weighting of relative lesion severity) for tagged, necropsied sea otters from Monterey Peninsula and Big Sur coast study sites, central California.

[ "0", none; "1”, mild,;"2", moderate; "3”, severe. For all fields "U” = unknown, "Hx”, histopathology; "N/A", not applicable; "Pres.”, presumed; "Conf.”, confirmed.

Carcass condition: "Fr", fresh,; "Mod", moderately decomposed; "Adv", advanced decomposition; "Mum/Frag", mummified/fragmented.]

\begin{tabular}{|c|c|c|c|c|c|c|c|c|c|c|c|c|c|c|c|c|c|c|c|c|}
\hline $\begin{array}{c}\text { BRD } \\
\text { number }\end{array}$ & Area & Sex & 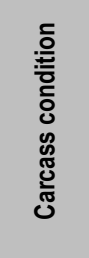 & 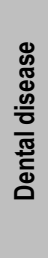 & 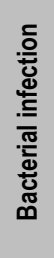 & 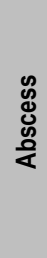 & $\begin{array}{l}\frac{n}{10} \\
\frac{0}{0} \\
\infty\end{array}$ & 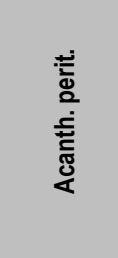 & 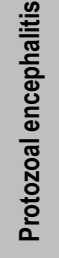 & 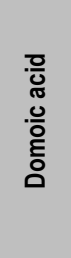 & 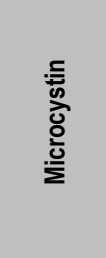 & 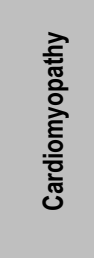 & 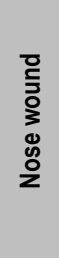 & 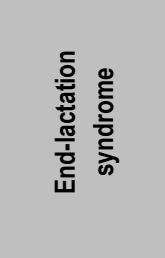 & 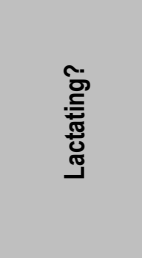 & 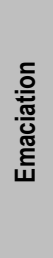 & 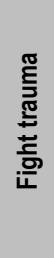 & 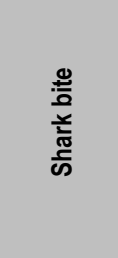 & 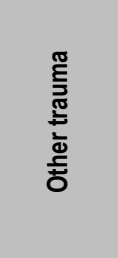 & 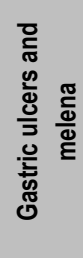 \\
\hline 1090 & BSR & $\mathrm{F}$ & $\mathrm{Fr}$ & 2 & 3 & 3 & 3 & 0 & 0 & 0 & $\mathrm{U}$ & 0 & 1 & 0 & 0 & 0 & 0 & 0 & $\begin{array}{l}3 \text { (recent } \\
\text { surgery) }\end{array}$ & 0 \\
\hline 1106 & BSR & $\mathrm{F}$ & Adv & $\mathrm{U}$ & $\mathrm{U}$ & U & U & 0 & 0 & $\mathrm{U}$ & $\mathrm{U}$ & 0 & $\mathrm{U}$ & $\begin{array}{l}3 \text { (pres. } \\
\text { based on } \\
\text { findings and } \\
\mathrm{Hx} \text { ) }\end{array}$ & Y (Scant) & 3 & $\mathrm{U}$ & $\mathrm{U}$ & $\mathrm{U}$ & 2 \\
\hline 1069 & BSR & F & $\mathrm{Fr}$ & 0 & 3 & 3 & 3 & 0 & 0 & $0-1$ & U & 0 & 3 & 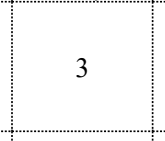 & $\begin{array}{l}\text { Y (Lots, \& } \\
\text { prom. } \\
\text { mammary } \\
\text { glands) }\end{array}$ & 3 & 0 & 0 & 0 & 3 \\
\hline 1074 & BSR & $\mathrm{F}$ & $\mathrm{Fr}$ & 3 & 0 & 0 & 0 & 0 & 0 & 0 & 0 & 0 & 3 & 0 & $\begin{array}{l}\text { No, but } \\
\text { mammary } \\
\text { tissue } \\
\text { prominent }\end{array}$ & 3 & 0 & 0 & 0 & 3 \\
\hline 1139 & BSR & F & $\mathrm{Fr}$ & 0 & 3 & 3 & 3 & 0 & 0 & 3 & 0 & 0 & 3 & $\begin{array}{l}1 \text { (Died of } \\
\text { bacterial \& } \\
\text { resp. dz.) }\end{array}$ & $\begin{array}{l}\text { Yes (milk } \\
\text { collected) }\end{array}$ & 3 & 0 & 0 & 0 & Y \\
\hline 1089 & BSR & F & $\mathrm{Fr}$ & 1 & 3 & 3 & 3 & $\begin{array}{l}1(\mathrm{Re}- \\
\text { solving) }\end{array}$ & 0 & 3 & 0 & 2 & 2 & 0 & $\mathrm{~N}$ & 3 & 0 & 3 (Pres.) & 0 & 3 \\
\hline 1093 & BSR & M & $\mathrm{Fr}$ & 0 & 3 & 3 & 3 & 0 & 0 & $0-1$ & $\begin{array}{l}\text { Poss. } \\
\text { contrib. }\end{array}$ & 0 & 0 & N/A & N/A & 1 & 0 & 0 & $\begin{array}{l}3 \text { (recent } \\
\text { surgery) }\end{array}$ & Y \\
\hline 1094 & BSR & M & Adv & 1 & $\mathrm{U}$ & U & $\mathrm{U}$ & 0 & $\mathrm{U}$ & $\mathrm{U}$ & $\mathrm{U}$ & $\mathrm{U}$ & 2 & N/A & N/A & 0 & 2 & 3 (Conf.) & 0 & 0 \\
\hline 1033 & MON & $\mathrm{F}$ & $\begin{array}{l}\text { Mum/ } \\
\text { Frag }\end{array}$ & 0 & $\mathrm{U}$ & $\mathrm{U}$ & U & $\mathrm{U}$ & 0 & $\mathrm{U}$ & $\mathrm{U}$ & $\mathrm{U}$ & $\mathrm{U}$ & $\mathrm{U}$ & $\mathrm{U}$ & $\mathrm{U}$ & $\mathrm{U}$ & $\mathrm{U}$ & $\mathrm{U}$ & $\mathrm{U}$ \\
\hline 1064 & MON & $\mathrm{F}$ & $\mathrm{Adv}$ & $\mathrm{U}$ & $\mathrm{U}$ & U & U & 0 & 0 & $\mathrm{U}$ & $\mathrm{U}$ & $\mathrm{U}$ & $\mathrm{U}$ & N/A & N/A & 2 & $\mathrm{U}$ & $\mathrm{U}$ & $\mathrm{U}$ & 2 to 3 \\
\hline 1051 & MON & $\mathrm{F}$ & Mod & 0 & $\mathrm{U}$ & $\mathrm{U}$ & $\mathrm{U}$ & 0 & 0 & 2 & $\mathrm{U}$ & $\begin{array}{c}0 ? \\
\text { (Not } \\
\text { noted) }\end{array}$ & 0 & 0 & $\begin{array}{l}\text { No, but } \\
\text { prominent }\end{array}$ & 0 & 0 & 3 & 0 & $\mathrm{U}$ \\
\hline
\end{tabular}




\begin{tabular}{|c|c|c|c|c|c|c|c|c|c|c|c|c|c|c|c|c|c|c|c|c|}
\hline $\begin{array}{c}\text { BRD } \\
\text { number }\end{array}$ & Area & Sex & 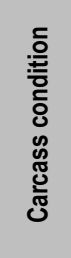 & 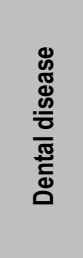 & 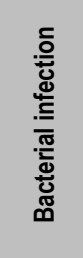 & 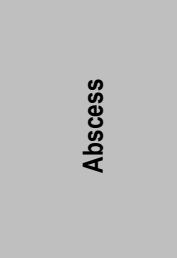 & 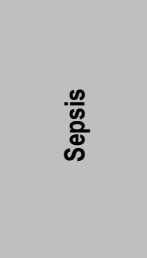 & 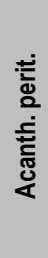 & 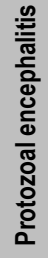 & 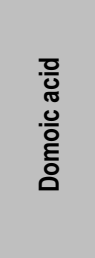 & 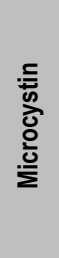 & 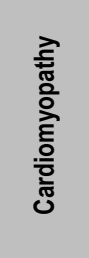 & 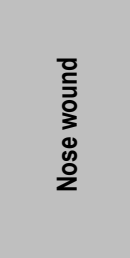 & 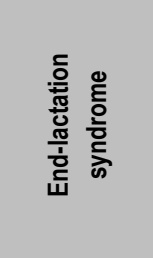 & 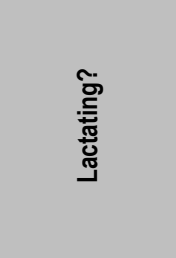 & 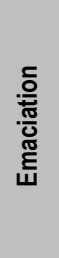 & 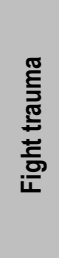 & 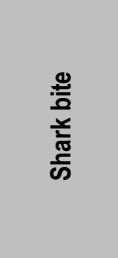 & 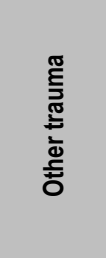 & 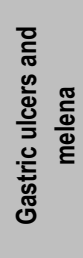 \\
\hline & & & & & & & & & & & \multicolumn{10}{|c|}{$\begin{array}{l}\text { mammary } \\
\text { glands }\end{array}$} \\
\hline 1155 & MON & $\mathrm{F}$ & $\mathrm{Fr}$ & 0 & 3 & 0 & 3 & 0 & 0 & $1-2$ & $\mathrm{U}$ & 0 & 2 & $\begin{array}{l}1 \text { (Poss. as } \\
\text { contrib. } \\
\text { factor) }\end{array}$ & No & 3 & 0 & 0 & 0 & 3 \\
\hline 1042 & $\mathrm{MON}$ & $\mathrm{F}$ & Adv & 1 & $\mathrm{U}$ & $\mathrm{U}$ & $\mathrm{U}$ & 0 & 0 & 0 & $\mathrm{U}$ & $\mathrm{U}$ & $\mathrm{U}$ & $\begin{array}{l}1 \text { (Possible, } \\
\text { as contrib. } \\
\text { factor) }\end{array}$ & Y (Scant) & 3 & $\mathrm{U}$ & 0 & 0 & 3 \\
\hline 1037 & MON & $\mathrm{F}$ & Mod & 1 to 2 & 1 & 1 & 0 & 0 & 0 & 3 & 0 & 3 & 2 & 1 (contrib.) & $\begin{array}{l}\text { No, but } \\
\text { mammary } \\
\text { tissue } \\
\text { prominent }\end{array}$ & 2 & $\mathrm{~N}$ & 0 & 0 & 1 \\
\hline 1016 & $\mathrm{MON}$ & $\mathrm{F}$ & $\mathrm{Fr}$ & 1 & 2 & 2 (Localized) & 0 & 2 & 0 & $\mathrm{U}$ & $\mathrm{U}$ & 0 & 2 & $\begin{array}{c}1 \text { (coming } \\
\text { out of ELS) }\end{array}$ & $\begin{array}{l}\text { N (small, flat, } \\
\text { atrophied } \\
\text { mammary } \\
\text { glands) }\end{array}$ & 0 & 0 & 0 & $\begin{array}{l}3 \text { (Boat } \\
\text { strike) }\end{array}$ & 0 \\
\hline 1109 & MON & $\mathrm{F}$ & $\mathrm{Fr}$ & 1 & 1 to 2 & $\begin{array}{l}1 \text { to } 2 \text { (Perit., } \\
\text { seg. enteritis) }\end{array}$ & $\begin{array}{c}1 \text { to } 2 \\
\text { (Poss. } \\
\text { chronic } \\
\text { Bartonella) }\end{array}$ & 2 & 0 & $\mathrm{U}$ & $\mathrm{U}$ & $0^{*}$ & 1 to 2 & $\begin{array}{l}\text { N/A (old, } \\
\text { not } \\
\text { cycling?) }\end{array}$ & $\mathrm{N}$ & 0 & 0 & 3 (Pres.) & 0 & $\mathrm{Y}$ \\
\hline 1024 & $\mathrm{MON}$ & M & $\mathrm{Fr}$ & 3 & 3 & 1 to 2 (teeth) & 3 & 0 & 0 & 1 to 2 & $\mathrm{U}$ & 1 to 2 & $\begin{array}{c}1(\mathrm{D} / \mathrm{T} \\
\text { abnormal } \\
\text { locomot.) }\end{array}$ & N/A & N/A & 2 & $0 *$ & $0 *$ & $\begin{array}{l}3 ? \\
(* \text { Prior } \\
\text { boat } \\
\text { strike, } \\
\text { pres. })\end{array}$ & 2 \\
\hline
\end{tabular}




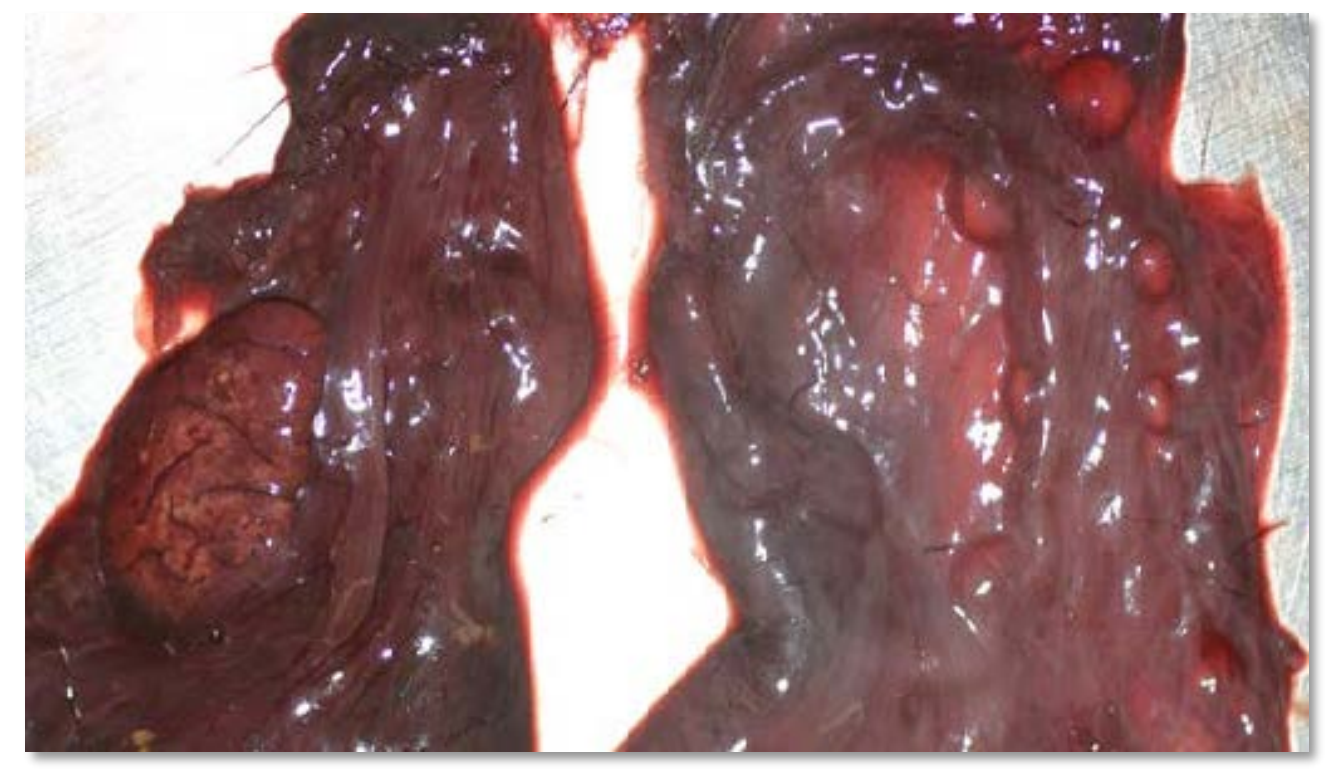

Figure 55. Photograph showing normal sea otter ovary (left), and missing ovary, (right). Note that a normal, wavy, undulating fallopian tube is still visible, just to the right of the thin flap of tissue where the ovary should be (right center). Photograph by Melissa Miller, California Department of Fish and Wildlife.

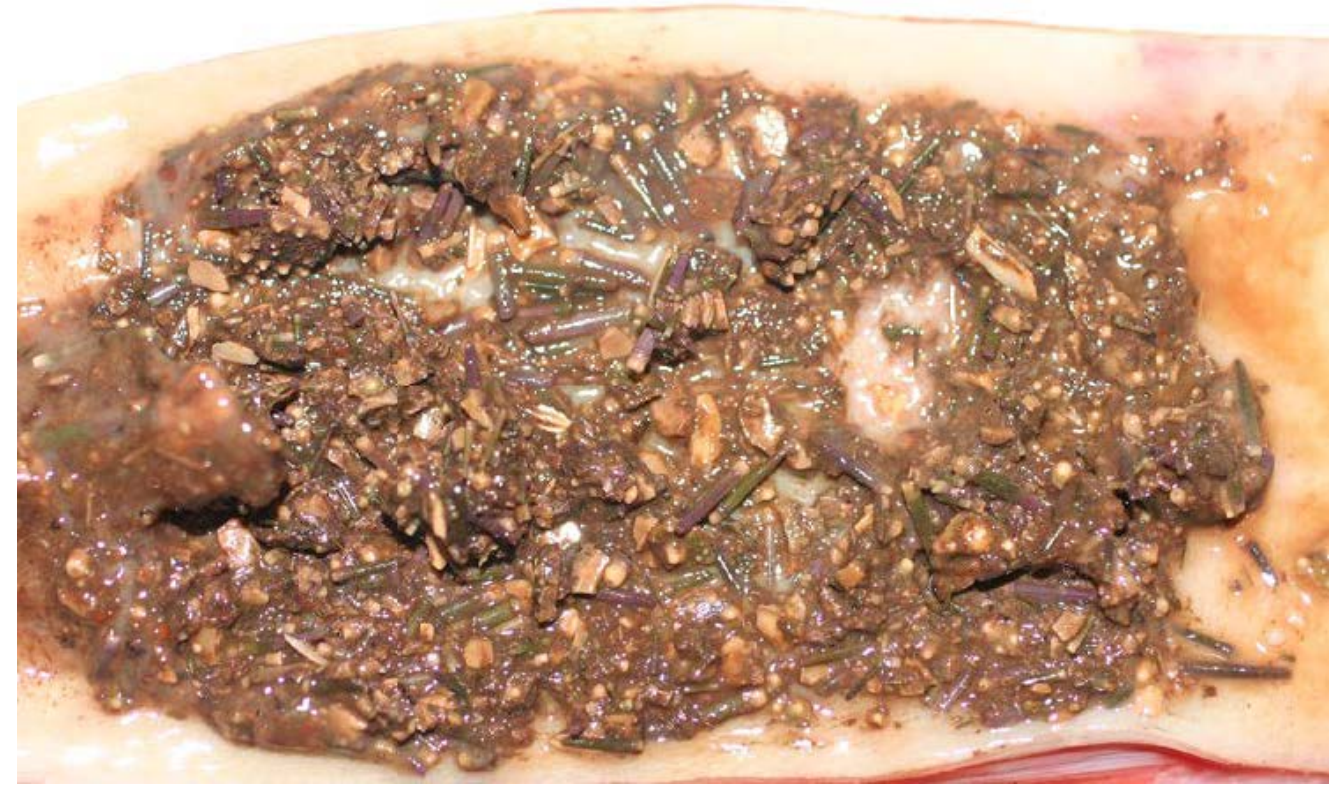

Figure 56. Photograph showing intestinal content of sea otter providing insight on this animal's "last meal"fragments of sea urchin exoskeleton and spines. Photograph by Melissa Miller, California Department of Fish and Wildlife. 


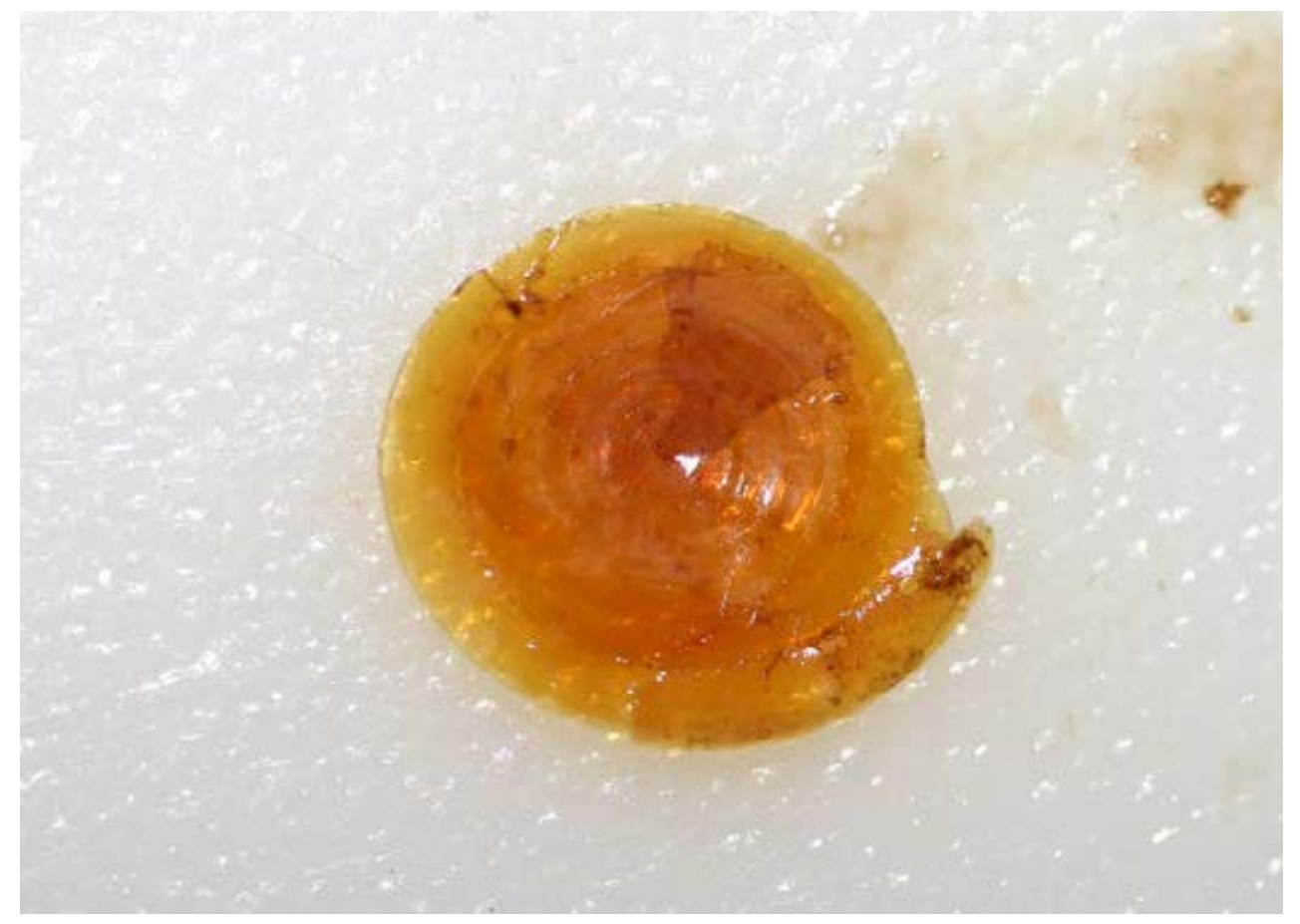

Figure 57. Photograph showing Tegula operculum from a sea otter that had been eating snails. Photograph: by Melissa Miller, California Department of Fish and Wildlife.

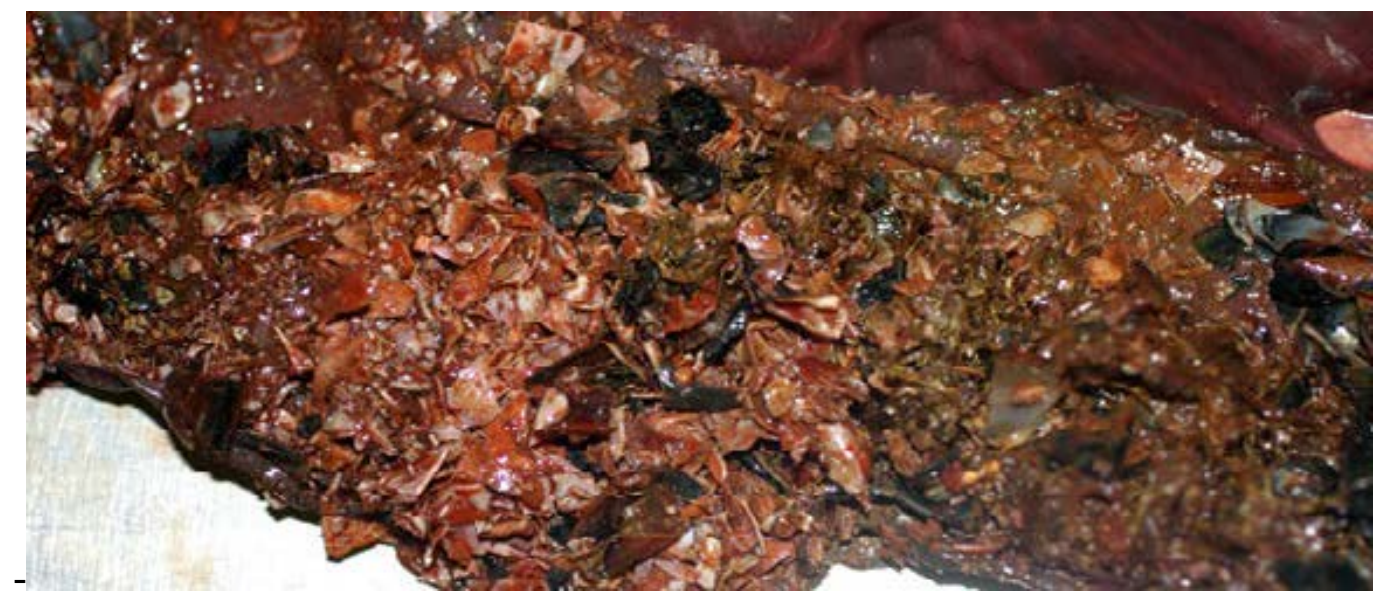

Figure 58. Photograph showing intestine of a sea otter with a colon impaction. The content is tightly packed and contains fragments of Cancer crab carapace and mussel shells. Photograph by Melissa Miller, California Department of Fish and Wildlife. 


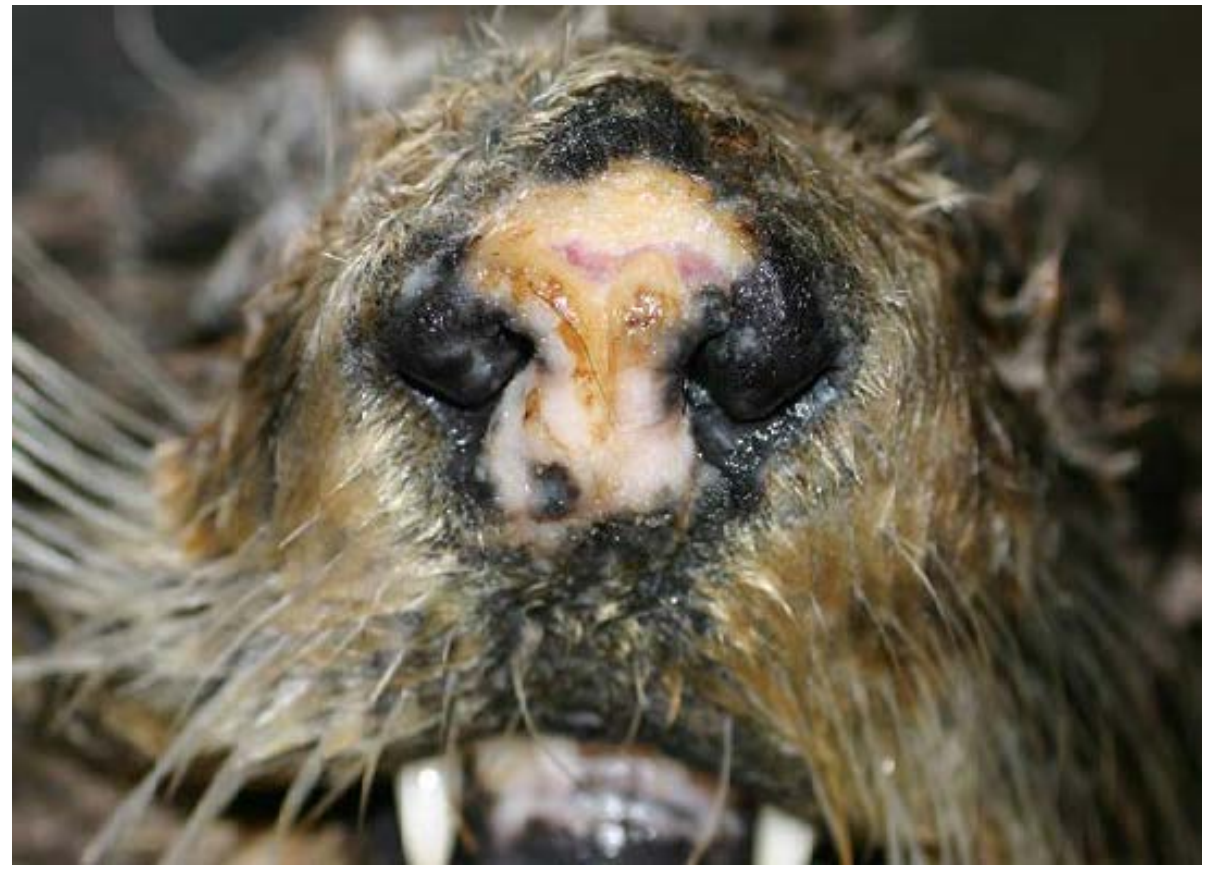

Figure 59. Photograph showing sea otter with a mild nose wound. Photograph by Melissa Miller, California Department of Fish and Wildlife.

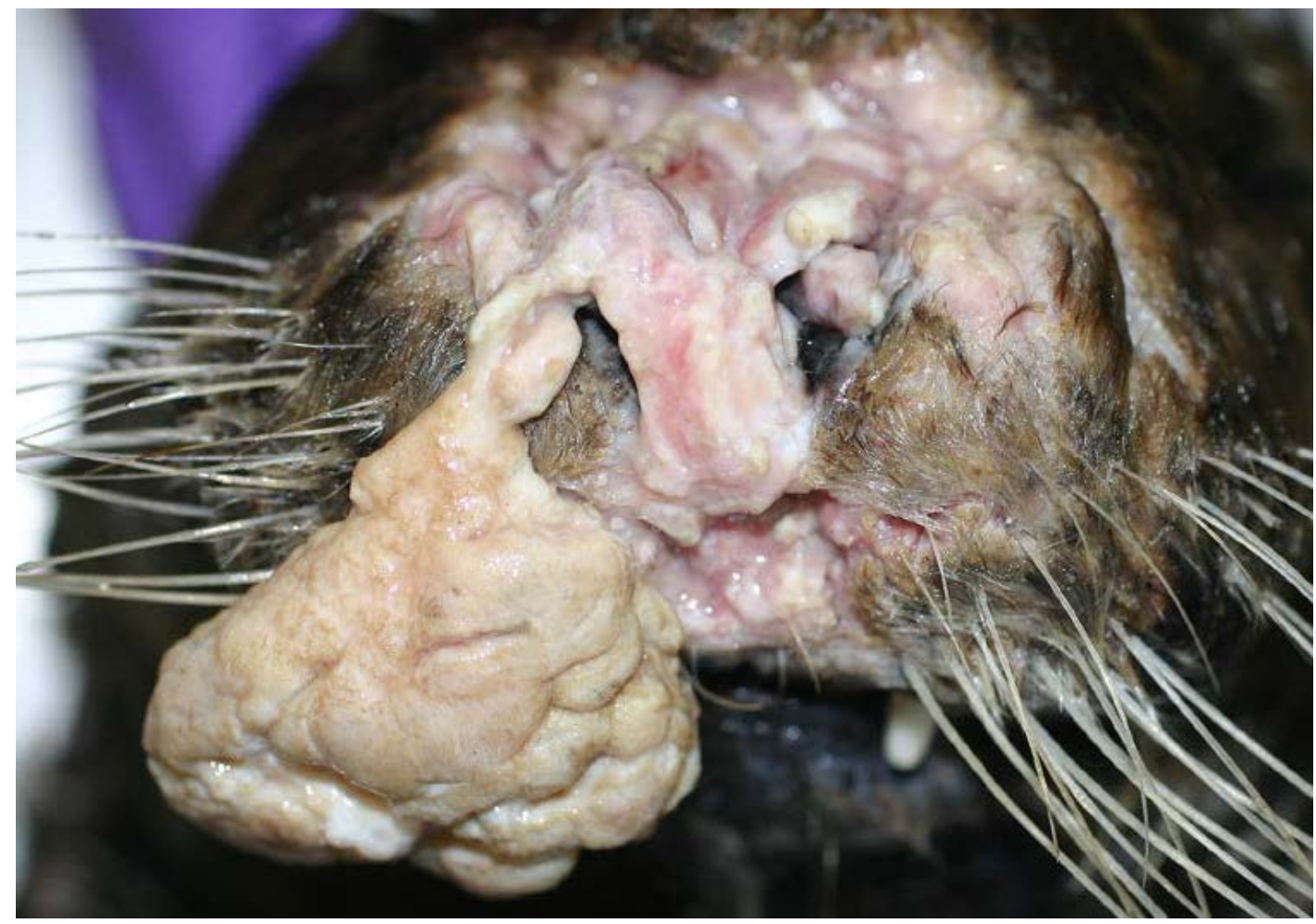

Figure 60. Photograph of sea otter with a severe nose wound. Photograph by Melissa Miller, California Department of Fish and Wildlife. 


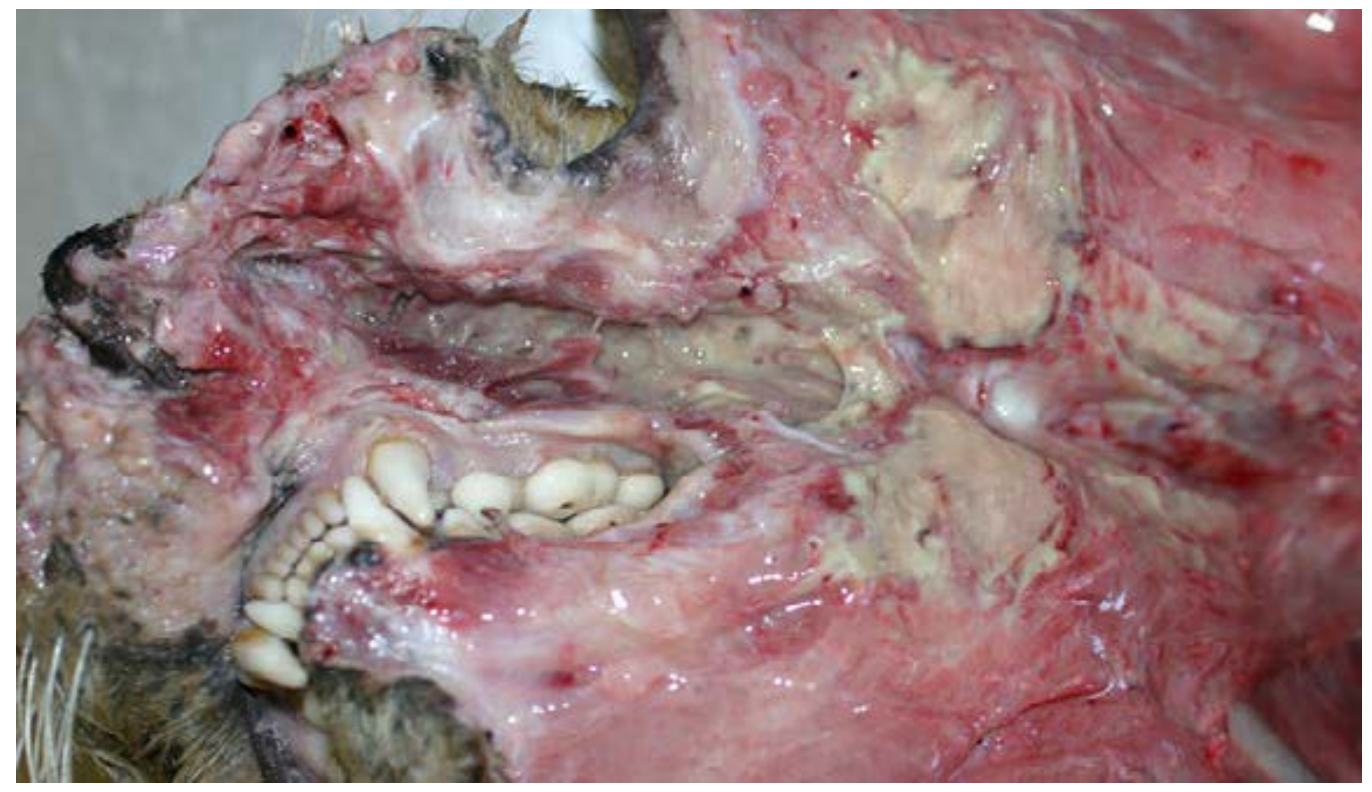

Figure 61. Photograph showing sea otter bacterial infections after mating-related trauma, which otters sometimes develop. These infections can travel away from the original bite location along tissue planes. Photograph by Melissa Miller, California Department of Fish and Wildlife.

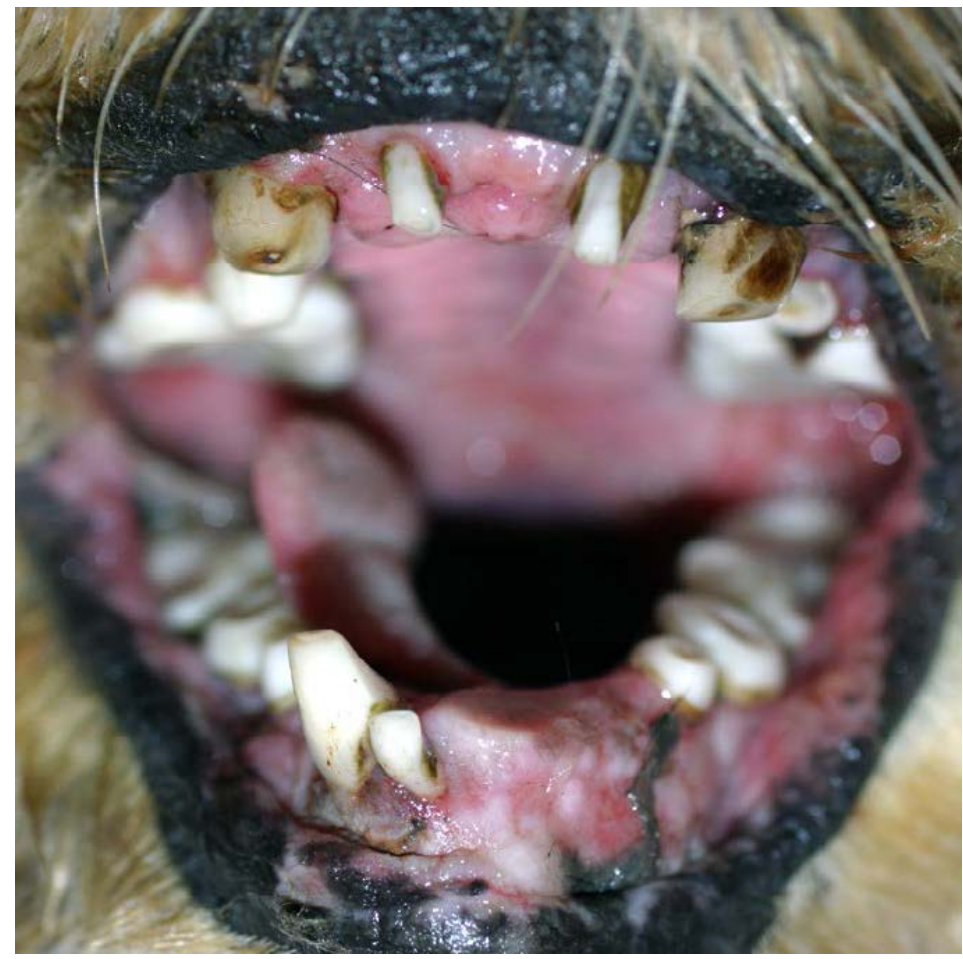

Figure 62. Photograph showing old sea otter with severely worn and missing teeth. Otter also had a partially healed mandible fracture. Note increased thickening of the mandible at the site of the fracture (bottom center). Photograph by Melissa Miller, California Department of Fish and Wildlife. 

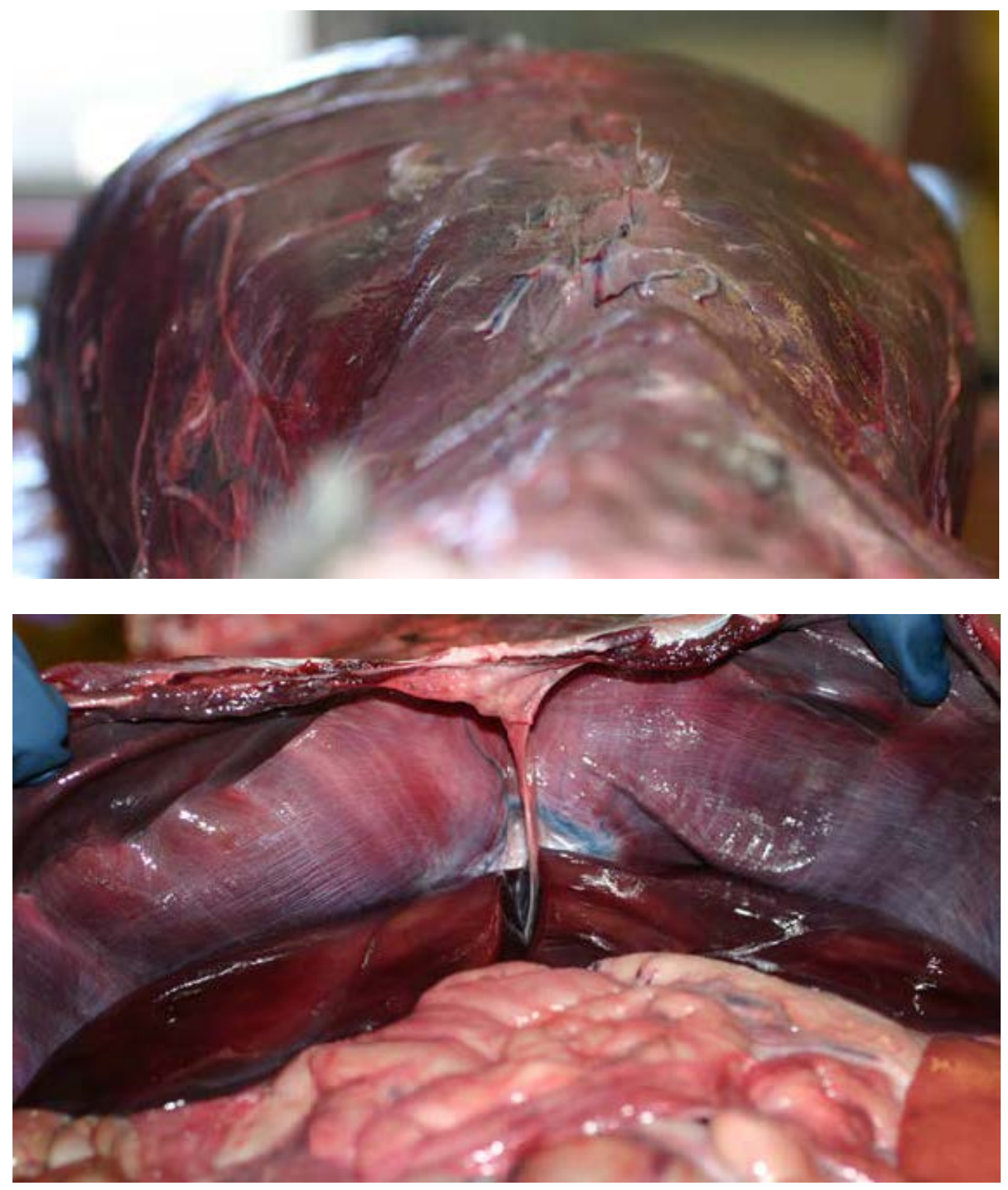

Figure 63. Photographs showing sea otter with a chest full of pressurized air or gas (pneumothorax), characterized by an abnormal, "barrel-shaped" chest (top photograph), and a diaphragm that is pushed back toward the abdomen (bottom photograph). This pneumothorax was associated with a bacterial infection. Pneumothorax is rapidly fatal because it impairs breathing. Photographs by Melissa Miller, California Department of Fish and Wildlife. 

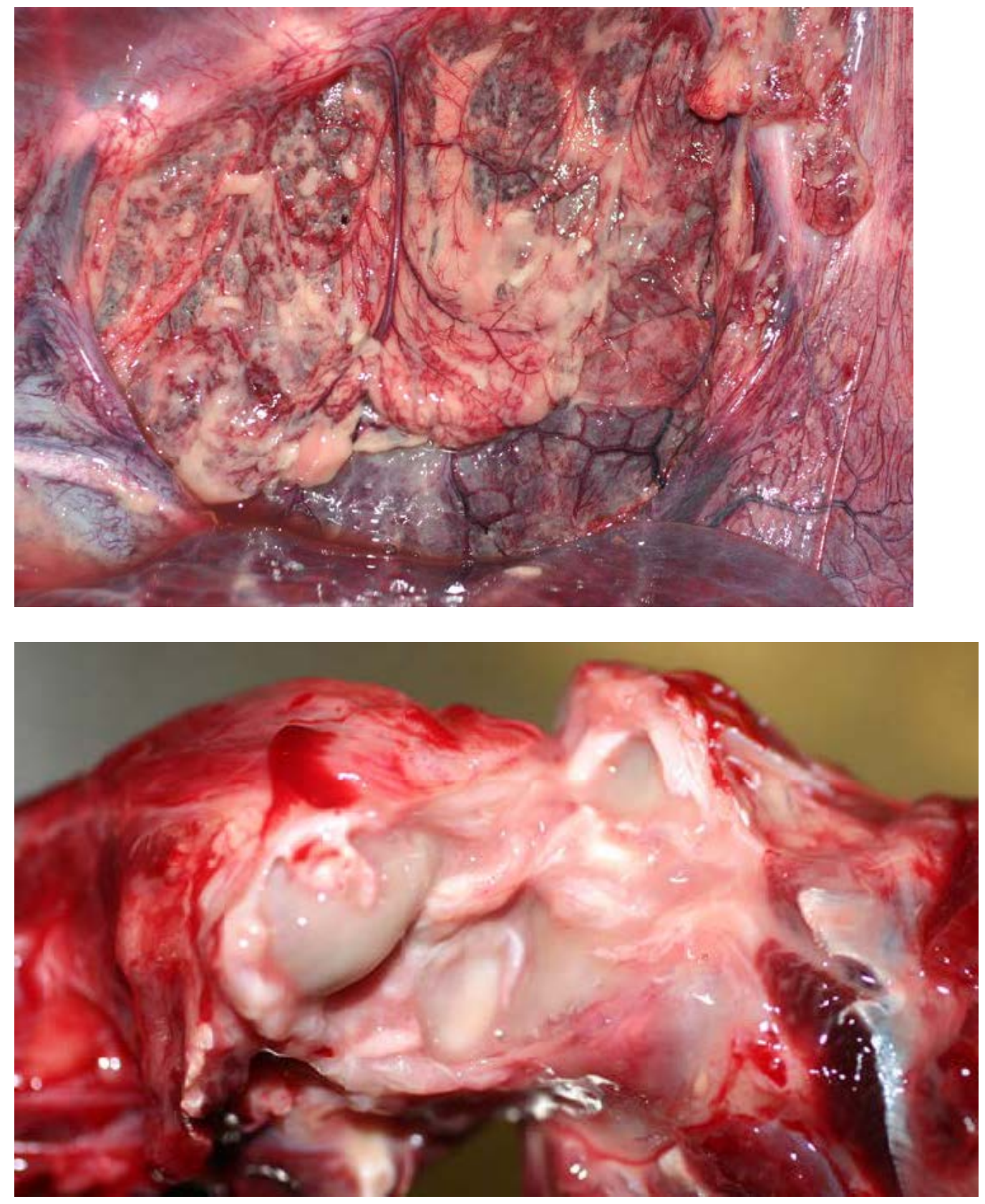

Figure 64. Photographs of same sea otter as in figure 63, showing a severe bacterial infection of the chest cavity (top photograph) and a knee joint (bottom photograph). Photographs by Melissa Miller, California Department of Fish and Wildlife. 

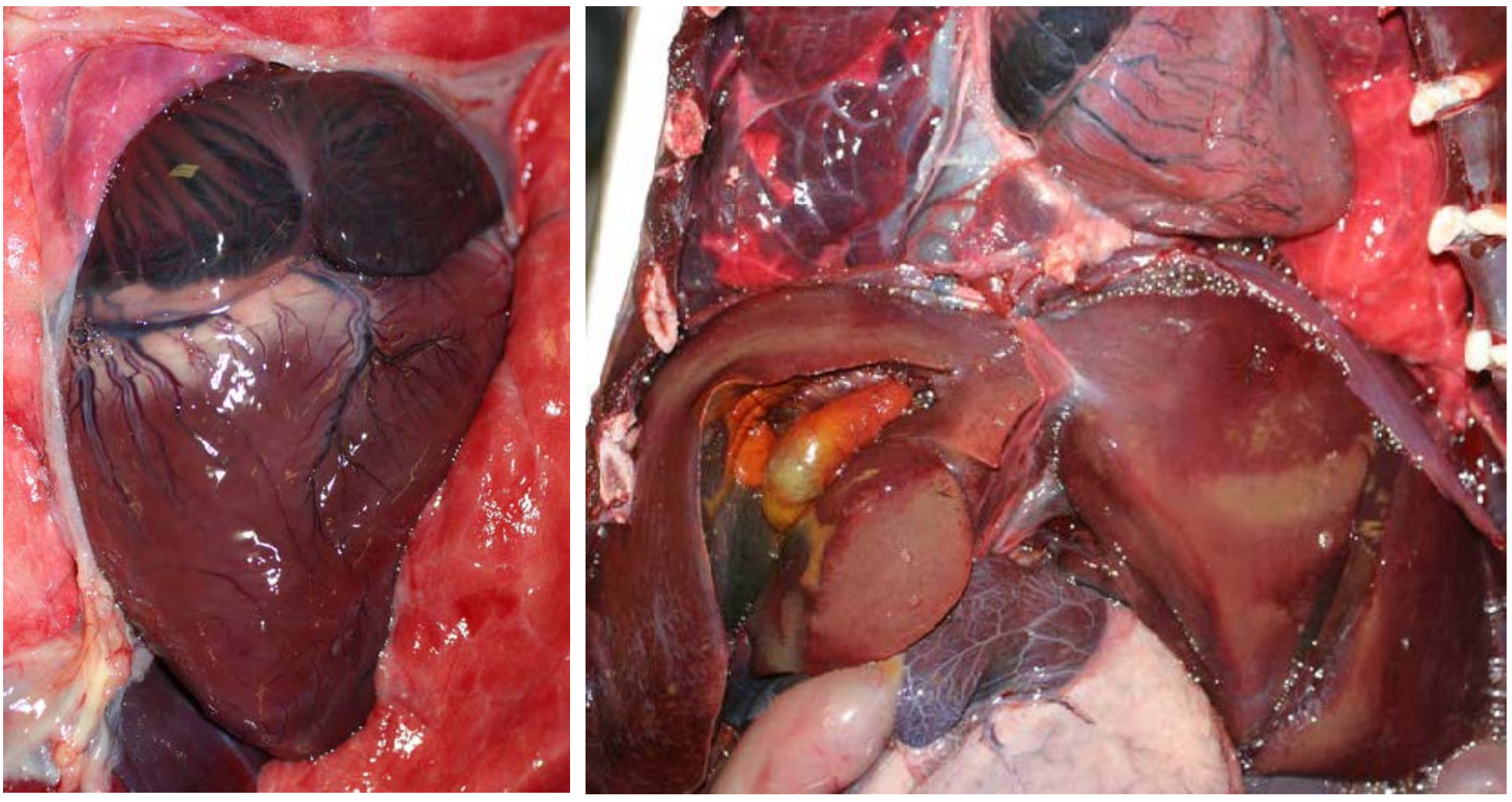

Figure 65. Photographs showing normal sea otter heart (left photograph), compared with the heart of an otter with cardiomyopathy (right photograph, upper right center). Photographs by Melissa Miller, California Department of Fish and Wildlife. 


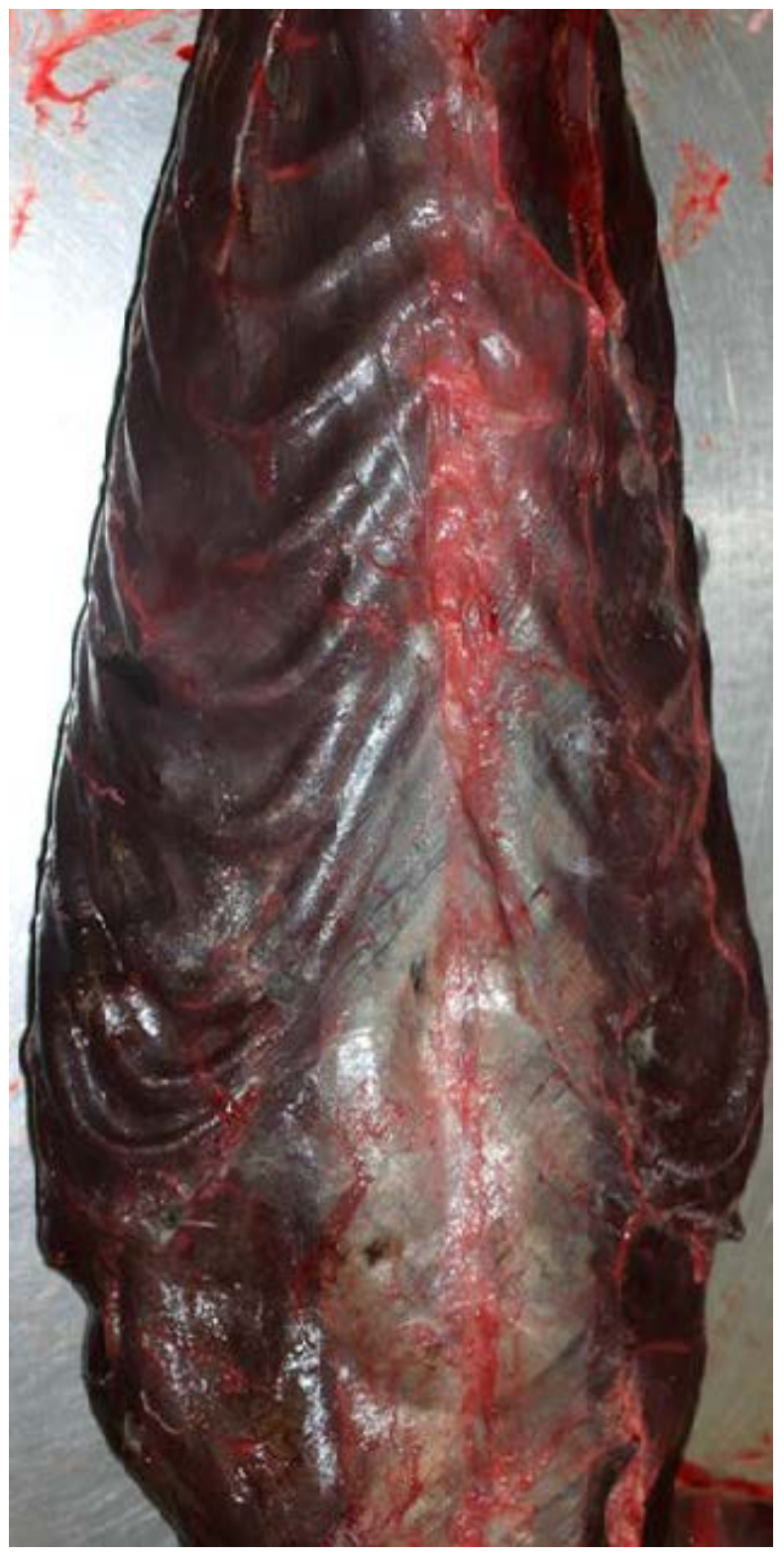

Figure 66. Photograph showing severely emaciated sea otter, typical of adult females dying with endlactation syndrome. Photograph by Melissa Miller, California Department of Fish and Wildlife. 


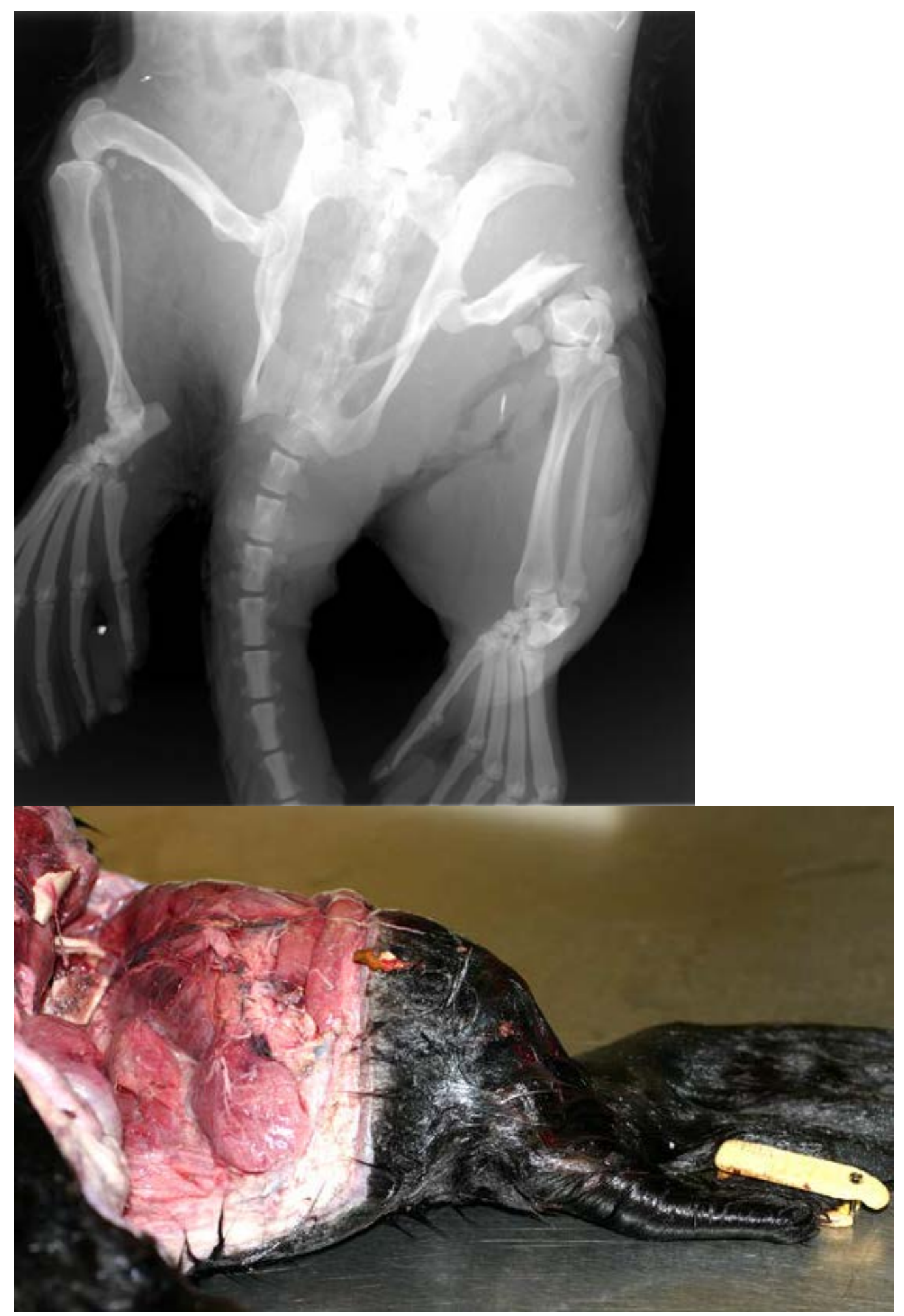

Figure 67. Photographs showing sea otter with a deep laceration and femur fracture due to boat propeller strike, as indicated by radiograph (top photograph) and gross photo (bottom photograph). Radiograph also shows passive integrated transponder tag (center right) and a flipper tag (bottom left). Photographs by Melissa Miller, California Department of Fish and Wildlife. 

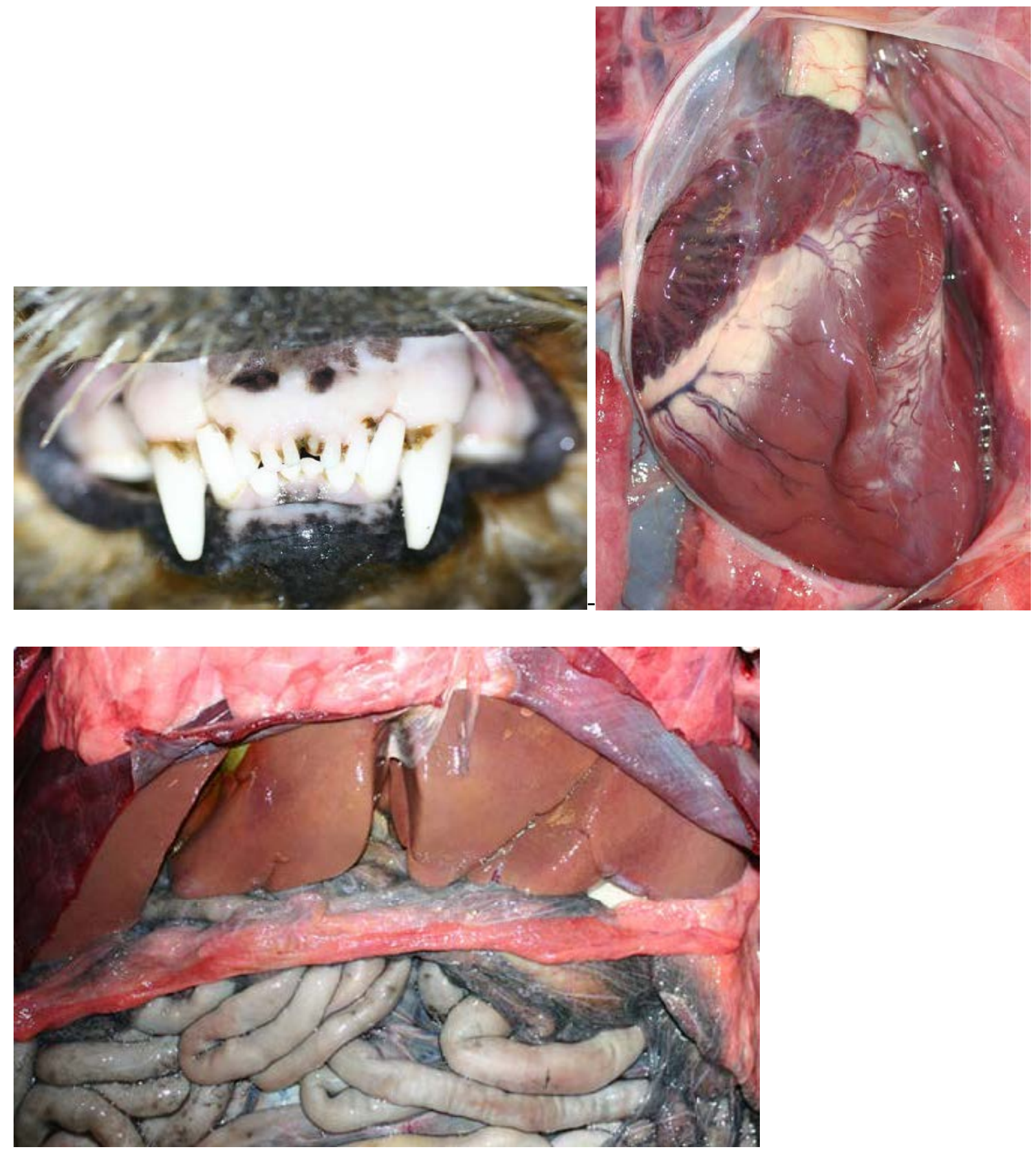

Figure 68. Photographs showing lesions suggestive of severe blood loss in sea otter due to trauma or other causes-Pale gums (top right photograph), small heart with collapsed atria and ventricles (top right photograph) and small, pale liver (bottom photograph). Photographs by Melissa Miller, California Department of Fish and Wildlife. 

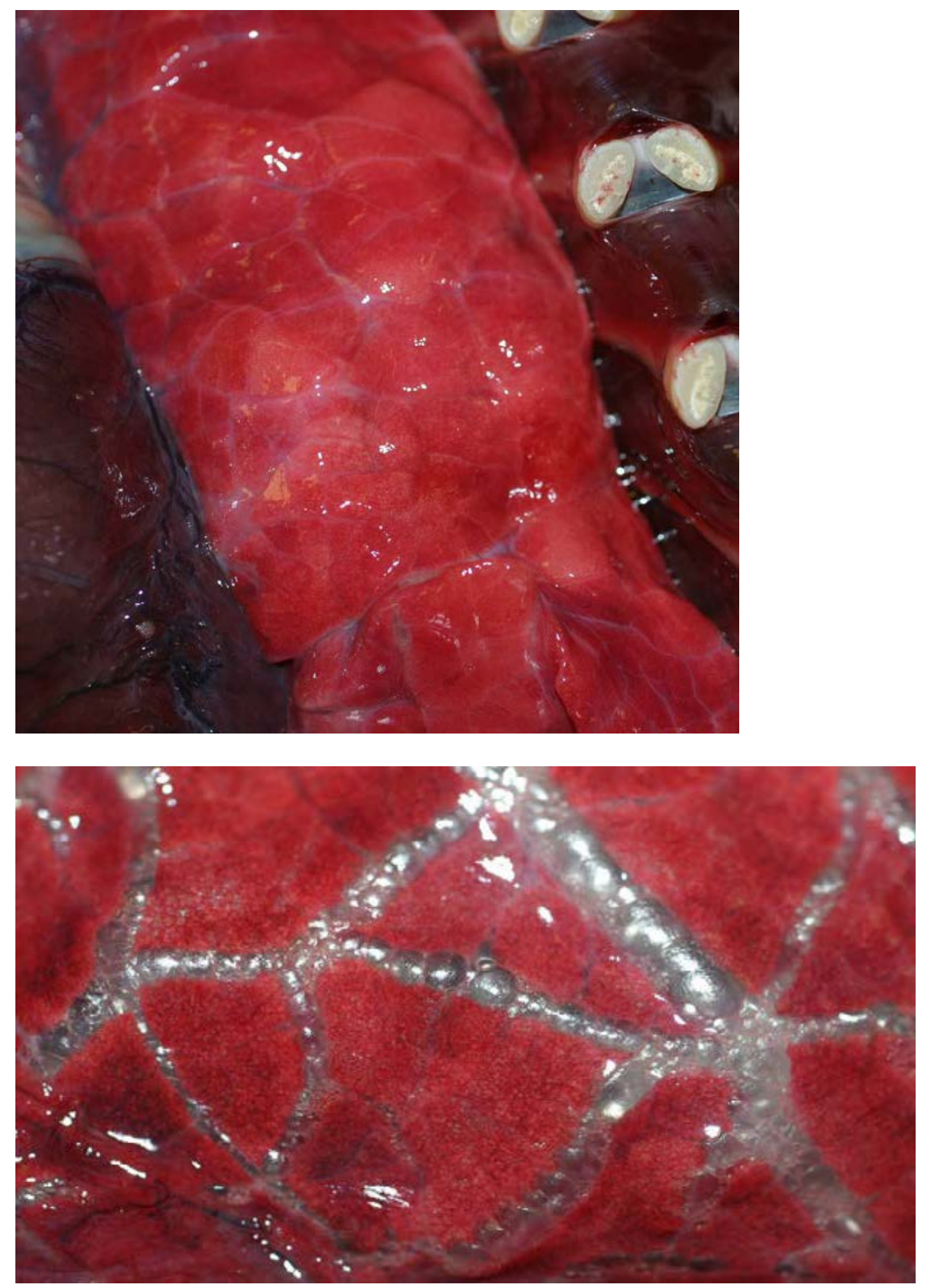

Figure 69. Photographs showing normal lung tissue (top photograph), compared with an otter with severe pulmonary emphysema (bottom photograph). This non-specific lesion typically is associated with an animal that has been breathing hard or abnormally prior to death. Photographs by Melissa Miller, California Department of Fish and Wildlife. 

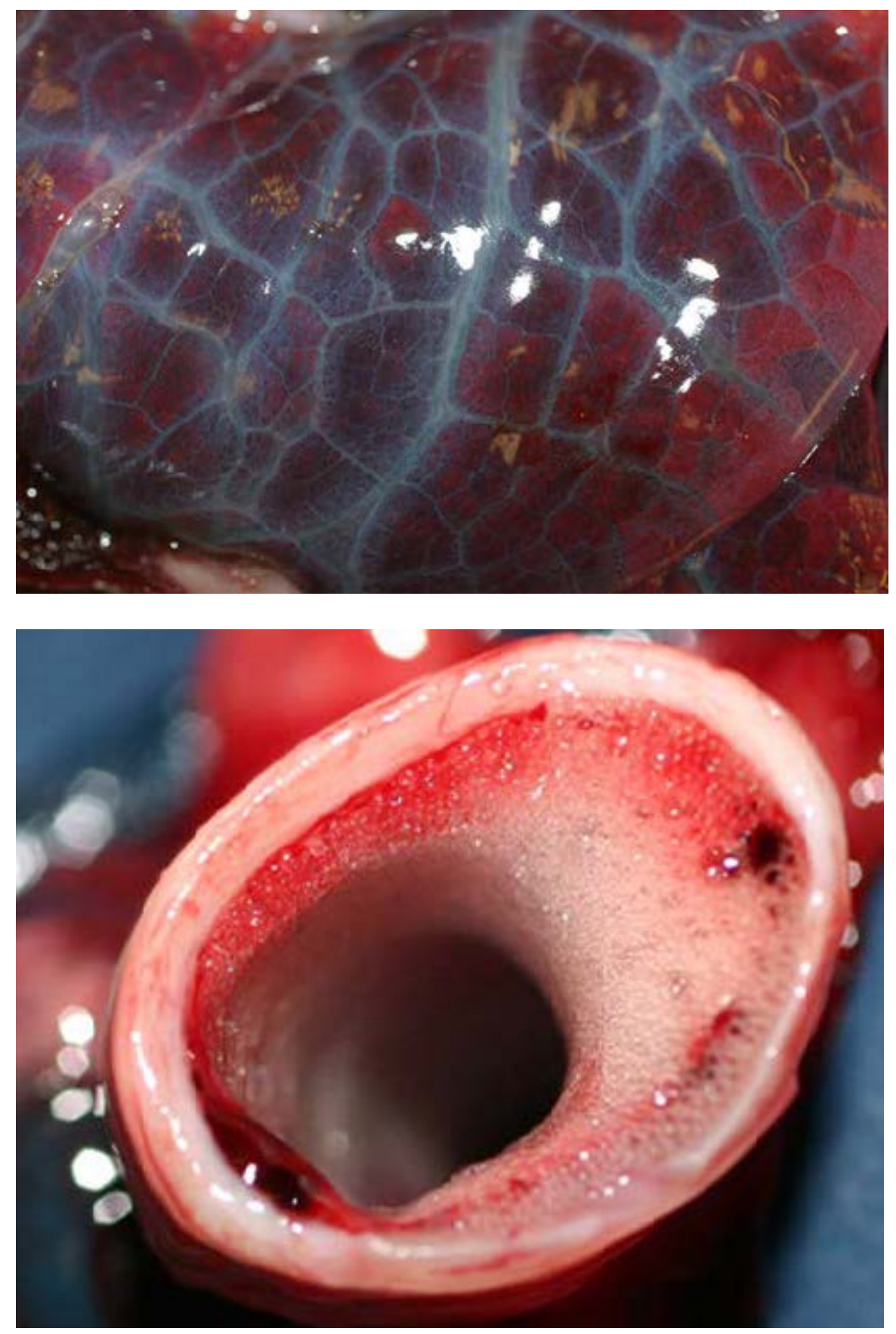

Figure 70. Photographs showing pulmonary edema, characterized by expansion of the spaces between pulmonary lobules by clear or blue-tinged fluid (top photograph), and build-up of pink or white froth in the trachea and bronchi (bottom photograph). Depending on the circumstances, this lesion may indicate primary drowning, terminal drowning (for example, aspiration of seawater near the time of death in an animal that is dying of other causes), heart failure, or other health problems. Photographs by Melissa Miller, California Department of Fish and Wildlife. 


\title{
Chapter 11. Synthesis and Conclusions
}

\author{
M. Tim Tinker ${ }^{1,2}$
}

\section{General Conclusions}

The various modules of the Big Sur-Monterey population study reported in the preceding chapters represent the culmination of one of the most expansive studies of sea otter biology ever conducted. The breadth of topics covered and the diversity of results are complex, and distilling all this information down to a few simple conclusions is no easy task. All the analyses presented in this report were conducted with the aim of testing one or more of the primary hypotheses, often using multiple lines of inquiry. Considered together, the various lines of investigation encompassed by this study generally were consistent with respect to their degree of support (or lack of support) for each of the four primary hypotheses, as described here.

1. Sea otters living in areas adjacent to human population centers and areas heavily impacted by runoff or sewage (for example, Monterey) are more likely to be exposed to pathogens and toxins of public health importance than those in more pristine areas (for example, Big Sur). This hypothesis was not supported by the results of our study. An epidemiological analysis of Toxoplasma gondii infections (chapter 9) indicated that sea otters in the highly impacted site were significantly less likely to be exposed than were otters from the pristine area. Necropsies of study animals that died during the study indicated that the frequency of the domoic acid exposure (a biotoxin produced from diatom blooms) as a contributing cause of death was approximately equal between the two study sites (occurring in 50-percent of recovered carcasses at Big Sur and 44-percent of recovered carcasses at Monterey; chapter 10). Gene expression analysis indicated no significant differences between sites in patterns indicative of physiological responses to pathogens or toxins, with the exception of elevated response to organic contaminants in sea otters from Big Sur in 2008 (possibly due to effects of Big Sur wildfires that year; chapter 3). We did not conduct laboratory tests of blood contaminant levels, owing to funding constraints (although blood samples have been archived to permit such analyses in the future), so we cannot rule out the possibility that there may have been differences in exposure to specific contaminants that were consistent with this hypothesis; however, physical exams and blood diagnostic tests showed no evidence of health effects that would suggest such a pattern, and the minor differences in health parameters that were reported indicated more abnormalities in sea otters from Big Sur, the pristine site (chapter 2).

\footnotetext{
${ }^{1}$ U.S. Geological Survey.

${ }^{2}$ University of California, Santa Cruz.
} 
2. Patterns of survival and causes of death will differ between heavily impacted and pristine environments, indicating differences in pathogen and toxin exposure. This hypothesis was not supported by our results. The comprehensive analysis of sea otter survival and weaning success showed that sea otters from the pristine site (Big Sur) had lower age-specific survival rates than did sea otters from the heavily impacted (Monterey) study site, and female weaning success rates showed a similar pattern (chapter 8 ). These differences were explained almost entirely by the differences in resource abundance and body condition of animals at the two sites; after controlling for the effect of age-specific body mass, there were no significant differences in survival rates between the two sites. In terms of causes of death of study animals (chapter 10), necropsies indicated that the same suite of causal factors were evident at both sites in roughly equal proportions, with the exception of boat strikes which only occurred at the Monterey site ( 2 out of 9 cases).

3. Environmental risk factors will vary between sites, corresponding to the differing land-use patterns. This hypothesis was for the most part not supported by our results, with some important caveats. As discussed in hypothesis 1, the health assessments (chapter 2) and gene expression analyses (chapter 3) suggested no consistent differences in environmental risk factors, with the exception of up-regulation of certain genes in sea otters captured at Big Sur in 2008 suggestive of increased exposure to organic contaminants. Epidemiological analysis (chapter 9) indicated that exposure to the protozoal parasite Toxoplasma gondii was significantly greater at Big Sur than at Monterey. Thus, our results indicate variation in environmental stressors across sites, and over time; however, the differences were not clearly attributable to differences in human population densities or land-use patterns.

4. Sea otters from high-density populations (and [or] areas that have been occupied longer) will have lower rates of foraging success compared to sea otters from lowdensity populations (and [or] areas that have been more recently occupied) due to prey resource depletion, and these patterns will be indicated by (a) greater percentage of time spent feeding, (b) more pronounced individual diet specialization, (c) poorer body condition, and (d) lower survival rates of adults and pups. This hypothesis was well supported by data collected in the current study and in previous similar studies. Sea otters at Big Sur and Monterey study sites (both of which have supported high-density populations for many years) had relatively low rates of energy gain while feeding as compared to low-density, growing populations in California, Washington, British Columbia, Alaska, and Russia (chapter 6). Big Sur sea otters had slightly lower energy intake rates than did otters in Monterey, and also spent slightly more time feeding (chapter 5) and had slightly greater levels of diet specialization (chapter 6), although these latter metrics were high at both sites as compared to low-density populations. A comparison of body condition and survival rates across six sites in California (chapters 7 and 8, respectively) showed that lower foraging success in high-density sea otter populations was indicated by poorer body condition and decreased survival, and pup weaning success rates, with strongly significant correlations among all of these parameters. 
Based on the hypothesis tests described here, not all of our predictions were supported by empirical datasets, requiring a reevaluation of some of our assumptions about factors driving trends in sea otter abundance in central California. The enormous scope and inter-disciplinary nature of this project, and the extensive sample sizes available from both the current study and from previous similar studies conducted over the past 15 years, allow us to update our understanding of southern sea otter population biology. Four general conclusions about the sea otter populations of central California, and the factors driving trends in abundance, have emerged from this work:

1. Density-dependent population regulation driven by per-capita resource abundance is the most significant factor currently limiting population growth in the center part of the range (approximately from the Monterey Peninsula to Estero Bay);

2. Spatial and temporal variation in environmental and anthropogenic stressors also can affect sea otter health, based on previous research (for example, Miller and others, 2002; Johnson and others, 2009; Miller and others, 2010), but patterns of variation are complex and are not simply a function of proximity to human populations;

3. Exposure to environmental stressors (either natural or anthropogenic in origin) does not act independently of resource limitation; and

4. Sea otter populations are structured at small spatial scales, and the processes that regulate population abundance (including density-dependent resource abundance) also occur locally.

\section{Density-Dependent Population Regulation}

Density-dependent resource limitation appears to be the dominant factor driving variation in reproductive success and survival of sea otters in central California. Sea otters had high survival/reproductive success in low-density populations near the edge of the range where prey resources were abundant (for example, San Nicolas Island, Santa Barbara Channel), and low survival/reproductive success in high-density populations near the center of range (Big Sur, Monterey, and San Simeon) where per-capita resource abundance was low. The hypothesis that per-capita resource abundance was depleted in the latter areas was supported by multiple, independent lines of evidence including low forage success, high percentage of time spent feeding, and poor body condition. The differences in estimated survivorship schedules and reproductive success between sea otters in Monterey and Big Sur and sea otters in San Nicolas Island were explained almost entirely by differences in body condition (chapter 8), and the resulting estimates correctly predicted observed population growth rates at these areas. Thus, sea otters in Big Sur seem to be at a carrying capacity determined by resource abundance; however, the phrase "resource limitation" sometimes causes confusion for those unfamiliar with the language and concepts of population ecology, so it is worth correcting a few common misunderstandings about this concept:

1. "Resource limitation" does not mean that there is a paucity of invertebrates or lower productivity in a given area; indeed, high-density sea otter populations tend to occur in areas of high productivity (as measured by invertebrate recruitment and growth rates) such as the Monterey Peninsula. Resource limitation implies that there is decreased per-capita availability of preferred, high-energy prey (for example, large red urchins), leading to increased reliance on lower-quality prey by many individuals, with consequences for one or more population vital rates.

2. Resource limitation does not mean that all sea otters will be equally affected by low food abundance. To the contrary, the emergence of individual prey specialization in 
resource-limited populations (Tinker, Bentall, and Estes, 2008) means that different individuals will be differently affected by competition for specific prey types, leading to increased variation in foraging success and body condition. This variation means that many individuals will be in very poor condition but some individuals will be in very good condition.

3. Resource limitation does not imply that starvation will be a common cause of death; it does imply that average demographic rates will vary as a function of per-capita resource abundance, until at some point birth rates and death rates are equal and the population reaches equilibrium abundance. The proximate causes of death for animals in a resource-limited population can be highly variable, including infectious disease, intra-specific aggression, intoxication, and many other pathological conditions.

"Starvation" per se may occur rarely, or not at all.

\section{Variation in Environmental Stressors}

Environmental stressors affecting sea otter health appear to vary both temporally and spatially within the sea otters' range, and results presented throughout this report suggest that this variation is more complex than can be explained by a simple "dirty compared to pristine" axis, or simply by proximity to human activities. For example, Toxoplasma gondii infection risk was highest for female sea otters in the pristine Big Sur site, and much lower for females in the highly impacted Monterey site (chapter 9). We reported evidence for variation in environmental health threats associated with natural stressors (fires, oil seeps, harmful algal bloom toxins; chapter 3) and human-caused stressors (anthropogenic pollution associated with bacterial infections; chapter 10). Exposure to some of these stressors may indicate variation in natural or anthropogenic sources, as well as geographic characteristics of watersheds and marine habitats, but also can be mediated by ecological interactions between sea otters and their prey. For example, in the case of $T$. gondii infections, sea otters that specialized on consuming marine turban snails had a risk of infection $42 \times$ higher than otters that specialized on other prey types, irrespective of their location. Although protozoal infections varied significantly among sites, other stressors appeared to be more ubiquitous throughout the sea otter range; for example, domoic acid intoxication was determined to be a contributing cause of death in otters at both sites, indicating the fact that the diatom blooms that produce these toxins are widespread and not associated with point-source pollution (Lefebvre and others, 2002).

\section{Synergistic Interactions between Resource Limitation and Environmental Stressors}

Just as importantly, the impact of environmental stressors on health and survival seems to be related to individual physiological condition and nutritional status. End-lactation syndrome (ELS) was determined to be a major cause of mortality for females in this study. Females that died of ELS often were exposed to other stressors or factors that contributed to their negative outcome (for example, domoic acid poisoning, bacterial infections), but also were characterized by extremely poor body condition at the end of a pup dependency period. In the high-density populations of central California, entering the end-lactation period was associated with a $3.6 \times$ increase in mortality risk for females (chapter 8), but this was not the case for females in the lowdensity population at San Nicolas, where females had much higher foraging success (chapter 6), spent less time feeding (chapter 5), and were in much better body condition (chapter 7). These findings suggest that resource limitation and exposure to environmental stressors are likely synergistic, because females are less capable of coping with exogenous stressors when they are in poor condition, particularly at the end of lactation when body reserves have been exhausted. 


\section{Population Structure}

Analysis of home-range behavior and individual movements of tagged sea otters in this study (and supported by previous analyses) indicates that the southern sea otter population is structured demographically at relatively small spatial scales. Put another way, there is very limited mixing of the demographically relevant components of the population (adult and sub-adult females) between habitat areas only 50 kilometers apart. Many of the factors affecting sea otter survival and driving population trends also can vary at small spatial scales; for example, relative abundance and productivity of invertebrate prey populations or point sources of pollution. The result of these two facts is that population regulation is, for the most part, a local rather than a regional process. Because of this, the question, "what factor is limiting the sea otter population in California", is inappropriate, and should be replaced by the question, "what factors are currently most important in limiting population growth in area X?"

\section{Resource Management Considerations}

The results and conclusions presented in this report and summarized in the preceding paragraphs are important to resource management and conservation strategies. Simple, universal explanations for population trends are unlikely to be fruitful, owing to the fact that population regulation occurs locally rather than regionally, and that environmental stressors and densitydependent processes also vary extensively throughout the range (and over time). Conservation research and management actions could instead be tailored to specific geographic areas and population threats. One example of this approach is the recently initiated (and Coastal Conservancy-supported) project in Elkhorn Slough, designed to inform habitat improvement and restoration actions to support sea otters within this particular estuary. Emerging threats that affect specific geographic areas at present include shark-bite mortality in the Estero Bay-Pismo Beach area (Tinker and others, 2016) and microcystin intoxication in the Monterey Bay area (Miller and others, 2010). Some stressors may occur more broadly (such as domoic acid intoxication; Kreuder and others, 2005), and these factors may benefit from coordinated conservation efforts at regional scales.

Second, it is increasingly clear that much of the geographic center of the sea otter's range seems to be at (or near) carrying capacity, and management actions probably would not increase population densities in these areas. Understanding the causes of mortality in these areas is valuable, as lessons learned often are broadly applicable; however, conservation actions focused on areas that are still well below carrying capacity (that is, areas nearer the range peripheries) would likely improve the potential for population growth. Moreover, the recovery of southern sea otters to the optimum sustainable population level identified for California (U.S. Fish and Wildlife Service, 2012) will require range expansion to the north and south of the current distribution; efforts to facilitate or accelerate the rate of range expansion may have a greater impact on the overall rate of recovery than any other conservation action.

Third, prioritization of conservation and management actions based on rigorous demographic sensitivity analyses could maximize the potential for recovery. Such analyses (often referred to as "Population Viability Analyses", or PVA models) can be used to assess the magnitude of expected benefits associated with particular actions. For example, actions to decrease mortality in an area dominated by juvenile males will have negligible effects on population recovery, but a small decrease in mortality in a female-dominated area near the range periphery could have enormous effects. The estimates of vital rate parameters and dispersal distance statistics presented in this report (chapters 8 and 4, respectively) provide the basic ingredients needed to develop such PVA models using well-established techniques. It is becoming increasingly evident 
that these models must appropriately factor in population structure, movement behavior, and range expansion to provide realistic results.

Finally, the Big Sur-Monterey population study provided a model for how collaborative, multidisciplinary research can make progress in answering challenging conservation questions involving highly complex ecological interactions. Recovery of sea otters from their near-extinction in the North Pacific fur trade in many ways represents a great conservation success story; however, the southern sea otter population still only occurs in a part of its former range, remains threatened, and is still well below the optimal sustainable population level identified by US Fish and Wildlife Service (U.S. Fish and Wildlife Service, 2012). Questions about factors affecting the slow process of population recovery have been difficult to resolve because they require untangling myriad, complicated interactions between sea otters and their ecosystems. Success in the "untangling process" requires expertise in physiology, behavior, animal health, molecular ecology (for example, stable isotope and gene expression methods), veterinary pathology, parasitology, epidemiology, and quantitative population biology. There is no single research laboratory or agency that has expertise in even one-half of those disciplines; however, all these skills are represented by the collaboration of scientists and experts that contributed to this study (a group loosely referred to as the "Southern Sea Otter Research Alliance"). The result of this collaboration has been substantial progress in our understanding sea otter ecology, and this progress has enabled us to provide the best available science to resource managers. The ongoing work by this group, bolstered by new collaborations with experts from other fields, should continue to provide tangible results that can benefit conservation of sea otters and their ecosystems. 


\section{References Cited}

Johnson, C.K., Tinker, M.T., Estes, J.A., Conrad, P.A., Staedler, M., Miller, M.A., Jessup, D.A., and Mazet, J.A.K., 2009, Prey choice and habitat use drive sea otter pathogen exposure in a resource-limited coastal system: Proceedings of the National Academy of Sciences of the United States of America, v. 106, p. 2242-2247.

Kreuder, C., Miller, M.A., Lowenstine, L.J., Conrad, P.A., Carpenter, T.E., Jessup, D.A., and Mazet, J.A.K., 2005, Evaluation of cardiac lesions and risk factors associated with myocarditis and dilated cardiomyopathy in southern sea otters (Enhydra lutris nereis): American Journal of Veterinary Research, v. 66, p. 289-299.

Lefebvre, K.A., Bargu, S., Kieckhefer, T., and Silver, M.W., 2002, From sanddabs to blue whales - The pervasiveness of domoic acid: Toxicon, v. 40, p. 971-977.

Miller, M.A., Gardner, I.A., Kreuder, C., Paradies, D.M., Worcester, K.R., Jessup, D.A., Dodd, E., Harris, M.D., Ames, J.A., Packham, A.E., and Conrad, P.A., 2002, Coastal freshwater runoff is a risk factor for Toxoplasma gondii infection of southern sea otters (Enhydra lutris nereis): International Journal for Parasitology, v. 32, p. 997-1006.

Miller, M.A., Kudela, R.M., Mekebri, A., Crane, D., Oates, S.C., Tinker, M.T., Staedler, M., Miller, W.A., Toy-Choutka S., Dominik, C. Hardin, D., Langlois, G., Murray, M., Ward, K., and Jessup, D.A., 2010, Evidence for a novel marine harmful algal bloom-Cyanotoxin (microcystin) transfer from land to sea otters: PLoS One, v. 5, p. e12576.

Tinker, M.T., Hatfield, B.H., Harris, M.D., and Ames, J.A., 2016. Dramatic increase in sea otter mortality from white sharks in California: Marine Mammal Science, v. 32, p. 309-326.

Tinker, M.T., Bentall, G., and Estes, J.A., 2008, Food limitation leads to behavioral diversification and dietary specialization in sea otters: Proceedings of the National Academy of Sciences of the United States of America, v. 105, p. 560-565.

Tinker, M.T., Doak, D.F., and Estes, J.A., 2008,Using demography and movement behavior to predict range expansion of the southern sea otter. Ecological Applications, v. 18, p. 1781-1794.

Tinker, M.T., Doak, D.F., Estes, J.A., Hatfield, B.B., Staedler, M.M., and Bodkin, J.L., 2006, Incorporating diverse data and realistic complexity into demographic estimation procedures for sea otters: Ecological Applications, v. 16, p. 2293-2312.

U.S. Fish and Wildlife Service, 2010, Southwest Alaska distinct population segment of the northern sea otter (Enhydra lutris kenyoni) - Draft recovery plan: U.S. Fish and Wildlife Service, Region 7, Anchorage, Alaska.

U.S. Fish and Wildlife Service, 2012. Final supplemental environmental impact statement on the translocation of southern sea otters: U.S. Fish and Wildlife Service, Ventura Fish and Wildlife Office, Ventura, California, 348 p. 


\section{Acknowledgments}

We would like to thank the California Coastal Conservancy and the U.S. Fish and Wildlife Service (especially Lilian Carswell) for making this research possible. The Monterey Bay Aquarium was a key collaborator on this project assisting not only with captures and carcass recovery, but by also providing daily monitoring and data collection efforts for the Monterey part of the study. We wholeheartedly thank the Monterey Bay Aquarium for their excellent work as collaborators, and we specifically acknowledge Dr. Mike Murray, Andy Johnson, Michelle Staedler, Gena Bentall, Teri Nicolson, Jessica Fujii, Marissa Young, and Chris Harold.

We thank our collaborators from the California Department of Fish and Wildlife, especially Mike Harris, Colleen Young, Laird Henkel, Dr. Melissa Miller, Francesca Batac, Erin Dodd, Jessica Kunz, Frank Wilhelm, Witold Piekarski, RC Mitchell, Dr. Dave Jessup (Ret.), and Jack Ames (Ret.) for their support during capture events and tracking, and for conducting necropsies of deceased study animals. We are grateful for the support of the U.S. Geological Survey Western Ecological Research Center and Alaska Science Center; specifically, Keith Miles, Tom Kimball, Steve Schwarzbach (Ret.), Brian Hatfield, Ben Weitzman, Anne Meckstroth, Dan Esler, Jim Bodkin (Ret.), Dan Monson, George Esslinger, Kim Kloecker, and Christine Alfano. We acknowledge the University of California, Santa Cruz; specifically, Mike Kenner, Dr. Max Tarjan, Dr. Nicole Thometz, and Dave Benet, who were all key players in various research support roles. We also thank the University of California, Davis, and the Oil Wildlife Care Network, especially Dr. Tristan Burgess, Dr. Christine Kreuder-Johnson, and Kyra Mills-Parker for their field assistance and expertise with sea otter disease processes. We thank the Seattle Aquarium, specifically Lesanna Lahner (now of SR3), for her excellent work as a veterinarian on this project.

We would especially like to thank Mark Readdie and Gage Dayton of Natural Reserve System at the University of California, Santa Cruz, for their long-term logistical support of this project by providing a remote field station and base of operations at the Big Creek Reserve in Big Sur, California. We thank the Ventana Wildlife Society, specifically Joe Burnett and Sayre Flannagin, for working with us to resolve telemetry conflicts with California Condors. We would like to thank the California Highway Patrol, particularly Officer Ben, for dutifully looking after, and ensuring the safety of our research personnel and volunteers as they tracked otters in the remote stretches of the Big Sur Coast. Our gratitude goes out to the California Department of Transportation for their outstanding work during many hazardous road situations, including several mudslides - their work helped keep our people safe. We also acknowledge support from California State Parks, specifically Limekiln State Park, for providing us with access for beach transfers of gear, personnel, and study animals. We are grateful to the Esalen Institute, which provided our researchers with access to their property to locate our study animals. We would like to thank many additional researchers and biologists, including Nicole LaRoche, Justine Grajski, Norma Vasquez, Nicole Brandt, Carolyn Miller, Caroline Cummings, and dozens of interns and volunteers without whom this work would not be possible. Finally we would like to thank the people of Big Sur, who embraced our research program and made us feel at home. 

Publishing support provided by the U.S. Geological Survey Science Publishing Network, Tacoma Publishing Service Center

For more information concerning the research in this report, contact the Director, Western Ecological Research Center

U.S. Geological Survey

3020 State University Drive

Modoc Hall, Room 4004

Sacramento, California 95819

https://www.werc.usgs.gov/ 
흘 